\title{
THE MOLECULAR PATHOGENESIS OF HAEMANGIOMA
}

\author{
By
}

Anasuya Vishvanath

\begin{abstract}
A thesis
submitted to the Victoria University of Wellington in fulfilment of the requirements for the degree of Doctor of Philosophy in Cell and Molecular Biology
\end{abstract}

Victoria University of Wellington 2008 



\section{ABSTRACT}

Haemangioma is a primary tumour of the microvasculature characterised by active angiogenesis and endothelial cell (EC) proliferation followed by slow regression or involution whereby the newly formed blood vessels are gradually replaced by fibrofatty tissue. These developmental changes have been arbitrarily divided into the proliferative, involuting and involuted phases. The cellular and molecular events that initiate and regulate the proliferation and spontaneous involution of haemangioma remain poorly understood.

This study examined the expression of a number of genes known to be associated with angiogenesis. These include members of the signal transducers and activators of transcription (STAT) protein family of transcription factors, STAT-3 and STAT-1, and the endothelial receptor tyrosine kinases, VEGFR-1 and VEGFR-2. While STAT-3, STAT-1 and VEGFR-1 expression was detected in all phases of haemangioma, VEGFR-2 expression was found to be abundant only during the proliferative phase and decreased with ongoing involution.

In this study the cellular structures that form capillary-like outgrowths in an in vitro haemangioma explant model were characterised as haemangioma-derived mesenchymal stem cells (HaemDMSCs) while the cells obtained directly from dissociated proliferative haemangioma tissue were defined as haemangioma-derived endothelial progenitor cells (HaemDEPCs). This investigation showed that although the vascular endothelial growth factor (VEGF), a key growth factor for ECs, was able to maintain HaemDEPCs morphology and immunophenotype for a limited period, these cells eventually differentiated into HaemDMSCs, which subsequently differentiated into adipocytes. Furthermore, while VEGF induced significant capillary-like sprouting from tissue explants, both capillary-like sprouting and HaemDMSCs proliferation was inhibited by the addition of AG490, a Janus kinase (JAK) inhibitor which has also been shown to inhibit the STAT protein pathway. These findings indicate that the 
development and differentiation of a progenitor cell and a stem cell population underlies the aethiopathogenesis of haemangioma and that VEGF and STAT signalling is involved in the programmed life-cycle of haemangioma.

The in vitro explant model for haemangioma offers an opportunity to study and identify novel treatment options for haemangioma. Interferon-alpha (IFN $\alpha$ ) has been used to treat steroid-resistant haemangioma but is associated with serious side-affects. The tumour necrosis factor (TNF)-related apoptosis-inducing ligand (TRAIL) has been shown to specifically induce apoptosis of cancer cells while sparing normal cells. As IFN $\alpha$ has previously been shown to sensitise cells to TRAIL-induced apoptosis, this study examined the efficacy of low dose IFN $\alpha$ in combination with TRAIL in the in vitro explant model and also in the purified HaemDMSCs. Results showed that combining IFN $\alpha$ with TRAIL led to synergistic inhibition of capillary-like outgrowth. These results indicate that IFN $\alpha$ in combination with TRAIL serves as a potential treatment option for haemangioma. In contrast, HaemDMSCs were protected from TRAIL-induced killing. These cells were found to express high levels of the decoy receptors, osteoprotegerin (OPG) and decoy receptor 2 (DcR2). Increased OPG expression was also detected in the extracellular matrix and in the conditioned medium of HaemDMSCs. From these findings, we postulate that the increased level of extracellular OPG by HaemDMSCs is a stress response induced by their in vitro expansion and that secreted OPG functions as a protective shield preventing TRAIL action.

The empirical and unsatisfactory nature of the current therapies for haemangioma underscores the importance of a scientific approach to this common tumour. A better understanding of the molecular mechanisms that govern haemangioma is of both clinical and biological interest as it may provide vital information with therapeutic potential for haemangioma and also for other angiogenesis-dependent conditions. 


\section{ACKNOWLEDGEMENTS}

The research presented in this dissertation has been extensive and trying, but also exciting, instructive, and fun. Without help, support, and encouragement from several persons, I would never have been able to finish this work.

To my supervisors, Dr Darren Day and Dr Swee Tan. I am extremely grateful for your constant availability, enthusiasm, encouragement, critical discussion and guidance throughout this project.

My sincere appreciation and gratitude to Dr Helen Brasch, Ms Elizabeth (Liz) Jones and other members of staff at the Department of Pathology, Hutt Hospital.

A special thank you to all the patients (and their parents) who were treated at the Centre for Study \& Treatment of Vascular Birthmarks, Hutt Hospital, who have willingly permitted utilisation of their samples that made this study possible.

To Dr Tim Sargent, Ms Anja Wilmes, Dr John Miller and especially Dr Pisana Rawson. I thank you all for the useful discussions and in-laboratory tips over the years.

To my close friend, Nitasha. Thank you for your loyalty, friendship and making me believe, even on the worst days, that 'Things CAN only get better!'

To my wonderful family, my father, Vishvanath, my mother, Anita and my sister, Supriya. Thank you for your patience, kind words and your belief in my ability to complete this $\mathrm{PhD}$.

This project was funded by research grants from the Wellington Medical Research Foundation, the Surgical Research Trust, the Reconstructive Plastic Surgery Research Foundation and the Cancer Society (Wellington Division). 


\section{CONTENTS}

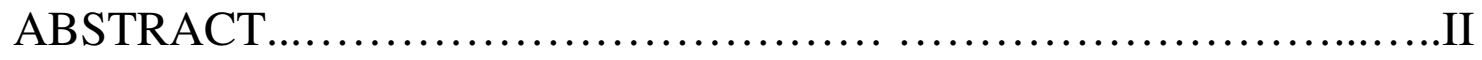

ACKNOWLEDGEMENT ............................................IV

TABLE OF CONTENTS ...............................................

LIST OF FIGURES ..............................................XX

LIST OF TABLES ........................................XXVII

ABBREVIATIONS ...........................................XXVIII

\section{CHAPTER 1: GENERAL INTRODUCTION...............1}

1.1 HAEMANGIOMA..................................................1

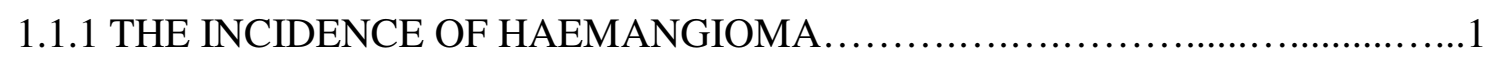

1.1.2. CLINICAL FEATURES....................................................

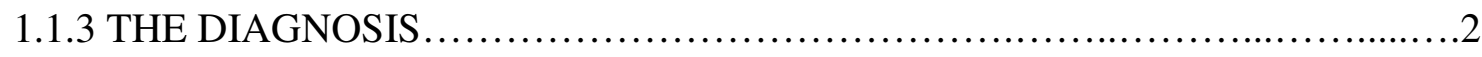

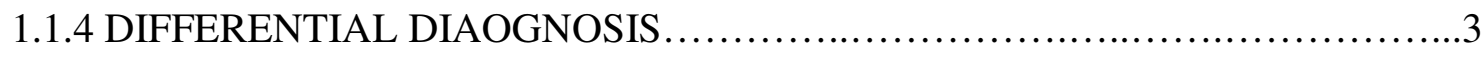

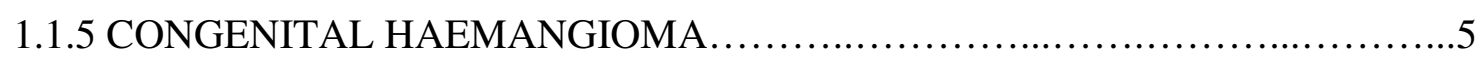

1.1.6 COMPLICATIONS OF HAEMANGIOMA.....................................

1.1.7 HEAD AND NECK HAEMANGIOMA.......................................6

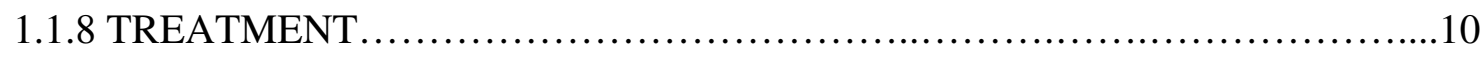

1.1.9 GENETICS IN HAEMANGIOMA...........................................11

1.1.10 THE HISTOPATHOLOGY ..................................................

1.2. CURRENT THEORIES FOR THE ORIGINS

OF HAEMANGIOMA...........................................15

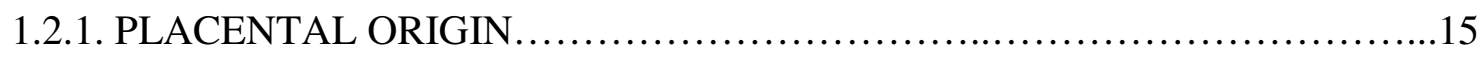


1.2.2. EXPANSION OF ENDOTHELIAL CELLS IN HAEMANGIOMA:

AN INTRINSIC OR EXTRINSIC DEFECT ? .17

1.2.2.1. AN INTRINSIC DEFECT? 18

1.2.2.1.1. Familial Studies. 18

1.2.2.1.2. Haemangioma-derived Endothelial Cell Studies. .19

1.2.2.2. AN EXTRINSIC DEFECT? 21

1.2.3. THE ANGIOGENESIS CONCEPT. .21

1.3. CELLULAR COMPONENTS OF HAEMANGIOMA .22

1.3.1 ENDOTHELIAL CELLS .22

1.3.2 MAST CELLS. .25

1.3.2.1. ANGIOGENIC ROLES FOR MAST CELLS IN HAEMANGIOMA. .26

1.3.2.2. ROLE OF MAST CELLS IN APOPTOSIS .27

1.3.3 OTHER COMPONENTS. .27

1.3.3.1 MACROPHAGES AND MONOCYTES .27

1.3.3.2. PERICYTES. .29

1.3.3.3. PROTEASES. .30

\subsection{CYTOKINES AND GROWTH FACTORS IN}

HAEMANGIOMA 30

1.4.1. VASCULAR ENDOTHELIAL GROWTH FACTOR .30

1.4.2. VASCULAR ENDOTHELIAL GROWTH FACTOR RECEPTORS. 31

1.4.3. TIE RECEPTORS AND THEIR ANGIOPOIETIN LIGANDS. 31

1.4.4 FIBROBLAST GROWTH FACTOR-2 .32

1.4.5 INSULIN GROWTH FACTOR-2. 32 
1.4.6.1 PLATELET DERIVED GROWTH FACTOR, TRANSFORMING

GROWTH FACTOR- $\beta$ AND INTERLEUKIN-6. .33

1.5. EXTRACELLULAR MARKERS 33

1.5.1 INTERSTITIAL MARKERS ........................................... 34

1.5.2 BASAL LAMINA MARKERS ............................................ 34

1.5.2.1 COLLAGEN TYPE IV ................................................. 34

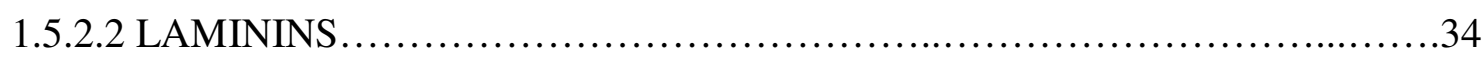

1.5.3 COLLAGEN TYPE VIII................................................ 35

1.5.4 VITRONECTIN ....................................................... 35

1.6. CELL ADHESION MOLECULES.................................35

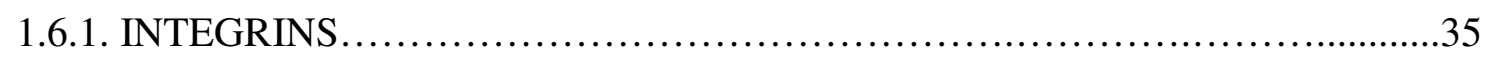

1.7. THE INVOLUTION OF HAEMANGIOMA.......................36

1.7.1. APOPTOTIC PROTEINS IDENTIFIED IN HAEMANGIOMA..................36

1.7.1.1. THE BCL-2 FAMILY PROTEINS..................................... 36

1.7.1.2. APOLIPOPROTIEN J............................................ 37

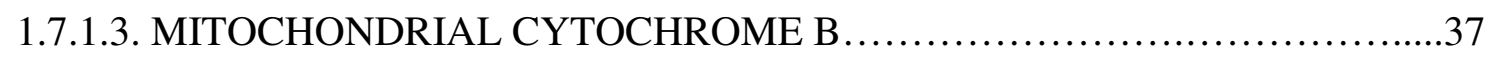

1.8. VASCULAR MORPHOGENESIS AND MAINTENANCE........37

1.8.1. THE MAMMALIAN VASCULAR SYSTEM...............................

1.8.2. DEVELOPMENT OF THE MAMMALIAN VASCULAR SYSTEM.............38

1.8.2.1 VASCULOGENESIS AND ANGIOGENESIS ...........................40

1.8.3. THE ANGIOGENIC CASCADE........................................45

1.8.4. ANGIOGENIC FACTORS IN BLOOD VESSEL DEVELOPMENT..............46

1.9. ROLE OF STAT PROTEIN IN TUMOURIGNESIS...............49 
1.9.1 STAT PROTEINS.

1.9.2 MECHANISMS OF STAT ACTIVATION ...............................50

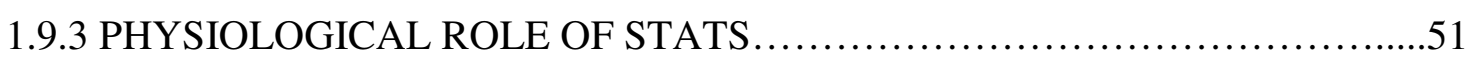

1.9.4 STAT-3 IN HUMAN ONCOGENESIS AND REGULATION

OF APOPTOSIS

1.9.5 STAT-1 IN GROWTH RESTRAINT, PROMOTER OF

APOPTOSIS AND TUMOUR SUPPRESSION. .53

1.10 AIM OF THIS PROJECT. 54

1.10.1 OBJECTIVES 54

CHAPTER 2: METHODS. 55

2.1 GENERAL METHODS..............................................55

2.1.1 QUANTITATIVE REAL TIME PCR ...................................55

2.1.1.1 AN INTRODUCTION TO QUANTITATIVE REAL TIME PCR................55

2.1.1.2 RNA ISOLATION AND QUANTITATION .............................58

2.1.1.2.1 RNA extraction from snap frozen tissue biopsies............................58

2.1.1.2.2. RNA extraction from cell cultures.........................................59

2.1.1.3 RNA QUANTIFICATION .............................................93

2.1.2 cDNA SYNTHESIS ..................................................59

2.1.3 PRIMER DESIGN AND RECONSTITUTION ...........................60

2.1.4 THE QUANTITATIVE REAL-TIME PCR ASSAY .........................65

2.1.5 HAEMANGIOMA SPECIMENS......................................66

2.1.6 CELL CULTURE.......................................................66

2.1.6.1 CULTURE FACILITY SET-UP...................................66

2.1.6.2 CELL LINES USED IN THIS STUDY ..................................67

2.1.6.2.1 HeLa cells.....................................................67

VIII 


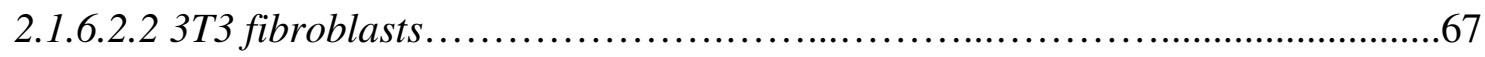

2.1.6.2.3 Human vascular endothelial cells......................................67

2.1.6.2.4 Haemangiendothelioma cells........................................68

2.1.6.3. STANDARD CELL CULTURE TECHNIQUES ............................68

2.1.7 IMMUNOHISTOCHEMISTRY ......................................69

2.1.7.1 PREPARATION OF SLIDES ..........................................69

2.1.7.2 PARAFFIN EMBEDDED TISSUE SECTIONS ........................69

2.1.7.3 CELL CULTURE PREPERATIONS .....................................70

2.1.8 STAINING PROCEDURES................................................ 70

2.1.8.1 HISTOCHEMISTRY ............................................. 70

2.1.8.2 FLOURESCENT IMMUNOHISTOCHEMISTRY .........................70

2.1.8.2.1 Paraffin embedded tissue sections......................................74

2.1.8.2.2 Cultured cells..................................................74

2.1.8.3 COUNTER STAINING OF THE NUCLEUS .............................75

2.1.8.4 COLOURIMETRIC VISUALISATION WITH DAB $\ldots \ldots \ldots \ldots \ldots \ldots \ldots \ldots . \ldots . \ldots . \ldots . \ldots$

2.1.8.5 NEGATIVE CONTROLS ........................................... 76

2.1.8.6 IMAGE ANALYSIS ................................................ 77

2.1.9 WESTERN BLOTTING........................................... 77

2.1.9.1 SAMPLE PREPARATION FOR WESTERN BLOTS ........................77

2.1.9.1.1 Tissue lysate..................................................... 77

2.1.9.1.2 Cell lysate.......................................................

2.1.9.2 PROTEIN DETERMINATION.......................................... 78

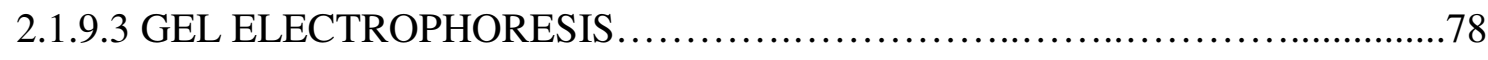

2.1.9.4 WESTERN BLOT TRANSFER ............................................ 79

2.1.9.5 IMMUNOSTAINING OF WESTERN TRANSFERRED

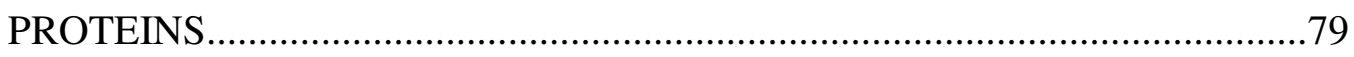

2.1.9.6 ANALYSIS OF WESTERN TRANSFERRED PROTEINS ........................80 
2.2 METHODS FOR CHAPTERS 3 AND 6.........................81

2.2.1 PRELIMINARY EXPERIMENTS .......................................... 81

2.2.2 GENE EXPRESSION CHANGES IN HAEMANGIOMA.....................81

2.2.2.1 QUANTITATIVE REAL TIME PCR ......................................81

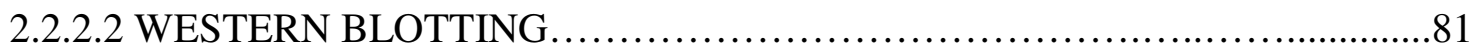

2.2.2.3 FLOURESCENT IMMUNOHISTOCHEMISTRY ..........................82

2.2.2.4 COLOURIMETRIC VISUALISATION WITH DAB ............................82

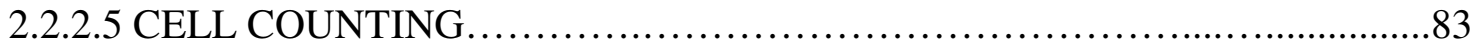

2.2.2.5.1 STAT-3 and PCNA expression in haemangioma...................................83

2.2.2.5.2 STAT-3 and CycA expression by MCs in haemangioma ................................83

2.2.2.5.3 p-STAT-3 (Tyr 705) and PCNA expression in haemangioma..........................83

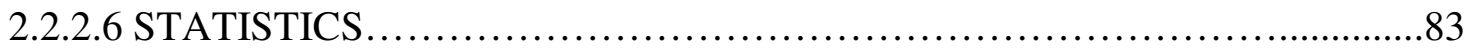

2.3 METHODS FOR CHAPTER 4................................85

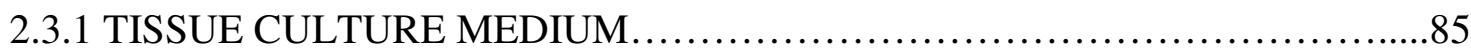

\subsubsection{HAEMANGIOMA TISSUE SPECIMENS AND}

SAMPLE PREPARATION ................................................. 85

2.3.3 GEL PREPARATION AND IN VITRO TISSUE CULTURE.....................85

2.3.4 MODIFICATION OF THE IN VITRO TISSUE CULTURE MODEL..............86

2.3.5 QUANTITATION OF OUTGROWTH FROM TISSUE PIECES................87

2.3.6 ISOLATION AND CULTURE OF HAEMANGIOMA

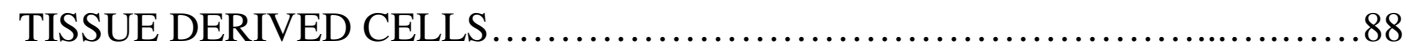

2.3.7 PRIMARY CELL CULTURE CHARACTERISATION .........................89

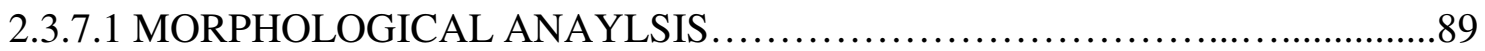

2.3.7.1.1 Change in cell morphology........................................... 89

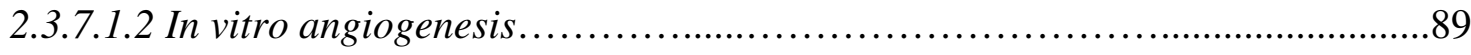

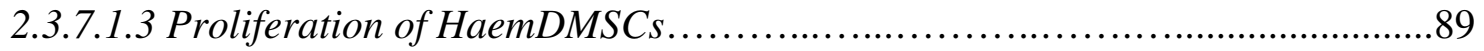


2.3.8.1 HaemDMSCs mRNA CHARACTERISATION .............................89

2.3.8.2 HaemDMSCs DIFFERENTIATION EXPERIMENTS ..........................90

2.3.9 IMMUNOFLUORESCENT CHARACTERISATION OF HaemDMSCs...........90

2.3.10 DIFFERENTIATION OF HaemDMSCs..................................91

2.3.10.1 OSTEOGENIC DIFFERENTIATION ....................................91

2.3.10.2 ADIPOGENIC DIFFERENTIATION .................................91

2.3.10.3 ENDOTHELIAL CELL DIFFERENTIATION...............................92

2.3.11 CELL CULTURE HISTOCHEMISTRY ...................................92

2.3.11.1 ALIZARIN RED STAINING....................................92

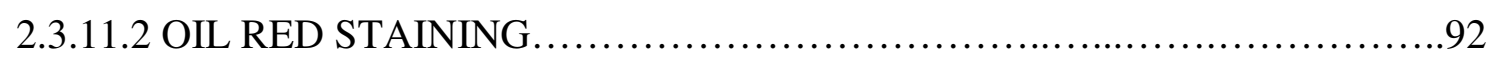

2.3.12 EFFECTS OF VEGF AND AG490 ON HaemDMSCs......................93

2.3.12.1 VEGF CELL PROLIFERATION ASSAY ...............................93

2.3.12.2 EFFECT OF AG490 ON HaemDMSCS PROLIFERATION

BY BrdU LABELLING............................................93

2.3.12.3 EFFECT OF AG490 ON IN VITRO CAPILLARY-LIKE FORMATION

AND QUANTITATION.............................................94

2.3.13 WESTERN BLOTTING........................................... 95

2.3.13.1 EFFECT OF VEGF, AG490 AND IFN $\alpha$-2b ON p-STAT-3 PROTEIN

EXPRESSION ..................................................95

2.3.13.2 DETECTION OF VEGF IN THE CONDITIONED MEDIA

OF HaemDMSCs..................................................96

2.3.14 IMMUNOCYTOCHEMISTRY .......................................97

2.3.14.1 EFFECT OF VEGF, IFN $\alpha$-2b AND ADIPOCYTIC DIFFERENTIATION

CONDITIONS ON STAT PROTEIN EXPRESSION ............................97

2.3.15 IMMUNOHISTOCHEMISTRY .................................... 98 
2.3.16 ISOLATION OF ENDOTHELIAL CELLS AND ENDOTHELIAL

PROGENITOR CELLS FROM PROLIFERATING HAEMANGIOMAS........99

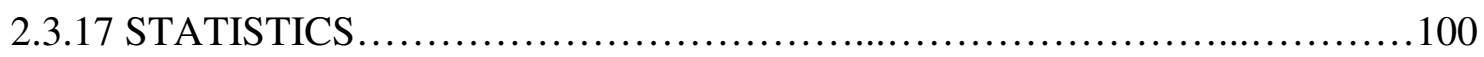

2.4 METHODS FOR CHAPTER 5..................................101

2.4.1 QUANTITATIVE REAL TIME PCR ..................................... 101

2.4.2 FLOURESCENT IMMUNOHISTOCHEMISTRY .........................101

2.4.3 EFFECT OF INTERFERON AND TRAIL ON HAEMANGIOMA

CAPILLARY-LIKE GROWTH......................................... 102

2.4.4 EFFECT OF IFN $\alpha-2 b$ AND TRAIL ON HaemDMSCs.......................102

2.4.5 WESTERN BLOTTING .............................................. 103

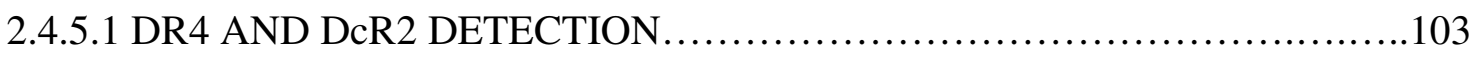

2.4.5.2 OPG DETECTION.................................................. 104

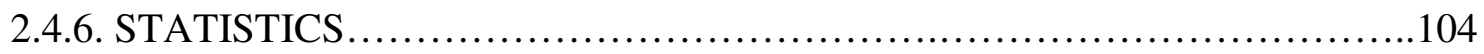

\section{CHAPTER 3: ROLE OF STAT PROTEINS AND VEGFR-2 IN HAEMANGIOMA.......................105}

3.1 INTRODUCTION...................................................105

3.2 AIMS..$\ldots \ldots \ldots \ldots \ldots \ldots \ldots \ldots \ldots \ldots \ldots \ldots \ldots \ldots \ldots \ldots \ldots \ldots \ldots \ldots \ldots \ldots \ldots \ldots \ldots \ldots$

3.3 RESULTS............................................................107

3.3.1 PRELIMINARY EXPERIMENTS....................................... 107

3.3.2 GENE EXPRESSION CHANGES IN HAEMANGIOMA......................107

3.3.2.1 CHANGE IN mRNA EXPRESSION................................... 107

3.3.2.2 DIFFERENCES IN PROTEIN EXPRESSION BY

WESTERN BLOTTING OF HAEMAGIOMA SPECIMENS ...............112

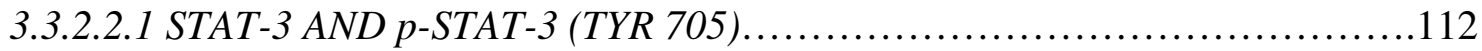

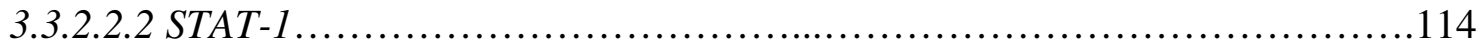




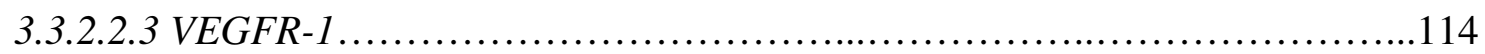

3.3.2.3 IMMUNOHISTOCHEMICAL ANALYSIS .............................116

3.3.2.3.1 STAT-3 EXPRESSION .............................................. 116

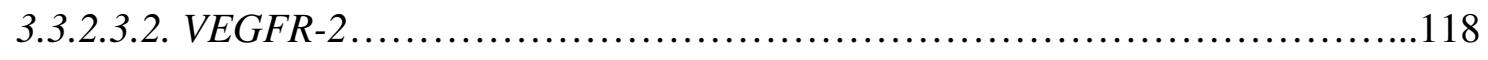

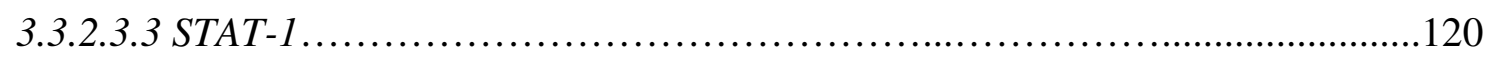

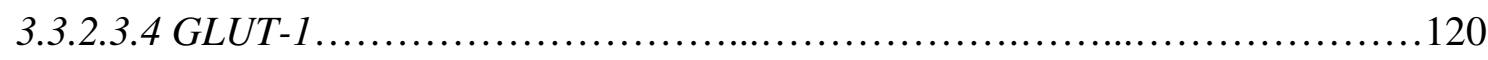

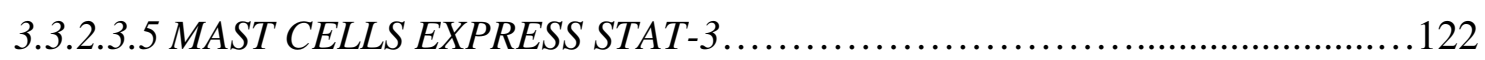

3.3.2.4 STAT-3 AND p-STAT-3 (TYR 705) IMMUNOLOCALISATION................124

3.3.2.5 STAT-3 PROTEIN EXPRESSION CORELLATES WITH

PROLIFERATION .............................................. 127

3.3.2.6 p-STAT-3 (TYR 705) EXPRESSION DOES NOT CORELLATE

WITH PROLIFERATION.......................................... 130

3.4 DISCUSSION......................................................132

3.4.1 IS VEGFR-2, STAT-3, p-STAT-3 (Tyr 705) SIGNALLING INVOLVED

IN HAEMANGIOMA PROLIFERATION?.........................................

3.4.2 IS STAT1 INVOLVED IN HAEMANGIOMA INVOLUTION?...............136

3.4.3 LIMITATIONS OF THIS STUDY.................................. 136

\section{CHAPTER 4: ROLE OF STEM CELLS IN} HAEMANGIOMA.

4.1 INTRODUCTION..............................................139

4.2 AIMS............................................................141

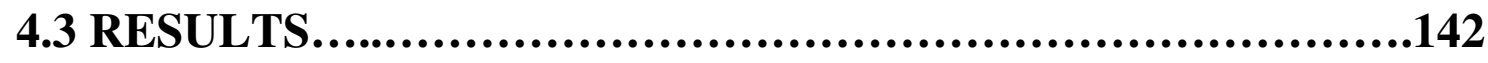

4.3.1 MORPHOLOGICAL OBSERVATIONS OF THE IN VITRO

HAEMANGIOMA CULTURE......................................... 142

4.3.2 MODIFICATION OF THE IN VITRO EXPLANT MODEL....................146 
4.3.3 PURIFICATION AND CHARACTERISATION OF THE

CAPILLARY-LIKE OUTGROWTHS................................... 146

4.3.3.1 CELL CULTURE CONDITIONS ....................................... 146

4.3.3.2 MORPHOLOGY AND GROWTH CHARACTERISTICS......................147

4.3.3.3 mRNA CHARACTERISATION....................................... 150

4.3.3.4 IMMUNOHISTOCHEMICAL CHARACTERISATION......................153

4.3.3.5 MULTILINEAGE DIFFERENTIATION POTENTIAL OF HaemDMCSs....158

4.3.4 ROLE OF VEGF AND STAT SIGNALLING IN VITRO......................163

4.3.4.1 EFFECT OF VEGF AND AG490 ON CAPILLARY-LIKE

OUTGROWTH...................................................163

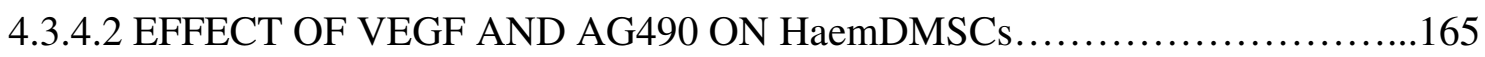

4.3.5 OSTEOPROTEGERIN EXPRESSION IN HAEMANGIOMA....................174

4.3.6 EFFECT OF INTERFERON AND DIFFERENTIATION MEDIA

ON STAT PROTEIN EXPRESSION IN HaemDMSCs.......................179

4.3.7 ENDOTHELIAL PROGENITOR CELLS IN HAEMANGIOMA..................181

4.4 DISCUSSION..........................................................189

4.4.1 CHARACTERISATION OF HAEMANGIOMA EXPLANT

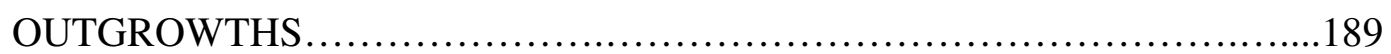

4.4.2 A ROLE FOR VEGF AND STAT SIGNALLING IN

HAEMANGIOMA IN VITRO ........................................... 191

4.4.3 ROLE OF OSTEOPROTEGERIN IN HAEMANGIOMA......................193

4.4.4 THE ROLE OF MSCs AND EPCs in HAEMANGIOMA......................196

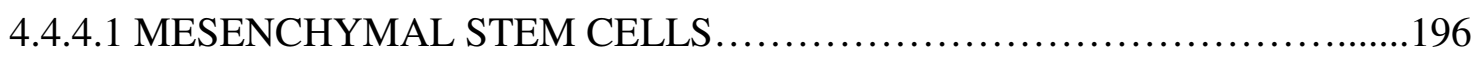

4.4.4.2 ENDOTHELIAL PROGENITOR CELLS ............................. 198

4.4.4.3 PROPOSED MODELS FOR HAEMANGIOMA.......................... 199

4.4.4.3.1 The Original Model................................................... 199

4.4.4.3.2 An Alternative Model......................................................201 
4.5.5 ROLE OF INTERFERONS AND STAT PROTEIN IN

\section{CHAPTER 5: TRAIL - A POTENTIAL TREATMENT FORHAEMANGIOMA?

5.1 INTRODUCTION .215

5.2 AIMS. .220

5.3 RESULTS. 221

5.3.1 mRNA EXPRESSION OF TRAIL AND ITS RECEPTORS. .221

5.3.2 PROTEIN EXPRESSION OF TRAIL AND ITS RECEPTORS .222

5.3.3 EFFECT OF INTERFERON AND TRAIL ON CAPILLARY-LIKE

GROWTH. .224

5.3.4 EFFECT OF INTERFERON AND TRAIL ON THE HaemDMSCs. .224

5.3.5 mRNA AND PROTEIN EXPRESSION OF TRAIL AND ITS RECEPTORS

IN HaemDMSCs .227

5.3.6 HaemDMSCs SECRETE OPG INTO THE CULTURE MEDIUM AND

OPG ANTIBODY SPECIFICITY .233

5.4 DISCUSSION. 236

5.4.1 THE EFFECT OF TRAIL AND INTERFERON ON HAEMANGIOMA

CAPILLARY-LIKE OUTGROWTH. .236

5.4.2 OPG ANTIBODY SPECFICITY 240 


\section{CHAPTER 6: CYCLOPHILIN A IN}

HAEMANGIOMA..............................................243

6.1 INTRODUCTION..............................................243

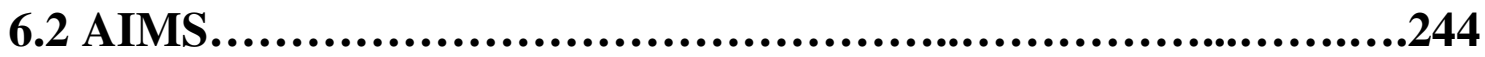

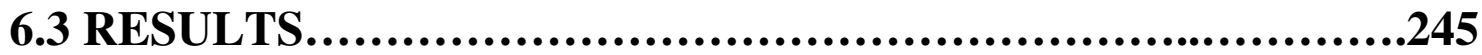

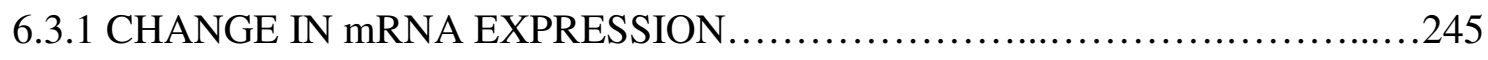

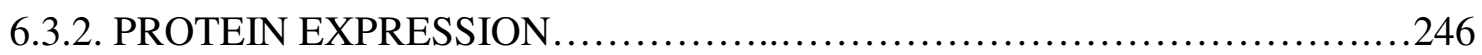

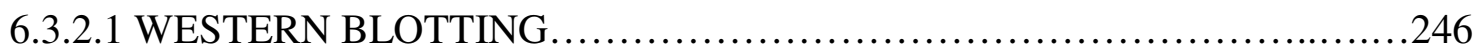

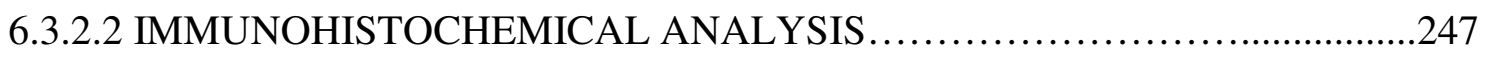

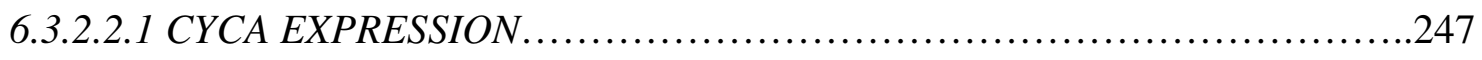

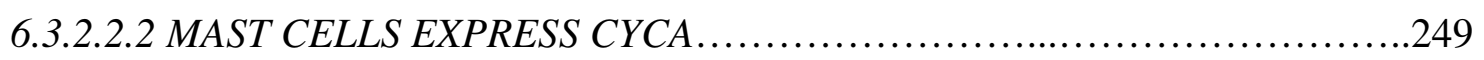

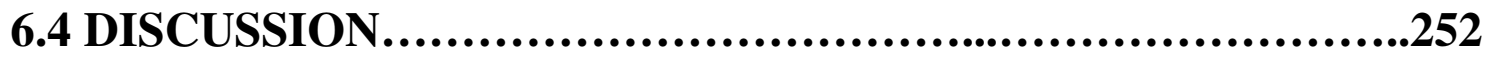

\section{CHAPTER 7: KEY FINDINGS AND FINAL}

CONCLUSIONS.......................................255

7.1 THE SIGNIFICANCE OF THIS RESEARCH...................255

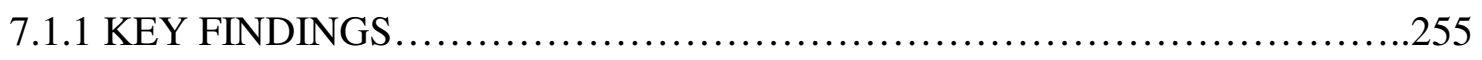

7.1.1.1 VEGF, VEGFRs AND STAT PROTEINS IN HAEMANGIOMA..............255

7.1.1.2 THE USE OF TRAIL AS TREATMENT FOR HAEMANGIOMA.............257

7.1.1.3 THE IDENTIFICATION AND ROLE OF STEM CELLS IN

HAEMANGIOMA PROGRESSION.

7.1.1.4 HOW DO THE FINDINGS FROM THIS STUDY FIT IN WITH THE

CURRENT KNOWLEDGE OF HAEMANGIOGENESIS?...................258

7.1.1.5 THE OVERALL SIGNIFICANCE OF THIS RESEARCH........................262 


\section{APPENDIX 1: CHAPTER 3..........................267}

\subsection{PRELIMINARY EXPERIMENTS...............................267}

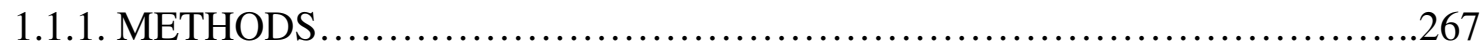

1.1.1.1 QUANTITATIVE REAL TIME PCR .................................267

1.1.1.2 IMMUNOHISTOCHEMICAL OPTIMISATION ...........................268

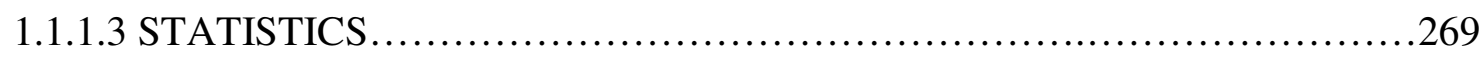

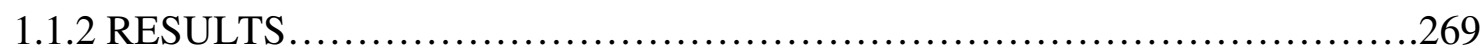

1.1.2.1 PCR QUALITY CONTROL .........................................269

1.1.2.2 VALIDATION OF AN APPROPRIATE INTERNAL REFERENCE

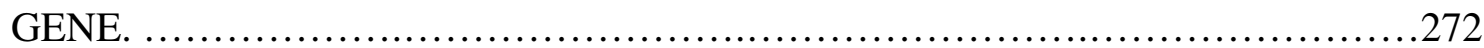

1.1.2.3 IMMUNOHISTOCHEMICAL OPTIMISATION...........................275

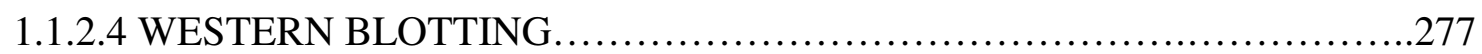

1.1.2.4.1 Lysate preparation, transfer conditions and antibody specificity.............277

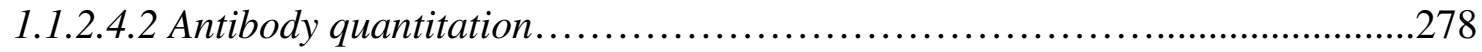

1.2 GENE EXPRESSION CHANGES IN HAEMANGIOMA........280

APPENDIX 2: CHAPTER 4........................284

2.1 PURIFICATION OF HAEMANGIOMA-DERIVED

ENDOTHELIAL CELLS.......................................289

APPENDIX 3: CHAPTER 5..........................292 
4.1.1 RNA Precipitation .294

4.1.2 PCR Amplification. 294

4.1.3 PCR Reaction Using Taq DNA Polymerase .294

4.1.4 Agarose Gel Electrophoresis .295

4.1.5 Purification Of DNA Fragments From Agarose Gels...........................296

4.1.6 Acetone Protein Precipitation...........................................296

\subsection{STOCK SOLUTIONS, BUFFERS AND OTHER RECIPES......297}

4.2.1 Phosphate buffered saline (PBS) 0.15 M pH 7.4 ..........................297

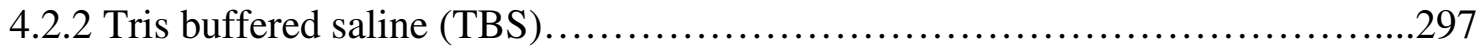

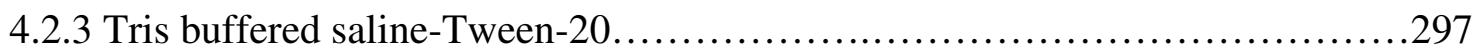

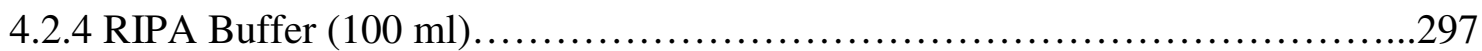

4.2.5 For Coomassie R-250 staining .....................................298

4.2.6 Western blot transfer buffer...........................................298

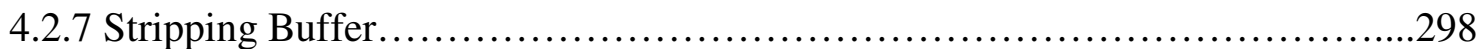

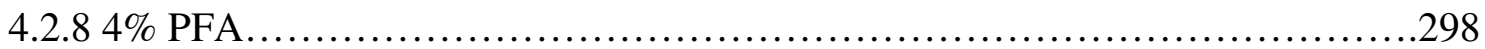

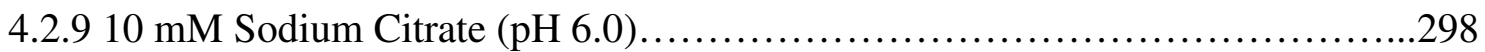

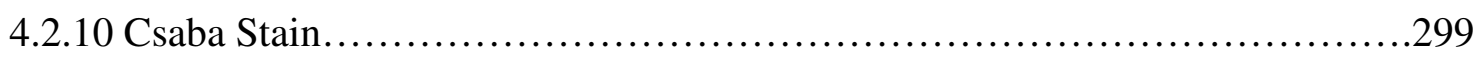

4.2.11 Coating culture dishes with $1 \%$ gelatin.................................299

4.2.12 AG490 stock solution................................................299

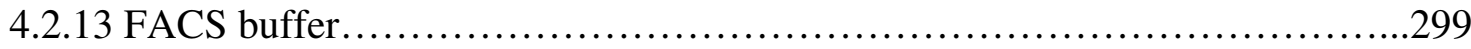

4.2.14 Alizarin Red Staining Solution...........................................299

4.2 .15 (A) Oil Red O stock stain...............................................

(B) Oil Red O working solution.................................... 300

4.2.16 Glycerin Jelly Mounting Medium......................................300 
4.2.18 10\% SDS Polyacrylamide Gels........................................300

4.2.19 4\% Stacking Gel.................................................... 301

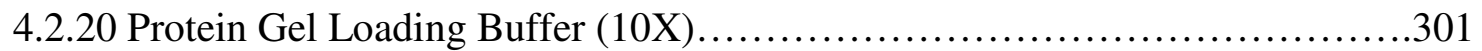

4.2.21 1X SDS Page Running Buffer (For 1 Litre)......................................

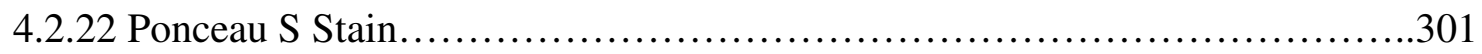

4.2.23 Western blotting apparatus........................................... 301

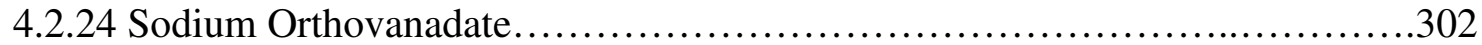

REFERENCES......................................304 


\section{LIST OF FIGURES}

\section{CHAPTER 1}

Figure 1.1: Natural progression of haemangioma on the right cheek and upper lip of a female patient. 4

Figure 1.2: Not all strawberries are haemangioma....................................

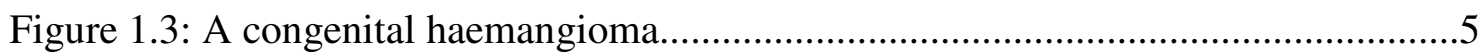

Figure 1.4: A bilateral cervico-facial haemangioma....................................................

Figure 1.5: A proliferating haemangioma on the left parotid region...................8

Figure 1.6: Natural progression of haemangioma near the right eye $\ldots \ldots \ldots \ldots \ldots \ldots \ldots \ldots . .8$

Figure 1.7: A patient with PHACES syndrome with segmental haemangioma associated with a sternal cleft........................................

Figure 1.8: Histopathological features of haemangioma at different phases of development.............................................13

Figure 1.9: A haemangioma on the elbow of a 13-month old girl......................14

Figure 1.10: The basic structure of the blood vessel walls...........................38

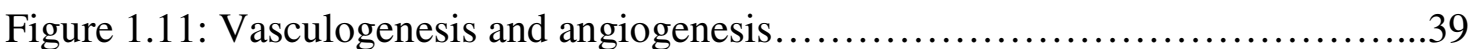

Figure 1.12: Roles of the vascular endothelial growth factor (VEGF)/VEGFRs) and the angiopoietin/Tie-2 systems in embryonic vascular development.........40

Figure 1.13: Growth factors and their receptors implicated in different stages of vasculogenesis and angiogenesis.

Figure 1.14: A model for the regulation of the vascular endothelium by VEGF, Ang-1 and Ang-2 .44

Figure 1.15: Schematic representation of the angiogenic process...................45

Figure 1.16: The role of cytokines and cell-cell interactions in blood vessel formation. 
Figure 1.17: Schematic representation of STAT activation via

JAK activation

\section{CHAPTER 3}

Figure 3.1: Proposed model for the role of STAT-3, p-STAT-3 (Tyr 705), STAT-1

and VEGFR-2 in haemangioma. 105

Figure 3.2: Changes in STAT-3 mRNA expression........................... 108

Figure 3.3: Changes in VEGFR-2 mRNA expression...........................108

Figure 3.4: Changes in STAT-1 mRNA expression.

Figure 3.5: Correlation between STAT-3 and VEGFR-2 mRNA expression in proliferating, involuting and involuted haemangiomas.

Figure 3.6: Western blotting for STAT-3 and p-STAT-3 (Tyr 705)....

Figure 3.7: Western blotting for STAT-1 and VEGFR-1 expression in proliferating, involuting and involuted haemangioma lesions.... 115

Figure 3.8: Immunohistochemical localisation of STAT-3 and PCNA in paraffin embedded haemangioma biopsies

Figure 3.9: Immunohistochemical localisation of VEGFR-2 in haemangioma. 119

Figure 3.10: Immunohistochemical localisation of STAT-1 in haemangioma.

Figure 3.11: Intense GLUT-1 immuoreactivity in a proliferating haemangioma.

Figure 3.12: Mast cells present in proliferating and involuting haemangiomas express STAT-3.

Figure 3.13: Confocal serial optical sections through a STAT-3 and PCNA dual labelled haemangioma section.

Figure 3.14: Confocal images through a p-STAT-3 (Tyr 705) and PCNA

Dual-labelled proliferating haemangioma section 126

Figure 3.15: STAT-3 expression in proliferating haemangioma. 128

Figure 3.16: STAT-3 expression in involuting haemangioma 128 
Figure 3.17: p-STAT-3 (Tyr 705) expression does not correlate with

proliferation

\section{CHAPTER 4}

Figure 4.1: Haemangioma explant model.

Figure 4.2: Morphological characteristics of the haemangioma explant model.

Figure 4.3: Morphological characterisation of HaemDCs

Figure 4.4: Population doubling time of HaemDCs

Figure 4.5: Immunohistochemical localisation of vimentin, $\alpha$-SMA and

GLUT-1 on HaemDCs.

Figure 4.6: Immunohistochemical localisation of vWF and CD34 on

HUVECs, HaemDCs and 3T3 fibroblasts....

Figure 4.7: Immunohistochemical localisation of VEGFR-2 and VEGFR-1

on HUVECs, HaemDCs and 3T3 fibroblasts 155

Figure 4.8: Western blotting for VEGF-A.

Figure 4.9: Picrosirius red stained section of an involuting haemangioma tissue section

Figure 4.10: Differentiation potential of HaemDMCs.

Figure 4.11: Validation of adipocytic and osteocytic differentiation of HaemDMCs 162

Figure 4.12: Western blot verification of VEGFR-1 and STAT-3 expression by HaemDMSCs

Figure 4.13: Effect of AG490 and VEGF on capillary-like outgrowth

from proliferating haemangioma explants.

Figure 4.14: VEGF stimulates proliferation of HUVECs but not

HaemDMSCs 166 
Figure 4.15: VEGF does not induce p-STAT-3 (Tyr 705) expression

in HaemDMSCs .168

Figure 4.16: Immunolocalisation of p-STAT-3 (Tyr 705), p-STAT-1 (Tyr 701)

and p-STAT-5 (Tyr 694) in response to VEGF 170

Figure 4.17: The effect of AG490 on HaemDMSCs....

Figure 4.18: Immunohistochemical localisation of vWF and OPG on

HUVECs and OPG on HaemDMSCs. 176

Figure 4.19: Confocal immunohistochemical localisation of vWF and OPG in haemangioma.

Figure 4.20: Immunohistochemical localization of vWF and PCNA on paraffin embedded haemangioma tissue biopsies

Figure 4.21: IFN $\alpha-2 b$ induces an increase in p-STAT protein expression and nuclear translocation in HaemDMSCs 180

Figure 4.22: EPC-like cells obtained from a proliferating haemangioma. 182

Figure 4.23: Immunohistochemical localisation of vWF and OPG on HaemDEPCs. 182

Figure 4.24: Change in HaemDEPC morphology and immunophenotype on day 5 in culture in vitro 184

Figure 4.25: Immunohistochemical localisation of VEGFR-1 and VEGFR-2 on HaemDEPCs 184

Figure 4.26: Adipocytic differentiation of the MSCs transdifferentiated from HaemDEPCs. 186

Figure 4.27: HaemDEPCs maintain their endothelial phenotype when cultured in VEGF. 188

Figure 4.28: An Alternative Model For Haemangioma... .205

Figure 4.29: The Final Model For Haemangioma. .211 


\section{CHAPTER 5}

Figure 5.1: Activation of the cell-extrinsic and cell-intrinsic apoptosis

pathways by TRAIL

Figure 5.2: Confocal immunohistochemical localisation of TRAIL-Rs in haemangioma.

Figure 5.3: Effect of TRAIL and IFN on haemangioma cultures, in vitro 226

Figure 5.4: Differences in mRNA levels for TRAIL and TRAIL receptors in haemangioma tissue and HaemDMSCs .228

Figure 5.5: Confocal immunohistochemical localisation of TRAIL-Rs on HaemDMSCs

Figure 5.6: Confocal immunohistochemical localisation of

OPG in haemangioma.

Figure 5.7: DR4 and DcR1 protein expression in haemangioma...

Figure 5.8: OPG protein expression in HaemDMSCs conditioned media, in HaemDMSCs and in a proliferative haemangioma tissue biopsy 235

\section{CHAPTER 6}

Figure 6.1: Changes in CycA mRNA expression.... 245

Figure 6.2: Western blotting for CycA in haemangioma. .246

Figure 6.3: Immunohistochemical localisation of CycA in haemangioma. 248

Figure 6.4: MCs in proliferating and involuting haemangiomas express CycA 251

Figure 6.5: A role for $\mathrm{CycA}$ in haemangioma progression .253 


\section{CHAPTER 7}

Figure 7.1: A model proposed for the development of haemangioma based on the speculative ideas and findings from this investigation. .263

\section{APPENDIX 1: CHAPTER 3}

Figure 1.1: An example of the cycling numbers obtained during a typical real-time PCR amplification of serially diluted PCR template. 270

Figure 1.2: The melt-curve obtained at the end of PCR amplification with serially diluted STAT-1 template

Figure 1.3: qRT-PCR primer pair efficiencies. 271

Figure 1.4: Validation of an appropriate internal house-keeping gene (Experiment 1). .273

Figure 1.5: Validation of an appropriate internal house-keeping gene (Experiment 2)

Figure 1.6: Immunohistochemical localisation of STAT-3, p-STAT-3 (Tyr 705) and STAT-1 on HeLa cells 276

Figure 1.7: Immunohistochemical localisation of VEGFR-2 on EOMA cells and GLUT-1 on placenta tissue sections.

Figure 1.8: Antibody specificity by Western blotting.... .279

Figure 1.9: Correlation between STAT-3, STAT-1 and VEGFR-2 mRNA expression in proliferating, involuting and involuted haemangiomas. .280

Figure 1.10: STAT-3 expression correlates with proliferation in proliferating haemangiomas .281

Figure 1.11: STAT-3 expression correlates with proliferation in involuting haemangiomas .282 


\section{APPENDIX 2: CHAPTER 4}

Figure 2.1: Haemangioma explant model (1)................................284

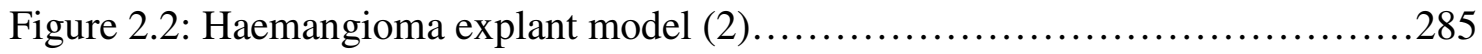

Figure 2.3: Phase contrast images showing the growth of a different cell type from haemangioma explants. 285

Figure 2.4: qRT-PCR results showing mRNA expression patterns for Collagen I, -II, -III, -VIII, Laminin-V, IGF-2 and VEGF-2 in HaemDMSCs and their original biopsy of origin 286

Figure 2.5: VEGF does not induce p-STAT-3 (Tyr 705) expression in HaemDMSCs 288

Figure 2.6: $\mathrm{CD} 31^{+}$cells isolated from two proliferating haemangioma biopsies 290

\section{APPENDIX 3: CHAPTER 5}

Figure 3.1: OPG protein expression in the media of HaemDMSCs conditioned over a 72-hour period. 


\section{LIST OF TABLES}

Table 1: Primer sequences and cDNA amplicon size (in $b p$ )

used in this study....

Table 2: Primary antibodies used in this study, their dilutions

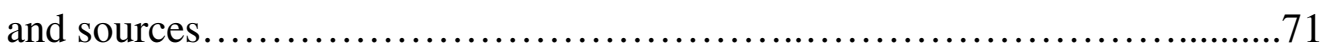

Table 3: Secondary antibodies used in this study, their dilutions

and sources

.73

Table 4: $\Delta$ Ct values for STAT-3, VEGFR-2 and STAT-1 obtained from

proliferating, involuting and involuted haemangioma biopsies.....

Table 5: The total number and percentage of STAT-3 positive MCs in

proliferating and involuting haemangioma.

Table 6: mRNA characterisation of HaemDCs 152

Table 7: PPAR- $\gamma$ and osteonectin mRNA expression

Table 8: Expression of TRAIL and TRAIL receptors mRNA in haemangioma.

Table 9: CycA expression by MCs in haemangioma.

\section{APPENDIX 2: CHAPTER 4}

Table 2.1: mRNA characterization of HaemDCs

\section{APPENDIX 3: CHAPTER 5}

Table 3.1: Differences in mRNA levels for TRAIL and TRAIL receptors in haemangioma tissue and HaemDMSCs. 


\section{ABBREVIATIONS}

\begin{tabular}{|c|c|}
\hline ANG & Angiopoietin \\
\hline APES & 3-amino-propyltriethoxysilane \\
\hline ApoJ & Apolipoprotein $\mathbf{J}$ \\
\hline $\mathrm{BCA}$ & Bicinchoninic acid \\
\hline $\mathrm{BM}$ & Basement membrane \\
\hline bp & Base pairs \\
\hline BrdU & 5-bromo-2-deoxyuridine \\
\hline BSA & Bovine serum albumin \\
\hline CA II & Carbonic anhydrase II \\
\hline CL & Cell lysate \\
\hline Col & Collagen \\
\hline $\mathrm{Ct}$ & Cycle threshold \\
\hline CVS & Chorionic villus sampling \\
\hline CycA & Cylophilin A \\
\hline DAB & Diaminobenzidine \\
\hline DAPI & 4',6-diamino-2-phenylindole \\
\hline DISC & Death inducing signalling complex \\
\hline DMSO & Dimethyl sulfoxide \\
\hline DTT & Dithiothreitol \\
\hline EBM & Endothelial basal medium \\
\hline $\mathrm{EC}$ & Endothelial cell \\
\hline ECGS & Endothelial cell growth supplement \\
\hline $\mathrm{ECM}$ & Extracellular matrix \\
\hline EDTA & Ethylenediaminetetraacetic acid \\
\hline EGF & epidermal growth factor \\
\hline EM & Electron microscopic \\
\hline EMT & Endothelial to mesenchymal transdifferentiation \\
\hline EPC & Endothelial progenitor cell \\
\hline ERK & Extracellular signal regulated kinase \\
\hline FADD & Fas-associated death domain \\
\hline FCS & Foetal calf serum \\
\hline FGF & Fibroblast growth factor \\
\hline FLT-4 & Fms-related tyrosine kinase \\
\hline GAPDH & Glyceraldehyde 3 phosphate dehydrogenase \\
\hline
\end{tabular}




\section{GLUT-1}

$\mathrm{H}_{2} \mathrm{O}_{2}$

$\mathrm{H} \& \mathrm{E}$

HaemDCs

HaemDEC

HaemDEPC

HaemDMCs

HaemDMSC

HGF

HPRT

HUVEC

IAP

ICAM-1

IFN

IGF

IHC

ISG

JAK

IL-6

Lam

LDS

LeY

M

MC

$\min$

MCP-1

$\mathrm{MeC}$

MMP

MPC

MSC

mt Cyt $b$

$\mathrm{NaBH}_{4}$

$\mathrm{NaF}$

$\mathrm{Na}_{2} \mathrm{~B}_{4} \mathrm{O}_{7}$

$\mathrm{nm}$

$\mathrm{NaO}_{3} \mathrm{~V}$
Glucose transporter-1

Hydrogen peroxide

Haematoxylin and eosin

Haemangioma explant-derived cells

Haemangioma-derived endothelial cell

Haemangioma-derived endothelial progenitor cell

Haemangioma-derived mesenchymal cells

Haemangioma-derived mesenchymal stem cell

Hepatocyte growth factor

Hypoxanthine phosphoribosyltransferase

Human vascular endothelial cell

Inhibitor of apoptosis protein

Intercellular adhesion molecule-1

Interferon

Insulin growth factor

Immunohistochemistry

Interferon stimulated gene

Janus kinase

Interleukin-6

Laminin

Lithium dodecyl sulphate

Lewis $\mathrm{Y}$ antigen

Medium

Mast cell

Minutes

Monocyte chemoattractant protein-1

Mesenchymal cell

Matrix metalloprotease

Magnetic particle concentrator

Mesenchymal stem cell

Mitochondrial cytochrome b

Sodium borohydride

Sodium fluoride

Sodium tetra borate (Boric acid)

Nanometer

Sodium orthovanadate 


\begin{tabular}{|c|c|}
\hline OPG & Osteoprotegerin \\
\hline PBS & Phosphate buffered saline \\
\hline PCNA & Proliferating cell nuclear antigen \\
\hline PCR & Polymerase chain reaction \\
\hline PDGF & Platelet-derived growth factor \\
\hline Penstrep & Penicillin-Streptomycin \\
\hline PFA & Paraformaldehyde \\
\hline PI & Propidium iodide \\
\hline PMSF & Phenylmethylsulphonyl fluoride \\
\hline PPAR $\gamma$ & Peroxisome proliferator-activated receptor- $\gamma$ \\
\hline PPIase & Peptidylprolyl cis-trans-isomerase \\
\hline PVDF & Polyvinylidine difluoride \\
\hline qRT-PCR & Quantitative real-time polymerase chain reaction \\
\hline RANK & Receptor activator of nuclear factor- $\kappa \mathrm{B}$ \\
\hline RANKL & Receptor activator of nuclear factor- $\kappa \mathrm{B}$ ligand \\
\hline $\mathrm{RICH}$ & Rapidly involuting congenital hemangioma \\
\hline RTK & Receptor tyrosine kinase \\
\hline SDS & Sodium dodecyl sulphate \\
\hline sec & Seconds \\
\hline s.e.m & Standard error of the mean \\
\hline siRNA & Short interfering RNA \\
\hline SMA & Smooth muscle actin \\
\hline $\mathrm{SMC}$ & Smooth muscle cell \\
\hline STAT & Signal transducer and activator of transcription \\
\hline STAT-3 + MC & STAT-3 positive mast cell \\
\hline STAT-3 - MC & STAT-3 negative mast cell \\
\hline TBS & Tris buffered saline \\
\hline TBST & Tris buffered saline - Tween \\
\hline TGF & Transforming growth factor \\
\hline TIMP & Tissue inhibitor of metalloproteinase \\
\hline Tm & Melting temperature \\
\hline TNF & Tumour necrosis factor \\
\hline TRAIL & Tumour necrosis factor related apoptosis inducing ligand \\
\hline TL & Tissue lysate \\
\hline VEGF & Vascular growth factor \\
\hline VEGFR & Vascular growth factor receptor \\
\hline
\end{tabular}


VLA

vWF

XIAP
Very late antigen

Von Willebrand factor

X-chromosome-linked inhibitor of apoptosis protein 


\section{CHAPTER 1: GENERAL INTRODUCTION}

\subsection{HAEMANGIOMA}

In 1982, Mulliken and Glowacki proposed to classify vascular anomalies into haemangioma or vascular malformations based on their biology (Mulliken \& Glowacki, 1982). The International Society for the study of Vascular Anomalies adopted this classification for vascular anomalies in 1996 (Enjolras \& Mulliken, 1997).

The term haemangioma refers to a rapidly growing vascular tumour of infancy (Mulliken \& Glowacki, 1982) commonly known as strawberry naevus (Vikkula et al., 1998). It is a benign tumour of the microvasculature in which there is initial excessive angiogenesis (new blood vessel growth) followed by slow spontaneous regression of the newly formed vessels within five to 12 years (Mulliken \& Glowacki, 1982; Mulliken, 1988). Haemangioma does not occur during adolescence or adulthood (Mulliken, 1988).

\subsubsection{THE INCIDENCE OF HAEMANGIOMA}

Haemangioma is the most common tumour of infancy affecting up to $12 \%$ of Caucasians but is uncommon in other races (Mulliken, 1988; Hildano \& Nakajima, 1972). An incidence of $0.8 \%, 1.4 \%$ and $3 \%$ has been reported in Japanese (Hildano \& Nakajima, 1972), Black (Pratt, 1953), and Asian Indian (Tan, 2001), respectively. The incidence increases to $23 \%$ in premature infants with a birth weight less of than $1000 \mathrm{~g}$ (Amir et al., 1996). Girls are three times more affected than boys (Hildano \& Nakajima, 1972).

\subsubsection{CLINICAL FEATURES}

Haemangioma is distinct from vascular malformations. $70-90 \%$ of haemangioma appear by the fourth week of life (Mulliken, 1988; Simpson, 1959) with 30-40\% being noticed at birth, usually as a pink macule, blanched area or telangiectasia surrounded by 
a pale halo (Hildano \& Nakajima, 1972; Payne et al., 1966). The majority of lesions present as single localised cutaneous tumours in the head and neck region $(60 \%)$ with the remainder being found in the trunk $(25 \%)$ or the extremities $(15 \%)$ (Finn et al., 1983).

Haemangioma may grow to an extensive tumour in a local area or proliferate simultaneously at multiple sites in the body (Mulliken, 1988). They have been found in lymph nodes, thymus, spleen, liver, gastrointestinal tract, gallbladder, pancreas, adrenal glands, urinary bladder and lung (Edmondson, 1956; Cooper \& Bolande, 1965; Burmanet et al., 1967; Holden \& Alexander, 1970; Cohen \& Myers, 1986). Cases of haemangioma have also been reported in the brain, meninges and spinal cord (Cooper \& Bolande, 1965; Burman \& Mnasell, 1967; Cohen \& Myers, 1986).

\subsubsection{THE DIAGNOSIS}

The diagnosis of haemangioma is well described (Mulliken, 1988; Pratt, 1953; Tan, 2001). This tumour usually first noticed as a pink macule (Figure 1.1-A), typically exhibits rapid growth during infancy, out of proportion to the child's growth. This rapid proliferation for the first 8-12 months is the hallmark of haemangioma. During this proliferative phase, the tumour assumes a bright crimson colour (hence the term strawberry naevus), is tense on palpation and is often tender to touch (Figure 1.1-B and -C) (Tan, 2001).

Following the proliferative phase, the tumour enters the involuting phase in which tumour growth is proportional to that of the child. During this phase, the lesion shrinks and softens. The crimson colour begins to fade becoming dull purple; the surface exhibits a mottled, greyish mantle with tiny white flecks (Figure 1.1-D). These cutaneous signs of involution start centrally and spread to the periphery. This takes at least 1-5 years. Once the haemangioma enters the involuted phase, which may take up to 10-12 years, its full life-cycle is spent (Takahashi et al., 1994). The involved skin by haemangioma exhibits mild atrophy, has a wrinkled quality with few telangiectic 
vessels and is slightly paler than the surrounding skin. There is often fibro-fatty tissue residuum with resultant contour deformity (Figure 1.1-E) (Tan, 2001). However, each lesion exhibits a spectrum of developmental changes, clinically and histologically (Mulliken, 1998; Tan, 2001).

$50 \%$ of haemangioma show complete regression by the time the child is five years of age, and over $70 \%$ by the age of seven (Pratt, 1953; Simpson, 1959). Normal skin is restored in $50 \%$ of patients when involution is complete. Studies have also shown that the gender, race of the patient, size and site of the lesion, duration of the proliferative phase and clinical appearance of the lesion do not influence the rate of regression (Simpson, 1959; Finn et al., 1983).

About $10-20 \%$ of lesions require treatment during the proliferative phase because they threaten life or function, or cause tissue distortion or destruction (Takahashi et al., 1994; Mulliken et al., 1995). Although all haemangioma eventually involute without treatment, they are disfiguring and may cause psychological distress, so intervention is required in a large number of patients (Tan, 2001).

\subsubsection{DIFFERENTIAL DIAGNOSIS}

'Not all haemangioma look like strawberries' (Mulliken, 1998). In the literature, almost all cutaneous vascular anomalies, at some point, have been called haemangioma. Terminological and clinical confusion between haemangioma and vascular malformations has been responsible for improper diagnosis, illogical treatment and misdirected research efforts (Mulliken \& Glowacki, 1982; Mulliken et al., 1995). An example of the similarity between a capillary-venous malformation that can look remarkably similar to a haemangioma that involves the skin and subcutaneous tissue is shown in Figure 1.2. 

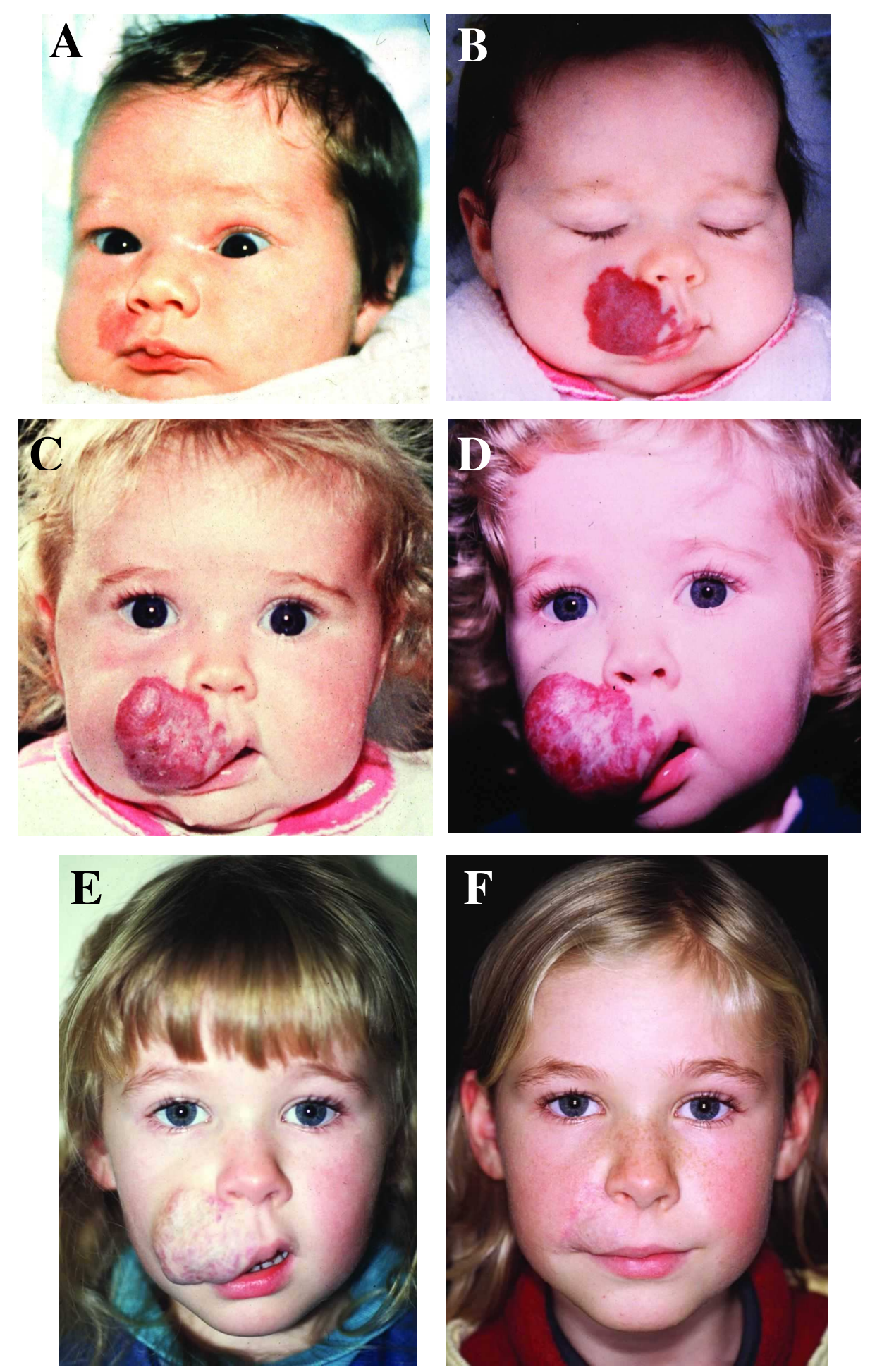

Figure 1.1: Natural progression of haemangioma on the right cheek and upper lip of a female patient. In Panels A-C, the haemangioma is in the proliferative phase. The lesion initially a pink macule noticed soon after birth then grew rapidly out of proportion of the growth of the child to assume a bright crimson colour. Photographs were taken 2 days after birth (A), at 1 month (B) and at 8 months (C). Panel $\mathbf{D}$ shows the lesion in the involuting phase at 18 months. During this phase, the lesion began to shrink and soften. The crimson colour changed to a dull purple and the surface exhibited a mottled, greyish mantle with tiny flecks. These cutaneous signs of involution began centrally and slowly spread to the periphery. Panel $\mathbf{E}$ shows the lesion at 6 years when it had fully involuted. The involved skin that remained had a wrinkled quality with mild atrophy and hypopigmentation and few telangiectic vessels. The fibro-fatty tissue residuum gave rise to distortion and contour deformity (E). Panel $\mathbf{F}$ shows the child 9 months after surgical excision of the residuum (F) (Courtesy of Dr Swee.T.Tan). 

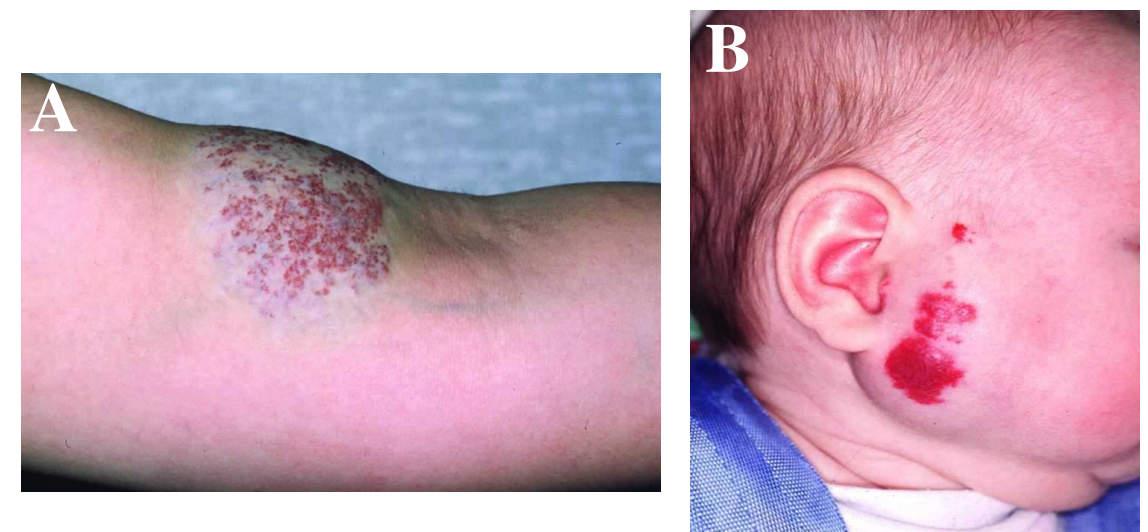

Figure 1.2: Not all strawberries are haemangioma. (A) A capillary-venous malformation behind the knee of a 1-year old child mimicking the appearance of a haemangioma. Unlike haemangioma, this lesion was present at birth and grew proportionately with the child. (B) A haemangioma in the right parotid region involving overlying skin and subcutaneous tissue in a 7-month old child. Spontaneous regression is expected in this lesion as in all haemangioma (Courtesy of Dr Swee.T.Tan).

\subsubsection{CONGENITAL HAEMANGIOMA}

There have been rare instances in which a fully-grown haemangioma is present at birth. These are known as congenital haemangioma (Boon et al., 1996). Vikkula et al (1998) suggested that the trigger for these lesions occured in utero. A subset of these lesions undergo rapid involution, usually within the first 14 months of life, leaving either atrophic or excess skin (Tan, 2001) (Figure 1.3) and are called rapidly involuting congenital hemangioma (RICH). There is also another type of congenital haemangioma called non-involuting congenital haemangioma $(\mathrm{NICH})$. These lesions are present at birth but do not undergo post-natal regression (Mulliken \& Enjolras, 2004). Some of the lesions have required steroid therapy or surgery because of their threat to life or function (Boon et al., 1996).
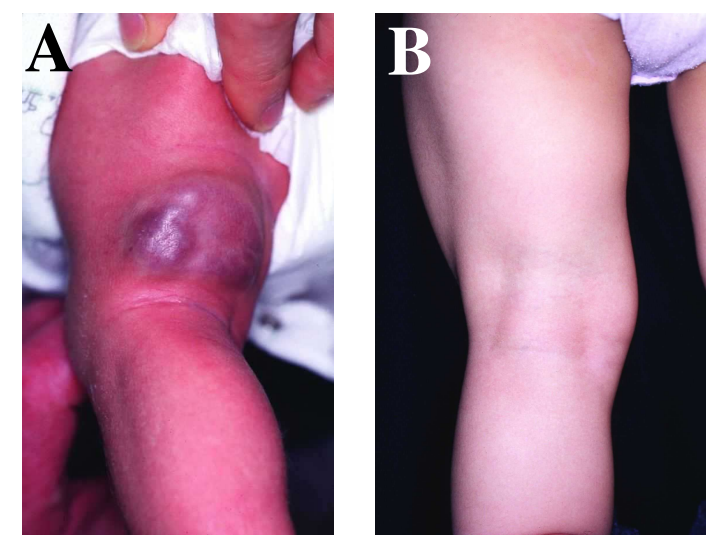

Figure 1.3: A congenital haemangioma. Panel A shows the lesion on the left thigh of a 3-week old infant. Panel $\mathbf{B}$ shows the lesion at 14 months. By this time, spontaneous complete involution had occurred leaving behind slight hypopigmented skin. (Courtesy of $\mathrm{Dr}$ Swee.T.Tan). 


\subsubsection{COMPLICATIONS OF HAEMANGIOMA}

The rapidly proliferating haemangioma may cause ulceration, obstruction of vital organs, tissue distortion or destruction, cardiac failure and skeletal changes (Tan, 2001).

Ulceration occurs in 5\% of cases (Margileth \& Museles, 1965) and is usually observed at the height of the proliferative phase (Bowers et al., 1960). Ulcerated haemangioma are painful and is sometimes accompanied by secondary infection resulting in extensive necrosis and destruction of facial soft tissues (Tan, 2001). The incidence of ulceration is much higher in segmental hemangioma (Waner et al., 2003)

A growing haemangioma can cause obstruction and tissue distortion as they encroach on a critical anatomical structure. For example, obstruction of the visual axis may cause deprivation amblyopia and the failure to develop binocular vision (Tan, 2001). Obstruction of the external auditory meatus can occur with haemangioma involving the parotid region (Figure 1.4-A and Figure 1.5-A). Hearing loss results if the lesion continues to grow beyond one year, when auditory conduction is necessary for normal speech development (Tan, 2001). Cardiac failure and skeletal changes such as overgrowth of the facial skeleton have also been described (Tan, 2001).

\subsubsection{HEAD AND NECK HAEMANGIOMA}

Haemangioma of the head and neck has been identified to favour certain sites on the face (Waner et al., 2003). The importance of anatomic location is now well recognised as a major factor in determining whether complications such as visual axis

occlusion, airway involvement, ulceration or permanent disfigurement may occur (Metry et al., 2001; Garzon et al., 2000; Zide et al., 1997; Haik et al., 1994). Waner et al (2003) categorised haemangioma into segmental and focal lesions. 
Focal lesions are localised and tumour-like and most commonly involve the mid cheek, upper lip and upper eyelid (Waner et al., 2003). An example of a focal lesion is depicted in Figure 1.6.

Segmental lesions are more widespread and plaque-like. The segmental distribution of these lesions is described as frontonasal, maxillary or mandibular and corresponds to the embryological mesenchymal prominences of the head and face. Ulceration and airway obstruction are more common in segmental lesions (Waner et al., 2003). The female to male ratio of patients with segmental lesions is 5.7:1, almost twice that of patients with focal lesions (Waner et al., 2003). Figures 1.1, 1.2-B, 1.4 and 1.5 show segmental haemangioma lesions.
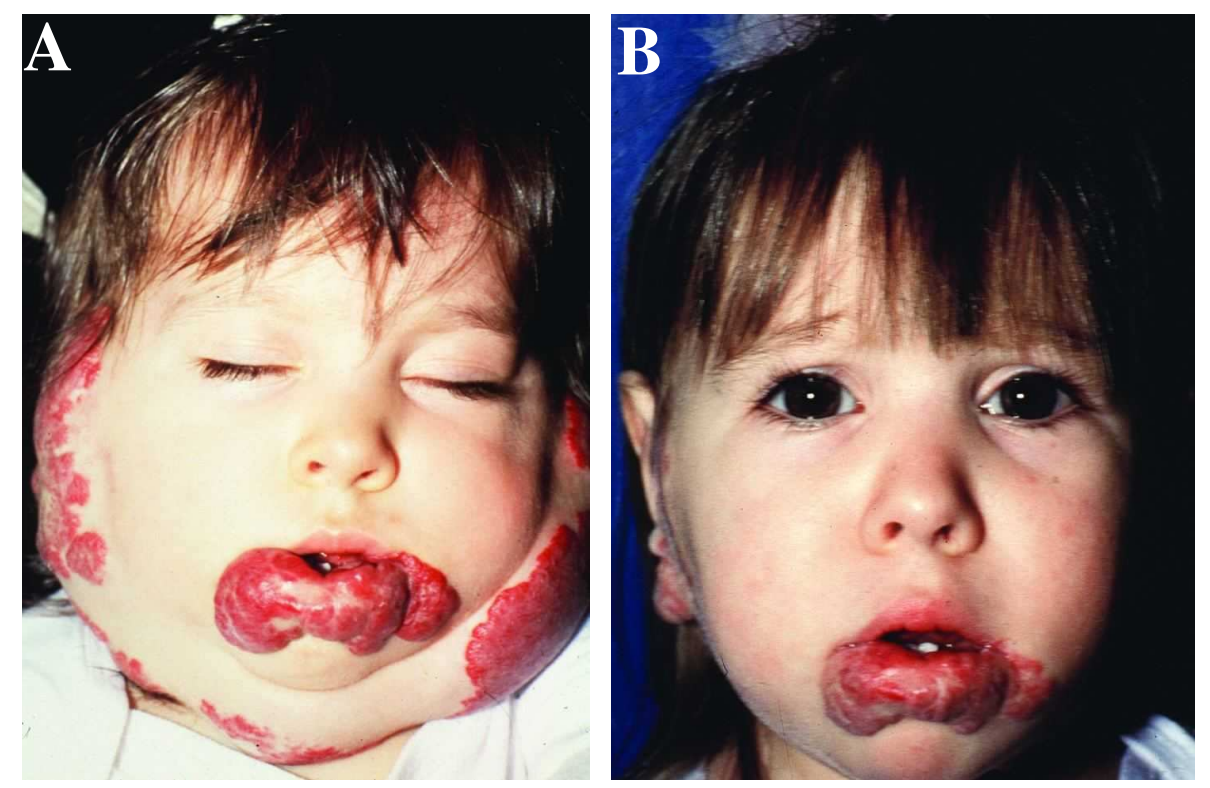

Figure 1.4: A bilateral cervico-facial haemangioma. Panel A shows the lesion on a 1year old girl with the 'beard' distribution, impinging on the upper airway. In Panel $\mathbf{B}$, accelerated regression had occurred after a 9-month course of IFN $\alpha$-2a therapy. Photograph was taken at age 1 year and 10 months (B) (Courtesy of Dr Swee.T.Tan). 

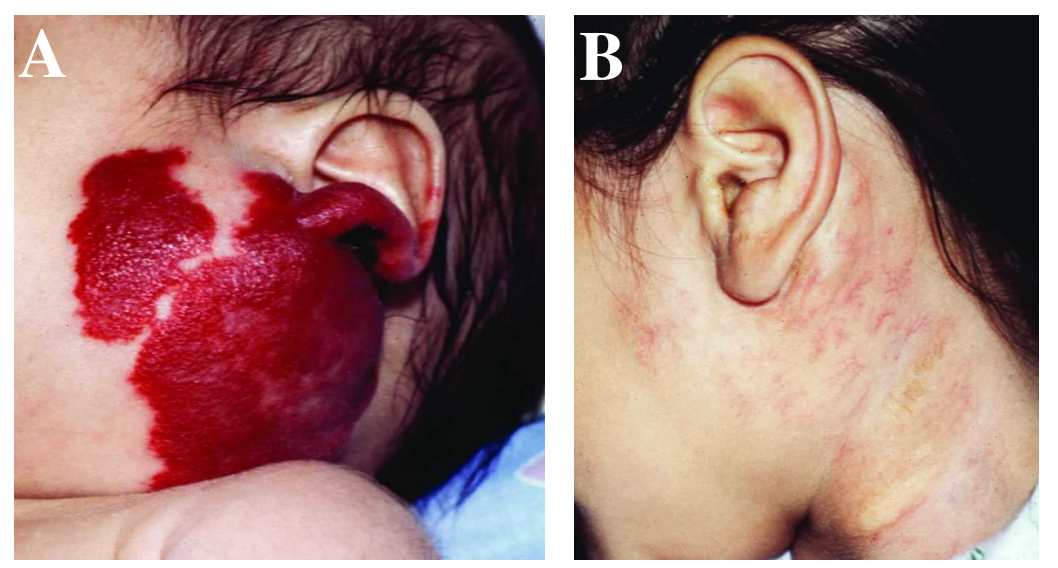

Figure 1.5: A proliferating haemangioma on the left parotid region. Panel A shows a proliferating haemangioma in a 2-month old female infant with ulceration and obstruction of the auditory meatus. In Panel $\mathbf{B}$, the lesion showed accelerated regression following systemic steroid therapy. Photograph was taken at 4 years and 3 months. At this stage, involution was complete leaving behind thin, atrophic and hypopigmented skin with some degree of fatty residuum (Courtesy of Dr Swee.T.Tan).
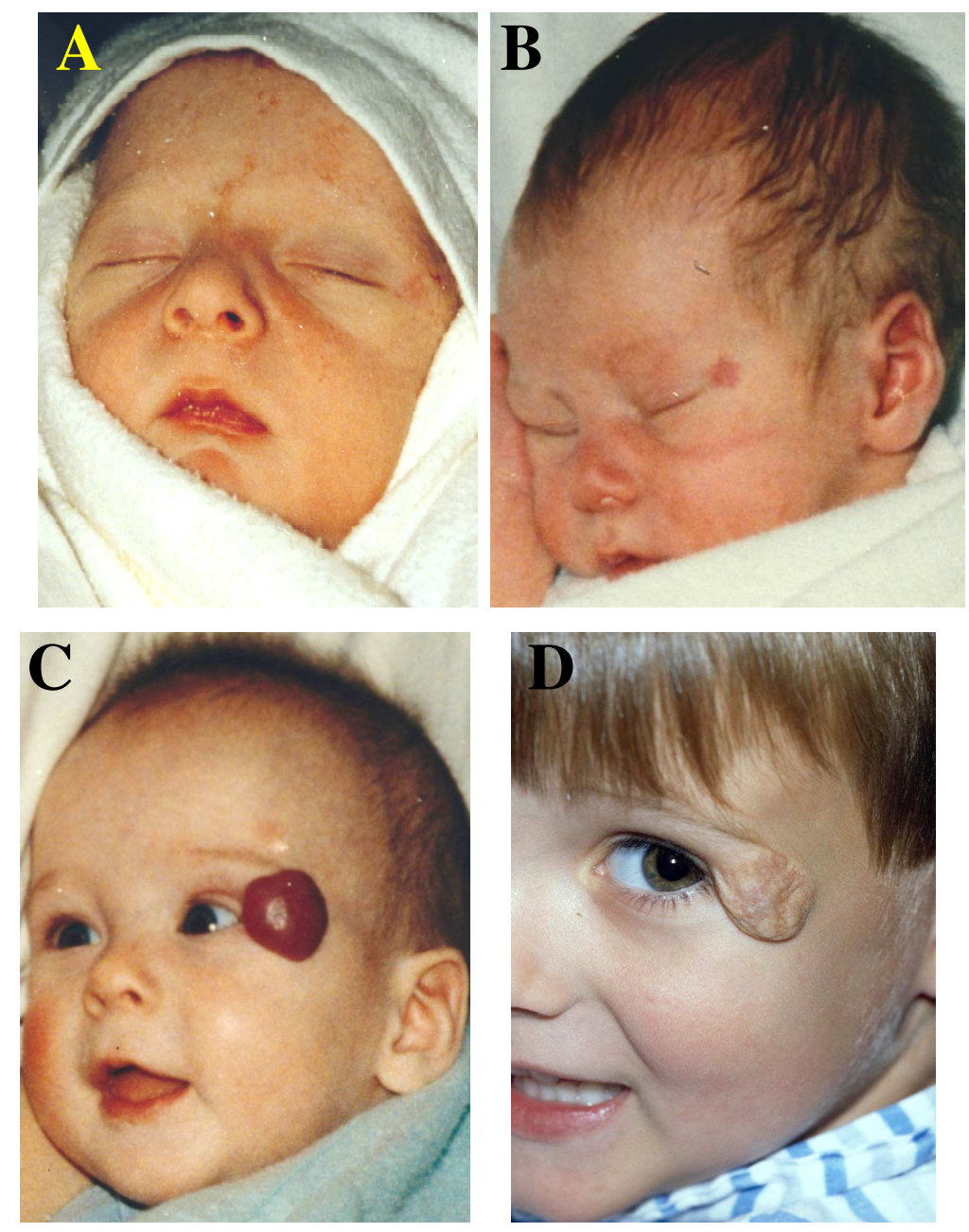

Figure 1.6: Natural progression of haemangioma near the right eye. The lesion initially a pink macule noticed soon after birth (A) became more prominent at 2 weeks of age (B) then grew rapidly to assume a bright crimson colour (C). Photograph was taken at 2 months $(\mathbf{C})$. At 4 years, the lesion had fully involuted $(\mathbf{D})$. The remaining fibro-fatty tissue residuum was then surgically removed (Courtesy of Dr Swee.T.Tan). 
Segmental haemangioma have been associated with a clinically recognised set of structural malformations and anomalies designated as PHACE or PHACES syndrome (Frieden et al., 1996; Reese et al., 1993). This association is characterised by posterior fossa anomalies, haemangioma that are typically plaque-like and segmental in the cervicofacial area, arterial anomalies, cardiac and aortic arch defects, eye anomalies and sternal clefts or supraumblical raphes. Brain abnormalities such as Dandy-Walker syndrome and cerebrovascular arterial anomalies have also been described (Adams \& Lucky, 2006). A child with PHACES syndrome with segmental haemangioma associated with a sternal cleft is shown in Figure 1.7.
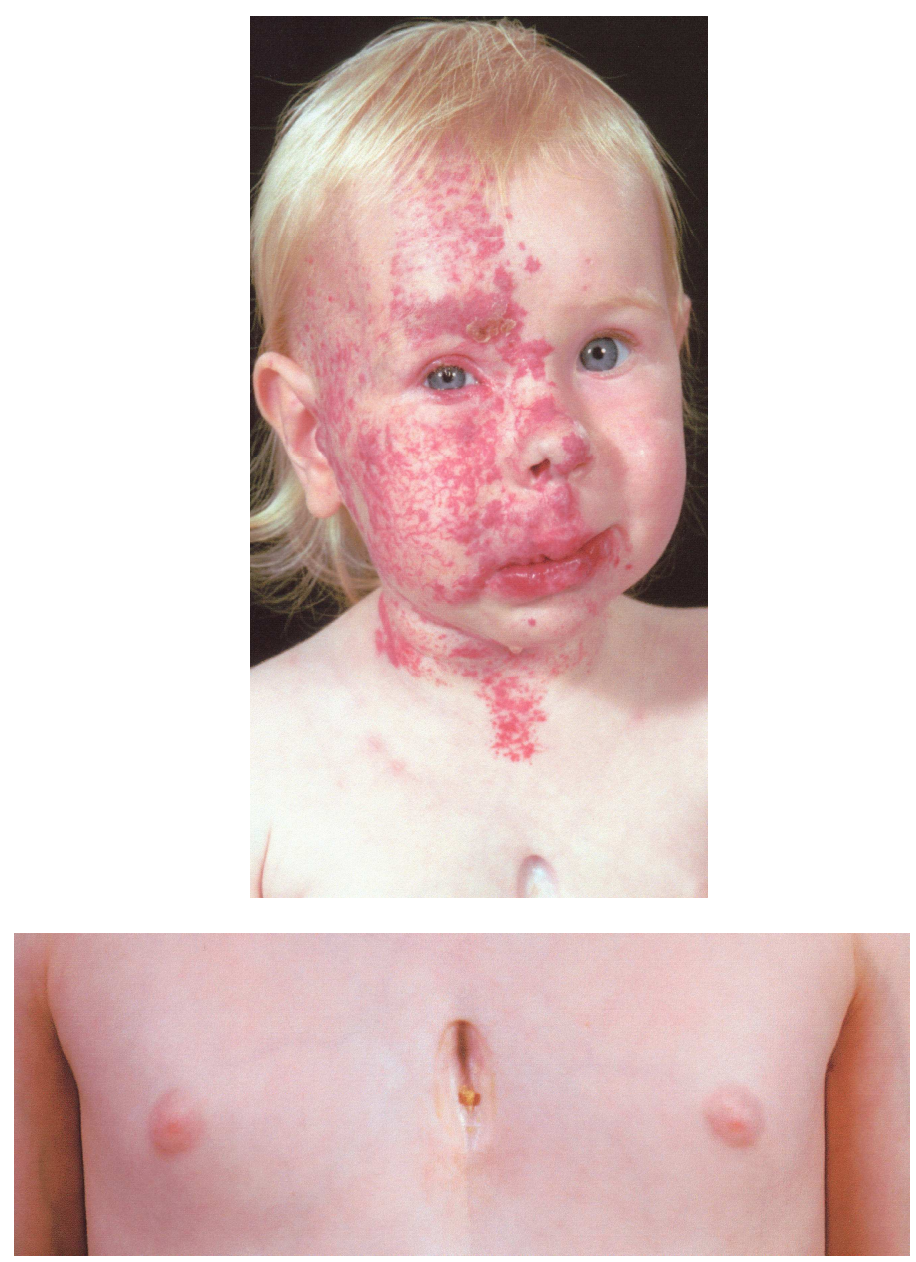

Figure 1.7: A patient with PHACES syndrome with segmental haemangioma associated with a sternal cleft. (Courtesy of Dr Swee.T.Tan). 


\subsubsection{TREATMENT}

The majority of haemangioma are harmless cutaneous lesions that require no intervention and should be left to involute spontaneously (Margileth \& Museles, 1965). However, treatment is necessary during the proliferative phase in $10-20 \%$ of cases because the tumour threatens life or function, causes tissue distortion and destruction (Takahashi et al., 1994; Mulliken et al., 1995). Various therapeutic modalities have been described, but pharmacological therapy with high dose systemic or intralesional corticosteroids is the first line of treatment (Mulliken et al., 1995). Corticosteroids arrest growth in 30-90\% of cases, however, side effects such as delayed skeletal growth, depressed immune function and personality changes limit their usefulness, particularly when prolonged treatment is required (Boon et al., 1999).

In life-threatening cases that do not responded to steroids, IFN treatment has been used (Figure 1.5) (Ezekowitz et al., 1992; Chang et al., 1997). Both IFNa-2a and IFN $\alpha-2 b$ are given subcutaneously with an average duration of 2 to 6 months (Drolet et al., 1999; Garmendía et al., 2001). The reported efficacy varies according to its definition in various series and ranges between 30-70\% (Garmendía et al., 2001). However, its use has been limited due to reports of severe neurotoxicity in the form of spastic diplegia in up to $20 \%$ of patients treated with IFN $\alpha-2 \mathrm{a}$ and in about $5 \%$ with IFNa-2b (Barlow et al., 1998; Worle et al., 1999; Dubois et al., 1999). Chemotherapy has also been used to treat life-threatening, steroid-resistant haemangioma. Cyclophosphamide was used successfully in three infants with life-threatening, steroidresistant haemangioma (Hurvitz et al., 1986). A few reports on the use of vincristine also exist (Fawcett et al., 2004). Other strategies that have been utilised, with varying degrees of success, include embolisation, surgery, laser therapy and sclerotherapy (Enjolras et al., 1990; Jackson et al., 1993; Tan, 2001). Surgery is considered an effective treatment for localised problematic proliferating haemangioma (Tan, 2001). 


\subsubsection{GENETICS IN HAEMANGIOMA}

Although a family history has been noted in $10 \%$ of affected individuals (Margileth \& Museles, 1965; Cheung et al., 1997), it is generally regarded that haemangioma does not have a hereditary basis (Tan, 2001). However, being a Caucasian female with a positive family history apparently lowers the threshold for haemangiogenesis wherein extragenetic factors can trigger the appearance of haemangioma (Vikkula et al., 1998).

\subsubsection{HISTOPATHOLOGY}

The pathogenesis of haemangioma is not well understood. The life cycle of haemangioma has been arbitrarily divided into three phases: (i) the proliferative phase, (ii) the involuting phase and (iii) the involuted phase (Enjolras \& Mulliken, 1997; Takahashi et al., 1994, Tan, 2001). These phases describe the gross morphological changes associated with tumour onset, growth, and subsidence.

In the early proliferative phase, the lesions are highly cellular and consist of syncytial masses of plump ECs with tiny lumens (Tan, 2001). Occasional mitotic figures can be seen but the nuclei do not exhibit pleomorphism (Figure 1.8-A). Later in the proliferative phase, vascular channels are less compact and capillary-sized lumens can be seen lined with plump, mitotically active ECs and pericytes (Figure 1.8-B) (Vikkula et al., 1998; Glukhova et al., 1991). Multilamination of the basement membrane (BM) and accumulation of other cellular elements including mast cells (MCs), macrophages, fibroblasts, plasma cells, and interstitial cells are also seen (Vikkula et al., 1998; Gonzalez-Crussi et al., 1991; Glowacki et al., 1982; Pasyk et al., 1984; Dethlefsen et al., 1986; Takahashi et al., 1994; Tan et al., 2004). As the haemangioma progresses to the involuting phase, the vascular lumens dilate, ECs flatten and the cellular elements are gradually replaced by fibro-fatty tissue, giving the haemangioma a lobular architecture (Figure 1.8-C). Progressive fibrosis is seen in the 
late involuting phase (Figure 1.8-D and -E). In the involuted phase, marked fibrosis and a few thick-walled channels lined with flat endothelium are seen (Figure 1.8-F). A fully involuted haemangioma contains few capillary-like feeding vessels, and possess draining veins with flattened endothelia that lie in a stroma of fatty tissue containing collagen and reticulin fibres. 

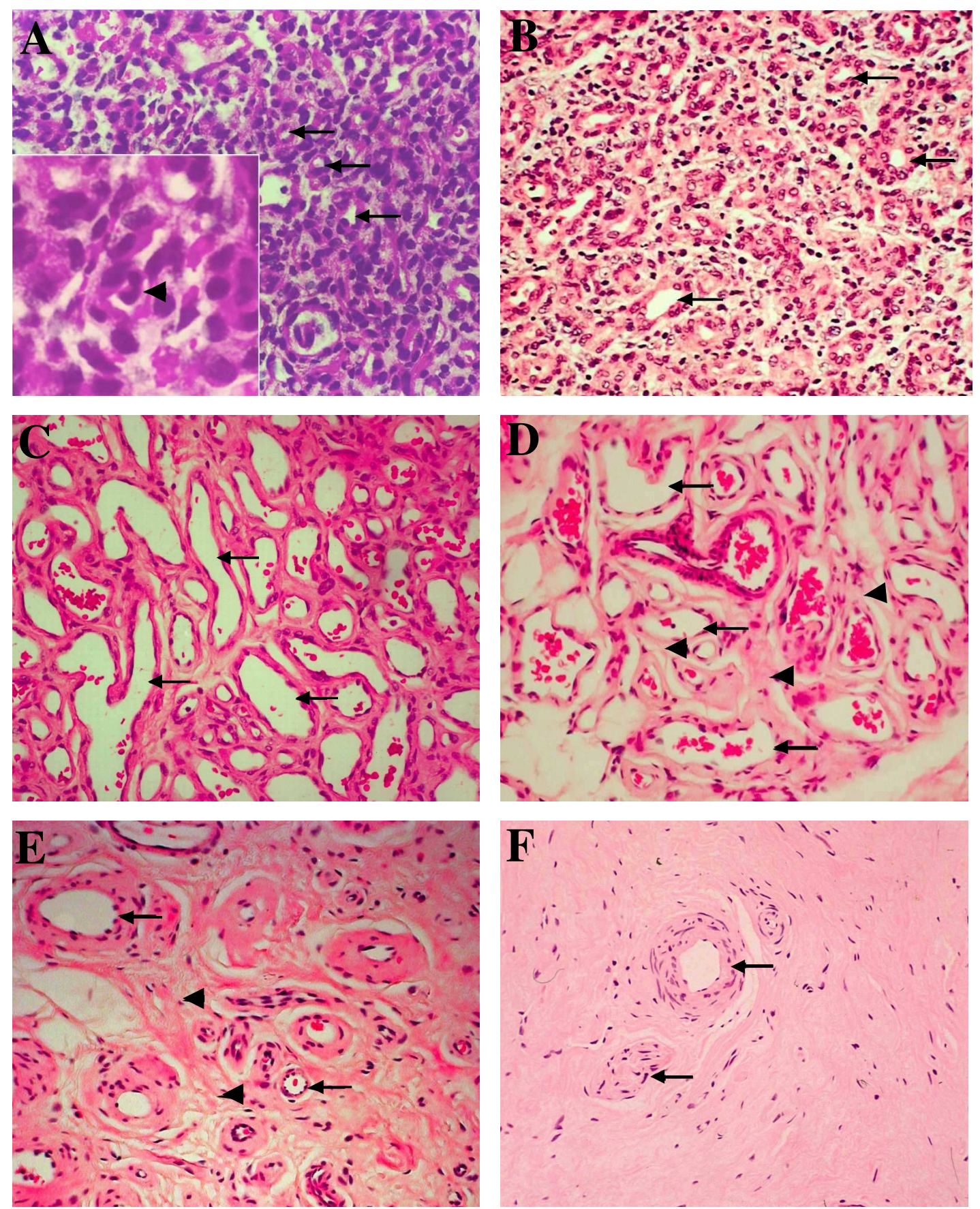

Figure 1.8: Histopathological features of haemangioma at different phases of development. A haemotoxylin and eosin (H\&E) stained haemangioma specimen from an 18-day old infant, showing proliferating endothelial masses with tiny lumens (arrows) (A). Occasional mitotic figures (insert: arrowhead) can be seen but nuclei do not exhibit pleomorphism (A insert). Later in the proliferative phase, vascular channels are less compact and capillary-sized lumens (arrows) can be seen lined with plump ECs in a specimen from a 5-month old child (B). In the early involuting phase, the vascular channels (arrows) are larger but there are still areas of endothelial proliferation (right upper corner) in this specimen from an 8-moth old child $(\mathbf{C})$. With further involution, the cellular elements are gradually replaced by fibro-fatty tissue (arrowheads). Progressive fibrosis is seen in this specimen taken from a 2 years and 9 months old child (D). Increasing fibrosis (arrowheads) with reduced cellularity occurs in the late involuting phase at 3 years and 6 months $(\mathbf{E})$. Fibrosis replaced the cellular elements and few thick walled channels remain, lined with flat endothelium (arrows) in a specimen from an 8 years and 6 months old child $(\mathbf{F})$. Original magnifications: $214 \mathrm{X}$ (insert: 640X) (Courtesy of Dr Swee.T.Tan). 
Studies have also shown that although all samples predominantly exhibit the histological features characteristic of either the proliferative, involuting or involuted phases, distinct areas displaying proliferation or varying degrees of involution are evident in each lesion. This indicates a continuous spectrum of structural changes during the natural progression of haemangioma (Tan, 2001) (Figure 1.9).

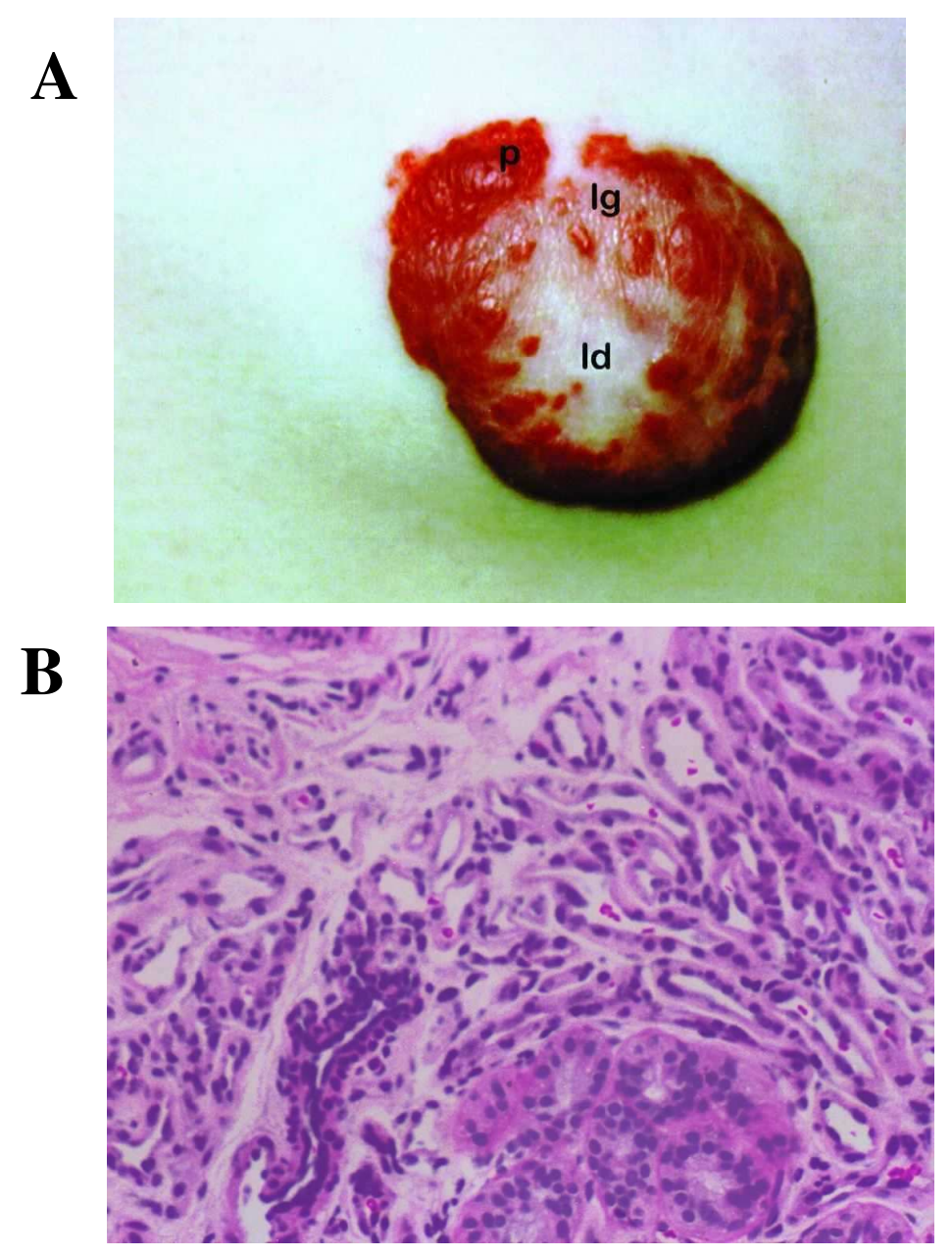

Figure 1.9: A haemangioma on the elbow of a 13-month old girl. The image in panel A, shows the 'Proliferative' (P), 'involuting' (Ig), and 'involuted' (Id) regions representing the different stages within a single lesion. Panel $\mathbf{B}$ shows an H\&E stained tissue section of the biopsy taken from A clearly showing defined regions of proliferation (lower right) and involution (upper left). Original magnification: 214X (Courtesy of Dr Swee.T.Tan). 


\subsection{CURRENT THEORIES ON THE ORIGINS OF HAEMANGIOMA}

\subsubsection{PLACENTAL ORIGIN}

North et al (2001) recently identified the expression of a distinct constellation of tissue-specific markers, co-expressed in both haemangioma and placental microvessels. They found that a set of placenta-associated vascular antigens including FcyRII and Lewis $\mathrm{Y}$ antigen (LeY) and placenta marker proteins including merosin and the glucose transporter-1 (GLUT-1) were expressed in 66 haemangioma lesions isolated from patients aged between 22 days and seven years.

It is now known that with the exception of GLUT-1 and merosin being present in brain microvessels, none of the other placenta-associated markers are expressed in the microvessels of normal skin. GLUT-1 is widely expressed early in development, but foetal endothelial GLUT-1 expression rapidly disappears except in microvessels of developing neuroepithelial tissues (Froehner et al., 1998; Harik et al., 1993; Mantych et al., 1993a ; Mantych et al., 1993 ). In the mature brain, a component of the blood brain barrier, necessitates high GLUT-1 expression for adequate blood:brain glucose transport (Pardridge et al.,1990). In the placenta, GLUT-1 expression is required throughout gestation for adequate maternal-foetal glucose transfer (Jansson et al., 1993), independent of developmental stage (Hahn et al., 1995). Thus, North et al (2001) proposed that high GLUT-1 expression by haemangioma may permit tumour growth by providing fuel for mitosis.

Intense expression of merosin in a broad, continuous, band-like pattern encircling lesional capillaries in GLUT-1 positive haemangioma during all stages of proliferation and involution has been reported (Martin-Padura et al., 1995; North et al., 2001). Merosin expression is normally restricted to the nervous system, eyes (Villanova et al., 1996) and placenta (Voit et al., 1994). The merosin $\alpha 2$ chain, one of ten distinct 
laminin chains, is also present in certain nonvascular BM, including those of placental trophoblast, schwann cells, striated muscle and skin (Engvall et al., 1990; Leivo et al., 1988; Sewry et al., 1996). The heterogeneity of laminin subtypes in the BM and the highly restricted expression of merosin suggests tissue-specific and developmental stage-specific functional diversity. Merosin is essential for stability and survival of fused myoblasts in vitro, by inhibiting apoptosis (Vachon et al., 1996). Therefore, a role for merosin in the growth of haemangioma by inhibiting apoptosis has been suggested (North et al., 2001).

The persistent expression of LeY and FcyRII in the endothelium of haemangioma and placental vessels is of unclear functional significance. LeY is a glycosylated oligosaccharide, expressed on the cell surface in a tissue-specific and stage-specific manner. It is important in cell-cell recognition and adhesion during development. Its expression has been associated with malignant transformation and progression (Lloyd et al., 1987; Zhu et al., 1995; Wakabayashi et al., 1995) and also apoptosis (Hiraishi et al., 1993; Minamide et al., 1995). Fc $\gamma$ RII is a low affinity Fc receptor normally expressed in macrophages, Langerhan cells, platelets and other leukocytes (Sedmak et al., 1991; Pulford et al., 1986). Its restricted expression to placental endothelium and its ability to bind only immunoglobulins suggest that it may be uniquely advantageous in placenta perhaps assisting clearance of immune complexes and/or maternal-foetal immunoglobulin transport (Sedmak et al., 1991; Lang et al., 1993).

North et al (2001) suggested that this unique phenotype shared between haemangioma and the placental foetal microvessels may be due to haemangioma ECs that originated from either invading angioblasts and aberrantly differentiated towards the placental microvascular phenotype. Alternatively, the ECs could have come from embolised placental cells or precursors that were dislodged into foetal circulation during gestation or at birth. Given the previously identified increased incidence of 
haemangioma in infants exposed to chorionic villus sampling (CVS) (Burton et al., 1995), North et al (2001) suggested that embolic placental ECs, already committed to the placental phenotype (GLUT-1/merosin/LeY/FcRII) could have reached foetal tissues from the chorionic villi through the right to left shunts of normal foetal circulation. In addition, Marchuk (2001) suggested that the local placental injury that usually occurs in CVS could result in increased intravascular shedding of placental cells from the chorionic villi into the foetal circulation. Furthermore, if the embolus contained a single EC or only a small number of ECs, this would be compatible with the clonality results of Boye et al (2001) (see section 1.2.2.1.2, page 19).

In contrast, the findings of Bree et al (2001) did not support the hypothesis that the placental trophoblast was the cell of origin for haemangioma as they lacked a villous architecture and did not express known placental trophoblastic markers. These investigators found no immunoreactivity for the trophoblastic markers, including human placental lactogen, placental alkaline phosphatase, and cytokeratins 7, 8, and 17, in 12 haemangioma tissue specimens from patients aged five days to two years.

\subsubsection{EXPANSION OF ENDOTHELIAL CELLS IN HAEMANGIOMA:} AN INTRINSIC OR EXTRINSIC DEFECT?

The process of foetal vascular development and angiogenesis is complex with several receptor tyrosine kinases (RTKs) and their ligands, namely angiopoietin-1 (Ang-1), angiopoietin-2 (Ang-2) and VEGF as known modulators (Cohen, 2002). Alteration in this system, at either the molecular or chromosomal level, has been suggested to account for the unregulated growth of blood vessels seen in haemangioma. In this paradigm, the rapid growth of these tumours in the postnatal period may be explained by the loss of angiogenic inhibitors derived from the placenta or mother (North et al., 2002). This idea has lead to another hypothesis for the highly proliferating ECs seen in haemangioma as being derived from angioblasts, aberrantly switched to a 
placental phenotype by somatic mutations and/or other abnormal inductive influences (an intrinsic defect). Furthermore, Bielenberg et al (1999) found that the epidermis overlying proliferating haemangioma showed altered expression of angiogenic factors. They suggested that the underlying defect may be extrinsic to the vascular ECs, which were responding to an aberrant exogenous growth signal (an extrinsic defect).

\subsubsection{AN INTRINSIC DEFECT?}

This theory suggests that the dramatic changes in endothelial behaviour in haemangioma may be caused by somatic mutations in a single EC which results in the disruption of normal controlled EC growth (Berg et al., 2001; Boye et al., 2001; Walter et al., 2002).

\subsection{Familial Studies}

Although current evidence points towards the sporadic nature of haemangioma, a small number of families have been identified, wherein haemangioma and other vascular lesions segregated as a highly penetrant, autosomal dominant trait. Familial studies have suggested that the genetic alteration may be a somatic mutation or a common predisposing allele with incomplete penetrance (Vikkula, 2000; Tan, 2001). Families with suggestive autosomal dominant inheritance with incomplete penetrance and variable phenotypic expression have been identified (Blei et al., 1998). These families represent, (i) subtypes of rare mutations causing autosominal dominantly inherited haemangioma; (ii) kindreds in which a common low penetrant mutation shows autosomal dominant pattern of inheritance; or (iii) families with random aggregation of a common genetic trait. In gene linkage studies involving three kindreds with familial haemangioma, Blei et al (1998) found linkage to the 5q chromosome. Walter et al (1999) further mapped this region to be 5q31-33. Candidate genes involved in blood vessel growth within this region include fibroblast growth factor-4 (FGF-4), plateletderived growth factor- $\beta$ (PDGF- $\beta$ ) and fms-related tyrosine kinase-4 (FLT-4). In 
addition, a subsequent study by Berg et al (2001) found evidence for loss of heterozygosity on chromosome $5 q(p<0.05)$ in three out of six haemangiomas. They suggested that the deletion of a gene or genes in this chromosomal region may be caused by an intrinsic endothelial alteration rather than an external stimulus and that this somatic mutation may be the cause of sporadic haemangioma.

\subsection{Haemangioma-derived Endothelial Cell Studies}

Boye et al (2001) purified ECs from proliferating haemangioma (HaemECs) obtained from eight female patients and found that these HaemECs were clonal. These authors identified a non-random $\mathrm{X}$ chromosome inactivation pattern for the human androgen receptor gene locus and proposed a clonal origin for these cells. In contrast, the non-endothelial stromal cells isolated from the same lesions, were not clonal. These results demonstrated that clonality was restricted to the ECs within the lesion. They hypothesised that EC clonality may be due to somatic mutations in the genes of the ECs but not in other cell types constituting haemangioma. These investigators also suggested that although stromal cells and the overlying epidermis of haemangioma exhibited properties, including aberrant expression of angiogenic factors (Bielenberg et al., 1999; Berard et al., 1997), the ECs would not be expected to develop as clones of a single founder cell if they were merely responding to an externally derived angiogenic signal.

Boye et al (2001) found that HaemECs exhibited increased proliferation and migration in response to VEGF as compared to normal ECs. Furthermore, VEGFinduced migration in HaemDECs was stimulated instead of being inhibited by the angiogenesis inhibitor, endostatin. They suggested that alterations in the signalling pathways downstream of the VEGF receptor in HaemDECs, may be the result of somatic mutations that converted the inhibitory effect of endostatin to one of stimulation.

Walter et al (2002) also identified significant skewing toward one allele in haemangioma lesions. These authors performed clonality assays on DNA isolated from 
haemangioma tissue subjected to gross dissection without the selective step of EC growth in culture and thereby avoided changes in cell composition that could occur with cell cultures.

Therefore, these studies provide strong support for the hypothesis that the clonal expansion and the abnormal proliferation of haemangioma is the result of one or more somatic mutations in key factors involved in angiogenesis. Although formal proof of this hypothesis awaits validation, Walter et al (2002) identified missense mutations in two VEGF receptors (VEGFRs), VEGFR-2 and VEGFR-3, which were only found in the haemangioma tissue and not in the patient matched normal skin. Therefore, these authors suggested that EC proliferation was the result of deregulated VEGF signalling.

There have also been reports on experimentally induced haemangioma lesions in mice. Williams et al (1989) showed that the injection of mouse ECs expressing the polyoma middle-T oncogene into adult mice resulted in the development of tumours that resembled haemangioma. These lesions were composed of host-derived ECs, apparently recruited by the injected oncogenic cells (Williams et al., 1989). In contrast, Boye et al (2001) showed no evidence for additional EC recruitment by the original tumour ECs.

Li et al (2003) recently identified CD146 to be a possible marker for haemangioma ECs due to its reduced expression in these cells compared to the surrounding pericyte-like cells, and also to normal ECs. Studies have also shown that the ECs in haemangioma showed altered expression of several other angiogenesisrelated molecules, such as E-selectin, TIE-2, VEGF, FGF-2 and insulin growth factor (IGF) (Kraling et al., 1996; Yu et al., 2001; Ritter et al., 2001). These findings together with the down-regulation of CD146 expression suggest that haemangioma may be a consequence of a genetic alteration in haemangioma ECs (Li et al., 2003). 


\subsubsection{AN EXTRINSIC DEFECT?}

The extrinsic hypothesis invokes a defect in the local environment, which produces a signal or stimulus that causes haemangioma. For example, Berard et al (1997) found that stromal cells isolated from proliferating haemangioma and cultured in vitro released VEGF. Transcripts for the VEGFRs, VEGFR-1 and VEGFR-2 are abundantly expressed in proliferating haemangioma (Yu et al., 2001), suggesting the high expression of these receptors in these lesions. Therefore, increased VEGF produced by stromal cells could accelerate the growth of haemangioma.

Bielenberg et al (1999) found a dramatic alteration in expression of IFNs in the epidermis overlying haemangioma. Compared with the epidermis of normal skin, there was a dramatic down-regulation of IFN $\beta$ in the epidermis overlying proliferating phase haemangioma. However, gradual restoration of IFN $\beta$ was observed as the lesion progressed to the involuting and involuted phases, with near-normal levels detected by five to 11 years of age. This dramatic down-regulation of IFN $\beta$ expression coinciding with the proliferating phase of haemangioma provides another example of a possible extrinsic alteration in haemangioma.

\subsubsection{THE ANGIOGENESIS CONCEPT}

The concept that tumours are 'angiogenic dependent' was first proposed by Folkman and suggests new insights into the life cycle of haemangioma (Folkman, 1997; Folkman \& Cotran, 1976). Angiogenic molecules are known to act on ECs and pericytes to initiate the formation of capillary networks (Risau et al., 1988; Folkman \& Klagsburn, 1987; Zetter, 1988; Breier et al., 1992; Pepper et al., 1992; Montesano et al., 1986; Mignatti et al., 1989). This process is tightly regulated by inhibitors of EC growth, which help to maintain the microvasculature in a quiescent state under physiological conditions (Mignatti et al., 1989; Risau, 1995; Risau, 1997; Montesano et $a l .$, 1986) 
The angiogenesis concept proposes that capillaries are normally prevented from growing by physiological regulation of angiogenic factors. These factors are found in neoplastic tissue but are also present in normal tissue where neovascularisation does not occur (Folkman, 1996; Risau et al., 1988; Montesano et al., 1986). Release of stored angiogenic factors occurs in short bursts of capillary proliferation during wound healing, and in female, ovulation and menstruation (Augustin et al., 1995; Klauber et $a l .$, 1997). The rapidly growing haemangioma is reminiscent of capillary proliferation seen during wound healing and the neovascularisation associated with tumour growth. Amongst the plethora of angiogenic regulators, VEGF, VEGFRs, Ang-1, Ang-2 and their RTKs, TIE-1 and TIE-2, play crucial roles in maintaining the balance between vascular growth and regression (Ferrara et al., 1992; Fong et al., 1995; Shalaby et al., 1995; Suri et al., 1996; Carmeliet et al., 1996; Hanahan, 1997; Maisonpierre et al., 1997). Other factors such as FGF-2, transforming growth factor- $\beta$ (TGF- $\beta$ ), PDGF and interleukin-6 (IL-6) are highly relevant as is the extracellular matrix (ECM) (Folkman \& Klagsbrun, 1987; Tomanek and Schatteman, 2000; Haas et al., 1999). Some of these factors are now known to be involved in both the proliferation and involution of haemangioma (Takahashi et al., 1994; Jang et al., 1998; Chang et al., 1999; Bielenberg et al., 1999) and are discussed in section 1.4, page 30.

\subsection{CELLULAR COMPONENTS OF HAEMANGIOMA}

\subsubsection{ENDOTHELIAL CELLS}

The hallmark of a growing haemangioma is the proliferating ECs forming syncytial masses with or without luminal formation (Tan et al., 2000a). Occasional mitotic figures are seen but the nuclei do not exhibit pleomorphism (Mulliken, 1988). As the lesion progresses, the later stages of the proliferating tumour consists of less compact vascular channels and capillary-sized lumens lined with highly cellular plump, proliferating ECs organised into lobules within a limiting membrane of reticulin fibres 
(Mulliken, 1988). Confocal microscopy showed the three-dimensional images of these densely disorganised tortuous networks of vessels in proliferating haemangioma compared with normal tissues (Takahashi et al., 1994).

The ECs within the growing lesion are proliferating as identified by tritiated thymidine autoradiography (Mulliken \& Glowacki, 1982) and by immunohistochemical staining for proliferating cell nuclear antigen (PCNA) (Tan et al., 2000a; Tan, 2001). Studies showed that the majority of ECs in the proliferative and early involuting phase expressed PCNA but this number decreased with the on-going involution of haemangioma. PCNA staining in pericytes and MCs during the proliferative have also been identified (Hasan et al., 2000a).

Electron microscopic (EM) studies showed that the ECs in haemangioma exhibited characteristics of intracellular activity such as a convoluted nuclear membrane, swollen mitochondria, membranes of rough endoplasmic reticulum, and clusters of free ribosomes (Dethlefsen et al., 1986; Hopfel-Kreiner, 1980). The luminal surface of these ECs exhibited thin projections whereas the basal side had thicker, club like projections (Dethlefsen et al., 1986; Mulliken, 1988). In contrast, the lumen in involuting haemangioma contained EC remnants, often lined by only one or two ECs with signs of endothelial discontinuity and vessel degradation. EM of involuted haemangioma showed that the vessels had reverted to a more normal architecture and contained single layers of ECs surrounded by pericytes. From these findings Mulliken (1988) suggested that with development, haemangioma becomes more organised, as the lobular components were separated by fibrous septae that contained large calibre feeding and draining vessels.

ECs express specific markers that are used in their identification in vivo and in culture. Some of these include von Willebrand Factor (vWF), CD31 (Belloni \& Tressler, 1990; DeLisser et al., 1994), CD34 (Krause et al., 1996), VEGFR-1 and 
VEGFR-2 (Rettig et al., 1992; Eichmann et al., 1993; Quinn et al., 1993; Peters et al., 1993; Mustonen \& Alitalo, 1995).

Studies have shown that although the ECs in haemangioma expressed CD31, vWF and urokinase in all phases, the levels of expression varied between phases. Takahashi et al (1994) showed that CD31 expression was significantly higher in the involuting phase compared to the involuted phase and vWF expression was higher in the proliferative and involuting phases but not in the involuted phase. Furthermore, Tan et al $\left(2001_{\mathrm{a}}\right)$ found that not every vessel in the involuted phase expressed these proteins. These findings are consistent with the current knowledge of these markers, i.e., they are not always expressed by ECs (Parums et al., 1990; Kuzu et al., 1992). Irregular vWF expression has also been identified in capillaries and tumour vessels (Garlanda \& Dejona, 1997).

The glycoprotein, E-selectin is an EC-specific leukocyte adhesion molecule, involved in angiogenesis and is important in capillary tube formation (Nguyen et al., 1993). High expression of E-selectin and VE-cadherin has been identified in the proliferative phase of haemangioma (Kraling et al., 1996).

Expression of other endothelial markers such as CD34, intercellular adhesion molecule 1 (ICAM-1) and the very late antigen (VLA) integrin have also been identified in haemangioma. Martin-Padura et al (1995) showed that these markers were expressed in haemangioma to similar levels as in normal ECs. Therefore, they suggested that the abnormal EC proliferation in the proliferating lesion was more related to the release of local factors and fibrinolytic agents, rather than an altered endothelial phenotype.

GLUT-1 has recently been described as a marker for haemangioma. North et al (2000) showed that the ECs of haemangioma from all phases expressed GLUT-1 while GLUT-1 immunoreactivity was absent in other vascular lesions. 
Li et al (2003) described the transmembrane glycoprotein, CD146 as another marker for haemangioma. This molecule is also considered a marker for ECs (Bardin et al., 1996). It is involved in cell adhesion (Bardin et al., 2001) and in the regulation, paracellular permeability and in signal transduction of ECs (Bardin et al., 2001; Anfosso et al., 1998; Anfosso et al., 2001). Li et al (2003) found significantly low CD146 expression by the ECs of haemangioma biopsies from all phases of development. In contrast, the pericyte-like cells surrounding the endothelium in haemangioma showed strong immunoreactivity. Therefore, they suggested that CD146 expression may be useful in distinguishing HaemDECs from normal ECs (Li et al., 2003). Their studies also showed that CD146 expression differed from previously described markers such as merosin, GLUT-1, FcyRII and LeY (North et al., 2001; North et al., 2000), in that, the reduced CD146 expression in haemangioma tissue was maintained even after the HaemDECs were isolated and cultured in vitro ( $\mathrm{Li}$ et al., 2003). On the other hand, while GLUT-1 and FcyRII were strongly expressed in fresh or formal in fixed haemangioma tissue, their levels of expression became indistinguishable from that of normal ECs in culture (Li et al., 2003).

\subsubsection{MAST CELLS}

MCs are highly heterogeneous in terms of morphology, function and metabolic products (Katz et al., 1991; Qu et al., 1995). These cells are implicated in a variety of biological responses. They phagocytose, process antigens, produce cytokines and release a variety of preformed (e.g., histamine, proteoglycans and proteases) and newly formed (e.g., leukotrienes and prostaglandins) physiological mediators (Frangogiannis et al., 1998). They carry an array of adhesion molecules, immune response receptors and other surface molecules that allow them to react to multiple specific and nonspecific stimuli (Metcalfe et al., 1997). MCs are involved in immediate hypersensitivity reactions, host responses to parasites and neoplasms, immunologically non-specific inflammatory and fibrotic conditions, angiogenesis, tissue remodelling and wound 
healing (Gordon et al., 1990; Meininger \& Zetter, 1992; Schwartz, 1994; Metcalfe et al., 1997).

MCs are abundant within haemangioma tissues (Mulliken, 1988; GonzalezCruss et al, 1991; Glowacki et al., 1982; Pasyk et al., 1984). While Gonzalez-Cruss et al (1991) and Glowacki et al (1982) found MCs to be abundant in the proliferative phase, Takahashi et al (1994) and Tan et al (2000a) showed that they were most prominent in the involuting phase, less in the involuted phase and least in the proliferating phase. Tan et al $\left(2000_{\mathrm{a}}\right)$ demonstrated that the proportion of proliferating MCs was highest in the proliferative and lowest in the involuted phase.

All MCs contain the specific glycosylated serine protease, tryptase $\left(\mathrm{MC}_{\mathrm{T}}\right)$ while a subgroup of MCs also express neutral protease like chymase $\left(\mathrm{MC}_{\mathrm{TC}}\right)$ (Schwartz, 1994; Metcalfe et al., 1997). Tan et al (2000a) showed that although haemangioma biopsies contained both $\mathrm{MC}$ types, the proportion of $\mathrm{MC}_{\mathrm{TC}}$ increased from the proliferative through the involuting to the involuted phases (Tan et al., 2000a). This correlation of $\mathrm{MC}_{\mathrm{TC}}$ with ongoing involution, paralleled that of progressive deposition of the $\mathrm{ECM}$, indicated by increasing fibrosis and fatty deposition (Ruger et al., 1994; Tan et al., $\left.2000_{\mathrm{a}}\right)$. In addition, Hasan et al $\left(2000_{\mathrm{b}}\right)$ found that although MC numbers increased four-fold following steroid treatment, the predominance of the histamine phenotype was unaltered.

\subsubsection{ANGIOGENIC ROLES FOR MAST CELLS IN HAEMANGIOMA}

The promotion of neovascularisation by MCs products, such as FGF-2 has been known for some time (Norrby et al., 1990; Norrby et al., 1994). MC accumulation has been correlated with normal and pathological angiogenesis (Meininger \& Zetter, 1992). It has been suggested that MC products can act directly on ECs to stimulate their migration and/or proliferation or may act by degrading connective tissue matrix to provide space for neovascular sprouts (Meininger \& Zetter, 1992). Qu et al (1995) identified positive staining for FGF-2 within MCs of the proliferative phase but not in 
the later phases of haemangioma and suggested that MCs may contribute to the growing haemangioma by releasing this polypeptide (Qu et al., 1995)

An anti-angiogenic role for MCs in haemangioma has also been suggested. Tan et al (2004) postulated that although MCs were implicated with the development of haemangioma, MC secretion of angiogenic inhibitors such as IFN $\alpha, \operatorname{IFN} \beta$, IFN $\gamma$, and TGF- $\beta$ may be involved in the regression of these lesions. However, they proposed that the involvement of MCs in the life cycle of haemangioma was complex and involved stimulators of angiogenesis in the proliferative phase but inhibitors in later phases.

\subsubsection{ROLE OF MAST CELLS IN APOPTOSIS}

Apolipoprotein $\mathbf{J}$ (ApoJ) is implicated in a variety of biological processes including membrane remodelling and apoptosis (Wong et al., 1994). Hasan et al (2000a) showed ApoJ staining within the MCs of haemangioma by tryptase and alcian bluesafranin (Csaba) double staining. They showed that expression of ApoJ in MCs was localised to the cytoplasmic granules which were also detected in the adjacent EC and capillary lumens. During the proliferative phase, only a small proportion of MCs were positive for ApoJ, while the proportion of ApoJ positive MCs increased with the involution of haemangioma. Due to the localisation of the ApoJ protein in MCs granules, these authors proposed that this protein may be synthesised and/or released by MCs. They also found ApoJ positive granules in the ECs and capillary lumens. Therefore, these authors suggested that MC secretion of ApoJ, an apoptotic modulator, may be involved in haemangioma involution.

\subsubsection{OTHER CELLULAR COMPONENTS}

\subsubsection{MACROPHAGES AND MONOCYTES}

All adult haematopoietic cells originate in the bone marrow and are derived from pluripotent haematopoietic stem cells (Buckland, 2002). Two major lineages then diverge in the bone marrow into the lymphoid and myeloid lineages. The common 
lymphoid progenitor differentiates into $\mathrm{B}$ and $\mathrm{T}$ cells and the common myeloid progenitor differentiates to form the cells of the granulocyte/macrophage and the megakaryocyte/erythroid lineages including monocytes and macrphages (Akashi et al., 2000). Monocytes then exit the peripheral circulation, enter tissues and differentiate into several cell types with varying functions. Macrophages, the major tissue-resident phagocytic cells of the immune system, are derived from monocytes (Rutherford et al., 1993; Sunderkotter et al., 1994).

Tissue macrophages are important mediators of physiological and pathological angiogenesis (Folkman \& Klagsbrun, 1987; Isik et al., 1996; Dipietro \& Polverini, 1993a ; Dipietro \& Polverini, 1993 ; Leibovich et al., 1987; Leibovich \& Wiseman, 1988; Thakral et al., 1979; Hunt et al., 1984; Polverini \& Leibovich, 1984; Koch et al., 1986; Leung et al., 1989). They are important in the induction of new blood vessel growth during wound repair, inflammation and tumour growth (Sunderkotter et al., 1994; Leibovich et al., 1987). They produce soluble mediators such as FGF-2, TGF- $\alpha$ and $-\beta$, PDGF and VEGF (Dipietro \& Polverini, 1993b). Once activated, macrophages can also modulate the ECM composition and induce EC migration and proliferation (Sunderkotter et al., 1994; Porras-Reyes et al., 1991; Sunderkotter et al., 1994).

Tan (2001) suggested an angiogenic role for macrophages in haemangioma. This was supported by results that showed increased macrophage infiltration during the proliferative phase but reduced numbers in the involuting and involuted phases.

Monocyte infiltration in haemangioma is suggested to be due to release of cellderived chemotactic factors (Isik et al., 1996). One candidate glycoprotein is the monocyte chemoattractant protein-1 (MCP-1) that is specific for monocytes and macrophages in vitro (Sunderkotter et al., 1994; Yoshimura et al, 1989; Rollins et al., 1990). ECs, SMCs, fibroblasts and macrophages can produce MCP-1 (Isik et al., 1996; Koch et al., 1992). Isik et al (1996) showed increased expression of MCP-1 mRNA by 
ECs, SMCs and macrophages in the proliferative phase of haemangioma. They also showed that nine out of ten involuting lesions did not express mRNA for this molecule.

Ritter et al (2006) identified that haematopoietic cells of the myeloid lineage constituted a significant proportion of the cells in hemangiomas of the proliferative phase and suggested that these cells may be involved in promoting haemangioma possibly through expression of cytokines in response to hypoxia/ischemia.

These authors also observed that haemangioma ECs co-expressed a number of myeloid markers, demonstrating yet another unique feature of these cells. From these findings, Jia et al (2007) hypothesised that monocytes may be the precursors of haemangioma ECs.

\subsubsection{PERICYTES}

Interactions between ECs and pericytes are known to be important in the maintenance of vascular integrity (Furchgott \& Zawadski, 1980). Pericytes control EC proliferation (Nehls \& Drenckhahn, 1991; Orlidge \& D’Amore, 1987) and serve as guiding structures, aiding the outgrowth of ECs (Nehls et al., 1992). Pericytes also regulate capillary blood flow via a contractile mechanism (Paweletz \& Knierim, 1989; Tilton et al., 1979a; Tilton et al., 1979b). Although pathological neovascularisation is associated with pericyte loss (Kuwabara \& Cogan, 1963), failure of pericyte development during the angiogenic process results in the regression of the newly formed blood vessels (Lindahl et al., 1997; Hirschi et al., 1999).

Plump mitotically active pericytes line the microvessels of proliferating haemangioma (Takahashi et al., 1994; Mulliken, 1988; Tan, 2001). Takahashi et al (1994) localised smooth muscle actin (SMA) to the cytoplasm of pericytes in all phases of haemangioma. They found that SMA expression was significantly higher in the involuting phase than in the proliferative and involuted phases. From their studies, Takahashi et al (1994) proposed an inhibitory role of pericytes in haemangioma. 


\subsubsection{PROTEASES}

Takahashi et al (1994) showed increased expression of type IV collagenase during the proliferative phase of haemangioma. The expression of urokinase was also significantly higher during the proliferative and involuting phases than the involuted phase. High molecular weight (>150 kDa) metallomatrix proteases (MMPs) have been detected in the urine of patients with proliferating haemangioma (Marler et al., 2000). These investigators suggested that proteolysis categorised by MMPs and type IV collagenase was required to provide space for the proliferating cells in haemangioma (Takahashi et al., 1994; Marler et al., 2000) and that the differentiating cells signalled an influx of MCs along with the autocrine induction of tissue inhibitor of metalloproteinase-1 (TIMP-1) expression. Furthermore, Takahashi et al (1994) showed that TIMP-1, while not detected during the proliferative phase, was readily detected in the pericyte cytoplasm of microvessels during the involuting phase.

TIMP-1 is well recognised for its role as an inhibitor of endothelial invasion into the yolk sac membrane and as a negative regulator of angiogenesis, by inhibiting angiogenic proteases, such as type IV collagenase (Takahashi et al., 1996; Mignatti et al., 1989; Takigawa et al., 1990). Therefore, Takahashi et al (1994) suggested that TIMP-1 expression along with the presence of MC secreted angiogenic inhibitors, such as IFNs and TGFs, (Friesel et al., 1987; Gordon et al., 1990; Antonelli-Orildge et al., 1989) can result in the down-regulation of haemangiogenesis.

\subsection{CYTOKINES AND GROWTH FACTORS IN HAEMANGIOMA}

\subsubsection{VASCULAR ENDOTHELIAL GROWTH FACTOR}

VEGF is an important angiogenic cytokine that induces EC proliferation and differentiation (Risau W., 1995; Brown LF et al., 1996). Sources of VEGF include 
tumour cells, ECs and MCs. These cells rapidly release VEGF, apparently from a preformed pool (Boesiger et al., 1998). Elevated serum levels of VEGF have been detected in patients with proliferating haemangioma compared with those with involuted lesions (Zhang et al., 2005). Although in situ hybridisation and DNA microarray analysis showed low levels of VEGF mRNA in haemangioma (Ritter et al., 2001; Yu et al., 2001), immunohistochemical studies confirmed the presence of VEGF protein within the cytoplasm of ECs and pericytes during the proliferative phase (Takahashi et al., 1994; Tan, 2000a $)$. Furthermore, Hasan et al (2000b) found that VEGF transcript levels were not altered in steroid-induced involuting haemangioma in vivo and suggested that steroids act by modulating other angiogenic factors.

\subsubsection{VASCULAR ENDOTHELIAL GROWTH FACTOR RECEPTORS}

VEGF interacts with cells mainly through two RTKs, VEGFR-1 and VEGFR-2. VEGFR-1 signalling is essential for vascular remodelling during the early stages of embryogenesis (Fong et al., 1995; Carmeliet et al., 1996 ). VEGFR-2 is expressed primarily on ECs and plays a pivotal role in angiogenesis, by inducing EC differentiation and vasculogenesis in both normal and tumour tissue (Millauer et al., 1996; Quin et al., 1993). VEGFR-2 signalling also regulates proliferation and survival of ECs (Zachary \& Gliki, 2001). While Brown et al (1996) showed high levels of VEGFR-1 and VEGFR-2 mRNA in proliferating haemangioma but not in involuting haemangioma, Yu et al (2001) showed that HaemDECs expressed increased mRNA levels for these receptors when in culture.

\subsubsection{TIE RECEPTORS AND THEIR ANGIOPOIETIN LIGANDS}

High expression of TIE-1, TIE-2 and Ang-2 but low expression of Ang-1 has been identified in HaemDECs (Yu et al., 2001). They showed that TIE-2 mRNA and protein was up-regulated in response to Ang-1 in HaemDECs which also correlated 
with increased survival of these cells. The authors confirmed increased TIE-2 expression in proliferating haemangioma by in situ hybridisation.

Yu et al (2001) also showed that Ang-2 mRNA was down-regulated in response to serum in HaemDECs, but not in normal ECs. They suggested that the regulation of the Ang/TIE system was altered in haemangioma. In addition, a mutation in the TIE-2 gene has been detected in haemangioma (Wang et al., 2004).

\subsubsection{FIBROBLAST GROWTH FACTOR-2}

The production and metabolism of FGF-2 is considered to be involved in the pathogenesis of haemangioma (Takahashi et al., 1994; Bielenberg et al., 1999; Tan, 2001). Studies have shown increased expression of FGF-2 mRNA and protein in the proliferative phase of haemangioma (Takahashi et al., 1994; Chang et al., 1997). In addition, Tan (2001) identified positive staining for FGF-2 in MCs of both the proliferating and involuting phases. It has been suggested that FGF-2 may be synthesised by MCs and/or by other cells and stored in MCs (Tan, 2001). In addition, Hasan et al (2000) showed that the high urinary levels of FGF-2 noted in individuals with proliferating haemangioma (Dosquet et al., 1998) was unaltered following successful steroid treatment as were the levels of FGF-2 mRNA transcripts within the lesion.

\subsubsection{INSULIN GROWTH FACTOR-2}

Another recently identified potential regulator of haemangioma growth is IGF-2. DNA microarray studies showed high expression of IGF-2 mRNA in proliferative lesions but low levels in involuting lesions. IGF-2 may be a reasonable putative regulator of haemangioma proliferation. As it is a known mitogen, IGF-2 can suppress apoptosis and regulate angiogenesis (Volpert et al., 1996). Ritter et al (2002) showed that IGF-2 protein expression was localised to the tumour vessels and that the addition of recombinant IGF-2 protein stimulated vascular sprouting from explanted 
haemangioma tissue. They also found that that IGF-2 expression was more correlated with the proliferative phase of haemangioma than VEGF. These authors suggested that high IGF-2 levels could increase expression of VEGF, thereby resulting in an increasingly favourable local environment for EC proliferation.

These results support a placental origin of haemangioma since IGF-2 is highly expressed and is important during embryonic development (Grupen et al., 1997; Demeestere et al., 2004). Furthermore, as IGFs induce IFN $\gamma$ expression (Tu et al., 1999), Ritter et al (2001) suggested that sustained levels of IGFs could result in a shift in the balance of regulators in favour of involution.

\subsubsection{OTHER FACTORS}

\subsubsection{PLATELET DERIVED GROWTH FACTOR, TRANSFORMING GROWTH} FACTOR- $\beta$ AND INTERLEUKIN-6

Hasan et al (2000 $)$ identified decreased transcription of PDGF, TGF- $\beta 1$, TGF$\beta 2$ and IL-6 mRNA following successful steroid therapy for haemangioma. Therefore, these authors suggested that the angiostatic effects of steroids were in part due to the down-regulation of these factors.

\subsection{EXTRACELLULAR MARKERS}

The attachment of cells to the ECM is crucial for tissue integrity. Cells attach either directly to components of the collagen-rich interstitial matrix or to the BM, a more distinct sheath of the ECM that surrounds many kinds of tissue (Gumbiner BM, 1996). Mulliken (1988) found that multilaminated BMs which is a hallmark of a haemangioma in the proliferative phase, persisted through to the involuted phase.

Jang et al (1998) showed that in addition to growth factors, the ECM composition of a growing haemangioma was different to those undergoing spontaneous involution (see section 1.5.4, page 35). 


\subsubsection{INTERSTITIAL MARKERS}

Tan et al $\left(2000_{\mathrm{a}}\right)$ showed that the interstitial collagen types I, III and V (Col-I, III, -V) were present in the multilaminated BM, in the perivascular and in the stromal regions of haemangioma in all phases. They also found that collagen deposition increased in the stromal area with progression of haemangioma. These authors suggested that this pattern of collagen deposition was concomitant with the decreasing cellular content but increasing fibrotic nature of the later phases (Tan et al., 2000a).

\subsubsection{BASAL LAMINA MARKERS}

\subsubsection{COLLAGEN TYPE IV}

Tan et al $\left(2000_{\mathrm{a}}\right)$ showed that collagen type IV protein was present throughout the multilaminated $\mathrm{BM}$ in all three phases of haemanigoma. In contrast, Ritter et al (2002) identified increased expression of collagen type IV mRNA and protein during the proliferative phase which decreased in the involuting phase. These authors suggested that the reduced levels of collagen type IV in the involuting and involuted phases are an effect, rather than a cause of involution (Ritter et al., 2002).

\subsubsection{LAMININS}

11 different heterotrimeric assembly forms of laminin are known, each consisting of a unique combination of $\alpha, \beta$ and $\gamma$ chains (Aumailley \& Smith, 1998; Tan, 2001; Tan et al., 2000a).

Tan et al (2000a) showed the presence of $\alpha_{1}, \beta_{2}$ and $\gamma_{1}$ chains but the absence of the $\alpha_{2}, \beta_{1}$ and $\gamma_{2}$ in the $\mathrm{BM}$ of microvessels in all three phases. From these findings together with the presence of the basement membrane-specific, heparan-sulphate proteoglycan perlecan (Noonan \& Hassell, 1993) and the three-chain partially helical molecule collagen type IV (Sado et al., 1998) in all phases of haemangioma, the authors concluded that the BM of haemangioma contained the same major constituents as normal vascular BMs. 


\subsubsection{COLLAGEN TYPE VIII}

Collagen type VIII (Col-VIII) has been detected in the ECM of haemangioma in all phases as well as within the MCs (Tan et al., 2000a). Its intracellular localisation to MCs only in the early proliferative phase led to the hypothesis that Col-VIII may be synthesised by MCs during this phase but secreted into the pericellular and stromal region during involution (Tan et al., 2000a). Furthermore, Tan et al (2004) showed that the addition of Col-VIII antibodies to haemangioma cultures resulted in the disruption of newly developed capillary-like vessels. These results provide evidence for a functional role of Col-VIII in haemangioma.

\subsubsection{VITRONECTIN}

Plasma-borne molecules include fibronectin and vitronectin. Jang et al (1998) found that only vitronectin showed a marked difference in the deposition pattern in haemangioma. They found that haemangioma in the proliferative phase had extensive deposition of vitronectin in the subendothelial space, while those in the involuting phase did not. Furthermore, the vessels located in the adjacent normal dermis lacked vitronectin deposition. Therefore, these authors suggested that vitronectin deposition may serve as a marker for an actively proliferating lesion.

\subsection{CELL ADHESION MOLECULES}

\subsubsection{INTEGRINS}

High expression of the cell adhesion molecules, the $\alpha \mathrm{v} \beta 3$ and $\alpha 5 \beta$ integrins, have been reported in proliferating haemangioma with decreased abundance in the involuting and involuted phases (Ritter et al., 2002; Nguyen et al., 2004). Nguyen et al (2004) found that involuting lesions strongly expressed another integrin, $\beta 4$. They showed that while untreated proliferating lesions lacked $\beta 4$ expression, proliferative lesions treated with IFN $\alpha$ re-expressed $\beta 4$. This re-expression clinically corresponded to 
the involution of haemangioma. Therefore, Nguyen et al (2004) suggested that both integrin molecules were involved in transducing mitogenic signals from ECM to the cell interior, which would either stimulate or inhibit proliferation of haemangioma.

\subsection{THE INVOLUTION OF HAEMANGIOMA}

The most curious biological characteristic of haemangioma is its spontaneous involution. Apoptosis is considered an important regulatory event in the involution of haemangioma (Razon et al., 1998; Iwata et al., 1996; Mancini \& Smoller, 1996). In haemangioma, apoptosis begins prior to one year of age, increases five-fold during the involuting phase, and reaches a maximum at two years (Razon et al., 1998). Iwata et al (1996) showed co-expression of the apoptosis-related antigen, LeY (also considered a tumour associated antigen) with the endothelial markers vWF, CD31 and CD34 and confirmed that at least some of the apoptotic cells in haemangioma are ECs.

\subsubsection{APOPTOTIC PROTEINS IDENTIFIED IN HAEMANGIOMA}

\subsubsection{THE BCL-2 FAMILY PROTEINS}

Bcl-2 and Bax protein expression have been implicated in the pathogenesis of haemangioma. Wang et al (2003) showed that Bax expression was higher in haemangioma compared to other vascular malformations and normal skin $(p<0.01)$ and that Bcl-2 was negative in all phases. However, other investigators have reported Bcl-2 expression in the proliferative phase and Bax in the involuting phase (Nakamura, 2000; Cheng et al., 1999; Zhang et al, 2003; Dyduch et al, 2004).

Mancini \& Smoller (1996) showed decreased Bcl-2 expression with age of the tumour. They found that the decrease in proliferation and Bcl-2 expression was detected much later in lesions, which exhibited a higher degree of vascularisation. Therefore, they suggested that such lesions would undergo longer periods of growth before entering the involuting phase. 


\subsubsection{APOLIPOPROTEIN J}

ApoJ is a secreted glycoprotein for which many functions have been proposed (Jenne et al., 1992) including apoptosis (Wong et al., 1993). It is consistently detected in apoptotic cell death paradigms such as the de novo regression of the prostate and other hormone-dependent tissues and tumours (Tenniswood et al., 1992; Wong et al., 1993; French et al, 1993; Wong et al, 1994) and in the involuting mammary gland (Lee et al., 1996).

Hasan et al $\left(2000_{\mathrm{a}}\right)$ found increased expression of ApoJ mRNA in involuting and involuted haemangioma while expression was undetected in proliferative lesions. Immunohistochemical analysis demonstrated increased positive granular staining for ApoJ localised to the MCs and the flattened endothelium of the involuting phase. However, the surrounding fibrofatty tissue was devoid of ApoJ. However, Tan, (2001) found that increased ApoJ expression was not associated with steroid-induced involution of haemangioma.

\subsubsection{MITOCHONDRIAL CYTOCHROME B}

Mitochondrial genes are associated with cell senescence and are proposed to play an important role in apoptosis (Zamzami et al., 1997). Hasan et al (2001) were the first to report increased expression of mitochondrial cytochrome $\mathrm{b}$ (mt Cyt $b$ ) in spontaneous and steroid-induced involution of haemangioma. They also found that expression was low or completely absent in proliferative and involuted samples.

\subsection{VASCULAR MORPHOGENESIS AND MAINTENANCE}

\subsubsection{THE MAMMALIAN VASCULAR SYSTEM}

The mammalian vascular system is composed of interconnected channels that distribute oxygenated blood via arteries and capillaries (Sadler, 1985). These vessels 
consist of three basic layers, viz., intima, media and adventitia (Figure 1.10) (Schoen \& Cotran, 1999). The intima is the innermost layer consisting of a single layer of ECs and a thin underlying layer of connective tissue called the internal elastic lamina. The media is mainly composed of SMCs. Arteries contain abundant elastic matrix to accommodate arterial pressure but veins have only a thin media with thinner SMC layers. Capillaries lack media but are lined with scattered pericytes. The adventitia is the outermost layer composed of mainly connective tissue through which the vessels contact the surrounding matrix (Tan, 2001).

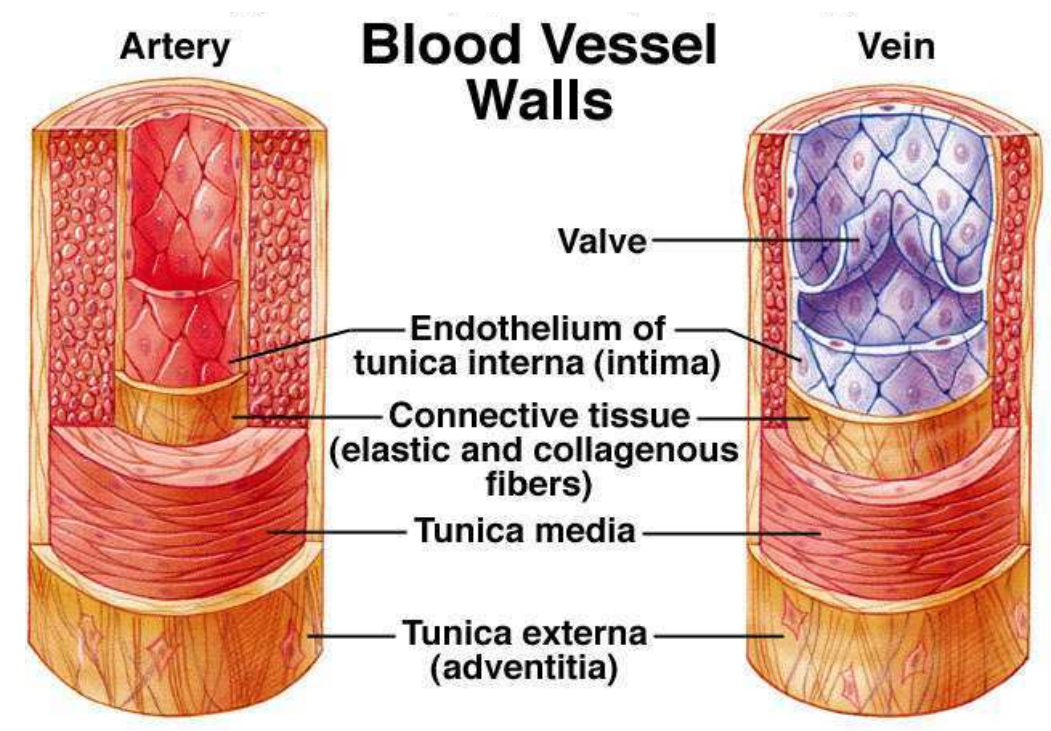

Figure 1.10: The basic structure of the blood vessel walls. Intima, media and adventitia are the three basic layers of the vessel wall of arteries, capillaries, veins and lymphatic vessels. Intima consists of a single layer of ECs and a thin underlying layer of connective tissue composed of elastic and collagen fibres. The media in the arteries and veins contain multiple layers of SMCs and elastic matrix. Capillaries lack media and are invested by scattered pericytes. The adventitia is mostly composed of connective tissue by which the vessels make contact with the ECM (Image reproduced from Sheir et al., 2004).

\subsubsection{DEVELOPMENT OF THE MAMMALIAN VASCULAR SYSTEM}

Early in embryonic development, vascular channels are formed by the differentiation of mesodermal cells into haemangioblasts (Folkman \& D'Amore, 1996; Risau, 1995; Sadler, 1985). This de novo differentiation leads to the formation of blood islands, which are small aggregations or clumps of haematopoeitic cells. While the cells 
at the borders, differentiate into angioblasts and EC precursors that would eventually line the lumen, the cells in the center differentiate into blood cells (Risau, 1997). These islands fuse together to form the primitive vascular channels, i.e., the primary capillary plexus. This process is called vasculogenesis (Risau, 1988).

The plexus continues to undergo remodelling which involves the generation of more small and large vessels and also regression of some vessels. Some pruning steps take place such as lumen formation, peri-endothelial support cell recruitment and sprouting (Nakamura, 1988). These processes are collectively known as angiogenesis (Risau, 1988). This angiogenic processes ultimately lead to the formation of differentiated blood vessels in the early embryo (Risau, 1988). These processes are also involved with the angiogenic response in the adult (Tan, 2001).

The mesenchymal cells (MeCs) differentiate into SMCs (Nakamura, 1988; Kirby \& Waldo, 1995). These cells migrate toward the evolving endothelial tubes and differentiate further into mural SMCs in veins and arteries, and into pericytes in the capillaries (Tan, 2001).

A

Angioblasts (haemangioblasts)

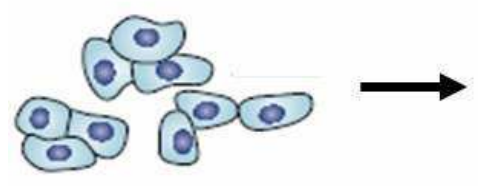

Coalescence of cells
B

Vasculogenesis

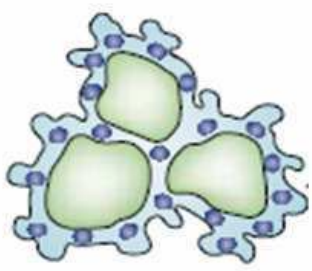

Primitive vasculogenic network
C

Angiogenesis

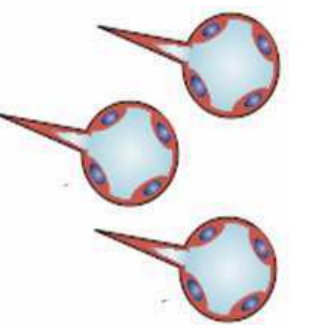

Sprouting

Figure 1.11.: Vasculogenesis and angiogenesis. Schematic overview of vasculogenesis and angiogenesis, showing coalescence of endothelial-cell precursors (angioblasts and haemangioblasts) and differentiation into ECs (A), which form the primitive vasculogenic networks (vasculogenesis) (B). Remodelling of these networks occurs through angiogenesis $(\mathbf{C})$, which involves sprouting resulting in the formation of microvessels. (Image redrawn from Hendrix et al., 2003). 


\subsubsection{VASCULOGENESIS AND ANGIOGENESIS}

Vasculogenesis is the process by which new blood vessels are formed by the differentiation and migration of endothelial progenitor cells to form interconnecting capillaries. Endothelial and haematopoietic cells share a common progenitor, the haemangioblast (Figures 1.12 and 1.13). These cells form aggregates in the developing yolk sac (also called the blood islands) and ablation of the transcription factor SCL present in these cells leads to failure in haematopoiesis and EC development (Robb et al., 1995; Robb et al., 1996; Sinclair et al., 1999). Angiogenesis is the generation of new blood vessels from pre-existing blood vessels. The signals that regulate the migration and organisation of cells into tubes during vasculogenesis and angiogenesis are complex and few of the factors involved in these processes have been identified (Figures 1.12 and 1.13).

A

Mesodermal cell

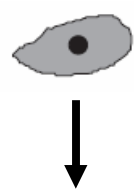

Haemangioblast

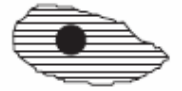

VEGF

Haematopoietic

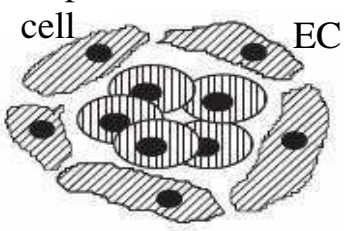

Vasculogenesis

B

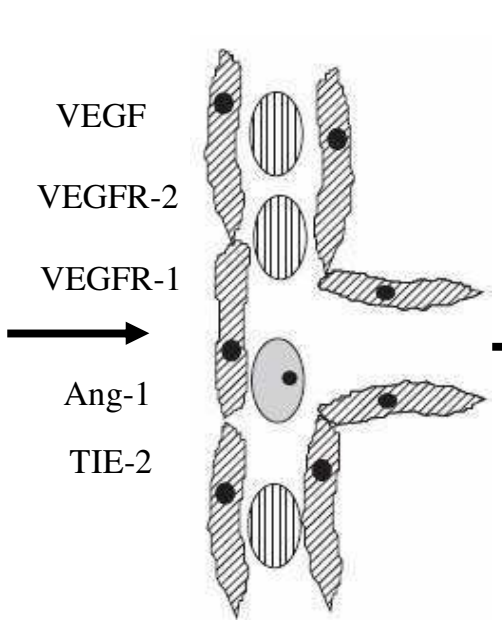

Angiogenesis

Intussusception

Remodelling
C

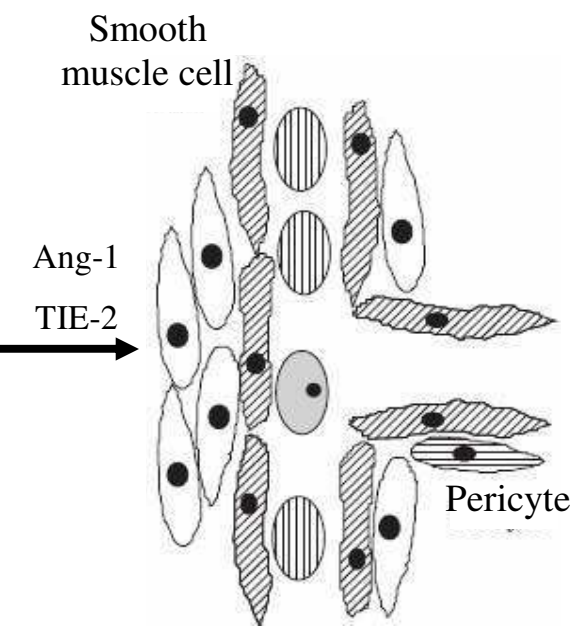

Figure 1.12: Roles of the vascular endothelial growth factor (VEGF)/VEGFRs) and the angiopoietin/TIE-2 systems in embryonic vascular development. (A) VEGF, VEGFR-1 and VEGFR-2, are critically involved in the formation of the primitive vascular system from differentiating mesodermal progenitors (vasculogenesis). (B) During the subsequent processes of angiogenesis and remodelling, VEGF and the VEGF receptors cooperate with the Ang-1/TIE-2 system. (C) The latter is also involved in the maturation of the developing vasculature, by mediating interactions between ECs and perivascular support cells. (Image redrawn from Breier, 2000). 
Gene targeting experiments have provided insights into the functions of VEGF and VEGFRs during embryonic vascular development. VEGF is critically involved in early stage vascular development. Formation of blood vessels was found to be severely impaired in mice that lack VEGF, resulting in embryonic death around day 10 (Carmeliet et al., 1996; Ferrara et al., 1996). Other early stage processes of vascular development such as vasculogenesis, large vessel formation, capillary sprouting and the remodelling of the yolk sac vasculature are affected in VEGF knockout embryos. The severe haploid insufficient phenotype indicates that VEGF acts in a strictly doesdependent manner to maintain angioblast differentiation and survival during early stage development. However, the vasculature becomes increasingly independent from VEGF after birth (Gerber et al., 1999). Inactivation of VEGFR-2 also causes embryonic lethality around mid-gestation but the phenotypes of such mice are distinct from those lacking VEGF, thus indicating that both these factors exert different functions (Breier, 2000). Mice lacking functional VEGFR-2 show complete failure of vasculogenesis, EC differentiation and haematopoiesis (Shalaby et al., 1995), consistent with its expression in the putative common precursor of endothelial and haematopoietic cells, the haemangioblast (Kataoka et al., 1997). VEGFR-1 deficient mouse embryos have abnormally enlarged blood vessels (Fong et al., 1995). Fong et al (1999) found that VEGFR-1 and VEGFR-2 appears to exert opposite activities, in that VEGF-2 signalling was required for the emergence of mature ECs, while VEGFR-1 negatively regulated the commitment of angioblasts during vasculogenesis. VEGFR-3 was however identified as non important for vasculogenesis but plays a role in the remodelling of the vasculature and in the formation of lymphatic vessels (Veikkola et al., 1999).

The angiopoietins, Ang-1 and Ang-2, also play a substantial role in angiogenesis (Hanahan, 1997). Ang-1, a chemotactic factor for ECs, supports and promotes vessel development (Figures 1.12, 1.13 and 1.14). Ang-1 is secreted from cells in the vicinity of developing blood vessels, and is considered as the agonistic TIE-2 ligand because 
Ang-1 and TIE-2 knockout mice show a very similar phenotype. The targeted inactivation of Ang-1 or TIE-2 resulted in defective vascular development and subsequent embryonic death (Sato et al., 1995; Suri et al., 1996). In contrast to mice deficient for VEGF or the VEGFRs, vasculogenesis proceeds normally in Ang-1 and TIE-2 deficient mouse embryos, indicating that this signalling system functions downstream of the VEGF/VEGFR system. The most prominent defects in Ang-1 or TIE-2 deficient mouse embryos are the absence of heart trabeculae, the failure of remodelling of the yolk sac vasculature and the absence of capillary sprouts in the neural tube. The heart trabeculation phenotype indicates that Ang-1 and TIE-2 are involved in the recruitment of perivascular cells.

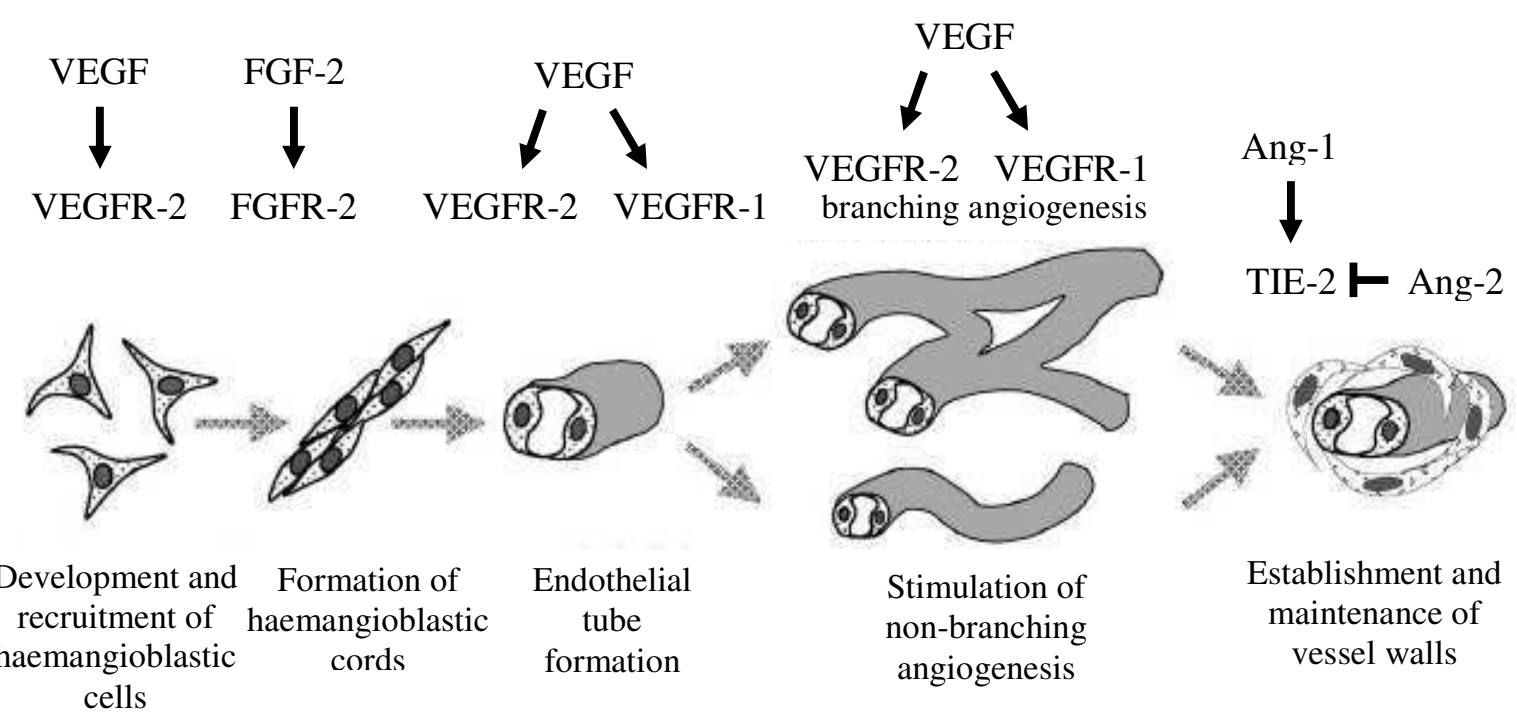

Figure 1.13: Growth factors and their receptors implicated in different stages of vasculogenesis and angiogenesis. Key: Ang-1 and -2 refer to angiopoietin-1 and -2 and TIE-2 to the angiopoietin receptor; FGF-2 is fibroblast growth factor-2 and FGFR is its receptor; VEGF is vascular endothelial growth factor and VEGFR-1 and VEGFR-2 its receptors. (Image redrawn from Charnock-Jones et al., 2004)

The Ang-1/TIE-2 system mediates vessel maturation from simple endothelial tubes into more elaborate vascular structures composed of several cell types, and the maintenance of those vessels via the cell-cell and cell-matrix associations that produce 
them. Consequently, Ang-1 may also help preserve EC quiescence. Moreover, Ang-1 also stimulates the formation of EC sprouts in vitro, and it acts synergistically with VEGF in this assay (Koblizek et al., 1998). Therefore, it is likely that Ang-1 and VEGF co-opperate to stimulate capillary sprouting in the developing organism. Based on the failure of blood vessels to interact properly with the underlying matrix or periendothelial supporting cells in Ang-1 deficient mouse embryos, it has been proposed that Ang-1 activity stabilizes blood vessels (Davis et al., 1999) (Figure 1.14).

Ang-2 on the other hand, although analogous to Ang-1, it completely inhibits Ang-1 induced kinase activation of TIE-2 receptor on ECs (Witzenbichler et al., 1998). Inhibition of TIE-2 kinase activity does not block EC proliferation and angiogenesis, but rather facilitates it (Hanahan, 1997). Focal expression of Ang-2 evidently blocks the Ang-1/TIE-2 signal, resulting in a loosening of this tight vascular structure and thereby exposing the ECs to activating signals from angiogenesis inducers, including VEGF. If VEGF (or another angiogenesis inducer) is present, the ECs become activated to migrate and proliferate, producing new capillary sprouts and in turn tubes. Furthermore, Ang-2 is uniformly expressed at high levels during vascular regression. This uniform presence of Ang-1 allows a shift in the local balance of Ang-1/Ang-2 back in favour of Ang-1, to effect maturation and stabilization of the newly formed vessels. Thus, there appears to be a collaboration between VEGF, Ang-2, and Ang-1 to elicit angiogenesis with vascular regression being associated with very high-level expression of Ang-2 in the absence of the activating (survival) signal from VEGF. This results in preventing the Ang-1 signal leading to catastrophic detachment from matrix and support cells, most likely with consequent apoptosis (Hanahan, 1997) (Figure 1.14) 


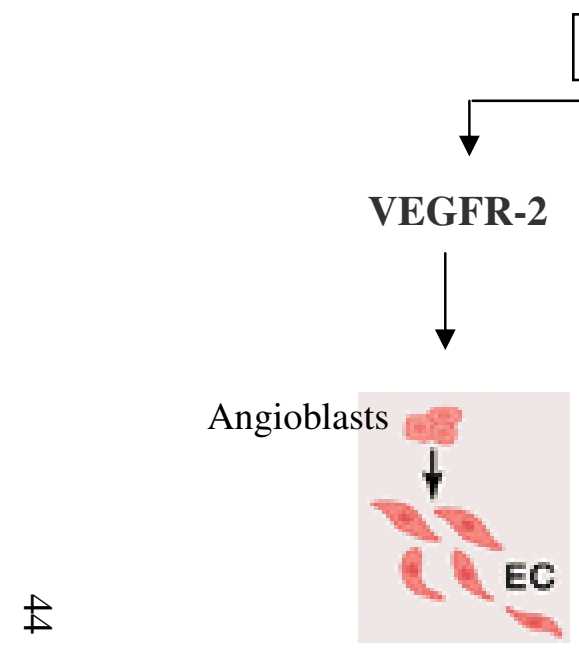

Birth, migration and proliferation of ECs

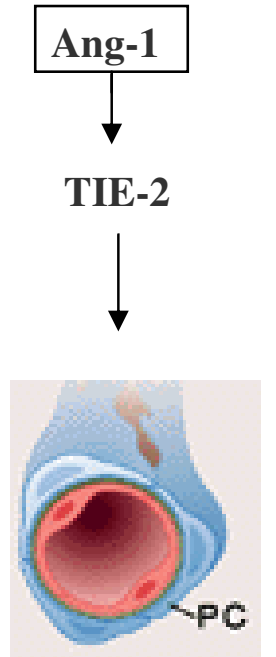

Tube formation, endothelial cell-cell

interactions

\section{VEGFR-1}

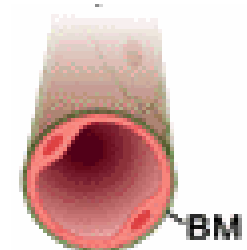

$B M$

Recruit and interact with periendothelial support cells;

Maintain vessel integrity and quiescence
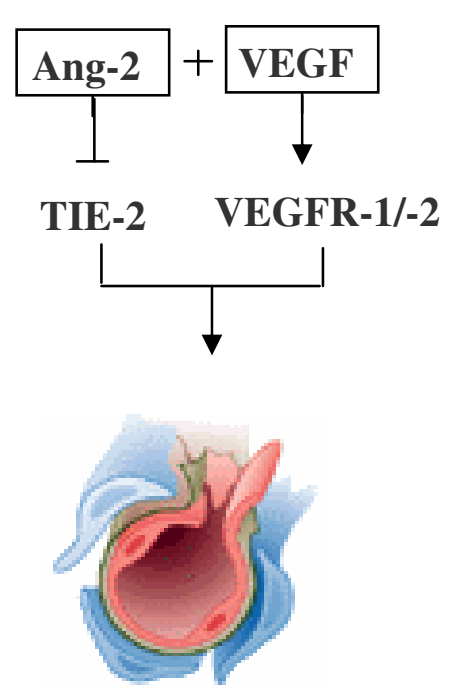

Loosening of matrix contacts and support cell interactions; Allow access and responsiveness to angiogenic inducers

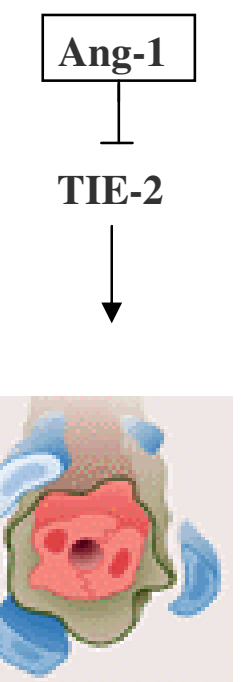

Loss of structure and matrix contacts; Absence of growth survival signals; Apoptosis?

Figure 1.14. A model for the regulation of the vascular endothelium by VEGF, Ang-1 and Ang-2. All three ligands bind to their respective receptors and elicit distinct downstream cellular responses. VEGF binding to VEGFR-2 sends a classical proliferation signal. When first activated in embryogenesis, this interaction induces the birth and proliferation of ECs. In contrast, VEGF binding to VEGFR-1 elicits endothelial cell-cell interactions and capillary tube formation. Ang-1 binding to TIE-2 receptor recruits and maintains the association of peri-endothelial support cells (pericytes, smooth muscle cells, myocardiocytes), thus solidifying and stabilising a newly formed blood vessel. Ang-2 binds and blocks kinase activation in ECs. The Ang-2 negative signal causes vessel structures to loosen, reducing EC contact with matrix and disassociating peri-endothelial support cells. This loosening appears to render the ECs more accessible and responsive toward the angiogenic inducer VEGF (and other inducers). Ang-2 is expressed at uniformly high levels in vascular regression (e.g., in nonproductive ovarian follicles); the lack of VEGF co-expression suggests that loosening of cell-matrix interactions in the absence of a growth or survival signal elicits EC death, likely by apoptosis. Other factors may also play into these distinctive states (Image redrawn from Hanahan, 1997) 


\subsubsection{THE ANGIOGENIC CASCADE}

The angiogenic process of vascular sprouting involves a cascade of events that include: (1) vasodilation and an increase in vascular permeability; (2) activation of proteases leading to degradation of the $\mathrm{BM}$; (3) increase in EC proliferation; (4) migration of the ECs (probably towards a angiogenic stimulus); (5) assembly of ECs to form a tube with a lumen; (6) Recruitment of pericytes and MeCs to the outside of the capillary to form a stable vessel and finally (7) vessel maturation which involves inhibitory signals and signals required for the reconstitution and formation of the BM (Figure 1.15) (Tomanek et al., 2000; Hirschi et al., 1999).

A
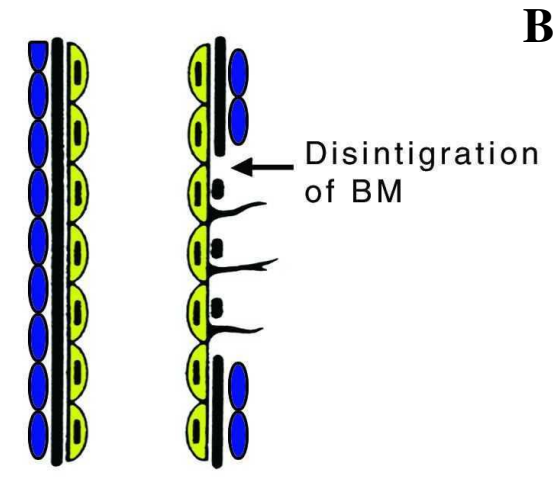

B

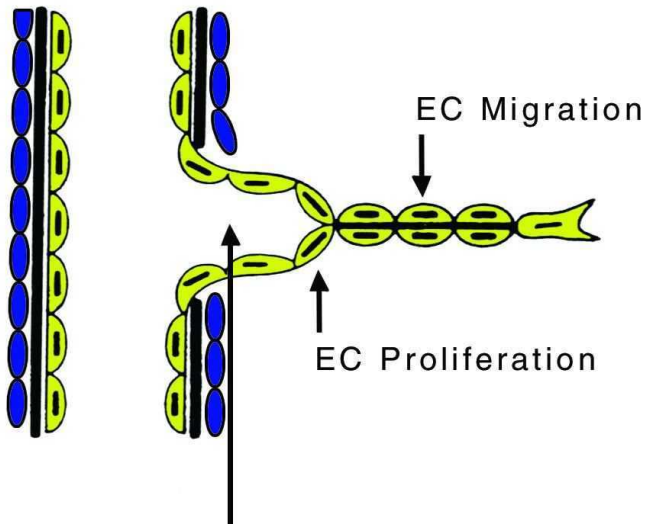

C

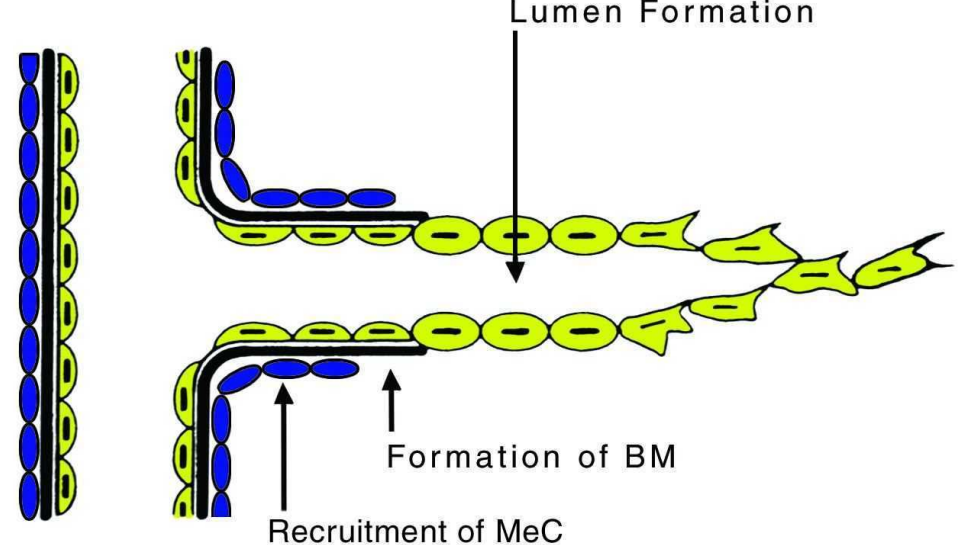

Figure 1.15: Schematic representation of the angiogenic process. EC activation subsequent to exposure of an angiogenic stimulus is followed by BM degredation and the extension of the cytoplasmic processes in the direction of the stimulus (A). This is followed by EC migration into the surrounding matrix and formation of capillary sprouts. EC proliferation occurs just behind the migrating front and lumen formation begins in the proximal region of the sprout $(\mathbf{B})$. Sprout maturation occurs with recruitment of MeCs to form peri-endothelial cells, reconstitution and formation of new BM which coincides with the cessation of cell migration and proliferation (C). (Courtesy of Dr Swee. T. Tan.) 


\subsubsection{ANGIOGENIC FACTORS IN BLOOD VESSEL DEVELOPMENT}

Central to vasculogenesis and angiogenesis are the ECs. These cells migrate, proliferate and assemble into tubes with tight cell-cell connections to contain blood. $\mathrm{MeCs}$ are recruited then differentiate into the peri-endothelial support cells (pericytes for capillaries, SMCs for larger vessels and myocardiocytes in the heart), which provide maintenance and modulatory function to the vessels (Hanahan, 1997; Tomanek \& Schatteman, 2000). The establishment and remodelling of blood vessels is controlled by paracrine and autocrine signals that mainly involve the binding of protein ligands to their respective RTKs expressed by vascular ECs (Folkman \& Shima, 2000; Hanahan, 1997; Hanahan and Folkman, 1996) (Figure 1.16).

The generation of angioblasts from the mesoderm and the formations of ECs from these mesodermal cells during vasculogenesis or from pre-existing capillaries during angiogenesis (Folkman et al., 1997; Tomanek \& Schatteman, 2000) requires the action of the FGF family and in particular FGF-2 (Folkman \& D'Amore, 1996; Flamme \& Risau, 1992) (Figure 1.16).

VEGF is also an important regulator of both vasculogenesis and angiogenesis. Although both VEGFR-1 and VEGFR-2 are expressed on angioblasts and the ECs that arise from them, only VEGF binding to VEGFR-2 leads to EC proliferation (Shalaby et al., 1995) (Figure 1.16). In contrast VEGF binding to VEGFR-1 is required for endothelial cell-cell interactions and capillary tube formation (Hanahan, 1997). Although disruption of the VEGFR-1 signal permits differentiation of ECs, it results in the formation of thin-walled vessels with a larger than normal diameter. Therefore, VEGFR-1 is said to be important with the later stags of vasculogenesis (Fong et al., 1995). 
Vasculogenesis

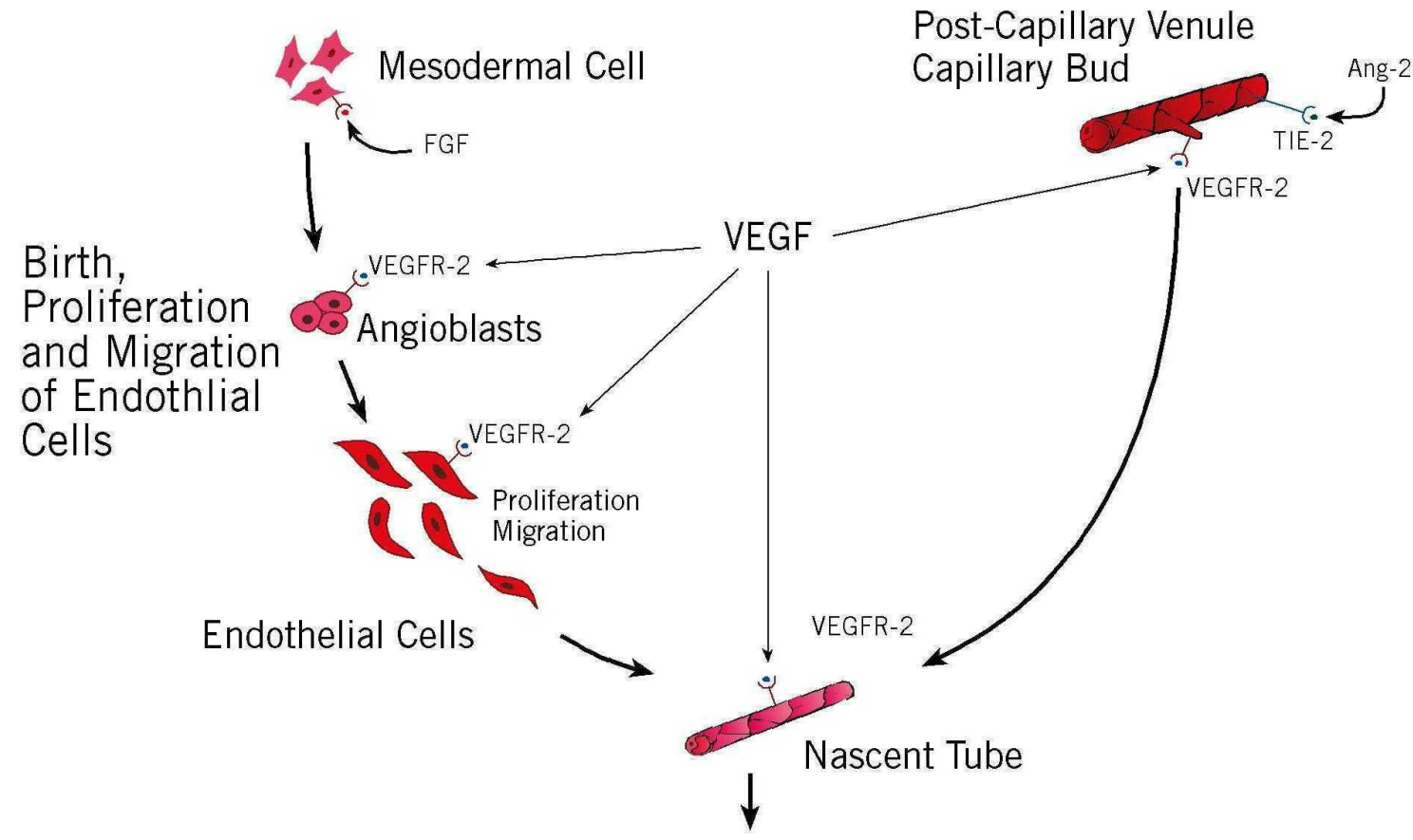

Angiogenesis

Post-Capillary Venule g-2
Capillary Bud

Recruitment of

Mesenchymal Cells

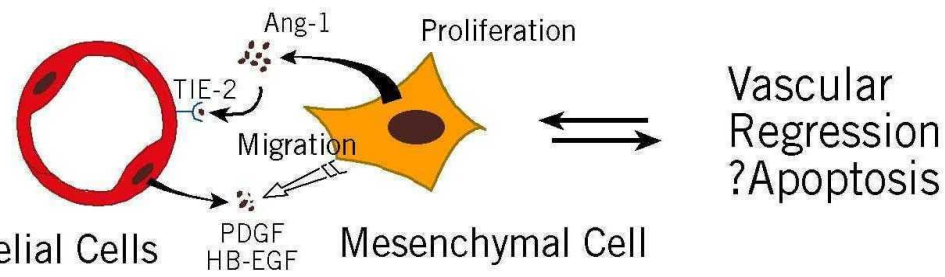

Endothelial Cells $\begin{gathered}\text { PDGF } \\ \text { HB-EGF }\end{gathered}$

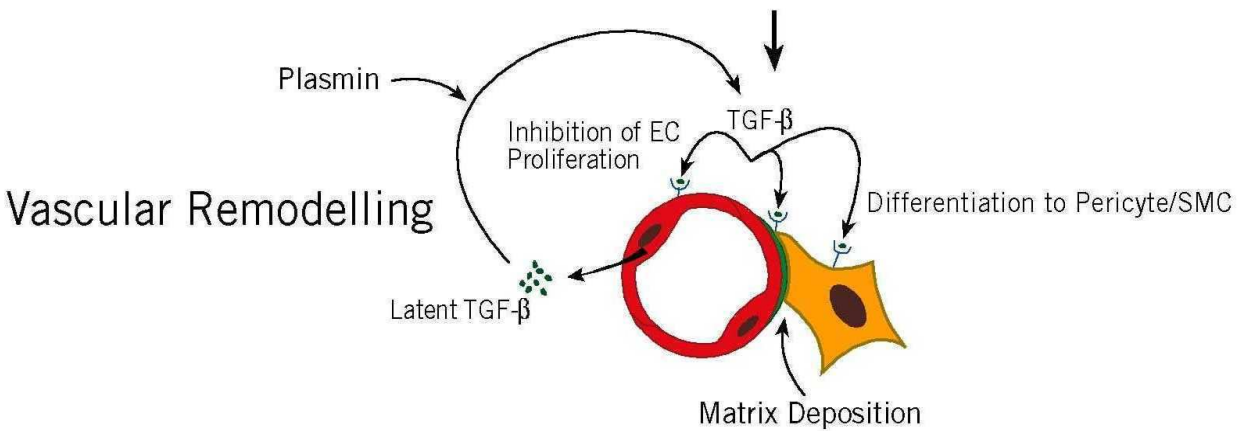

Destabilisation of Blood Vessels

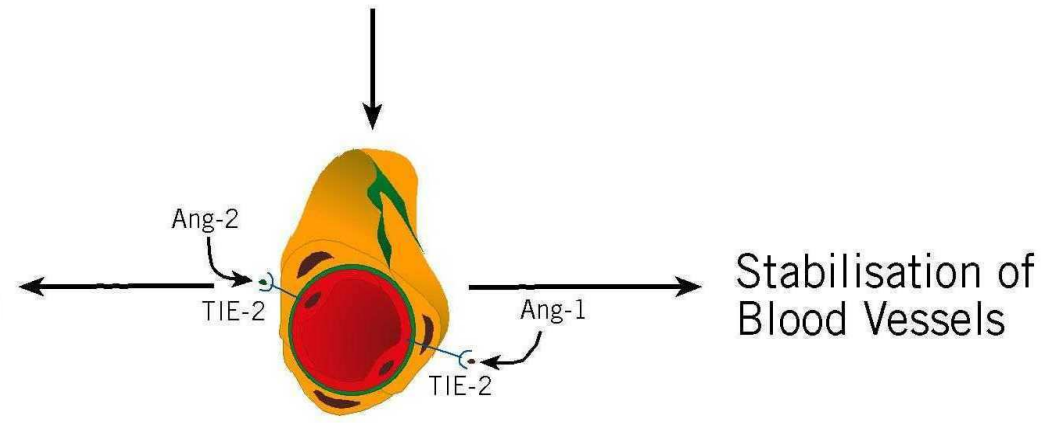

Mature Blood Vessels

Figure 1.16: The role of cytokines and cell-cell interactions in blood vessel formation (vasculogenesis and angiogenesis) is a culmination of a number of processes involving a variety of molecules and extensive interactions between a number of cell types (see text for details) (Reproduced with permission from Tan, 2001) 
Ang-1 produced by MeCs is the chemotactic factor for ECs and also supports and promotes vessel development. Ang-1 binding to TIE-2 activates a signal from the ECs, which recruits undifferentiated MeCs to the forming vessel (Folkman et al., 1997; Hanahan, 1997). Its signal is also required to maintain EC association with the differentiated peri-endothelial support cells, thereby stabilising the newly formed blood vessel (Hanahan, 1997) (Figure 1.16). Ang-2 on the other hand, is known as a natural antagonist of Ang-1. Ang-2 over expression blocks the stabilising signal of Ang-1 because Ang-1 can no longer induce TIE-2 receptor activation on ECs (Witzenbichler et al., 1998). However, inhibition of TIE-2 kinase activity does not block EC proliferation and angiogenesis, instead facilitates it (Hanahan, 1997). This happens because the Ang2 negative signal causes vessel structures to become loosened by the reduction of EC contact with the matrix and dissociation of peri-endothelial support cells. This loosening appears to render the ECs more accessible and responsive to VEGF (and other inducers) (Hanahan, 1997). It has also been suggested that high Ang-2 expression, loosening of cell-matrix interactions together with the absence of growth factors such as VEGF results in EC death most likely by apoptosis (Hanahan, 1997). Other modulators, some of which have been described in section 1.4 (page 30) also play into these distinctive states of angiogenesis (Plank \& Sleeman, 2003).

Once the primitive vessel tubes are formed, they are remodelled to form the mature vessel bed. This remodelling involves the formation of the medial layer consisting of SMCs in large vessels and pericytes in the microvasculature, deposition of the BM and induction of tissue specific specialisation (Tan, 2001). Upon contact of EC with MeCs, the EC-derived TGF- $\beta$ is activated from its latent state (Hirschi et al., 1999; Folkman \& D'Amore, 1996). The active TGF- $\beta$ leads to the up-regulation of myosin and SMA which causes the MeCs to assume the morphology of pericytes or SMCs (Folkman \& D’Amore, 1996). Failure of pericyte formation has been shown to cause regression of the naked nascent endothelial tubes (Folkman \& D'Amore, 1996). EC- 
$\mathrm{MeC}$ contact also suppresses cell proliferation and stabilises the mature vasculature (Tan, 2001) (Figure 1.16).

In addition to inducing differentiation of MeCs into pericytes or SMCs, the activated form of TGF- $\beta$ also inhibits EC proliferation and stimulates matrix deposition (Hirschi et al., 1999). Thus, a lack of local TGF- $\beta$ production could permit increased proliferation of ECs. Furthermore, as TGF- $\beta$ alters integrin expression, the poor vascular integrity in the TIE-2/Ang-null mice could be explained by the lack of ECSMC contacts and lack of TGF- $\beta$ (Folkman \& D'Amore, 1996).

\subsection{ROLE OF STAT PROTEIN IN TUMOURIGNESIS}

\subsubsection{STAT PROTEINS}

STAT proteins comprise a family of transcription factors that consists of seven different members: STAT-1, -2, -3, -4, -5A, -5B, and -6 (Darnell, 1997). Abundantly produced in many cell types, they are activated by over 40 ligands (Calo et al., 2003). These include a series of extracellular signalling proteins such as cytokine, growth factors, and hormones that bind to specific cell-surface receptors (Horvath, 2000). Aberrant activation of STAT signalling can lead to the deregulation of crucial signalling pathways. In this way, STATs take part in tumourigenenic cell transformation (Bowman et al., 2000).

Cytokines that activate STATs include, type I cytokines (interleukins, neutrophic factors, and hormones) and type II cytokines (IFNs). Growth factors also activate STAT proteins via their receptors. These include epidermal growth factor (EGF), hepatocyte growth factor (HGF), PDGF and VEGF (Korpelainen et al., 1999; Bartoli et al., 2000). 


\subsubsection{MECHANISMS OF STAT ACTIVATION}

STAT proteins become activated by tyrosine phosphorylation of critical tyrosine residues. These residues are Tyr 701 for STAT-1, Tyr 690 for STAT-2, Tyr 705 for STAT-3, Tyr 693 for STAT-4, Tyr 694 for STAT-5 and Tyr 641 for STAT-6 (Becker et al., 1998). The best-studied pathway for STAT activation is through the Janus kinases (JAKs). JAKs are constitutively associated with cytokine receptors. Ligand binding leads to dimerisation of the receptor and activation of the JAKs. These kinases once activated can trans-phosphorylate the critical tyrosine residues within the intracytoplasmic portion of the receptor chains to provide docking sites for the STAT proteins (Briscole et al., 1996). STATs then make contact with the receptor through their SH2 domain. On binding, the JAKs subsequently tyrosine phosphorylate the 'docked' STAT at conserved tyrosine residues (Figure 1.17). Upon tyrosine phosphorylation, the STAT proteins dissociate from the receptor subunit and form dimers by virtue of reciprocal-SH2 phosphotyrosine interactions i.e., the $\mathrm{SH} 2$ domain of one STAT molecule binds with the SH2 domain of a second STAT molecule containing the phosphotyrosine (Chatterjee-Kishore et al., 2000; Darnell, 1997).

STATs can form homodimers (most commonly) as well as heterodimers (Bromberg, 2001). Dimeric STATs are further phosphorylated, this time on serine, which makes it an active transcription factor. STAT dimers then translocate to the nucleus, bind to specific DNA-binding elements and activate gene transcription. Therefore, while tyrosine phosphorylation activates the STAT proteins, nuclear translocation and DNA binding is essential for it to effect gene regulation (Yahata et al., 2003). Since STAT proteins lack a nuclear localisation signal, chaperone proteins have been suggested to assist nuclear translocation of STATs (Johnson et al., 1998). 


\subsubsection{PHYSIOLOGICAL ROLE OF STATS}

STATs play important roles in programming gene expression in biological events as diverse as embryonic development, organogenesis, regulation of cell growth and apoptosis (Horvath, 2000). Evidence for this comes from cell culture experiments, animal experiments and clinical observations on human cancer cells (Bromberg, 2001). While in normal cells ligand-dependent activation of STATs is a transient process, lasting for several minutes to several hours, many cancerous cell lines and tumors exhibit persistently tyrosine phosphorylated and thus constitutively activated STAT proteins. Furthermore, deregulated STAT protein signalling contributes to tumour development by bringing about the loss of immune surveillance (Bromberg, 2002).

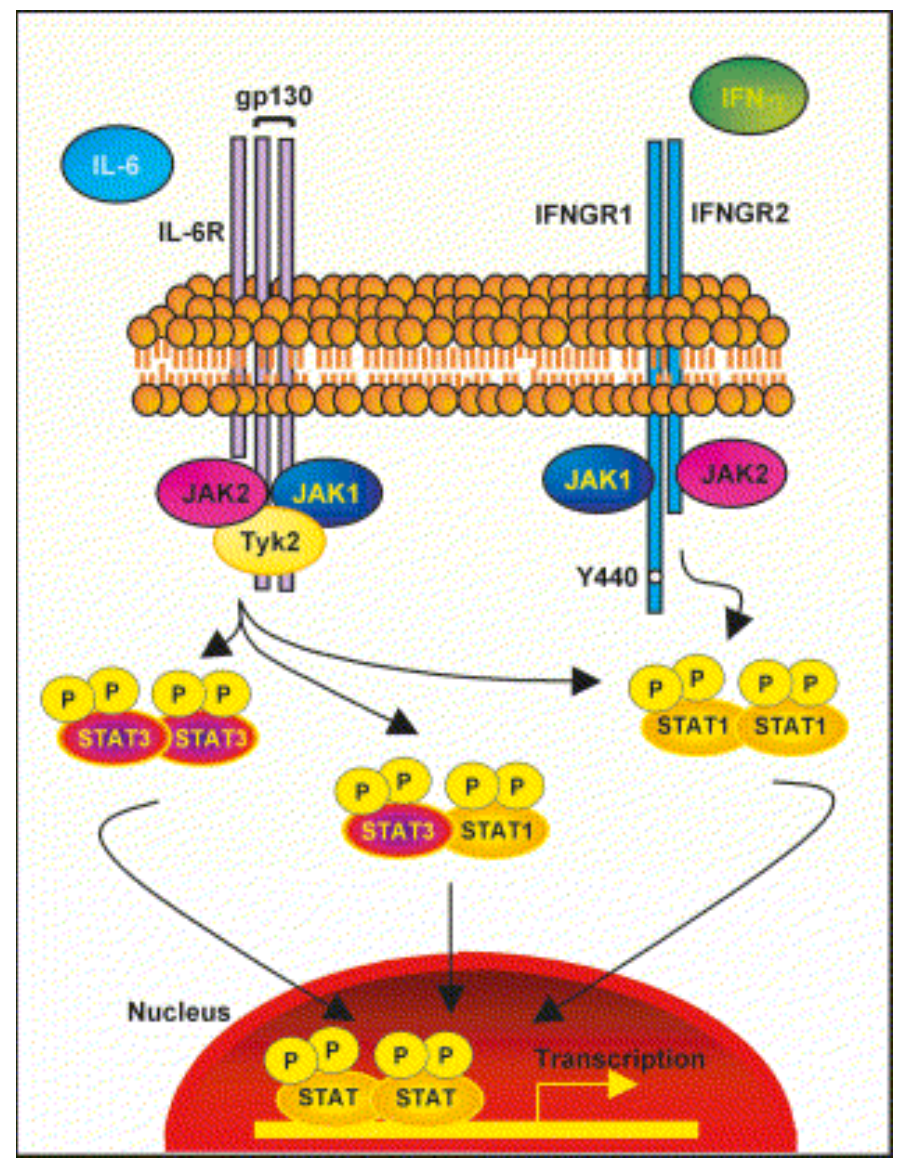

Figure 1.17: Schematic representation of STAT activation via JAK activation. STAT-3 and STAT- 1 activation is induced via signalling though IL-6 and IFN $\gamma$ receptors. See text for details (Reproduced from Kerr et al., 2003). 
STAT-3 activation is involved in early embryogenesis (Akira, 2000, Takeda et $a l .$, 1997), in migration of skin epidermic cells and in skin remodelling during wound healing (Sano et al., 1999). It is required for the inactivation of the macrophages and the inhibition of inflammatory cytokines in the T-helper cell response (Takeda et al., 1999). STAT-3 activation is also required to maintain the pluripotent state of proliferating embryonic stem cells and for macrophage differentiation (Hirano et al., 2000).

\subsubsection{STAT-3 IN HUMAN ONCOGENESIS AND REGULATION OF}

\section{APOPTOSIS}

Persistent activation of STAT-3 plays an important role in preventing apoptosis in many large tumours and cell lines including lymphomas, leukemias, mycoses fungoides (Nielson et al., 1999), multiple myeloma, brain (Bonni et al., 1997; Ihara et al., 1997), prostate, breast, lung, and head and neck cancers (Bromberg et al., 2001). Studies have shown that the suppression of apoptosis by STAT-3 was due to its ability to induce expression of apoptosis suppressor proteins such as Bcl-2 and Bcl-xl (CatlettFalcone et al., 1999; Buettner et al., 2002).

Various studies have shown that STAT-3 activation was required and sufficient to mediate cellular transformation (Bromberg et al., 1998; ; Turkson et al., 1998; Bromberg et al., 1999; Ram et al., 2000). A possible connection between STAT-3 and tumourigenesis is its association with c-Jun, the first discovered nuclear proto-oncogene (Bromberg, 2001).

Recently, Chan et al (2004) demonstrated that STAT-3-deficient mice were resistant to skin tumour development and suggested that STAT-3 was required for epithelial carcinogenesis by maintaining the survival of DNA-damaged stem cells and mediating the proliferation necessary for the clonal expansion of these cells during tumour promotion (Chan et al., 2004). In addition, Zong et al (2000) found that endogenous IGF stimulation resulted in increased STAT-3 activation. Studies have also 
shown that the stable dimerisation of STAT-3, caused by engineered mutations, was sufficient to transform NIH 3T3 cells (Bromberg et al., 1999). All these findings have accentuated the importance of STAT-3 activation in inducing cell proliferation (Bromberg et al., 1999).

\subsubsection{STAT-1 IN GROWTH RESTRAINT, PROMOTER OF} APOPTOSIS AND TUMOUR SUPPRESSION

STAT-1 is increasingly being established as a tumour suppressor since its activation is associated with growth arrest (Durbin et al., 1996). Kaplan et al (1998) showed that STAT-1 deficient mice were more susceptible to tumour development and that the STAT-1-/- genotype coupled with the removal of the tumour suppressor gene, p53, greatly increased spontaneous tumour formation (Kaplan et al., 1998).

STAT-1 is now recognised as the principal target of IFNs (Schindler et al., 1992; Darnell et al., 1994; Stark et al., 1998). Studies have shown that IFNa or IFN $\gamma$ treatment does not inhibit the proliferation of STAT-1 deficient fibroblasts and that STAT-1 deficient ostoesarcoma-derived cells do not undergo apoptosis in response to IFN $\gamma$ or the tumour necrosis factor (TNF) (Su et al., 1997; Sahni et al., 1999; Migone, 1995). In addition, the defective growth of tumour cells in response to IFN $\gamma$ has been attributed to STAT-1 activation (Chin et al., 1997; Kumar et al., 1997).

The involvement of STAT-1 in growth arrest and apoptosis in many cell types, seems to be due to its capacity to induce expression of pro-apoptotic mediators such as caspases and TRAIL (Choi et al., 2003; Chin et al, 1997; Kumar et al., 1997) while decreasing the expression of anti-apoptotic modulators such as Bcl-2 and Bcl-xl (Stephanou et al., 2000). 


\subsection{AIM OF THIS PROJECT}

The overall aim of this thesis is to study the cellular development of haemangioma and the signalling pathways that are involved in the proliferation and involution of haemangioma. The identification of novel treatment agents that may be associated with fewer side effects was also investigated. Studying the cellular processes involved in the biology of haemangioma will provide a better understanding of the formation of microvessels. This is of importance for both haemangioma growth, and also for angiogenesis associated with other proliferating tumours, as inhibiting the formation of new blood vessels is an important therapeutic target for reducing tumour growth.

\subsubsection{OBJECTIVES}

The specific objectives of this study were (1) to identify changes in expression of key transcription factors and RTKs involved in cellular proliferation and apoptosis during the natural progression of a haemangioma; (2) to study the expression patterns of TRAIL and its receptors in proliferating, involuting and involuted haemangioma and to elucidate the role of TRAIL receptor signalling in modulating haemangioma progression; (3) the utilisation of the newly developed in vitro model to evaluate the use of TRAIL as a potential treatment option for haemangioma; (4) the purification, culture and characterisation of haemangioma-derived cells in vitro; and (5) the identification of novel genes or cell types which may be important modulators of haemangioma development. 


\section{CHAPTER 2: METHODS}

\subsection{GENERAL METHODS}

\subsubsection{QUANTITATIVE REAL TIME PCR}

\subsubsection{AN INTRODUCTION TO QUANTITATIVE REAL TIME PCR}

Quantitative real-time polymerase chain reaction (qRT-PCR) assay was used in this study to quantify changes in mRNA expression. The basic principles, advantages, limitations and the special kinetics of qRT-PCR have been well adequately covered in numerous reviewed articles (Bustin, 2000; Giulietti et al., 2001; Yin et al., 2001; Rose'Meyer et al., 2003). However, a brief introduction to qRT-PCR, the detection method used in this study and the limitations are described below.

qRT-PCR is now widely used to measure differences in mRNA expression due to its outstanding accuracy, broad dynamic range and sensitivity. It is also fast, highly reproducible and requires minimal amount of RNA (Freeman et al., 1999; Heid et al., 1996). qRT-PCR analysis allows direct quantification of specific PCR products during the exponential phase (i.e., at linearity) of the reaction, also taking into account the special amplification kinetics of each target.

For this study, the SYBR green dye was used as the reporter dye. This dye fluoresces on binding to double stranded DNA. Therefore, there is an increase in fluorescence of dye with the accumulation of PCR product which is reported after every cycle. Quantification is achieved by determining the cycle at which the fluorescence increases above an arbitrary threshold. This threshold can be manually changed for each experiment if necessary. The PCR cycle at which the fluorescence exceeds the chosen threshold, due to the linear amplification of the specific product is reported as the $\mathrm{Ct}$ value (Yin et al., 2001). For most genes, an end point or plateau is reached in less than 40 cycles (depending on transcript abundance), whereby no further amplification can 
take place due to competitive PCR effects. $\Delta \mathrm{Ct}$ values refer to the relative mRNA transcript levels for a particular candidate gene, whereby the $\mathrm{Ct}_{\mathrm{x}}$ value for the reference or housekeeping gene is subtracted from the candidate gene $\mathrm{Ct}_{\mathrm{y}}$ in relation to the total mRNA concentration and is given by the equation $\Delta \mathrm{Ct}=\mathrm{Ct}_{\mathrm{y}}-\mathrm{Ct}_{\mathrm{x}}$. This means, a single Ct difference $(\Delta \mathrm{Ct}=1)$ represents a two-fold difference in the amount of specific target (Livak \& Schmittgen, 2001). Therefore, Ct values decrease linearly with increasing input target quantity and this can be used as a quantitative measurement of the input target (Giulietti et al, 2001).

The limitation of SYBR Green I real time detection is that it does not differentiate between specific target and non-specific product. To overcome this limitation, DNA melt curves were analysed at the end of all qRT-PCR amplifications performed in this study. These are graphs obtained by plotting fluorescence as a function of temperature as the thermal cycler heats through the dissociation temperature of the product. The shape and position of this curve are functions of the GC/AT ratio, length and sequence and can be used to differentiate amplified products separated by less than $2^{\circ} \mathrm{C}$ in melting temperature. Analysis of melting curves can therefore distinguish desired from undesired products and also extend the dynamic range of template quantification (Ririe et al., 1997) (see Appendix 1, Figure 1.2, page 270)

Relative quantification was the method chosen to quantify changes in mRNA levels in this study. Because this method quantifies the expression of the target gene with respect to a stably expressed reference gene (housekeeper gene), selection of an appropriate house keeping gene becomes important. House keeping genes are necessary for cell survival. The mRNA synthesis of these genes is considered to be stable between tissues and under different experimental treatments. However, numerous studies have shown that this is not always the case (Zhang \& Snyder et al, 1992; Bhatia et al., 1994; Bereta and Bereta 1995; Chang et al., 1998; Thellin et al., 1999; Zhong \& Simons, 1999; Schmittgen \& Zakrajsek, 2000; Selvey et al., 2001). In addition, the copy number 
of the house keeping gene and the target gene should be similar so that measurements can be made on the same linear scale (Roche LC technical note 15/2002).

Quantification using a true reference gene allows for the normalisation of differences in the amount of amplifiable RNA or cDNA in individual samples generated by (i) different amounts of starting material, (ii) the quality of the starting material, (iii) enzymatic efficiency; and (iv) differences in RNA preparation and cDNA synthesis. This is because the reference gene is exposed to the same preparation steps as the gene of interest. When a single house keeping gene is used as a reference, differences in gene expression ratios may reflect regulation of the house keeping gene rather than the gene under investigation. Therefore, relating gene expression to several housekeepers in parallel has been suggested (Schmid et al., 2003).

Some commonly used housekeeping genes include cyclophilin A (CycA) (Bjarnason et al., 1998), glyceraldehyde 3 phosphate dehydrogenase (GAPDH) (Petersen et al., 1990), hypoxanthine phosphoribosyltransferase (HPRT) (Foss et al., 1998) and 18SrRNA (Finnegan et al., 1993). These genes were validated for use in this study (see Appendix 1, section 1.1.2.2, page 272). 


\subsubsection{RNA ISOLATION AND QUANTITATION}

RNA from snap-frozen haemangioma tissue biopsies (1-2 mg of frozen tissue) and cell cultures (approximately $1 \times 10^{5}$ viable cells) was isolated by a modified protocol using a combination of TRIzol reagent (Invitrogen, Auckland, New Zealand) and the High pure RNA tissue Kit (Roche diagnostics, New Zealand, Ltd).

\subsection{RNA extraction from snap frozen tissue biopsies.}

Frozen haemangioma tissue was ground to a powder in liquid nitrogen and added to $750 \mu 1$ of Trizol. Samples were left in TRIzol overnight at $4^{\circ} \mathrm{C} .350 \mu \mathrm{l}$ of choloroform was then added, mixed for $15 \mathrm{sec}$ and left at room temperature for $10 \mathrm{~min}$. The tubes were centrifuged at $4^{\circ} \mathrm{C}$ for $15 \mathrm{~min}$ at $13,000 \mathrm{X} \mathrm{g}$ and the aqueous phases (approx 500 $\mu 1)$ containing the RNA were transferred into clean eppendorf tubes to which an equal volume of $70 \%$ ethanol was added. The entire mixture was then applied to the upper reservoir of an assembled High Pure filter and collection tube, and spun in a benchtop centrifuge at room temperature for $15 \mathrm{sec}$ at $8,000 \mathrm{Xg}$. The flowthrough was discarded and the filter and collection tube re-assembled. Any contaminating DNA was removed by adding $90 \mu \mathrm{l}$ of DNAse I incubation buffer (1 M Nacl, $20 \mathrm{mM}$ Tris-HCL, $10 \mathrm{mM}$ $\mathrm{MnCl}_{2} \mathrm{pH}$ 7.0) with $10 \mu \mathrm{l}$ of DNAse I onto the glass filter fleeces of the upper reservoir of each filter tube, and incubating for $15 \mathrm{~min}$ at $15-25^{\circ} \mathrm{C}$. Next, $500 \mu \mathrm{l}$ of wash buffer I (5 M guanidine hydrochloride, $20 \mathrm{mM}$ Tris-HCL, ethanol, $\mathrm{pH}$ 6.6) was added to the upper reservoir and spun for $15 \mathrm{sec}$ at $8,000 \mathrm{X} \mathrm{g}$. The flowthrough was discarded and $500 \mu \mathrm{l}$ of wash buffer II (20mM NaCl, $2 \mathrm{mM}$ Tris-HCL, ethanol, $\mathrm{pH} 7.5)$ was added, and spun for $15 \mathrm{sec}$ at $8,000 \mathrm{X} \mathrm{g}$. After discarding the flow-through, a final wash step using $300 \mu \mathrm{l}$ of wash buffer II was carried out and the filter tubes were inserted into sterile $1.5 \mathrm{ml}$ eppendorf tubes. $50 \mu \mathrm{l}$ of elution buffer (nuclease-free, sterile, double distilled water) was applied to the upper reservoir of the filter tubes and incubated at 
room temperature for $10 \mathrm{~min}$. RNA was eluted by centrifugation for $1 \mathrm{~min}$ at 13,000 X g. RNA was stored at $-80^{\circ} \mathrm{C}$.

\subsection{RNA extraction from cell cultures}

To extract RNA from cell cultures, the culture media was removed from the dish and $1 \mathrm{ml}$ of TRIzol was pipetted directly into the culture dishes containing approximately $1 \times 10^{5}$ cells. The culture dish was swirled vigorously and RNA was purified from the TRIzol reagent as described in section 2.1.1.2.1 (page 58).

\subsubsection{RNA QUANTIFICATION}

The quantity and purity of each RNA extraction was determined using a NanoDrop ND-1000 spectrophotometer (NanoDrop Technologies, DE). The absorbance was measured at $260 \mathrm{~nm}$ from $2 \mu \mathrm{l}$ of samples. RNA concentration was determined using the extinction coefficient for RNA of $40 \mu \mathrm{g} / \mathrm{ml}$ and calculated using the following formula.

\section{Concentration $(\mathrm{ng} / \mu \mathrm{l})=\mathrm{A}_{260} \times$ dilution $\mathrm{x} 40$}

The ratio of the absorbance at $260 / 280 \mathrm{~nm}$ was used to determine the purity of the RNA. Pure RNA has a ratio between 1.9-2.1. Early in the study the quality of the RNA samples were also determined by electrophoresis on agarose gels and by visualisation of the integrity of the $18 \mathrm{~S}$ and $28 \mathrm{SrRNA}$ bands.

\subsection{2 cDNA SYNTHESIS}

cDNA was synthesised using a Superscript III cDNA synthesis kit (Invitrogen). cDNA was synthesised using either random hexamers or Oligo $(\mathrm{dT})_{20}$ as primers. Each reaction contained approximately $400 \mathrm{ng}$ of RNA, $500 \mu \mathrm{M}$ dGTP, dCTP, dATP, dTTP and $2.5 \mu \mathrm{M}$ Oligo(dT) $)_{20}$ or $250 \mathrm{ng}$ of random hexamer primers. The RNA and primer mix was first denatured for $5 \mathrm{~min}$ at $65^{\circ} \mathrm{C}$ followed by incubation on ice for $1 \mathrm{~min}$. A 
reaction mixture containing $1 X$ First-Strand buffer (50 mM Tris-HCL, $\mathrm{pH} 8.3,75 \mathrm{mM}$ KCL, $3 \mathrm{mM} \mathrm{MgCl}$ ), 5 mM DTT, 200 U SuperScript III and 40 U RNaseOUT, was then added to each tube to a final volume of $20 \mu \mathrm{l}$ and incubated for 1 hour (hr) at $50^{\circ} \mathrm{C}$. cDNA was stored at $-20^{\circ} \mathrm{C}$ until required.

Random hexamers primed cDNA templates were used in the housekeeping gene validation experiments where $18 \mathrm{SrRNA}$ was used as the internal reference gene and Oligo(dT $)_{20}$ was used for all subsequent amplifications.

\subsubsection{PRIMER DESIGN AND RECONSTITUTION}

PCR primers for this study were designed using the Oligo 6 software (Version 6.02, Molecular Biology Insights Inc., MN). Gene sequence information was obtained using nucleotide databases (www.ncbi.nlm.gov) and primer sequences were assessed for specificity using non-reduntant basic local alignment search tools (www.ncbi.nlm.nih.gov/BLAST). Where possible, primers were designed to be intron spanning to differentiate between amplification from contaminating DNA and true cDNA amplified PCR product. A number of parameters were taken into account when the primer pairs were designed:

- $\quad$ The amplicon size ranged between 80-250 bp

- All primers were designed to have a GC content of 50-60\%.

- $\quad$ All primer pairs had high melting temperatures (Tm of approximately $\left.68^{\circ} \mathrm{C}\right)$ with the difference in $\mathrm{Tm}$ being not more than $2^{\circ} \mathrm{C}$ apart.

- $\quad$ Primers with stable 3' ends, stable hairpins with 5' overhangs, duplexes with 3' overlaps and mismatches at the 3' end were avoided as best as possible.

All the primers were synthesised by Invitrogen. Primers were reconstituted in 10 $\mathrm{mM}$ Tris, $\mathrm{pH} 8.0,0.1 \mathrm{mM}$ EDTA to a final concentration of $250 \mu \mathrm{M}$. A working concentration stock was then prepared for each primer pair containing $10 \mu \mathrm{M}$ of 
forward and reverse primer in a single tube, diluted in distilled water $\left(\mathrm{dH}_{2} \mathrm{O}\right)$. Primer sequences and product sizes are given in Table 1.

\begin{tabular}{|c|c|c|}
\hline Gene & $\begin{array}{r}\text { Primers: sense (+) } \\
\text { Anti-sense (-) }\end{array}$ & Product size \\
\hline STAT-3 & $\begin{array}{l}\text { (+) 5'-GCAGAAAGATACGACTGAGG- 3'; } \\
\text { (-) 5'-GCAGATCACCCACATTCACT- 3'; }\end{array}$ & $180 b p$ \\
\hline STAT-1 & $\begin{array}{l}\text { (+) 5'-GGC AAA GAG TGA TCA GAA ACA A- 3' } \\
\text { (-) 5'-GTT CAG TGA CAT TCA GCA ACT CT- 3' }\end{array}$ & $118 b p$ \\
\hline VEGFR-2 & $\begin{array}{l}\text { (+) 5' -ATTCCATGTCTCGGGTCCATT- 3' } \\
\text { (-) 5' -CTTGCTGTCCCAGGAAATTCT- 3' }\end{array}$ & $107 b p$ \\
\hline CycA & $\begin{array}{l}\text { (+) 5' -GGGGAGAAAGGATTTGGCTA- 3' } \\
\text { (-) 5' -ACATGCTTGCCATCCACCC- 3' }\end{array}$ & $259 b p$ \\
\hline 18SrRNA & $\begin{array}{l}\text { (+) 5' -CTT AGA GGG ACA AGT GGC G- 3' } \\
\text { (-) 5' -ACG CTG AGC CAG TCA GTG TA- 3' }\end{array}$ & $110 b p$ \\
\hline GAPDH & $\begin{array}{l}\text { (+) 5' -TAAAGGGCATCCTGGGCTACACT- 3’; } \\
\text { (-) 5' -TTACTCCTTGGAGGCCATGTAGG- 3' }\end{array}$ & $200 b p$ \\
\hline HPRT & $\begin{array}{l}\text { (+) 5' -CCC CAC GAA GTG TTG GAT ATA A- 3' } \\
(-) \text { 5' -GTC AAG GGC ATA TCC TAC AAC A- 3' }\end{array}$ & $82 b p$ \\
\hline
\end{tabular}

Table 1: Primer sequences and cDNA amplicon size (in $b p$ ) used in this study. 


\begin{tabular}{|c|c|c|c|}
\hline Gene & $\begin{array}{l}\text { Primers: sense (+) } \\
\text { Anti-sense (-) }\end{array}$ & Product size & Reference \\
\hline CD29 & $\begin{array}{l}(+) 5^{\prime} \text {-TCCAACCTGATCCTGTGTC-3' } \\
(-) 5^{\prime} \text {-TCGTTGTTCCCATTCACTG-3' }\end{array}$ & $88 b p$ & Gambero et al., 2006 \\
\hline CD133 & $\begin{array}{l}(+) 5^{\prime} \text {-GCATTGGCATCTTCTATGGTT-3' } \\
(-) 5^{\prime} \text {-CGCCTTGTCCTTGGTAGTGT-3' }\end{array}$ & $170 b p$ & Liu et al., 2006 \\
\hline CD44 & $\begin{array}{l}(+) 5^{\prime} \text {-AGAAGGTGTGGGCAGAAGAA-3' } \\
(-) 5^{\prime} \text {-AAATGCACCATTTCCTGAGA-3' }\end{array}$ & $116 b p$ & Liu et al., 2006 \\
\hline CD90 & $\begin{array}{l}(+) 5^{\prime} \text {-CGCTCTCCTGCTAACAGTCTT-3' } \\
(-) 5^{\prime} \text {-CAGGCTGAACTCGTACTGGA-3' }\end{array}$ & $143 b p$ & Liu et al., 2006 \\
\hline $\operatorname{PPAR} \gamma$ & $\begin{array}{l}(+) 5^{\prime} \text {-CACAAGAACAGATCCAGTGGTTGCAG-3' } \\
(-) 5^{\prime} \text {-AATAATAAGGTGGAGATGCAGGCTCC-3' }\end{array}$ & $101 b p$ & Redonnet et al., 2002 \\
\hline Osteonectin & $\begin{array}{l}(+) 5^{\prime} \text {-AGA AGC TGC GGG TGA AGA A-3' } \\
(-) 5^{\prime}-\text { TGC CAG TGT ACA GGG AAG ATG-3' }\end{array}$ & $127 b p$ & Yi-xiong et al., 2006 \\
\hline VEGFR-1 & $\begin{array}{l}(+) 5^{\prime} \text {-TAC AAA GAA ATA GGG CTT CTG ACC-3' } \\
(-) 5^{\prime} \text {-ATG GCC TCT AAG TAA TTT GAC TGG-3' }\end{array}$ & $144 b p$ & Hong et al., 2006 \\
\hline
\end{tabular}

Table 1: Primer sequences and cDNA amplicon size (in $b p$ ) used in this study (contd). 


\begin{tabular}{|c|c|c|}
\hline Gene & $\begin{array}{r}\text { Primers: sense (+) } \\
\text { Anti-sense (-) }\end{array}$ & Product size \\
\hline TRAIL & $\begin{array}{l}\text { (+) 5' -GAG AAC CTC TGA GGA AAC CATT- 3' } \\
(-) \text { 5' -TTT CAT GGA TGA CCA GTT CAC C- 3' }\end{array}$ & $256 b p$ \\
\hline DR4 & $\begin{array}{l}\text { (+) 5' -TGT ACG CCC TGG AGT GAC AT- 3' } \\
(-) \text { 5' -CAC CAA CAG CAA CGG AAC AA- 3', }\end{array}$ & $115 b p$ \\
\hline DR5 & $\begin{array}{l}\text { (+) 5' -CAA CGC TTC CAA CAA TGA- 3' } \\
(-) \text { 5'- ACG TGC CTT CTT TAC ACT GA- 3' }\end{array}$ & $119 b p$ \\
\hline DcR1 & $\begin{array}{l}(+) \text { 5' -CCT GCA CCA TGA CCA GAG A- 3' } \\
(-) \text { 5' -TGG CAC CAA ATT CTT CAA CA- 3', }\end{array}$ & $168 b p$ \\
\hline DcR2 & $\begin{array}{l}\text { (+) 5' - GCG GAG GAG ACA GTG ACC A- 3' } \\
(-) \text { 5' - CCA GGA ACT CGT GAAGGA CA- 3' }\end{array}$ & $227 b p$ \\
\hline OPG & $\begin{array}{l}\text { (+) 5' -GTC TTT GGT CTC CTG CTA- 3' } \\
(-) \text { 5' -ACA CTC TCT GCG TTT ACT TT- 3' }\end{array}$ & $151 b p$ \\
\hline
\end{tabular}

Table 1: Primer sequences and cDNA amplicon size (in $b p$ ) used in this study (contd). 


\begin{tabular}{|c|c|c|}
\hline Gene & $\begin{array}{l}\text { Primers: sense (+) } \\
\text { Anti-sense (-) }\end{array}$ & Product size \\
\hline CD31 & $\begin{array}{l}\text { (+) 5' -CAG TGT CCC CAG AAG CAA AT- 3' } \\
(-) \text { 5' -CTC CGA TGA TAA CCA CTG CAA- } 3\end{array}$ & $88 b p$ \\
\hline GLUT-1 & $\begin{array}{l}\text { (+) 5' -CAG CCT GTG TAT GCC ACC ATT- 3' } \\
(-) \text { 5' -TAG CGC GAT GGT CAT GAG TAT- 3' }\end{array}$ & $155 b p$ \\
\hline CD45 & $\begin{array}{l}\text { (+) 5' -TGT CTT TGA ACT GAG ACA TTC CA- 3' } \\
\text { (-) 5' -TGC TCC ACA CTC CAG TTT GTA T- 3' }\end{array}$ & $83 b p$ \\
\hline Collagen-I & $\begin{array}{l}\text { (+) 5' -CACTGGGTTCGGAGGAGAGT- 3' } \\
(-) \text { 5' -CGGCACAAGGGATTGACAC- 3' }\end{array}$ & $84 b p$ \\
\hline Collagen-II & $\begin{array}{l}\text { (+) 5' -ATGGAGACTGGCGAGACTTG- 3' } \\
\text { (-) 5' -TGGACAGCAGGCGTAGGA- 3' }\end{array}$ & $199 b p$ \\
\hline Collagen-III & $\begin{array}{l}\text { (+) 5' -GCGGATAGAGATGTCTGGAA- 3' } \\
\text { (-) 5' -GAGGCTGTGGGCAAAC- 3' }\end{array}$ & $157 b p$ \\
\hline Collagen-VIII & $\begin{array}{l}\text { (+) 5' -CTCCTCAGATGCCACCAC- 3' } \\
\text { (-) 5' -TTCTTTGCCTTTCTTGGGTA- 3' }\end{array}$ & $209 b p$ \\
\hline Laminin-V & $\begin{array}{l}\text { (+) 5' -AGGGCAACCAGATGAGCA- 3' } \\
\text { (-) 5' -AGGAAGACAGCCGAGGAGAT- 3', }\end{array}$ & $211 b p$ \\
\hline IGF-2 & $\begin{array}{l}\text { (+) 5' -CCGTGCTTCCGGACAACTTC- 3' } \\
\text { (-) 5' -TGGACTGCTTCCAGGTGTCA- 3' }\end{array}$ & $72 b p$ \\
\hline VEGF & $\begin{array}{l}\text { (+) 5' -TGA GGA GTC CAA CAT CAC CAT- 3' } \\
\text { (-) 5' -TGT GCT GTA GGA AGC TCA TCT- 3' }\end{array}$ & $88 b p$ \\
\hline
\end{tabular}

Table 1: Primer sequences and cDNA amplicon size (in $b p$ ) used in this study (contd). 


\subsubsection{THE QUANTITATIVE REAL-TIME PCR ASSAY}

qRT-PCR analysis was performed using the Platinum SYBR Green qPCR supermix UDG (Invitrogen) appropriately diluted to a final reaction volume of $20 \mu \mathrm{l}$. Each reaction contained SYBR Green I, 1.5 U/ml Taq DNA polymerase, $20 \mathrm{mM}$ TrisHCL (pH 8.4), $50 \mathrm{mM} \mathrm{KCL,} \mathrm{3mM} \mathrm{MgCl}_{2}, 200 \mu \mathrm{M}$ dGTP, dCTP, dATP and dTTP, 1 U/ml UDG, $0.1 \mu \mathrm{M}$ gene-specific primers with $1 \mu \mathrm{l}$ of template (reverse transcribed cDNA or purified PCR product) and $10 \mathrm{nM}$ fluorescein (Invitrogen). All reactions were performed in duplicate. Controls included a no template (water) control and amplification from an equivalent amount of RNA that had not been reverse transcribed. Amplification was achieved with an initial denaturation at $95^{\circ} \mathrm{C}$ for $30 \mathrm{sec}$, followed by 35 cycles of denaturation at $95^{\circ} \mathrm{C}$ for $15 \mathrm{sec}$, annealing at $60^{\circ} \mathrm{C}$ for $20 \mathrm{sec}$ and extensions at $72^{\circ} \mathrm{C}$ for $20 \mathrm{sec}$. Data was collected from both the annealing and extension steps. A melt-curve analysis was performed at the end of every amplification via a temperature gradient from 65 to $95^{\circ} \mathrm{C}$ in $0.5^{\circ} \mathrm{C}$ increment steps measuring fluorescence at each temperature for a period of $30 \mathrm{sec}$. The melt-curve analysis was performed to confirm the absence of spurious amplification products and primer dimers. Agarose gel electrophoresis was also performed on selected amplifications from each run to confirm that products of the expected size were amplified. Amplification products and the $\mathrm{Ct}$ values were detected using an iQ qPCR system and associated software (Bio-Rad Laboratories Pty Ltd, Auckland, New Zealand). The cycle numbers were calculated from areas of exponential amplification of the traces. The amount of mRNA expression was determined relative to a chosen internal house keeping gene included with each run. In some cases, the relative fold change in expression was also calculated using the formula.

Fold change in expression $=2^{-\Delta \mathbf{C t}}$ 


\subsubsection{HAEMANGIOMA SPECIMENS}

All haemangioma specimens were obtained according to a protocol approved by the Wellington Ethics Committee. Part of each specimen was snap-frozen in liquid nitrogen for subsequent mRNA and protein analysis while the remainder was fixed in paraformaldehyde prior to wax embedding for immunohistochemistry. The haemangioma biopsies were categorised clinically into proliferating, involuting and involuted phases and the assignments confirmed by histological analysis as previously described $\left(\operatorname{Tan}_{\mathrm{a}}\right.$ et al., 2000).

\subsubsection{CELL CULTURE}

\subsubsection{CULTURE FACILITY SET-UP}

All plasticware (except pipette tips and eppendorfs) and scalpels were obtained sterile from the manufacturers. The pipette tips, eppendorfs, and glass bottles, were sterilized by autoclaving at $120^{\circ} \mathrm{C}$ for $20 \mathrm{~min}$ before use. Spatulas and microforceps were wiped with $70 \%$ ethanol to prevent contamination. All handling of the tissues and transfer of all liquids were conducted under asceptic conditions in a sterile Email Air Handling Class II Biological safety cabinet (AES Environmental Pty LTD, Auburn Australia). Latex gloves were used at all times to prevent contamination.

Several different cell lines were used in the present project. Cell cultures were grown in a Heracell incubator (Kendro Laboratory Prodects GmbH, Hanau, Germany) at $37^{\circ} \mathrm{C}$ in humidified air with $5 \% \mathrm{CO}_{2}$; herein referred to as the cell culture incubator. All cell line stocks were stored at $-80^{\circ} \mathrm{C}$ using $1 \mathrm{ml}$ cryotubes (Greiner Bio-one, Frickenhausen, Germany) at $1 \times 10^{6}$ cells $/ \mathrm{ml}$ in $10 \%$ DMSO (dimethyl sulfoxide) and 90\% heat-inactivated foetal calf serum (FCS) (Gibco-BRL, Invitrogen). 


\subsubsection{CELL LINES USED IN THIS STUDY}

\subsection{HeLa cells}

HeLa cells are an immortalized adherent cervical cancer cell line isolated from Henrietta Lacks, who died from her cancer in 1951 (Master, 2002). HeLa cells were grown in Roswell Park Memorial Institute 1640 with Glutamine (RPMI 1640 + Glutamine, Gibco-BRL) medium supplemented with 10\% FCS and 2\% Penstrep (100 $\mu \mathrm{g} / \mathrm{ml}$ streptomycin sulfate and $100 \mu \mathrm{g} / \mathrm{ml}$ penicillin $\mathrm{G}$, Gibco-BRL), herein referred to as cell culture medium. Cells were maintained in either $25 \mathrm{~cm}^{2}$ or $75 \mathrm{~cm}^{2}$ sterile cell culture flasks (Greiner Bio-One) and were passaged by splitting the cells 1:10 twice weekly.

\subsection{T3 fibroblasts}

The mouse fibroblast cell line, $3 \mathrm{~T} 3$ fibroblasts, were obtained from Mr An Tan, Malaghan Institute of Research, Wellington, New Zealand. This is an immortalized cell line originally established from primary mouse embryonic fibroblast cells by two scientists called George Todaro and Howard Green in 1962 (Todaro \& Green, 1963). $3 \mathrm{~T} 3$ cells were grown in cell culture medium and maintained in a similar way as decribed for HeLa cells. 3T3 cells were passaged by splitting the cells 1:5 twice weekly.

\subsection{Human vascular endothelial cells}

Human vascular endothelial cells (HUVECs) were a kind gift from Dr Sarah Gunningham, Christchurch School of Medicine and Health Sciences, Otago University. HUVECs were grown in RPMI 1640 + glutamine, 10\% FCS, $2 \%$ Penstrep and $30 \mu \mathrm{g} / \mathrm{ml}$ endothelial cell growth supplement (ECGS, Sigma-Aldrich, New Zealand). Cells were passaged 1:3 when the cells reached $80 \%$ confluencey. 


\subsection{Haemangiendothelioma cells}

The mouse derived haemangiendothelioma cell line (EOMA) was purchased from the American Type Culture Collection (ATCC). Cells were obtained frozen and thawed as described in section 2.1.6.3. EOMA cells were grown in cell culture medium and passaged by splitting the cells 1:5 twice weekly.

\subsubsection{STANDARD CELL CULTURE TECHNIQUES}

When required, cells frozen at $-80^{\circ} \mathrm{C}$ were thawed in a water bath at $37^{\circ} \mathrm{C}$. Cells were then transferred to $15 \mathrm{ml}$ centrifuge tubes (Greiner Bio-one) containing $10 \mathrm{ml}$ of pre-warmed cell culture medium and centrifuged at $300 \mathrm{X} \mathrm{g}(1000 \mathrm{rpm})$ for $5 \mathrm{~min}$ at room temperature. Following removal of the supernatant, the cell pellet was resuspended in fresh cell culture medium and transferred to culture flasks for expansion.

To passage cells, the medium was removed and the cells were rinsed with sterile phosphate buffered saline (PBS) (Appendix 4, section 4.2.1). To dissociate the cells, an appropriate volume of pre-warmed trypsin-ethylenediaminetetraacetic acid (EDTA) (Invitrogen) (Final concentration of $0.05 \%-0.02 \%$, respectively) was added, enough to cover the surface of the dish and the flasks were returned to the incubator for $2-5 \mathrm{~min}$, or until the cells had detached. Media containing 5\% FCS was then added at three times the volume of trypsin and cells were collected and transferred to $15 \mathrm{ml}$ polypropylene centrifuge tubes. Cells were centrifuged at $300 \mathrm{X} \mathrm{g}(1000 \mathrm{rpm})$ for $5 \mathrm{~min}$ at room temperature. The supernatant was discarded, the cell pellet resuspended in fresh cell culture medium, and an appropriate volume of the cell suspension was transferred into new culture dishes.

When required the percentage of viable cells were counted after trypsinisation by the trypan blue dye exclusion using a haemocytometer. The pellet obtained after centrifugation following trypsinisation was resuspended in 500-1000 $\mu$ l of fresh cell culture medium. A $10 \mu \mathrm{l}$ sample of the suspended cells was diluted in $10 \mu 1$ of $0.02 \%$ 
trypan blue (Invitrogen) in PBS and $10 \mu \mathrm{l}$ of this mixture was loaded into the groove of the haemocytometer. Only the cells which excluded the trypan blue dye were counted and the percentage of viable cells determined.

\subsubsection{IMMUNOHISTOCHEMISTRY}

\subsubsection{PREPARATION OF SLIDES}

For immunohistochemistry (IHC), slides were first cleaned with 70\% ethanol, dried and submerged in a freshly prepared $2 \%$ solution of 3-aminopropyltriethoxysilane (APES, Sigma) prepared in acetone for $1 \mathrm{~min}$. Slides were then transferred to a large tank of water to wash off any remaining APES and dried overnight in an oven at $37^{\circ} \mathrm{C}$. The poly-cationic nature of APES allows interaction with the anionic sites of tissue sections resulting in strong adhesion, thus preventing loss of sections during the staining procedures (Howard \& Bethell, 2001).

\subsubsection{PARAFFIN EMBEDDED TISSUE SECTIONS}

Although paraformaldehyde (PFA) fixation provides good tissue preservation and high resolution for light microscopy, it crosslinks proteins so strongly that antigenic sites become obscured thereby inhibiting or distorting immunoreaction. Unmasking of hidden antigenic epitopes was carried out using non-enzymatic methods as described in the following sections. $5 \mu \mathrm{m}$ sections of formalin fixed paraffin embedded tissues were cut using a Reichert-Jung Biocut microtome (Leica Instruments, Nussloch, Germany), stretched in a $48^{\circ} \mathrm{C}$ water bath, transferred onto APES-coated glass slides and dried at room temperature overnight. Prior to immunostaining, paraffin embedded haemangioma biopsies were de-paraffinised in xylene ( 2 changes, 5 min each), re-hydrated through a graded ethanol $(100 \%, 95 \%, 85 \%$ and $70 \%)$ series followed by a final rinse in $\mathrm{dH}_{2} \mathrm{O}$ for $5 \min$. 


\subsubsection{CELL CULTURE PREPERATIONS}

Cultured cells were plated onto $13 \mathrm{~mm}$ diameter coverslips (BDH, Palmerson North, New Zealand) which were cleaned and stored in a sealed container in $70 \%$ ethanol until needed. Prior to plating, the coverslips were flame-sterilised, and four coverslips were placed in each $35 \mathrm{~mm}$ diameter cell culture dish (Greiner Bio-one). The dishes containing the coverslips were then placed under UV light for 15 minutes.

After trypsinisation, approximately 1000-5000 cells were plated onto each coverslips in a total volume of $20 \mu \mathrm{l}$ and allowed to attach for 15 minutes. The appropriate culture medium was then added to the dish and the cells were left overnight in the incubator.

\subsubsection{STAINING PROCEDURES}

\subsubsection{HISTOCHEMISTRY}

For histochemical analysis, tissue sections were stained with Mayer's haematoxylin and eosin (H\&E) (Luna, 1992). Slides were deparaffined in xylene (2 changes, 5 min each), rehydrated through a graded ethanol series and rinsed in $\mathrm{dH}_{2} \mathrm{O}$. The slides were submerged in haematoxylin for 2 min and washed in running tap water for $5 \mathrm{~min}$ before counterstaining with eosin for $1 \mathrm{~min}$. They were then dehydrated in $95 \%$ alcohol and absolute alcohol ( 2 changes for $5 \mathrm{~min}$ ), cleared in xylene ( 2 changes for 3 min each) and mounted in D.P.X (BDH Chemicals Ltd, Poole, United Kingdom).

\subsubsection{FLOURESCENT IMMUNOHISTOCHEMISTRY}

Sensitive detection systems were employed to facilitate the immunolocalisation of some proteins. Sources and dilutions for the primary and secondary antibodies used in this study are given in Tables 2 and 3, respectively. Antibodies used in this study were raised in either, a mouse, a rabbit or a goat but are know to specifically recognise the human antigen. 


\begin{tabular}{|c|c|c|c|c|}
\hline Marker & Species & $\begin{array}{l}\text { Antibody } \\
\text { dilution }\end{array}$ & $\begin{array}{l}\text { Technique } \\
\text { employed }\end{array}$ & Source of primary antibody \\
\hline STAT-1 & Mouse & $\begin{array}{l}1: 50 \\
1: 100 \\
1: 1000\end{array}$ & $\begin{array}{l}\text { IHC-P } \\
\text { IHC-C } \\
\text { WB }\end{array}$ & Cell Signalling, MA \\
\hline STAT-3 & $\begin{array}{l}\text { Rabbit } \\
\text { Mouse } \\
\text { Mouse }\end{array}$ & $\begin{array}{l}1: 30 \\
1: 100 \\
1: 1000\end{array}$ & $\begin{array}{l}\text { IHC-P,IHC-C } \\
\text { IHC-C } \\
\text { WB }\end{array}$ & Cell Signalling \\
\hline STAT-5 & Mouse & $1: 100$ & IHC-C & Invitrogen \\
\hline VEGFR-2 & Rabbit & $\begin{array}{l}1: 30 \\
1: 1000\end{array}$ & $\begin{array}{l}\text { IHC-P,IHC-C } \\
\text { WB }\end{array}$ & Cell Signalling \\
\hline CycA & Rabbit & $\begin{array}{l}1: 100 \\
1: 1000\end{array}$ & $\begin{array}{l}\text { IHC-P } \\
\text { WB }\end{array}$ & $\begin{array}{l}\text { BIOMOL Research Laboratories } \\
\text { Inc, PV }\end{array}$ \\
\hline GAPDH & Mouse & $1: 1000$ & WB & Abcam Limited, Cambridge, UK \\
\hline DR4 & Mouse & $\begin{array}{l}1: 100 \\
1: 1000\end{array}$ & $\begin{array}{l}\text { IHC-P,IHC-C } \\
\text { WB }\end{array}$ & Imgenex, SD \\
\hline DR5 & Mouse & $1: 200$ & IHC-P,IHC-C & Imgenex \\
\hline DcR1 & Rabbit & $1: 100$ & IHC-P,IHC-C & Imgenex \\
\hline DcR2 & Rabbit & $\begin{array}{l}1: 200 \\
1: 1000\end{array}$ & $\begin{array}{l}\text { IHC-P,IHC-C } \\
\text { WB }\end{array}$ & Imgenex \\
\hline OPG & Mouse & $5 \mu \mathrm{g} / \mathrm{ml}$ & $\begin{array}{l}\text { IHC-P,IHC-C } \\
\text { WB }\end{array}$ & R\&D systems, $\mathrm{MN}$ \\
\hline vWF & Rabbit & $1: 200$ & IHC-P,IHC-C & Dako Corp, Glostrup, Denmark \\
\hline GLUT-1 & Rabbit & $1: 100$ & IHC-P,IHC-C & Dako Corp \\
\hline VEGFR-1 & Goat & $\begin{array}{l}5 \mu \mathrm{g} / \mathrm{ml} \\
0.2 \mu \mathrm{g} / \mathrm{ml}\end{array}$ & $\begin{array}{l}\text { IHC-C } \\
\text { WB }\end{array}$ & R\&D systems \\
\hline
\end{tabular}

Table 2: Primary antibodies used in this study, their dilutions and sources. 


\begin{tabular}{|c|c|c|c|c|}
\hline Marker & $\begin{array}{l}\text { Primary } \\
\text { antibody }\end{array}$ & $\begin{array}{l}\text { Antibody } \\
\text { dilution }\end{array}$ & $\begin{array}{l}\text { Technique } \\
\text { employed }\end{array}$ & Source of primary antibody \\
\hline $\begin{array}{l}\text { p-STAT-3 } \\
\text { (Tyr 705) }\end{array}$ & $\begin{array}{l}\text { Rabbit } \\
\text { Mouse }\end{array}$ & $\begin{array}{l}1: 50 \\
1: 1000\end{array}$ & $\begin{array}{l}\text { IHC-P, IHC-C } \\
\text { WB }\end{array}$ & Cell Signalling \\
\hline $\begin{array}{l}\text { p-STAT-1 } \\
\text { (Tyr 701) }\end{array}$ & Goat & $5 \mu \mathrm{g} / \mathrm{ml}$ & IHC-C & Santa Cruz Biotechnology, CA \\
\hline $\begin{array}{l}\text { p-STAT-5 } \\
\text { (Tyr 695) }\end{array}$ & Rabbit & $1: 50$ & IHC-C & Cell Signalling \\
\hline Vimentin & $\begin{array}{l}\text { Mouse } \\
\mathrm{mAb}\end{array}$ & $1: 100$ & IHC-C & Dako Corp \\
\hline$\alpha$-SMA & $\begin{array}{l}\text { Mouse } \\
\text { mAb }\end{array}$ & $1: 100$ & IHC-C & Dako Corp \\
\hline VEGF & Mouse & $5 \mu \mathrm{g} / \mathrm{ml}$ & WB & R\&D systems \\
\hline PCNA & Mouse & $1: 50$ & IHC-P & Chemicon International, CA \\
\hline CD34 & Mouse & $1: 50$ & IHC-C & Dako Corp \\
\hline
\end{tabular}

Table 2: Primary antibodies used in this study, their dilutions and sources (contd). Key: IHC-C-Immunohistochemistry on cultured cells, IHC- Immunohisotchemistry on paraffin embedded tissue sections, FACS-Flow cytometry cell sorting, WB-Western blotting. 


\begin{tabular}{|c|c|c|}
\hline Secondary antibody & Antibody dilution & Source of antibody \\
\hline Biotinylated sheep anti-goat IgG & $1: 50-\mathrm{IHC}-\mathrm{C}$ & Sigma \\
\hline $\begin{array}{l}\text { Sheep anti-goat Streptavidin } \\
\text { AlexaFluor } 555 \text { conjugated IgG }\end{array}$ & $\begin{array}{l}1: 250-\mathrm{IHC}-\mathrm{C} \\
1: 2500-\mathrm{FACS}\end{array}$ & Invitrogen \\
\hline $\begin{array}{l}\text { Sheep anti-rabbit } \mathrm{Cy} 3 \text { conjugated } \\
\text { IgG }\end{array}$ & 1:250-IHC-P, IHC-C & Sigma \\
\hline $\begin{array}{l}\text { Sheep anti-mouse AlexaFluor } 488 \\
\text { conjugated } \operatorname{IgG}\end{array}$ & 1:250-IHC-P, IHC-C & Invitrogen \\
\hline $\begin{array}{l}\text { Sheep anti-goat AlexaFluor } 488 \\
\text { conjugated IgG }\end{array}$ & 1:1000-WB & Invitrogen \\
\hline Biotinylated goat anti-rabbit IgG & 1:200-IHC-P & Sigma \\
\hline $\begin{array}{l}\text { Sheep anti-rabbit digoxigenin } \\
\text { (DIG) conjugated IgG }\end{array}$ & $1 \mu \mathrm{g} / \mathrm{ml}-\mathrm{IHC}-\mathrm{P}, \mathrm{IHC}-\mathrm{C}$ & Chemicon \\
\hline $\begin{array}{l}\text { Sheep anti-mouse } \quad \text { DIG } \\
\text { conjugated IgG }\end{array}$ & 1:25- IHC-P, IHC-C & Roche Diagnostics \\
\hline $\begin{array}{l}\text { Sheep anti-mouse Fluorescein } \\
\text { (FITC) conjugated IgG }\end{array}$ & $\begin{array}{l}\text { 1:100- IHC-P, IHC-C } \\
1: 1000-\mathrm{WB}\end{array}$ & Sigma \\
\hline $\begin{array}{l}\text { Sheep anti-DIG Rhodamine } \\
\text { conjugated IgG }\end{array}$ & $0.5-\mu \mathrm{g} / \mathrm{ml}-\mathrm{IHC}-\mathrm{P}, \mathrm{IHC}-\mathrm{C}$ & Roche Diagnostics \\
\hline $\begin{array}{l}\text { Biotinylated horse anti-mouse } \\
\text { IgG }\end{array}$ & 1:200-IHC-P & Vector Laboratories \\
\hline
\end{tabular}

Table 3: Secondary antibodies used in this study, their dilutions and sources. Key: IHCC-Immunocytochemistry, IHC- Immunohisotchemistry, FACS-Flow cytometry cell sorting, WB-Western blotting. 


\subsection{Paraffin embedded tissue sections}

Following deparaffinisation and rehydration, slides were rinsed in $\mathrm{dH}_{2} \mathrm{O}$ and then in tris buffered saline (TBS, pH 7.5, Appendix 4) containing 0.1\% Tween-20 (BDH Chemicals) (TBST, Appendix 4) for 5 min each. To unmask cross-linked antigenic epitopes, the tissue sections were immersed in coplin jars containing boiling $10 \mathrm{mM}$ sodium citrate (pH 6.0) for $10 \mathrm{~min}$, except for VEGFR-2 staining for which a $1 \mathrm{mM}$ EDTA (pH 8.0) solution was used. The coplin jars were placed in a pressure cooker containing boiling water and the lid of the cooker was loosely placed on top to contain the heat. The coplin jars were removed from the cooker and left to cool to room temperature for $40 \mathrm{~min}$ on the bench. The slides were then rinsed in TBST ( 2 changes, 5 min each). Autofluorescence from the tissue sections was quenched by washing the slides in three changes of freshly prepared $0.5 \%(\mathrm{w} / \mathrm{v})$ sodium borohydride $\left(\mathrm{NaBH}_{4}\right)$ solution in TBS for 15 min each. Slides were then washed in TBST for 5 min and blocked with either $5 \%$ bovine serum albumin (BSA) or $5 \%$ goat serum prepared in TBST for $60 \mathrm{~min}$ at room temperature. This was followed by incubation with primary antibodies diluted in TBST containing either $1 \%$ BSA or $1 \%$ goat serum at $4{ }^{\circ} \mathrm{C}$ overnight. Controls included the omission of the primary antibodies. The next day, sections were washed in TBST ( 3 changes, 5 min each) before incubation with the secondary antibodies diluted in TBST containing 1\% BSA or 1\% goat serum for 2 hours at room temperature. Slides were washed in TBST ( 3 changes, 5 min each) and the cell nuclei were counterstained with 4',6-diamino-2-phenylindole (DAPI; Boerhinger Mannheim Biochemicals, IN) and mounted in Antifade (Vectorshield, Burlingame, CA) or ProLong ${ }^{\circledR}$ Gold Antifade (Invitrogen) (see section 2.1.8.3, page 75).

\subsection{Cultured cells}

Coverslips with the attached cells were removed from the culture medium and washed in TBS before being fixed. Cells were fixed in an ice cold solution of methanol 
and acetone $(1: 1, \mathrm{v} / \mathrm{v})$ for $10 \mathrm{~min}$ at $-20^{\circ} \mathrm{C}$ except where indicated. Fixed cells were washed in TBST (2 changes, 5 min each). Boiling $10 \mathrm{mM}$ sodium citrate, $\mathrm{pH} 6.0$ or 1 mM EDTA, pH 8.0 (for VEGFR-2 staining) solution was poured over the coverslips and allowed to cool on the bench top for at least $30 \mathrm{~min}$. Coverslips were washed in TBST ( 3 changes, 5 min each) and non-specific binding sites were blocked for $60 \mathrm{~min}$ at room temperature in TBST containing 5\% BSA. Coverslips were then incubated in the respective primary antibodies diluted in TBST containing 1\% BSA overnight at $4^{\circ} \mathrm{C}$. The next day, the coverslips were rinsed in TBST and incubated in the respective secondary antibodies diluted in TBST containing 1\% BSA for at least $1 \mathrm{hr}$ at room temperature in a humidified chamber. Coverslips were washed in TBST ( 3 changes, 5 min each) and the cell nuclei were counterstained with DAPI or propidium iodide (PI) (Sigma) (see section 2.1.8.3) and mounted in Antifade onto clean micorscope slides. In some cases, a post fixation wash in $4 \%$ PFA for 2 min was included after antibody incubation and prior to mounting in Antifade.

\subsubsection{COUNTER STAINING OF THE NUCLEUS}

DAPI was used to label the nucleus in fluorescently labelled samples. DAPI stock $\left(100 \mu \mathrm{g} / \mathrm{ml}\right.$ prepared in PBS, and stored in the dark at $\left.-20^{\circ} \mathrm{C}\right)$ was diluted immediately before use in TBST to a final concentration of $20 \mu \mathrm{g} / \mathrm{ml}$. Tissue sections and cell culture samples were counterstained in DAPI for $10 \mathrm{~min}$ after fluorescent labelling, washed and mounted in Antifade.

For some experiments, the nuclei were stained with PI $(10 \mu \mathrm{g} / \mathrm{ml})$. PI stock was prepared in PBS to a final concentration of $2 \mathrm{mg} / \mathrm{ml}$ and stored in the dark at $4^{\circ} \mathrm{C}$ until required.

\subsubsection{COLORIMETRIC VISUALISATION WITH DAB}

Diaminobenzidine (DAB) colorimetric visualisation of immunostaining was 
used to detect STAT-1 expression in haemangioma. STAT3, STAT-1 and CycA immunoreactive MCs were detected using $\mathrm{DAB}$ staining in conjunction with Csaba (alcian blue-safranin solution) staining. Five specimens each of proliferating, involuting and involuted haemangiomas were processed as for fluorescent immunohistochemistry, except the treatment with $\mathrm{NaBH}_{4}$ was replaced with a 30 min incubation in $3 \%$ hydrogen peroxide $\left(\mathrm{H}_{2} \mathrm{O}_{2}\right)$ prepared in methanol and slides were blocked with TBST containing 5\% horse serum for $60 \mathrm{~min}$ at room temperature. Slides were incubated with either mouse anti-STAT-1, mouse anti-STAT-3 or rabbit anti-CycA primary antibodies followed by detection with a sensitive 3-step biotin-streptavidin/immunoperoxidase technique. Bound primary antibodies were detected by incubating the sections with either biotinylated anti-mouse or anti-rabbit antibodies for $1 \mathrm{hr}$ at room temperature and then washed in TBST (3 changes, 5 min each). The Vecta stain Elite ABC reagent containing peroxidase-conjugated streptavidin (Vector Labs, CA) was then added to the section and incubated for $30 \mathrm{~min}$ at room temperature. All incubations were carried out in a humidified chamber. The sections were washed in TBST ( 3 changes, 5 min each) and immunoreactivity was detected by incubation with $0.05 \%$ 3'3'-diaminobenzidine tetrahydrochloride (Sigma), 0.01\% $\mathrm{H}_{2} \mathrm{O}_{2}$ in $50 \mathrm{mM}$ Tris HCL, pH 7.5 from 5-20 min. Stained sections were immersed in Csaba stain (see Appendix 4, section 4.2.10, page 299) for 15 min to identify MCs (Tan et al., 2000). The sections were then rinsed thoroughly in tap water, dehydrated in tertiary butyl alcohol, cleared in xylol (BDH Chemicals) and mounted in D.P.X.

\subsubsection{NEGATIVE CONTROLS}

All immunostaining experiments included control sections which were processed in the same way as the test sections but excluded incubation with the primary antibody. For the DAB stained sections, the control slides were incubated with the DAB substrate for the same length of time as the test slides. 


\subsubsection{IMAGE ANALYSIS}

Immunostained slides were viewed using an Olympus AX70 photomicroscope (Olympus Opticals Co., Hamburg, Germany) equipped with appropriate narrow band fluorescence filters. Images were captured using an Olympus DP70 CCD camera. Some slides were visualised using a Leica TCS 4D confocal laser-scanning microscope fitted with a krypton/argon laser (Leica Lasertechnik, Heidelberg, Germany). For presentation purposes, images were background corrected and overlaid to produce dual colour images using Adobe Photoshop versions CS2 software.

\subsubsection{WESTERN BLOTTING}

\subsubsection{SAMPLE PREPARATION FOR WESTERN BLOTS}

\subsection{Tissue lysate}

Snap frozen tissue biopsies were weighed and crushed to a powder in liquid nitrogen and transferred to a sterile $1.5 \mathrm{ml}$ eppendorf containing freshly prepared lysis buffer (50 mM Tris-Hcl pH 6.8 containing 5 mM EDTA, $2.5 \mathrm{mM} \mathrm{NaF}, 2 \mathrm{mM} \mathrm{NaO} 3 \mathrm{~V}, 2$ $\mathrm{mM}$ PMSF and $2 \% \mathrm{SDS}$ ) at $100 \mathrm{mg} / \mathrm{ml}$. Activation of $\mathrm{Na}_{3} \mathrm{VO}_{4}$ is required before use

(see Appendix 4, section 4.2.2, page 297). Tissue was further homogenised with a plastic pestle (Sigma) and boiled in a dry heat block for $10 \mathrm{~min}$ at $100^{\circ} \mathrm{C}$. Lysates were then spun in a microcentrifuge at $14,000 \mathrm{rpm}$ for $15 \mathrm{~min}$ at $4^{\circ} \mathrm{C}$. Supernatants were transferred to fresh eppendorf tubes and stored at $-20{ }^{\circ} \mathrm{C}$ until required for protein analysis.

\subsection{Cell lysate}

To prepare cell lysates for Western blotting, the cell culture media was first removed. The cells were rinsed with PBS and covered with $0.2 \mathrm{ml}$ of freshly prepared lysis buffer. The cells were then mechanically detached from the cell culture dish using 
a rubber cell scrapper and spun in a microcentrifuge at $14,000 \mathrm{rpm}$ for $15 \mathrm{~min}$ at $4^{\circ} \mathrm{C}$.

From this point the cell lysates were processed in the same way as the tissue lysates.

HeLa cell lysates were also prepared for Western blots. The cells were grown to $95 \%$ confluence in cell culture media in $100 \mathrm{~cm}^{2}$ tissue culture dishes. The media was then replaced with fresh cell culture media containing IFN $\alpha-2 b$ (Schering-Plough Ltd, New South Wales, Australia) at 10,000 U/ml and returned to the incubator for $10 \mathrm{~min}$. Cells were then lysed and lysates were prepared for Western blotting as described above.

\subsubsection{PROTEIN DETERMINATION}

Protein concentrations in tissue and cell culture lysates were determined using the bicinchoninic acid (BCA) protein assay kit (Pierce Biotechnology, Inc., WA). Serial dilutions of the BSA standard were prepared in lysis buffer ranging from $2 \mathrm{mg} / \mathrm{ml}$ to 15 $\mu \mathrm{g} / \mathrm{ml}$ and $25 \mu \mathrm{l}$ of each diluted BSA standard was added in duplicate into a 96-well plate. $25 \mu \mathrm{l}$ of each lysate was also analysed in duplicate along with $25 \mu \mathrm{l}$ of lysis buffer alone without protein. The BCA working reagent was made up as per manufacturer's instructions, as 50 parts solution A to 1 part solution B and $200 \mu 1$ was pipetted into each well. The solutions in the wells were mixed and the plate incubated for $30 \mathrm{~min}$ at $37^{\circ} \mathrm{C}$. Colour development was measured in a VersaMAX multiwell plate spectrophotometer (Molecular Devices, CA). The absorbance was measured at a wavelength of $562 \mathrm{~nm}$, blanked against lysis buffer alone containing no protein. Results were analysed with the Softmax Pro software programme (Molecular Devices) and the protein concentrations determined using a linear fit for the protein standards by plotting absorbance against concentration in $\mu \mathrm{g} / \mathrm{ml}$.

\subsubsection{GEL ELECTROPHORESIS}

All reagents for protein electrophoresis were purchased from Invitrogen except where indicated. Samples were prepared for electrophoresis according to 
manufacturer's instructions. Briefly, $5 \mu \mathrm{l}$ NuPage ${ }^{\circledR}$ LDS sample buffer $(4 \mathrm{X}), 2 \mu 1$ NuPage ${ }^{\circledR}$ reducing agent $(10 \mathrm{X})$ with the appropriate volume of lysate was made up to $20 \mu \mathrm{l}$ with deionised water. Samples were heated for $10 \mathrm{~min}$ at $70^{\circ} \mathrm{C}$ in a heat block, centrifuged and loaded into each lane of precast $4-12 \%$ gradient MES NuPAGE ${ }^{\mathrm{TM}}$ BisTris gels alongside $7 \mu$ of Sea Blue ${ }^{\circledR}$ Plus-2 pre-stained standard for size determination. The gel was run in 1XNuPage MES running buffer in a XCell SureLock ${ }^{\mathrm{TM}}$ Mini-Cell electrophoresis unit for $1 \mathrm{hr}$ at 120 volts.

\subsubsection{WESTERN BLOT TRANSFER}

Following electrophoresis, the gel was soaked in Western blot transfer buffer (Appendix 4, section 4.2.6, page 298) for $10 \mathrm{~min}$. An immobilon low fluorescence PVDF (polyvinylidine difluoride) membrane $(0.2 \mu \mathrm{m}$ pore size, Millipore Corporation, MA) was pre-soaked in methanol for $2 \mathrm{~min}$, then $1 \mathrm{~min}$ in $\mathrm{dH}_{2} \mathrm{O}$ and $5 \mathrm{~min}$ in transfer buffer before being placed onto the gel for transfer. The materials for the transfer were stacked in transfer buffer in a Western transfer apparatus (Appendix 4, section 4.2.23, page 301), avoiding air bubbles between the layers. The tank was filled with freshly prepared, ice cold transfer buffer, and the black side of the assembled cassette was placed nearest to the black electrode Transfer was carried at $4^{\circ} \mathrm{C}$ in a miniTRANSBLOT Cell transfer tank (BioRad Laboratories) for 6 hours at 100 volts onto the PVDF membrane.

\subsubsection{IMMUNOSTAINING OF WESTERN TRANSFERRED PROTEINS}

After protein transfer, the gels were stained with Comassie Blue and destained (see Appendix 4, section 4.2.5, page 298). Transfer efficiency was determined by eye on examining the level of protein that remained in the gel post transfer. 95-99\% transfer efficiency of was assumed when all of the highest molecular weight markers of the protein ladder was successfully transferred onto the membrane. Following protein transfer, the membranes were rinsed in $\mathrm{dH}_{2} \mathrm{O}$ and blocked, protein side up, in TBST 
containing 5\% non-fat milk for $1 \mathrm{hr}$ at room temperature. All membrane incubations were performed on a rocker platform at room temperature unless otherwise specified. The blocking agent was removed and the membranes were washed in TBST ( 3 changes, 5 min each) followed by incubation with the respective primary antibodies. All primary antibodies were diluted 1:1000 in TBST containing 5\% non-fat milk and incubated overnight at $4^{\circ} \mathrm{C}$. The next day, the PVDF membranes were rinsed in TBST ( 3 changes, 5 min each) before incubation with secondary antibodies. Primary antibody binding was detected using either anti-mouse or anti-goat AlexaFluor 488 conjugated antibodies or with anti-rabbit $\mathrm{Cy} 3$ conjugated secondary antibodies. All secondary antibodies were diluted 1:1000 in TBST and incubated for $1 \mathrm{hr}$ at room temperature. Following secondary antibody incubation, the membranes were rinsed in TBST ( 3 changes, 5 min each). Membranes were then scanned using a Fujifilm FLA-5100 imaging system (Fuji Photo Film Co Ltd., Tokyo, Japan).

\subsubsection{ANALYSIS OF WESTERN TRANSFERRED PROTEINS}

Image analysis and densitometry was performed using Image $\mathrm{J}$ software (National Institute of Health, MD). To obtain the band densities, the total area under the histogram for each band measurement was first calculated to obtain a band value. The same calculation was made on an equivalent area on the blot containing no band and was used as the background value. The background value was then subtracted from the band value to obtain a final band density measurement. This value was used for statistical analysis.

\subsubsection{GENERAL MOLECULAR BIOLOGY TECHNIQUES}

See Appendix 4, section 4.1 (page 294). 


\subsection{METHODS FOR CHAPTERS 3 AND 6}

\subsubsection{PRELIMINARY EXPERIMENTS}

Methods pertaining to all preliminary experiments can be found in Appendix 1, section 1.1.1 (page 267)

\subsubsection{GENE EXPRESSION CHANGES IN HAEMANGIOMA}

\subsubsection{QUANTITATIVE REAL TIME PCR}

RNA was extracted from each of eight randomly selected proliferating, involuting and involuted haemangioma specimens. Oligo $(\mathrm{dT})_{20}$ primed cDNA was synthesised and qRT-PCR was performed for STAT-3, STAT-1, VEGFR-2, CycA and GAPDH according to sections 2.1.2 and 2.1.4 (pages 59 and 65). mRNA expression levels were determined relative to the internal reference gene GAPDH using the formula:

$$
\Delta \mathrm{Ct}=\mathrm{Ct}_{(\mathrm{TEST} \mathrm{GENE})}-\mathrm{Ct}_{(\mathrm{GAPDH})}
$$

The relative fold change in mRNA expression was calculated using the formula:

$$
\text { Fold change in expression }=2^{-\Delta \mathrm{Ct}}
$$

(Wang et al., 2006; Miracle et al., 2006; Ortiga-Carvalho et al., 2005)

The data is expressed as the relative fold change in mRNA levels \pm s.e.m.

\subsubsection{WESTERN BLOTTING}

Western blotting was performed as described in section 2.1.9 (page 77). Total protein was extracted and quantified from five proliferating, four involuting and five involuted haemangiomas biopsies and quantified using the $\mathrm{BCA}$ protein assay kit (section 2.1.9.2, page 78) and $50 \mu \mathrm{g}$ of total protein was separated on a 4-12\% MES 
NuPage gradient gel. Lysate $(50 \mu \mathrm{g})$ from HeLa cells treated with IFN $\alpha-2 b$ was included as a positive control. Following electrophoresis and transfer, membranes were blocked and incubated with primary antibodies against STAT-3, p-STAT-3 (Tyr 705), STAT-1, VEGFR-1, CycA and GAPDH at $4^{\circ} \mathrm{C}$ overnight followed by incubation with AlexaFluor 488 conjugated anti-mouse or anti-goat secondary antibodies. CycA immunoreactivity was detected by incubating the membranes with $\mathrm{Cy} 3$ conjugated antirabbit antibodies. Membranes were then washed and scanned on a fluorescent scanner.

\subsubsection{FLOURESCENT IMMUNOHISTOCHEMISTRY}

Sources and dilutions of the primary and secondary antibodies are given in Chapter 2, Tables 2 and 3, respectively and the general protocol used for immunostaining in section 2.1.8.2.1 (page 74). To facilitate the immunolocalisation of some proteins however, a 3-step detection system was employed as described in the text below.

Paraffin embedded haemangioma tissue sections (five from each phase) were immunostained with primary antibodies against VEGFR-2, STAT-3, p-STAT-3 (Tyr 705), CycA and GLUT-1. Staining for PCNA was undertaken in conjunction with that for STAT-3 and p-STAT-3 (Tyr 705) by incubating both primary antibodies together. Immunoreactivity for VEGFR-2, STAT-3 and p-STAT-3 (Tyr 705) was detected using an anti-rabbit DIG conjugate followed by an anti-DIG-Rhodamine conjugate for visualisation. Bound PCNA antibodies were detected using an anti-mouse FITC conjugate. Bound CycA and GLUT-1 antibodies were detected using an anti-rabbit Cy3 conjugate.

\subsubsection{COLORIMETRIC VISUALISATION WITH DAB}

DAB staining was used to detect STAT-1 expression in haemangioma. Following immunolabelling, the STAT-1 stained tissue sections were counterstained with the Csaba stain as described in section 2.1.8.4 (page 75). To identify MC 
expression of STAT-3 and CycA, DAB staining in conjunction with Csaba staining was performed as described in section 2.1.8.4 (page 75).

\subsubsection{CELL COUNTING}

\subsection{STAT-3 and PCNA expression in haemangioma}

A minimum of 600 cells were counted from a typical 'proliferative' area (densely packed blood vessels with small lumens lined by plump ECs), an 'involuting' area (larger vessel lumens with flattened endothelia) and an 'involuted' area of each immunolabelled tissue section. Cells that stained positive for STAT-3, PCNA and STAT-3+PCNA were tabulated and the data plotted as the number of positively stained cells/unit area.

\subsection{STAT-3 and CycA expression by MCs in haemangioma}

At least $200 \mathrm{MCs}$ were counted from proliferating and involuting specimens (four samples per phase). The data for STAT-3 and CycA staining within MCs is plotted as total number of MCs that were positive and negative for STAT-3 and $\mathrm{CycA} /$ unit area. The average number of positively stained MCs in each phase was evaluated statistically.

\subsubsection{3 p-STAT-3 (Tyr 705) and PCNA expression in haemangioma}

Five proliferative and three involuting specimens were counted as described in section 2.2.2.5.1. All cells that stained for p-STAT-3 (Tyr 705), PCNA and those that stained for both p-STAT-3 (Tyr 705) and PCNA were tabulated and the data from individual samples were plotted as histograms. 'Proliferative' areas from proliferating specimens and 'involuting' areas from involuting specimens were analysed from each section. 


\subsubsection{STATISTICS}

All statistical analysis for qRT-PCR data was performed using the R package ( $\mathrm{R}$ Development Core Team., 2005). The linear mixed effects method of analysis was used to allow for repeated measures on subjects (Pinheiro \& Bates, 2000) and for each transcript, a sequential bonferroni adjustment was made for multiple testing. Data is represented as relative fold changes in mRNA expression \pm standard error of the mean (s.e.m). SPSS software (version 12.0) was used to calculate the Spearman rank correlation coefficients between STAT-3 and VEGFR-2, STAT-3 and STAT-1 and between STAT-1 and VEGFR-2 mRNA expression. The MC data sets were analysed with the Binary Logistic Regression test using SPSS. The non-parametric KruskalWallis one-way ANOVA by ranks test was applied to the band densities obtained from the immunoblotting data sets. The multiple comparisons test was then applied to determine where significant changes occurred. For all the statistical tests, significant changes were accepted at $p<0.05$. 


\subsection{METHODS FOR CHAPTER 4}

\subsubsection{TISSUE CULTURE MEDIUM}

MCDB 131 powder medium (Sigma) containing trace elements and L-glutamine was reconstituted with $1.18 \mathrm{~g} / \mathrm{L}$ sodium bicarbonate (Sigma) to a final volume of 1 litre with $\mathrm{dH}_{2} \mathrm{O}$. To this, $2 \%$ Penstrep and $2.5 \mu \mathrm{g} / \mathrm{ml}$ Amphotericin B (Sigma) were added. The mixture was agitated vigorously to ensure complete solubilisation of the additives and the solution was filter sterilized through a $0.2 \mu \mathrm{M}$ filter (Greiner Bio-One). The medium was either used immediately or stored at $4^{\circ} \mathrm{C}$ for up to eight weeks.

\subsubsection{HAEMANGIOMA TISSUE SPECIMENS AND SAMPLE PREPARATION}

Six proliferating and two involuting haemangioma biopsies were obtained according to protocols approved by the Wellington Ethics Committee. The specimens were kept moist and cleansed of all blood clots with PBS. Pieces of fresh operative haemangioma tissue were cut at room temperature into 1-mm explants and submerged in disinfecting media which was made up of MCDB 131 medium containing $1.18 \mathrm{~g} / \mathrm{L}$ sodium bicarbonate, $10 \%$ Penstrep $(500 \mu \mathrm{g} / \mathrm{ml}$ streptomycin sulfate, $500 \mu \mathrm{g} / \mathrm{ml}$ penicillin G), $25 \mu \mathrm{g} / \mathrm{ml}$ Amphotericin B and $2 \mathrm{mM}$ L-glutamine (Gibco-BRL). Disinfection was carried out on a rocking platform for at least 2 hours at room temperature.

\subsubsection{GEL PREPARATION AND IN VITRO TISSUE CULTURE}

$25 \mathrm{ml}$ of the tissue culture medium prepared as described in section 2.3.2 was transferred to a $50 \mathrm{ml}$ centrifuge tube (Greiner Bio-One) containing $75 \mathrm{mg}$ of sheep fibrinogen I (Sigma) to a final concentration of $3 \mathrm{mg} / \mathrm{ml}$. The tube was mixed gently for $2 \mathrm{~min}$ and $6 \mathrm{ml}$ was transferred aseptically into $15 \mathrm{ml}$ conical tubes, to which $0.5 \mathrm{U} / \mathrm{ml}$ 
of human thrombin (Serva Electrophoresis GmbH, Heidelberg, Germany) was added. The tube was then mixed by inverting and $400 \mu \mathrm{l}$ of the mixture was added into each well of a 24-well tissue culture plate (Greiner Bio-One). The plates were left in the hood for $20 \mathrm{~min}$ at room temperature to allow gel formation after which a piece of the haemangioma tissue specimen was placed on the centre of each gel. The tissue pieces were allowed to attach to gel surfaces for $20 \mathrm{~min}$ in the laminar hood. A second aliquot of fibrinogen-thrombin-medium mixture was then added so that the tissue fragment was sandwiched between two layers of fibrin gel (total volume $0.8 \mathrm{ml}$ ). Following gel formation, $1.5 \mathrm{ml}$ of supplemented MCDB 131 medium was added to each well. The plates were incubated at $37^{\circ} \mathrm{C}$ in $3 \% \mathrm{CO}_{2} / 97 \%$ air in a humidified environment using a Heracell incubator (Kendo Laboratory Products).

This model was used to test the effect of AG490 on capillary-like outgrowth. From a stock solution of $1 \mathrm{mM}$ AG490 (Sigma) dissolved in 100\% DMSO (Sigma, Appendix 4 section 4.2.12, page 299), AG490 was diluted into both gel sandwiches and also into the liquid medium overlaying the cultures at the concentrations indicated. In addition, control cultures were treated with the same volume of DMSO as that used for the highest concentration of AG490. At least six cultures were used for each treatment type.

\subsubsection{MODIFICATION OF THE IN VITRO TISSUE CULTURE MODEL}

This model was used for the purification of the outgrowing cells from haemangioma explants. Following disinfection, the haemangioma explants were cultured as described above in section 2.3.3, except for a few modifications. These are, (i) $10 \%$ FCS (Gibco-BRL) was added to the tissue culture medium before use, (ii) 350 $\mu \mathrm{l}$ of the fibrinogen-MCDB 131-thrombin mixture was placed in each well of 24-well culture plate and the tissue explants were placed into the gel before the gel formed. Therefore, only one layer of gel was used for each explant. After gel formation, $500 \mu 1$ 
of the supplemented MCDB 131 containing 10\% FCS was added to cover the gel in each well and the cultures were allowed to grow at $37^{\circ} \mathrm{C}$ under a $3 \% \mathrm{CO}_{2} / 97 \%$ air humidified environment. The culture medium was replaced every 3-4 days until the explant was used for dissociation.

This model was used to test the effect of VEGF and AG490 on haemangioma capillary-like outgrowth. Following three days of culture, only those explants which exhibited capillary-like outgrowth were selected. Culture media was removed and replaced with $500 \mu \mathrm{l}$ of serum free MCDB 131 media containing 2\% Penstrep. Following 24 hours of serum starvation, culture medium was removed once more and replaced with fresh serum free media containing either $50 \mathrm{ng} / \mathrm{ml}$ of VEGF (Sigma) and/or $50 \mu \mathrm{M}$ of AG490. Cultures were maintained for a period of six days with the drug. Images of each explant were photographed before and after drug treatment for analysis.

\subsubsection{QUANTITATION OF OUTGROWTH FROM TISSUE PIECES}

Capillary-like outgrowth was recorded during the culture period by capturing images with an Olympus IX51 inverted microscope (upgraded to IX71) fitted with phase contrast objectives and a color view 1 camera (Tokyo, Japan). For most tissue pieces, the region of outgrowth from the original tissue piece exceeded the field view at X4 magnification. Therefore, a set of overlapping images were taken for each explant and Adobe Photoshop CS2 was used to generate a single image to include the entire region of outgrowth for analysis. Neovascular area was calculated by applying a grid (150 pixels/150 pixels) to each image and counting the boxes containing three or more vessels. The capillary-like outgrowth is expressed as a ratio of the area occupied by the neovessels to the area of haemangioma tissue from which they emanated (Hasan et al., 2003; Hasan et al., 2000). 


\subsubsection{ISOLATION AND CULTURE OF HAEMANGIOMA TISSUE- DERIVED CELLS}

Within 10-15 days of culture, significant growth of cells from the tissue explants was observed. To purify these outgrowths from the haemangioma tissue explants, the culture medium was first removed. Sterilized (autoclaved and cleaned with $70 \%$ ethanol) forceps were used to remove the original tissue piece from the gel. Removing the tissue piece did not disturb the outgrowths which remained in the gel. The gel containing the outgrowths were then scraped off the well and digested in serum free RPMI media containing $1 \mathrm{mg} / \mathrm{ml}$ elastase (Worthington Biochemicals Corp, $\mathrm{NJ}$ ) at $37^{\circ} \mathrm{C}$ for 2-5 min or until the gel was completely dissolved. The RPMI medium-elastase mixture was filter sterilized through a $0.2 \mu \mathrm{M}$ filter and warmed to $37^{\circ} \mathrm{C}$ before being used. On an average, the gels from three wells of a 24-well plate were digested in $2 \mathrm{ml}$ of dissociation media. Once dissolved, enzyme activity was quenched by the addition of 3-4 $\mathrm{ml}$ of cell culture media (10\% FCS, 2\% penstrep in RPMI 1640 media) and the tubes were centrifuged at room temperature for $5 \mathrm{~min}$ at $300 \mathrm{X} \mathrm{g}(1000 \mathrm{rpm})$. The supernatant was then discarded and the cell pellet resuspended in $500 \mu \mathrm{l}$ of fresh cell culture medium. The percentage of viable cells was determined by trypan blue dye exclusion and seeded at a density of $1 \times 10^{4}$ viable cells $/ \mathrm{cm}^{2}$ in tissue culture flasks. Cells were incubated at $37^{\circ} \mathrm{C}$ in $97 \%$ humidity with $95 \%$ air and $5 \% \mathrm{CO}_{2}$ along with the other cell lines. The day after dissociation, the cell culture media was replaced with fresh media to remove non-attached, dead cells and cell debris. Following this, cultures were fed every 3-4 days and passaged 1:3 weekly or as required.

In addition, using previously described methods with a few modifications, the isolation of ECs from human haemangioma biopsies was also attempted (see section 2.3.16, page 99). This was to be used as a patient matched comparative cell line. 


\subsubsection{CHARACTERISATION OF HAEMANGIOMA CAPILLARY- LIKE OUTGROWTHS}

\subsubsection{MORPHOLOGICAL ANALYSIS}

\subsection{Change in cell morphology}

To detect changes in morphology with passage number, the haemangioma explant-derived cells (HaemDCs) were photographed at each passage using an Olympus IX51 inverted microscope (upgraded to IX71) fitted with phase contrast objectives and a color view 1 camera (Tokyo, Japan).

\subsection{In vitro angiogenesis}

To test capillary-like formation in vitro approximately 3000 HaemDCs were resuspended in $300 \mu \mathrm{l}$ of cell culture media containing $(3 \mathrm{mg} / \mathrm{ml}$ fibrinogen and $5 \mathrm{U} / \mathrm{ml}$ thrombin) and plated into 24-well culture dishes. The gel matrix was then overlayed with $500 \mu \mathrm{l}$ of cell culture media and maintained in $95 \%$ air, $5 \% \mathrm{CO}_{2}$ at $37{ }^{\circ} \mathrm{C}$. HUVECs were also plated in parallel for comparison in cell culture media supplemented with $30 \mu \mathrm{g} / \mathrm{ml}$ ECGS.

\subsection{Proliferation of HaemDCs}

HaemDCs were plated at a density of $1 \times 10^{4}$ cells per well into 24 -well plates in cell culture medium. Cell numbers were counted using a haemocytometer at days 0, 2, 4, 6 and 9 and cell viability was assessed by the ability of the cells to exclude the trypan blue dye.

\subsubsection{QUANTITATIVE REAL-TIME PCR}

\subsubsection{HaemDCs mRNA CHARACTERISATION}

RNA was isolated from approximately $1 \times 10^{5}$ HaemDCs and qRT-PCR was performed as described in the General Methods (sections 2.1.1.2.2, 2.1.2 and 2.1.4, 
pages 59 and 65). For mRNA characterisation experiments, qRT-PCR was performed for CD29, CD44, CD90, CD133, CD31, VEGFR-1, VEGFR-2, CD45, GLUT-1, Collagen-I, Collagen-II, Collagen-III, Collagen-VIII, Laminin-V, IGF-2 and VEGF. Primer sequences are listed in Table 1 (page 62). Changes in gene expression are reported relative to a GAPDH as $\triangle \mathrm{Ct}$. Based on cell morphology and mRNA expression profiles, the HaemDCs were characterised as haemangioma-derived mesenchymal cells (HaemDMCs)

\subsubsection{HaemDMCs DIFFERENTIATION EXPERIMENTS}

Following adipocytic and osteocytic differentiation, mRNA was extracted from differentiated and undifferentiated cells and qRT-PCR was performed for PPAR $\gamma$ and osteonectin using GAPDH as the internal reference gene. Changes in gene expression between treatment groups are reported as $\triangle \triangle \mathrm{Ct}$ values \pm s.e.m.

\subsubsection{IMMUNOFLUORESCENT CHARACTERISATION OF HaemDMCs}

Immunofluorescent staining of HaemDMCs, HUVECs and 3T3 fibroblasts was performed as described in section 2.1.8.2.2 (page 74). Fixed cells were incubated with primary antibodies overnight at $4^{\circ} \mathrm{C}$ against either $\alpha$-SMA, vimentin, vWF, GLUT-1, CD34, OPG, VEGFR-1 or VEGFR-2. Immunoreactivity was detected using either an anti-mouse AlexaFluor 488 conjugate or an anti-rabbit $\mathrm{Cy} 3$ conjugate. To detect bound VEGFR-2 antibodies, coverslips were incubated with an anti-rabbit DIG conjugate followed by an anti-DIG-Rhodamine conjugate. VEGFR-1 immunoreactivity was detected using an anti-goat biotin conjugate followed by incubation with an AlexaFluor 555 streptavidin conjugate. All antibodies were diluted in TBST containing 1\% BSA in a humidified chamber. For the EC specific markers, CD34, vWF, VEGFR-1 and VEGFR-2, HUVECs and 3T3 fibroblasts were stained in parallel as positive and negative controls, respectively. For all other markers, negative controls were included in 
each staining procedure and consisted of HaemDMCs cultured on coverslips and processed in the same way as the test with the omission of the primary antibody.

Immunofluorescent detection of OPG was performed on confluent HaemDMCs cultured for at least eight days as described above. Dual staining was performed on HUVECs for vWF and OPG by incubating both primary antibodies together in TBST containing $1 \% \mathrm{BSA}$ overnight at $4^{\circ} \mathrm{C}$. OPG and vWF immunoreactivity was detected using anti-mouse AlexaFluor 488 and anti-rabbit Cy3 conjugated antibodies, respectively.

\subsubsection{DIFFERENTIATION OF HaemDMCs}

HaemDMCs at passage 3 were plated at $2 \times 10^{4}$ cells $/ \mathrm{cm}^{2}$ onto $13-\mathrm{mm}$ diameter coverslips in 35-mm culture dishes and cultured in cell culture medium. At 90\% - 95\% confluence, cells were differentiated into adipocytes or osteocytes in specific induction media. Differentiation media was replaced every 2-3 days.

\subsubsection{OSTEOGENIC DIFFERENTIATION}

Osteogenic differentiation was induced by culturing HaemDMCs for a minimum of 14 days in osteogenic medium containing 10\% FCS, 2\% Penstrep, $100 \mathrm{nM}$ Dexamethasone (Mayne Pharma Ltd, Victoria, Australia), $0.2 \mathrm{mM}$ Ascorbic acid (Sigma) and $10 \mathrm{mM} \beta$-glycerophosphate (Sigma) in RPMI $1640+$ glutamine media.

Alizarin Red (BDH Laboratories chemicals group, Poole, UK) was used to detect calcium deposition indicative of osteogenesis on both uninduced and induced cells (Tropel et al., 2004; Lee et al., 2004; Sutherland et al., 2005).

\subsubsection{ADIPOGENIC DIFFERENTIATION}

Adipogenic differentiation was induced by culturing HaemDMCs in adipogenic medium containing 10\% FCS, 2\% Penstrep, $100 \mathrm{nM}$ Dexamethasone, $0.2 \mathrm{mM}$ Ascorbic acid, $10 \mathrm{mM} \beta$-glycerophosphate, $5 \mu \mathrm{g} / \mathrm{ml}$ insulin (Sigma) and $60 \mu \mathrm{M}$ indomethecin 
(Sigma) in RPMI 1640 + glutamine media. Cells were cultured in adipogenic medium for a minimum of 21 days and stained with Oil-Red O (Sigma) to detect lipid laiden adipocytes (Tropel et al., 2004; Lee et al., 2004; Sutherland et al., 2005).

\subsubsection{ENDOTHELIAL CELL DIFFERENTIATION}

To induce EC differentiation, confluent HaemDMCs were cultured in RPMI medium containing 2\% Penstrep and either 2\% FCS or $10 \%$ FCS along with $50 \mathrm{ng} / \mathrm{ml}$ of VEGF (Oswald et al., 2004). After 14 days of induction, cells were immunostained for EC specific markers, vWF, CD34 and VEGFR-2 as described in section 2.3.9 (page 90).

\subsubsection{CELL CULTURE HISTOCHEMISTRY}

\subsubsection{ALIZARIN RED STAINING}

Following osteocytic differentiation, cells were fixed with $4 \%$ PFA for 5 min and washed thoroughly in tap water. Cells were stained with Alizarin Red solution (pH 4.2, see Appendix 4, section 4.2.14, page 299) for $5 \mathrm{~min}$. After removal of excess dye, the coverslips were dipped in acetone (20 dips) followed by a mixture of acetone-xylene (1:1) solution and finally in xylene alone. Coverslips were then mounted in D.P.X mounting medium.

\subsubsection{OIL RED STAINING}

Following adipocytic induction, cells were fixed in 4\% PFA for 5 min and then thoroughly rinsed in tap water. Following a 5 min wash in $60 \%$ isopropanol, the cells were stained in a working solution of Oil Red O (see Appendix 4, section 4.2.15, page 300) for $15 \mathrm{~min}$. Excess stain was then washed off by rinsing in $60 \%$ isopropanol. Cell nuclei were then lightly stained using Harris's haematoxylin and rinsed in $\mathrm{dH}_{2} \mathrm{O}$ before being mounted in glycerine jelly (see Appendix 4, section 4.2.16, page 300). 
As HaemDMCs differentiated into adipocytes and osteocytes (see Chapter 4), the cells are here in referred to as haemangioma-derived mesenchymal stem cells (HaemDMSCs).

\subsubsection{EFFECTS OF VEGF AND AG490 ON HaemDMSCs}

\subsubsection{VEGF CELL PROLIFERATION ASSAY}

The effect of VEGF on proliferation of HaemDMSCs and HUVECs was determined using the colorimetric EZ4U cell proliferation assay, according to manufacturer's instructions (Biomedica, Vienna, Austria). Briefly, 5000 cells in $50 \mu 1$ of RPMI media containing 0.1\% FCS (for HaemDMSCs) or 2\% FCS (for HUVECs) were seeded into 96-well plates and allowed to attach overnight. The next day, the cells were treated with $50 \mu 1$ of fresh media containing various concentrations of VEGF for the times indicated. Fresh media $(50 \mu 1)$ containing VEGF was added to the HaemDMSCs cultures every 48 hours to maintain the VEGF concentration. At the end of the culture period, substrate was dissolved in $2.5 \mathrm{ml}$ of activator and pre-warmed to $37^{\circ} \mathrm{C} .20 \mu \mathrm{l}$ of this solution was added to each well and the cells were incubated at $37^{\circ} \mathrm{C}$ for 2 hours. Colour development was measured in a VersaMAX multiwell plate spectrophotometer. The absorbance was measured at wavelengths of 492 and $620 \mathrm{~nm}$ and blanked against wells containing medium but no cells. Results were analysed with the Softmax Pro software programme. Data is plotted as the mean absorbance value obtained from three wells per treatment group \pm s.e.m.

\subsubsection{EFFECT OF AG490 ON HaemDMSCS PROLIFERATION BY BrdU}

\section{LABELLING}

$5 \times 10^{5}$ HaemDMSCs were plated onto $35 \mathrm{~mm}$ culture dishes in RPMI media containing 5\% FCS and 2\% Penstrep and allowed to adhere for 6 hours. Media was then replaced with fresh media containing $100 \mu \mathrm{m}$ or $50 \mu \mathrm{m}$ of AG490 diluted into the 
media from a stock solution (see Appendix 4, section 4.2.12, page 299) and cells were returned to the incubator for 17 hours (an overnight incubation). Cells were given two pulses of BrdU (5-bromo-2-deoxyuridine, $20 \mathrm{nmol}$, Sigma) to achieve a final concentration of $10 \mu \mathrm{M}$. BrdUwas added at 3 hours intervals for the last 7 hours of a 24 hr incubation period with AG490. Cells were then trypsinized, pelleted and resuspended in $1 \mathrm{ml}$ of TBS. Following a second centrifugation step, cells were resuspended in 300 $\mu \mathrm{l}$ of TBS. Cells were then fixed in $70 \%$ ethanol by slowly adding $700 \mu$ of ice cold ethanol $(100 \%)$ to the tube containing the cells while the tube was being vortexed. This was to avoid clumping of the cells on adding the fixative. Cells were fixed overnight at $4^{\circ} \mathrm{C}$. Fixed cells were washed in cold TBS and digested in $0.5 \%$ Triton-X in $2 \mathrm{M} \mathrm{HCL}$ for $30 \mathrm{~min}$ at room temperature. Washed cells were treated with $0.1 \mathrm{M} \mathrm{Na}_{2} \mathrm{~B}_{4} \mathrm{O}_{7}(\mathrm{pH}$ 8.5) to neutralise the samples and incubated with a biotin conjugated anti-BrdU antibody $\left(1: 100\right.$, Invitrogen) overnight at $4^{\circ} \mathrm{C}$. The next day, cells were washed in TBS and incubated an AlexaFluor 555 streptavidin conjugate for 2 hours at room temperature. All antibodies were diluted in TBST containing 1\% BSA. At least $1 \times 10^{4}$ cells were analysed for fluorescence per experimental condition on a FACScan (Becton Dickinson \& Co, MA). To determine antibody specificity, BrdU unpulsed HaemDMSCs $\left(1 \times 10^{4}\right.$ cells $)$ were also incubated with the anti-BrdU antibodies and treated in the same way as the experimental samples. The fraction of cells in $\mathrm{S}$ phase was determined from the number of cells that had incorporated BrdU using the CellQuest software (Becton Dickinson \& Co).

\subsubsection{EFFECT OF AG490 ON IN VITRO CAPILLARY-LIKE FORMATION AND QUANTITATION}

To test the effect of AG490 on capillary-like formation by HaemDMSCs in vitro, $3 \times 10^{5}$ cells $/ \mathrm{ml}$ were resuspended in RPMI culture medium containing $5 \%$ FCS, $2 \%$ Penstrep and $3 \mathrm{mg} / \mathrm{ml}$ fibrinogen along with either DMSO alone (control cultures) or AG490 at various concentrations. Immediately after adding $0.5 \mathrm{U} / \mathrm{ml}$ of thrombin to 
the cell-media mixture, $300 \mu$ l containing approximately $1 \times 10^{5}$ cells were plated into each well of a 24-well culture dish. Culture plates were maintained in the laminar flow hood until gel formation occurred after which the gel was overlayed with $500 \mu$ of RPMI media containing either vehicle alone (DMSO) or AG490 at the required doses. Cultures were maintained at $37^{\circ} \mathrm{C}$ in $5 \% \mathrm{CO}_{2} / 97 \%$ air for 12 days. During the culture period, half the media was replaced with fresh media containing the drugs at $72 \mathrm{hr}$ intervals.

Capillary-like formation was quantified using the Image $\mathbf{J}$ software (National Institute of Health, MD). A counting frame $(1000 \mu \mathrm{m} / 1000 \mu \mathrm{m})$ was applied to each image and the measure tool was used to calculate total capillary-like length. Cell nuclei present in each counting frame were counted using the cell counter tool.

\subsubsection{WESTERN BLOTTING}

\subsubsection{EFFECT OF VEGF, AG490 AND IFNa-2b ON p-STAT-3 PROTEIN EXPRESSION}

$1 \times 10^{6}$ HaemDMSCs were plated onto $100 \mathrm{~cm}^{2}$ culture dishes in cell culture medium and left to attach overnight. Culture media was then replaced with fresh media containing either $50 \mathrm{ng} / \mathrm{ml}$ of VEGF, $50 \mu \mathrm{m}$ AG490 or 10,000 U /ml of IFN $\alpha-2 \mathrm{~b}$ and the dishes were returned to the incubator for the times indicated. For the combination treatment of AG490 and VEGF, the cells were pre-treated with AG490 for one hr prior to adding VEGF for an additional 45 min. Following drug treatments, cells were lysed and subjected to Western blotting as described in section 2.1.9 (page 77). $50 \mu \mathrm{g}$ of total protein from HaemDMSCs lysates and HeLa cell lysate (IFN $\alpha-2 b$ treated for $10 \mathrm{~min}$ ) was separated by electrophoresis and transferred onto PVDF membranes. The membranes were then blocked and incubated with mouse anti-p-STAT-3 (Tyr 705) and mouse anti-GAPDH primary antibodies. Anti-mouse AlexaFluor 488 conjugated secondary antibodies were used for detection. Following fluorescence scanning, the 
membranes were stripped for 30 min (see Appendix 4, section 4.2.7 for the stripping buffer recipe, page 298) at room temperature and re-scanned to ensure signal from the p-STAT-3 protein bands were lost. Membranes were then probed with mouse antiSTAT-3 and goat anti-VEGFR-1 primary antibodies followed by incubation with antimouse and anti-goat AlexaFluor 488 conjugated secondary antibodies and scanned. Image analysis and densitometry was performed using the Image $\mathbf{J}$ software.

\subsubsection{DETECTION OF VEGF IN THE CONDITIONED MEDIA OF HaemDMSCs}

To detect if VEGF is secreted into the conditioned media by HaemDMSCs, $1 \times 10^{6}$ HaemDMSCs were plated onto $100 \mathrm{~cm}^{2}$ culture dishes. On day 3, culture media was replaced with $10 \mathrm{ml}$ of fresh serum free RPMI culture media containing $2 \%$ Penstrep. 24 hours later, the media was discarded and replaced with fresh serum free media. The cells were cultured for 96 hours after which the conditioned medium was collected in fresh tubes and the cells were overlayed with fresh cell culture medium containing 10\% FCS and 2\% Penstrep. HaemDMSCs were cultured for an additional 3 days and lysed as described in section 2.1.9.1.2 (page 77). Conditioned medium was collected and centrifuged to remove cell debris or particulate matter. The supernatant was then transferred to a pre-rinsed centricon-20 (Millipore Pty Ltd, Australia) and concentrated by centrifugation $4000 \mathrm{x} \mathrm{g}$ for $15 \mathrm{~min}$ to $200 \mu \mathrm{l}$ (final volume). Concentrated medium was then precipitated using the ProteoExtract protein precipitation kit (Chemicon) as per manufacturer's instructions. Briefly, four volumes (800 $\mu \mathrm{l}$ ) of precipitant was added to $200 \mu \mathrm{l}$ of concentrated HaemDMSCs conditioned media and precipitated overnight at $-20^{\circ} \mathrm{C}$. The next day, precipitated protein was collected by centrifugation at $14,000 \mathrm{rpm}$ for $10 \mathrm{~min}$. The pellet was washed, resuspended in $50 \mu \mathrm{l}$ of lysis buffer and quantitated as described in section 2.1.9.2 (page 78). Approximately $40 \mu \mathrm{g}$ of total protein from HaemDMSCs conditioned media, 100 $\mu \mathrm{g}$ of haemangioma tissue, $100 \mu \mathrm{g}$ of HaemDMSCs lysate was added to protein gel 
loading buffer (10 X stock) (Appendix 4, section 4.2.20, page 301) in $1.5 \mathrm{ml}$ microcentrifuge tubes to obtain a final concentration of at least $1 \mathrm{X}$ loading buffer along with $2.5 \mathrm{mM} \beta$-mercaptoethanol. Samples were boiled for $10 \mathrm{~min}$ at $100^{\circ} \mathrm{C}$ on a heated dry block and subjected to electrophoresis on $1.5 \mathrm{~mm}$ thick, $10 \%$ SDS polyacrylamide gels using a 4\% stacking gel (Appendix 4, sections 4.2.18 and 4.2.19, pages 300 and 301) in a Miniprotean cell (BioRad Laboratories) along with $10 \mu 1$ of Magic Mark prestained molecular weight markers (Invitrogen). An additional gel containing HaemDMSCs and haemangioma tissue lysate, (100 $\mu \mathrm{g}$ each) was run for the negative control. Gels were run for $120 \mathrm{~min}$ at $100 \mathrm{~V}$ in SDS running buffer (see Appendix 4, section 4.2.21, page 301).

Following gel electrophoresis and immunotransfer, the membranes were incubated with anti-mouse VEGF and anti-mouse GAPDH antibodies followed by antimouse AlexaFluor 488 conjugated secondary antibodies. The negative control membranes were incubated with secondary antibodies only. Antibody detection was analysed by scanning the membranes on a fluorescent scanner.

\subsubsection{IMMUNOCYTOCHEMISTRY}

\subsubsection{EFFECT OF VEGF, IFN $\alpha$-2b AND ADIPOCYTIC DIFFERENTIATION CONDITIONS ON STAT PROTEIN EXPRESSION}

$1 \times 10^{5}$ HaemDMSCs were plated onto sterile $13 \mathrm{~mm}$ diameter coverslips in cell culture medium and allowed to attach overnight. Culture media was removed and replaced with fresh media containing either $50 \mathrm{ng} / \mathrm{ml}$ VEGF, 10,000 U/ml IFNa-2b or adipocytic differentiation media and the dishes were returned to the incubator for the times indicated. Culture media was then removed and the dishes were rinsed in PBS. Cells were fixed in $4 \%$ PFA for 5 min at $4^{\circ} \mathrm{C}$. After washing in PBS ( 2 changes, $5 \mathrm{~min}$ ), cells were permeabilised in $10 \%$ acetic acid prepared in $100 \%$ methanol for 10 min at $20^{\circ} \mathrm{C}$ (Ma et al., 2005). Cells were then washed in PBS and subjected to antigen 
retrieval with $10 \mathrm{mM}$ sodium citrate $(\mathrm{pH} \mathrm{6)}$ and blocking as described in section 2.1.8.2.2 (page 74). Coverslips were incubated with rabbit anti-p-STAT-3 (Tyr 705), rabbit anti-p-STAT-5 (Tyr 694), goat anti-p-STAT-1(Tyr 701), mouse anti-STAT-3 or mouse anti-STAT-5 primary antibodies. p-STAT-3 and p-STAT-5 expression was detected using an anti-rabbit-DIG conjugate followed by either an anti-DIG FITC conjugate or an anti-DIG rhodamine conjugate, while p-STAT-1 expression was detected using an anti-goat biotin conjugate and an AlexaFluor 488 strepavidin conjugate. STAT-3 and STAT-5 immunoreactivity was detected using an anti-mouse AlexaFluor 488 conjugate. Cell nuclei were stained using either PI $(10 \mu \mathrm{g} / \mathrm{ml})$ or DAPI $(20 \mu \mathrm{g} / \mathrm{ml})$ as described in section 2.1.8.3 (page 75) and the coverslips were mounted in Prolong Gold Antifade reagent (Invitrogen) on pre-cleaned glass slides.

Image analysis of p-STAT-5 (Tyr 694) nuclear translocation was performed using Image $\mathbf{J}$ software. At least 20 cells from each treatment group were identified and their nuclear boundaries traced. The intensity of p-STAT-5 (Tyr 694) immunoreactivity within these defined areas was measured and used as an index of STAT translocation (Ma et al., 2005).

\subsubsection{IMMUNOHISTOCHEMISTRY}

Fluorescent immunohistochemical staining was performed on paraffin embedded haemangioma tissue sections (six from the proliferative phase and five from the involuting and involuted phases) as described in section 2.1.8.2.1 (page 74). Antigen retrieval was performed by immersing the slides in boiling $10 \mathrm{mM}$ sodium citrate $(\mathrm{pH}$ 6.0) for $10 \mathrm{~min}$. Sections were incubated with rabbit anti-vWF, mouse anti-PCNA and mouse anti-OPG primary antibodies. Bound primary antibodies were detected with antirabbit Cy3 and anti-mouse AlexFluor 488 conjugated secondary antibodies. 


\subsubsection{ISOLATION OF ENDOTHELIAL CELLS AND ENDOTHELIAL PROGENITOR CELLS FROM PROLIFERATING HAEMANGIOMAS}

Approximately $1 \mathrm{~g}$ of proliferating haemangioma tissue was cut into $1 \mathrm{~mm}^{2}$ and disinfected for at least 4 hours as described in section 2.3.2 (page 85). Tissue pieces were then pelleted by centrifugation for $5 \mathrm{~min}$ at $300 \mathrm{X} \mathrm{g}$ and resuspended in serum free RPMI medium. Following a second centrifugation step, the tissue was resuspended and digested in serum free RPMI media containing $0.5 \mathrm{mg} / \mathrm{ml}$ Collagenase P (Roche) and $2 \%$ Penstrep for $30 \mathrm{~min}$ in a water bath maintained at $37^{\circ} \mathrm{C}$. The digested tissue was then passed through a $45 \mu \mathrm{m}$ cell strainer (Greiner Bio-one) and the collagenase activity was quenched by adding FCS to $10 \%$ of the total volume. The undigested tissue was transferred to a fresh $50 \mathrm{ml}$ falcon tube using the digestion medium, gently homogenised in a glass homogeniser and then allowed to digest for an additional 30 min. The cells were strained and the collagenase activity quenched as above. The single cell suspension was washed in FACS buffer (Appendix 4, section 4.2.13, page 299) and counted using a haemocytometer. The ECs were isolated with CD31 coated magnetic dynal beads (Invitrogen) using approximately five beads per cell. The required volume of coated beads was added to the cells and the final volume was made up to $300 \mu l$ with FACS buffer and transferred to a FACS tube. The cell bead mixture was then incubated at $4^{\circ} \mathrm{C}$ on a tube rotator for $1 \mathrm{hr}$. The FACS tube was placed into a magnetic particle concentrator (MPC) and the beads were allowed to collect to the magnet. The remaining FACS buffer containing the unbound fraction was removed. The tube was then removed from the MPC and the beads were resuspended in fresh cell culture media and plated onto $1 \%$ gelatine coated culture dishes (see Appendix 4, section 4.2.11, page 299).

The bead purification step was omitted in later experiments due to low yield. From then on the entire single cell suspension obtained after digestion was directly plated onto $1 \%$ gelatine coated dishes and cultured in RPMI media containing $20 \%$ FCS, 2\% Penstrep and $15 \mu \mathrm{g} / \mathrm{ml}$ ECGS (Sigma; Appendix 4, section 4.2.17, page 300). 


\subsubsection{STATISTICS}

To test for an effect of VEGF and AG490 on capillary-like outgrowth a 2-way ANOVA was used. To test for an effect of various concentrations of AG490 on capillary-like outgrowth and for the effect of VEGF on HUVEC and HaemDMSCs proliferation, a 1-way ANOVA was used. All ANOVA tests were followed by Bonferroni's post hoc test to determine where significant differences between experimental groups occured. For these experiments, the statistical tests were performed on the data from replicate wells set up with biopsies from a single patient. Therefore, since all the samples tested came from a single patient and were performed on the same day, a normal distribution can be assumed. This allows the use of the parametric method of statistical testing.

Statistical analysis on p-STAT-5 nuclear translocation was performed using the non-parametric Mann-Whitney $U$ test. For all other data sets, the Kruskil-Wallis by ranks test was performed followed by multiple comparisons to determine where significant effects occurred. For these data, although the cell line used was consistent, normal distribution cannot be assumed as the experiments were performed on different days. Therefore, non-parametric statistical tests by ranks were applied. For all statistical tests, differences with $p$ values of $<0.05$ were considered significant. 


\subsection{METHODS FOR CHAPTER 5}

\subsubsection{QUANTITATIVE REAL TIME PCR}

RNA was extracted from frozen haemangioma tissue and from HaemDMSCs as described previously in the section 2.1.1.2 (page 58). Oligo (dT) $)_{20}$ primed cDNA was synthesised and qRT-PCR was performed for TRAIL, DR4, DR5, DcR1, DcR2 and OPG according to sections 2.1.1.2.1, 2.1.2 and 2.1.4 (pages 58, 59 and 65). Primer sequences are listed in Table 1 (page 63). Changes in TRAIL and TRAIL-Rs mRNA expression between phases are expressed relative to that for GAPDH as $\triangle \mathrm{Ct}$ values. Changes in gene expression between haemangioma tissue samples and HaemDMSCs are expressed as $\triangle \triangle \mathrm{Ct}$ values \pm s.e.m.

\subsubsection{FLOURESCENT IMMUNOHISTOCHEMISTRY}

Four proliferative paraffin embedded haemangioma biopsies were immunostained as described in section 2.1.8.2.1 (page 74). All slides except those which were stained for DR5 were subjected to heat induced antigen retrieval by boiling in $10 \mathrm{mM}$ sodium citrate $(\mathrm{pH}$ 6.0) for $10 \mathrm{~min}$. No antigen retrieval was required for DR5 detection. All slides were blocked in TBST containing 5\% BSA for 60 min which was followed by incubation with primary antibodies overnight at $4^{\circ} \mathrm{C}$ against either mouse anti-DR4, mouse anti-DR5, rabbit anti-DcR1, rabbit anti-DcR2 or mouse antiOPG. The next day, the slides were washed and incubated with secondary antibodies as follows, (i) for DR4 detection, slides were incubated with an anti-mouse DIG conjugate followed by an anti-DIG Rhodamine conjugate, (ii) for DR5 and OPG detection, slides were incubated with an anti-mouse AlexaFluor 488 conjugate and (iii) for DcR1 and DcR2 detection, slides were incubated with an anti-rabbit DIG conjugate followed by an anti-DIG FITC conjugate (see Tables 2 and 3 for antibody dilutions, pages 71 and 73). 
Immunofluorescent staining of HaemDMSCs was performed as described in section 2.1.8.2.2 (page 74). Fixed cells were incubated with primary and secondary antibodies antibodies as described above except for DR4 detection. Following incubation with anti-DR4 antibodies, coverslips were washed and incubated with an anti-mouse AlexaFluor 488 conjugate.

All slides and coverslips were counterstained with either DAPI or PI (see section 2.1.8.3, page 75). Negative controls were included in each staining experiment in which the primary antibody was omitted.

\subsubsection{EFFECT OF INTERFERON AND TRAIL ON HAEMANGIOMA}

\section{CAPILLARY-LIKE OUTGROWTH}

Proliferative haemangioma biopsies were cultured as described in section 2.3.3 (page 85). For drug treatments, TRAIL (250 ng/ml, BIOMOL) and IFN $\alpha-2 b$ (4600 U/ml and 23000 U/ml; Schering-Plough Pty Ltd, Wellington, New Zealand) was dissolved in the fibrinogen-thrombin-medium mixture and in the supplemented medium. A minimum of six wells were used for each treatment type and 18 wells were used as the untreated control cultures. Microvessel outgrowth was analysed on day 9 in culture as a ratio of the area occupied by the neovessels to the area of haemangioma tissue (section 2.3.5, page 87) from which they emanated (Hasan et al., 2003; Hasan et al., 2000) and is expressed as $\%$ Control \pm s.e.m.

\subsubsection{EFFECT OF IFN $\alpha-2 b$ AND TRAIL ON HaemDMSCs}

The effect of IFN $\alpha-2 b$ and TRAIL on HaemDMSCs was determined using the colorimetric EZ4U cell proliferation assay, according to the manufacturer's instructions (Biomedica). Briefly, 5000 cells were seeded in $50 \mu 1$ of RPMI media containing $0.1 \%$ FCS into each well of a 96-well plate and allowed to attach overnight. The next day, the cells were treated with $50 \mu \mathrm{l}$ of fresh media containing either TRAIL (250 ng/ml) 
and/or IFN $\alpha-2 b(4600 \mathrm{U} / \mathrm{ml}$ and $23000 \mathrm{U} / \mathrm{ml})$. Fresh media $(50 \mu \mathrm{l})$ containing the drugs were added to each well after 48 hours. Following 96 hours of incubation with the drugs, the EZ4U substrate was dissolved in $2.5 \mathrm{ml}$ of activator and prewarmed to $37^{\circ} \mathrm{C}$. $20 \mu \mathrm{l}$ of this solution was added to each well and the cells were incubated at $37^{\circ} \mathrm{C}$ for 2 hours. Absorbance was read at 492 and $620 \mathrm{~nm}$. The experiment was performed with HaemDMSC isolates from three separate patients and the results are expressed as $\%$ Control \pm s.e.m.

\subsubsection{WESTERN BLOTTING}

\subsubsection{DR4 AND DcR2 DETECTION}

Protein was extracted from snap frozen haemangioma biopsies of the same patients that were used for the in vitro explant model, as described in section 2.1.9.1.1 (page 77). Protein from HaemDMSCs was extracted using the same protocol with the following exceptions. $100 \mathrm{~cm}^{2}$ tissue culture dishes containing HaemDMSCs at $90 \%$ confluence were used from each patient. Culture media was removed and the cells were rinsed once with PBS. $50 \mu \mathrm{l}$ lysis buffer was added to each plate and the cells were mechanically scrapped off the dish using a rubber cell scrapper. Cell lysate was transferred to a new eppendorf tube. From here on, cell lysates were treated in the same way as tissue lysates. $50 \mu \mathrm{g}$ of total protein from cell and tissue lysate was subjected to Western blotting as described in section 2.1.9 (page 77). Immunoblots were incubated with either mouse anti-DR4 or rabbit anti-DcR2 along with mouse anti-GAPDH primary antibodies followed by incubation with anti-mouse and/or anti-rabbit AlexaFluor 488 conjugated secondary antibodies. Antibody binding was detected as described in section 2.1.9.5 (page 79). The negative control membranes were treated in the same way as the test with the omission of primary antibody incubation. 


\subsubsection{OPG DETECTION}

To detect OPG protein, HaemDMSC conditioned media and cell lysates were prepared as described in section 2.3.13.2 (page 96). Following gel electrophoresis and immunotransfer, the membranes were incubated with anti-mouse OPG and anti-mouse GAPDH antibodies followed by anti-mouse AlexaFluor 488 conjugated secondary antibodies. The negative control membranes were incubated with secondary antibodies only. Antibody detection was analysed by scanning the membranes on a fluorescent scanner.

\subsubsection{STATISTICS}

To test for significant changes in TRAIL and TRAIL receptor mRNA expression between phases, the R package (R Development Core Team., 2005) was used. The linear mixed effects method of analysis was used to allow for repeated measures on subjects (Pinheiro \& Bates, 2000) and for each transcript, a sequential Bonferroni adjustment was made for multiple testing. To test for changes in mRNA expression between HaemDMSCs and haemangioma tissue biopsies, the nonparametric Friedmann's test for paired samples was applied to the $\triangle \triangle \mathrm{Ct}$ values obtained for each gene. To test for an effect of TRAIL and IFN $\alpha-2 b$ on capillary-like outgrowth and on HaemDMSCs proliferation, a 2-way ANOVA was used. As multiple ANOVA tests were performed for each treatment, sequential bonferroni adjustments were applied to the resultant $p$ values. Pairwise comparisons were performed between each treatment group using the Bonferroni's post hoc test. The Mann Whitney U test was applied to the band densities obtained from the immunoblotting data sets. Differences with $p$ values of $<0.05$ were considered significant. 


\section{CHAPTER 3: ROLE OF STAT PROTEINS AND VEGFR-2 IN HAEMANGIOMA}

\subsection{INTRODUCTION}

Given the high frequency of deregulated STAT-3 and STAT-1 signalling in tumors (see section 1.9, page 49) and the elevated levels of VEGF found in proliferating haemangiomas (Takahashi, et al., 1994; Tan et al., 2000a), a model for the involvement of these genes in haemangioma was proposed (Figure 3.1). This model suggests that increased expression of VEGFR-2, STAT-3 and the activated form of STAT-3, p-STAT-3 (Tyr 705) is associated with the proliferative phase whereas the involuting phase involves apoptosis induced by increased STAT-1 signalling.

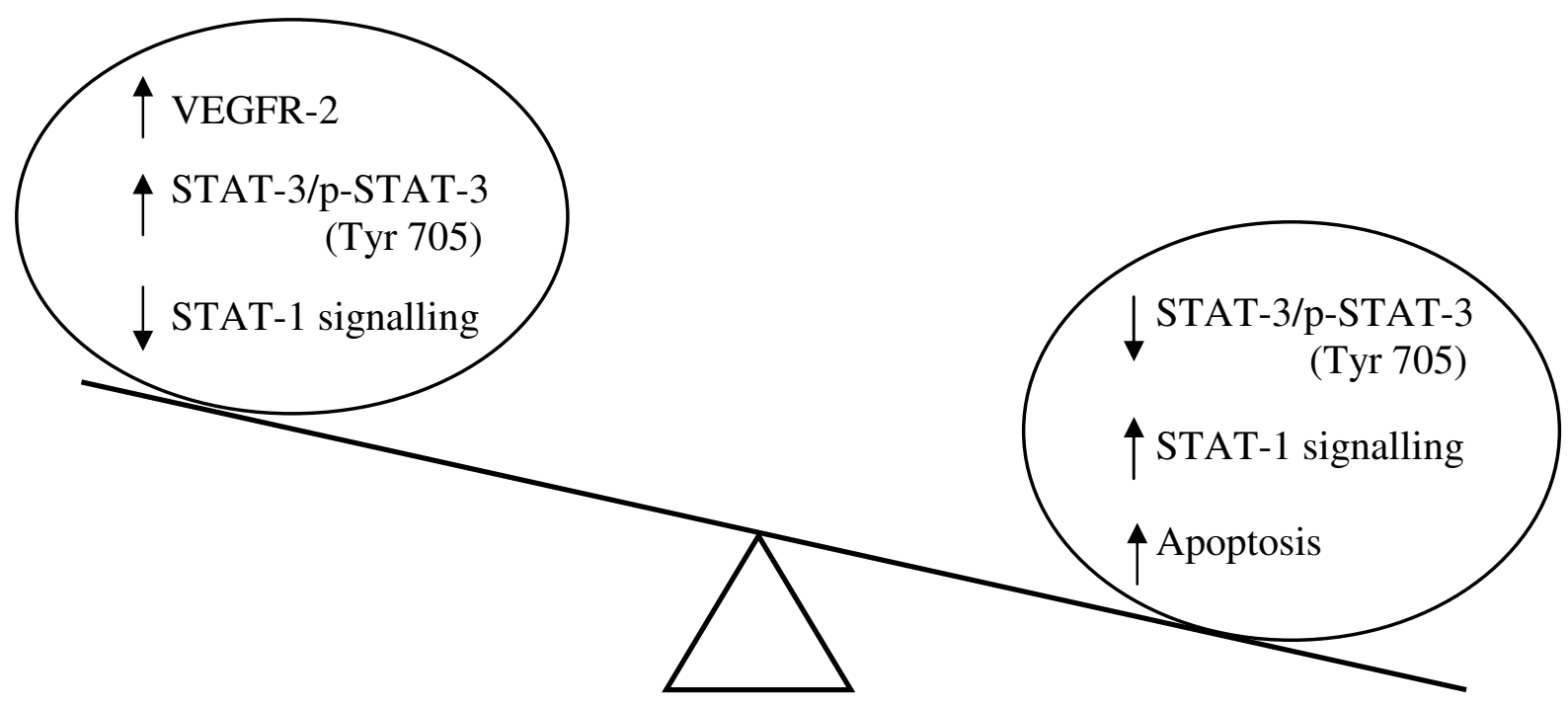

PROLIFERATIVE PHASE

INVOLUTING PHASE

Figure 3.1: Proposed model for the role of STAT-3, p-STAT-3 (Tyr 705), STAT-1 and VEGFR-2 in haemangioma. 


\subsection{AIMS}

The aim of this study was to test the model proposed in section 3.1 (Figure 3.1). This study investigated whether increased VEGFR-2, STAT-3 and p-STAT-3 (Tyr 705) expression was present during the proliferative phase of haemangioma and if increased STAT-1 expression was present during involution. qRT-PCR was used to determine changes in mRNA expression of STAT-3, STAT-1 and VEGFR-2 in proliferating, involuting and involuted haemangioma specimens. To correlate changes of mRNA with protein abundance, Western blotting and IHC procedures were used to examine protein expression, distribution and state of phosphorylation. 


\subsection{RESULTS}

\subsubsection{PRELIMINARY EXPERIMENTS}

Preliminary experiments were conducted to assess the amplification kinetics of the qRT-PCR amplification assays used in this study. All amplifications were linear over a 10,000-fold range and only primer sets with greater than $96 \%$ efficiency were used. Preliminary experiments determined that GAPDH was the most suitable internal reference gene to use in qRT-PCR of those tested (18SrRNA, HPRT and CycA), as its expression did not vary significantly between the phases of haemangioma when normalised to total RNA content (Appendix 1, section 1.1.2.2, page 272). The mean $\mathrm{Ct}$ value for GAPDH was 20 (s.e.m. = 1.0) using $25 \mathrm{ng}$ of input RNA.

In addition, specificity of the various antibodies used in this study was validated by immunohistochemical and Western blotting studies on cell lines and tissue sections known to express those proteins (see Appendix 1, section 1.1.2.3, page 275).

\subsubsection{GENE EXPRESSION CHANGES IN HAEMANGIOMA}

\subsubsection{CHANGE IN mRNA EXPRESSION}

The relative change in mRNA expression for STAT-3, STAT-1 and VEGFR-2 was determined for five proliferating, eight involuting and seven involuted haemangioma samples. Proliferating haemangioma specimens expressed more STAT-3 mRNA than involuting and involuted specimens, with a significant difference detected between proliferating and involuted lesions $(p<0.05$; Figure 3.2). Expression of VEGFR-2 mRNA was significantly greater in proliferating haemangioma compared with both involuting and involuted lesions $(p<0.05$ and $p<0.01$, respectively; Figure 3.3). No statistically significant difference in STAT-1 mRNA expression was detected between proliferating and involuting lesions, however, significantly lower levels were detected in involuted lesions compared to both, proliferative and involuting lesions $(p<$ 0.05, Figure 3.4) 


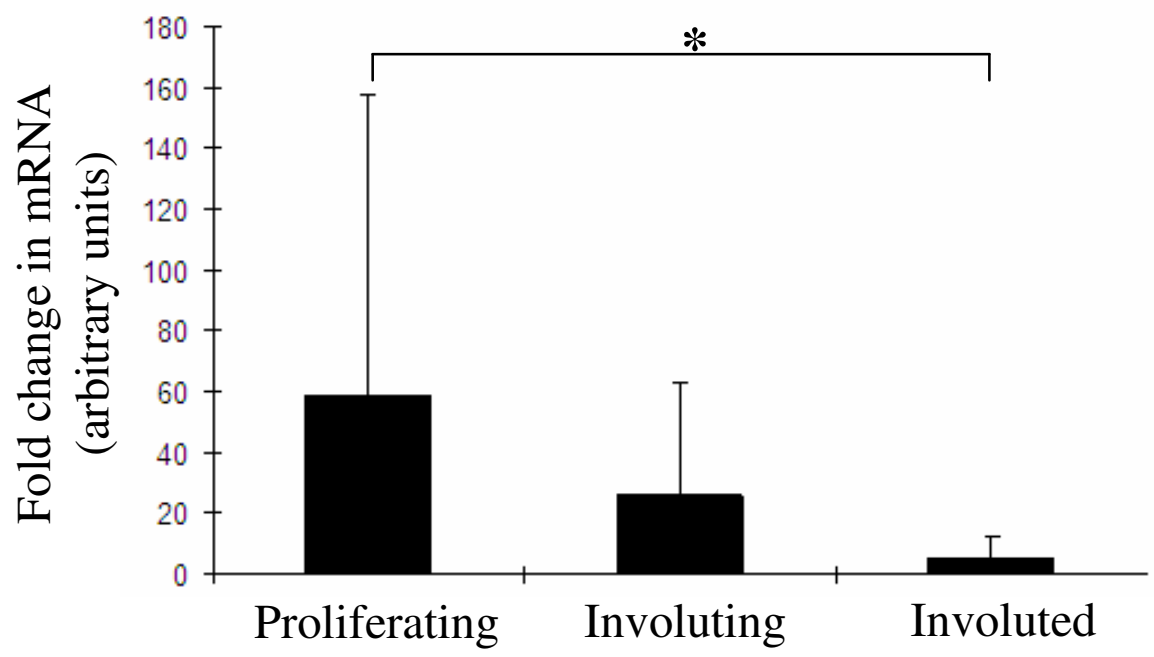

Figure 3.2: Changes in STAT-3 mRNA expression. The mRNA levels for STAT-3 were determined for five proliferating, eight involuting and seven involuted specimens relative to GAPDH. Proliferating haemangioma specimens expressed significantly higher levels of STAT-3 mRNA than involuted specimens $(* p<0.05)$.

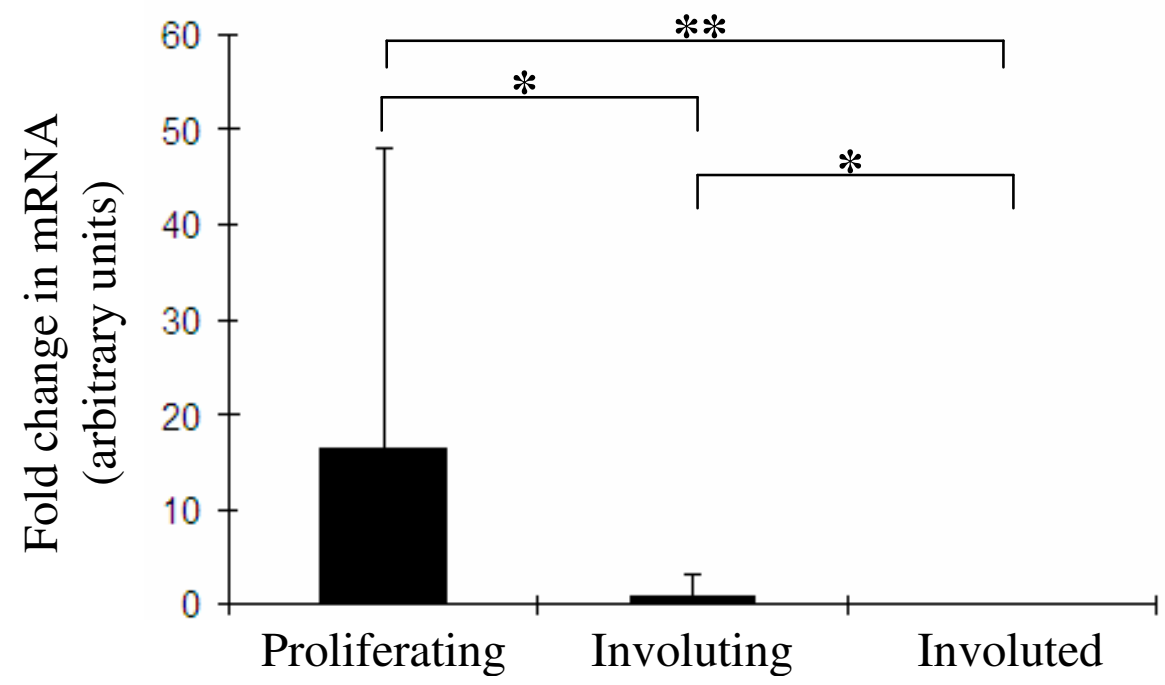

Figure 3.3: Changes in VEGFR-2 mRNA expression. VEGFR-2 mRNA levels were determined in the same samples as in Figure 3.2. Proliferating haemangioma specimens expressed highest levels of VEGFR-2 mRNA which decreased in the involuting and involuted specimens $(* p<0.05, * * p<0.01)$. 


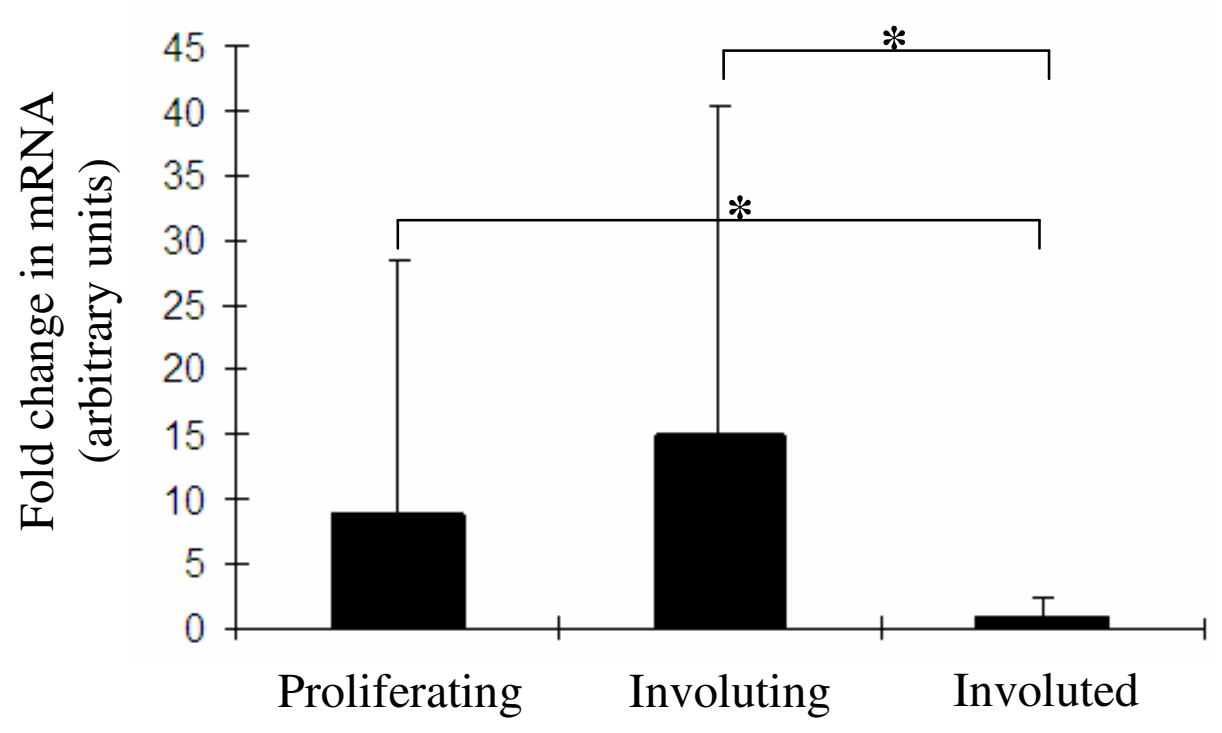

Figure 3.4: Changes in STAT-1 mRNA expression. mRNA levels for STAT-1 were determined in five proliferating, eight involuting and seven involuted specimens relative to GAPDH. Proliferating and involuting samples expressed more STAT-1 mRNA compared to involuted samples $(* p<0.05)$.

In addition, mRNA expression of VEGFR-2 and STAT-3 varied proportionately across all the three phases $(\mathrm{r}=0.820 ; p<0.001$; Figure 3.5). Graphs showing the correlation between STAT-3 with STAT-1 mRNA ( $r=0.692 ; p<0.001)$ and VEGFR-2 with STAT-1 mRNA $(\mathrm{r}=0.75 ; p<0.001)$ are given in Appendix 1, Figure 1.9, page 280. The raw $\Delta \mathrm{Ct}$ values obtained for STAT-3, VEGFR-2 and STAT-1 mRNA from proliferating, involuting and involuted haemangioma biopsies are given in Table 4, page 111.

As the method used in this study reports gene expression changes relative to the internal reference gene (GAPDH) using the same amount of input mRNA, it circumvents the possibility of identifying false changes in gene expression. 


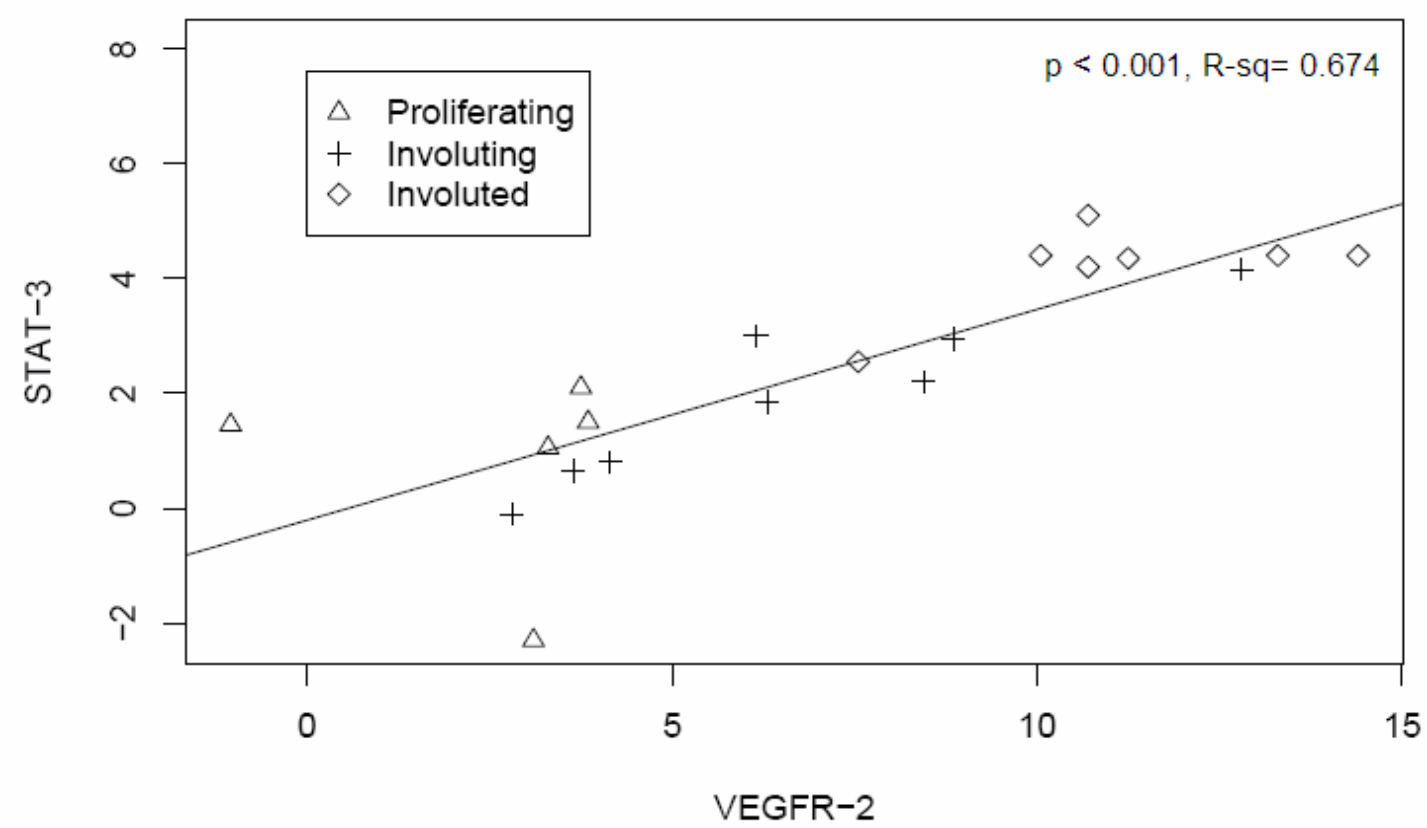

Figure 3.5: Correlation between STAT-3 and VEGFR-2 mRNA expression in proliferating, involuting and involuted haemangiomas. The mRNA levels for STAT-3 and VEGFR-2 were determined from haemangioma biopsies from the different clinical phases using qRT-PCR. The threshold value $(\mathrm{Ct})$ was determined for each gene and then expressed as a difference $(\Delta \mathrm{Ct})$ between that for the target gene and that for internal house-keeping gene, GAPDH. Low $\Delta \mathrm{Ct}$ values reflect greater mRNA expression. Proliferating haemangiomas $(\triangle)$ had greatest STAT-3 and VEGFR-2 expression compared with involuting $(+)$ and involuted $(\diamond)$ lesions. VEGFR-2 and STAT-3 mRNA expression were correlated (Spearman rho $r=0.893 ; p<0.001$ ). 


\begin{tabular}{|c|c|c|c|c|}
\hline & Phase & $\Delta$ STAT-3 & $\Delta$ VEGFR-2 & $\Delta$ STAT-1 \\
\hline Patient-1 & Proliferating & 1.1 & 3.3 & 5.2 \\
\hline Patient-2 & Proliferating & 2.1 & 3.8 & 4.9 \\
\hline Patient-3 & Proliferating & 1.5 & 3.6 & 4.7 \\
\hline Patient-4 & Proliferating & -2.3 & 3.1 & 3.6 \\
\hline Patient-5 & Proliferating & 1.45 & -1.1 & -1 \\
\hline Patient-6 & Involuting & 0.8 & 4.2 & -0.8 \\
\hline Patient-7 & Involuting & 1.85 & 6.3 & 2 \\
\hline Patient-8 & Involuting & 4.2 & 12.8 & 4.9 \\
\hline Patient-9 & Involuting & 0.65 & 3.7 & 1.8 \\
\hline Patient-10 & Involuting & 3 & 6.2 & 5.5 \\
\hline Patient-11 & Involuting & 2.95 & 8.9 & 4.5 \\
\hline Patient-12 & Involuting & 2.2 & 8.5 & 3.4 \\
\hline Patient-13 & Involuting & -0.1 & 2.8 & 0.7 \\
\hline Patient-14 & Involuted & 4.4 & 14.4 & 7.2 \\
\hline Patient-15 & Involuted & 4.4 & 13.3 & 7.9 \\
\hline Patient-16 & Involuted & 5.1 & 10.7 & 6.4 \\
\hline Patient-17 & Involuted & 4.4 & 10.1 & 8.6 \\
\hline Patient-18 & Involuted & 4.6 & 11.25 & 7.1 \\
\hline Patient-19 & Involuted & 2.6 & 7.6 & 3.8 \\
\hline Patient-20 & Involuted & 4.2 & 10.7 & 6.1 \\
\hline
\end{tabular}

Table 4: $\triangle \mathrm{Ct}$ values for STAT-3, VEGFR-2 and STAT-1 obtained from proliferating, involuting and involuted haemangioma biopsies. 


\subsubsection{DIFFERENCES IN PROTEIN EXPRESSION BY WESTERN BLOTTING OF}

\section{HAEMANGIOMA SPECIMENS}

Antibody specificity was confirmed by using HeLa cells as a positive control. Immunoreactive bands detected on all immunoblots were at the expected molecular weights. In addition, negative controls in which the primary antibody was omitted, showed minimal staining. Therefore, all immunoreactivity observed in this study was presumed to be due to specific labelling of the primary antibodies. All band density analyses were made on the same gel.

\subsection{STAT-3 AND p-STAT-3 (TYR 705)}

To determine whether changes in STAT-3 mRNA expression correlated with changes in its protein abundance, the same samples used for mRNA analysis were analysed by Western blotting. Lysates prepared from four proliferating, three involuting and three involuted haemangioma biopsies were analysed for STAT-3 protein expression. As discussed in Chapter 1, p-STAT-3 (Tyr 705) is the activated form of STAT-3. Levels of p-STAT-3 (Tyr 705) were studied in five proliferating, three involuting and five involuted haemangioma biopsies. All samples showed STAT-3 and p-STAT-3 (Tyr 705) immunoreactive bands at $92 \mathrm{kDa}$ as expected (Wu et al., 2003). No statistically significant difference in STAT-3 protein abundance was found between proliferating, involuting and involuted specimens (Figure 3.6-A and -B).

Immunoblotting for p-STAT-3 (Tyr 705) showed that the amount of p-STAT-3 (Tyr 705) was greatest in proliferating haemangioma and decreased in involuting and involuted lesions, however, this difference in expression was not statistically significant $(p=0.23)$ as very little p-STAT-3 (Tyr 705) was detected (approximately 5\% of that of IFN $\alpha-2 b$ stimulated HeLa cells) (Figure 3.6-C and -D). 
A
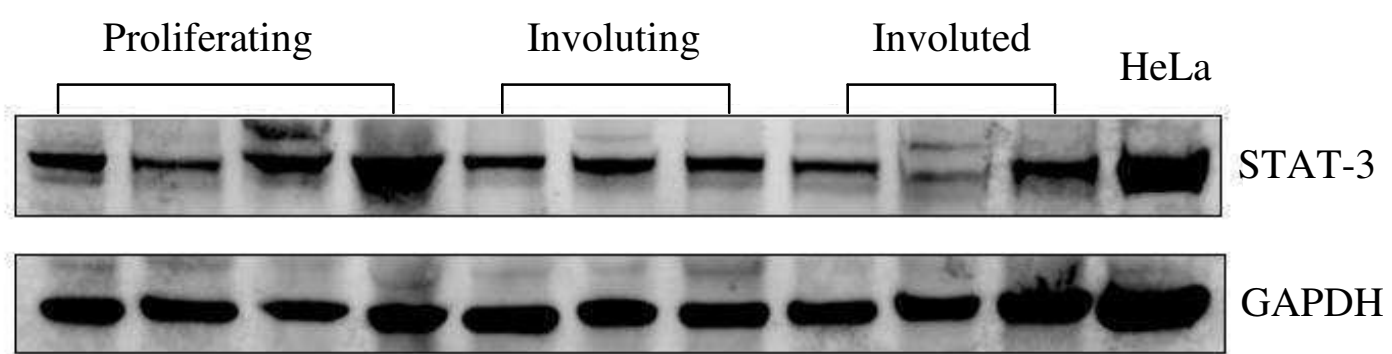

B

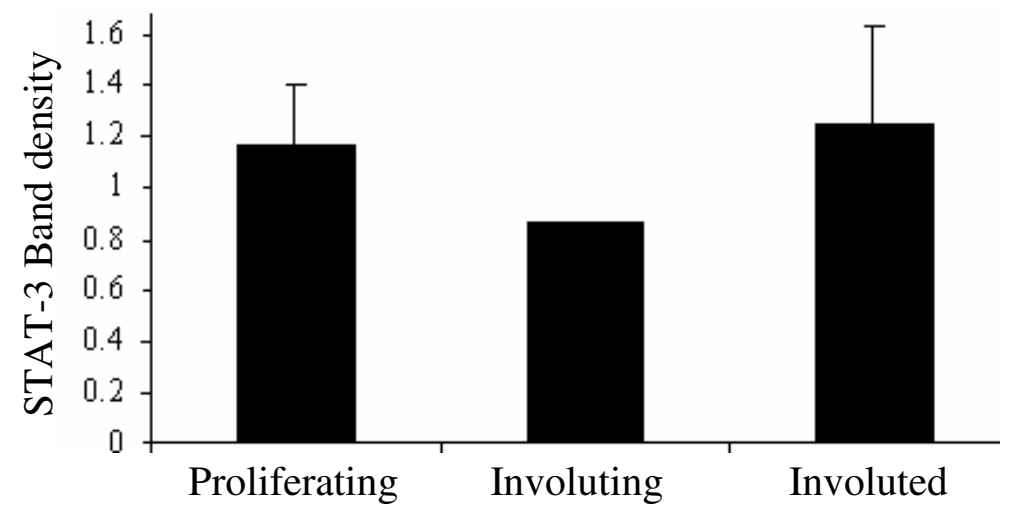

$\mathrm{C}$

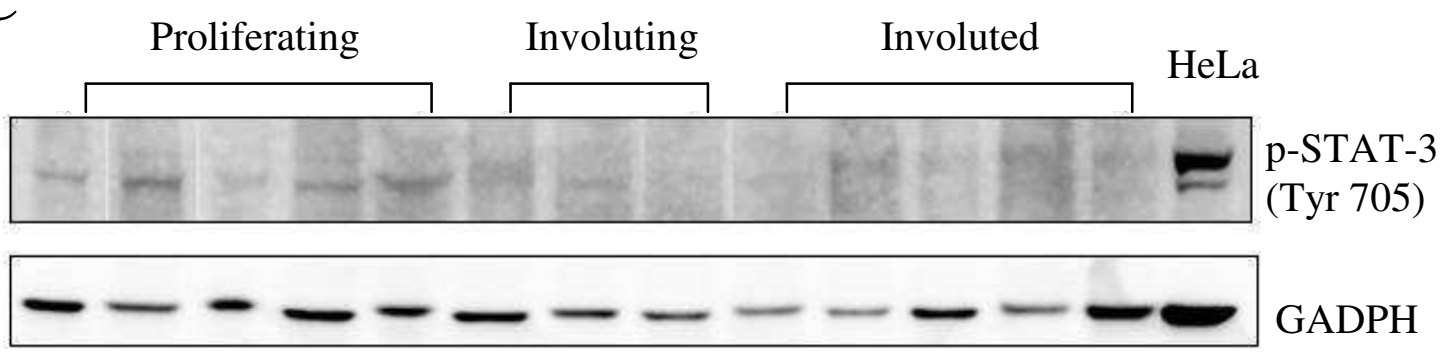

$\mathrm{D}$

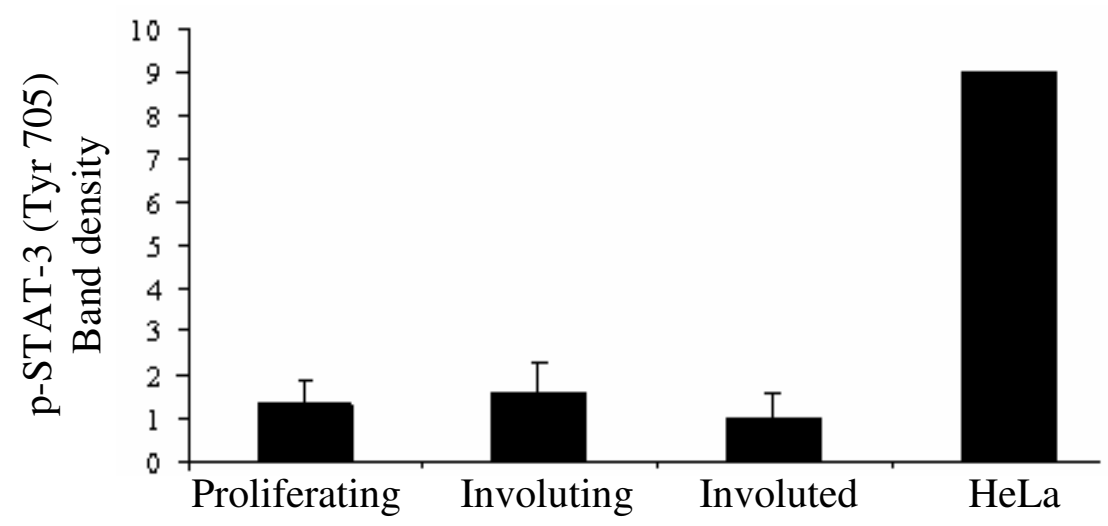

Figure 3.6: Western blotting for STAT-3 and p-STAT-3 (Tyr 705). $50 \mu \mathrm{g}$ of total protein from proliferating, involuting and involuted haemangioma lesions were subjected to immunoblotting as described in section 2.2.2.2. Membranes were incubated with either mouse anti-STAT-3 or mouse anti-p-STAT-3 (Tyr 705) along with mouse anti-GAPDH antibodies. With equal protein loaded from each sample (A), no statistically significant change in total STAT-3 protein expression was found between phases as determined by densitometry analysis $(\mathbf{B})$. Although abundance of p-STAT-3 (Tyr 705) decreased with phase $(\mathbf{C})$, this decrease failed to reach statistical significance. In addition, p-STAT-3 (Tyr 705) expression levels were significantly lower within haemangioma biopsies compared to IFNa-2b stimulated HeLa cells (D). 
STAT-1 protein expression was studied in five proliferating, three involuting and four involuted haemangioma biopsies. Immunoreactive bands were detected at approximately $90 \mathrm{kDa}$ as expected (Lemmink et al., 2001). Although there appeared to be a decrease in relative STAT-1 protein expression with ongoing involution (Figure 3.7-A), this change did not achieve significance (data not shown).

\subsection{VEGFR-1}

Five proliferating, three involuting and four involuted haemangioma biopsies were examined for VEGFR-1 protein expression. Immunoreactivity was detected at 180 $\mathrm{kDa}$ as expected (Takahashi \& Shibuya, 1997). No significant change in VEGFR-1 expression was detected between the lesions (Figure 3.7-B and -C). 
A
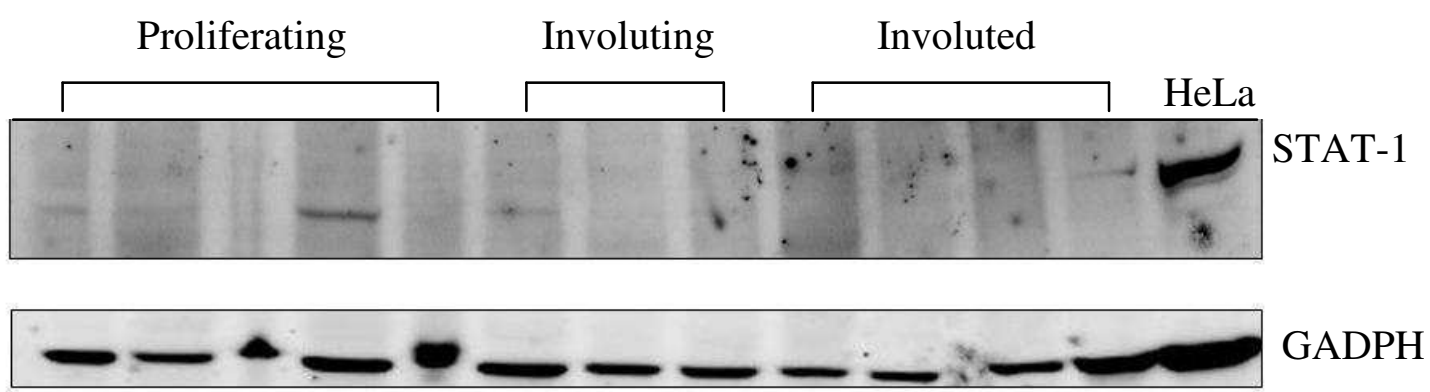

B

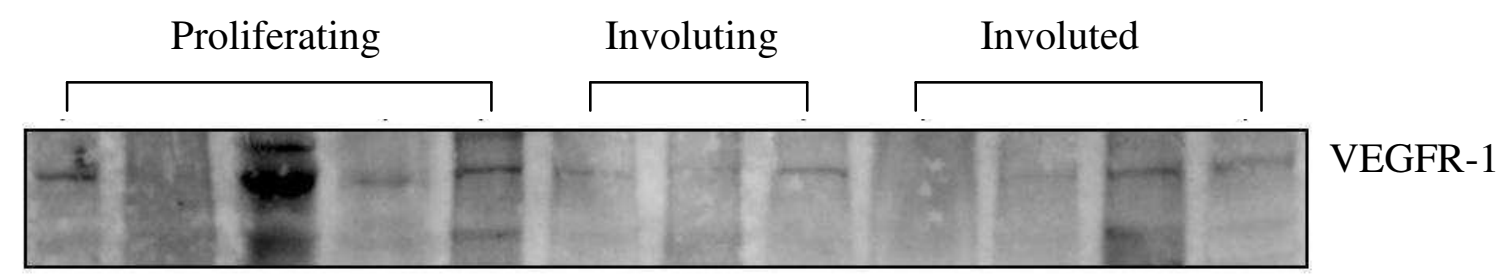

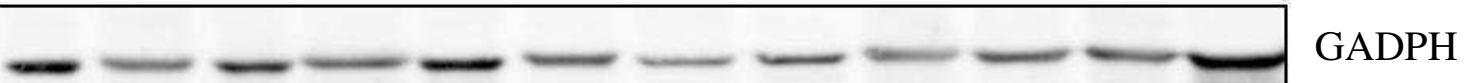

$\mathrm{C}$

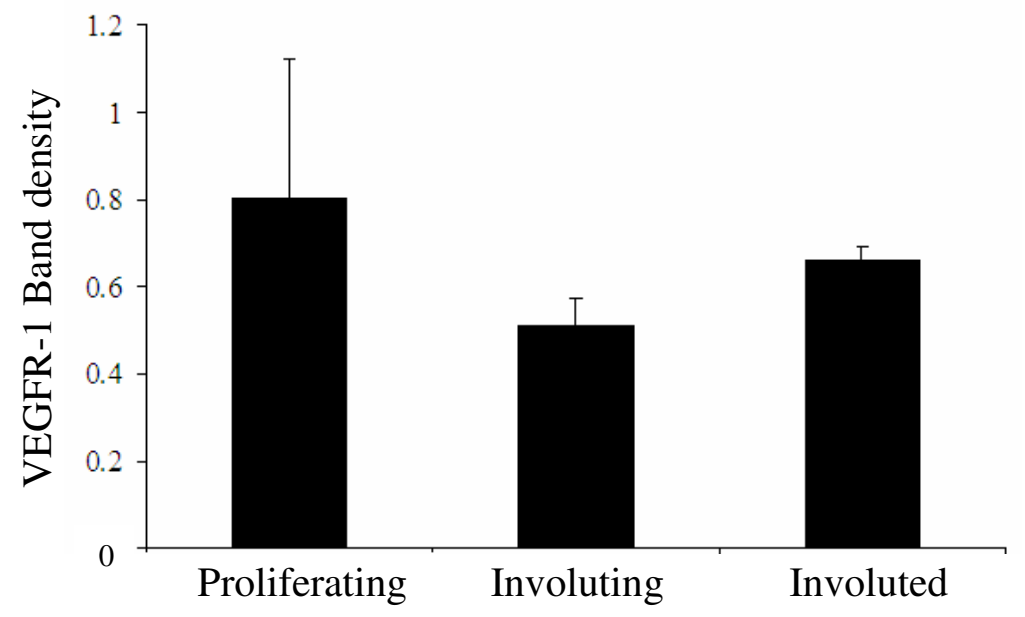

Figure 3.7: Western blotting for STAT-1 and VEGFR-1 expression in proliferating, involuting and involuted haemangioma lesions. $50 \mu \mathrm{g}$ of total protein from proliferating, involuting and involuted haemangioma lesions were subjected to immunoblotting as described in section 2.2.2.2. The membranes were blocked and probed with mouse anti-STAT-1 or goat anti-VEGFR-1 antibodies along with mouse anti-GAPDH antibodies. (A) STAT-1 expression within the haemangioma lesions was found to be significantly lower compared to IFN $\alpha-2 b$ stimulated HeLa cells (A). No statistically significant difference in STAT-1 (A) and VEGFR-1 (B) protein expression was found between proliferating, involuting and involuted specimens $(\mathbf{C})$. 


\subsubsection{IMMUNOHISTOCHEMICAL ANALYSIS}

\subsection{STAT-3 EXPRESSION}

Immunohistochemical staining of haemangioma specimens (five samples per phase) showed that STAT-3 was readily detectable in all three phases of haemangioma (Figure 3.8). Proliferating lesions showed highest STAT-3 expression in the plump ECs lining the densely packed blood vessels of the proliferative phase (Figure 3.8-A and D). Some of these cells were found to be actively proliferating as they co-expressed PCNA (Figure 3.8-A and -D, yellow arrows). Panels-B and -E show STAT-3 staining in the flattened endothelium lining the large dilated vessels and in the pericellular regions of the involuting phase. There were also fewer PCNA-positive cells present during the involuting phase (Figure 3.8-B and -E, yellow arrows). Minimal STAT-3 expression was detected in involuted lesions which were devoid of any proliferating PCNApositive cells. The same pattern of staining was observed when a different STAT-3 antibody was used.

The decrease in STAT-3 expression seen between phases in Figure 3.8 appears to be due to a decrease in cell number as apposed to a decrease in STAT-3 staining intensity per cell. 

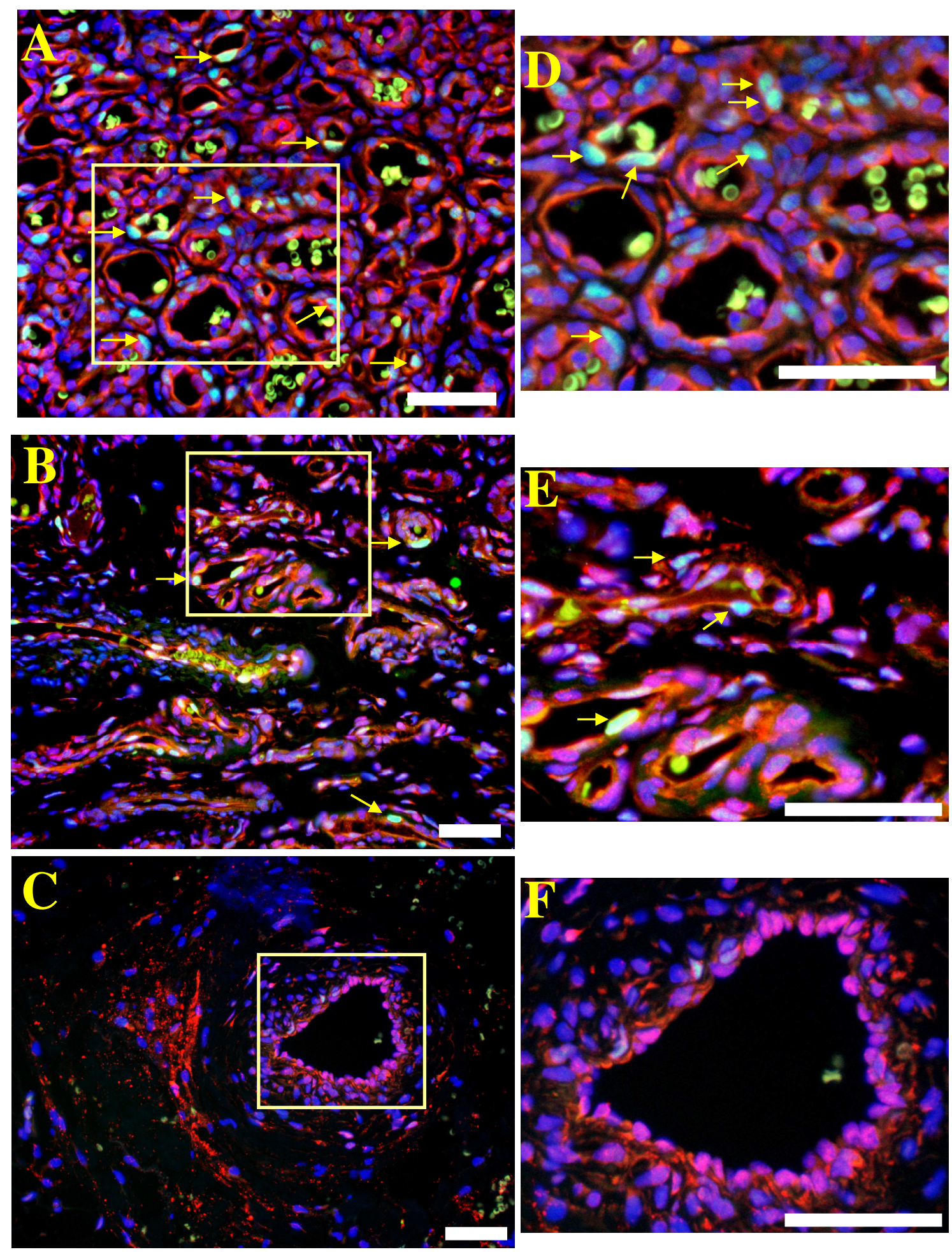

Figure 3.8: Immunohistochemical localisation of STAT-3 and PCNA in paraffin embedded haemangioma biopsies. Proliferating (A\&D), involuting $(\mathbf{B} \& \mathbf{E})$ and involuted $(\mathbf{C} \& \mathbf{F})$ haemangioma sections were stained with rabbit anti-STAT-3 and mouse anti-PCNA antibodies as described in section 2.2.2.3. Bound STAT-3 antibodies were detected using an anti-rabbit DIG conjugate and an anti-DIG-Rhodamine conjugate. Bound PCNA antibodies were detected using an anti-mouse FITC conjugate. Cell nuclei were counterstained with DAPI (blue). Panels D, E \& F are enlargements of the areas outlined in A, B \& C, respectively. Proliferating lesions (A\&D) contained the greatest number of PCNA stained nuclei (light blue staining, yellow arrows) and showed the greatest STAT-3 expression (red staining). Involuting (B\&E) and involuted lesions $(\mathbf{C} \& \mathbf{F})$ contained fewer PCNA positive cell nuclei, and showed less staining for STAT-3 protein. Red blood cells in the lumen of the blood vessels appear green, due to autofluorescence. Scale bars: $50 \mu \mathrm{m}$. 


\subsection{VEGFR-2}

Greatest VEGFR-2 immunoreactivity was detected in proliferating lesions, less in the involuting, and the least in the involuted specimens (Figure 3.9). Within the proliferative lesions, VEGFR-2 staining was restricted to the cells lining the blood vessels (Figure 3.9-A, white arrows). These cells have been characterised as plump ECs with "immature" morphology, that is, large nuclei and scant cytoplasm, lining small nascent vessels but also in the interstitial regions (Yu et al., 2004). Serial sections stained with anti-vWF antibody showed a similar staining pattern for vWF (Figure 3.9D) and VEGFR-2 (Figure 3.9-B). These results indicated that most of the VEGFR-2 positive cells also expressed vWF. However, not all vessels in the involuting haemangiomas expressed VEGFR-2 (Figure 3.9-B, yellow arrows) with almost negligible staining in the involuted lesions (Figure 3.9-C). Although the IHC technique is not considered to be quantitative, different staining intensities for VEGFR-2 were observed within a single field of view. Figure 3.9-B shows that within an involuting lesion, the small vessels continue to express high levels for VEGFR-2 (white arrows) while the larger, more established or 'mature' vessels show decreased immunoreactivity for VEGFR-2 (yellow arrows). 

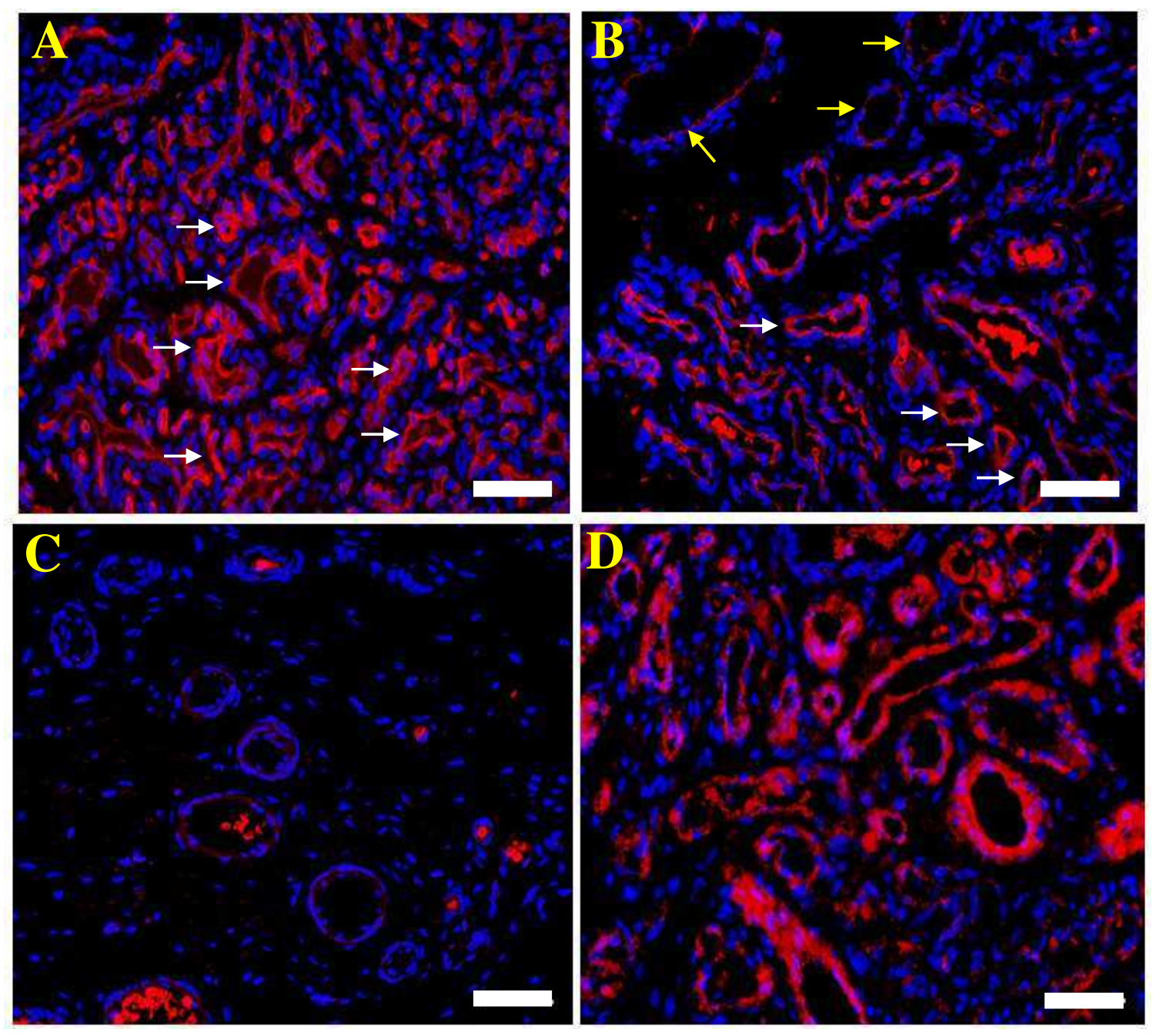

Figure 3.9: Immunohistochemical localisation of VEGFR-2 in haemangioma. Proliferating (A), involuting (B\&D) and involuted (C) haemangioma biopsies were stained with rabbit anti-VEGFR-2 antibodies (A-C) or rabbit anti-vWF antibodies (D) as described in section 2.2.2.3. Bound VEGFR-2 antibodies were detected using an antirabbit DIG conjugate and an anti-DIG-Rhodamine conjugate (A-C). vWF immunoreactivity was detected using an anti-rabbit $\mathrm{Cy} 3$ conjugate (D). Red blood cells in the lumen of the blood vessels appear red, due to autofluorescence. Cell nuclei were counterstained with DAPI (blue). VEGFR-2 expression (red staining) was found to be the greatest in the proliferating lesions (A, white arrows) and less in involuting lesions (B, white arrows) with immunoreactivity localized to the cells surrounding the vessel lumens (white arrows). Note some large vessels in the involuting do not stain for VEGFR-2 (B; yellow arrows) whereas other smaller vessels in the vicinity still express VEGFR-2 (B; white arrows). There was negligible VEGFR-2 expression in involuted lesions (C). Immuonoreactivity for $\mathrm{vWF}$ (red) was also localised to the cells surrounding the blood vessel lumens (D) indicating that VEGFR-2 expression may be predominantly expressed by the ECs in haemangioma. Scale bars: $50 \mu \mathrm{m}$. 
The anti-STAT-1 antibody used in this study did not yield a detectable signal on immunofluorescently labelled tissue sections. Therefore, STAT-1 immunoreactivity was visualised using a peroxidase conjugated secondary antibody in combination with $\mathrm{DAB}$ as the enzyme substrate.

STAT-1 immunoreactivity was present in the plump ECs lining the vessels as well as in the interstitium of the proliferative phase (Figure 3.10-A) and decreased in the involuting phase (Figure 3.10-B). The difference in STAT-1 expression between the proliferative and involuting phase may be merely a consequence of decreased cellular content in the involuting lesions as compared with proliferating lesions. Involuted lesions (Figure 3.10-C) showed almost negligible STAT-1 immunoreactivity, which was similar in staining intensity to the negative control (Figure 3.10-D). Some MCs in the proliferative and involuting phases also expressed STAT-1 (Figure 3.10- A and -B, black arrows).

STAT-1 protein abundance was also analysed by Western blotting (see section 3.3.2.2.2, Figure 3.7-A, page 115). From a total of 12 samples, only one proliferative lesion exhibited a strong immunopositive band for STAT-1. Week bands were detected from two other proliferating lesions and one involuting lesion. The IHC and Western blotting results therefore suggest that haemangioma express low levels of STAT-1.

\subsection{GLUT-1}

GLUT-1 has been identified as an immunohistochemical marker expressed in all three phases of haemangioma but not in other vascular malformations (North et al., 2000). Some of the haemangioma biopsies used in this study were stained with rabbit anti-GLUT-1 antibodies. An example of GLUT-1 immunoreactivity on haemangioma samples is shown in Figure 3.11. 


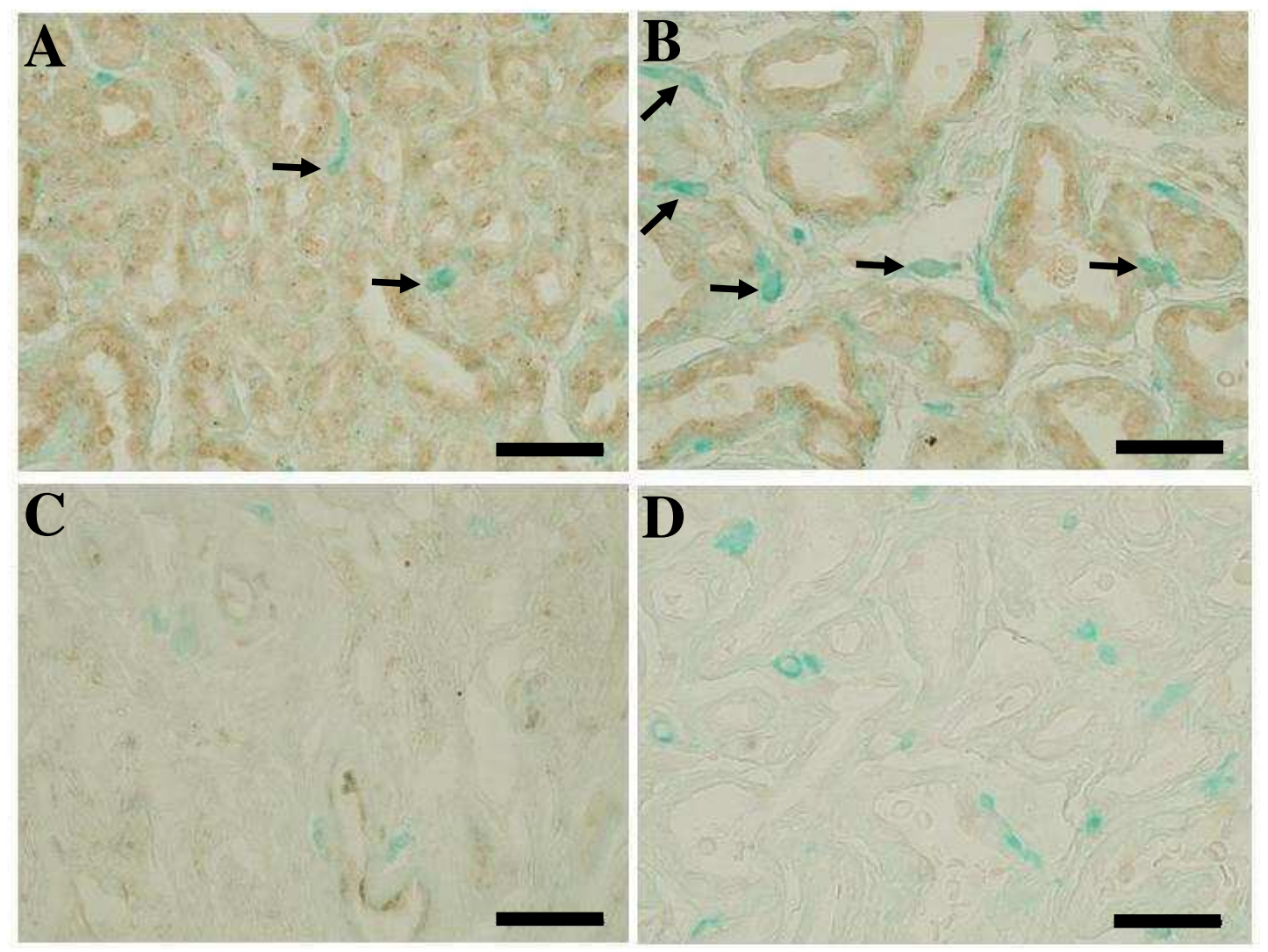

Figure 3.10: Immunohistochemical localisation of STAT-1 in haemangioma. Proliferating (A), involuting (B) and involuted (C) haemangioma tissue sections were stained with anti-STAT-1 antibodies and counterstained with Csaba to identify MCs (light blue) as described in section 2.2.2.4. Most STAT-1 immunoreactivity (brown staining) was detected in the ECs surrounding the blood vessels of the proliferative phase (A) and the involuting phase (B). Some STAT-1 expression was also detected in the MCs (light blue staining, black arrows). Weak STAT-1 expression was present in the involuted phase with only a few positively stained flattened endothelia (C). An involuting haemangioma section used as the negative control showed no non-specific staining of the STAT-1 antibody (D). Note the increase in MC numbers in the involuting phase $(\mathbf{B} \& \mathbf{D})$. Immunoreactivity was detected with $\mathrm{DAB}$ and visualised using bright field microscopy. Scale bars: $50 \mu \mathrm{m}$.
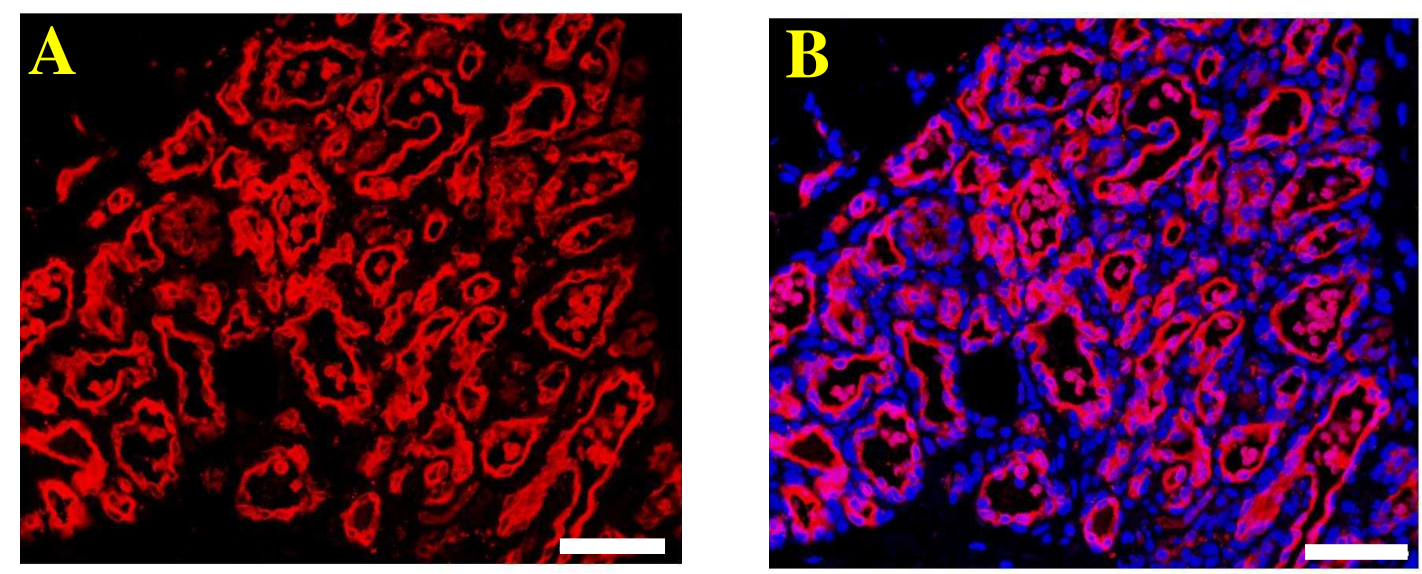

Figure 3.11: Intense GLUT-1 immuoreactivity in a proliferating haemangioma. Sections were stained with rabbit anti-GLUT-1 antibodies as described in section 2.2.2.3. Bound GLUT-1 antibodies were detected with an anti-rabbit Cy3 conjugate (red). (A) GLUT-1 immunoreactivity (red staining) was detected on the cells that surround the blood vessels. Panel B shows the overlayed image of the DAPI stained cell nuclei (blue) and the GLUT-1 positive vessels (red). Scale bars: $50 \mu \mathrm{m}$. 


\subsection{MAST CELLS EXPRESS STAT-3}

Csaba staining was used to identify MCs (Rüger et al., 1994) and showed that there was a three-fold increase in MC numbers in involuting haemangiomas compared with proliferating haemangiomas, and that the MCs were predominantly of biogenic amine phenotype throughout the development of haemangioma (Figures 3.12). These findings are consistent with those previously reported (Tan et al., 2000a, Tan, 2001).

This study showed that MCs in both the proliferative and involuting phases expressed STAT-3 protein. Of 200 MCs counted, those that stained positive for STAT-3 (STAT-3 + MCs) and negative (STAT-3 - MCs) were identified in the proliferative and involuting phases. Although the total number of MCs was higher in the involuting phase than the proliferative phase, the proportion of STAT-3 expressing MCs was highest during proliferative phase and decreased in the involuting phase (Figure 3.12-C and Table 5). This change was found to be statistically significant with $p<0.05$.

Developmental Phase Number of STAT-3 + MCs $\quad \%$ STAT-3 + MCs

Proliferative

$195.33 \pm 6.6(n=30)$

90.5

Involuting

164. $5 \pm 5.5(n=10)$,

76.1

Proliferative vs Involuting $\quad p<0.05$

Table 5: The total number and percentage of STAT-3 positive MCs in proliferating and involuting haemangioma. Values are mean \pm s.e.m; $n$ indicates the average number of fields counted at a magnification of X40. 

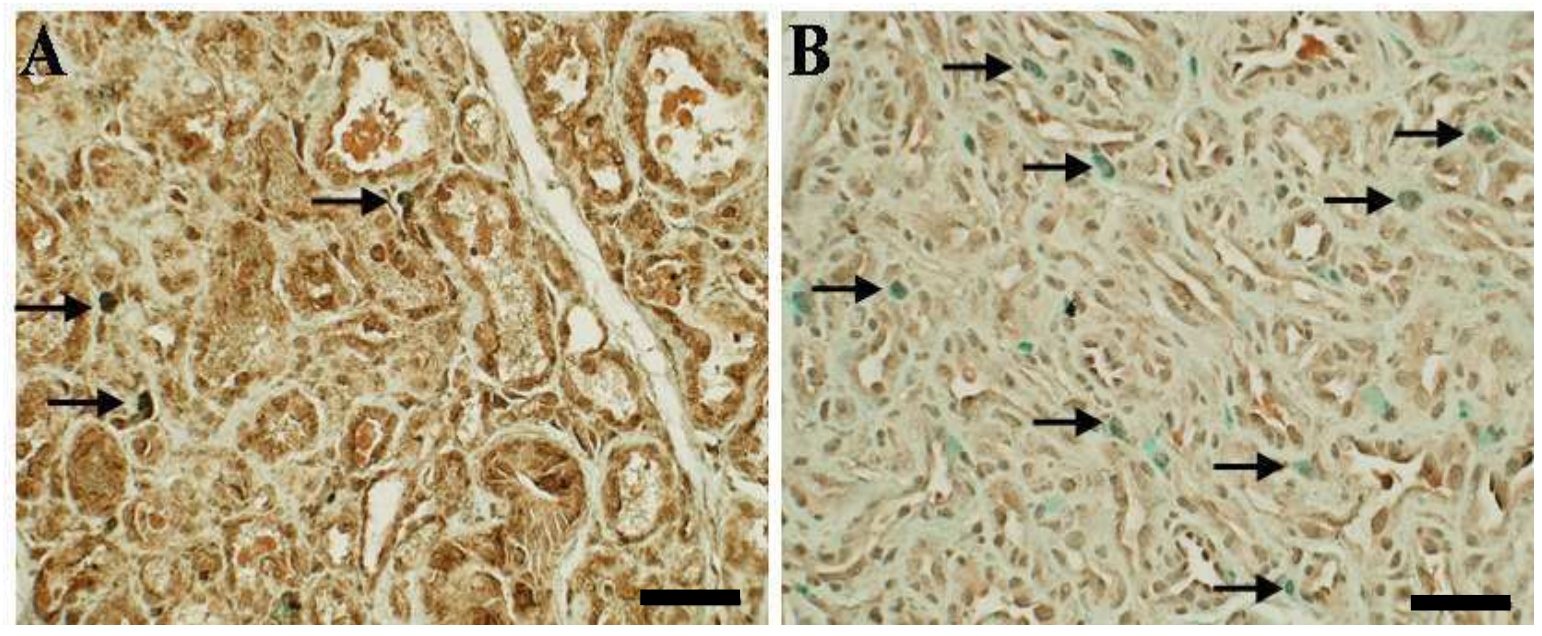

$\mathrm{C}$

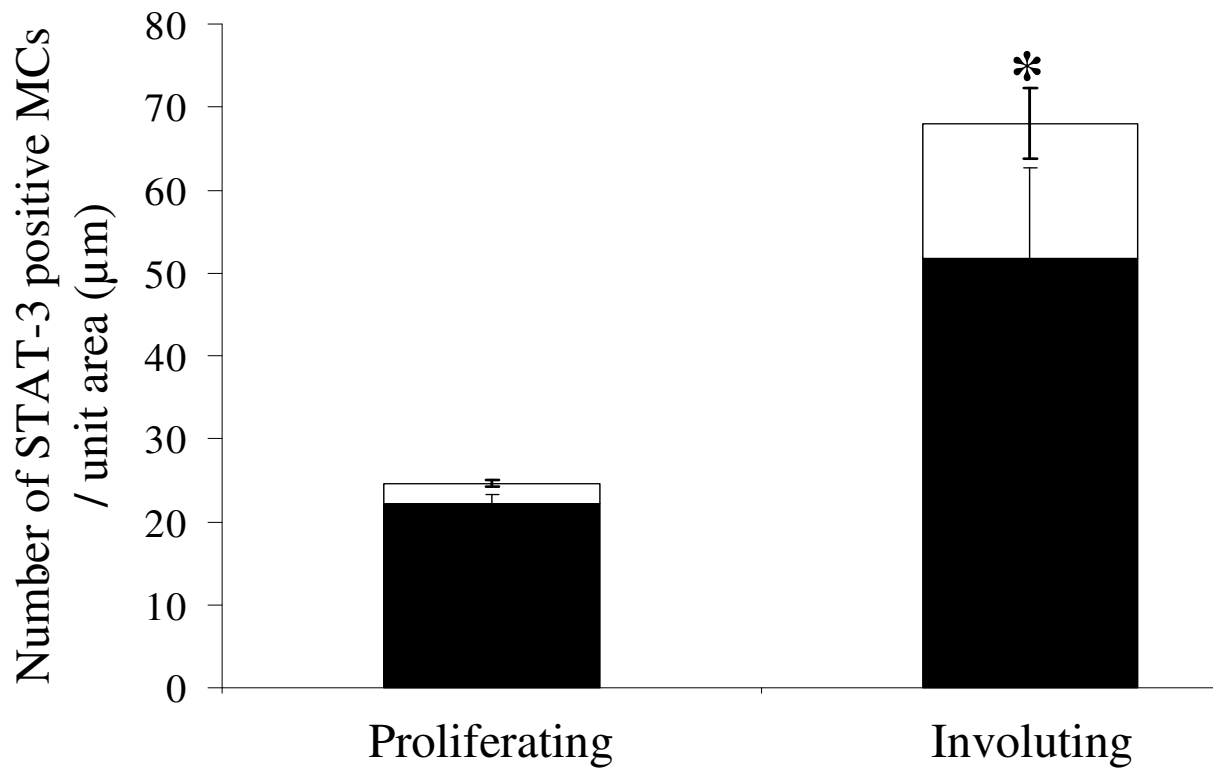

Figure 3.12: Mast cells present in proliferating and involuting haemangiomas express STAT-3. STAT-3 immunoreactivity was detected by DAB staining (brown) and counterstained with Csaba to identify the MCs (light blue). Double labelling of a proliferating (A) and an involuting (B) haemangioma with STAT-3 antibodies and Csaba stain demonstrated the presence of STAT-3 protein within MCs (black arrows). (C) The black columns show STAT-3 positive MCs and the white columns show STAT-3 negative MCs. Fewer MCs were present in proliferating lesions per unit area compared to involuting lesions which contained a three-fold increase in the number of MCs per unit area. However, 90\% of MCs in proliferating lesions expressed STAT-3 while only $76 \%$ of MCs expressed STAT-3 in the involuting phase. This change reached statistical significance with $* p<0.05$. Scale bars: $50 \mu \mathrm{m}$. 


\subsubsection{STAT-3 AND p-STAT-3 (TYR 705) IMMUNOLOCALISATION}

Confocal microscopy was used to examine the intracellular localisation of STAT-3 and p-STAT-3 (Tyr 705) within proliferating haemangioma biopsies. Tissue sections were double labelled with PCNA and STAT-3 or p-STAT-3 (Tyr 705). STAT3 expression was detected in the cytoplasm of both proliferating and non-proliferating cells (Figure 3.13, red staining). While immunoreactivity for p-STAT-3 (Tyr 705) was localised to the nucleus across all haemangioma tissue sections examined, p-STAT-3 (Tyr 705) staining was detected in both proliferating (PCNA positive) (Figure 3.14) and non-proliferating cells (PCNA negative) (data not shown). Figure 3.14 shows a region on a proliferating haemangioma tissue section where p-STAT-3 (Tyr 705) expression was detected within the nuclear compartment of PCNA positive cells. 

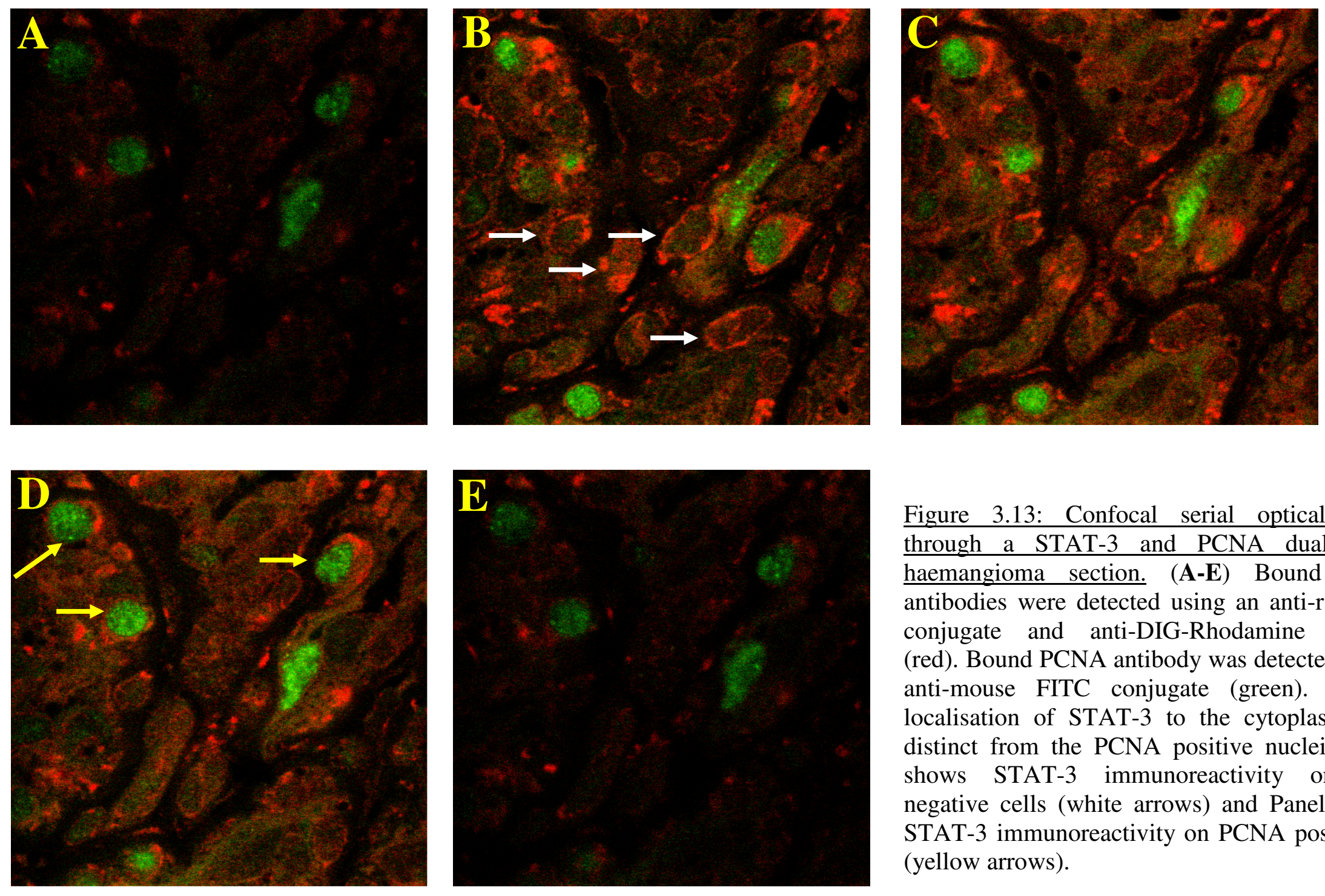

Figure 3.13: Confocal serial optical sections through a STAT-3 and PCNA dual labelled haemangioma section. (A-E) Bound STAT-3 antibodies were detected using an anti-rabbit DIG conjugate and anti-DIG-Rhodamine conjugate (red). Bound PCNA antibody was detected using an anti-mouse FITC conjugate (green). Note the localisation of STAT-3 to the cytoplasm clearly distinct from the PCNA positive nuclei. Panel B shows STAT-3 immunoreactivity on PCNA negative cells (white arrows) and Panel D shows STAT-3 immunoreactivity on PCNA positive cells (yellow arrows). 

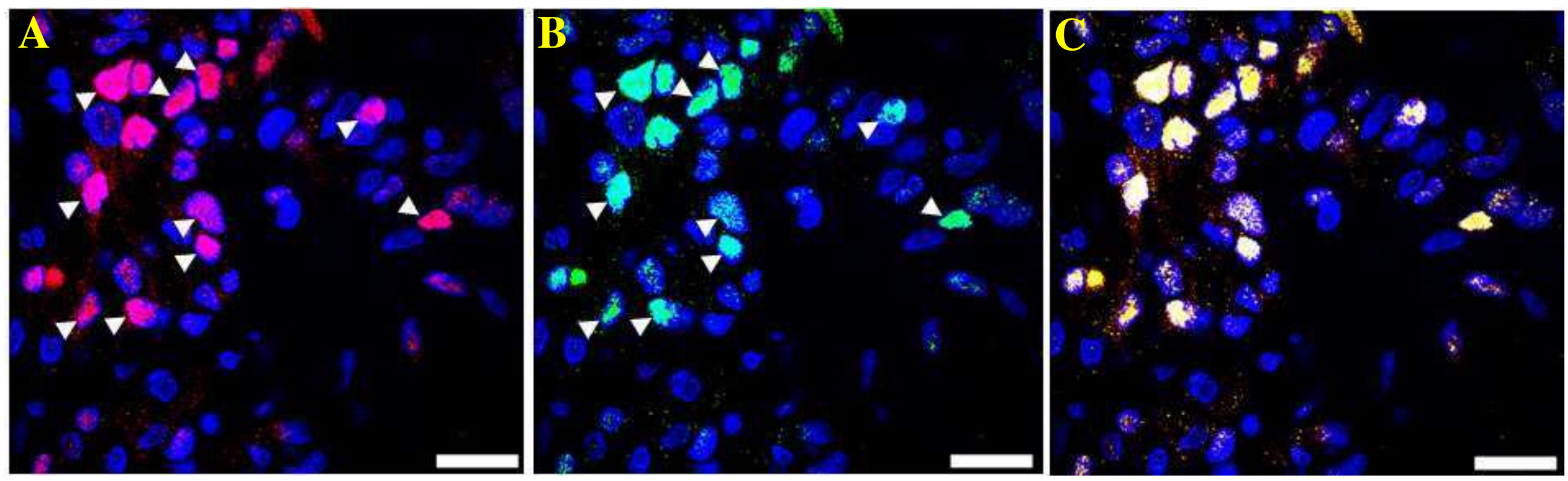

Figure 3.14: Confocal images through a p-STAT-3 (Tyr 705) and PCNA dual-labelled proliferating haemangioma section. (A) Bound p-STAT-3 (Tyr 705) antibodies were detected using an anti-rabbit DIG conjugate and an anti-DIG-Rhodamine conjugate (red). (B) Bound PCNA antibodies were detected using an anti-mouse FITC conjugate (green). Cell nuclei were counterstained with DAPI (blue). Panels A ands B show p-STAT-3 (Tyr 705) (white arrow heads, red) and PCNA (white arrow heads, green) staining within the cell nuclei, respectively. Panel $\mathbf{C}$ is the overlay of $\mathbf{A}$ and $\mathbf{B}$ showing co-localisation of pSTAT-3 and PCNA to the nucleus (yellow). Although in this image, p-STAT-3 (Tyr 705) appears to co-localise with PCNA, this was not always case in other regions of the same section and on other haemangioma tissue sections. Scale bars: $20 \mu \mathrm{m}$. 


\subsubsection{STAT-3 PROTEIN EXPRESSION CORELLATES WITH PROLIFERATION}

To investigate if STAT-3 expression correlates with proliferation, immunohistochemical staining for STAT-3 was performed in conjunction with PCNA on haemangioma tissue sections (three from each phase). PCNA is a nuclear antigen expressed maximally in the $S$ phase of the cell cycle (Kurki et al., 1986). In order to use IHC to make a quantitative measurement of protein abundance, different areas on the same slide were analysed. This eliminates variables relating to tissue or slide processing and preparation. Although the haemangioma biopsies used in this study were clinically assigned to proliferative, involuting or involuted phases, there existed regions within a single tissue section that exhibited the characteristics of each phase. Therefore, this provided an opportunity to adopt IHC staining to quantitatively measure the correlation of STAT-3 expression with proliferation. As described in section 2.2.2.5.1. (page 83), a 'proliferative' area (densely packed blood vessels with small lumens lined by plump ECs), an 'involuting' area (larger vessel lumens with flattened endothelium) and an 'involuted' area within the same tissue section was chosen using the DAPI channel. At least 600 cells were counted from each 'area' and the number of STAT-3-positive, PCNA-positive and those cells that expressed both STAT-3 and PCNA were tabulated and expressed as the number of positively stained cells per unit area (Figures 3.15 and 3.16). The results from additional lesions analysed are shown in Figures 1.10 and 1.11 in Appendix 1 (pages 281 and 282). The cells counted represent all positively stained cells across the lesions and not only the ECs. Results showed that STAT-3 and PCNA protein expression concurred with the mRNA study in that the proliferative areas of proliferating haemangiomas had more PCNA stained nuclei and the highest number of STAT-3 positive cells compared with the involuting and involuted areas on the same section. However, not all PCNA-positive cells expressed STAT-3 and vice versa. 


\section{Proliferating haemangioma}

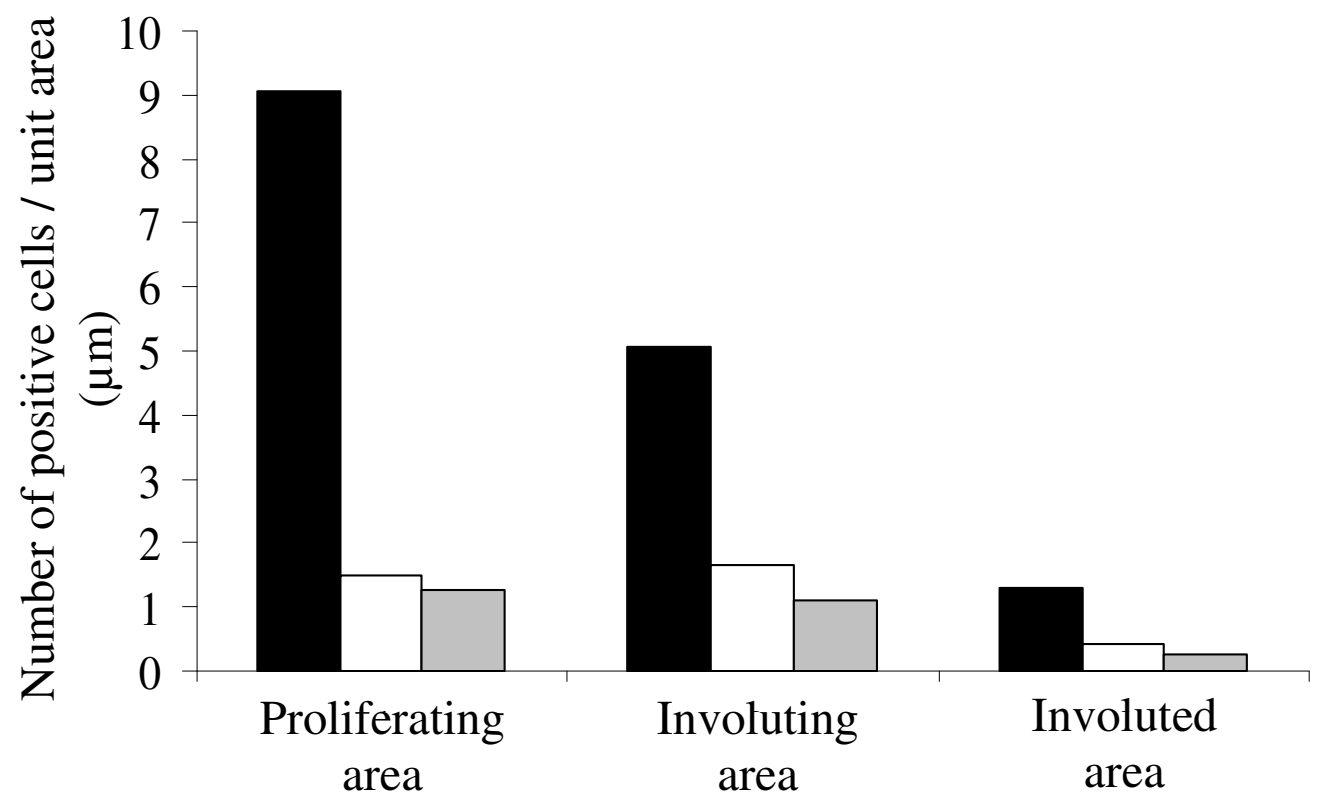

$\mathrm{B}$

\begin{tabular}{llll}
\hline & Proliferative area & Involuting area & Involuted area \\
\hline STAT-3 & $68 \%$ & $55.5 \%$ & $25.7 \%$ \\
PCNA & $15 \%$ & $25 \%$ & $11.3 \%$ \\
STAT3+PCNA & $7 \%$ & $10 \%$ & $4.5 \%$ \\
\hline
\end{tabular}

Figure 3.15: STAT-3 expression in different areas of proliferating haemangioma. (A) Results shown here are representative of three different lesions analysed. Black columns show STAT-3-positive cells, white columns show PCNA-positive cells and grey columns show the number of cells that stain positive for STAT-3 and PCNA (STAT3+PCNA). 'Proliferating' areas within a proliferating lesion contained the most number of STAT-3-positive cells which decreased in the 'involuting' and 'involuted' areas. No significant differences in the number of proliferating cells (PCNA-positive) between the 'proliferating' and 'involuting' areas suggests that the lesion on the whole is highly proliferative. 'Involuted' areas contained few STAT-3 and PCNA-positive cells. (B) Percentage of cells that stained positive for STAT-3, PCNA and those that expressed both STAT-3 and PCNA (STAT3+PCNA). 


\section{Involuting haemangioma}

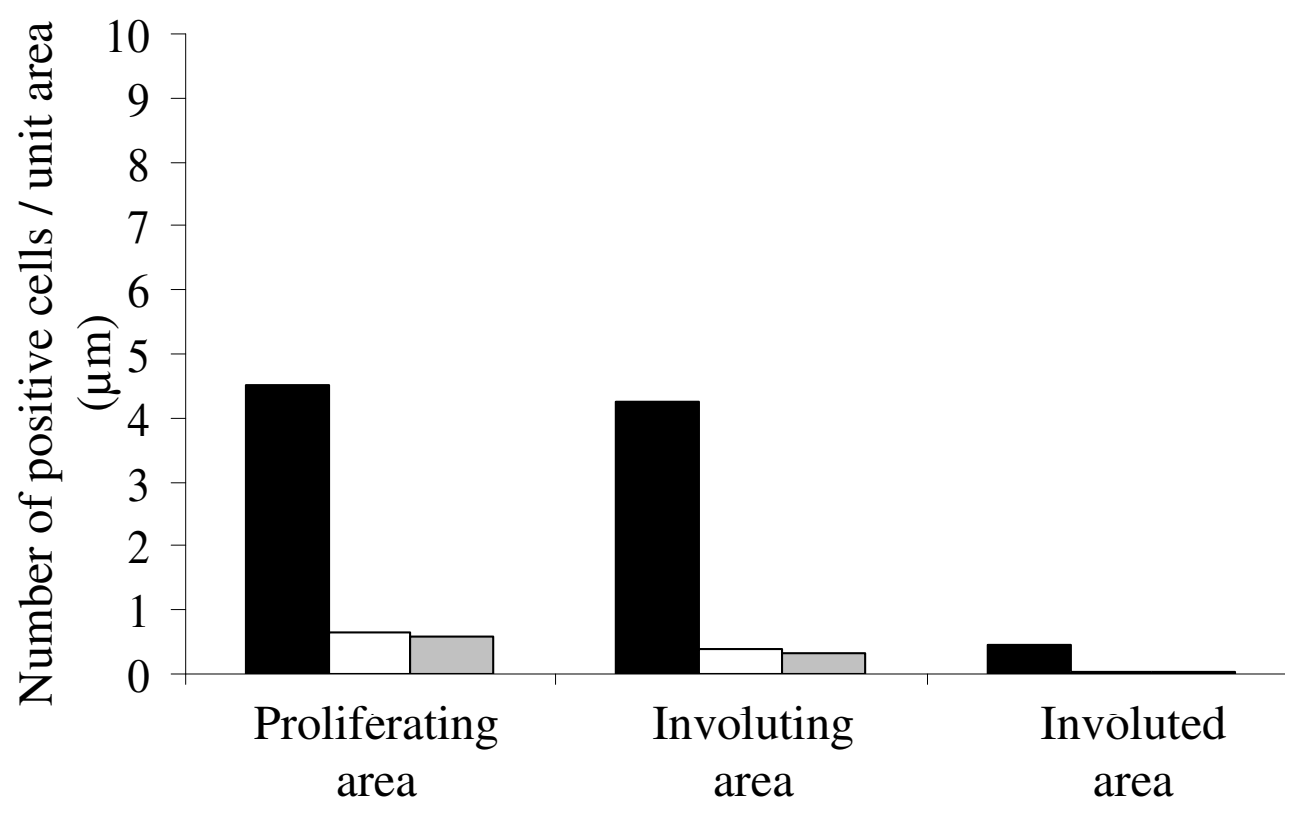

$\mathrm{B}$

\begin{tabular}{llll}
\hline & Proliferative area & Involuting area & Involuted area \\
\hline STAT-3 & $90 \%$ & $47.3 \%$ & $26.7 \%$ \\
PCNA & $13 \%$ & $4.5 \%$ & $2.4 \%$ \\
STAT3+PCNA & $12 \%$ & $3.6 \%$ & $1.9 \%$ \\
\hline
\end{tabular}

Figure 3.16: STAT-3 expression in different areas of involuting haemangioma. (A) Results shown here are representative of three different lesions analysed. Black columns show STAT-3-positive cells, white columns show PCNA-positive cells and grey columns show the number of cells that stain positive for STAT-3 and PCNA. 'Proliferating' areas within an involuting lesion contained the most number of STAT-3positive cells which decreased in the 'involuting' and 'involuted' areas. There were also fewer PCNA-positive cells in the 'involuting' area as compared to the 'proliferative' area. The 'involuted' areas were devoid of any PCNA expression. 'Involuted' areas contained very few STAT-3-positive cells. (B) Percentage of cells that stained positive for STAT-3, PCNA and those that expressed both STAT-3 and PCNA (STAT3+PCNA). 


\section{PROLIFERATION}

This study found that p-STAT-3 (Tyr 705) immunoreactivity was not evenly distributed across haemangioma tissue sections but was found in distinct but random regions. To investigate if p-STAT-3 (Tyr 705) expression correlates with proliferation, immunohistochemical staining for p-STAT-3 (Tyr 705) was performed in conjunction with PCNA on five proliferating and three involuting haemangioma tissue samples. Positively stained cells in three fields of view were counted as described in section 2.2.2.5.3 (page 83). The counted cells in Figure 3.17 include all p-STAT-3 (Tyr 705) and PCNA-positive cells and were not limited only to the ECs in the lesions. Proliferating lesions contained the highest number of PCNA-positive cells as compared to involuting lesions (Figure 3.17). However, there was no obvious correlation between PCNA expression and p-STAT-3 (Tyr 705) expression. Figure 3.17 shows that proliferating lesions contained more p-STAT-3 (Tyr 705) expressing cells than involuting lesions and that the decrease in p-STAT-3 (Tyr 705) did not correlate with PCNA expression. In addition, the staining pattern of p-STAT-3 (Tyr 705) did not correlate with either age or treatment regime of the patient. Therefore, these results indicate a role for p-STAT-3 (Tyr 705) in signalling pathways other than proliferation in haemangioma. 


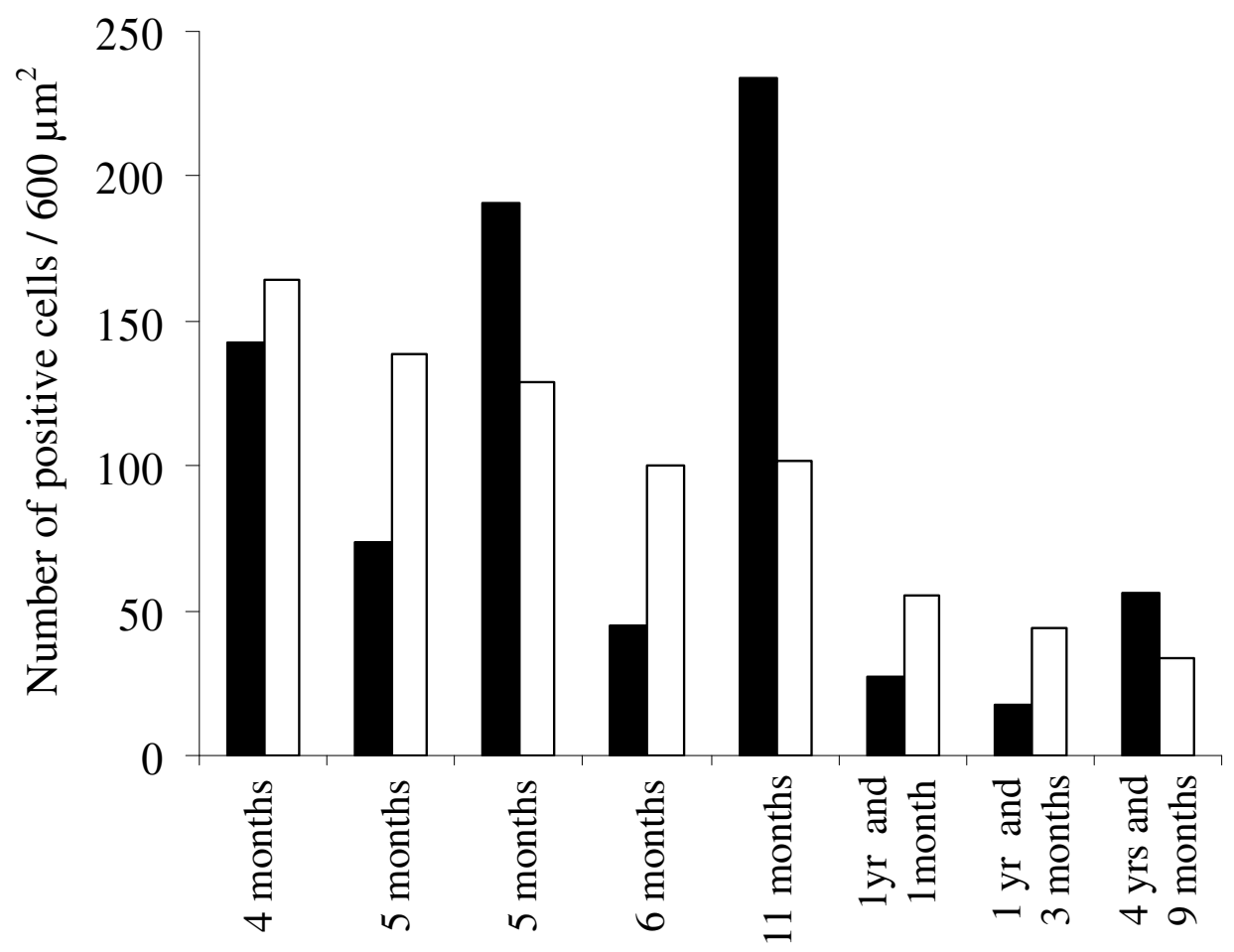

B

\begin{tabular}{lccc}
\hline Age & $\begin{array}{c}\text { p-STAT-3 } \\
\text { (Tyr 705) }\end{array}$ & PCNA & $\begin{array}{c}\text { \% p-STAT-3 (Tyr 705) } \\
+ \text { PCNA }\end{array}$ \\
\hline 4 months & 143 & 164 & 33 \\
5 months & 74 & 139 & 4.4 \\
5 months & 191 & 129 & 4.2 \\
6 months & 45 & 100 & 1.4 \\
11 months & 234 & 102 & 3.7 \\
1yr and 1 month & 27 & 55 & 2.5 \\
1yr and 3 months & 18 & 44 & 3.3 \\
4 yrs and 9 months & 56 & 34 & 14 \\
\hline
\end{tabular}

Figure 3.17: p-STAT-3 (Tyr 705) expression does not correlate with proliferation. pSTAT-3 (Tyr 705) and PCNA-positive cells were examined in proliferating and involuting haemangiomas as described in section 2.2.2.5.3. (A) Black columns show pSTAT-3 (Tyr 705)-positive cells and white columns show PCNA-positive cells. Proliferative lesions contained the highest number of PCNA-positive cells which decreased in the involuting phase. Although proliferating lesions contained more pSTAT-3 (Tyr 705)-positive cells, its expression did not correlate with PCNA expression in that p-STAT-3 (Tyr 705) was not expressed by the same cells that expressed PCNA. (B) Number of positively stained cells for p-STAT-3 (Tyr 705) and PCNA and the percentage of cells that expressed both p-STAT-3 (Tyr 705) and PCNA (p-STAT-3 (Tyr $705+$ PCNA) within three fields of view $\left(600 \mu \mathrm{m}^{2}\right)$. 


\subsection{DISCUSSION}

The proliferative phase of haemangioma is characterised by excessive angiogenesis (Mulliken et al., 1995). In section 3.1, a model was proposed in which increased VEGFR-2, STAT-3 and p-STAT-3 (Tyr 705) expression was hypothesised to be associated with haemangioma proliferation and increased STAT-1 expression with haemangioma involution. The following sections will discuss and relate the results of this study to the model described in section 3.1.

\subsubsection{IS VEGFR-2, STAT-3, p-STAT-3 (Tyr 705) SIGNALLING INVOLVED IN HAEMANGIOMA PROLIFERATION?}

Given that the growth of many tumours is dependent on VEGF/VEGR-2 signalling (Kim et al., 1993; Chen et al., 2004; Strawn et al., 1996, Drevs et al., 2000) and that VEGFR-2 expression is found to be three to five-fold higher in ECs of tumour vasculature compared with normal vasculature (Plate et al., 1994), this study sought to determine if expression of VEGFR-2 differed between the different phases of haemangioma.

Consistent with previous reports, this study found that proliferating haemangioma lesions expressed highest levels of VEGFR-2 mRNA which decreased in involuting and involuted lesions. IHC staining showed that VEGFR-2 protein concurred with mRNA levels, i.e., the plump ECs lining small nascent vessels in the proliferative phase strongly expressed VEGFR-2. While all the vessels stained positive in proliferating lesions, the number and intensity of VEGFR-2 positive blood vessels decreased in the involuting lesions, with the involuted lesions being completely devoid of any VEGFR-2 immunoreactivity. These results confirm that increased VEGFR-2 expression may be crucial for haemangioma proliferation. This pattern of VEGFR-2 expression is also consistent with the increasing evidence of its importance in tumour vascularisation, growth, and metastasis (Millauer et al., 1994; Bernatchez et al., 1999). 
VEGFR-2 activates STAT proteins (Bartoli et al., 2000; Bartoli et al., 2003). In particular, STAT-3 has been shown to be activated by EC receptors such as Ang-2 and VEGFR-2 (Korpelaine et al., 1999; Bartoli et al., 2000; Niculescu et al., 1999; Bartoli et al., 2003; Schaefer et al., 2002). While numerous studies have found STAT-3 activation in a variety of human tumours including breast, prostate, head and neck and ovarian cancer (Garcia et al., 2001; Grandis et al., 2000; Dhir et al., 2002; Song \& Grandis, 2000), other investigators have reported that STAT-3 activation was required and sufficient to mediate cellular transformation (Bromberg et al., 1998; Turkson et al., 1998; Bromberg et al., 1999; Ram et al., 2000). In addition, Chen et al (2004) showed that a strong a correlation existed between VEGF, VEGFR-2, STAT-3 and p-STAT-3 expression in ovarian epithelial carcinoma. A role for STAT-3 has also been identified in vasculogenesis as well as angiogenesis. Osugi et al (2002) showed that cardiacspecific activation of STAT-3 promoted vascular formation in the heart while Funamoto et al (2000) found that STAT-3 signalling could control vessel growth during cardiac remodelling. In addition, Valdembri et al (2002) found that STAT-3 activation induced vessel sprouting from chicken aorta rings. Chen et al (2007) have also recently reviewed the role of STAT-3 in angiogenesis.

This study found that proliferating haemangioma expressed highest levels of STAT-3 mRNA with decreased abundance in involuting and involuted lesions. In addition, a strong correlation between VEGFR-2 and STAT-3 mRNA expression was identified. This suggests that VEGFR-2 signalling may be important in driving EC proliferation through STAT-3 activation, although direct evidence of this relationship was not investigated at this stage of the study. A more functional role for STAT-3 is discussed in section 4.3.4 (page 163). Increased transcription of STAT-3 and VEGFR-2 mRNA is also indicative of the rapid turnover occurring in the proliferative phase. Double staining IHC results showed that 'proliferating' areas of haemangioma tissue sections taken from lesions in the proliferative and involuting phase contained the 
highest number of STAT-3 cells and also the highest proportion of proliferating nuclei (PCNA-positive) as compared to the involuting and involuted areas. IHC results also showed that some of these proliferating cells (PCNA-positive) also expressed STAT-3. These findings support the role of STAT-3 in haemangioma proliferation.

In addition, 90\% of MCs present in the proliferative phase expressed STAT-3, and although the number of MCs increased approximately three-fold in involuting lesions, only $70 \%$ of the MCs in the involuting phase expressed STAT-3. The promotion of neovascularisation by MC products such as FGF-2 has been known for some time (Norrby, 1994; Norrby et al., 1990). The positive staining for STAT-3 in MCs in both proliferative and involuting lesions suggests that STAT-3 may be synthesised and stored by MCs. A similar role for MCs in the synthesis and/or storage of other angiogenic proteins has been previously described (Tan, 2001; Tan et al., 2000, Tan et al., 2004).

Although IHC showed that the number of STAT-3 expressing cells was highest in the proliferative phase and that fewer STAT-3 expressing cells were present in involuting and involuted lesions, Western blotting showed that abundance of STAT-3 protein per cell remained unaltered between the proliferating, involuting and involuted lesions. This discrepancy may be due to the basic biology of these lesions. Through-out this study, involuting and involuted lesions contained more fibro-fatty deposits and lysates from these lesions contained less total protein per mg of tissue as compared to proliferating lesions. Therefore, larger volumes of protein lysate from the involuting and involuted lesions were loaded on the gels during Western blotting to maintain equal protein loading. These results mean that although the number of STAT-3 expressing cells is highest in the proliferative phase compared to the involuting and involuted phases, total STAT-3 protein abundance per cell does not change with the phases. Interestingly, Yang et al (2005) showed that unphosphorylated STAT-3 can also drive 
gene expression of proteins such as MET and MRAS, both of which are present at high levels in many cancers.

Phosphorylation of STAT-3 at tyrosine-705 is generally considered to be a prerequisite for STAT-3 activation in tumours (Shi et al., 2002). This tyrosine residue is constitutively phosphorylated during viral-induced angiogenesis and transformation in Kaposi's sarcoma (Burger et al., 2005). Of particular importance to this study is the fact that this residue is phosphorylated by VEGF signalling. Bartoli et al (2000) showed that STAT-3 (Tyr 705) phosphorylation occurred within 60-90 min of VEGF stimulation in bovine ECs. In addition, Johnson et al (1998) showed that STAT protein nuclear translocation was required for their transcriptional ability.

Consistent with the conventional model of p-STAT-3 (Tyr 705) activation in oncogenesis, this study identified that proliferative haemangioma contained more pSTAT-3 (Tyr 705) protein than involuting and involuted lesions. Interestingly, although p-STAT-3 (Tyr 705) immunoreactivity was nuclear in all phases, its expression was not restricted to proliferating (PCNA positive) cells. Although unexpected, these results can be explained by the involvement of p-STAT-3 (Tyr 705) in cellular processes other than proliferation, such as differentiation and apoptosis (Deng et al., 2000; Zhao et al., 2002). Although expression of p-STAT-3 (Tyr 705) expression was initially thought to be associated with the proliferation of haemangioma, the results from this study indicate a role for p-STAT-3 (Tyr 705) in the involution of haemangioma. This hypothesis is discussed further in other sections of this thesis (see section 4.3.6, page 179).

VEGFR-1 protein expression was also investigated in haemangioma biopsies in proliferating, involuting and involuted haemangioma biopsies. This receptor was studied because it was found to be expressed by HaemDMSCs (section 4.3.3.4, page 153). VEGFR-1 expression in haemangioma is discussed in Chapter 4. 


\subsubsection{IS STAT-1 INVOLVED IN HAEMANGIOMA INVOLUTION?}

STAT-1 is considered a tumour suppressor as it inhibits growth and acts as a pro-apoptotic factor (Calo et al., 2003). STAT-1 activation is required for the antiproliferative effects of IFN $\alpha$ as well as the apoptotic effect of TNF $\alpha$ (Bromberg et al., 1996; Kumar et al., 1997). The loss of STAT-1 signalling enhances oncogenesis (Kaplan et al., 1998).

Due to the involvement of STAT-1 in growth arrest and apoptosis in many cell types (Chin et al, 1996; Chin et al, 1997; Kumar et al., 1997), a role for STAT-1 signalling in the involution of haemangioma was proposed (Figure 3.1). STAT-1 mRNA and protein expression patterns were investigated in this study. qRT-PCR results showed that proliferating and involuting haemangiomas expressed more STAT-1 mRNA than involuted lesions. This decrease in mRNA levels correlated with a decrease in STAT-1 protein expression. IHC showed that proliferating lesions contained more STAT-1 protein primarily localised to the cells lining the blood vessels and also in some of the interstitial cells. Although IHC detected STAT-1 protein in proliferating and involuting haemangioma, Western blotting showed that the overall expression of STAT-1 was low. These results suggest that even though STAT-1 is classically considered to be a tumour suppressor (Kaplan et al., 1998) and a regulator of apoptosis (Chin et al., 1997; Kumar et al., 1997), it may not be an important factor in regulating the biology of haemangioma.

\subsubsection{LIMITATIONS OF THIS STUDY}

The major limitation of this study is that the samples available consisted of only formalin-fixed paraffin embedded haemangioma tissue sections and fresh snap frozen tissue biopsies. These biopsies were taken from different individuals at different developmental ages. In addition, although each lesion was clinically categorised as being proliferative, involuting or involuted, it is known that any individual 
haemangioma lesion can exhibit characteristics typical of all three phases (Tan, 2001). Although each of the biopsies predominantly represented one phase of haemangioma (i.e., either proliferating, involuting or involuted) it is possible that the tissue sample contained regions from the other phases as well. This becomes important during qRTPCR and Western blotting studies when gene expression analyses are done on snap frozen tissue samples. With IHC, however, it is possible to visually identify and discriminate between the different phases.

A second limitation of this study is that the results obtained in this chapter by qRT-PCR, IHC and Western blotting techniques identify correlations in gene expression. Ideally, experiments need to be performed on an appropriate cell line derived from haemangioma in order to provide functional evidence for the importance of VEGF and STAT signalling in these lesions. 


\section{CHAPTER 4: ROLE OF STEM CELLS IN HAEMANGIOMA}

\subsection{INTRODUCTION}

The precise causes for haemangioma remain unknown, however, considerable progress has been made towards understanding the cellular and molecular events that govern its life cycle. The early proliferating tumour is characterised by excessive angiogenesis (Takahashi et al., 1994; Kraling et al., 1996; Bielenberg et al., 1999). Several angiogenic factors such as VEGF, FGF-2, PDGF, TGFs and IL-6 (Folkman \& Klagsbrun, 1987; Folkman, 1997) are now known to be important regulators of haemangioma development (Hasan et al., 2000b; Takahashi et al., 1994; Chang et al., 1999). In addition to increased expression of receptors crucial in angiogenesis, genetic alterations and somatic mutations in VEGFRs have been reported (Walter et al., 2002; Blei et al., 1998; Walter et al., 1999; Nguyen et al., 2004; Yu et al., 2001; Ritter et al., 2002). Recently, the presence of endothelial progenitor cells (EPCs), mesenchymal stem cells (MSCs) and clonal expansion of ECs with aberrant properties have been identified in proliferating haemangioma (Yu et al., 2004a; Yu et al., 2006; Yu et al., 2001, Li et al., 2003, Khan et al., 2006; Boye et al., 2001; Walter et al., 2002).

Primary cultures derived from haemangioma are useful models for in vitro studies because it allows the addition of factors to the medium or components of the medium to be changed and their effects evaluated. However, as a single haemangioma lesion consists of a highly heterogeneous population of cells, the purification and establishment of a primary cell line is not straightforward and may often contain contamination with other cell types. Other disadvantages of using a cell culture model include the formation of abnormal cell architecture and changes in gene expression (Kivell, 2003). 
A scientific approach to the study of the pathogenesis of haemangioma has been hampered by the absence of a suitable model for haemangioma. Previous experimental models include the induction of 'haemangioma' in mice by transgenic ECs (DuboisStringfellow et al., 1994), ECs that have been transformed by the polyoma middle T oncogene (Sage, 1992; Williams et al., 1989) or ECs obtained from murine (Meininger et al., 1995) and human haemangioma (Boye et al., 2001). ECs have also been successfully cultured from human haemangioma on plasma clots (Mulliken et al., 1982). The limiting factor of these models is the absence of other cellular elements and ECM characteristics of human haemangioma as well as the necessity of plasma (Tan, 2001). Therefore, these models are not ideal for investigating the effect of modulating molecules.

In 2000, Tan et al $\left(2000_{\mathrm{b}}\right)$ developed a novel human in vitro model for haemangioma, which overcomes some of the deficiencies associated with the above systems. In this model, freshly obtained haemangioma biopsies are embedded between two fibrin gel layers and cultured in serum free conditions. Within days, a complex network of capillary-like outgrowth emanates from the tissue fragment. In addition, the serum free condition of this model allows for agents such as growth factors, drugs or antibodies to be added to the cultures so that their effect on capillary-like outgrowth can be studied. However, one limitation of this model is that cell-specific signal transduction pathways, in response to drugs or other factors added to the medium, cannot be easily investigated. 


\subsection{AIMS}

The capillary-like outgrowth emanating from the haemangioma biopsy in the explant model has previously been characterised as ECs (Tan et al., 2000 ; Ritter et al., 2000). As these cells exhibit an aggressive proliferative and migratory characteristic, it is hypothesised that these cells are involved in driving the proliferation and involution of haemangioma. The aim of this study was to purify, characterise and establish primary cultures of these cells in order to further elucidate the mechanisms regulating the programmed biological behaviour of haemangioma. A functional role for the importance of VEGF and STAT signalling in haemangioma was also investigated using the in vitro explant model and the purified cultures. 


\subsection{RESULTS}

\subsubsection{MORPHOLOGICAL OBSERVATIONS OF THE IN VITRO}

HAEMANGIOMA CULTURE

Proliferative haemangioma tissue fragments were cultured using a previously described method (Tan et al., 2000 $0_{\mathrm{b}}$ ). 90-95\% of the explants gave rise to a complex array of microvessels emanating from its perimeter within 3-4 days. Early on in this study, involuting and involuted haemangiomas were also cultured. However, outgrowths from these cultures had fewer sprouts, which were not seen until after 10-12 days in culture. This difference in growth rates between proliferative, involuting and involuted haemangiomas has been reported previously (Tan et al., 2000 ${ }_{\mathrm{b}}$ ). The capillary-like outgrowth is shown in Figure 4.1. Additional images can be found in Appendix 2, Figure 2.1, page 284.

Several other observations were made from these explanted cultures. The capillary-like structures were often noted to emanate from the tissue perimeter and follow a circular path surrounding the original tissue piece (Figure 4.2-A and Appendix 2, Figure 2.2, page 285). This ring like formation was sometimes seen even before any sprouting had occurred (Figure 4.2-B). Removal of the original tissue piece was followed by the disruption and the eventual disintegration of the newly formed outgrowths (Figure 4.2-C). 95\% of the cultures gave rise to the cell type shown in Figure 4.1 and Figure 4.2-A. However, in 4-5\% of the cultures, a different cell type was seen to grow out, sometimes along with another cell type (Figure 4.2-D and Appendix 2, Figure 2.3, page 285). This cell type was also seen to rapidly proliferate and form a sheet-like layer within the fibrin gel (Figure 4.2-E). Morphologically, these cells look similar to ECs, however, confirmation of this requires immunohistochemical analysis, which was not performed during this study. In addition, occasionally both cell types grew out from some explants (Figure 4.2-F). 

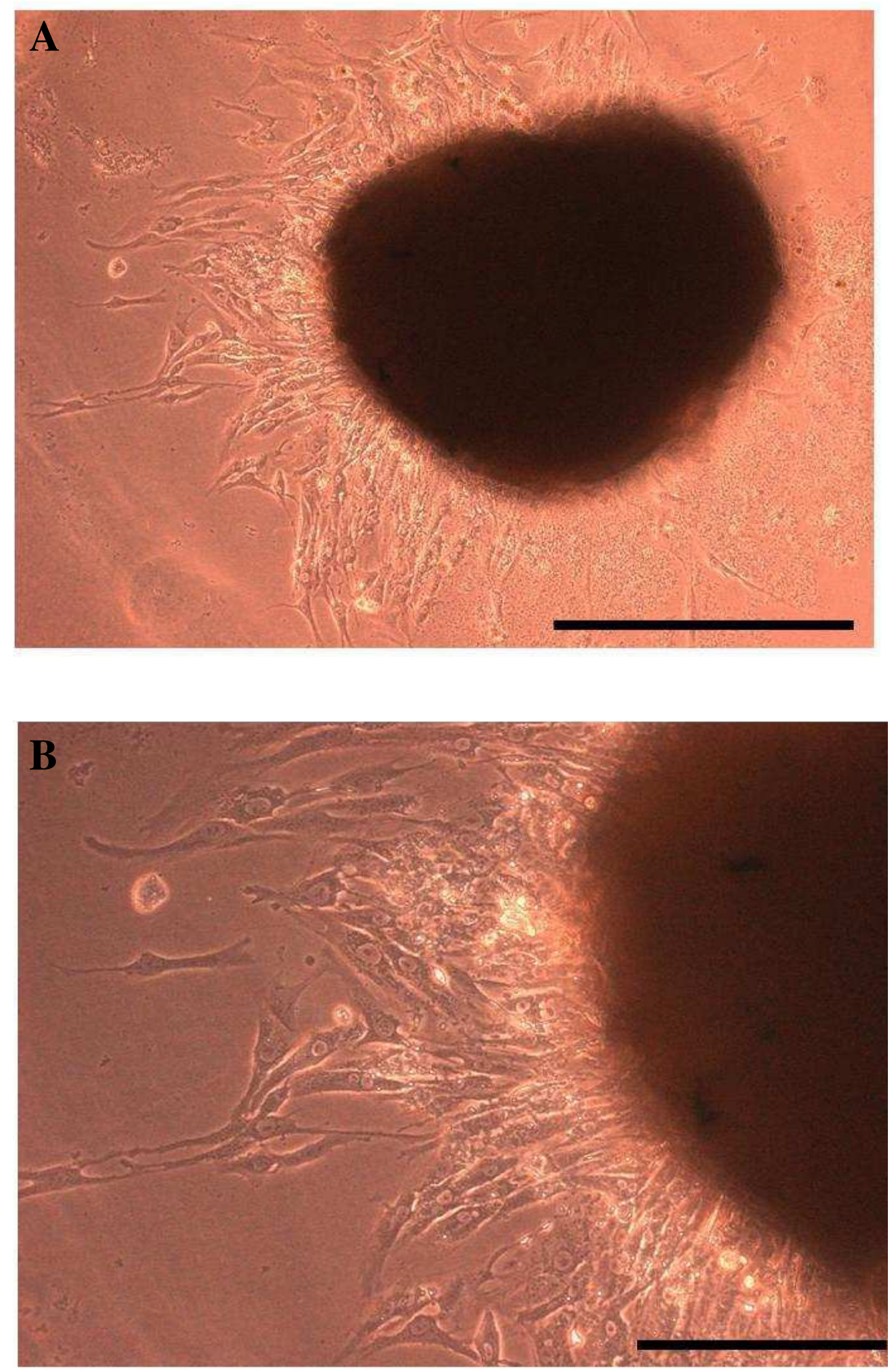

Figure 4.1: Haemangioma explant model. Phase contrast microscopy of the explant cultures at high magnification showing the individual cell structures growing out from the haemangioma tissue explant. Scale bars: (A) $500 \mu \mathrm{m}$, (B) $200 \mu \mathrm{m}$. 
Figure 4.2: Morphological characteristics of the haemangioma explant model. Proliferating haemangioma biopsies were cultured as described in section 2.3.3. (A) Capillary-like like structures that emanated from the explanted tissue followed a circular pattern of growth surrounding the original tissue. Photograph was taken on day 14 in culture. (B) A ring like clearing was sometimes observed around the explant even before any capillary-like growth. Photograph was taken on day 2 in culture. (C) Capillary-like structures did not survive when the original haemangioma tissue piece was removed. Photograph was taken on day 2 after the tissue piece was removed. (D) 4-5\% of cultured proliferating haemangioma explants gave rise to another cell type emanating from its surface. These cells exhibited the morphology similar to ECs. Photograph was taken on day 16 in culture. (E) When present, the second cell type continued to grow forming a sheet like layer in the gel matrix. (F) Some explants gave rise to two cells types with different morphologies emanating from the tissue surface. Photograph was taken on day 7 of culture. Scale bars: (A,B,C\&E) $-1000 \mu \mathrm{m},(\mathrm{D} \& \mathrm{~F})-200 \mu \mathrm{m}$, respectively. 

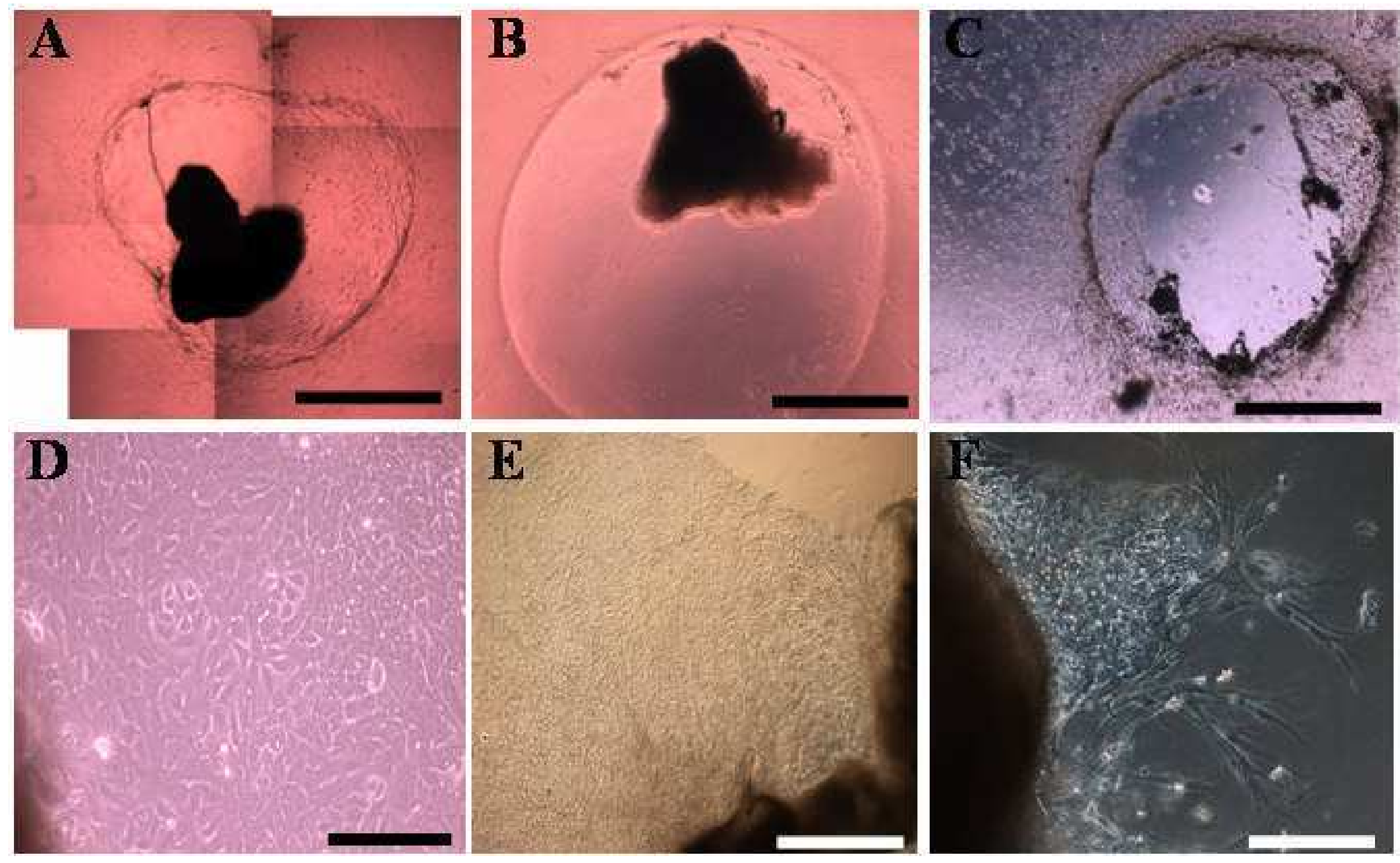


\subsubsection{MODIFICATION OF THE IN VITRO EXPLANT MODEL}

In addition to culturing haemangioma explants as described by Tan et al $\left(2000_{\mathrm{b}}\right)$, a modification of this system was also used in this study. Preliminary experiments showed that this system (see section 2.3.4, page 86) yielded capillary-like sprouts within the same time frame as the sandwich model. The main difference between the two systems is that the modified system consisted of the haemangioma tissue piece embedded into a single gel layer which was then covered with liquid medium. This modified system offered several advantages.

In testing for the effects of various agents on capillary-like outgrowth, this model, (i) allowed easy access of the agent to the explanted haemangioma biopsy; (ii) was more economical as it required less culture media and agents to be added; and (iii) was less time consuming and tedious. This system was also more suited for the purification of the capillary-like outgrowths as lower volumes of the fibrin gel used was easily digested with elastase.

\subsubsection{PURIFICATION AND CHARACTERISATION OF THE}

\section{CAPILLARY-LIKE OUTGROWTHS}

\subsubsection{CELL CULTURE CONDITIONS}

Haemangioma explant-derived primary cells (HaemDCs) were isolated from explanted cultures of six proliferating and two involuting haemangioma biopsies (see section 2.3.6, page 88 for details on the isolation procedure). The adherent cells were culture-expanded in RPMI 1640 + L-glutamine medium supplemented with $10 \%$ FCS and $2 \%$ antibiotics.

Although previous studies have shown that primary cell cultures often require special growth conditions such as the use of high serum concentrations (20\%) and additional supplements including ECGS and FGF-2, these factors were not required for the survival and proliferation of HaemDCs. Preliminary experiments showed that 
although there was no indication of apoptosis or necrosis when the cells were maintained in medium containing $1-2 \%$ FCS, the rate of proliferation was slow. Cells cultured in medium containing 0-0.1\% FCS survived for approximately 6-8 days after which the cells showed signs of senescence and eventually death (data not shown).

Coating the cell culture dish with a protein substrate to aid cell attachment is also a common practice for primary cell culture. Preliminary experiments determined that although the haemangioma-derived cell cultures attached well to all the substrates tested in this study including $10 \mu \mathrm{g} / \mathrm{cm}^{2}$ Fibronectin (Sigma), $1 \%$ Gelatine (commercially available from the super-market), Collagen (Sigma) and Poly-D-lysine (kindly provided by Dr Bronwyn Kiwell, Victoria University of Wellington), their use was found to be unnecessary and was therefore discontinued.

\subsubsection{MORPHOLOGY AND GROWTH CHARACTERISTICS}

Phase contrast microscopy of HaemDCs demonstrated a homogeneous population of fibroblast-like, spindle-shaped cells at passage 1 (Figure 4.3-B), which formed colonies when grown at low density. In later passages (> passage 5), the cells began to display a broadened, flat morphology (Figure 4.3-C), which is typical of MSCs (Tropel et al., 2004). With the appearance of the flattened star-shape morphology, the cultures grew exponentially, requiring weekly passages. They could be expanded for more than 15 doublings without any more changes in morphology and cryopreservation did not affect their growth or morphology (data not shown).

The HaemDCs also exhibited angiogenesis-like characteristics in vitro. When plated back into a fibrinogen-media gel matrix, the cells formed capillary-like structures (Figure 4.3-D), which continued to develop with time and resulted in substantial formation of capillary-like structures by day 20 in culture (Figure 4.3-E). HUVECs, which are normal ECs, did not form these capillary-like structures even after 20 days in culture (Figure 4.3-F). 
Figure 4.3: Morphological characterisation of HaemDCs. (A) Phase contrast microscopy of a proliferating haemangioma explant in culture with capillary-like outgrowths emanating from the original tissue piece. (B) Cells purified from the solubilised gel matrix and cultured in vitro exhibited fibroblast-like spindle shape morphology at passage 1. (C) At passage 5, cells appeared flat and broadened. (D) The purified cells formed capillary-like like structures when cultured in a 3-dimensional medium based gel matrix which continued to develop with time (image shown is after 15 days in culture) (E). (F) HUVECs did not form capillary-like structures when plated into the medium-based gel matrix. Scale bars: (A) $500 \mu \mathrm{m}$, (B-F) $250 \mu \mathrm{m}$. 

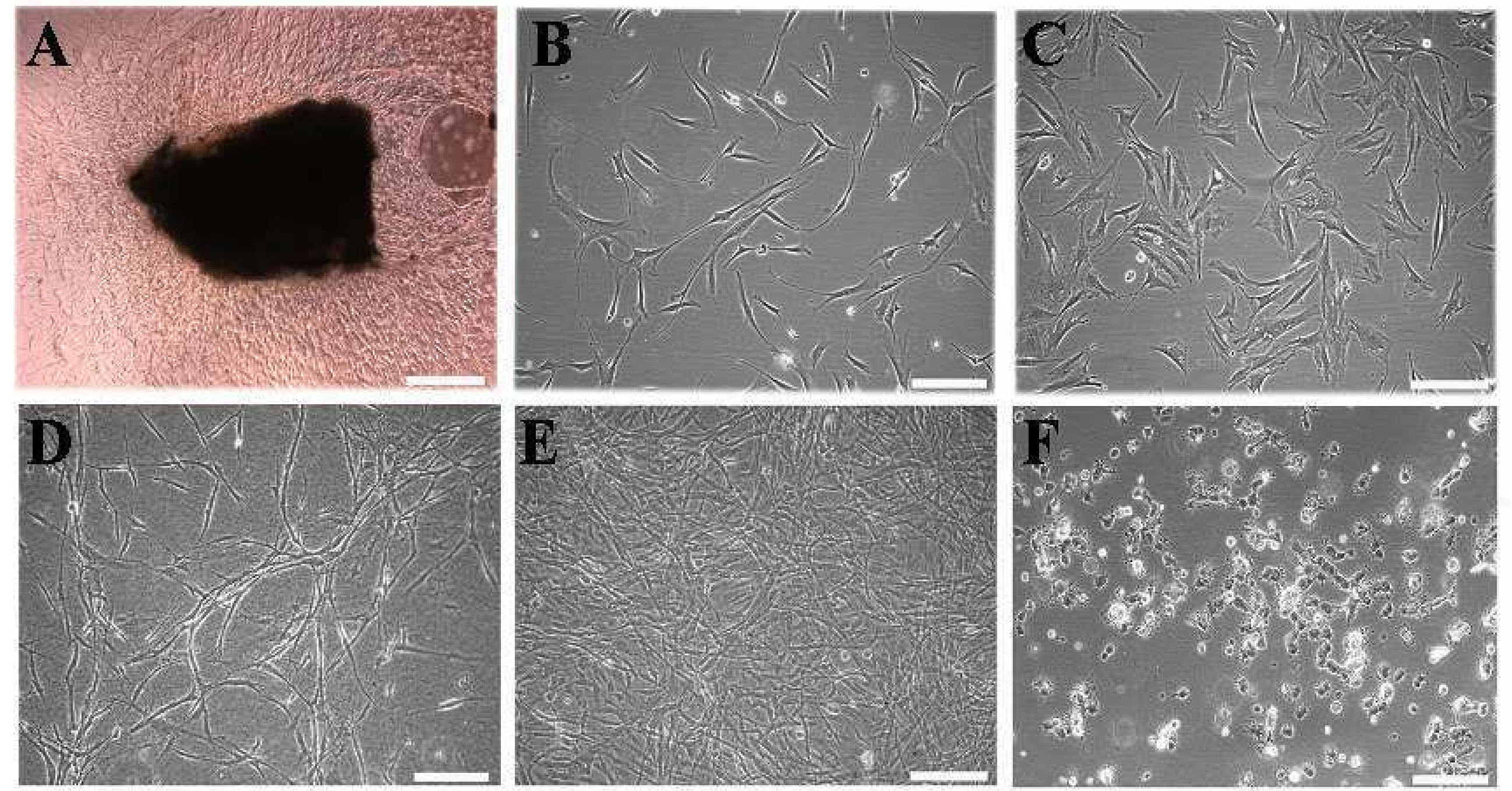
To examine the population doubling time of these primary cultures, cell isolates from two proliferative and one involuting haemangioma were plated at the same density and grown under identical conditions. The cultures derived from the proliferative lesions were assayed at passage 5 while the cultures from the involuting lesions were at passage 2. Cell numbers were determined on days 0,2, 4, 6 and 9 and doubling time was found to be approximately 4 days (Figure 4.4).

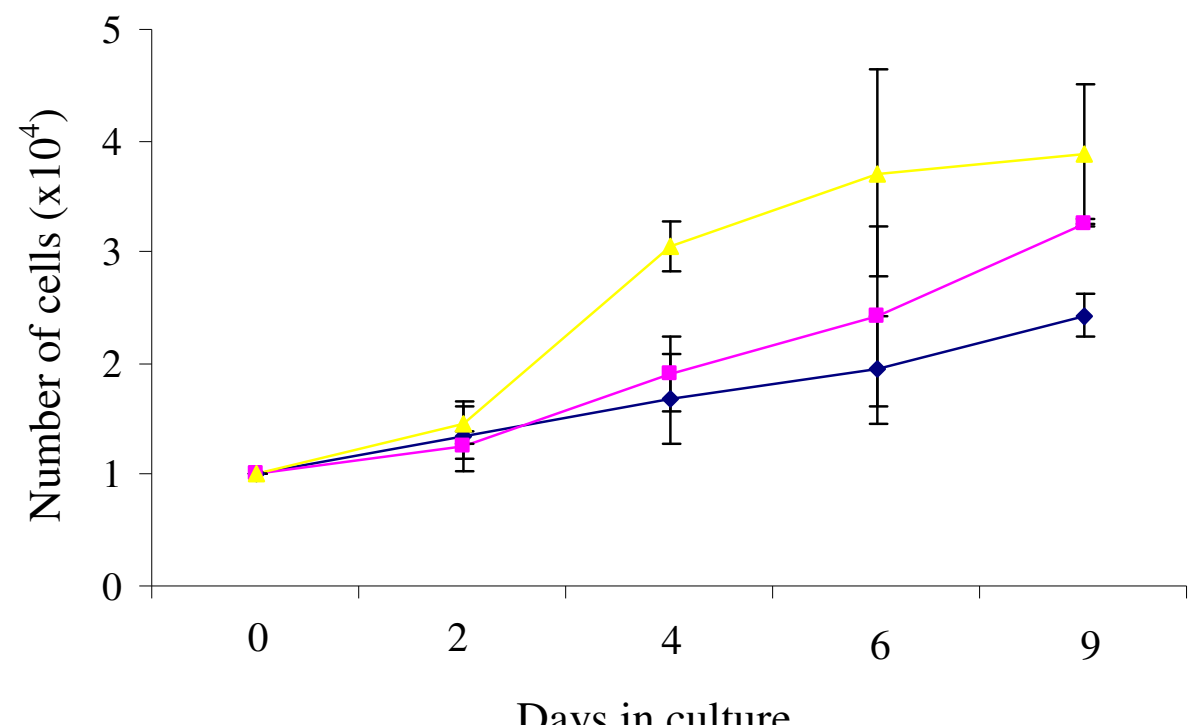

Figure 4.4: Population doubling time of HaemDCs. Cell isolates derived from two proliferative haemangioma lesions (pink and blue line) and one involuting lesion (yellow line) were plated into 24-well tissue culture plates at a density of $1 \times 10^{4}$ cells/well. Cell numbers were counted at days 0,2, 4, 6 and 9 and plotted as number of cells $\times 10^{4}$. The results are presented as the mean \pm s.e.m of triplicate determinations.

\subsubsection{3 mRNA CHARACTERISATION}

To determine whether the HaemDCs isolated from cultured explants were mesenchymal, as suggested by their morphology and growth characteristics, qRT-PCR for lineage-specific transcripts was undertaken. Analysis of mRNA transcripts was assessed by analysing the threshold cycle $(\mathrm{Ct})$ obtained for each gene by qRT-PCR from HaemDCs isolated from four proliferating haemangiomas and one involuting haemangioma relative to the house-keeping gene, GAPDH. HUVECs and 3T3 
fibroblasts were also analysed in parallel for comparison. Table 6 shows that the HaemDCs expressed transcripts for the mesenchymal markers, CD29, CD44 and CD90, but did not express transcripts for the EC markers, CD31 and VEGFR-2, or the haematopoietic antigen, CD45. Low level expression of transcripts for VEGFR-1 was detected in the HaemDCs tested. The HaemDCs also expressed mRNA for GLUT-1 which is the marker used to differentiate haemangiomas from other vascular malformations (North et al., 2000). Although HUVECs expressed transcripts for CD29 and CD44, CD90 mRNA was not detected. In addition, while HUVECs expressed transcripts for the EC markers VEGFR-2 and CD31, 3T3 fibroblasts did not express any of the above markers with the exception of CD44. These results indicate that the HaemDCs isolated from haemangioma explants express a distinct profile of mRNA transcripts for a range of MSC markers that is different from both normal ECs and fibroblasts.

In addition, qRT-PCR showed that HaemDCs isolates from four different patients expressed transcripts for VEGF, Collagen-I, -II, -III and VIII but not LamininV. Low levels of IGF-2 mRNA were also detected. In contrast, 3T3 fibroblasts did not express mRNA for any of these genes. An example of the typical mRNA expression profile obtained for these markers in HaemDCs and their corresponding biopsies of origin is given in Appendix 2, Figure 2.4, page 286. The raw $\triangle \mathrm{Ct}$ values (relative to GAPDH) for all cell lines analysed for these markers can be found in Appendix 2, Table 2.1, page 287 . 


\begin{tabular}{|c|c|c|c|c|c|c|c|c|c|c|c|}
\hline & & & & $\begin{array}{l}\text { Stem cell } \\
\text { marker }\end{array}$ & & ISC ma & & & EC markers & & $\begin{array}{l}\text { matopoieti } \\
\text { antigen }\end{array}$ \\
\hline Phase & HaemDCs & Passage & GLUT-1 & CD133 & CD29 & CD44 & CD90 & VEGFR-1 & VEGFR-2 & CD31 & CD45 \\
\hline Prolif & Patient A & 3 & ND & - & 2.9 & 4.4 & 5.2 & 10.1 & - & - & - \\
\hline Prolif & Patient A & 9 & ND & - & 2.9 & 3.7 & 4.6 & 9.8 & - & - & - \\
\hline Prolif & Patient B & 3 & ND & - & 1.9 & 3 & 2.8 & ND & - & - & - \\
\hline Prolif & Patient $\mathrm{C}$ & 3 & 7 & - & 1 & 2.5 & 2.2 & 10.2 & - & - & - \\
\hline Prolif & Patient D & 3 & 5.5 & - & 1.2 & 3.7 & 2.6 & ND & - & - & - \\
\hline \multirow[t]{3}{*}{$\operatorname{Ig}$} & Patient F & 3 & 7.9 & - & 2.2 & 3.2 & 2.4 & 9.9 & - & - & - \\
\hline & HUVECS & 12 & 9.7 & - & 2.3 & 3.2 & - & 9.8 & 9.6 & 2.2 & - \\
\hline & 3T3 Fibroblasts & & - & - & - & 4.1 & - & - & - & - & - \\
\hline
\end{tabular}

Table 6: mRNA characterisation of HaemDCs. mRNA expression for GLUT-1, the stem cell marker, CD133, the MSC markers, CD29, CD44 and CD90, the EC markers, CD31, VEGFR-1 and VEGFR-2 and the haematopoietic antigen, CD45 was analysed in HaemDCs isolated from four proliferating haemangioma lesions (Patient A-D), one involuting haemangioma lesion (Patient F), HUVECs and 3T3 fibroblasts. RNA was extracted from $1 \times 10^{5}$ cells, reverse transcribed into cDNA and analysed by qRT-PCR as described in section 2.3.8.1. Expression of transcripts was determined relative to GAPDH $(\triangle \mathrm{Ct}$ ). The table gives the $\triangle \mathrm{Ct}$ values obtained for each gene. HaemDCs expressed mRNA for a number of markers characteristic of MSCs including CD29, CD44 CD90 but did not express mRNA for the EC markers, CD31, VEGFR-1 and VEGFR-2 or the hematopoietic marker, CD45. HaemDCs and HUVECs expressed mRNA for the the haemangioma antigen, GLUT-1. None of the cell lines expressed mRNA for the stem cell marker, CD133. Key: '-' shows no amplification and therefore absence of mRNA transcript; ND indicates not determined. 


\subsubsection{IMMUNOHISTOCHEMICAL CHARACTERISATION}

Immunofluorescent labelling of lineage-specific markers revealed that all cells stained for vimentin (Figure 4.5-A) and GLUT-1 (Figure 4.5-B) while only some cells were positive for $\alpha$-SMA (Figure 4.5-C). In addition, the HaemDCs did not express any of the commonly used EC markers, vWF, CD34 or VEGFR-2 (Figure 4.6 and Figure 4.7). As positive and negative controls in the same experiment, immunostaining for vWF, CD34 and VEGFR-2 was carried out on HUVECs and 3T3 fibroblasts, respectively. Immunoreactivity for VEGFR-1 was detected on HaemDCs and HUVECs but not on $3 \mathrm{~T} 3$ fibroblasts (Figure 4.7). VEGFR-1 expression by HaemDCs was confirmed by Western blotting (Figure 4.12).

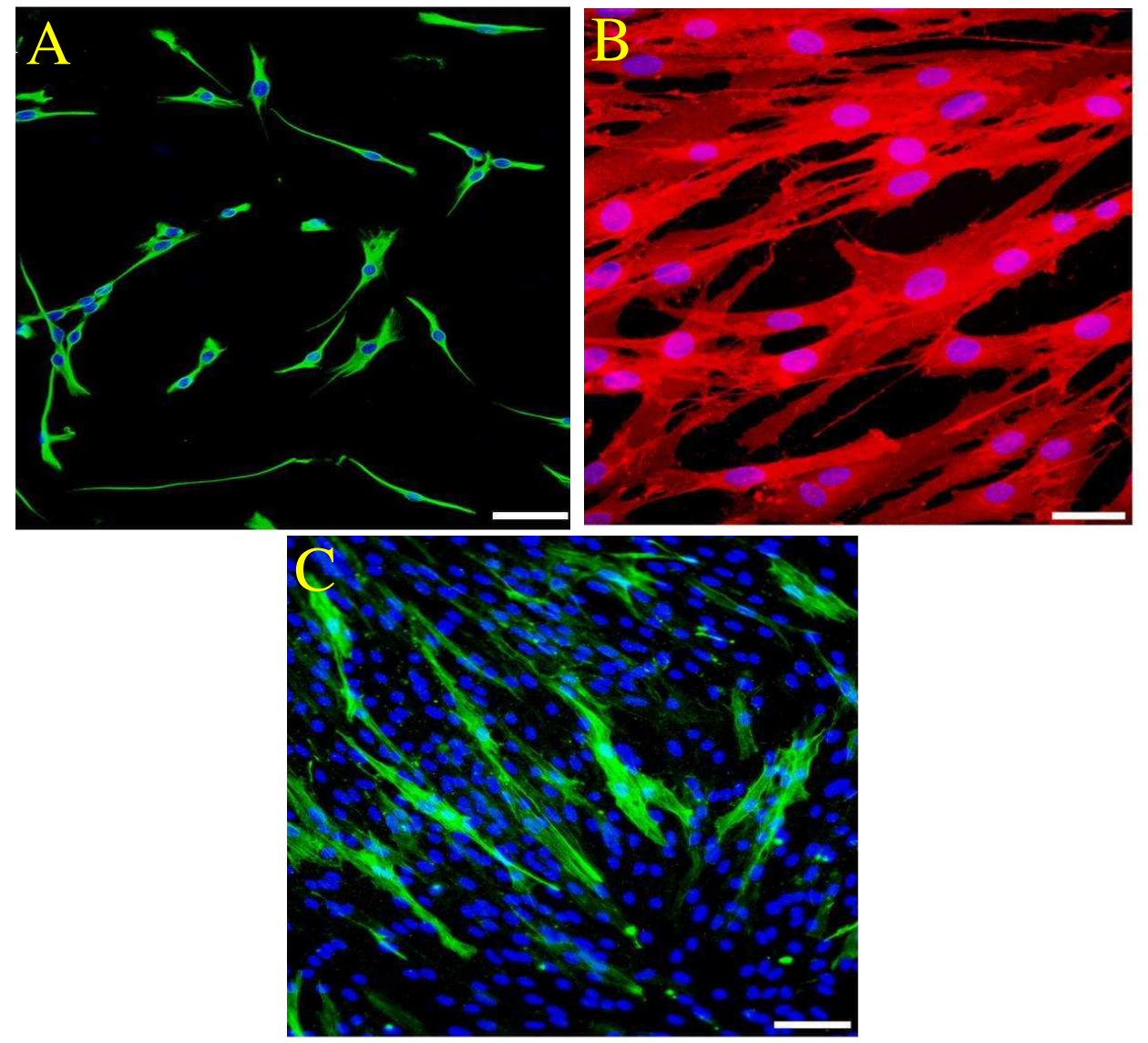

Figure 4.5: Immunohistochemical localisation of vimentin, $\alpha$-SMA and GLUT-1 on HaemDCs. HaemDCs were fixed and immunostained for vimentin, $\alpha$-SMA and GLUT1 as described in section 2.3.9. Vimentin and $\alpha$-SMA expression was detected using anti-mouse AlexaFluor 488 conjugated antibodies (green staining) and GLUT-1 expression was detected with an anti-rabbit $\mathrm{Cy} 3$ conjuate (red staining). Cell nuclei were counterstained with DAPI (blue). All HaemDCs expressed the MSC marker, vimentin (A) and the haemangioma marker, GLUT-1 (C) while only some cells showed immunoreactivity for another MSC marker, $\alpha$-SMA (B). Scale bars: (A-B) $100 \mu \mathrm{m},(\mathrm{C})$ $50 \mu \mathrm{m}$. 

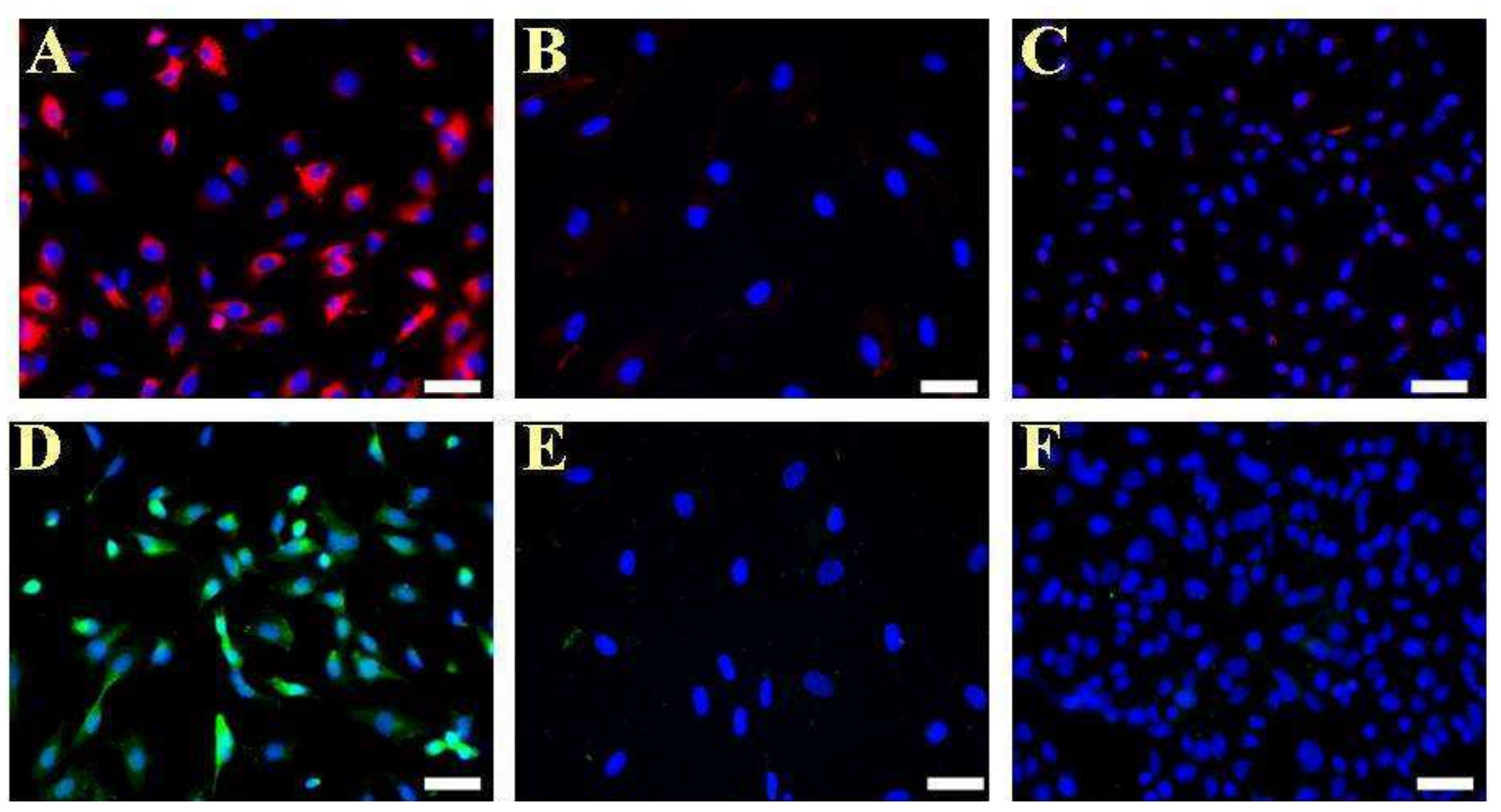

Figure 4.6: Immunohistochemical localisation of vWF and CD34 on HUVECs, HaemDCs and 3T3 fibroblasts. HUVECs (A\&D), HaemDCs (B\&E) and 3T3 fibroblasts $(\mathbf{C} \& \mathbf{F})$ were fixed and labelled with rabbit anti-vWF (A-C) and mouse anti-CD34 (D-F) antibodies as described in section 2.3.9. Bound antibodies were detected using either an anti-rabbit $\mathrm{Cy} 3$ (red) or an anti-mouse AlexaFluor 488 conjugate (green). Cell nuclei were counterstained with DAPI (blue). HUVECs were used as a positive control and expressed both vWF (A) and CD34 (D) while no immunoreactivity was detected for vWF and CD34 on the HaemDCs $(\mathbf{B} \& \mathbf{E}$, respectively) and the cell line used as a negative control, the 3T3 fibroblasts $(\mathbf{C} \& \mathbf{F}$, respectively). Scale bars: $50 \mu \mathrm{m}$. 

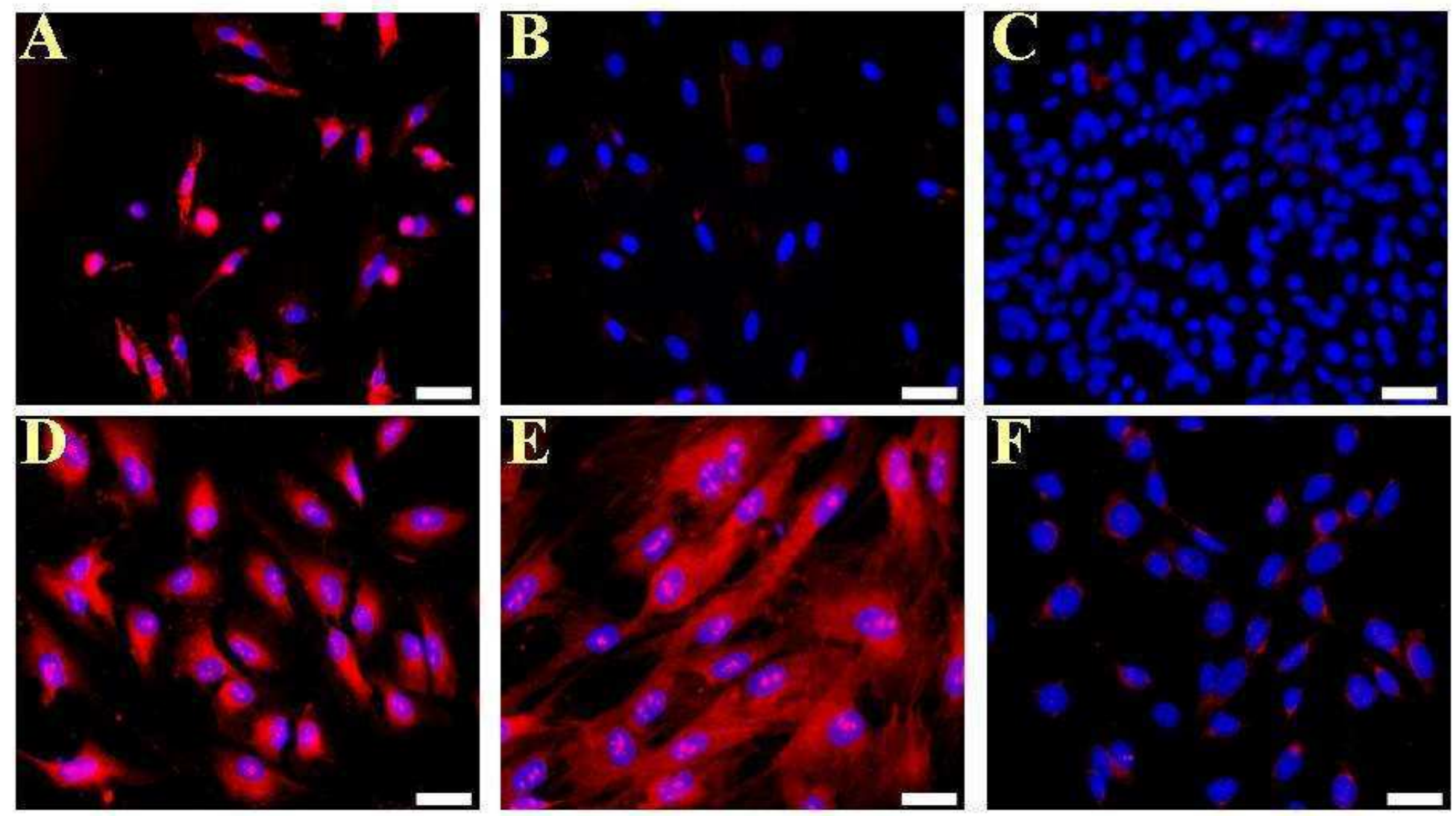

Figure 4.7: Immunohistochemical localisation of VEGFR-2 and VEGFR-1 on HUVECs, HaemDCs and 3 T3 fibroblasts. HUVECs (A\&D), HaemDCs $(\mathbf{B} \& \mathbf{E})$ and 3 T3 fibroblasts $(\mathbf{C} \& \mathbf{F})$ were fixed and labelled with rabbit anti-VEGFR-2 (A-C) and goat anti-VEGFR-1 antibodies (D-F). Bound antibodies were detected using either an anti-rabbit DIG conjugate followed by an anti-DIG-Rhodamine (red) conjugate or an anti-goat biotin conjugate followed by an AlexaFluor 555 streptavidin conjugate (red). Cell nuclei were counterstained with DAPI (blue). HUVECs used as a positive control, expressed both VEGFR-2 (A) and VEGFR-1 (D). HaemDCs and 3 T3 fibroblasts did not express VEGFR-2 (B\&C, respectively). Positive staining for VEGFR-1 was detected on HaemDCs (E) but was absent on the negative control cell line, 3 T3 fibroblasts (F). Scale bars: (A-C) $50 \mu \mathrm{m}$, (D-F) $20 \mu \mathrm{m}$. 
Taken together, the qRT-PCR and IHC data supports the assertion that the primary cultures obtained from the capillary-like outgrowths from haemangioma explants are of mesenchymal origin and are now referred to as haemangioma-derived mesenchymal cells (HaemDMCs)

Although the HaemDMCs were found to express VEGF and collagen mRNA, protein expression was absent. Western blotting (Figure 4.8) and immunofluorescence staining (data not shown) of HaemDMCs showed that the cells neither expressed nor secreted VEGF. $50 \mathrm{ng}$ and $25 \mathrm{ng}$ of purified recombinant $\mathrm{VEGF}_{165}$ along with total protein from HaemDMCs conditioned media $(40 \mu \mathrm{g})$, HaemDMCs $(100 \mu \mathrm{g})$ and proliferative haemangioma tissue $(100 \mu \mathrm{g})$ lysates were subjected to Western blotting under reducing conditions. The resulting immunoblot was probed with an anti-VEGF antibody that recognises three isoforms of VEGF-A, VEGF $\mathrm{VE}_{121}, \mathrm{VEGF}_{165}$ and $\mathrm{VEGF}_{189 .}$ Immunoreactive bands corresponding to a molecular weight of $23 \mathrm{kDa}$ were detected only with the purified VEGF protein (Figure 4.8).

In addition, picrosirius red staining of HaemDMCs showed no collagen fibres either in the cells themselves or in their ECM (data not shown). An involuting haemangioma section was used as a positive control and showed expression of collagen fibers (Figure 4.9). Staining of collagen fibres with picrosirius red allows the visualisation of individual collagen fibres on the basis of double refraction of light against a black background, when epipolarisation microscopy is used (Junqueira et al., 1979). 


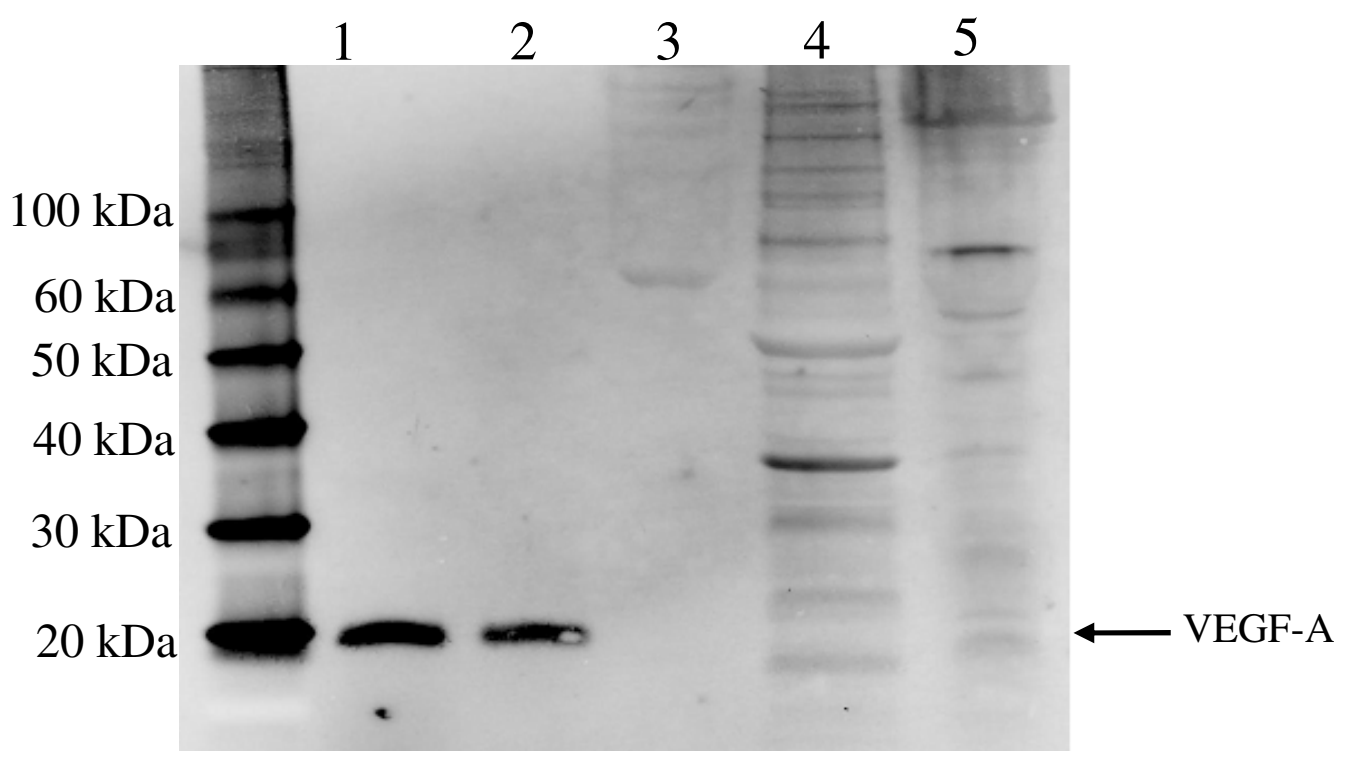

Figure 4.8: Western blotting for VEGF-A. Total protein extracted from the conditioned media of HaemDMCs, from HaemDMCs and from proliferating haemangioma tissue was subjected to Western blotting as described in section 2.3.13.2. Lane: 1) $50 \mathrm{ng}$ of purified $\mathrm{VEGF}_{165}$; 2) $25 \mathrm{ng}$ of purified $\mathrm{VEGF}_{165}$; 3) $40 \mu \mathrm{g}$ of total protein from the medium of HaemDMCs conditioned over a 96 hour period; 4) $100 \mu \mathrm{g}$ of total protein from HaemDMCs and 5) $100 \mu \mathrm{g}$ of total protein from a proliferative haemangioma tissue sample. Following electrophoresis and transfer onto a PVDF membrane, the membrane was blocked and probed with an antibody that recognises the $\mathrm{VEGF}_{121}$, $\mathrm{VEGF}_{165}$ and $\mathrm{VEGF}_{189}$ isoforms of VEGF-A. Specific immunoreactivity was detected at $23 \mathrm{kDa}$ from the purified VEGF protein but was absent on the other samples (black arrow).

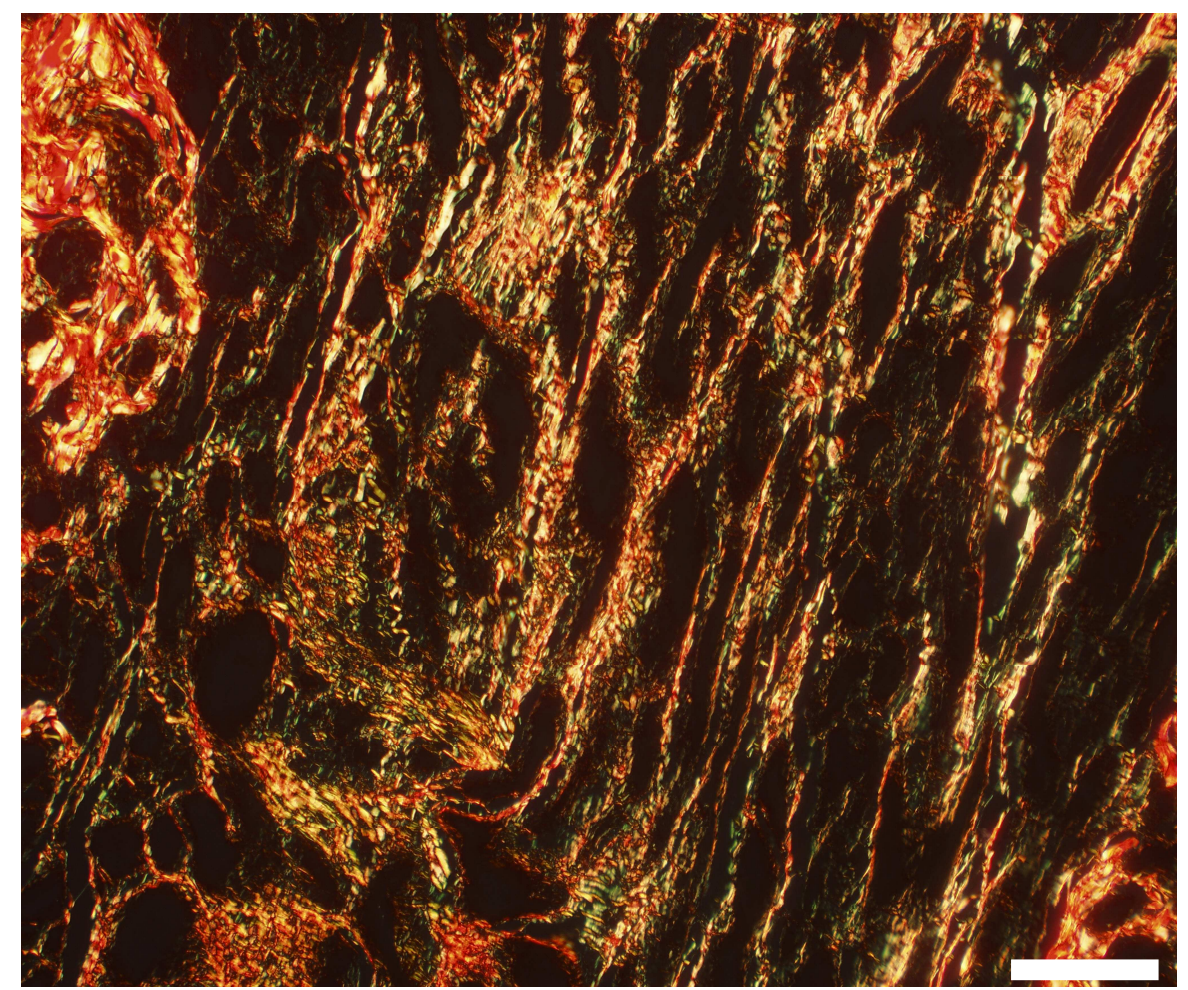

Figure 4.9: Picrosirius red stained section of an involuting haemangioma tissue section. Under a polarised light source, the haemangioma tissue section stained with picrosirius red showed the presence of collagen fibers. In the image above, thick fibers appear red or yellow while thin fibers appear green. Scale bar: $500 \mu \mathrm{m}$. 


\subsubsection{MULTILINEAGE DIFFERENTIATION POTENTIAL OF HaemDMCs}

To determine the multilineage capacity of HaemDMCs cutured cells were differentiated toward the adipogenic and osteogenic lineages using lineage-specific induction factors. To induce adipogenesis, cells were cultured in medium containing Dexamethasone, ascorbic acid, $\beta$-glycerophosphate, insulin and indomethecin. Differentiation into adipocytes was detected within 15 days of induction and continued to accumalate over time (Figure 4.10-D). A significant fraction of the cells contained multiple, intracellular lipid-filled droplets that accumulated Oil-Red O (Figure 4.10-E). No lipid droplets were observed in undifferentiated HaemDMCs (Figure 4.10-A). Adipocytic differentiation potential of HaemDMCs was maintained even after 10 passages, indicating their phenotypic stability and their ability to undergo self-renewal in vitro.

Differentiation into osteoblasts was induced in vitro by treating cells with ascorbic acid, $\beta$-glycerophosphate and Dexamethasone. To confirm osteogenic differentiation, calcification of the ECM was assessed using Alizarin Red stain. While no calcification was observed in undifferentiated cells (Figure 4.10-A) the HaemDMCs readily differentiated into osteocytes. Figures $4.10-\mathrm{B}$ and $-\mathrm{C}$ show mineralization of the ECM produced by cultured HaemDMCs that was revealed by Alizarin Red staining. Consistent with previous findings, differentiation required the cells to be plated at high confluencey (greater than $2 \times 10^{4}$ cells $/ \mathrm{cm}^{2}$ ) (Tropel et al., 2004).

HaemDMCs isolated from proliferating and involuting haemangioma biopsies showed similar osteogenic differentiation potential (Figure 4.10-B and -C, respectively). However, HaemDMCs isolated from proliferating haemangiomas readily differentiated into adipocytes by day 15 (Figure 4.10-E) while HaemDMCs derived from involuting lesions took approximately 60 days to produce any lipid vacuoles (Figure 4.10-F).

In contrast, HaemDMCs isolated in this study did not differentiate into ECs or express EC-like markers when cultured in the presence of VEGF (data not shown). 
Confirmation of differentiation into adipocytes and osteoblasts was investigated by qRT-PCR analysis of transcripts for the peroxisome proliferator-activated receptor (PPAR- $\gamma$ ), a key regulatory gene of adipogenesis (Camp et al., 2002), and osteonectin, a gene expressed by osteoblasts (Jundt et al., 1987). Table 7 shows the expression levels of these genes in mesenchymal cell isolates from five proliferating and one involuting haemangioma. Gene expression was determined relative to GAPDH $(\triangle \mathrm{Ct})$ in undifferentiated versus differentiated cells $(\triangle \triangle \mathrm{Ct})$. Expression of PPAR- $\gamma$ increased as expected (approximately 16-fold) while that for osteonectin did not alter significantly. Differentiation experiments were replicated with HaemDMCs isolates from five different proliferative biopsies and two involuting biopsies. These data are consistent with the HaemDMCs being haemangioma-derived mesenchymal stem cells (HaemDMSCs), which are here after referred to as such. An example of the typical change in mRNA expression for osteonectin and PPAR $\gamma$, before and after induction, from one experiment is shown in Figure 4.11.

\begin{tabular}{ccccc}
\hline Phase & HaemDMCs & Passage & $\triangle \triangle \mathrm{Ct}$ PPAR- $\gamma$ & $\triangle \triangle \mathrm{Ct}$ Osteonectin \\
\hline Prolif & Patient A & 3 & 4.7 & 3.4 \\
Prolif & Patient B & 3 & 4.3 & -1.5 \\
Prolif & Patient C & 3 & 5.5 & 1.7 \\
Prolif & Patient D & 3 & 4.9 & -0.2 \\
Prolif & Patient E & 3 & 2.9 & -1 \\
Ig & Patient F & 3 & 3.3 & -0.4 \\
\hline & Mean $( \pm$ s.e.m.) & & $4 \pm 0.4$ & $0.3 \pm 0.8$ \\
\hline
\end{tabular}

Table 7: PPAR- $\gamma$ and osteonectin mRNA expression. HaemDMCs isolated from five proliferating (Patient A-E) and one involuting haemangioma (Patient F) were differentiated into adipocytes and osteoblasts. qRT-PCR was performed to identify changes in PPAR- $\gamma$ and osteonectin mRNA expression in undifferentiated cells versus differentiated cells $(\triangle \triangle \mathrm{Ct})$ 
Figure 4.10: Differentiation potential of HaemDMCs. HaemDMCs purified from a proliferating (B,D\&E) and an involuting haemangioma $(\mathbf{C} \& \mathbf{F})$ were plated onto glass coverslips at $2 \times 10^{4}$ cells $/ \mathrm{cm}^{2}$ and cultured in osteocytic or adipocytic differentiation media as described in section 2.3.9. HaemDMSCs purified from proliferating $(\mathbf{B})$ and involuting $(\mathbf{C})$ lesions differentiated into osteocytes after 14 days of induction as depicted by Alizarin Red staining. HaemDMCs also differentiated into lipid laiden adipocytes. Phase contrast image of adipocyte formation in culture by HaemDMCs (D). Cytoplasmic lipid vacuoles were identified by staining with Oil Red O (E\&F). HaemDMCs from proliferative lesions (E) more readily differentiated into adipocytes than HaemDMCs from involuting lesions (F). Panel $\mathbf{E}$ shows increased adipocyte formation by HaemDMCs from a proliferative lesion after only 21 days of induction while Panel $\mathbf{F}$ shows adipocyte formation by HaemDMCs from an involuting lesion after 50 days in induction media. Undifferentiated HaemDMCs islolated from a proliferating haemangioma showed no staining with Alizarin Red and Oil Red O (A). Scale bars: $50 \mu \mathrm{m}$. 

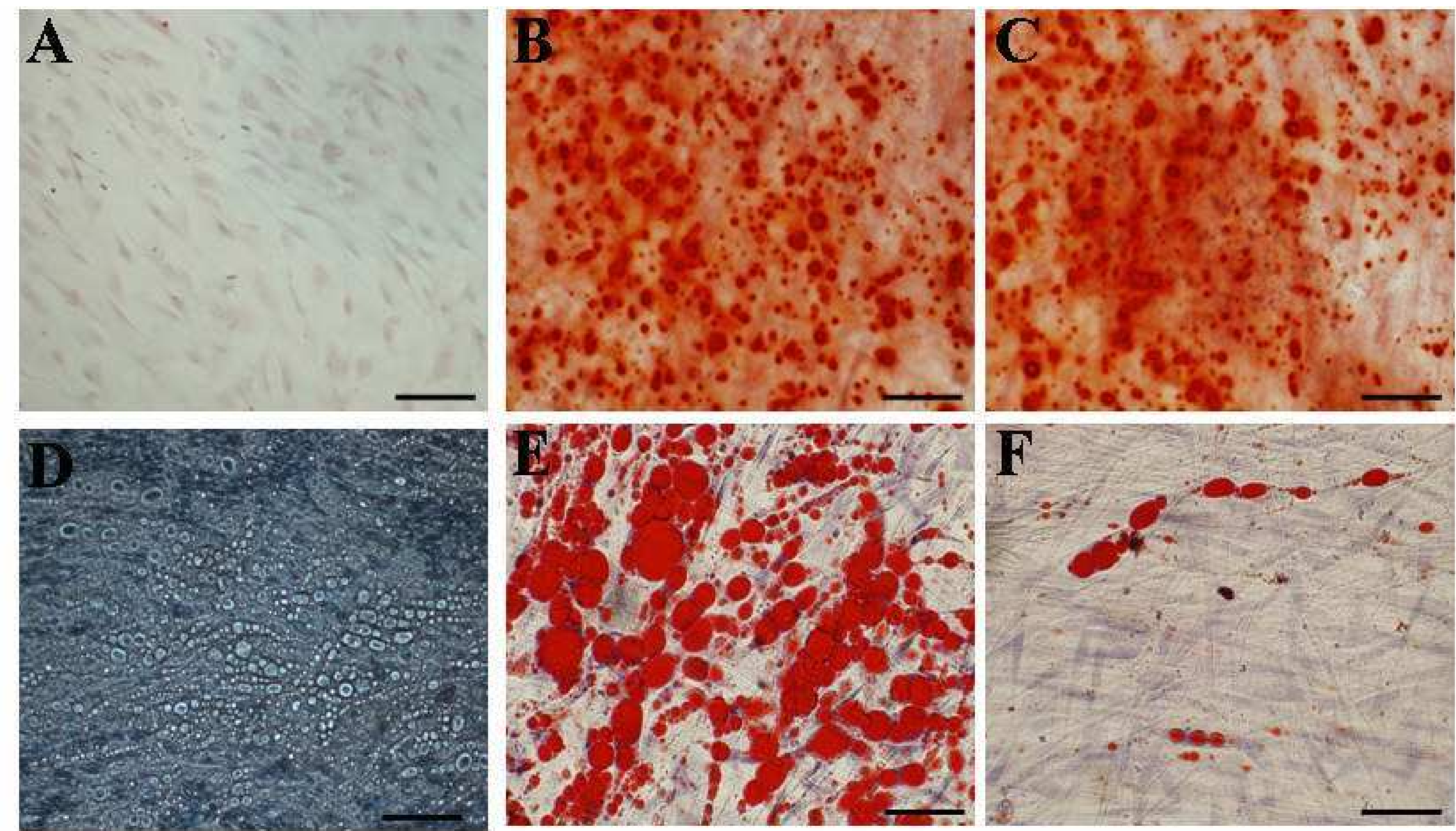


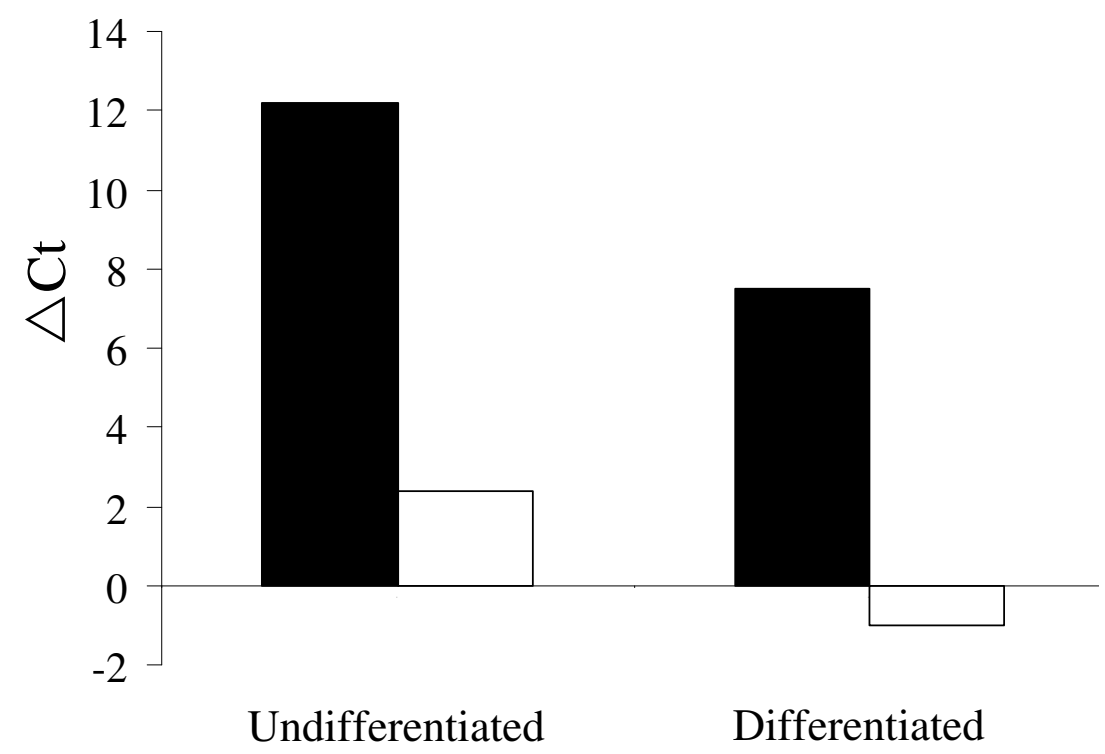

Figure 4.11: Validation of adipocytic and osteocytic differentiation of HaemDMSCs. Differentiation into adipocytes and osteocytes was validated by qRT-PCR performed for PPAR $\gamma$ and osteonectin, respectively. Changes in mRNA expression are expressed relative to that for GAPDH (the internal reference gene) as $\triangle \mathrm{Ct}$ values. Black columns indicate PPAR $\gamma$ expression and white columns indicate osteonectin expression. An increase in mRNA expression was observed for both genes in differentiated HaemDMSCs compared to undifferentiated cells. A lower $\triangle \mathrm{Ct}$ indicates higher gene expression while a higher $\triangle \mathrm{Ct}$ indicates low expression.

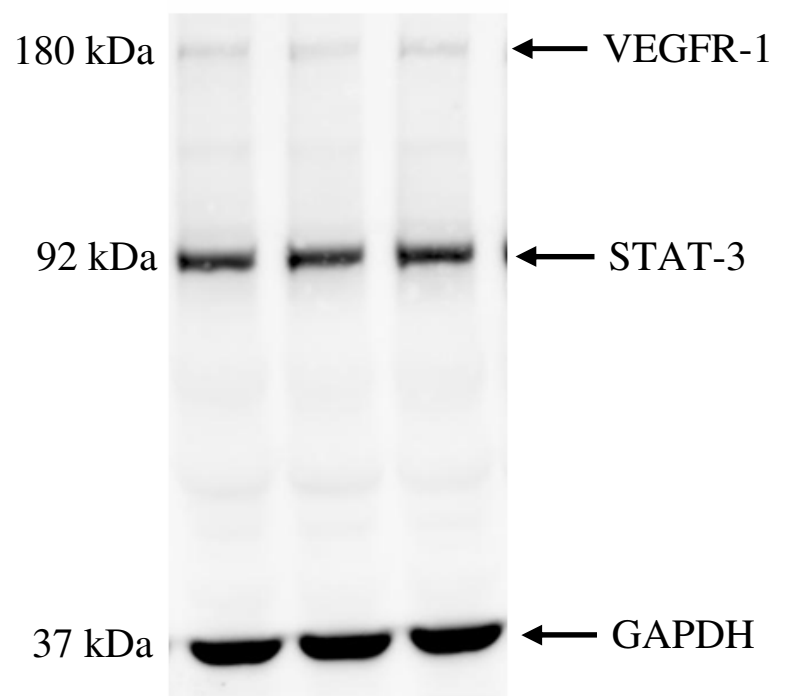

Figure 4.12: Western blot verification of VEGFR-1 and STAT-3 expression by HaemDMSCs. Western blotting was performed as per section 2.3.13.1. Each lane contains $50 \mu \mathrm{g}$ of total protein from HaemDMSCs. Following electrophoresis and transfer onto a PVDF membrane, the membrane was blocked with goat anti-VEGFR-1, mouse anti-STAT-3 and mouse anti-GAPDH antibodies followed by incubation with anti-goat and anti-mouse AlexaFluor 488 conjugated secondary antibodies. The resulting blot confirmed expression of VEGFR-1 and STAT- 3 by HaemDMSCs as signal was observed at the appropriate sizes of $180 \mathrm{kDa}$ and $92 \mathrm{kDa}$, respectively. GAPDH was included as the loading control. 


\subsubsection{ROLE OF VEGF AND STAT SIGNALLING IN VITRO}

\subsubsection{EFFECT OF VEGF AND AG490 ON CAPILLARY-LIKE OUTGROWTH}

The importance of VEGF and STAT signalling on 'microvessel' outgrowth was investigated using the explant model developed by Tan et al $\left(2000_{\mathrm{b}}\right)$ and also using the modified culture system developed in this study (section 2.3.4, page 86). Explants treated with $50 \mathrm{ng} / \mathrm{ml}$ of VEGF exhibited a higher degree of capillary-like outgrowth after six days in culture compared to controls $(p<0.01)$. This dramatic sprouting was mitigated by the addition of $50 \mu \mathrm{M}$ of AG490, an inhibitor of STAT protein phosphorylation (Huang et al., 2006; Andl et al., 2004), in the culture medium (Figure 4.13-A). Among the sprouting explants, the VEGF treated group was observed to be more advanced in terms of number and length of sprouts. In addition, cultures treated with AG490 and VEGF showed significantly less capillary-like growth than untreated cultures $(p<0.01)$ (Figure 4.13-A). This suggests that the inhibitory effect of AG490 can overcome the stimulatory effect of VEGF on capillary-like outgrowths. Figure 4.13A shows the average capillary-like outgrowth of explanted haemangioma tissue obtained from two separate patients. Duplicate wells containing an explanted tissue piece were used for each treatment.

Explants were also cultured with various concentrations of AG490 diluted into the gel matrix and in the overlying medium for a period of ten days. Addition of $10 \mu \mathrm{M}$ and $50 \mu \mathrm{M}$ AG490 at the beginning of the culture inhibited sprouting with significance achieved at $p<0.05$ and $p<0.001$, respectively (Figure 4.13-B). Results shown in Figure 4.13-B are representative of two separate experiments conducted with haemangioma biopsies obtained from two individual patients. Triplicate wells were used for each treatment with at least six wells used as control cultures. 


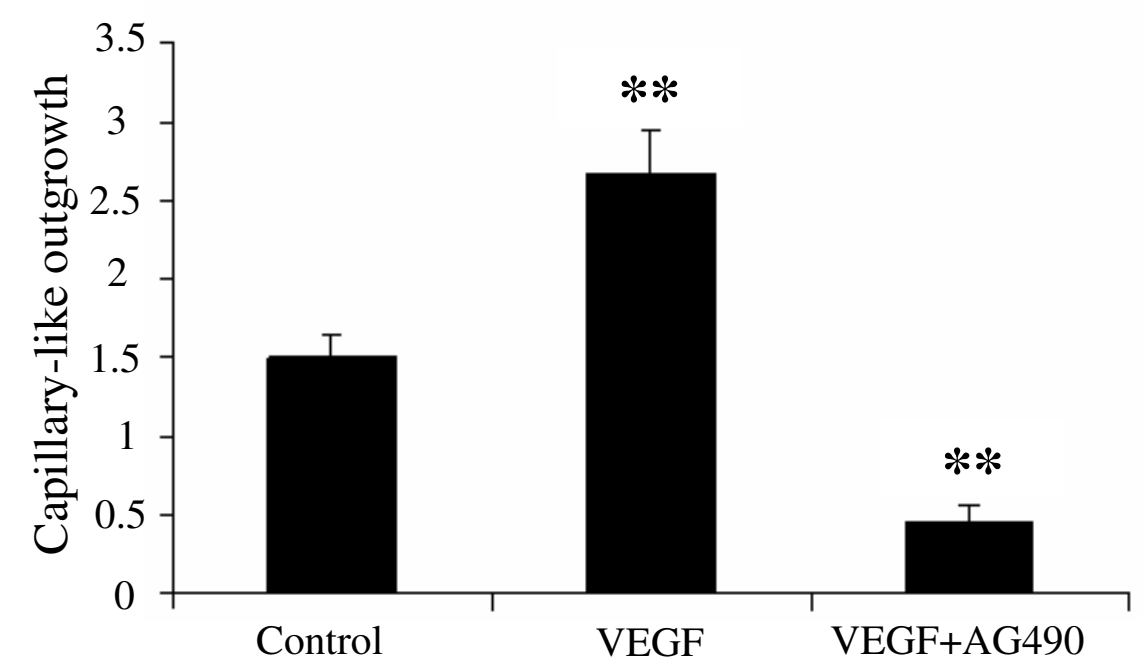

B

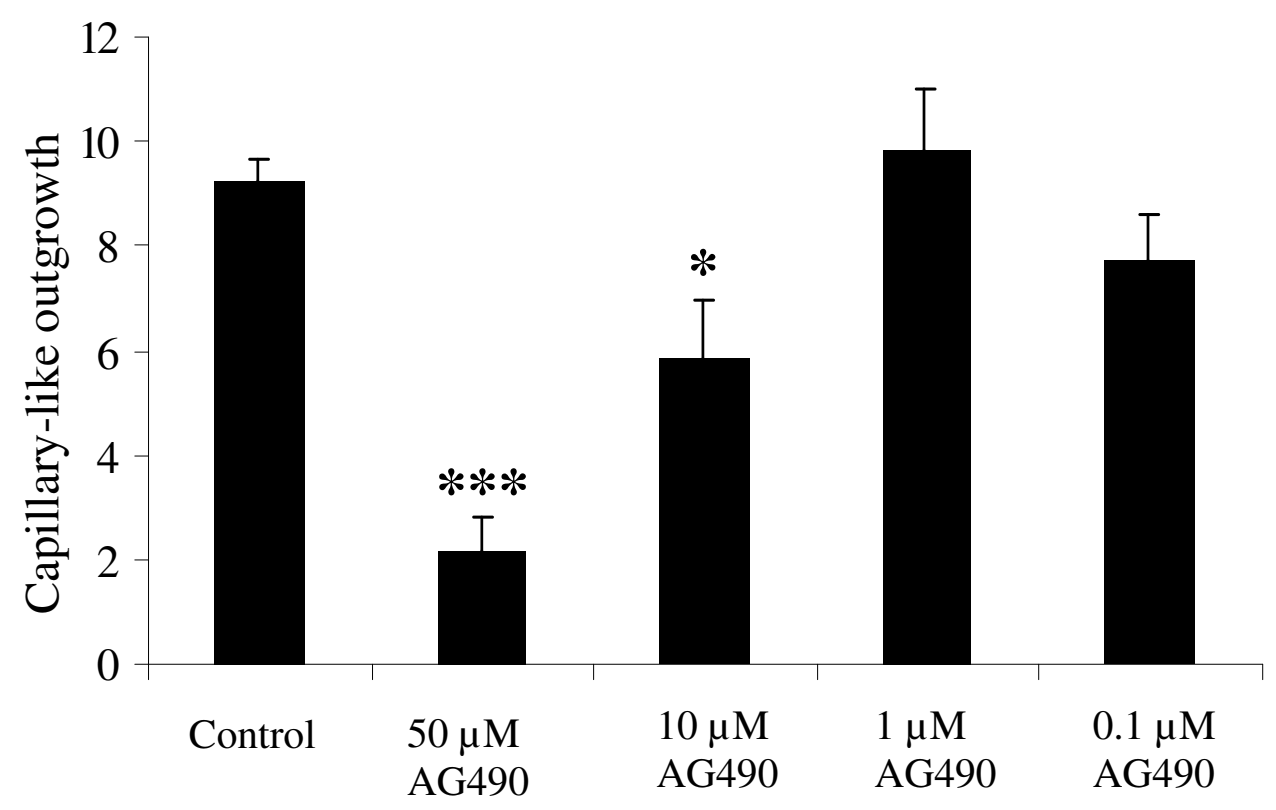

Figure 4.13: Effect of AG490 and VEGF on capillary-like outgrowth from proliferating haemangioma explants. Proliferating haemangioma explants were cultured as outlined in sections 2.3.3 and 2.3.4. In all experiments, DMSO was added to the untreated control cultures at the same volume as that used for the highest concentration of AG490. (A) Significant increase in capillary-like outgrowth was observed with the addition of VEGF $(50 \mathrm{ng} / \mathrm{ml})$ to the cultures above that of the controls. This stimulatory effect of VEGF was mitigated by including AG490 $(50 \mu \mathrm{M})$. In addition, including AG490 along with VEGF decreased capillary-like growth compared with untreated cultures. Capillary-like outgrowth was quantified 6 days post treatment. (B) Pieces of proliferating haemangioma biopsies were cultured in the presence of AG490 at various concentrations and capillary-like growth was assayed after 9 days in culture. Capillarylike outgrowth was significantly inhibited by $50 \mu \mathrm{M}$ and $10 \mu \mathrm{M}$ AG490. * represents significance at $p<0.05, * *$ at $p<0.01$ and $* * *$ at $p<0.001$. 


\subsubsection{EFFECT OF VEGF AND AG490 ON HaemDMSCs.}

To investigate if VEGF signalling through STAT proteins is involved in the proliferation of HaemDMSCs, cells were incubated with VEGF at various concentrations known to stimulate proliferation of ECs and the extent of proliferation was measured using the EZ4U assay. Although VEGF induced proliferation of HUVECs (Figure 4.14-A), it did not stimulate the proliferation of HaemDMSCs even at low serum concentrations (Figure 4.14-B). 
A

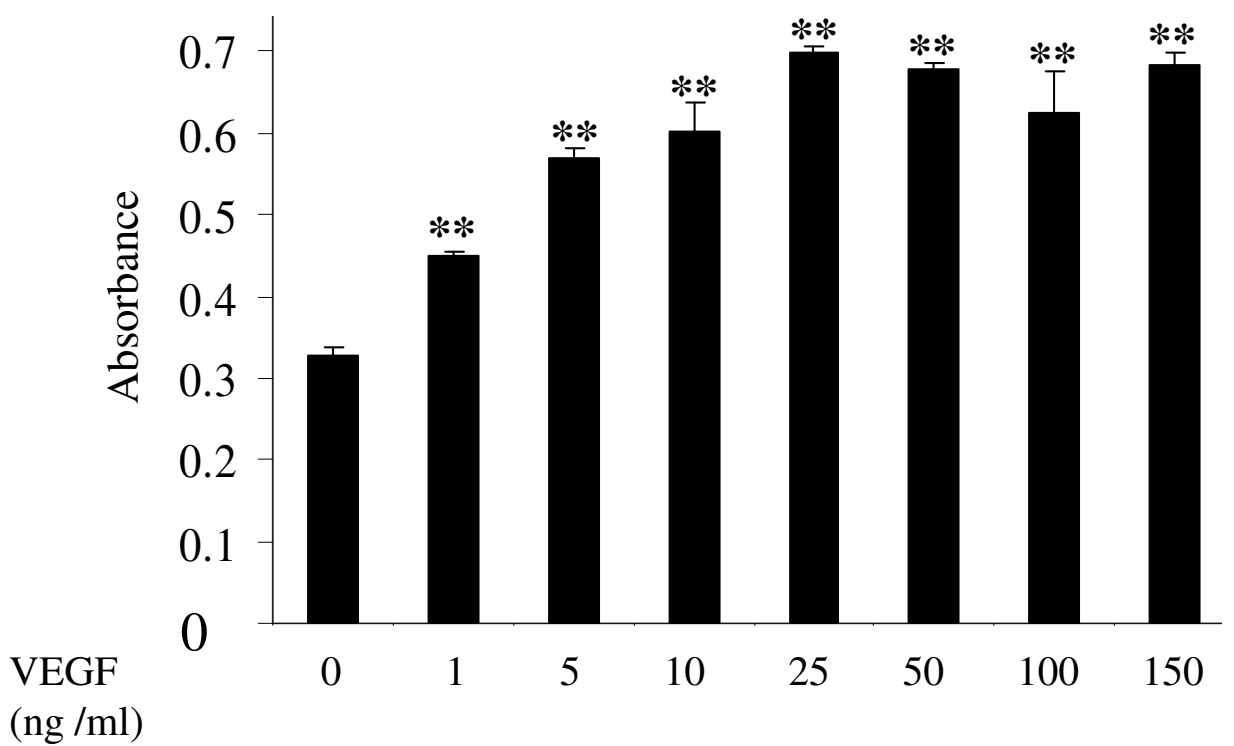

B

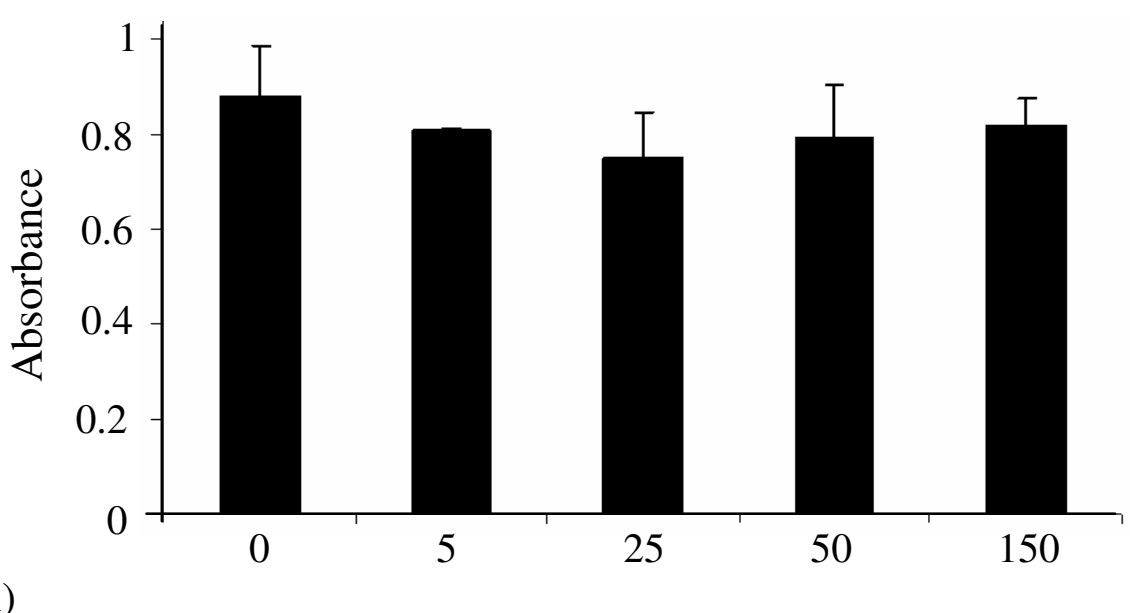

Figure 4.14: VEGF stimulates proliferation of HUVECs but not HaemDMSCs. HUVECs (A) and HaemDMSCs (B) were treated with the indicated concentrations of VEGF and the extent of cell proliferation was measured using the EZ4U assay as described in section 2.3.12.1. The increase in cell number was measured by the increase in the absorbance values of HUVEC cultures treated with various concentrations of VEGF for 72 hours (A). VEGF did not stimulate proliferation of HaemDMSCs proliferation as assayed from the absorbance readings $(\mathbf{B})$. Data shown in $(\mathbf{B})$ is representative of two separate experiments performed with HaemDMSCs isolates from two haemangioma patients set up in triplicate. HUVECs were cultured in RPMI medium containing 2\% FCS for $72 \mathrm{hrs}$ and HaemDMSCs in $0.1 \%$ FCS for 6 days. $* * p$ $<0.01$. 
In addition, treatment with VEGF neither induced p-STAT-3 phosphorylation (Figures 4.15) nor caused its nuclear translocation (Figure 4.16). Figure 4.15 shows that in comparison with IFN treated HeLa cells, unstimulated HaemDMSCs express very low levels of p-STAT-3 (Tyr 705) and that VEGF treatment does not significantly alter p-STAT-3 (Tyr 705) expression levels. Identical results were obtained when the experiment was performed on two separate occasions with HaemDMSCs isolates from two different haemangioma biopsies (from two patients).

In addition, no significant change in p-STAT-3 (Tyr 705) expression was noted when HaemDMSCs were treated with AG490 (50 $\mu \mathrm{M})$ for 24 hours (Figure 4.15). Immunoblotting for total STAT-3 and GAPDH protein confirmed equal protein loadings. 

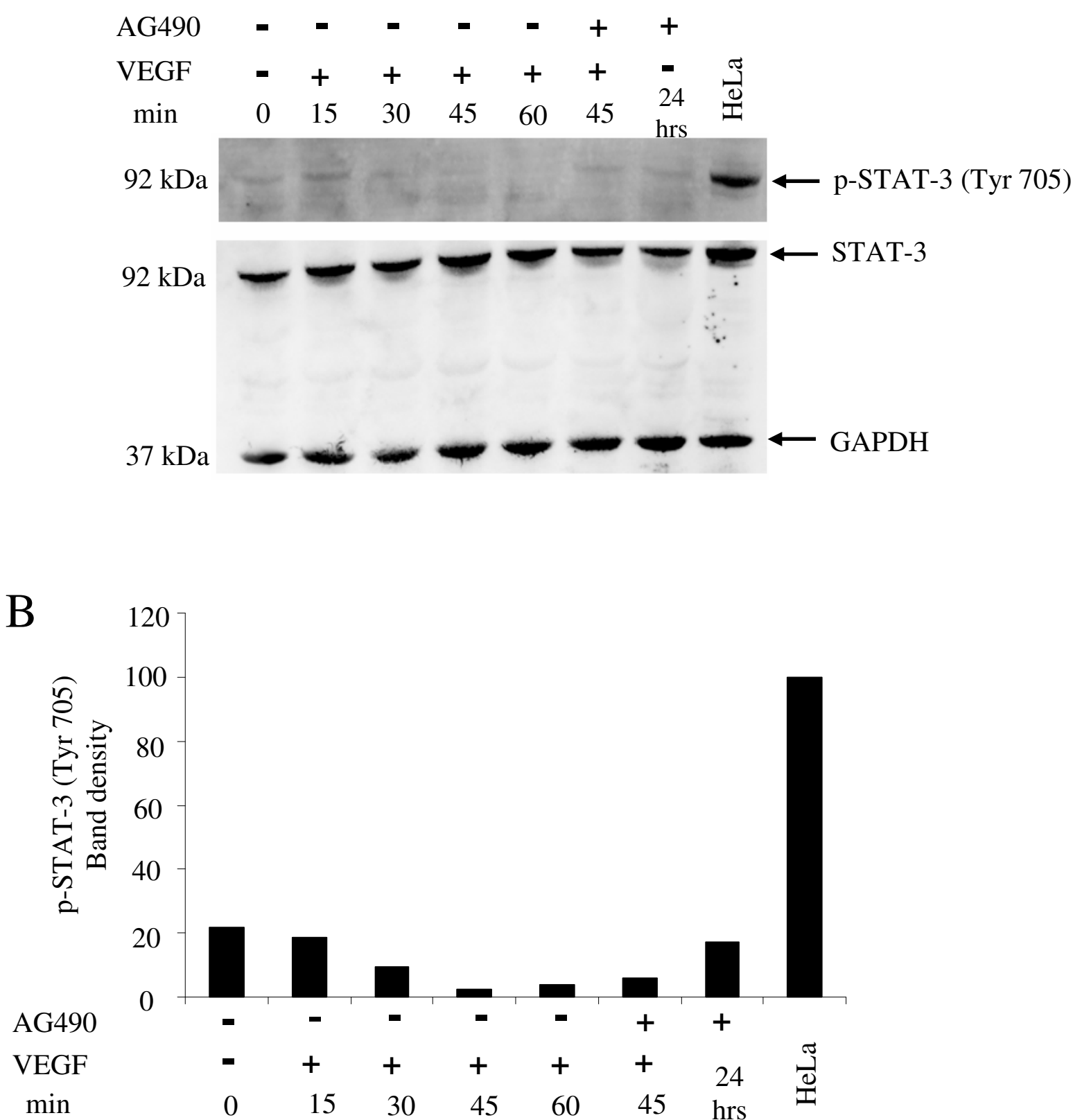

Figure 4.15: VEGF does not induce p-STAT-3 (Tyr 705) expression in HaemDMSCs. HaemDMSCs isolated from a proliferating haemangioma were treated with either VEGF $(50 \mathrm{ng} / \mathrm{ml})$, AG490 $(50 \mu \mathrm{M})$ alone or both for the indicated time periods and subjected to Western blotting as described in section 2.3.13.1. HeLa cells stimulated with IFN $\alpha-2 b(10,000 \mathrm{U} / \mathrm{ml})$ for $10 \mathrm{~min}$ were included as a positive control. Immunoblots were probed with mouse anti-p-STAT-3 (Tyr 705) and mouse antiGAPDH antibodies. (A) Western blot analysis showed that p-STAT-3 (Tyr 705) expression was not induced after VEGF stimulation in whole cell lysates. Membranes were then stripped and re-probed with an anti-STAT-3 antibody. The levels of STAT-3 and GAPDH expression were determined as a control for equivalent protein loading. (B) Graphical representation of p-STAT-3 (Tyr 705) expression by densitometry. In comparison with IFN treated HeLa cells, unstimulated HaemDMSCs expressed very low levels of p-STAT-3. The slight decrease in p-STAT-3 (Tyr 705) expression identified with VEGF treatment was not considered significant due to the low abundance of p-STAT-3 (Tyr 705) expression in the unstimulated cells to begin with. 
Localisation of p-STAT-3 (Tyr 705) in response to VEGF treatment was determined by confocal microscopy. No change in p-STAT-3 (Tyr 705) expression or localisation was detected with VEGF treatment (Figure 4.16-A). Expression pattern of p-STAT-1 (Tyr 701) and p-STAT-5 (Tyr 694) was also investigated in HaemDMSCs with and without VEGF treatment. Immunoreactivity for p-STAT-1 was observed to be cytoplasmic in both unstimulated and VEGF stimulated HaemDMSCs (Figure 4.16-A). Although immunoreactivity for p-STAT-5 was undetectable in resting HaemDMSCs, there was an increase in p-STAT-5 (Tyr 694) immunoreactivity within the nuclear compartment following a 30 min incubation with VEGF (Figure 4.16-A). p-STAT-5 (Tyr 694) immunoreactivity detected within the nuclei of HaemDMSCs after VEGF treatment was significantly greater than that detected in the unstimulated cells $(p<$ 0.001) (Figure 4.16-B). 


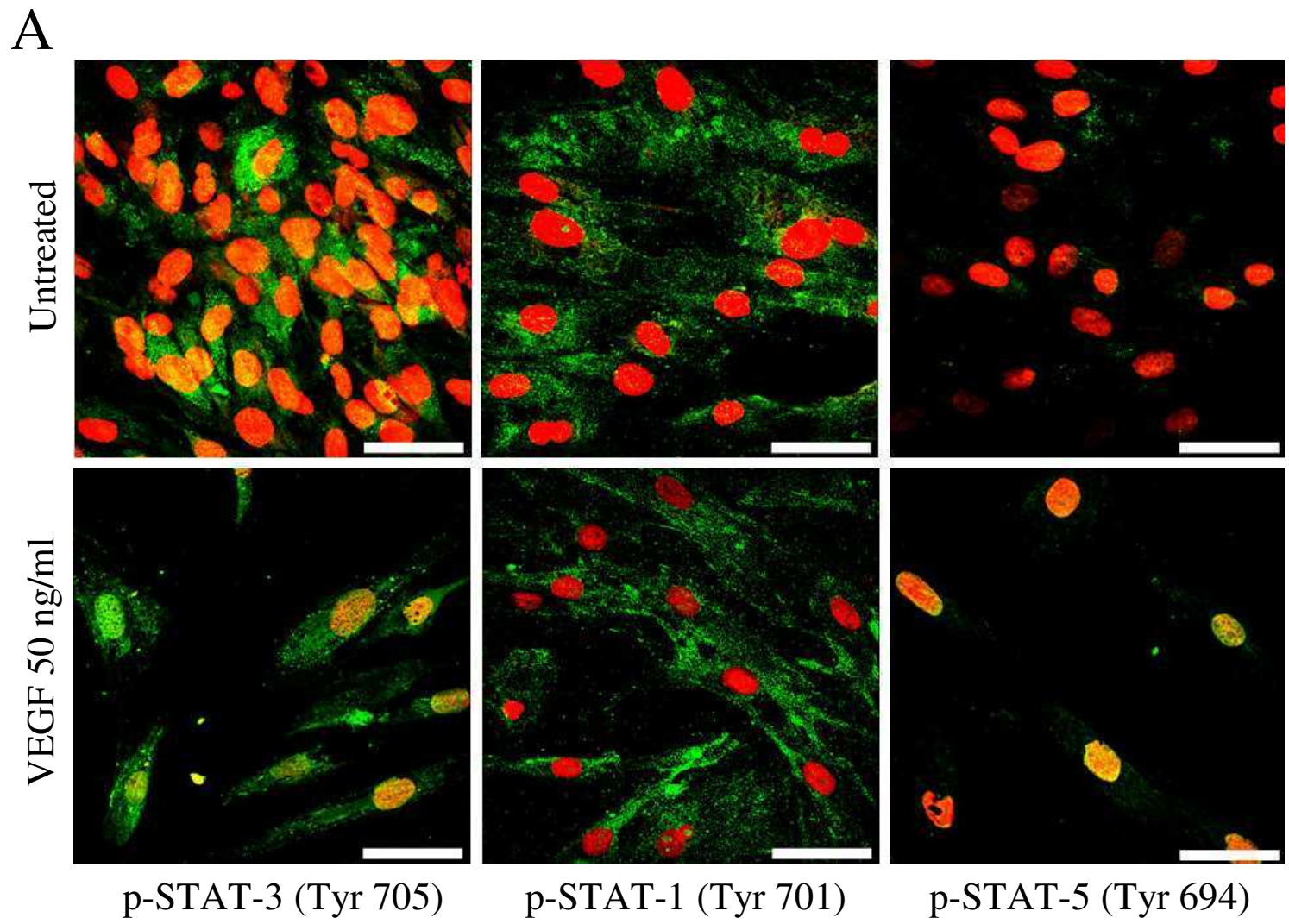

B

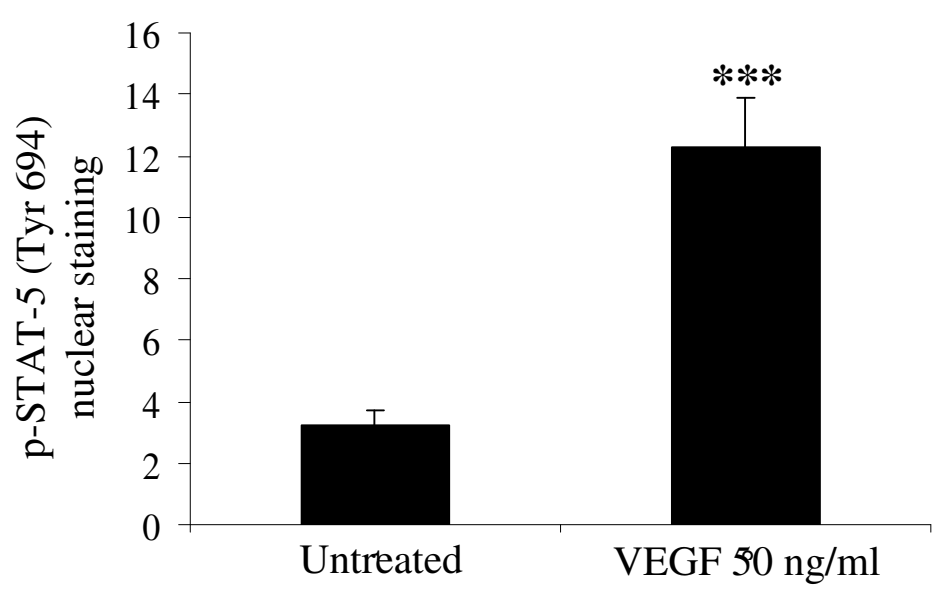

Figure 4.16: Immunolocalisation of p-STAT-3 (Tyr 705), p-STAT-1 (Tyr 701) and pSTAT-5 (Tyr 694) in response to VEGF. Panel A: Confocal microscopy was used to detect immunoreactivity for p-STAT proteins (green) in unstimulated HaemDMSCs (AC) and HaemDMSCs stimulated with VEGF $(50 \mathrm{ng} / \mathrm{ml})$ for $30 \mathrm{~min}(\mathbf{D}-\mathbf{F})$. Cells were fixed and stained as described in section 2.3.14.1. Cell nuclei were counterstained with PI (red). In untreated cells, immunoreactivity for p-STAT-3 (Tyr 705) (A) and p-STAT1 (Tyr 701) (B) was mainly cytoplasmic while staining for p-STAT-5 (Tyr 694) (C) was almost negligible. After $30 \mathrm{~min}$ of VEGF stimulation an increase in p-STAT-5 (Tyr 694) immunoreactivity was detected which was found to be predominantly within the nuclear compartment (yellow) (F). Overlapping of the green (p-STATs) and the red (nucleus) colors (yellow) indicates the presence of p-STAT proteins within the nuclei of HaemDMSCs. No significant change in p-STAT-3 (D) or p-STAT-1 (E) expression or localisation was detected. Panel B: Graphical representation of the increase in p-STAT5 (Tyr 694) expression detected within the nuclei of HaemDMSCs following VEGF treatment as determined by densitometry analysis, $*^{* *} p<0.001$. Scale bars: $50 \mu \mathrm{m}$ 
To test if STAT signalling is involved in HaemDMSCs proliferation, cells were treated with AG490 in the presence of the thymidine analogue, BrdU. Studies have shown that as BrdU is readily incorporated into the DNA of replicating cells and that quantifying BrdU incorporation can be a used as a measure of cell proliferation (Porstmann et al., 1985).

Interestingly, after 24 hours of treatment with AG490, the number of BrdU positive cells significantly decreased indicating a delay in the accumulation of cells in $S$ phase (Figure 4.17-A). A slight increase in the number of cells in G1 phase of the cell cycle, with minimal effects on G2/M phase of the cell cycle was also observed (data not shown). Results shown in Figure 4.17-A are representative of at least five individual experiments.

In addition, the effect of AG490 on vessel formation was investigated using the in vitro assay described in section 2.3.12.3 (page 94). A dose dependent decrease in vessel formation was observed when this assay was performed with medium containing both 5\% (Figure 4.17-B) and 1\% FCS (data not shown). The cultures in 5\% FCS were assayed after a period of 14 days while those in $1 \%$ FCS were analysed after 21 days. In addition to measuring vessel formation, cell nuclei were also counted for each treatment group using counting frames of $1000 \mathrm{~cm}^{2}$ (see section 2.3.12.3, page 94). A dose dependent decrease in cell number was observed with AG490 treatment (Figure 4.17-C). Figure 4.17-B and -C show the results from of two individual experiments. 
Figure 4.17: The effect of AG490 on HaemDMSCs. (A) AG490 inhibited proliferation of HaemDMSCs by stalling cell cycle progression. HaemDMSCs were treated with 100 $\mu \mathrm{M}$ or $50 \mu \mathrm{M}$ AG490 for $24 \mathrm{hrs}$ in culture media containing 5\% FCS. Cells were pulsed with BrdU for the last seven hours of treatment with AG490 and fixed in ethanol. Following incubation with an anti-BrdU antibody, antibody binding was analysed by flow cytometry. A minimum of $1 \times 10^{6}$ cells were analysed per experimental condition and the fraction of cells in $\mathrm{S}$ phase were determined by BrdU incorporation. Results obtained are representative of five individual experiments. (B) AG490 inhibited vessel formation of HaemDMSCs. $1 \times 10^{5}$ HaemDMSCs were plated into each well of a 24-well culture dish containing either vehicle alone (DMSO), $100 \mu \mathrm{M}$ AG490 or $50 \mu \mathrm{M}$ AG490 dissolved in the culture media. After 12 days in culture, vessel formation was assessed as described in section 2.3.12.3. A significant decrease in vessel formation was detected with AG490 at both $100 \mu \mathrm{M}$ and $50 \mu \mathrm{M}$. (C) AG490 inhibited proliferation of HaemDMSCs. The total cell nuclei within the same area as that used for assessing vessel formation was counted. Results showed that the number of cell nuclei also decreased with an increase in AG490 concentration. All values are expressed as the mean \pm s.e.m. Results shown in $(\mathbf{B})$ and $(\mathbf{C})$ are representative of two individual experiments set up in duplicate. Control cultures were set up in quadruplicates. $* p<$ $0.05, * * p<0.01$. 
A

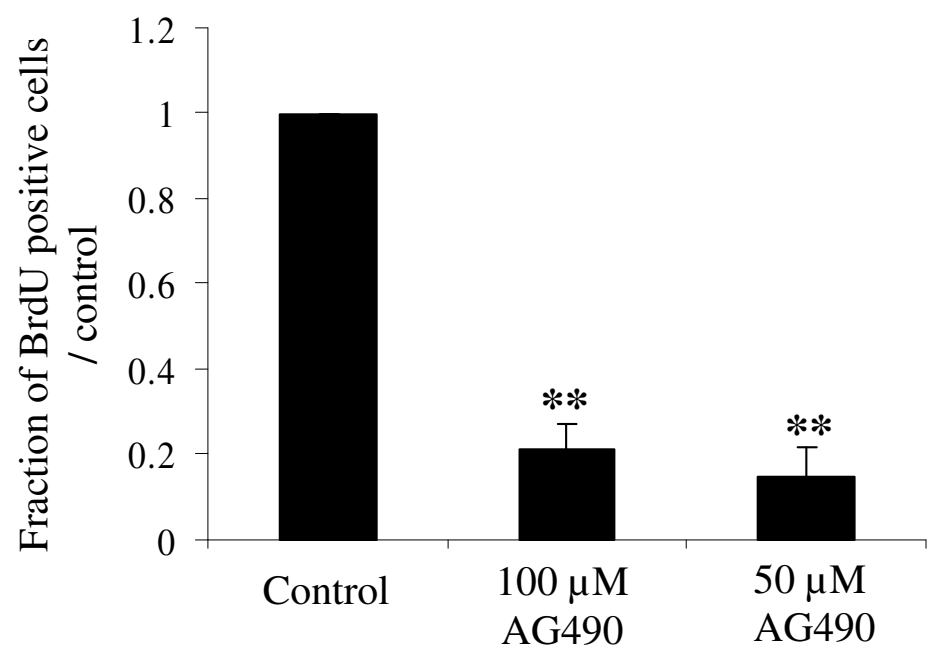

B

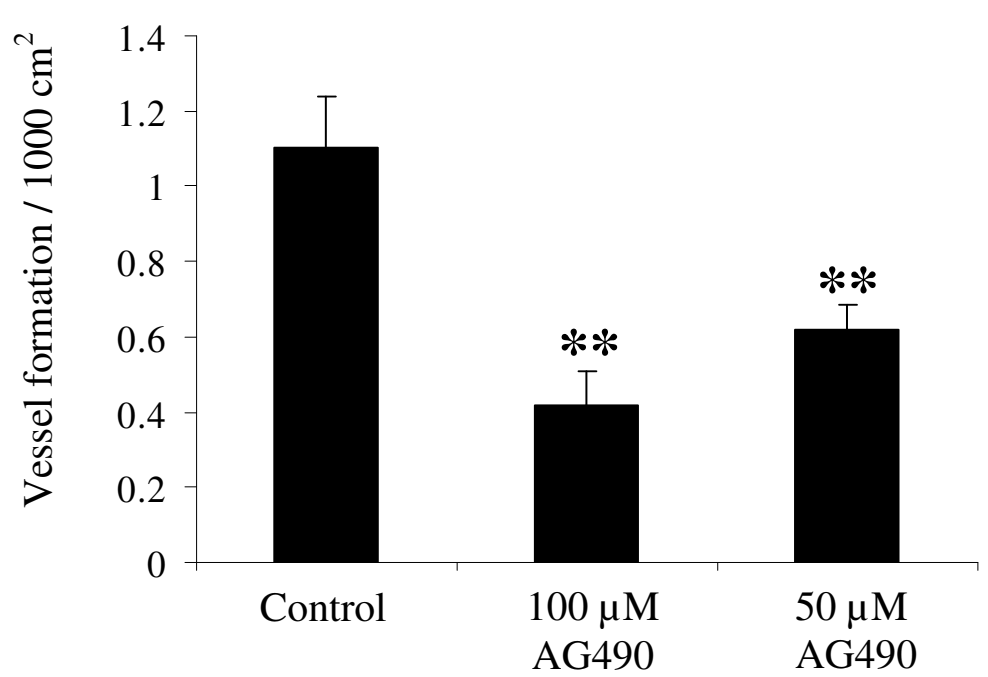

C

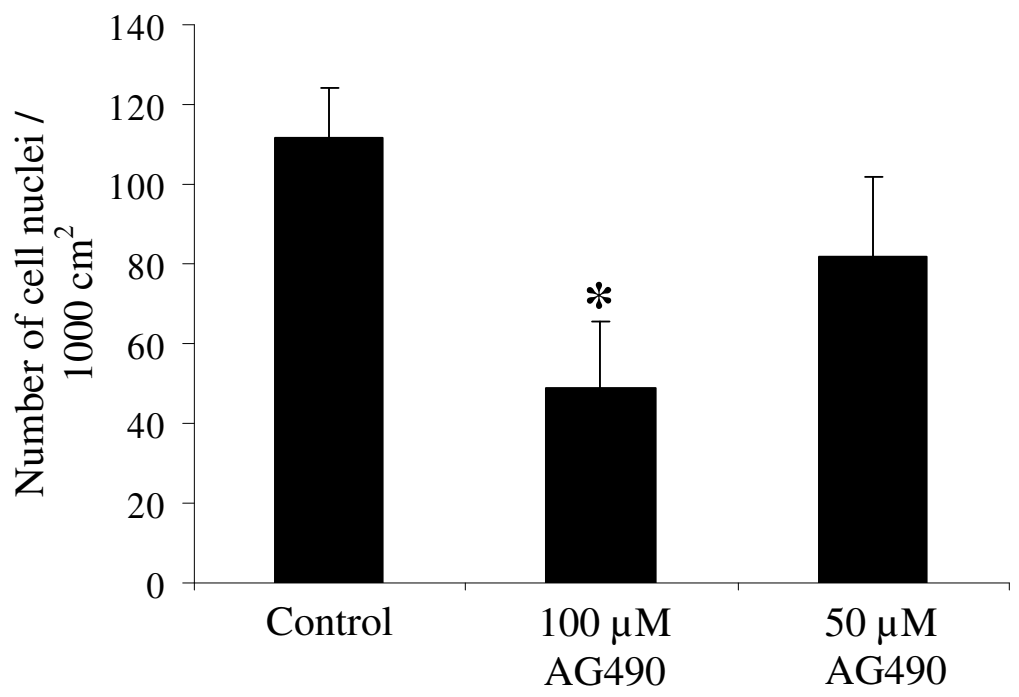




\subsubsection{OSTEOPROTEGERIN EXPRESSION IN HAEMANGIOMA.}

Osteoprotegerin (OPG) is involved in osteoclast differentiation and activation (Simonet et al., 1997; Tsuda et al., 1997; Fuller et al., 1998). See section 5.3 .5 (page 227) for more details on why OPG expression was investigated in HaemDMSCs. qRTPCR revealed that HaemDMSCs expressed high levels of OPG mRNA (Figure 5.4-A and Appendix 3, Figure 3.5). Immunohistochemical staining revealed that HaemDMSCs expressed large amounts of OPG protein in the ECM but not within the cells (Figure 4.18-D). Consistent with previous reports, OPG co-localised with vWF in HUVECs (Figure 4.18-C) (Zannettino et al., 2005). However, HUVECs expressed less OPG protein (Figure 4.18-B) than HaemDMSCs (Figure 4.18-B). Since HaemDMSCS expressed high levels of OPG protein, it was hypothesised that OPG might serve as a marker for MSCs within haemangioma tissues in vivo.

Immunohistochemical staining of haemangioma tissue sections showed that proliferating lesions contained more OPG positive cells (Figure 4.19-B, green staining) than involuting lesions (Figure 4.19-E) while involuted lesions contained no OPG staining cells (Figure 4.19-H). Dual immunostaining for vWF and OPG revealed that most vessels in the proliferating lesions expressed vWF but in a punctate manner (Figure 4.19-A, white arrows) and that these cells strongly expressed OPG (Figure 4.19$\mathrm{B}$, white arrows). Late proliferative and early involuting phases contained regions that exhibited punctuate vWF (Figure 4.19-D, white arrows) and high OPG staining (Figure 4.19-E, white arrows). These lesions also contained vessels that strongly expressed vWF (Figure 4.19-D, yellow arrows), which did not express OPG (Figure 4.19-E, yellow arrows). In addition, the vessels in the late involuting and involuted phases that strongly expressed vWF (Figure 4.19-G, yellow arrows) did not express OPG (Figure 4.19-H, yellow arrows). Dual staining for $\mathrm{vWF}$ and PCNA indicated that regions with punctate, weak vWF staining and high OPG expression also contained more PCNA 
positive cells than regions with strongly staining vWF and no OPG expression (Figure 4.20). 

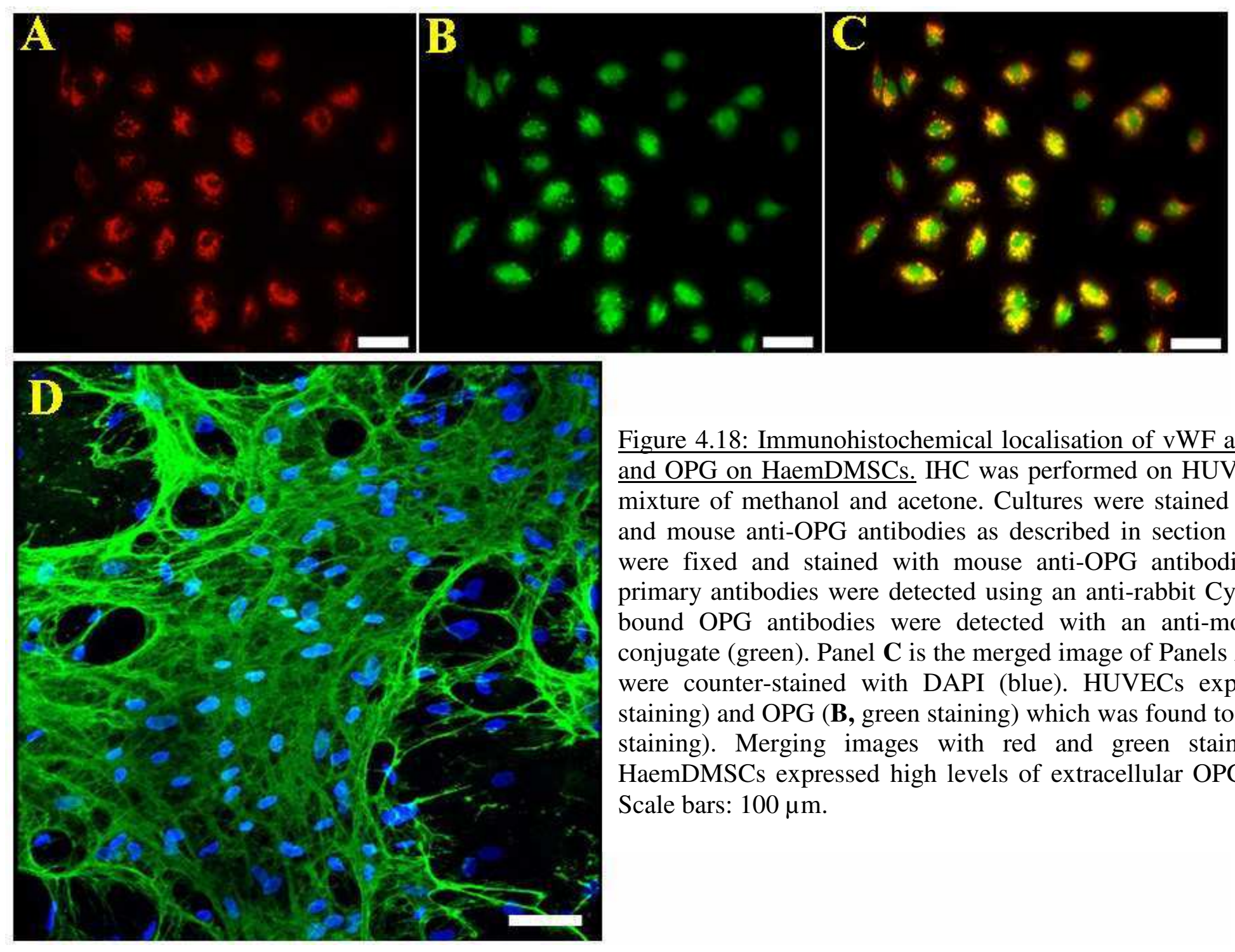

Figure 4.18: Immunohistochemical localisation of vWF and OPG on HUVECs and OPG on HaemDMSCs. IHC was performed on HUVECs (A-C) fixed in a mixture of methanol and acetone. Cultures were stained with rabbit anti-vWF and mouse anti-OPG antibodies as described in section 2.3.11. HaemDMSCs were fixed and stained with mouse anti-OPG antibodies (D). Bound vWF primary antibodies were detected using an anti-rabbit $\mathrm{Cy} 3$ conjugate (red) and bound OPG antibodies were detected with an anti-mouse AlexaFluor 488 conjugate (green). Panel $\mathbf{C}$ is the merged image of Panels A with $\mathbf{B}$. Cell nuclei were counter-stained with DAPI (blue). HUVECs expressed vWF (A, red staining) and OPG (B, green staining) which was found to colocalize $(\mathbf{C}$, yellow staining). Merging images with red and green staining appears yellow. HaemDMSCs expressed high levels of extracellular OPG (D, green staining). Scale bars: $100 \mu \mathrm{m}$. 

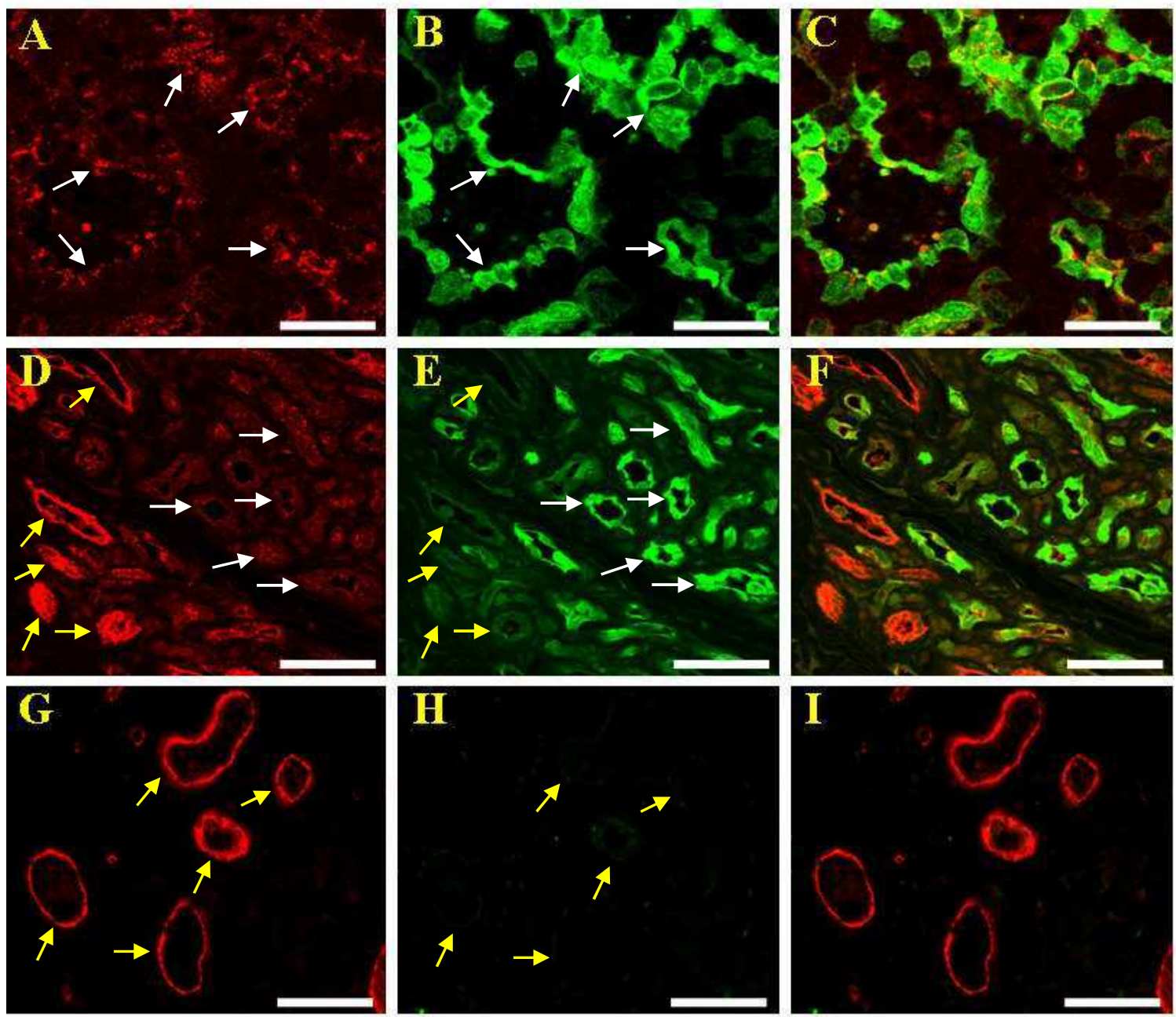

Figure 4.19: Confocal immunohistochemical localisation of VWF and OPG in haemangioma. Proliferating (A-C), involuting (D-F) and involuted (G-I) paraffin embedded haemangioma tissue sections were stained with rabbit anti-vWF (A,D\&G) and mouse anti-OPG (B,E\&H) antibodies. Bound vWF and OPG antibodies were detected using an anti-rabbit-Cy3 conjugate (red) and an anti-mouse AlexaFluor 488 conjugate (green), respectively. Panels $\mathbf{C}, \mathbf{F}$ and $\mathbf{I}$ are the merged images of Panels $\mathbf{A}$ with $\mathbf{B}, \mathbf{D}$ with $\mathbf{E}$, and $\mathbf{G}$ with $\mathbf{H}$, respectively. Most cells lining the blood vessels in proliferating lesions showed punctate immunoreactivity for $\mathrm{vWF}$ (A, white arrows). The same cells also showed strong staining for OPG (B, white arrows). Involuting lesions contained regions that showed punctate $\mathrm{vWF}$ staining (D, white arrows). These regions expressed high levels of OPG (E, white arrows). However, these lesions also contained vessels that showed intense immunoreactivity for vWF (D, yellow arrows) but were negative for OPG (E, yellow arrows). Vessels in involuted lesions that strongly stained for $\mathrm{vWF}$ (G, yellow arrows) were negative for OPG ( $\mathbf{H}$, yellow arrows). Scale bars: 50 $\mu \mathrm{m}$. 

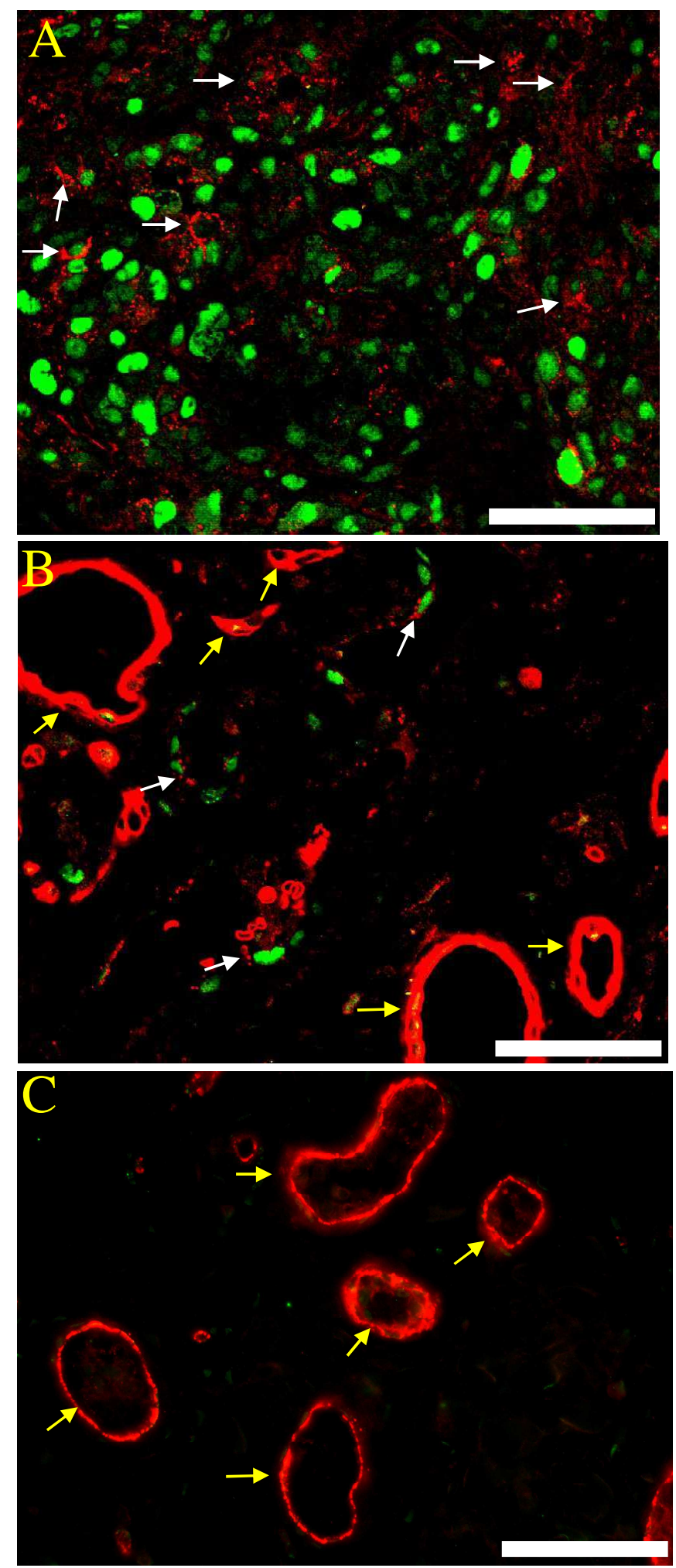

Figure 4.20: Immunohistochemical localization of $\mathrm{vWF}$ and PCNA on paraffin embedded haemangioma tissue biopsies. Proliferating (A-C), involuting (D-F) and involuted (G-I) haemangioma biopsies were stained with rabbit anti-vWF antibodies and mouse anti-PCNA antibodies. Bound $\mathrm{vWF}$ and PCNA antibodies were detected using an anti-rabbit-Cy3 conjugate (red) and an anti-mouse AlexaFluor 488 conjugate (green) respectively. (A) Proliferating lesions showed punctate immunoreactivity for vWF (white arrows) and contained the highest number of PCNA positive cells (green staining). (B) In involuting lesions, the vessels that exhibited punctate staining for vWF (white arrows) also contained some PCNA positive cells. But vessels that stained strongly for vWF (yellow arrows) did not contain any cells that express PCNA. (C) Vessels in involuted lesions that strongly expressed vWF were devoid of PCNA positive cells. Scale bars: $50 \mu \mathrm{m}$. 


\subsubsection{EFFECT OF INTERFERON AND DIFFERENTIATION MEDIA ON STAT PROTEIN EXPRESSION IN HaemDMSCs}

IFN $\alpha$-2b treatment significantly decreased proliferation of HaemDMSCs (see section 5.3.4, page 224). In addition, treatment of HaemDMSCs with 10,000 U/ml of IFN $\alpha-2 b$ for 15 min resulted in a dramatic increase in p-STAT-3 (Tyr 705) expression (Figure 4.21-A) accompanied by its nuclear translocation (Figure 4.21-B). In addition, increased expression and nuclear translocation above that of the untreated controls was seen for p-STAT-5 (Tyr 694) and p-STAT-1 (Tyr 701) (Figure 4.21-B) in IFN $\alpha-2 b$ treated cultures. These results lead us to the hypothesis that STAT signalling might be involved in haemangioma differentiation.

To test this hypothesis, STAT-3, STAT-5, p-STAT-3 (Tyr 705) and p-STAT-5 (Tyr 694) expression and localisation was analysed in HaemDMSCs treated with adipocyte induction media for $30 \mathrm{~min}, 4$ hours and 24 hours. No significant change in expression or localisation of p-STAT-3 (Tyr 705) and p-STAT-5 (Tyr 694) was observed in treated versus the untreated cells at all time points (Appendix 2, Figure 2.5, page 288).

In addition, the effects of IFN $\alpha-2 b$ and AG490 were tested on the rate of adipogenesis. HaemDMSCs were cultured with differentiation media containing either, $100 \mathrm{U} / \mathrm{ml}$ IFN $\alpha-2 b, 15000 \mathrm{U} / \mathrm{ml}$ of IFN $\alpha-2 b, 5 \mu \mathrm{M}$ AG490 or $20 \mu \mathrm{M}$ of AG490. These differentiation conditions were maintained for a period of 16 days with media being changed every 2-3 days. No significant increase in adipocyte formation was observed with either dosage of IFN $\alpha-2 b$ above that of the control cultures containing differentiation media alone. In addition, neither dose of AG490 was able to block lipidlaiden vacuole formation (data not shown). 

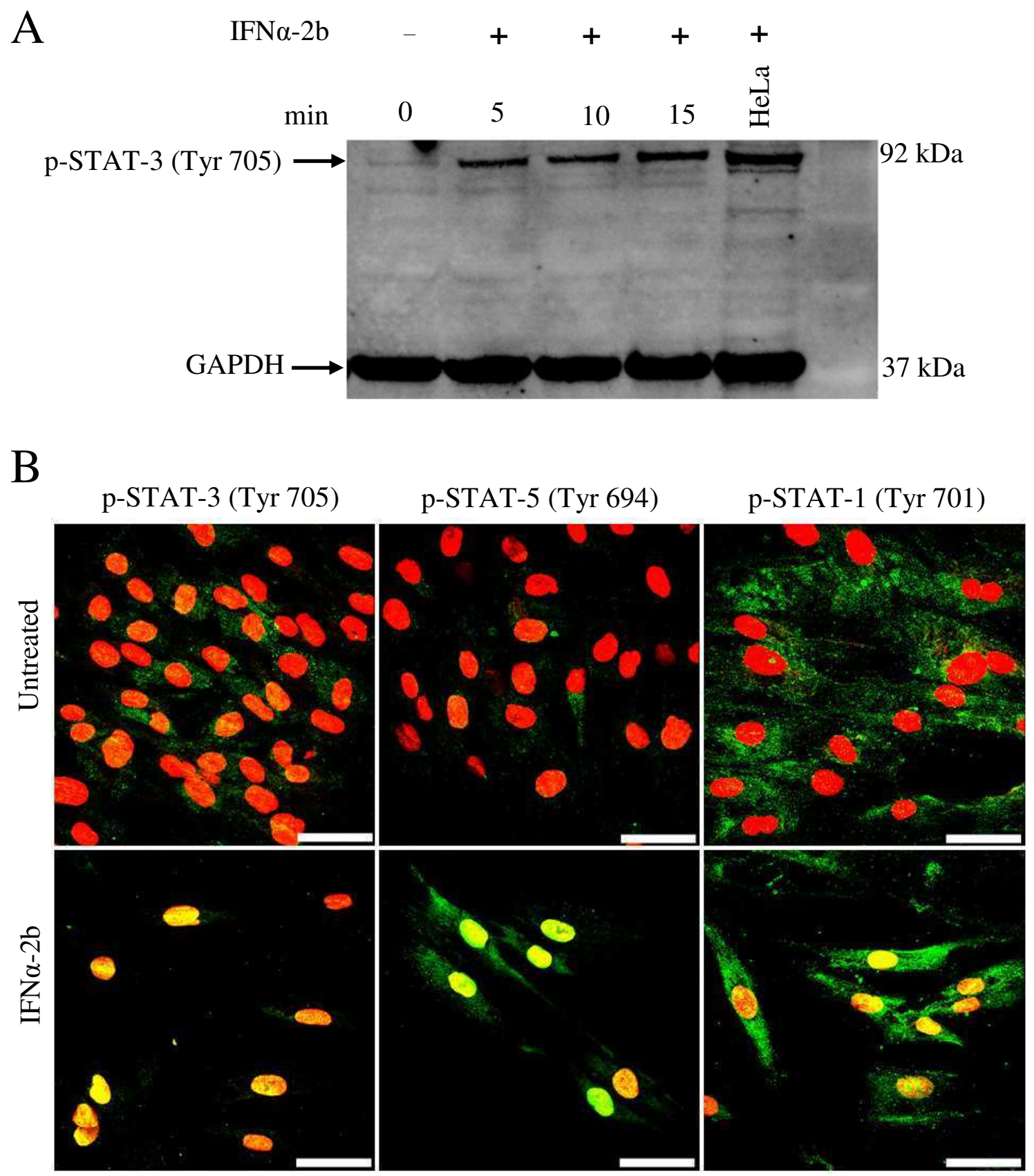

Figure 4.21: IFN $\alpha-2 b$ induces an increase in p-STAT protein expression and nuclear translocation in HaemDMSCs. (A) HaemDMSCs were treated with IFN $\alpha$-2b for the times indicated. Unstimulated and HaemDMSCs stimulated with $10,000 \mathrm{U} / \mathrm{ml}$ of IFN $\alpha$ $2 \mathrm{~b}$ for 5,10 and $15 \mathrm{~min}$ were lysed and subjected to Western blotting as described in section 2.3.13.1. While unstimulated HaemDMSCs expressed low levels of p-STAT-3 (Tyr 705), a time dependent increase in p-STAT-3 (Tyr 705) expression was detected with IFN $\alpha-2 b$ stimulation. (B) Confocal immunohistochemical localisation of p-STAT proteins (green) in HaemDMSCs stimulated with 10,000 U/ml IFNa-2b for 15 min (DF) in comparison with control unstimulated HaemDMSCs (A-C). Cell nuclei were counterstained with PI (red). Unstimulated HaemDMSCs expressed low levels of pSTAT3 (Tyr 705) (A), p-STAT-5 (Tyr 694) (B) and p-STAT-1 (Tyr 701) (C) (green) detected only in the cytoplasm. Following IFN $\alpha-2 b$ treatment, immunoreactivity for $p$ STAT3 (Tyr 705) (D), p-STAT-5 (Tyr 694) (E) and p-STAT-1 (Tyr 701) (F) was detected within the nuclei of HaemDMSCs (yellow). Overlapping of the green (pSTATs) and the red (nucleus) colors (yellow) indicates the presence of p-STAT proteins within the nuclei of HaemDMSCs. Some immunoreactivity for p-STAT-5 and p-STAT1 was also detected in the cytoplasm of the IFN $\alpha-2 b$ stimulated HaemDMSCs $(\mathbf{E} \& \mathbf{F}$, respectively). Scale bars: $50 \mu \mathrm{m}$. 


\subsubsection{ENDOTHELIAL PROGENITOR CELLS IN HAEMANGIOMA}

Using previously described methods with a few modifications, the isolation of ECs from human haemangioma biopsies was attempted. This was to be used as a patient matched comparative cell line. However, these attempts were futile as cell yield was low after the isolation step with CD31 coated beads. These cells did not proliferate well and died following the first passage (See Appendix 2, section 2.1, page 289)

However, good yield was obtained when the bead isolation was omitted. The morphology exhibited by the cells obtained from digested proliferating haemangioma tissues was distinct from HaemDMSCs in that these cells had round central bodies with some cytpolasmic projections (Figure 4.22). This morphology has been shown to be typical of EPCs (Ashara et al., 1997; Kalka et al., 2000). Immunohistochemical staining performed on these cells 48 hours after dissociation showed that the cells expressed typical EC markers, vWF (Figure 4.23), VEGFR-1 (Figure 4.25-A) and more importantly VEGFR-2 (Figure 4.25-B). These results are consistent with an EC phenotype. Therefore, these cells are here in referred to as haemangioma-derived EPCs (HaemDEPCs). 


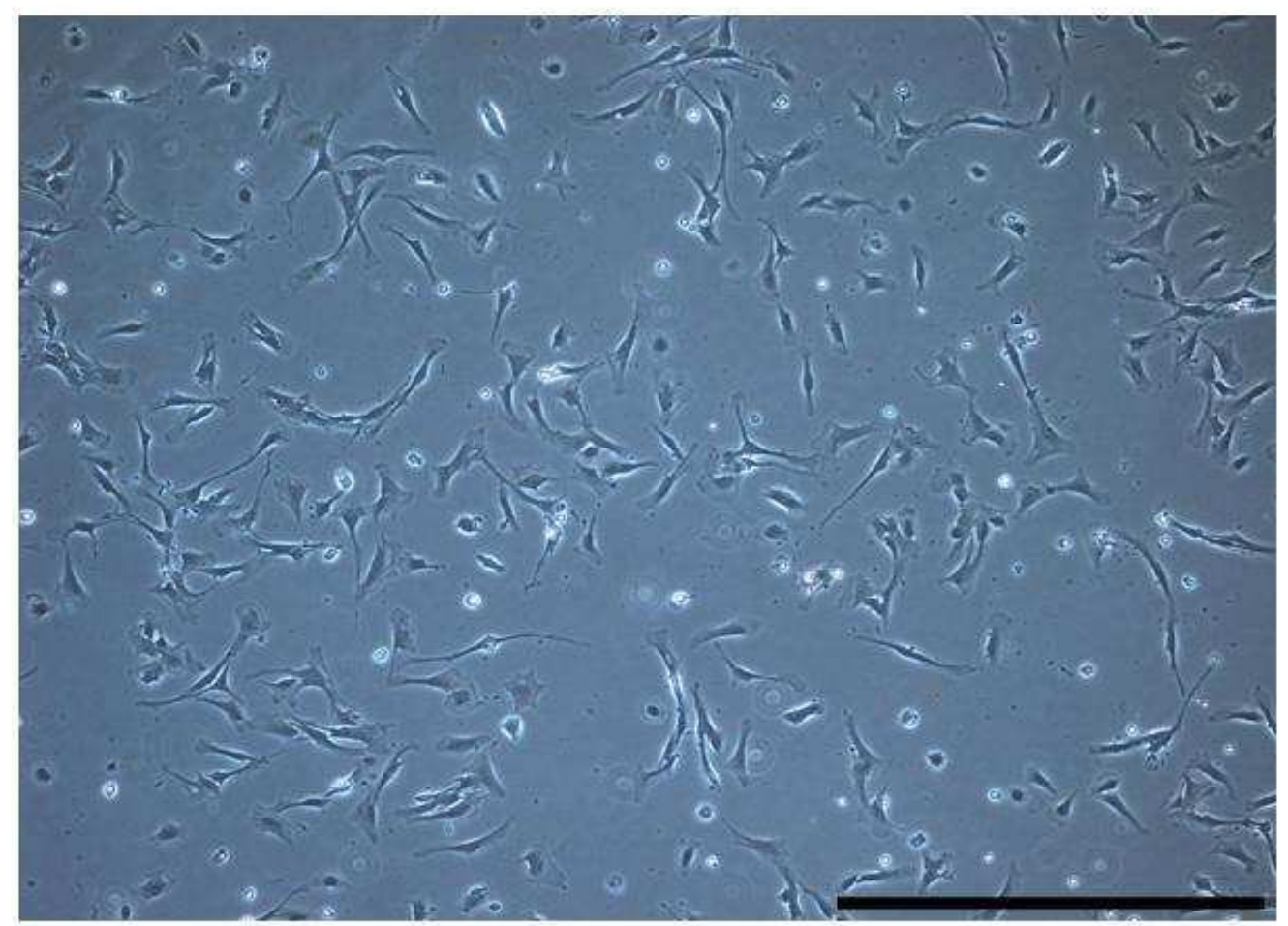

Figure 4.22: EPC-like cells obtained from a proliferating haemangioma. The cells obtained from collagenase treated proliferating haemangioma tissue were plated onto gelatin coated culture dishes. These cells exhibited EPC-like morphology i.e., they contained round central bodies with some cytplasmic projections. Photograph was taken on day 2 in culture. Scale bar: $500 \mu \mathrm{m}$.

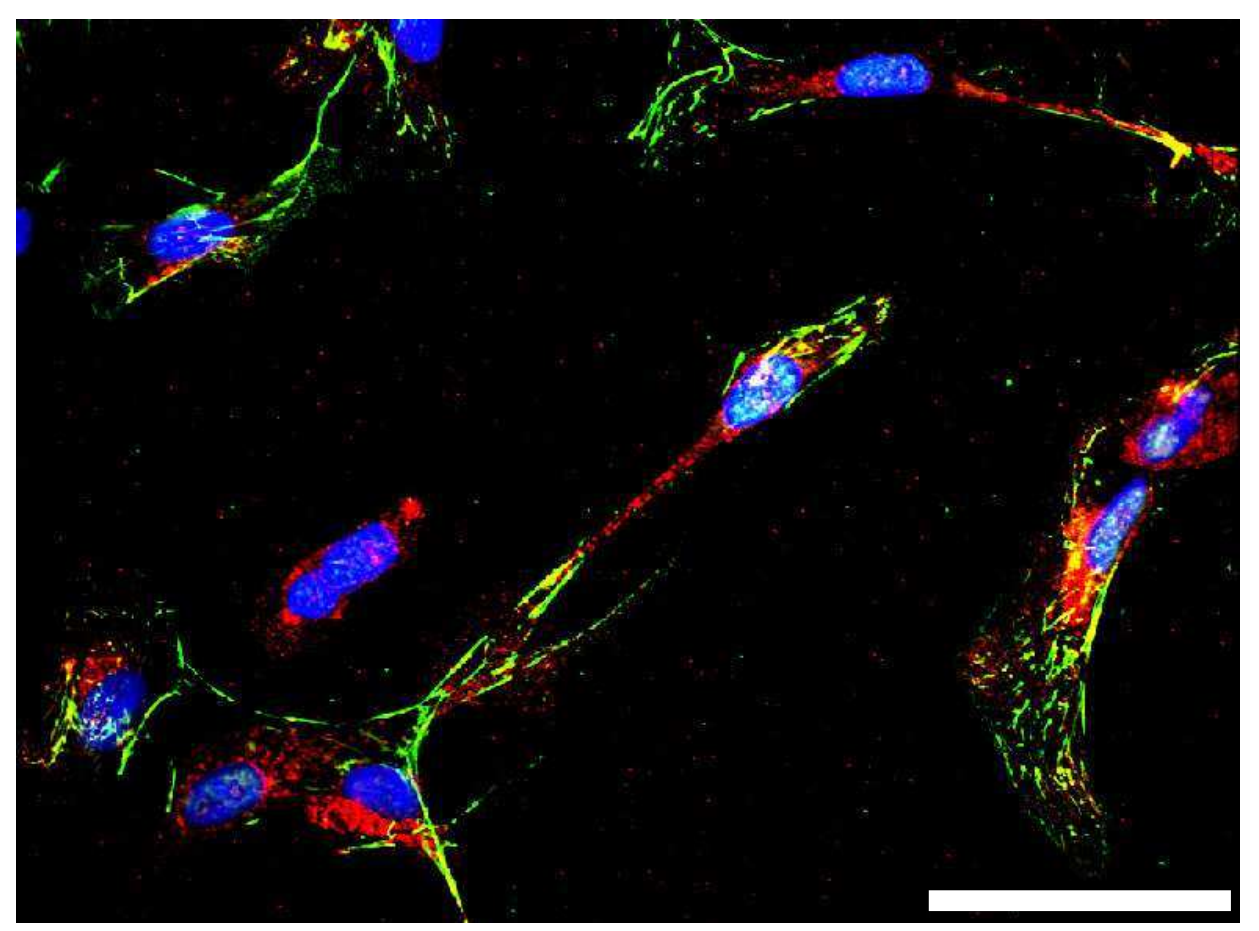

Figure 4.23: Immunohistochemical localisation of vWF and OPG on HaemDEPCs. On day 2 in culture, HaemDEPCs were fixed and stained with rabbit anti-vWF and mouse anti-OPG primary antibodies as described in section 2.3.9. Bound anti-vWF antibodies were detected with an anti-rabbit Cy3 (red) conjugate and bound anti-OPG antibodies were detected with an anti-mouse AlexaFluor 488 (green) conjugate. Cell nuclei were counterstained with DAPI (blue). Positive immunoreactivity was detected for vWF (red) and OPG (green) on all HaemDEPCs present on the coverslip. Scale bar: $50 \mu \mathrm{m}$. 
Interestingly, on day 5 in culture, HaemDEPCs had changed to a MSC-like morphology, i.e., they appeared flat and broadened (Figure 4.24-A). At this stage, immunohistochemical analysis showed that they no longer expressed vWF (Figure 4.24B) and VEGFR-2 (Figure 4.25-D). However, the cells continued to express VEGFR-1 (Figure 4.25-C) and high levels of extracellular OPG (Figure 4.24-B) which again is typical of HaemDMSCs as shown earlier (Figure 4.18-C). Furthermore, the MSC nature of the spontaneously differentiated cells was confirmed by the ability of the cells to form adipocytes (Figure 4.26). These results indicate that there is an EPC to MSC transdifferentiation process occurring in vitro. 

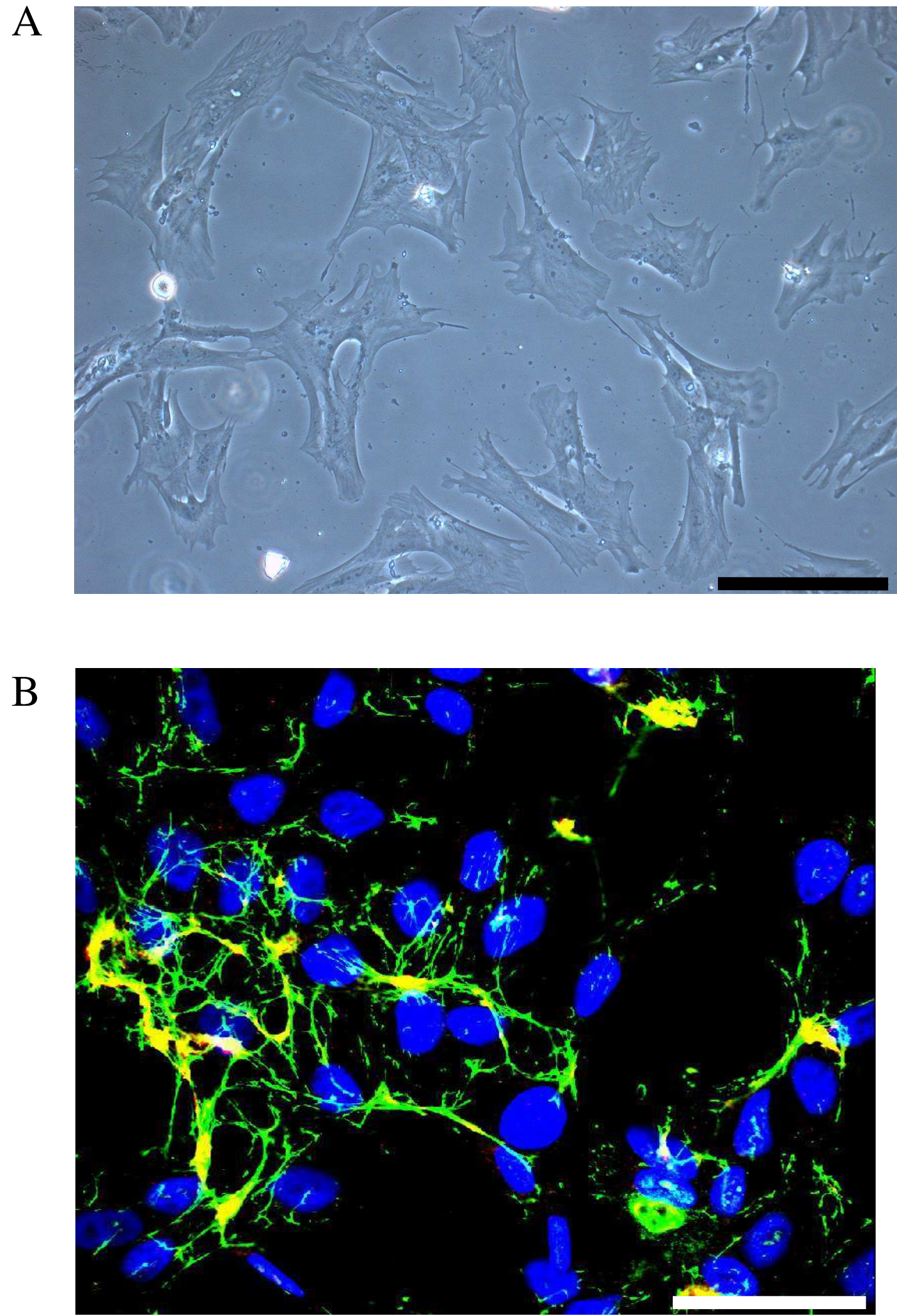

Figure 4.24: Change in HaemDEPC morphology and immunophenotype on day 5 in culture in vitro. (A) Phase contrast microscopy showing MSC like morphology, i.e., cells appeared flat and broadened. These cells were fixed and stained with rabbit antivWF and mouse anti-OPG primary antibodies as described in section 2.3.9. Bound antivWF antibodies were detected with an anti-rabbit $\mathrm{Cy} 3$ (red) conjugate and bound antiOPG antibodies were detected with an anti-mouse AlexaFluor 488 (green) conjugate. Cell nuclei were counterstained with DAPI (blue). At this point in culture, vWF expression (red) was lost while high levels of extracellular OPG staining was observed (B). Note the OPG staining pattern (green) is similar to that detected on HaemDMSCs (Figure 4.18).. Scale bars: (A) $100 \mu \mathrm{m}$, (B) $50 \mu \mathrm{m}$. 

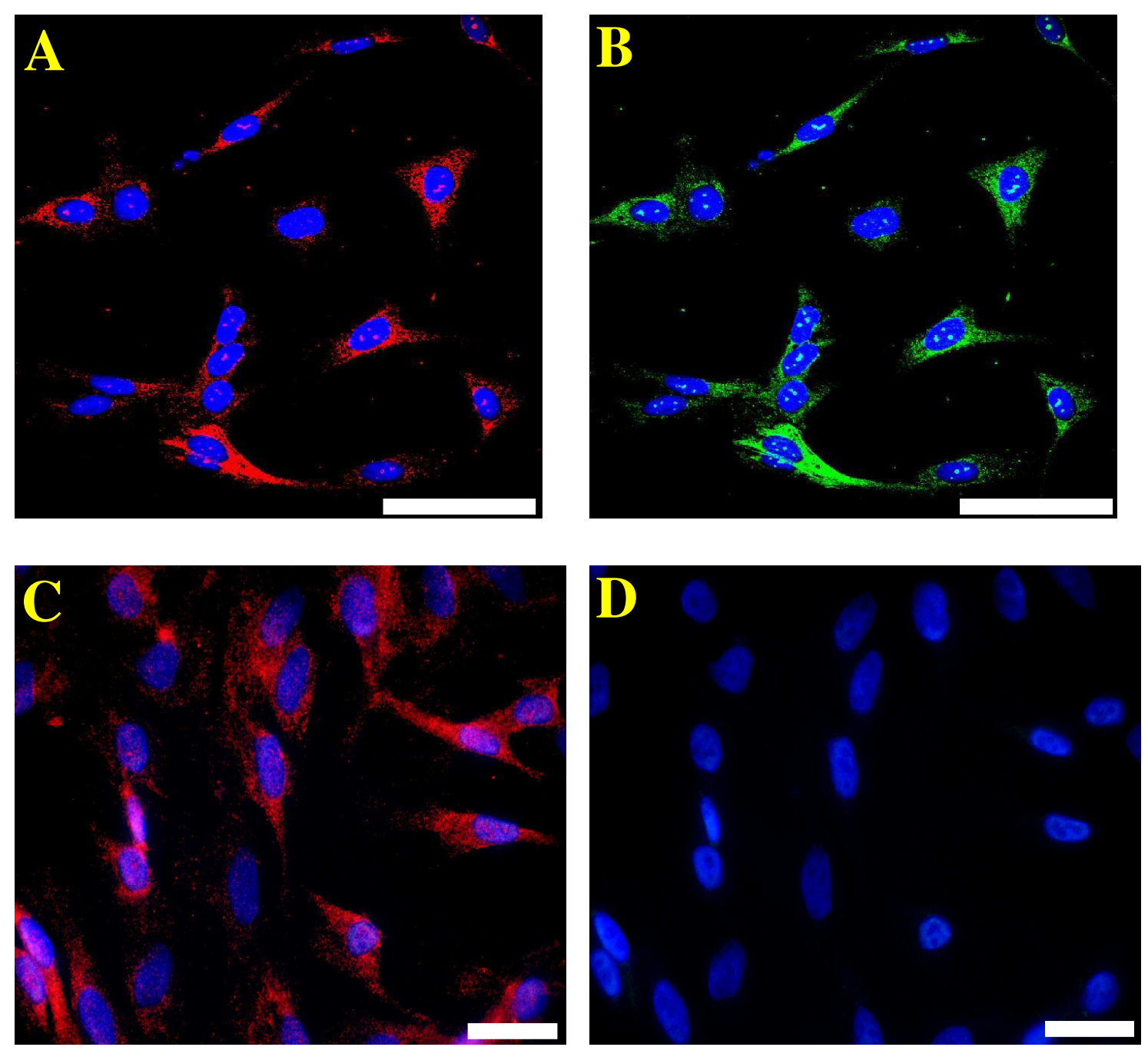

Figure 4.25: Immunohistochemical localisation of VEGFR-1 and VEGFR-2 on HaemDEPCs. HaemDMSCs were fixed and stained with goat anti-VEGFR-1 and rabbit anti-VEGFR-2 antibodies as described in section 2.3.9. Bound VEGFR-1 antibodies were detected with an anti-goat biotin conjugate followed by a Strepavidin 555 conjugtae (red) and bound VEGFR-2 antibodies were detected with an anti-rabbit DIG conjugate followed by an anti-DIG FITC conjugate (green). Cell nuclei were counterstained with DAPI (blue). HaemDEPCs expressed VEGFR-1 and VEGFR-2 on day 2 in culture (A\&B, respectively). The cells continued to express VEGFR-1 on day 5 (C) but lost VEGFR-2 expression by day 5 in culture (D). Scale bars: $20 \mu \mathrm{m}$. 


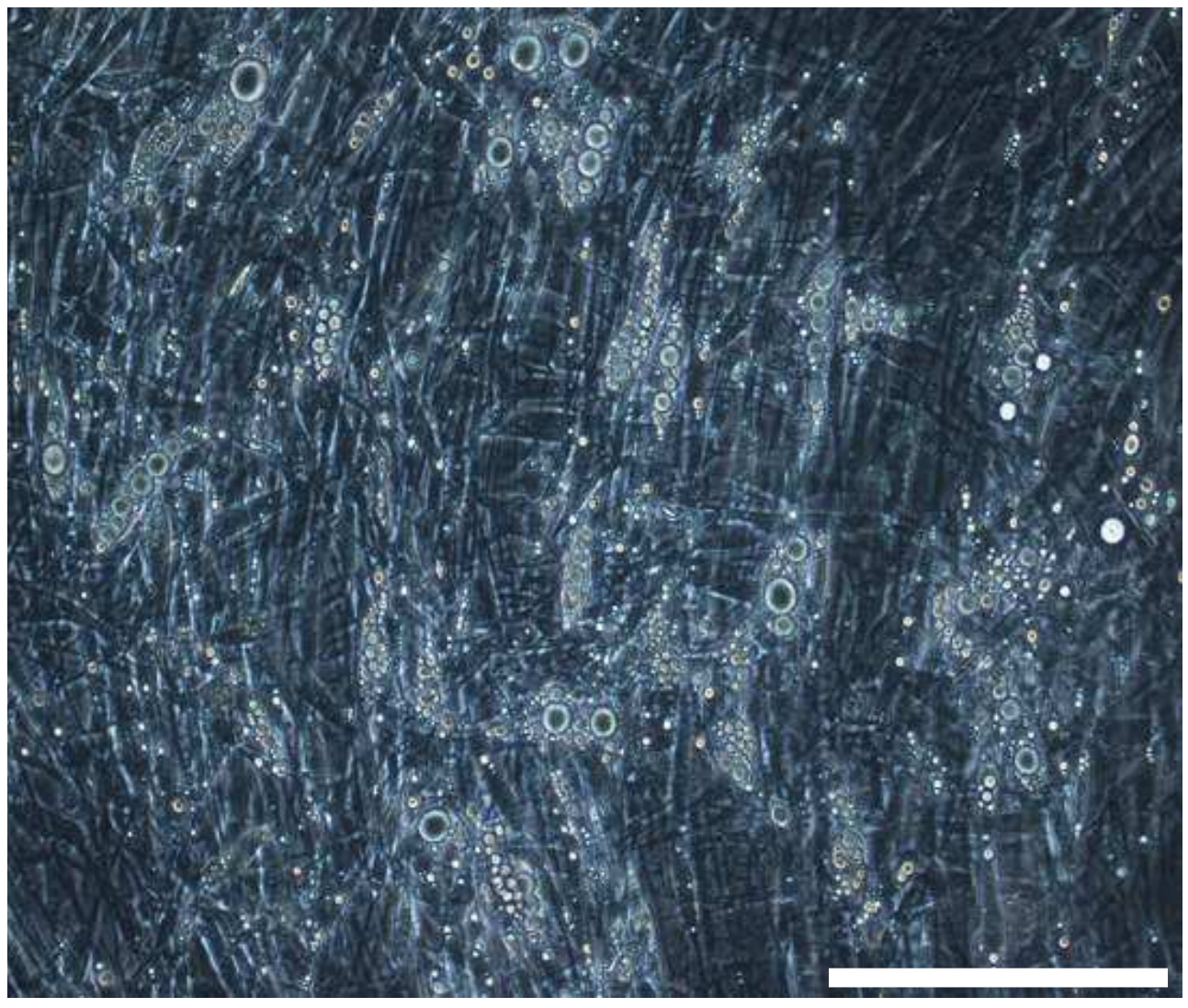

Figure 4.26: Adipocytic differentiation of the MSCs transdifferentiated from HaemDEPCs. The MSCs-like cells shown in Figure 4.24-A differentiated into adipocytes when cultured in adipocytic induction media as described in section 2.3.10.2. Lipid-laiden vacoules (circular bodies) appeared within two weeks of induction. Scale bar: $200 \mu \mathrm{m}$. 
In addition, when a second dish containing HaemDEPCs was cultured in media containing $50 \mathrm{ng} / \mathrm{ml}$ of VEGF from day 2 after dissociation, some cells continued to exhibit the EPC morphology (Figure 4.27-A). These cells expressed both vWF (Figure 4.27-B) and OPG (Figure 4.27-C). However, not all cells cultured in VEGF were able to maintain their EC phenotype as some cells present in the culture dish were morphologically and immunohistochemically similar to HaemDMSCs (data not shown). These results indicate that the spontaneous differentiation of HaemDEPCs to HaemDMSCs could be delayed with addition of VEGF thereby indicating the importance of this growth factor in HaemDEPCs survival and proliferation, but VEGF alone is not sufficient to retain the endothelial properties of these cells and that other growth factors such as FGF-2 and IGF-2 may be required. Furthermore, these results were replicated from a proliferating haemangioma biopsy obtained from a second patient. 

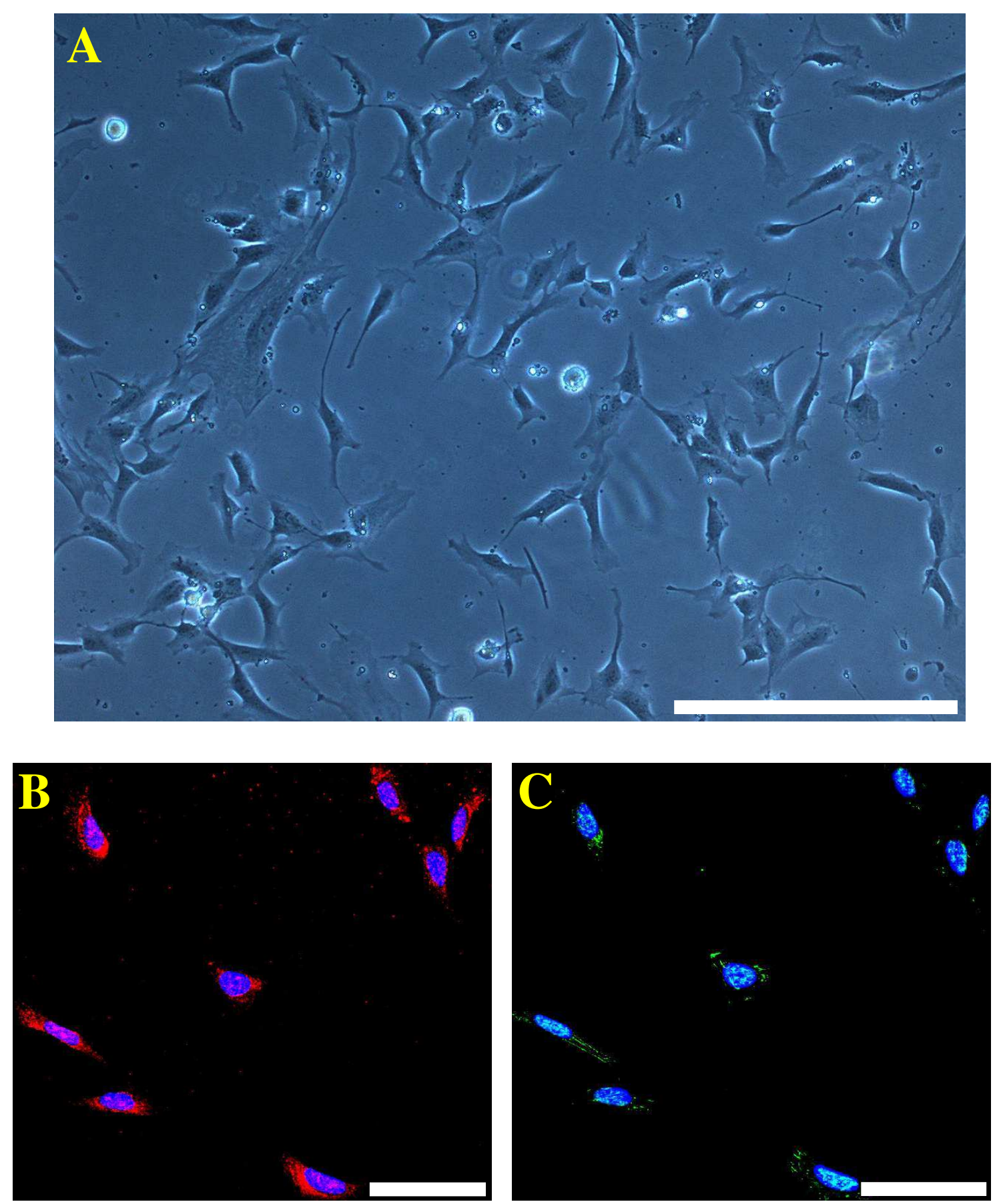

Figure 4.27: HaemDEPCs maintain their endothelial phenotype when cultured in VEGF. (A) Phase contrast microscopy showing HaemDEPCs maintained in $50 \mathrm{ng} / \mathrm{ml} \mathrm{of}$ VEGF for ten days. The cells appeared morphologically similar to those from day 2 after dissociation (Figure 4.22). Immunohistochemical staining for vWF (B) and OPG (C) on HaemDEPCs maintained in VEGF for ten days. The cells were fixed and stained with rabbit anti-vWF and mouse anti-OPG primary antibodies as described in section 2.3.9. Bound $\mathrm{vWF}$ antibodies were detected with an anti-rabbit $\mathrm{Cy} 3$ conjugate (red) and bound OPG antibodies were detected using an anti-mouse AlexaFluor 488 conjugate (green). Cell nuclei were counterstained with DAPI (blue). Cells expressed vWF (B, red) and some OPG ( $\mathbf{C}$, green). Note OPG expression is very different from that seen on HaemDMSCs (Figure 4.18-D).. Scale bars: (A) $500 \mu \mathrm{m}$, (B\&C) $50 \mu \mathrm{m}$. 


\section{CHAPTER 4.4: DISCUSSION}

\subsubsection{CHARACTERISATION OF HAEMANGIOMA EXPLANT OUTGROWTHS}

The lack of an ideal model system for haemangioma has led to empirical and unsatisfactory therapies (Mulliken et al., 1995; Tan et al., 2000 ). However, an in vitro model that mimics, as closely as possible, the features of haemangioma was developed (Tan et al., 2000 b) based on the models using the rat aortic rings (Nicosia \& Ottinetti, 1990) and human placenta (Brown et al., 1996). In this model, freshly resected haemangioma tissue placed into fibrin gels, develop outgrowths or 'microvessels' within days of culture. Although these outgrowths have been reported to be ECs (Tan et $a l ., 2000_{\mathrm{b}}$ ), other reports have shown that these outgrowing cells express EC-specific markers only in the area immediately adjacent to the explanted tissue (Ritter et al., 2002). This suggests that the cells may be dedifferentiating and down-regulating these proteins as they acquire a migratory phenotype.

During this study, a method was developed for the purification and culture of these haemangioma explant outgrowths in vitro. This study characterised these cells to be MSCs rather than ECs. MSCs derived from proliferating and involuting haemangiomas exhibited fibroblast-like morphology in early passages and a flat broadened morphology in later passages. qRT-PCR showed that the cells expressed mRNA transcripts for the MSC markers, CD29, CD44 and CD90 (Pittenger et al., 1999) but not CD133 which identifies haemopoetic precursor cells and EPCs (Hilbe W et al., 2004). The cells did not express mRNA transcripts for CD31, CD45 and VEGFR-2 and were not immunohistochemically positive for vWF, VEGFR-2 or CD31, indicating that they were not contaminated with cells of haematopoietic or endothelial origin. These results and the finding that the cells expressed mRNA and protein for the haemangioma marker, GLUT-1, confirmed that the cells were haemangioma-derived MSCs. 
Furthermore, consistent with previous reports, the HaemDMSCs expressed $\alpha$-SMA and vimentin, which have been used to characterise MSCs (Sutherland et al., 2005; Yu et al., 2006).

As no MSC-specific markers currently exist, rigorous identification of MSCs requires demonstration of their capability to differentiate along specific mesenchymal lineages. Consistent with previous reports of the presence of MSCs in haemangioma (Yu et al., 2006), the HaemDMSCs isolated in this study exhibited capacity for selfrenewal and the ability to differentiate into multiple mesenchymal cell lineages, adipocytes and osteoblasts, when cultured in specific induction media. The increase in osteonectin and PPAR $\gamma$ mRNA expression identified post-induction validated the differentiation of the HaemDMSCs into adipocytes and osteocytes, respectively. PPAR $\gamma$ is a key regulatory gene of adipogenesis (Camp et al., 2002) while osteonectin is considered a differentiation marker for bone cells, as its expression has been identified in osteoblasts, young osteocytes and also in osteoprogenitor cells (Jundt et al., 1987).

Similar to bone marrow-derived MSCs (Okuyama et al., 2006), the HaemDMSCs expressed mRNA and protein for VEGFR-1. However, unlike bonemarrow derived MSCs (Oswald et al., 2004), HaemDMSCs did not exhibit EC-like characteristics when cultured in high concentrations of VEGF. In addition, Yu et al (2001) showed that haemangioma-derived ECs did not express mRNA for VEGF. However, the HaemDMSCs isolated in this study expressed high levels of VEGF and IGF-2 mRNA, as identified by qRT-PCR. Both these factors are potent stimulators of angiogenesis known to be up-regulated during the proliferative phase of haemangioma in vivo (Takahashi et al., 1994; Ritter et al., 2002). These data suggest that the MSCs present in haemangioma may be an important source of angiogenic regulators that govern haemangioma progression. This study also found that HaemDMSCs expressed mRNA for Collagen -I, -II,-III and -VIII but did not express or secrete collagen protein when cultured in vitro. As these collagen fibres are present in all three phases of 
haemangioma (Tan et al., 2000 a), it may be postulated that in the in vivo environment, HaemDMSCs may be involved in synthesising some of the ECM proteins in haemangioma.

\subsubsection{A ROLE FOR VEGF AND STAT SIGNALLING IN} HAEMANGIOMA IN VITRO

As discussed in Chapter 3 of this thesis, VEGF and STAT protein signalling may be important in haemangioma proliferation. To obtain functional evidence for this, VEGF and STAT signalling was studied in both the haemangioma tissue explants and HaemDMSCs. To elucidate the importance of STAT protein signalling, a JAK/STAT inhibitor, AG490, was added to haemangioma explants and its effect on capillary-like outgrowth was studied. The precise role of VEGF detected in proliferative haemangioma (Takahashi et al., 1994; Tan et al., 2000a) is unknown. In this study, addition of VEGF to haemangioma explants stimulated significant sprouting of outgrowths from haemangioma tissues. This effect was mitigated by addition of AG490 along with VEGF in the cultures. In addition, incubating the explants with various concentrations of AG490 resulted in decreased levels of sprouting. These data suggest a model of haemangioma progression in which an important element is the relationship between VEGF and STAT proteins.

The role of VEGF and STAT protein signalling was also studied in the purified HaemDMSCs cultures. With respect to the VEGFRs, HaemDMSCs expressed only VEGFR-1 but not VEGFR-2, while normal ECs (HUVECs) expressed both receptors. This study found that while addition of VEGF significantly induced the proliferation of HUVECs, it had no effect on HaemDMSCs proliferation. These results were not surprising as previous studies have shown that the activation of VEGFR-2 was required for EC proliferation and migration, while the activation of VEGFR-1 resulted in EC differentiation (Neufeld et al., 1999). 
The observation that proliferation was substantially inhibited by AG490 confirms the importance of STAT protein signalling in HaemDMSCs proliferation. Furthermore, blocking STAT signalling reduced vessel formation of HaemDMSCs, when cultured in a 3-dimensional fibrin matrix in vitro. Similar to the decrease in vessel formation, a dose dependent decrease in cell number was observed with AG490 treatment. These results suggest that the decrease in vessel formation may simply be a secondary effect resulting from decreased cellular proliferation in the presence of AG490.

Various studies have shown that AG490 inhibited phosphorylation and activation of STAT-1, STAT-3 and STAT-5 (Zhang et al., 2000; Andl et al., 2004; Maziere et al., 2001; Hattori et al., 2001; Yamaura et al., 2003; El-Adawi et al., 2002; Banes et al., 2003), however, it is now known that AG490 can affect different members of the STAT family depending on the cell type. Andl et al (2004) showed that AG490 abolished the migration of EPCs by decreasing STAT-1 phosphorylation, while other investigators showed that it inhibited the proliferation of ovarian, breast, pancreatic and renal cell cancer cells by decreasing STAT-3 phosphorylation and STAT-3 DNA binding activity (Burke et al., 2001; Horiguchi et al., 2002; Huang et al., 2006). Mascareno et al (2001) showed that AG490 decreased Ang-2 mRNA expression in ischaemic hearts by inhibiting activation of STAT-5 and STAT-6 and Seki et al (2000) showed that AG490 blocked Ang-2 induced STAT-3 phosphorylation and SMC proliferation. The results obtained with AG490 in this study reveal the importance of STAT protein signalling in HaemDMSCs and in haemangioma. However, further investigation is required to identify the member(s) of the STAT family affected by AG490 in HaemDMSCs.

Studies in other cell culture systems showed increased phosphorylation and nuclear translocation of STAT-3, STAT-5 and STAT-1 following VEGF treatment (Lu et al., 2006; Bartoli et al., 2003; Yahata et al., 2003; Korpelainen et al., 1999). This 
study showed that HaemDMSCs expressed both STAT-3 and STAT-5 protein. Although a small fraction of STAT-3 was phosphorylated at the steady state, VEGF treatment failed to alter the levels and nuclear translocation of both p-STAT-3 (Tyr 705) and p-STAT-1 (Tyr 701). In contrast, increased expression of p-STAT-5 (Tyr 694) was detected within the nuclei of HaemDMSCs following VEGF stimulation.

It is now known that the outcome of STAT signalling is determined by its integration with other activated signal transduction pathways. For example, Korpelainen et al (1999) showed that although all three VEGFRs (VEGFR-1, VEGFR-2 and VEGFR-3) activated both STAT-3 and STAT-5, only VEGFR-1 signalling induced the expression of a cell cycle inhibitor protein, p21 in a STAT-5 dependent manner. They found that although VEGFR-2 and VEGFR-3 signalling suppressed p21 expression, their activity was overcome by increased STAT-5 activation.

This study found that VEGF did not induce HaemDMSCs proliferation or have an affect on p-STAT-3 (Tyr 705) and p-STAT-1 (Tyr 701) signalling. However, as VEGF induced the nuclear expression p-STAT-5 (Tyr 694) and since HaemDMSCs expressed VEGFR-1, it is interesting to speculate that VEGF signalling through pSTAT-5 is required for cellular processes other than proliferation such as cell migration or cell adhesion. Furthermore, since STAT-5 signalling is involved in cell differentiation (Dai et al., 2007; Hoshino \& Fujii, 2007) and adipogenesis (NanbuWakao et al., 2002), a role for STAT-5 as a mediator of haemangioma involution was tested in this study. These results are discussed in section 4.4.5 (page 212).

\subsubsection{ROLE OF OSTEOPROTEGERIN IN HAEMANGIOMA}

This is the first study to report expression of osteoprotegerin (OPG) in haemangioma. OPG, a member of the TNF receptor superfamily (Simonet et al., 1997) is a secreted glycoprotein that exists as a $60 \mathrm{kDa}$ monomer and a $120 \mathrm{kDa}$ disulfidelinked dimer. OPG is critical in the control of bone remodelling by inhibiting 
osteoclastogenesis. It does this by binding the receptor activator of nuclear factor- $\mathrm{KB}$ ligand (RANKL) and thereby preventing the interaction of receptor activator of nuclear factor-кB (RANK) and RANKL (Lacey et al., 1998; Yasuda et al., 1998). Transgenic mice over-expressing OPG have increased bone mass (Simonet et al., 1997) while OPG-deficient mice have decreased bone density (Bucay et al., 1998; Mizuno et al., 1998; Yun et al., 2001). Osteoclasts are responsible for the resorption of bone during normal bone metabolism and for the destruction of bone seen in a variety of pathologies, such as rheumatoid arthritis (Haynes et al., 2003)

In addition to bone, OPG mRNA expression is found in a number of tissues, including lung, heart, kidney, liver, stomach, intestine, brain, spinal cord and the thyroid gland (Simonet WS et al., 1997; Yasuda et al., 1998). This wide tissue distribution of OPG suggests its involvement in processes in addition to bone turnover. This notion is supported by the findings of Yun et al (2001) who showed that OPG regulated B cell maturation and development. Collin-Osdoby et al (2001) showed that OPG was involved in the inflammatory functions of ECs. Furthermore, Price et al (2001) showed that administration of OPG prevented vascular calcification induced by treatment with warfarin and supra-physiological doses of vitamin D in rats. Therefore, these studies provide evidence for the protective vascular effects of OPG. In addition, Hofbauer \& Schoppet (2004) have reviewed the links between OPG and vascular disease.

Consistent with previous reports that showed OPG mRNA and protein expression by cultured MSCs and marrow stromal cells (Le Blanc et al., 2004; NevilleWebbe et al., 2004), the HaemDMSCs isolated in this study expressed high levels of OPG mRNA and secreted abundant levels of OPG protein which appeared to be associated with the ECM. This extracellular pattern of OPG expression has also been noted in human osteoblast-like cells (Atkins et al., 2002). Because the HaemDMSCs expressed high levels of OPG, it was hypothesised that OPG may serve as a potential marker for MSCs within the haemangioma tissue in vivo. In addition, as various studies 
have shown that proliferating haemangioma contain ECs that express vWF, double labelling immunohistochemical analysis for vWF and OPG was performed in this study with an aim to differentiate between ECs and MSCs within haemangioma lesions of various developmental stages. High OPG expression was identified in the cells surrounding the blood vessels. These cells also exhibited weak (punctate) staining for vWF. These results suggest that these cells are either immature ECs (the punctate staining pattern may represent the immature state of the ECs) or perhaps MSCs which have the capacity to differentiate into ECs. Alternatively, it may be possible that the MSCs express low levels of vWF when present within the tissue but loose vWF expression when cultured in vitro. Findings to support this hypothesis include those of Nagaya et al (2004) who showed that MSCs expressed vWF and were involved in neovascularisation in the infarcted heart.

In addition, the regions with high OPG expression and punctate vWF staining also contained more PCNA-positive cells. This indicated that these regions were rapidly proliferating. Importantly, as the lesions progressed towards the involuting phase, OPG and PCNA expression decreased concomitant with increased vWF staining. High PCNA expression in proliferating lesions with decreased abundance in involuting lesions has already been shown in Chapter 3. These results indicate that OPG protein expression correlates with proliferation.

Because of the high levels of extracellular OPG staining identified on HaemDMSCs and the high OPG expression detected in haemangioma tissue sections, we hypothesise that in haemangioma, OPG acts as a survival factor and affords protection against apoptosis in vivo and in vitro. Previous studies have implicated OPG as a mediator of cell survival. Malyankar et al (2000) showed that purified OPG protected ECs from apoptosis induced by growth factor deprivation. Cross et al (2005) showed that OPG stimulated EC growth, as well as the formation of cord-like structures on a matrigel substrate to a similar extent as VEGF. In addition, the increased 
extracellular OPG secretion by HaemDMSCs could also be a stress response associated with the in vitro culture of these cells.

The cells isolated from dissociated tissue also expressed OPG at similar levels to HUVECs rather than the high extracellular levels described for HaemDMSCs. Together these data suggest that the dissociated tissue contains abundant EPCs rather than the HaemDMSCs. This discrepancy can be accounted for by the observation that the putative EPCs spontaneously differentiated in culture to form MSCs, and that the resulting haemangioma-derived MSCs were able to differentiate into adipocytes and osteoblasts as for the HaemDMSCs. It has been previously suggested that adipocytes and ECs have a common progenitor (Planat-Benard et al., 2004). This data supports this postulate as the EPCs identified in haemangioma were able to form adipocytes. We suggest that the EPCs are stem cells that give rise to MSCs which are able to form adipocytes and osteoblasts. While these putative endothelial stem cells are pluoripotent and are capable of forming self-renewing MSCs, the self-renewing potential of the endothelial stem/progenitor cells was not demonstrated in this study. The observation that VEGF treatment of HaemDEPCs was able to inhibit the spontaneous differentiation to MSCs suggests a role for VEGF in EC maturation in haemangioma.

\subsubsection{THE ROLE OF MSCs AND EPCs in HAEMANGIOMA}

To place the results of this study into context, an introduction to MSCs and EPCs is described in sections 4.4.4.1 and 4.4.4.2. In addition, several models for haemangioma progression were hypothesised from the findings of this study together

with the previous identification of EPCs and MSCs in haemangioma (Yu et al., 2004; Yu et al., 2006). These models are discussed in section 4.4.4.3.

\subsubsection{MESENCHYMAL STEM CELLS}

Stem cells are an attractive source of cells for tissue engineering because of their unique biological properties. Stem cells have been isolated from a variety of somatic 
tissues (Korbling, et al., 2003). MSCs represent a subset of these cells residing in the bone marrow. Other sources of MSCs included peripheral blood (Pittenger et al., 1999; Zvaifler et al., 2000), umbilical cord blood (Kim et al., 2004) and also connective tissue sites including the dermis, muscle, adipose, trabecular bone, and synovial membranes (Tuan et al., 2003). MSCs are also present in term cord blood (Zvaifler et al., 2000), in foetal organs (such as liver, BM, and kidney), the blood of preterm foetuses (Erices et al., 2000; Campagnoli et al., 2001; Almeida-Porada et al., 2002) and the placenta (Fukuchi et al., 2004).

MSCs can be expanded in the laboratory and can be induced to differentiate along multiple lineages giving rise to cartilage, bone, fat, muscle, ECs and vascular tissue in vitro and in vivo (Jiang et al., 2002; Abedin et al., 2004; Phinney, 2002; Devine et al., 2003), displaying the important properties of self-renewal and plasticity of stem cells.

One of the most interesting characteristics of MSCs is their ability to home to sites of tissue damage or inflammation (Devine et al., 2001). Homing of MSCs have been demonstrated in the settings of bone fracture, cerebral ischemia and the infarcted heart (Mosca et al., 2000; Devine et al., 2001; Devine et al., 2002; Price et al., 2006). Saito et al (2002) demonstrated that when MSCs were administered intravenously, these cells engrafted within regions of myocardial infarction and a distinct subpopulation participated in angiogenesis. Pittenger \& Martin (2004) showed that the "window" of MSC homing was limited i.e., if MSC administration was delayed until two weeks after infarction, no significant cardiac engraftment was observed and that most of the cells had returned to the bone marrow. In addition, Rombouts \& Ploemacher (2003) showed that the in vitro expansion of MSCs diminished the efficacy of injury-induced homing.

The possible involvement of MSCs in neovascularisation has been recently proposed due to their capacity to contribute to tumour angiogenesis in vivo (Reyes et al., 2002) and due to their plasticity to differentiate into endothelial-like cells (Reyes et al., 
2001). Tang et al (2003) showed that neovascularisation induced by MSCs involved secretion of paracrine factors by the MSCs themselves. This resulted in vessel sprouting and incorporation of MSCs into newly forming blood vessels. In addition, Silva et al (2004) found increased vascularity and improved cardiac function with the implantation of MSCs into chronically ischaemic myocardium. These authors showed that implanted MSCs homed to the site of injury and differentiated into both, ECs and SMCs, which formed the vessel walls.

MSCs have also been shown to differentiate into ECs when cultured in the presence of VEGF (Oswald et al., 2004). Wu et al (2005) showed that when MSCs were co-cultured with mature ECs, about $25 \%$ of the MSCs began to express KDR but not vWF. Their morphological appearance was also different from that of the adjacent mature ECs. Previous studies have shown that ECs go through different stages of differentiation. They initially express VEGFR-2, one of the earliest differentiation markers for ECs, which plays an important role in ECs growth and vasculogenesis, followed in sequence by TIE-2, TIE-1, VE-cadherin, CD34 and vWF (Vittet et al., 1996; Yamashita et al., 2000). Wu et al (2005) suggested that MSCs possess the milieudependent differentiation potential along endothelial lineage when co-cultured with mature ECs. These authors also found that the VEGFR-2-positive MSCs cells did not co-express CD105 or CD166. These results indicate that the cells lose the phenotypic characteristics of MSCs as they differentiate toward ECs. In addition, Wu et al (2005) showed that the addition of anti-VEGF antibodies inhibited the differentiation of MSCs, indicating the important role of VEGF in EC differentiation.

\subsubsection{ENDOTHELIAL PROGENITOR CELLS}

EPCs were first discovered by Ashara et al (1997) as a population of cells that co-expressed EC specific markers such as vWF (Shi et al., 1998) and VEGFR-2 (Peichev et al., 2000) along with the stem cell marker, CD133 (Fargeas et al., 2003). 
Since then, EPCs have been found in the bone marrow, blood circulation, foetal liver and skeletal muscle (Peichev et al., 2000; Majka et al., 2003).

EPCs are involved in tissue neovascularisation, post-natal organ generation, arteriogenesis and also tumour angiogenesis (Rafii et al., 2002; Rafii \& Lyden, 2003; Fuchs et al., 2001; Asahara et al., 1997; Al-Khaldi et al., 2003; Kocher et al., 2001 Orlic et al., 2001; Jackson et al., 2001; Kinnaird et al., 2004; Carmeliet \& Luttun, 2001). Incorporation of EPCs and MSCs into sites of physiological and pathological neovascularisation in vivo has also been reported (Asahara et al., 1997; Asahara et al., 1999; Shi Q et al., 1998). Studies have also shown that EPCs are highly proliferative. These cells form new blood vessels de novo as well as enhance neovascularisation when administered systemically (Rafii \& Lyden, 2003; Kalka et al 2000).

Tepper et al (2005) showed that hypoxia initiated a cellular cascade with localised migration and proliferation of bone marrow-derived EPCs in vitro and in vivo, which resulted in angiogenesis. In animal models of myocardial infarction, the injection of ex vivo expanded EPCs significantly improved blood flow and cardiac function (Kawamoto et al., 2001; Kocher et al., 2001). Furthermore, Lyden et al (2001) showed that impaired recruitment of bone marrow-EPCs blocked tumour angiogenesis and growth. Various studies also showed that terminally differentiated mature ECs did not improve neovascularisation (Kalka et al., 2000; Kocher et al., 2001; Hur et al., 2004).

\subsubsection{PROPOSED MODELS FOR HAEMANGIOMA}

\subsection{The Original Model}

One of the main findings from this study is that MSCs rapidly grow out from explanted haemangioma biopsies. This suggests that MSCs are present at high numbers in haemangioma. The initial clinical description of a promontory mark of haemangioma as 'an anemic nevi' or 'area of low blood flow' suggests that tissue hypoxia, a powerful stimulus for neovascularisation, may be involved (Al Buainian et al., 2003; Hamlat et 
al., 2005). A recent study has also reported that proliferating haemangiomas contain large numbers of haematopoietic cells of the myeloid lineage which are known to migrate to zones of hypoxia (Ritter et al., 2006). Additionally, Kleinman et al (2007) demonstrated that hypoxia-induced factors which are important for post natal vasculogenesis are upregulated in children with proliferating haemangiomas. These include hypoxia inducible factor- $1 \alpha$ (HIF-1 $\alpha)$, stromal cell-derived factor- $1 \alpha$ (SDF-1 $\alpha$ ), matrix metalloprotease-9 (MMP-9) and VEGF-A. Kleinman et al (2007) also suggested that even though overexpression of these factors can occur in normoxic tissues in certain types of cancers (Zhong et al., 1999), the lack of increased HIF-1 $\alpha$ expression in involuting haemangioma biopsies indicates that physiological mechanisms are active in haemangioma and that these lesions are truly hypoxic. There is also data to suggest that hypoxia may be the initial stimulus for chorangioma, a tumour of the placenta that is histologically similar to haemangioma (Ogino et al., 2003).

The original model proposes the recruitment of MSCs to the site of the haemangioma as a result of hypoxia. The recruited MSCs then express high levels of VEGF and other growth factors which act in an autocrine manner to induce MSC differentiation into ECs. This is supported by the findings of Annabi et al (2003) who showed that in a hypoxic environment MSCs secreted increased levels of VEGF that in turn induced MSC migration in an autocrine manner. They also showed increased expression of matrix metalloproteinase-1 (MMP-1) during hypoxia and suggested that this protein was involved in MSCs-mediated tube formation. However, the HaemDMSCs isolated in this study failed to differentiate and exhibit EC characteristics when cultured in the presence of $50 \mathrm{ng} / \mathrm{ml}$ VEGF along with low serum (2\%) and normal serum (10\%) concentrations. These culture conditions have been previously described for MSC differentiation into ECs (Oswald et al., 2004; Wu et al., 2005). These results suggest that HaemDMSCs either lose their ability to differentiate into ECs when in culture in vitro or that a second progenitor cell type is responsible for the origin 
of ECs in haemangioma. Based on these findings, a second model involving EPCs was hypothesised for haemangioma progression.

\subsection{An Alternative Model}

The ECs in early proliferating haemangioma are described as 'angioblastic' and are similar to embryonic rather than neonatal vascular ECs based on their morphology and protein expression patterns (Malan, 1974; Pack \& Miller, 1950; Dosanjh et al., 2000). These findings suggest that haemangioma contain primitive ECs. Increased levels of circulating EPCs have been noted in haemangioma patients (Kleinman et al., 2003). Furthermore, Yu et al (2004a) isolated EPCs from proliferating haemangioma by purifying a population of cells that expressed the EC marker, VEGFR-2 and the stem cell marker, CD133. They speculated that these EPCs were responsible for the uncontrolled clonal expansion of ECs in haemangioma.

The cellular pathways of EPC mobolisation and trafficking are becoming clear. HIF-1 $\alpha$, SDF-1 $\alpha$ and VEGF-A are now known to be capable of enhancing the mobilisation and recruitment of EPCs to hypoxic tissues during post natal vasculogenesis (Ceradini et al., 2004; Rafii et al., 2003; Kalka et al., 2000; Park et al., 2004; Forsythe et al., 1996). Increased expression of these mediators has been recently identified in proliferating haemangiomas (Kleinman et al., 2007).

In the alternative model for haemangioma (Figure 4.28), both MSCs and EPCs are recruited to the site of the haemangioma as a result of hypoxia during the proliferative phase. Both cell types express high levels of OPG that prevents them from undergoing apoptosis during this phase. The MSCs secrete growth factors such as VEGF which first induce EPC proliferation followed by EPC differentiation into mature ECs as the lesion progresses towards involution. The presence of EPCs together with the possibility that MSCs can then themselves differentiate into vascular smooth muscle cells now creates a perfect environment to form functional capillary networks in vivo. This phenomenon has been recently shown by Melero-Martin et al (2007). These 
authors found that implantation of EPCs with SMCs subcutaneously into immunodeficient mice resulted in the formation of an extensive blood vessel network within one week. Wu et al (2004) showed that EPC-derived ECs had the ability to assemble into a microvascular-like network when seeded on three-dimensional glycolic scaffolds along with SMCs.

The alternative model is based on results from other studies that have reported the differentiation of EPCs into mature ECs (Gehling et al., 2000; Peichev et al., 2000). In addition, studies have also reported the secretion of angiogenic factors such as VEGF by MSCs (Hamano et al., 2000; Villars et al., 2000). The VEGF family of polypeptides includes VEGF-A, VEGF-B, VEGF-C, VEGF-D, VEGF-E and PIGF (Korpelainen EI \& Alitalo K, 1998; Veikkola et al., 2000; Veikkola \& Alitalo, 1999). The VEGF-A gene can be alternatively spliced resulting in gene products corresponding to amino acids 121, 165, 189 and 206 main splice forms in humans. VEGF-A is expressed during MSC-derived osteoblastogenesis (Furumatsu et al., 2003; Mayer et al., 2005). Mayer et al (2005) showed that mRNA for $\mathrm{VEGF}_{165}, \mathrm{VEGF}_{121}$ and VEGFR-1 increased during osteogenenesis and also under hypoxic conditions. Furthermore, this secreted VEGF induced increased EC sprouting. Therefore, these authors suggested that human MSCs secreted VEGF-A as an angiogenic factor in a differentiation dependent manner. In addition, VEGF has been shown to mobilise EPCs from bone marrow (Asahara et al., 1997) and also cause EPC maturation to ECs (Ehrbar et al., 2005).

qRT-PCR results from this study showed that HaemDMSCs expressed VEGF and IGF-2 mRNA transcripts to similar levels as that seen in the original haemangioma tissue. Both these factors have been identified as modulators of haemangioma in the proliferative phase in vivo (Takahashi et al., 1994; Ritter et al., 2000). Since Yu et al (2001) found that the isolated ECs from proliferating haemangiomas expressed very low levels of VEGF transcript, we hypothesised that the HaemDMSCs may be the source for at least some of the angiogenic modulators of haemangioma. However, Western 
blotting experiments showed that the secreted isoforms of VEGF-A, VEGF 121 and VEGF $_{165}$ were not present in the conditioned medium of the cultured HaemDMSCs. In addition, Western blotting results showed that neither $\mathrm{VEGF}_{121}, \mathrm{VEGF}_{165}$ nor $\mathrm{VEGF}_{189}$ were detected within HaemDMSCs themselves or in proliferative haemangioma tissue.

Other studies that reported on the high expression of VEGF protein during the proliferative phase (Tan et al., 2000a ;akahashi et al., 1994) did not provide details on the member(s) of the VEGF family detected in their studies. Although, all members show some degree of sequence homology, the anti-VEGF antibody used in this study was specific for the $\mathrm{VEGF}_{121}, \mathrm{VEGF}_{165}$ and $\mathrm{VEGF}_{189}$ isoforms of VEGF-A. Therefore, these findings suggest that VEGF expression seen in haemangioma may be that of the other members of the VEGF family and not the VEGF isoforms of VEGF-A which were tested for, in this study.

$\mathrm{Yu}$ et al (2006) first speculated that MSCs were the source of adipocytes that appeared during involution of haemangioma. Consistent with this theory, this investigation found that MSCs rapidly grew out from explanted haemangioma tissue pieces and that these HaemDMSCs maintained their adipocytic and osteocytic differentiation properties in vitro (The Alternative Model; Figure 4.28). These findings further support the model in which MSCs are likely to be precursors of the adipocytes during involution of haemangioma in vivo. 
Figure 4.28: An Alternative Model For Haemangioma. This model suggests that there are two main cell types, the MSCs and EPCs which are involved in the pathogenesis of haemangioma. Both cell types may be recruited to the site of the haemangioma perhaps, in response to hypoxia. Both cell types express increased levels of OPG and exhibit punctate immunoreactivity for vWF indicating their immature state. High OPG expression prevents apoptosis during the proliferative phase. This model suggests that the MSCs are the main source for a variety of angiogenic modulators such as VEGF and IGF-2. These growth factors secreted by MSCs can in turn act on the recruited EPCs to enhance their survival, proliferation and also induce EPC maturation to ECs. This model also proposes that a yet-to-be identified signal is involved in inducing MSCs differentiation into the adpiocytes which is seen during involution and that when a proliferating haemangioma explant is cultured in vitro, it is the MSCs that rapidly grow out from the tissue piece. These HaemDMSCs maintain their ability to differentiate into adipocytes and osteocytes in vitro. The role of IFNs and STAT proteins in haemangioma is discussed further in section 4.4.5. 


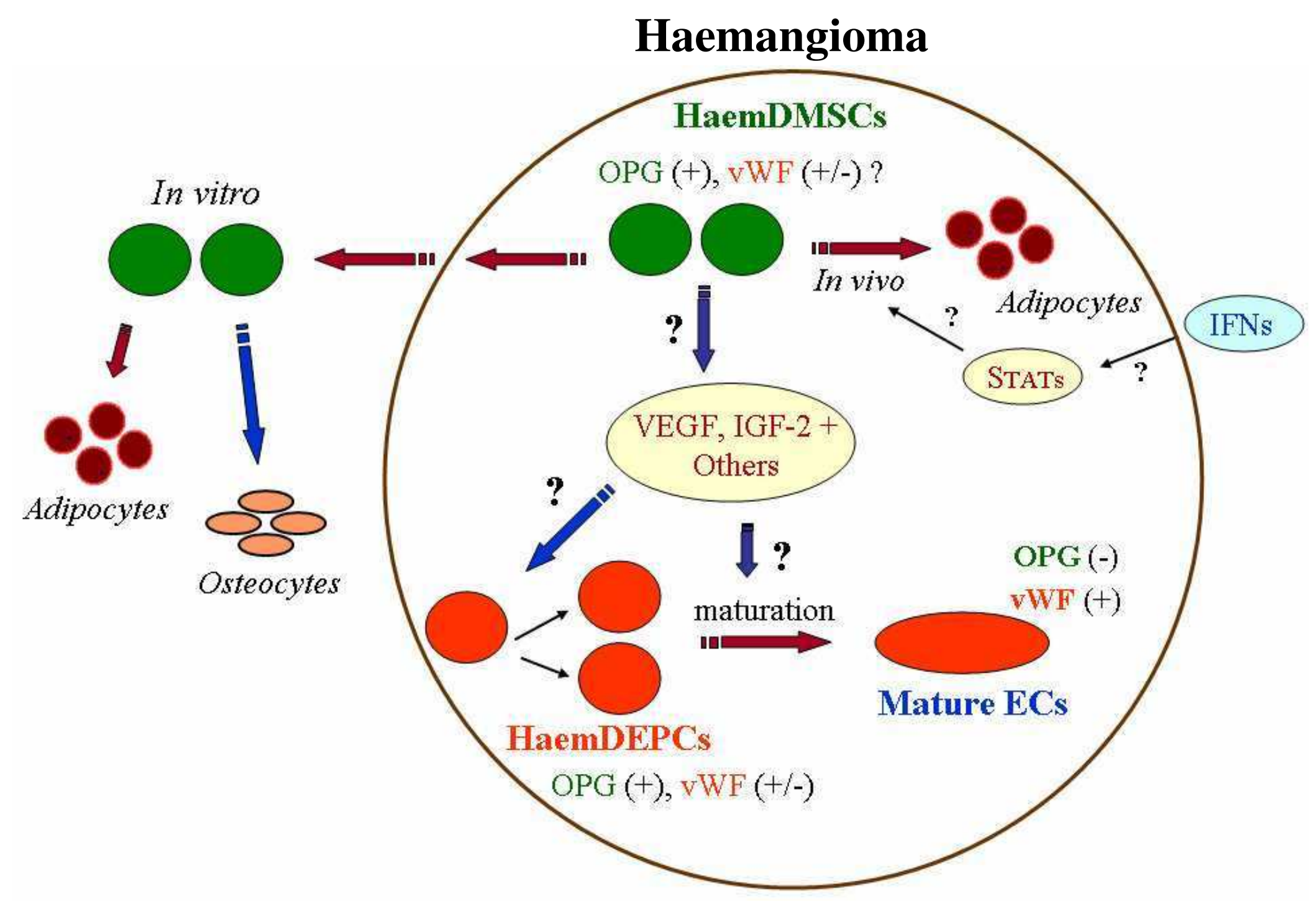




\subsection{The Final Model}

Towards the end of this study, tissue from a proliferating haemangioma was treated with collagenase to obtain a single cell suspension and the cells were cultured in vitro. These cells had a distinct appearance in that they had round central bodies with some cytoplasmic projections. This morphology has been shown to be typical of EPCs (Asahara et al., 1997; Quirici et al., 2001). After two days in culture, cells obtained directly from dissociated proliferative tissue biopsies were also immunophenotypically similar to EPCs as they stained for vWF, VEGFR-1 and VEGFR-2 which is consistent with previous findings (Ziegler et al., 1999). VEGFR-2 is one of the earliest markers expressed on EPCs. In addition, although these cells expressed OPG, the pattern of OPG expression was not extracellular as seen in MSCs, but was similar to the OPGvWF staining pattern seen on normal ECs or HUVECs.

Following continued culture, an interesting change in cell morphology to that of the flat, broadened shape of MSCs was noted within five days. The cells now lost vWF expression and exhibited the MSC-like extracellular pattern of OPG expression. Furthermore, these cells differentiated into adipocytes under appropriate conditions which confirmed their plasticity and mesenchymal characteristics. However, when the same cells were cultured in VEGF for eight days, some cells retained their EPC-like morphology and continued to express both $\mathrm{vWF}$ and OPG, while some others showed MSC morphology, did not express vWF and expressed high levels of extracellular OPG.

From these results, we propose a final model for haemangioma in which the HaemDEPCs represent the primary and most important component in a proliferating haemangioma (Figure 4.29). In this model, the high levels of VEGF present within the lesions during the proliferative phase are involved in EPCs survival, proliferation and/ or maturation of ECs. Results from this study also suggest that VEGF alone may not be enough to maintain the endothelial phenotype of the HaemDEPCs and that other growth factors such FGF-2 or IGF-2 may be required. High levels of both these growth factors 
have been identified during the proliferative phase and have been found to decrease with involution (Tan et al., 2000a ; Ritter et al., 2001). Khan et al (2006) also isolated HaemDEPCs from proliferative haemangioma biopsies and maintained them in culture medium containing various factors including VEGF, FGF-2, IGF-2, hydrocortisone, heparin, ascorbic acid, and cAMP. These authors did not provide any information on the consequence of removing these factors.

In this study, all the HaemDEPCs cultured in vitro spontaneously differentiated into MSCs in the absence of VEGF. We established this phenomenon as a transdifferentiation process as opposed to a situation in which one cell type outgrows another within the same culture dish. This was based on the following reasons. In the absence of VEGF, a change in cell morphology was observed to be uniform across the entire dish with every single cell exhibiting a flat, broadened shape within five days in culture. If two cell types were present and one cell type was outgrowing the other, the presence of both cell types would have been seen sometime during the two-five day culture period but this was not the case. In contrast, both cell types (EPCs and MSCs) were observed in the second situation examined in this study i.e., culturing HaemDEPCs in the presence of VEGF. This suggests that VEGF along with other factors may be required for HaemDEPCs to maintain their endothelial phenotype.

In the model shown in Figure 4.29, we hypothesise that involution of haemangioma is triggered by a decrease in the levels of VEGF (and other growth factors). The consequence of this is that the EPCs no longer survive and proliferate as ECs but transdifferentiate into MSCs that further differentiates into the adipocytes seen in the involuting and involuted phases of haemangioma. Furthermore, when haemangioma biopsies are cultured in vitro, we believe that it is the EPCs that migrate out but as they migrate further away from the tissue they are no longer exposed to the growth factors present with the original lesion (i.e., VEGF and others). This results in their transdifferentiation from EPCs to MSCs. It was these MSCs that were isolated 
from the in vitro haemangioma model and characterised in this study (see section 4.4.1, page 189). These migrating MSCs retain their ability to form both adipocytes and osteocytes in vitro. This is consistent with the findings of Ritter et al (2001) who identified that the sprouting outgrowths contained ECs only in the area immediately adjacent to the explant tissue whereas cells further away from the tissue no longer expressed these markers. The ECs in their study were characterized by their expression for CD31 and CD34 as well as for the positive staining for G. simplicifolia isolectin, a documented endothelial stain (Faraggiana et al., 1989; Sahagun et al., 1989; HansenSmith et al., 2001).

This kind of endothelial to mesenchymal transdifferentiation (EMT) occurs in response to TGF- $\beta$ signalling in the developing heart (Ramsdell \& Markwald, 1997; Boyer et al., 1999). Frid et al (2002) found that a small percentage of cells in EC preparations from bovine arteries can undergo EMT. Dvorin et al (2003) showed that in addition to ECs, EPCs were also capable of transdifferentiating into a mesenchymal phenotype, in response to TGF- $\beta$. Camenisch et al (2002) showed that once EMT took place, endothelial functions such as the ability to form tube-like structures and to adhere leukocytes in response to inflammatory signals, were lost.

Finally, Paruchi et al (2006) have shown that clonal ECs isolated from the pulmonary valves of a nine month-old infant exhibited EMT in response to TGF- $\beta$. However, they stated that the resulting MeCs failed to differentiate into adipocytes or osteocytes. In contrast, the HaemDMSCs isolated in this study, could undergo adipocytic differentiation under appropriate conditions. This suggests that these trandifferentiated cells are MSCs and exhibit MSC-like properties.

From these results we hypothesise that the cells obtained by the direct dissociation of a proliferating haemangioma biopsy are EPCs as they could differentiate from an endothelial phenotype expressing all the ECs markers into MSCs. Furthermore, the pattern of EC marker expression seen on the HaemDEPCs was 
consistent with that detected in vivo by immunohistochemical analysis of haemangioma tissue sections.

The results from this investigation also provide evidence for the role of VEGF in maintaining the endothelial characteristics of HaemDEPCs. Results showed that with the withdrawal of VEGF, the HaemDEPCs differentiated into HaemDMSCs that further differentiated into the adipocytes seen in involuting and involuted haemangioma.

Chapter 3 (section 3.3.2.2.3, page 114) described the expression of VEGFR-1 in all phases of haemangioma whereas VEGFR-2 expression decreased with involution. VEGFR-1 expression detected on both EPCs and MSCs isolated in this study indicates that its constant expression may be required for the differentiation processes occurring throughout the different developmental phases of haemangioma progression, i.e. EPCs to mature ECs and EPCs to MSCs to adipocytes. In contrast, VEGFR-2 expression detected only on EPCs suggests that activation of this receptor, in response to the high levels of VEGF, could be responsible for EPCs surivival and proliferation during the proliferative phase (The Final Model, Figure 4.29).

Finally, although CD133 expression by HaemDEPCs was not directly investigated in this study, the ability of these cells to differentiate from one cell type to another meets the criteria of a stem cell (Lovell \& Mathur, 2004). A point to note here is that CD133 expression is now known to be quickly down-regulated in culture (Wu et al., 2004). To our knowledge there have not been any previous reports of an EPC to MSC transdifferentiation process occurring in haemangioma. Therefore, these findings represent novel insights into the pathogenesis of haemangioma. In the final model, we hypothesise that the EPC to MSC differentiation process governs haemangioma biology (Figure 4.29). 
Figure 4.29: The Final Model For Haemangioma. In this model, the EPCs represent the primary component of a proliferating haemangioma. These cells express OPG which acts as a survival factor. They also show punctuate or weak immunoreactivity when immunostained for vWF. This punctuate staining may represent their immature developmental state. During the proliferative phase, high levels of growth factors, such as VEGF present in the lesion act on the EPCs to enhance their survival and induce their proliferation. VEGF may also play a role in EPC maturation into ECs. These mature ECs exhibit intense immunoreactivity when immunostained for vWF, seen as the lesion progresses towards involution. The decreasing concentrations of VEGF and other growth factors in the involuting phase results in the differentiation of the EPCs to MSCs. These MSCs can then further differentiate into the increasing numbers of adopicytes seen in the involuting and involuted phases of haemangioma. In addition, this model suggests that when proliferative haemangioma explants are cultured in vitro, the EPCs grow out but as they migrate further away from the original tissueand are thereby exposed to low VEGF concentrations, the cells transdifferentiate into MSCs. These HaemDMSCs maintain their ability to differentiate into adipocytes and osteocytes in vitro. Finally, although the findings from this investigation did support the notion that IFN $\alpha-2 b$ can accelerate adipogenesis, it would be interesting to further investigate the role of STAT proteins in haemangioma adipogenesis. 


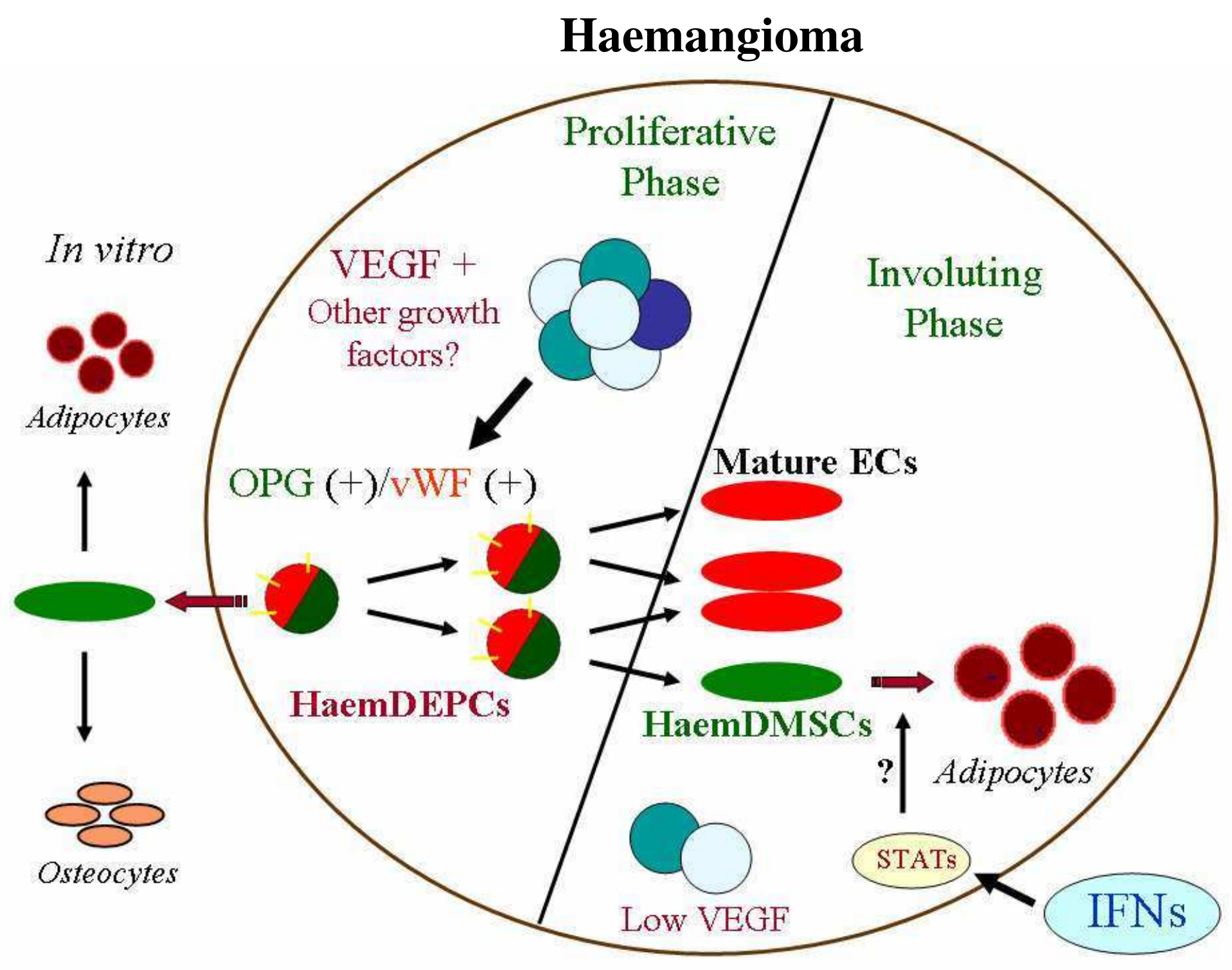




\subsubsection{ROLE OF INTERFERONS AND STAT PROTEINS IN}

\section{HAEMANGIOMA ADIPOGENESIS}

Results from this study also provide evidence for the involvement of STAT proteins and IFN signalling in HaemDMSCs (see section 4.3.6, page 179). The identification of increased expression of IFN stimulated genes together with the increased expression of IFN $\beta$ in the dermis overlying involuting and involuted haemangioma (Ritter et al., 2000; Bielenberg et al., 1999) suggests that IFN signalling may be important in driving haemangioma involution. As IFN $\alpha-2 b$ has been used as treatment for haemangioma, we hypothesise a model for haemangioma in which expression of IFNs in the overlying dermis of involuting and involuted haemangioma activates the STAT proteins which then causes and/or accelerates MSC adipogenesis (Figures 4.28 and 4.29). Several members of the STAT family play a role in the regulation of genes that contribute to the phenotype of the mature adipocyte (Stephens et al., 1996; Stephens et al., 1999; Floyd and Stephens, 2003).

In order to provide functional evidence for the role of IFNs and STAT proteins in adipogenesis, adipocytic differentiation of HaemDMSCs was induced in presence of IFN $\alpha-2 b(15,000 \mathrm{U} / \mathrm{ml})$ and AG490 $(20 \mu \mathrm{M})$. As both drugs inhibited HaemDMSCs proliferation (see section 4.3.4.2, page 165, for AG490 and Chapter 5, section 5.3.4, page 224 , for IFN $\alpha-2 b$ ), the doses selected for the differentiation experiments were chosen with the rationale being that the drugs should have a biological effect on their target proteins without significantly affecting cell proliferation. Failure to detect accelerated adipogenesis with IFN $\alpha-2 b$ may be due to several possibilities, (i) the physiological concentrations of IFNs in haemangioma is currently unknown therefore the arbitrarily chosen IFN $\alpha-2 b$ concentration used in the differentiation experiments of this study, may not have been high enough to induce adipogenesis above that of the control, (ii) this study did not examine the effects of other IFNs such as IFN $\beta$ and IFN $\gamma$ which also activate STAT proteins (Darnell, 1997; Durbin et al., 1996; Meraz et al., 
1996). Given that IFN $\beta$ expression has been detected in the dermis overlying involuting haemangioma (Bielenberg et al., 1999), it may be useful to test its effect on HaemDMSCs adipogenesis in future experiments. In addition, as stated in section 4.4.2. (page 191), AG490 affects different STAT proteins depending on the cell type. The identification of adipocytes in the presence of AG490 suggests that the particular STAT protein members that are involved in HaemDMSC adipogenesis are not inhibited by AG490. 


\section{CHAPTER 5: TRAIL - A POTENTIAL TREATMENT FOR HAEMANGIOMA?}

\subsection{INTRODUCTION}

Although most haemangiomas require no intervention (Margileth et al., 1965), treatment is necessary in 10 to $20 \%$ of cases during the proliferative phase because of their location, size, or behaviour (Takahashi et al., 1994; Mulliken et al., 1995). The mainstay treatment for haemangioma during the proliferative phase is pharmacologic therapy with glucocorticoids being the first line of treatment (Mulliken et al., 1995). However, not all lesions respond to steroids. IFN $\alpha$ is considered the second-line of treatment and is indicated for lesions that fail to respond to steroid therapy (Mulliken et al., 1995; White et al., 1991; Spiller et al., 1992; Blei et al., 1993; Ohlms et al., 1994), with accelerated regression seen in $90 \%$ of cases (Mulliken et al., 1995). However, IFN treatment is accompanied by serious side-effects (Vial \& Descotes, 1994; Barlow et al., 1998; Dubois et al., 1999). Most children given IFN $\alpha$ develop fevers for the first onetwo weeks of therapy (Tan, 2001). Reversible toxicoses such as transient neutropenia, anaemia and a five-fold increase in liver transaminases is also seen (Tan, 2001). Significant neurotoxicity also occurs with spastic diplegia in $10 \%$ of cases (Barlow et al., 1998). The neurological sequelae are arrested by cessation of treatment. In some cases they resolve completely but in others varying degrees of residual deficit persists (Tan, 2001).

The actual mechanism of action of IFNs in the regression of haemangioma is unknown. IFNs are a family of cytokines which are classified into two groups, type I (IFN $\alpha$ and IFN $\beta$ ) and type II (IFN $\gamma$ ). In addition to their anti-viral effects, IFNs exhibit pleiotropic biological activities, including anti-tumor, anti-proliferative and 
immunomodulatory effects (Morris and Zvetkova 1997; Gresser et al 1997; Stark et al., 1998) and transcriptionally regulate over 100 genes (Stark et al., 1998).

IFNs act as apoptosis-inducing cytokines on various cancer cell lines (ChawlaSarkar et al., 2003), including multiple myeloma, (Chen et al., 2001), melanoma (Chawla-Sarkar et al., 2001), and ovarian carcinoma (Morrison et al., 2002). Greater cell growth-inhibitory effects of IFN $\beta$ compared with IFN $\alpha$ has been shown consistently (Borden et al., 1982; Rosenblum et al., 1990; Johns et al., 1992). IFNs have also been shown to up-regulate the expression of different apoptosis-related proteins including death receptors and their respective ligands, caspases and several members of the Bcl-2 family in a variety of cells (Ossina et al., 1997; Spanaus et al., 1998). Transcriptional induction of TRAIL has been found to be one of the earliest events following IFN administration (Chen et al., 2001; Sato et al., 2001). IFN $\beta$ pre-treatment has been found to sensitise human melanoma cells to TRAIL-induced apoptosis (Chawla-Sarkar et al., 2002; Leaman et al., 2002), which were otherwise resistant to TRAIL treatment alone.

TRAIL is a member of the TNF superfamily, that induces rapid apoptosis in a wide variety of transformed cell lines, but appears to have little or no detectable toxicity on normal cells in vitro and in vivo (Walczak et al., 2000; Pitti et al., 1996; Walczak et al., 1999). TRAIL binds to four distinct membrane-bound receptors, DR4 (TRAIL-R1), DR5 (TRAIL-R2), DcR1 (TRAIL-R3), DcR2 (TRAIL-R4), and a soluble receptor, OPG. These receptors are expressed by tumour, transformed and normal cells (Griffith et al., 1999; Walczak et al., 1997; Green, 2000; Spierings et al., 2004). TRAIL induces apoptosis through the activation of DR4 and DR5, which engage the 'cell-extrinsic' apoptosis pathway (LeBlanc \& Ashkenazi, 2003; Kelly and Ashkenazi, 2004) (Figure 5.1). TRAIL binding, leads to the formation of the death inducing signalling complex (DISC) consisting of the apoptosis 'initiating' proteases, caspase- 8 and caspase-10, and an adaptor molecule, the Fas-associated death domain (FADD). Once recruited, the activated caspase- 8 and -10 in turn activate 'effector' caspases, including caspase-3, -6 
and -7, which then execute the cell's apoptotic demise (Sprick et al., 2000; Kischkel et al., 2000). In some cancer cell lines, TRAIL-induced caspase-3 activation is further augmented through engagement of the cell-intrinsic apoptosis pathway (LeBlanc et al., 2002; Deng et al., 2002; Ravi and Bedi, 2002) (Figure 5.1). In this case, caspase-8 or caspase-10 cleaves and activates the pro-apoptotic Bcl-2 family member Bid, which then interacts with two other pro-apoptotic members, Bax and Bak, to induce the release of cytochrome $c$ and Smac/Diablo from the mitochondria. Cytochrome $c$ together with Apaf-1 activates caspase-9, which contributes to further activation of caspase-3, -6 and 7. Smac/Diablo binds to inhibitor of apoptosis proteins (IAPs), such as X-chromosomelinked IAP (XIAP). This prevents the IAPs from physically binding caspase-3, hence promoting further caspase-3 activation. In certain cancer cells, Bax is essential to connect the extrinsic and intrinsic pathways. Loss of function mutations have been shown to render cells resistant to TRAIL. However, the resistance associated with Bax inactivation can be circumvented by pre-exposing the cells to chemotherapy, which upregulates levels of DR5 and Bak (Kelley \& Ashkenazi, 2004)

Binding of TRAIL to DcR1, DcR2 and OPG does not transduce the death signal as they do not contain a functional cytoplasmic death domain and are therefore considered as competitive decoys receptors for TRAIL (Griffith et al., 1998; Ashkenazi et al., 1999; Degli-Esposti, 1999; Ashkenazi \& Dixit, 1998). Interestingly, DcR1 and DcR2, as anti-apoptotic genes, are expressed in many normal tissues, but their expression are frequently lost in various types of human cancers (Ashkenazi \& Dixit, 1998; Kelley Ashkenazi, 2004).

In addition to inducing apoptosis in cancer cell lines (Mitsiades et al., 2001; Naka et al., 2002), recombinant soluble TRAIL shows remarkable efficacy against tumour xenografts of colon carcinoma (LeBlanc et al., 2002; Ashkenazi et al., 1999), breast carcinoma (Walczak et al., 1999), multiple myeloma cells (Mitsiades et al., 2001) and glioma cells (Fulda et al., 2002; Pollack et al., 2001). 
Furthermore, the potential for TRAIL to act synergistically with other anticancer therapies is of particular interest. TRAIL has been shown to act synergistically when combined with IFNa (Shigeno et al., 2003), genotoxic agents including doxorubicin, cisplatin or etoposide (Arizono et al., 2003; Bouralexis et al., 2003; Miao et al., 2003; Hotta et al., 2003; Jones et al., 2003; Xu et al., 2003), DNA-damaging drugs (Morrison et al., 2002; Ashkenazi et al., 1999), radiotherapy (Gong \& Almasan 2000; Chinnaiyan et al., 2000; Ramp et al., 2003), and cyclooxygenase-2 inhibitors (Totzke et al., 2003).

IFN $\alpha$ was found to sensitise cells to TRAIL-induced apoptosis through an upregulation of DR4 and DR5 (Oshima et al., 2001; Shigeno et al., 2003). In addition, Kumar-Sinha et al (2002) showed that a synergistic apoptotic response was achieved in breast cancer cells with the combined treatment of IFN $\alpha$ and TRAIL. In certain tumour cell lines, IFN induced TRAIL expression by the tumour cells themselves. This resulted in TRAIL-mediated apoptosis by an autocrine or paracrine mechanism (Chen et al., 2001; Oshima et al., 2001). Therefore, from these studies, Kumar-Sinha et al (2002) have suggested that IFNs may 'prime' cells for TRAIL induced apoptosis. 


\section{Cell-extrinsic}

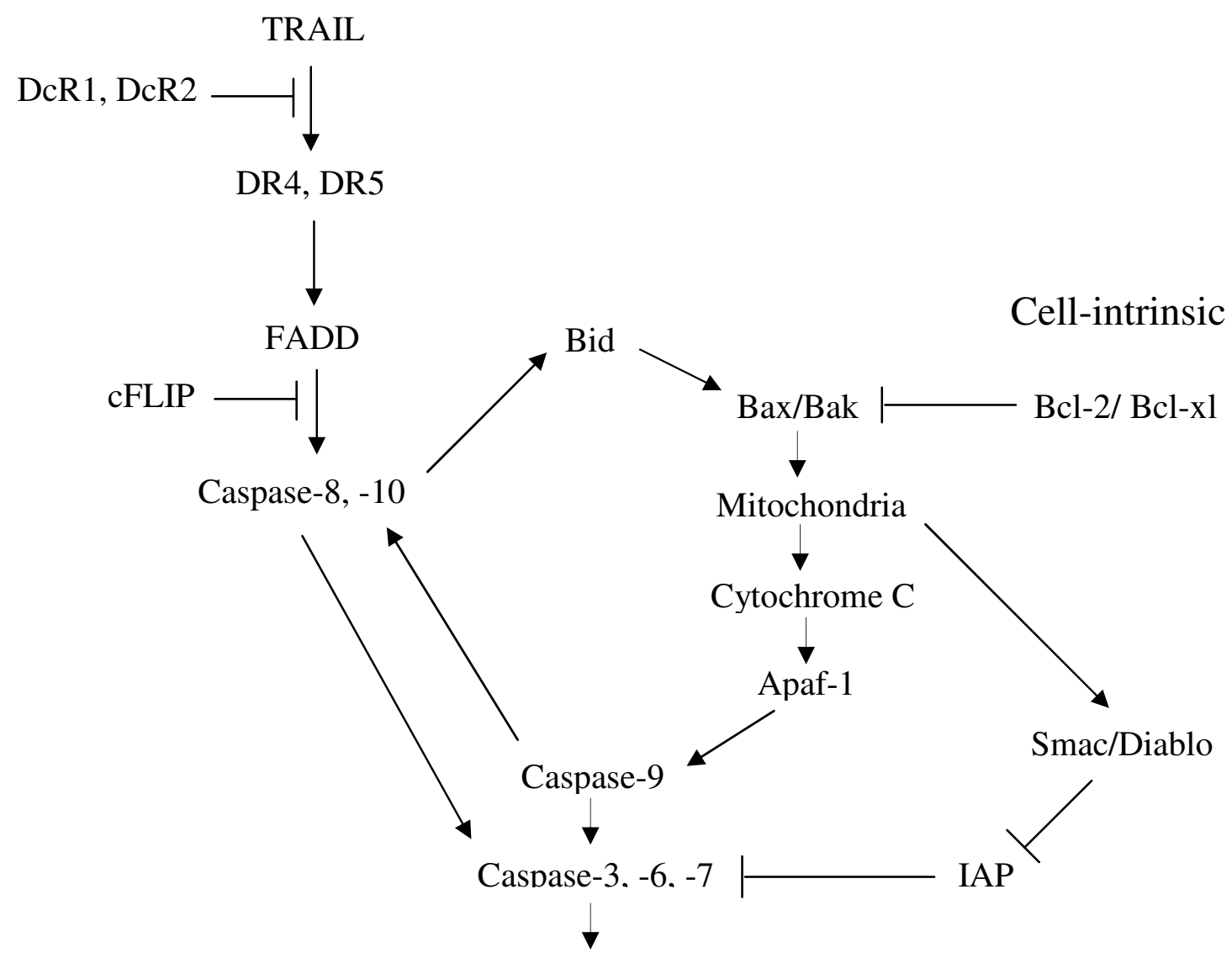

APOPTOSIS

Figure 5.1: Activation of the cell-extrinsic and cell-intrinsic apoptosis pathways by TRAIL (Image re-drawn from Ashkenazi \& Kelley, 2004). TRAIL binding to the death receptors (DR4 and DR5) activates the cell-extrinsic pathway and is sufficient to trigger apoptosis in some cell types, whereas in other cell types amplification of this pathway through the intrinsic pathway is needed to commit the cell to apoptosis. Crosstalk between these two pathways involves cleavage of Bid and subsequent activation of Bax and Bak. See text for details. 


\subsection{AIMS}

As described in section 5.1, various reports of the anti-tumour effects of IFNa have been documented. In addition, IFN $\alpha$ has been shown to be effective as an angiogenic inhibitor in a variety of angiogenic diseases (Gutterman, 1994). Furthermore, the high expression of IFN $\beta$ in the dermis overlying involuting and involuted haemangioma but not in the dermis overlying proliferating lesions, together with the efficacy of IFN $\alpha$ in the treatment of haemangioma, suggests that this cytokine may play an important role in haemangioma involution. Unfortunately, the significant side effects associated with high dose IFN therapy especially neurotoxocicity has limited its use for haemangioma (Tan, 2001). In this study, it was hypothesised that if a lower dose of IFN $\alpha$ shows improved efficacy in combination with another agent, it may be of greater clinical value.

Therefore, in this study the efficacy of the combined treatment of IFN $\alpha-2 b$ with TRAIL was investigated. mRNA levels of TRAIL and its receptors were investigated using qRT-PCR in proliferating, involuting and involuted haemangioma biopsies. IHC analyses of the TRAIL receptors (TRAIL-Rs) on paraffin embedded haemangioma tissue sections were performed. Using the in vitro model (described in Chapter 4), the effect of TRAIL and IFN $\alpha-2 b$ treatment on haemangioma capillary-like growth was investigated. 


\subsection{RESULTS}

\subsection{1 mRNA EXPRESSION OF TRAIL AND ITS RECEPTORS}

Relative changes in mRNA expression for TRAIL, DR4, DR5, DcR1, DcR2 and OPG was determined in 11 proliferating, 4 involuting and 6 involuted haemangioma samples relative to GAPDH as $\triangle \mathrm{Ct}$ values. The average $\triangle \mathrm{Ct}$ values obtained for each gene per phase are presented in Table 8.

TRAIL was abundantly expressed in all three phases of haemangioma at similar levels. The expression profile for TRAIL receptors was similar for the proliferating and involuted samples. DR4 mRNA was the most abundant and was expressed in all three phases at greater levels than DR5 (at least 30-fold more). Surprisingly, DR5 expression could not be detected in the involuting samples although it was detected in the proliferating and involuted specimens $(p<0.05)$ albeit at low levels. The decoy receptors DcR1, DcR2 and OPG were detected at low but comparable levels within all three phases.

\begin{tabular}{ccccccc}
\hline Phase & TRAIL & DR4 & DR5 & DcR1 & DcR2 & OPG \\
\hline $\begin{array}{c}\text { Proliferating } \\
(\mathrm{n}=11)\end{array}$ & $4.3 \pm 0.5$ & $8.1 \pm 0.2$ & $14.3 \pm 0.7$ & $9.5 \pm 0.7$ & $9.5 \pm 0.3$ & $10.7 \pm 0.5$ \\
$\begin{array}{c}\text { Involuting } \\
(\mathrm{n}=4)\end{array}$ & $5.2 \pm 0.3$ & $9.9 \pm 0.3$ & $>18$ & $13.6 \pm 0.3$ & $12.1 \pm 0.4$ & $11.7 \pm 0.7$ \\
$\begin{array}{c}\text { Involuted } \\
(\mathrm{n}=6)\end{array}$ & $3.4 \pm 1.4$ & $8.3 \pm 3.4$ & $13.6 \pm 5.6$ & $10.2 \pm 4.2$ & $10 \pm 4.1$ & $8.8 \pm 3.6$ \\
\hline
\end{tabular}

Table 8: Expression of TRAIL and TRAIL receptors mRNA in haemangioma. Relative expression levels of mRNA for TRAIL and TRAIL receptors in 11 proliferating, 4 involuting and 6 involuted specimens determined by qRT-PCR using GAPDH as the internal house-keeping reference gene. Values are expressed as the average $\triangle \mathrm{Ct}$ value obtained for each gene \pm s.e.m. Lesions from all phases expressed high levels of TRAIL mRNA and moderate levels of DR4, DcR1, DcR2 and OPG mRNA. Very low levels of DR5 mRNA were detected in all samples. 


\subsubsection{PROTEIN EXPRESSION OF TRAIL AND ITS RECEPTORS}

Immunohistochemical staining revealed a positive reaction for all five TRAIL receptors in proliferating haemangioma tissue sections. Confocal laser scanning showed that proliferating haemangioma lesions expressed low levels of DR4 (Figure 5.2-A, white arrows) and DR5 protein (Figure 5.2-B, white arrows), moderate levels of DcR1 protein (Figure 5.2-C, white arrows), high levels DcR2 (Figure 5.2-D, white arrows) and OPG protein (Figure 5.6-A and -B). While staining for DR4, DR5, DcR1 and DcR2 was essentially cytoplasmic in all lesions analysed (Figure 5.2, white arrows), immunoreactivity for OPG was detected in the cytoplasm (Figure 5.6, yellow arrows) as well as within the nuclear compartment in some cells (Figure 5.6, white arrows). Negative controls included immunostaining without primary antibody incubation and showed no staining throughout the study. In addition, antibody specificity for DR4 and DcR2 was determined by Western botting (Figure 5.7). As expected, immunoreactive bands were detected for DR4 and DcR1 at $55 \mathrm{kDa}$ and $32 \mathrm{kDa}$, respectively (Grataroli et al., 2002; Vindrieux et al., 2002). Unfortunately, antibody specificity for the anti-DR5 and anti-DcR1 antibodies could not be verified, as the antibodies did not perform well on Western blots. 

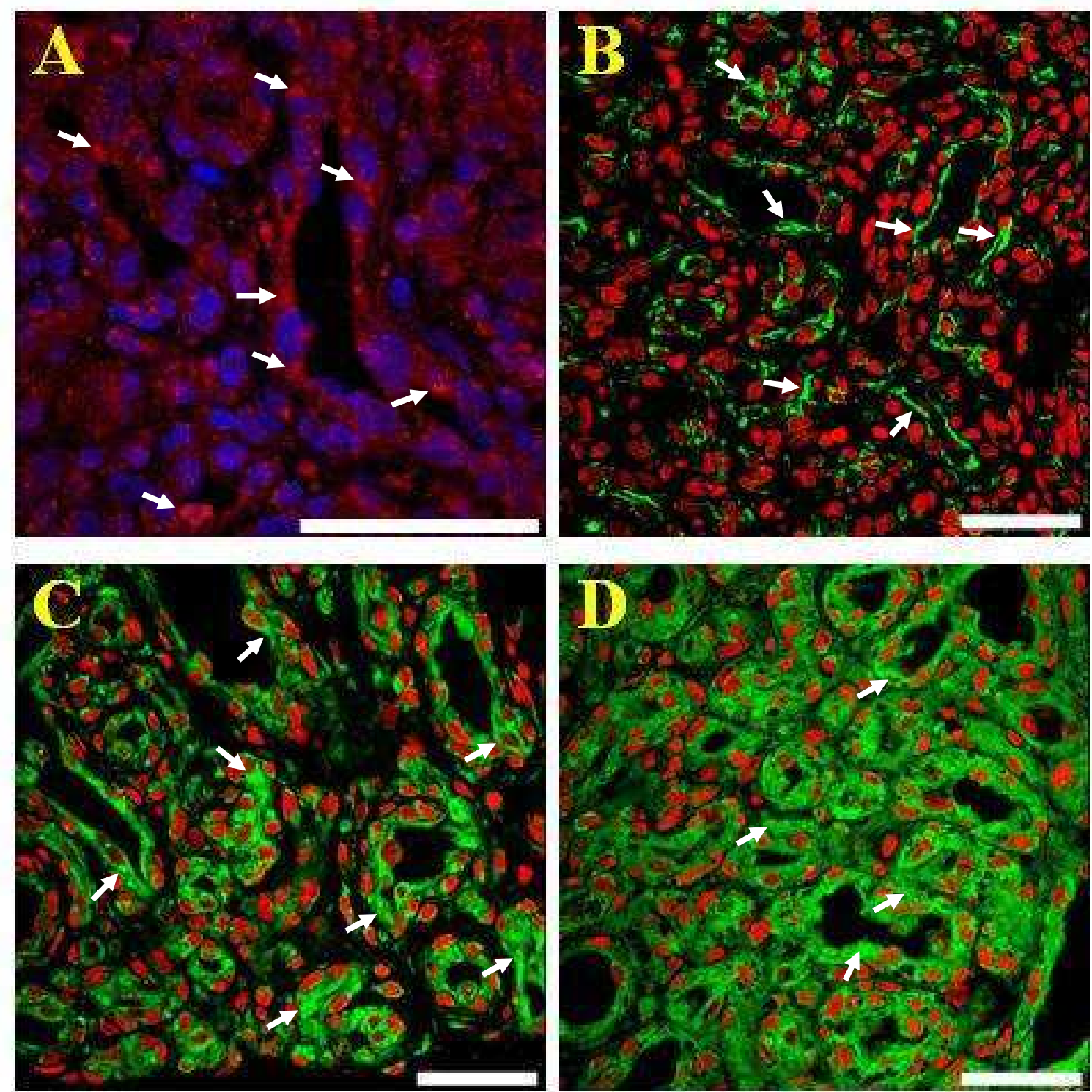

Figure 5.2: Confocal immunohistochemical localisation of TRAIL receptors in haemangioma. Paraffin embedded proliferating haemangioma tissue sections were stained with primary antibodies against DR4 (A), DR5 (B), DcR1 (C) and DcR2 (D) as described in section 2.4.2. Bound DR4 antibodies were detected using an anti-mouse DIG conjugate and an anti-DIG-Rhodamine conjugate (red). Bound DR5 antibodies were detected using an anti-mouse AlexaFluor 488 conjugate (green). Bound DcR1 and DcR2 antibodies were detected using an anti-rabbit-DIG and an anti-DIG-FITC conjugate (green). Cell nuclei were counterstained with either DAPI (blue) or PI (red). Positive immunoreactivity was detected for all four TRAIL receptors. Red staining indicates DR4 expression (Panel A), while green staining indicates DR5 (Panel B), DcR1 (Panel C) and DcR2 (Panel D) expression. White arrows show cytoplasmic staining detected for all receptors. Scale bars: $50 \mu \mathrm{m}$. 


\subsubsection{EFFECT OF INTERFERON AND TRAIL ON CAPILLARY-LIKE}

\section{GROWTH}

Freshly resected haemangioma tissue has been shown to develop outgrowths or 'microvessels' within days of placing in culture (Tan et al., 2000 b). Using this system, the effect of exogenously applied TRAIL $(250 \mathrm{ng} / \mathrm{ml})$ and a low and high dose of IFN $\alpha$ $2 \mathrm{~b}(4600 \mathrm{U} / \mathrm{ml}$ and $23000 \mathrm{U} / \mathrm{ml}$, respectively), singly and in combination was tested on proliferating haemangioma tissue explants isolated from five different patients (Figure 5.3-A). Haemangioma explants when grown in the absence of TRAIL and IFN under serum-free culture conditions grew well and gave rise to numerous microvessel-like outgrowths by nine days in culture. Figure 5.3-A graphically represents the extent of microvessel outgrowth for all five patients relative to the control (C). TRAIL treatment (T) alone had negligible effect on microvessel outgrowth in all five patients. Except for the tissue explants that were treated with TRAIL (T) alone, all other treatments resulted in significant inhibition of microvessel outgrowth compared to untreated control (C) cultures $(p<0.01)$. In addition, while low dose of IFN $\alpha-2 b\left(\mathrm{I}_{\mathrm{L}}\right)$ inhibited outgrowth $(p<$ 0.01), IFN $\alpha-2 b\left(\mathrm{I}_{\mathrm{L}}\right)$ when used in conjunction with TRAIL $\left(\mathrm{T}+\mathrm{I}_{\mathrm{L}}\right)$ resulted in a synergistic inhibition of microvessel outgrowth $(p<0.001)$ such that the level of inhibition was similar to that seen with high dose IFN $\alpha-2 b$ treatment $\left(\mathrm{I}_{\mathrm{H}}\right)$ alone. While cultures treated with high dose IFN $\alpha-2 b\left(I_{H}\right)$ showed reduced microvessel outgrowth $(p$ $<0.001)$, the combined treatment with TRAIL and high dose IFN $\alpha-2 b\left(\mathrm{~T}+\mathrm{I}_{\mathrm{H}}\right)$ reduced microvessel outgrowth further but not to a statistically significant extent.

\subsubsection{EFFECT OF INTERFERON AND TRAIL ON HaemDMSCs}

The EZ4U assay was used to test the effect of TRAIL $(250 \mathrm{ng} / \mathrm{ml})$ and IFN $\alpha-2 b$ (4600 U/ml and $23000 \mathrm{U} / \mathrm{ml}$ ) on HaemDMSC proliferation. No effect was detected at the end of a 48 hour incubation period. HaemDMSCs were also treated with IFN $\alpha-2 b$ and TRAIL for 96 hours in a similar manner as the tissue explants. Figure 5.3-B shows 
that similar to the tissue explants, treatment with TRAIL alone did not inhibit proliferation of HaemDMSCs. While IFN $\alpha-2 b$ treatment (low dose and high dose) significantly inhibited HaemDMSC proliferation compared to untreated cultures $(p<$ $0.01)$, the combined treatment of TRAIL with low dose of IFN $\alpha-2 b\left(T+I_{L}\right)$ did not show a synergistic effect on HaemDMSC proliferation (Figure 5.3-B) as that seen with the cultured tissue explants shown in Figure 5.3-A. All proliferation assays were performed on the purified cells from at least three different patients. Cultures were assayed in triplicate for each treatment type. 

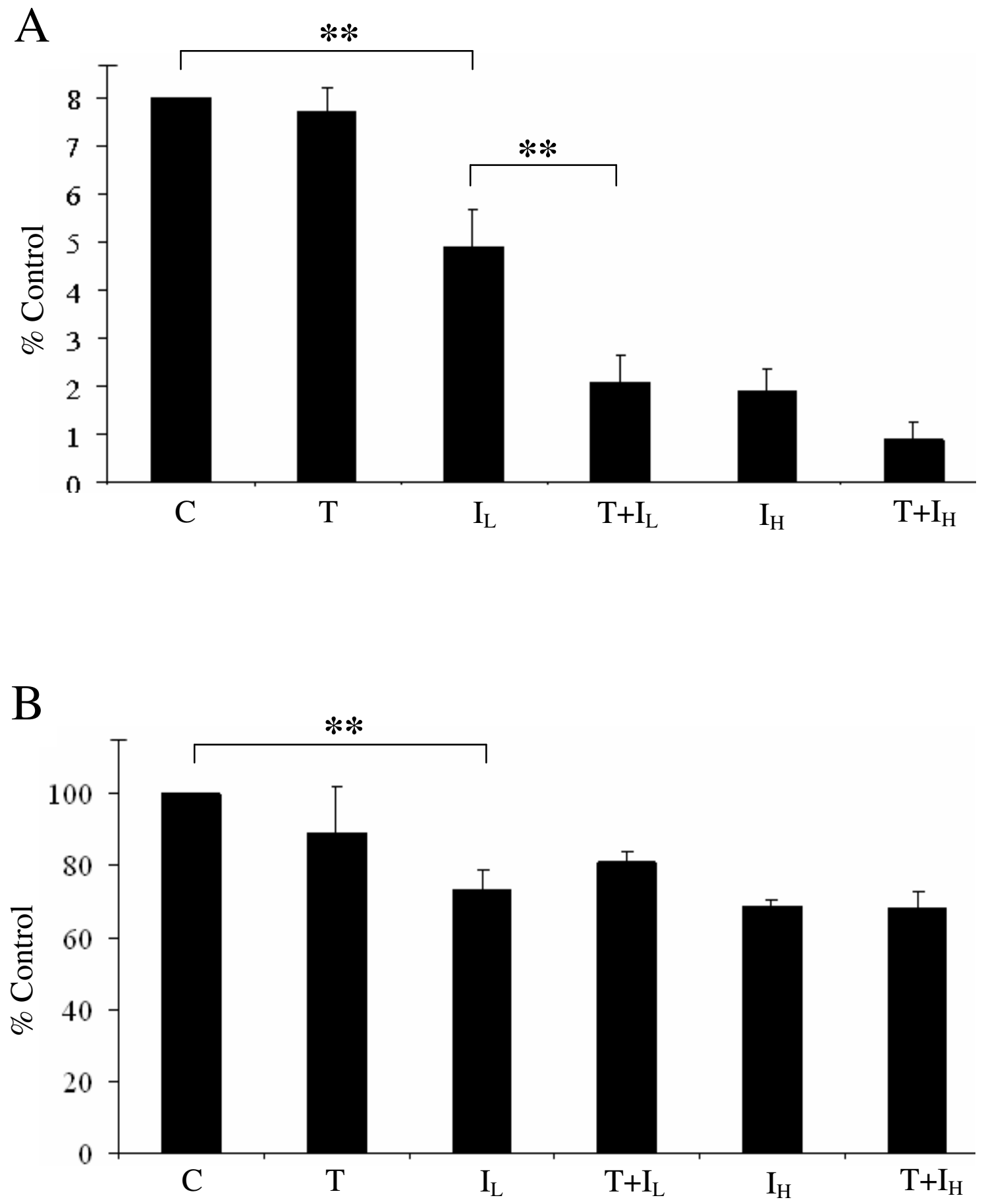

Figure 5.3: Effect of TRAIL and IFN $\alpha-2 b$ on haemangioma cultures, in vitro. The effect of $250 \mathrm{ng} / \mathrm{ml}$ TRAIL (T), $4600 \mathrm{U} / \mathrm{ml}$ IFN $\alpha-2 b\left(\mathrm{I}_{\mathrm{L}}\right)$ and $23000 \mathrm{U} / \mathrm{ml} \mathrm{IFN \alpha -2b}\left(\mathrm{I}_{\mathrm{H}}\right)$ was tested on haemangioma capillary-like outgrowth (A) and HaemDMSC proliferation (B). (A) Capillary-like outgrowth was calculated by the area occupied by the outgrowing vessels relative to the area of the tissue from which they emanated and is expressed as $\%$ Control. Data presented shows the average results obtained following nine days of treatment from five different proliferating haemangioma patients \pm s.e.m. (B) Extent of HameDMSC proliferation following 96 hours of treatment with TRAIL and IFN $\alpha-2 b$. Proliferation was assessed as a direct measure of absorbance and the results are presented as \% Control. Data shown in Panel B represent the average results obtained from three individual experiments performed with HaemDMSC isolates from three proliferating haemangioma patients \pm s.e.m. To detect statistically significant changes, a 2 -way ANOVA was applied to all data sets. ${ }^{* *}$ represents significance at $p<0.01$. 


\subsection{5 mRNA AND PROTEIN EXPRESSION OF TRAIL AND ITS RECEPTORS IN HaemDMSCs}

The difference in response to the combined treatment of TRAIL and IFN $\alpha-2 b$ on the tissue explants compared with the HaemDMSCs was speculated to be due to differences in TRAIL receptor expression. Using qRT-PCR, the expression profiles of mRNA for TRAIL and TRAIL receptors in HaemDMSCs and the tissue pieces from which they were isolated were compared. The difference in mRNA expression between the tissue and the cultured cells normalised to expression of GAPDH as $\triangle \mathrm{Ct}$ values (Figure 5.4-A) is graphically represented as the average $\triangle \Delta \mathrm{Ct}$ values for the six biopsies analysed (Figure 5.4-B). Small differences in expression of mRNA for DR4, DR5 and DcR1 were found but there was a large induction of OPG expression $(p<$ $0.001)$ and DcR2 $(p<0.01)$ decoy receptors in the HaemDMSCs. This increase in decoy receptor expression was also matched by a similar decrease in TRAIL expression $(p<0.001)$ by the cultured cells. The raw $\triangle \mathrm{Ct}$ values are listed in Table 3.1, Appendix 3, page 292 . 


\begin{tabular}{lcccccc}
\hline & TRAIL & DR4 & DR5 & DcR1 & DcR2 & OPG \\
\hline HaemTissue & 5.1 & 7.6 & 12.6 & 8.4 & 8.7 & 10.7 \\
HaemDMSCs & 15.9 & 11.3 & 14.7 & 10.9 & 5.7 & 4.3 \\
\hline
\end{tabular}

$\mathrm{B}$

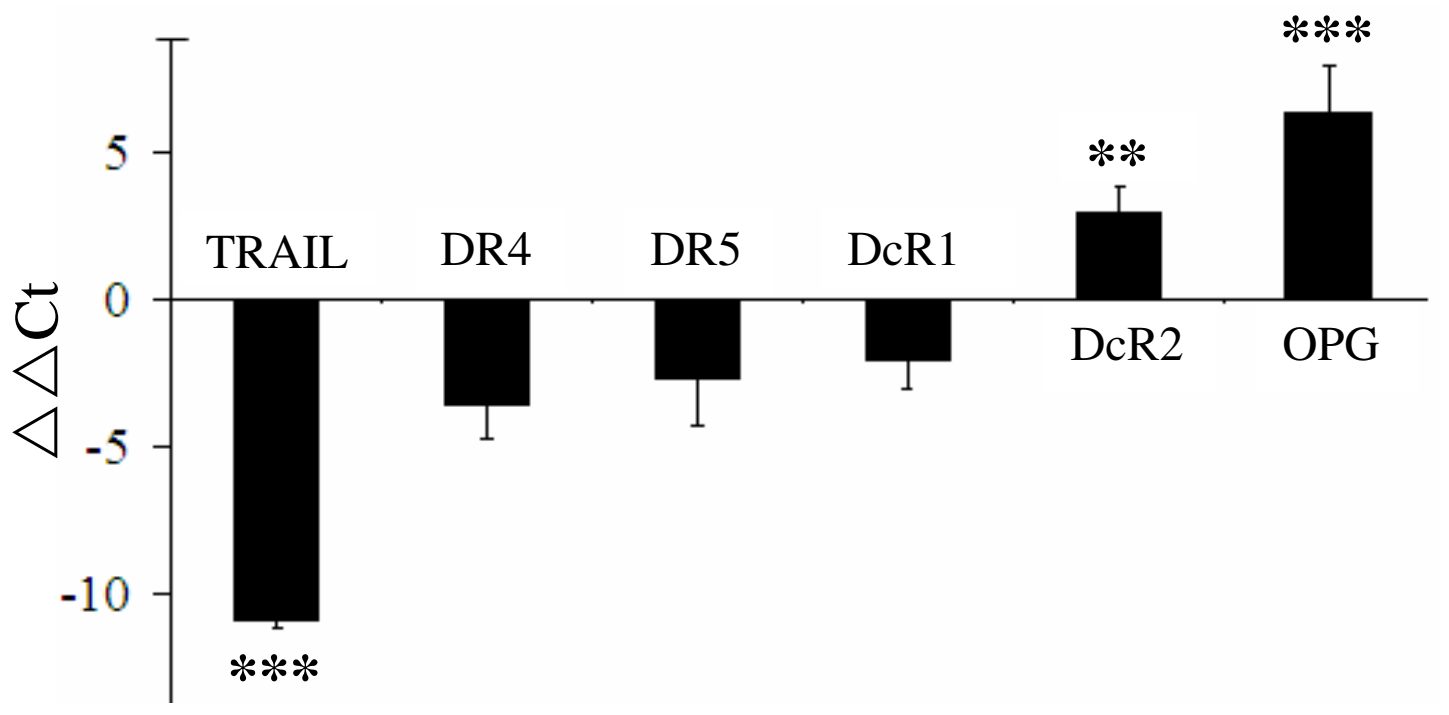

Figure 5.4: Differences in mRNA levels for TRAIL and TRAIL receptors in haemangioma tissue and HaemDMSCs. Relative mRNA levels for TRAIL and TRAIL receptors were determined by qRT-PCR from six proliferating haemangioma tissues and the corresponding HaemDMSC isolates from each tissue. (A) The table gives the average $\triangle \mathrm{Ct}$ values obtained for each gene from tissue pieces (HaemTissue) and cell isolates (HaemDMSCs). (B) Differences in TRAIL and TRAIL receptor expression between the HaemDMSC cell lines relative to tissues were determined as $\triangle \triangle \mathrm{Ct}$ values. Data presented shows the average $\triangle \triangle \mathrm{Ct}$ values obtained for each gene between tissue and cells. A low value indicates higher expression of the gene in the tissue while a high value shows higher mRNA expression of that gene by HaemDMSCs. Significant differences in gene expression between tissues and cells were detected using the Friedmann's non-parametric test for paired samples, where ** represents significance at $p<0.01$ and $* * *$ at $p<0.001$. 
IHC analysis showed strong positive cytoplasmic immunoreactivity for DR4, DR5 and DcR2 on HaemDMSCs (Figure 5.5-A, -B and -D, respectively). Low levels of DcR1 were also detected (Figure 5.5-C). As mentioned in Chapter 4, HaemDMSCs showed strong extracellular staining for OPG (Figure 5.6-C) which was clearly different from cytoplasmic (Figure 5.6-A and -B, yellow arrows) and nuclear staining (Figure 5.6-A and -B, white arrows) seen within the tissue lesion in vivo. In addition, OPG expression by HaemDMSCs was found to increase with time (data not shown).
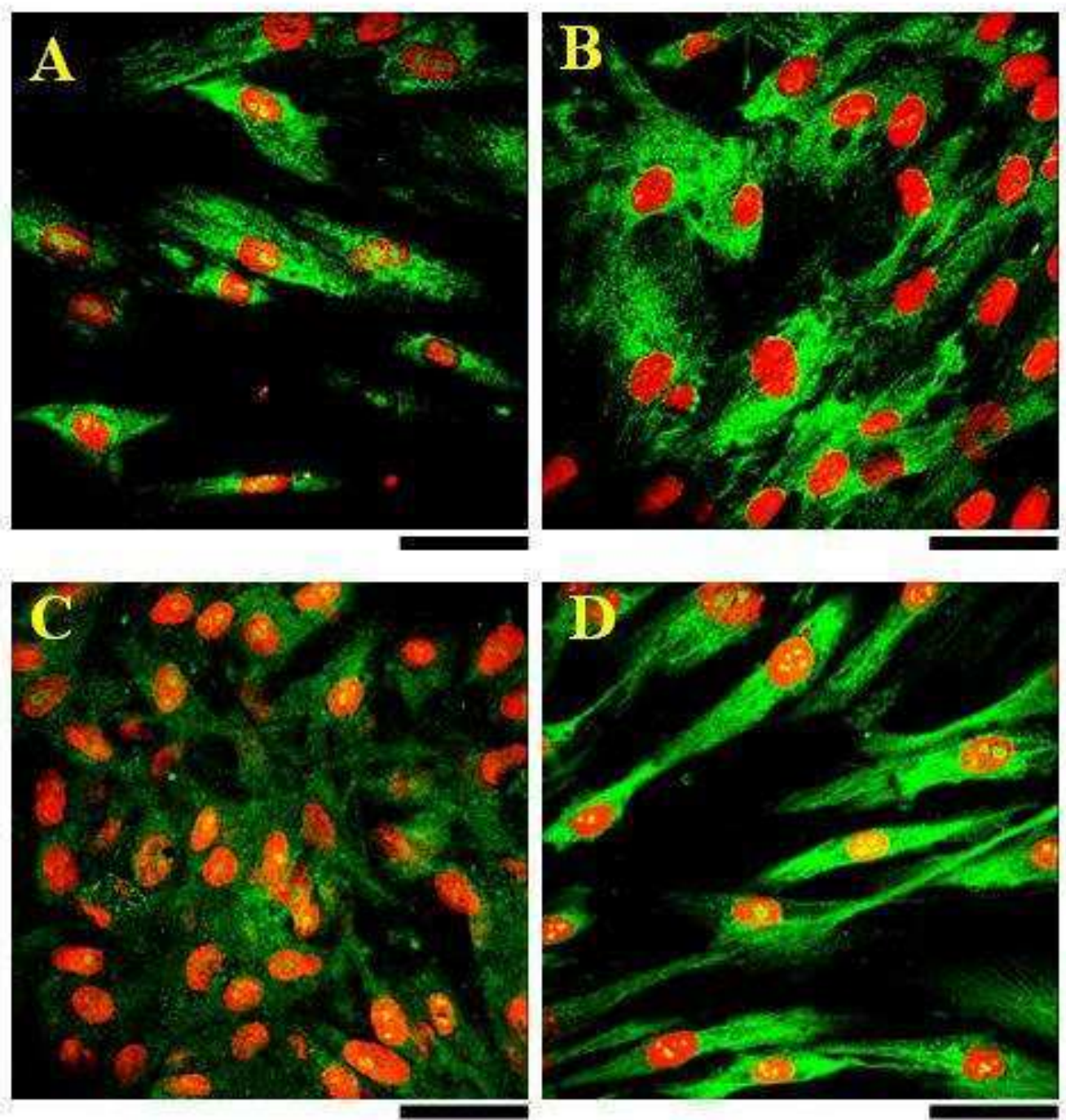

Figure 5.5: Confocal immunohistochemical localisation of TRAIL receptors on HaemDMSCs. Cells were fixed and stained with antibodies against DR4 (A), DR5 (B), DcR1 (C) and DcR2 (D) as described in section 2.4.2. Bound primary antibodies were detected using either an anti mouse AlexaFluor 488 conjugate (green) or a FITC conjugated anti-DIG secondary antibody (green). Cell nuclei were counterstained with PI (red). Green staining shows high expression for DR4, DR5 and DcR2 but low expression of DcR1 in HaemDMSCs. Scale bars: $50 \mu \mathrm{m}$. 

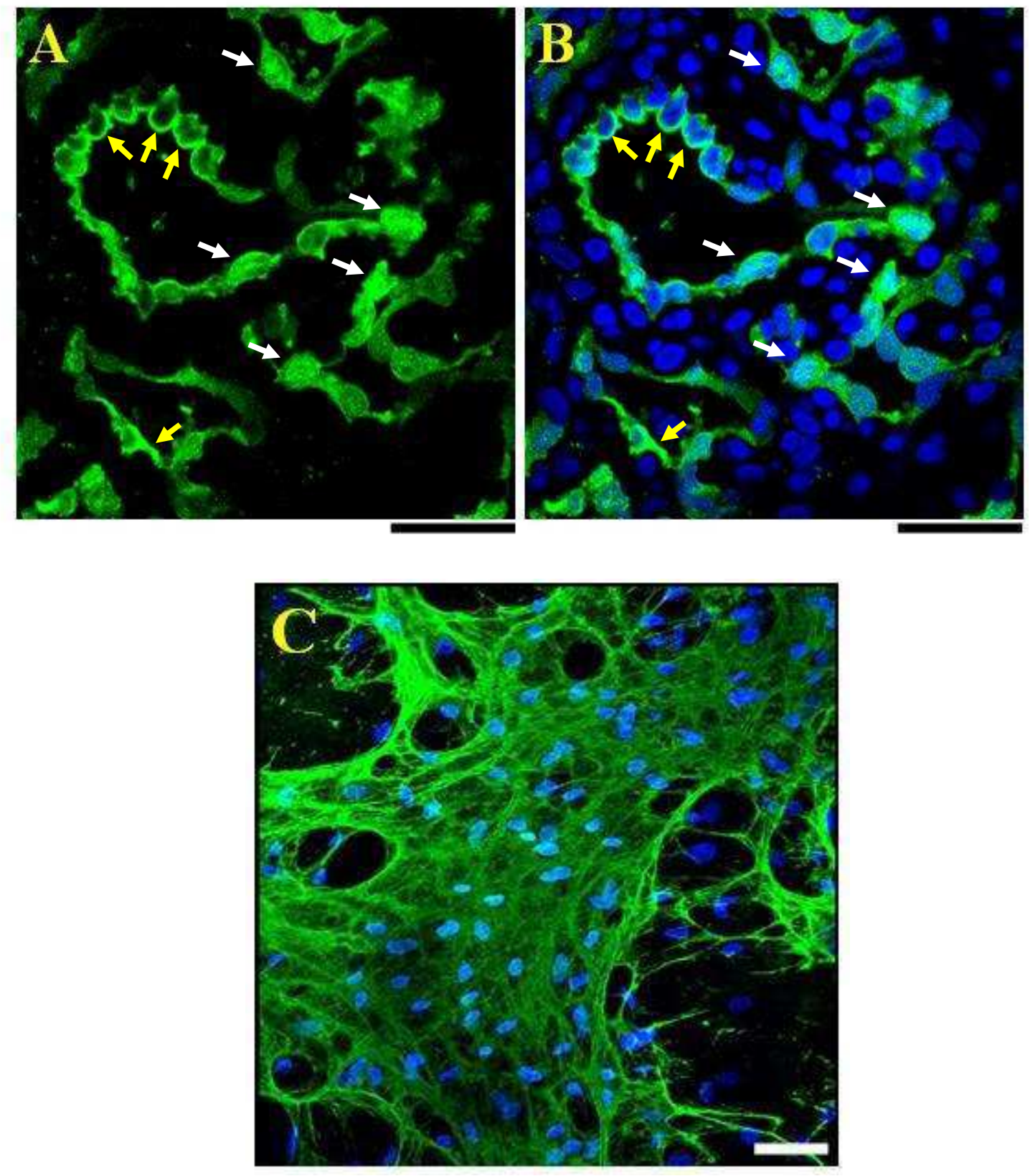

Figure 5.6: Confocal immunohistochemical localisation of OPG in haemangioma. Paraffin embedded proliferating haemangioma tissue sections (A\&B) and HaemDMSCs were stained with a mouse anti-OPG antibody as described in section 2.4.2. Immunoreactivity was detected using an anti-mouse AlexFluor 488 conjugate (green). Cell nuclei were counterstained with DAPI (blue). In proliferative haemangioma lesions (Panels A\&B), OPG expression was detected in the cytoplasm (yellow arrows) as well as within the nuclear compartment (white arrows). HaemDMSCs showed strong extracellular staining for OPG (Panel $\mathbf{C}$, green staining). Scale bars: $50 \mu \mathrm{m}$. 
To determine whether changes in DR4 and DcR2 mRNA expression correlated with changes in its protein abundance, the same samples were used for immunoblotting as described in section 2.4.5.1 (page 103). The immunoblots shown in Figure 5.7 clearly shows that although equal amounts of total protein $(100 \mu \mathrm{g} /$ well $)$ were loaded from each sample, HaemDMSCs expressed greater amounts of GAPDH protein compared to proliferating haemangioma tissues. No significant difference in total DR4 protein expression was detected between the cells and tissues (Figure 5.7-A). Consistent with mRNA studies, DcR2 protein expression was significantly higher in HaemDMSCs than in the tissues $(p<0.05$; Figure 5.7-B and $-\mathrm{C})$. 

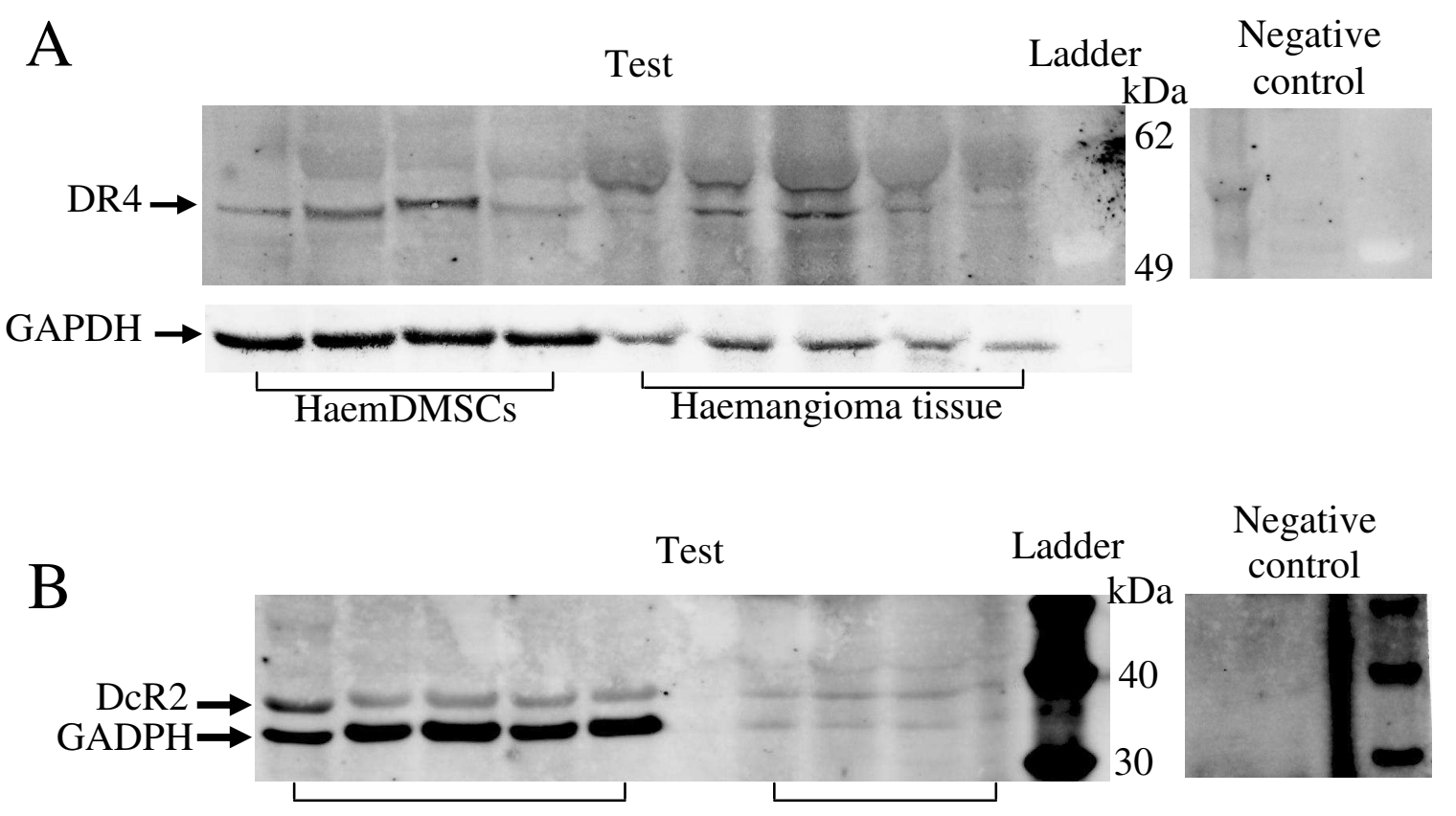

HaemDMSCs Haemangioma tissue

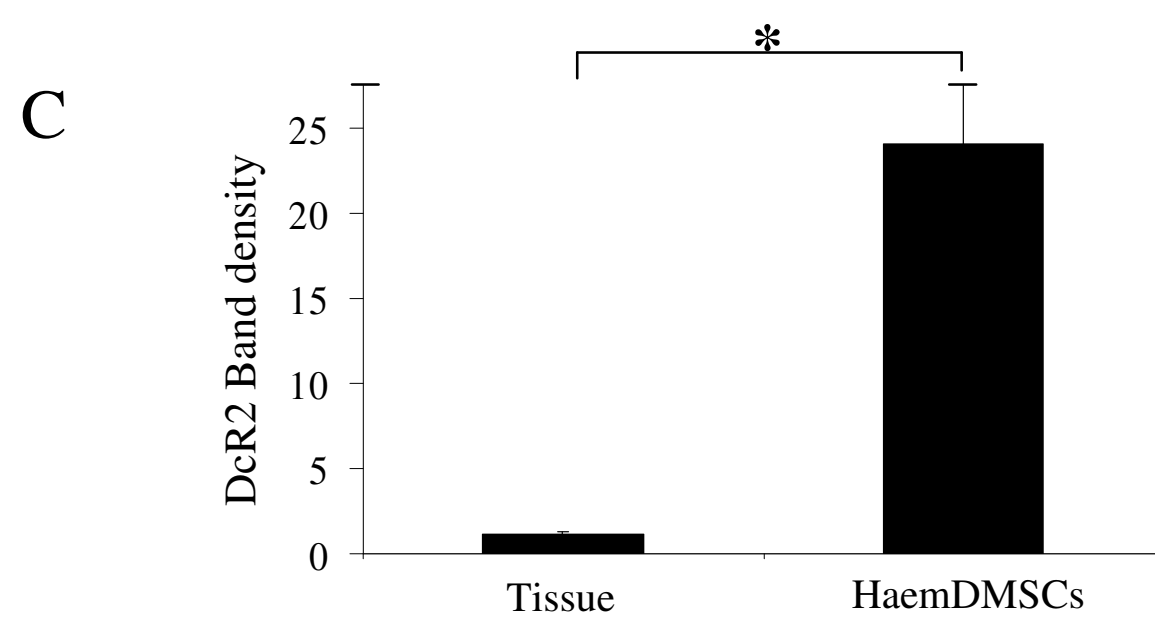

Figure 5.7: DR4 and DcR2 protein expression in haemangioma. DR4 (A) and DcR2 (B) protein expression was examined in proliferative haemangioma tissue biopsies and HaemDMSCs by Western blotting as described in section 2.4.5.1. Each gel contained $50 \mu \mathrm{g}$ of total protein extracted from haemangioma tissues from four patients and five HaemDMSCs cell lines. Membranes were incubated with mouse anti-DR4 or rabbit anti-DcR 2 antibodies followed by AlexaFluor 488 conjugated anti-mouse or anti-rabbit secondary antibodies. Immunoreactive bands were detected for DR4 and DcR2 at 55 $\mathrm{kDa}$ and $32 \mathrm{kDa}$, respectively, corresponding to the expected molecular weight for these proteins. Membranes were also probed with mouse anti-GAPDH antibodies. The immunoblots showed that HaemDMSCs expressed greater amounts of GAPDH protein than tissue biopsies. No change in total DR4 protein expression was found between cells and tissues. In contrast, HaemDMSCs expressed greater amounts of DcR2 protein than haemangioma biopsies. (C) Densitometry analysis of DcR2 band densities confirmed that the increase was significant with $p<0.05\left(^{*}\right)$. Negative controls were included in each experiment in which lysate from haemangioma tissue and HaemDMSCs were immunoblotted with secondary antibody only. No non-specific immunoreactive bands were detected on the negative control immunoblots therefore validating the specificity of the anti-DR4 and anti-DcR2 primary antibodies. 


\subsubsection{HaemDMSCs SECRETE OPG INTO THE CULTURE MEDIUM}

AND OPG ANTIBODY SPECIFICITY

To determine whether HaemDMSCs secrete and release OPG when cultured in vitro, Western blot analysis was performed using the MAB8051 anti-OPG antibody (R\&D Systems) as described in section 2.4.5.2 (page 104). Under reducing conditions, the immunoblots showed that the culture media conditioned over a 96 hour period, contained detectable levels of the monomeric form of OPG (60 kDa) (Figure 5.8-A, red arrow). In addition, a band at approximately $220 \mathrm{kDa}$ was also detected in the conditioned media of HaemDMSCs (Figure 5.8-A, black arrow). Both bands were detected when the experiment was repeated with conditioned media obtained over a 72 hour culture period (Appendix 3, Figure 3.1, page 293). In addition, no bands were detected when unconditioned media was analysed in the same way as conditioned media (Appendix 3, Figure 3.1, page 293). These results suggest that perhaps the $220 \mathrm{kDa}$ band represents a novel hyper-glycosylated form of OPG. The dimeric form of OPG has been shown to be $120 \mathrm{kDa}$ and was not detected in either experiment.

In addition, HaemDMSCs lysate and haemangioma tissue lysate were also run on the same gel and subjected to immunoblotting. Bands at both $60 \mathrm{kDa}$ and $220 \mathrm{kDa}$ were detected from HaemDMSCs lysate (Figure 5.8-A, red and black arrows, respectively). In order to determine the specificity of antibody binding, immunoblotting was performed with the omission of the primary antibody and incubation with secondary antibodies only. The resulting immunoblot showed no bands at either $60 \mathrm{kDa}$ or $220 \mathrm{kDa}$ using HaemDMSCs lysates (Figure 5.8-B). Furthermore, GAPDH immunoreactivity was not detected from the conditioned media (Figure 5.8-C, black arrow). These results indicate that the OPG protein detected in the media was not the result of HaemDMSCs lysing during the experiment

Waterman et al (2007) recently showed that the MAB8051 anti-OPG antibody recognises a $30 \mathrm{kDa}$ band in addition to OPG in breast cancer tissues and prostate 
cancer cell lines. Using MALDI-TOF, these authors identified that the $30 \mathrm{kDa}$ band corresponded to carbonic anhydrase II (CA II). In this study, only the haemangioma tissue lysates showed an immunoreactive band at $30 \mathrm{kDa}$ with this antibody (Figure 5.8, green arrow). Since neither the conditioned medium nor the HaemDMSCs lysates showed this $30 \mathrm{kDa}$ band, it was confirmed that the immunohistochemical studies performed for OPG on HaemDMSCs was not detecting CA in the cultured cells but was specific for OPG or at the least, a protein that contains an OPG domain. However, caution is required in interpreting results of OPG immunohistochemical analysis in paraffin embedded haemangioma tissue sections because of the non-specific binding of the MAB8051 anti-OPG antibody on haemangioma tissue lysate. 


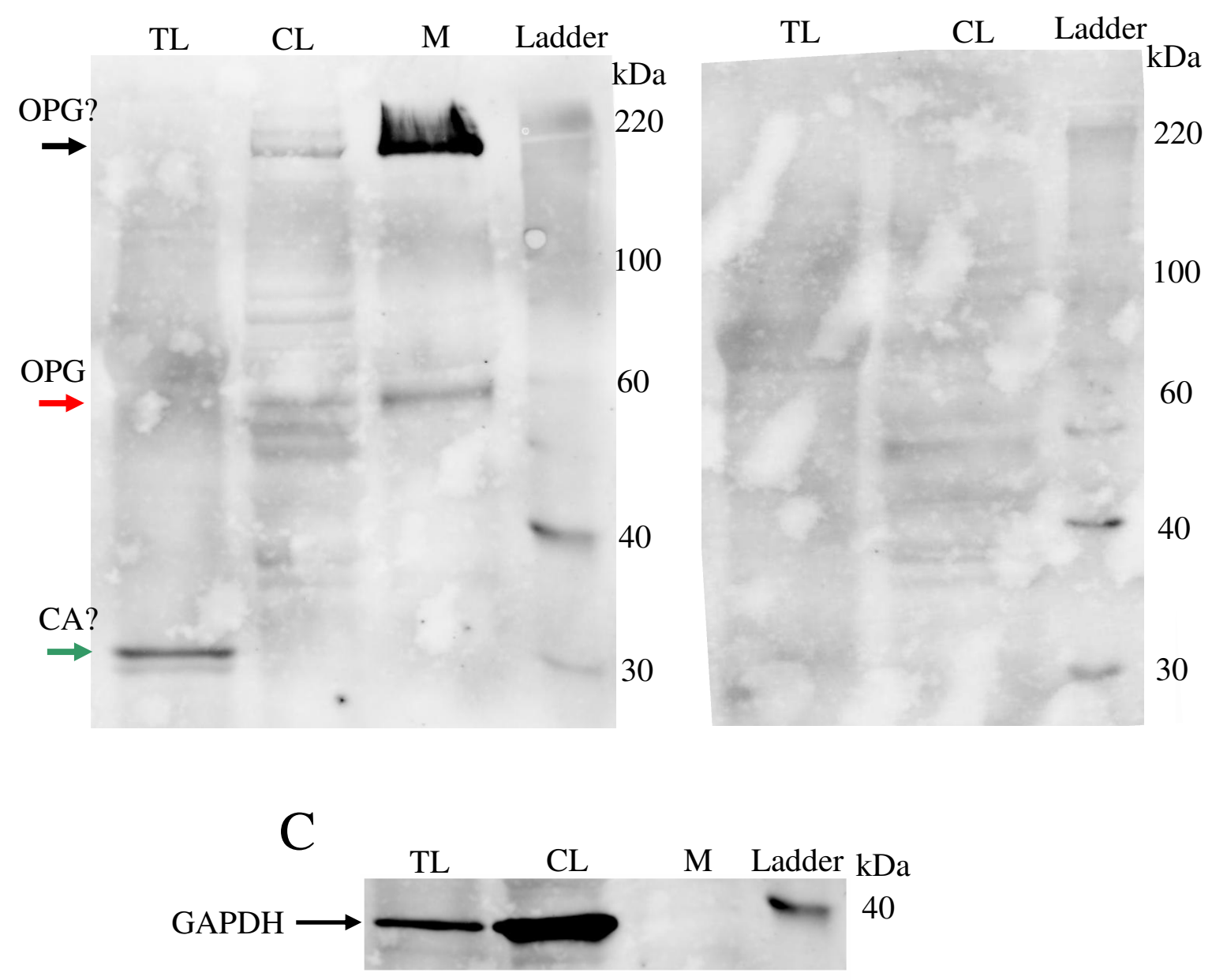

Figure 5.8: OPG protein expression in HaemDMSCs conditioned media, in HaemDMSCs and in a proliferative haemangioma tissue biopsy. (A) $40 \mu \mathrm{g}$ of total protein from the medium of HaemDMSCs conditioned over a 96 hour period (M), 100 $\mu \mathrm{g}$ of total protein from HaemDMSCs (CL) and proliferating haemangioma tissue lysate (TL) were analysed by SDS-PAGE and immunoblotted with the mouse anti-OPG MAB8051 primary antibody. (B) A negative control immunoblot containing lysate from HaemDMSCs (CL) and haemangioma tissue lysate (TL) was probed with secondary antibody only. The test sample showed immunoreactive bands at $60 \mathrm{kDa}$ (red arrow) and at $220 \mathrm{kDa}$ (black arrow) from the conditioned media and HaemDMSCs. These bands were absent in tissue lysate and on the negative control. In contrast, a band at 30 $\mathrm{kDa}$ was detected from tissue lysate (green arrow) which was absent in the conditioned media, the HaemDMSCs lysates and the negative control. (C) The test immunoblot was probed with anti-GAPDH antibodies. Absence of an immunoreactive band for GAPDH $(38 \mathrm{kDa})$ from conditioned media $(\mathrm{M})$ indicates that the band corresponding to OPG protein $(60 \mathrm{kDa}$, red arrow) from the media did not come from lysed cells. 


\subsection{DISCUSSION}

\subsubsection{THE EFFECT OF TRAIL AND INTERFERON ON}

HAEMANGIOMA CAPILLARY-LIKE OUTGROWTH

The cellular and molecular biology of haemangioma and its spontaneous and therapy-induced regression is still largely unknown. Understanding the molecular pathways that are active in these tumours could lead to the development of more effective therapies, as molecules that are involved in proliferation or involution could be targeted.

Recombinant TRAIL protein offers great promise as a cancer therapy as it shows the unique property to destroy several types of tumour cells but spares most normal cells (Smyth et al., 2003). TRAIL also induces the expression of a cohort of IFN stimulated genes (ISGs) including IFN $\beta$, caspase 7 and STAT-1 which are involved in apoptosis. This suggests the existence of molecular cross-talk between the TRAIL death receptor and IFN signalling pathways (Kumar-Sinha et al., 2002). Increased expression of ISGs has been identified during haemangioma involution. In addition, apoptosis has been shown to increase five-fold in haemangioma undergoing regression (Razon et al., 1998). It is therefore interesting to postulate that TRAIL receptor signalling may be a regulator of haemangioma progression, presumably through the induction of apoptosis of the ECs that form the microvasculature characteristic of haemangioma. Given the potential use of TRAIL in anti-cancer therapy, this study investigated the effects of TRAIL and IFN $\alpha-2 b$ on haemangioma capillary-like outgrowth when added singly and in combination. The expression of TRAIL and TRAIL-Rs in proliferating haemangioma biopsies and on HaemDMSCs was also investigated.

This study showed that although proliferating haemangioma expressed protein for the functional receptors for the TRAIL ligand, DR4 and DR5, TRAIL alone did not affect rate of capillary-like outgrowth. However, combining TRAIL with a low dose 
IFN $\alpha-2 b$ resulted in a synergistic inhibitory effect on capillary-like outgrowth. In addition, for some explants, this inhibition was similar to the inhibition caused by high dose IFN $\alpha-2 b$ alone. These results suggest the possibility of substituting high dose IFN $\alpha-2 b$ therapy with the combined treatment of TRAIL with a low dose of IFN $\alpha-2 b$, thereby possibly reducing the harmful side effects seen clinically with IFN treatment.

This study also investigated the effect of TRAIL and IFN $\alpha-2 b$ on HaemDMSCs proliferation. Although TRAIL and IFN $\alpha-2 b$ added singly inhibited HemDMSCs proliferation to a certain extent, the synergistic effect of TRAIL with IFN was not detected. qRT-PCR and immunohistochemical analysis showed that although the cells expressed DR4, DR5 and DcR1, the expression of DcR2 and OPG was greatly upregulated in the cultured cells.

The molecular mechanism by which DcR2 confers resistance to TRAIL-induced apoptosis remains unclear (Almasan \& Ashkenazi, 2003; Bouralexis et al., 2003; Davidovich et al., 2004). Various studies have found that DcR2 overexpression protected cells from TRAIL-induced apoptosis (Degli-Esposti et al., 1997; Marsters et $a l .$, 1997). In addition, decreased DcR2 expression caused due to the hypermethylation of the DcR2 gene has been identified in a number of tumours and tumour cell lines tumours including breast, lung, prostate, bladder, cervical cancer, ovarian, lymphoma, leukaemia and multiple myeloma and neuroblastomas (Shivapurkar et al., 2004; Van Noesel et al., 2002). Mérino et al (2006) showed that DcR2 impaired TRAIL-DISC processing and initiator caspase activation while Meng et al (2000) showed that the over-expression of DcR2 delayed p53-induced apoptosis in human colon cancer cells. Liu et al (2005) reported that while DcR2 over-expression in human lung cancer cells was protective against chemotherapeutic agent-induced apoptosis, silencing of DcR2 expression enhanced apoptosis. These findings support the possibility that the increased expression of DcR2 by HaemDMSCs may be to some extent protecting them from apoptosis. 
During this study, increased extracellular OPG deposition by HaemDMSCs was consistently detected by immunohistochemical studies. From these results, we hypothesise that HaemDMSCs secrete increased levels of OPG, which then acts as a protective shield around the cells preventing apoptosis. We also postulate that increased OPG production is a stress response that results from culturing these cells in vitro i.e. outside their normal environment. Previous studies have shown that the in vitro culture of primary cell lines can result in the formation of abnormal cell architecture and can cause significant changes in gene expression (Kivell, 2003). Kanzaki et al (2006) found that application of a cyclical tensile strain up-regulated OPG mRNA expression in human periodontal ligament cells (hPLDCs). Yamamoto et al (2006) also found that hPLDCs exposed to mechanical stress as hydrostatic pressure showed increased OPG mRNA expression compared to the untreated cells. In addition to increased OPG mRNA expression, Tang et al (2006) showed increased concentrations of OPG protein in the conditioned medium of MC3T3-E1 osteoblastic cells subjected to cyclic tensile strain. The presence of secreted OPG in the conditioned media of HaemDMSCs and the increased expression of OPG by HaemDMSCs are consistent with these findings.

In contrast, although immunohistochemical studies showed OPG expression within the haemangioma lesions (Figure 5.6), Western blotting analysis failed to detect an immunoreactive band at the correct size using haemangioma tissue lysates (see section 5.3.6, page 233). These data suggest that proliferating haemangioma lesions do not express high levels of OPG and this could explain why the combined treatment of IFN $\alpha-2 b$ and TRAIL resulted in the synergistic inhibition of capillary-like growth from explanted biopsies.

To date, the physiological functions of the multiple TRAIL-Rs remain obscure. Although various studies have suggested that the ratio of death and decoy receptors are critical in the regulation of the apoptotic pathway (Ashkenazi, 2002; Bouralexis et al., 2003), other investigators have shown that there is no correlation between TRAIL 
receptor expression and susceptibility to TRAIL-induced apoptosis (Keane et al., 1999; Wen et al., 2000; Knight et al., 2001). In addition, several other mechanisms of resistance to TRAIL have been proposed in different cell types including cFLIP, a caspase- 8 and a caspase-10 related molecule that lacks catalytic activity and acts as a competitive inhibitor of death receptor-mediated apoptosis (Tschopp et al., 1998; Leverkus et al., 2000). XIAP can also bind to caspase-3 and caspase-9 and inhibit their activity (LeBlanc \& Ashkenazi, 2003; Salvesen, 2002; MacFarlane et al., 2002). Furthermore, sensitivity of some cell types to TRAIL is hinged on the balance between pro-apoptotic (e.g., BAX and BAK) and anti-apoptotic (e.g. Bcl-2 and Bcl-xl) members of the Bcl-2 family (Kelly \& Ashkenazi, 2004).

There is mounting evidence to suggest that the interaction of OPG with TRAIL is biologically important, particularly in in vitro culture systems. OPG has been shown to protect serum-starved ECs and fibroblast-like synovial cells from TRAIL-triggered cell death (Pritzker et al., 2004; Miyashita et al., 2004). OPG also acts in a paracrine and autocrine manner by binding TRAIL to promote the survival of prostate cancer cells (Holen et al., 2002), breast cancer cells (Neville-Webbe et al., 2004), multiple myeloma cells (Shipman \& Croucher, 2003) and ameloblastoma cells (Sandra et al., 2006). Although further studies are required to elucidate the ability of OPG to counteract the biological activity of TRAIL in vivo, it is clear that the relative concentrations and the expression patterns of OPG in the local environment is a key determinant in TRAILmediated cell toxicity. Based on these findings, we hypothesise that the HaemDMSCs isolated in this study are resistant to TRAIL mediated apoptosis due to increased protein levels of OPG and DcR2. However, further experiments would be required to confirm this. This would include testing the effect of TRAIL on HaemDMSCs transfected with short interfering RNA (siRNA) for the OPG and DcR2 genes. 


\subsubsection{OPG ANTIBODY SPECIFICITY}

OPG is secreted as a covalent, disulfide-linked homodimer, which has been shown to be the predominant extracellular form (Simonet et al., 1997). While the homodimer has a molecular weight of $120 \mathrm{kDa}$, immunoblotting for OPG under reducing conditions shows an immunoreactive band at $60 \mathrm{kDa}$ corresponding to the OPG monomer (Hyanes et al., 2003). In addition to the monomerric and dimeric forms of OPG, alternative splicing of the OPG gene has been shown to give rise to three mRNA isoforms: mature mRNA and two splice variants (Morinaga et al., 1998). The splice variant sequences contain a premature stop codon and are predicted to encode truncated proteins of $30-40 \mathrm{kDa}$.

There have been conflicting reports on the specificity of the MAB 8051 antibody. This antibody was used in this study to detect OPG protein expression. Although Haynes et al (2003) showed that the antibody detected both, the monomeric and the dimeric forms of OPG, Waterman et al (2007) advised caution when using this antibody. Their studies showed that the antibody, in addition to OPG, also detected a 30 $\mathrm{kDa}$ band on immunoblots that corresponded to CA II. To determine the specificity of the MAB 8051 antibody, Western blotting was performed in this study with HaemDMSCs lysates, HaemDMSCs conditioned media and haemangioma tissue lysates.

Consistent with the findings of Waterman et al (2007), an immunoreactive band at $30 \mathrm{kDa}$ was detected when haemangioma tissue lysates were subjected to immunoblotting. However, this band was not detected using either HaemDMSC lysates or the HaemDMSC conditioned medium run on the same gel and therefore subjected to the same conditions as the tissue lysates. In contrast, the HaemDMSCs lysates and HaemDMSCs conditioned media showed the presence of an immunoreactive band at 60 $\mathrm{kDa}$ which corresponds to the molecular weight of the native OPG monomer. Furthermore this band was absent on haemangioma tissue lysates. However, 
haemangioma tissue lysates did not show any immunoreactive bands at the appropriate size. Therefore, we conclude that the immunohistochemical studies performed during this study using the MAB 8051 anti-OPG antibody may be non-specifically cross reacting to CA II within the haemangioma tissue sections.

CA II is a highly conserved enzyme which catalyses the hydration of carbon dioxide and the dehydration of bicarbonate. In addition to normal cells (Onji, 1999), increased CA II expression is detected in brain tumors (Parkkila et al., 1995), leukemia (Leppilampi et al., 2002), the tumour endothelium of melanoma, renal cell carcinoma, oesophageal and lung cancers (Yoshiura et al., 2005). CA II is considered an important factor for tumour cell growth as rapidly proliferating cells have a requirement for a high influx of bicarbonate to support their metabolic processes (Bianchi et al., 2005). Increased CA II expression has been reported in acidic and hypoxic conditions mimicking a tumour environment (Yoshiura et al., 2005). Furthermore, CA II inhibitors can inhibit the growth of cancer cell lines such as prostate and breast cancer cells and cell lines (Supuran \& Scozzafava, 2000; Mallory et al., 2005). Consistent with the function of this enzyme, the high expression of CA II identified within the proliferative phase seems logical, as most cells in this phase, being highly proliferative would require increased levels of CA II. In contrast, minimal proliferation in the involuting and involuted phases corresponds to reduced CA II expression.

However, it is important to note that the epitope recognised by the MAB 8051 anti-OPG antibody within haemangioma tissue sections still differentiated between the cells that exhibited punctuate vWF immunoreactivity and those that showed strong vWF immunoreactivity (see section 4.3 .5 , page 174 ). Therefore, this antibody may be useful in determining the 'maturity' of the ECs within haemangioma.

Finally, an additional immunoreactive band was detected with both HaemDMSCs lysates and HaemDMSCs conditioned medium and had a molecular weight of $220 \mathrm{kDa}$. Two groups have investigated the domains of OPG and their 
importance to function. These studies have shown that the first four domains of OPG (encompassing the TNF receptor-like region) were required for activity in an osteoclastogenesis assay while the last intra-chain disulfide bond was critical for OPG function (Simonet et al., 1997; Yun et al., 1998). Cundy et al (2002) reported a mutant form of OPG that resulted from the loss of an aspartate residue. They showed that this mutant form was secreted and was hyperglycosylated. It is known that glycosylation is important for correct folding, trafficking, stabilisation and protection from proteases, as well as for interaction with other molecules (Dwek, 1998). The primary sequence of OPG contains six potential glycosylation sites but the degree of glycosylation at each of these sites in vivo has not been investigated previously (Cundy et al., 2002).

Since the band at $220 \mathrm{kDa}$ was repeatedly detected on the immunoblots from two separate experiments performed during this study and was not detected when unconditioned media was anaylsed in the same way, we can conclude that this band may represent a novel glycosylated form of the OPG protein. However, deglycosylation experiments or carbohydrate detection methods would need to be performed to confirm this. We hypothesise that the protein at $220 \mathrm{kDa}$ being detected by the antibody must at least contain an OPG domain. 


\section{CHAPTER 6: CYCLOPHILIN A IN HAEMANGIOMA}

\subsection{INTRODUCTION}

CycA, a ubiquitously distributed protein, belongs to the immunophilin family (Galat, 1993) and is defined on the basis of its binding to the potent immunosuppressive drug cyclosporine A (Fruman et al., 1992; Liu et al., 1991). Intracellular CycA has peptidylprolyl cis-trans-isomerase (PPIase) activity, which is crucial for folding and isomerisation of many proteins. (Galat, 1993; Kofron et al., 1991). CycA also acts as a potent chemoattractant for monocytes (Sherry et al., 1992), neutrophils (Sherry et al., 1992; Xu et al., 1992), eosinophils (Xu et al., 1992) and T cells (Allain et al., 2002) in vitro. $\mathrm{Xu}$ et al (1992) showed that CycA also elicited an inflammatory response characterised by a rapid influx of neutrophils when injected in vivo.

Initially believed to exist solely as an intracellular protein, various studies have shown that CycA is secreted by cells in response to inflammatory stimuli (Sherry et al., 1992; Xu et al., 1992). Jin et al (2000) showed that CycA functioned as a secreted oxidative-stress-induced growth factor from rat SMCs and mediated extracellular signal regulated kinase (ERK1/2) activation and SMCs growth. They also found that recombinant CycA mimicked the effects of secreted CycA. Seko et al (2004) found that CycA was secreted by cardiac myocytes in response to hypoxia/reoxygenation and that CycA secretion resulted in the activation of the ERK1/2 pathway and increased Bcl-2 expression. These authors suggested a protective role for CycA against oxidative stressinduced apoptosis. In addition, Yang et al (2005) showed that CycA induced EC and SMC proliferation while Jin et al (2004) showed that it activated ECs in a proinflammatory manner. 


\subsection{AIMS}

In this study, CycA was first investigated as a house keeping gene. However, preliminary qRT-PCR results identified that CycA mRNA levels differed between the three developmental phases (see Appendix 1, section 1.1.2.2, page 272). Therefore, CycA expression was investigated as a test gene. qRT-PCR was used to measure changes in levels of CycA mRNA while Western blotting and IHC techniques were used to measure CycA protein abundance in proliferating, involuting and involuted haemangioma samples. 


\subsection{RESULTS}

\subsubsection{CHANGE IN mRNA EXPRESSION}

The relative change in mRNA expression for CycA was determined for proliferating, involuting and involuted haemangioma samples (eight samples per phase) by qRT-PCR as described in section 2.2.2.1 (page 81). Expression of CycA mRNA was highest in proliferating haemangiomas and decreased in involuting and involuted lesions with a significant difference detected between proliferating and involuting lesions $(p<$ 0.05) (Figure 6.1).

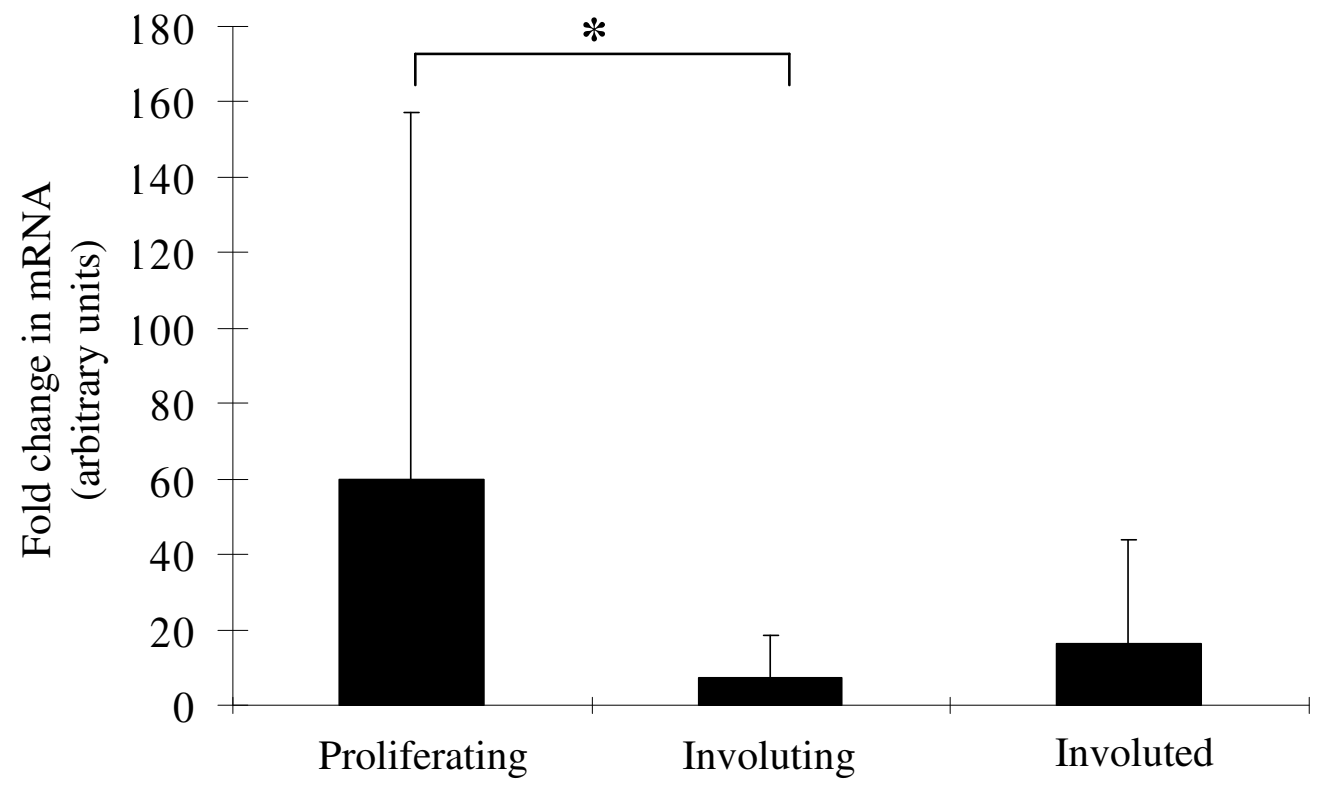

Figure 6.1: Changes in CycA mRNA expression. mRNA levels for CycA were determined for eight proliferating, eight involuting and eight involuted haeamgnioma samples relative to GAPDH. Proliferating samples expressed more CycA mRNA than involuting and involuted lesions. Significance was achieved between the proliferative and involuting phases $(* p<0.05)$. 


\subsubsection{PROTEIN EXPRESSION}

\subsubsection{WESTERN BLOTTING}

Some of the samples used for qRT-PCR were examined by Western blotting to identify if CycA protein abundance correlated with changes in mRNA expression. Figure 6.2 shows immunoreactive bands for $\mathrm{CycA}$ protein detected at $18 \mathrm{kDa}$, as expected (Hamilton \& Steiner, 1998), from four proliferating, three involuting, three involuted haemangioma biopsies and HeLa cells. Immunoblots were also analysed for GAPDH to confirm equal amounts of total protein. Although CycA mRNA levels varied with phase, no statistically significant difference in CycA protein expression was found between the specimens (Figure 6.2).
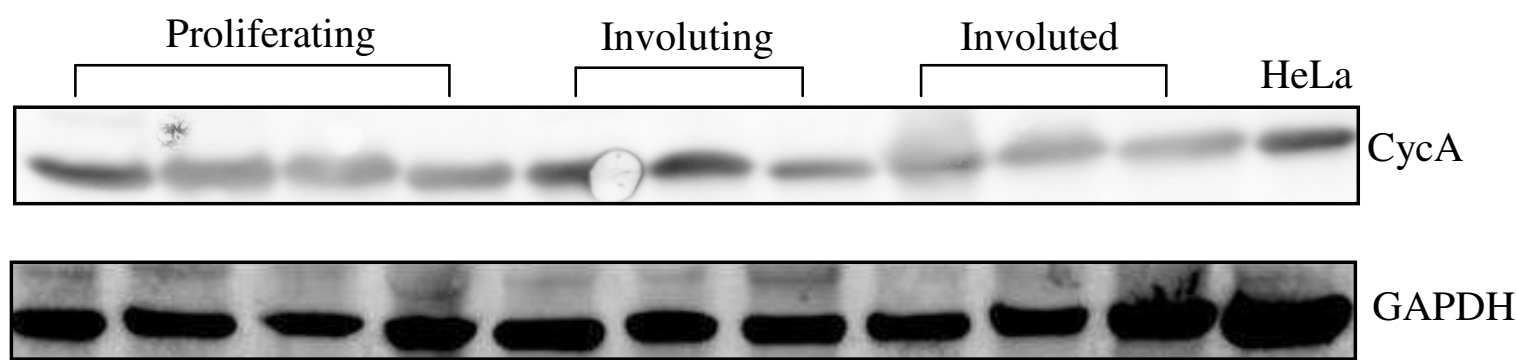

Figure 6.2: Western blotting for CycA in haemangioma. $50 \mu \mathrm{g}$ of total protein from proliferating, involuting and involuted haemangioma lesions were subjected to immunoblotting as described in section 2.2.2.2. Membranes were incubated with rabbit anti-CycA and mouse anti-GAPDH antibodies. With equal protein loaded from each sample (as determined by GAPDH immunoreactivity), no change in total CycA protein expression was found between phases. 


\subsubsection{IMMUNOHISTOCHEMICAL ANALYSIS}

\subsection{CycA EXPRESSION}

Immunohistochemical staining of haemangioma specimens (five samples per phase) showed that CycA was detectable in all phases of haemangioma (Figure 6.3, red staining). CycA protein was detected in the plump ECs of the proliferative phase (Figure 6.3-D, white arrows) and in the flattened ECs of the involuting (Figure 6.3-E, white arrows) and involuted phases (Figure 6.3-F, white arrows). In addition, the cells in the fibrofatty residuum of the involuting and involuted phases also expressed CycA (Figure 6.3-E and -F, respectively, yellow arrows). The decrease in CycA expression seen between phases in Figure 6.3 appears to be due to a decrease in cell number as opposed to a decrease in CycA staining intensity per cell. 

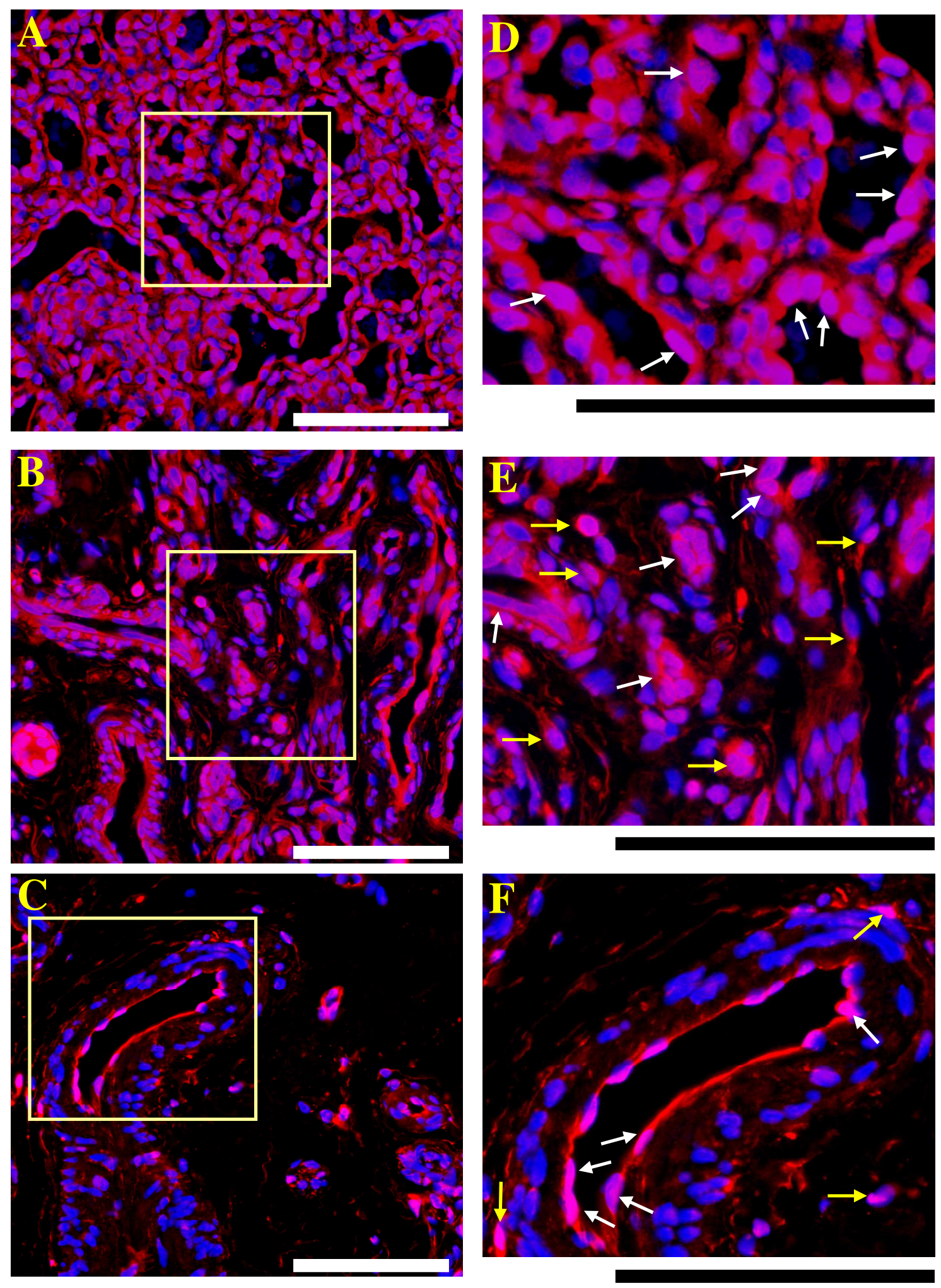

Figure 6.3: Immunohistochemical localisation of CycA in haemangioma. Proliferating $(\mathbf{A} \& \mathbf{D})$, involuting $(\mathbf{B} \& \mathbf{E})$ and involuted $(\mathbf{C} \& \mathbf{F})$ paraffin embedded haemangioma sections were stained with a rabbit anti-CycA antibody. Bound antibodies were detected using an anti-rabbit Cy3 conjugate (red). Cell nuclei were counterstained with DAPI (blue). Panels $\mathbf{D}, \mathbf{E} \& \mathbf{F}$ are the enlargements of the areas outlined in $\mathbf{A}, \mathbf{B} \& \mathbf{C}$, respectively. CycA expression (red staining) was detected in the plump ECs of the proliferative phase (D, white arrows) and also in the flattened ECs of the involuting and involuted phases $(\mathbf{E} \& \mathbf{F}$, respectively, white arrows). Involuting $(\mathbf{E})$ and involuted $(\mathbf{F})$ lesions also contained CycA positive cells in the interstitium (yellow arrows).

Scale bars: $50 \mu \mathrm{m}$. 


\subsection{MAST CELLS EXPRESS CycA}

To detect if $\mathrm{MCs}$ express $\mathrm{CycA}$, DAB colorimetric visualisation of CycA immunoreactivity in conjunction with Csaba staining was performed as described in section 2.2.2.4 (page 82). Of the $200 \mathrm{MCs}$ counted, those that stained positive for CycA $(\mathrm{CycA}+\mathrm{MCs})$ were identified in the proliferative and involuting phases. Consistent with earlier results (see section 3.3.2.3.5, page 122), a three-fold increase in the number of MCs was identified in involuting samples as compared to proliferating samples. However, the proportion of MCs expressing CycA did not vary with phase (Table 9, Figure 6.4)

Developmental Phase

$$
\text { Number of CycA + MCs \% CycA + MCs }
$$

Proliferative

$$
169 \pm 2.3(n=25),
$$

Involuting

$$
177 \pm 6.5(n=7)
$$

Proliferative vs Involuting $\quad p<0.1$

Table 9: CycA expression by MCs in haemangioma. CycA positive and negative MCs were counted from five samples of the proliferative and involuting phases as described in section 2.2.2.5.2. Of a total of $200 \mathrm{MCs}$ counted from each lesion, the values in the table gives the number of CycA positive $\mathrm{MCs}(\mathrm{CycA}+\mathrm{Mcs})$ and the percentage of CycA expressing $\mathrm{MCs}(\% \mathrm{CycA}+\mathrm{Mcs}$ ). Values are the mean (of five lesions) \pm s.e.m; $n$ indicates the total number of fields of view counted at a magnification of X40. 
Figure 6.4: MCs in proliferating and involuting haemangiomas express CycA. CycA immunoreactivity was detected by DAB staining (brown) and counterstained with Csaba to identify the MCs (light blue). Double labelling of a proliferating (A) and an involuting (B) haemangioma with CycA antibodies and Csaba stain demonstrated the presence of CycA protein within MCs (black arrows). In Panel B, the yellow arrows show few MCs that do not express CycA. (C) The black columns show CycA positive MCs and the white columns show CycA negative MCs. As identified earlier in this study, involuting lesions contained approximately three-fold more MCs per unit area as compared to proliferating lesions. However, approximately $80 \%$ of MCs present in proliferating and involuting lesions expressed CycA. Scale bars: $50 \mu \mathrm{m}$. 
A

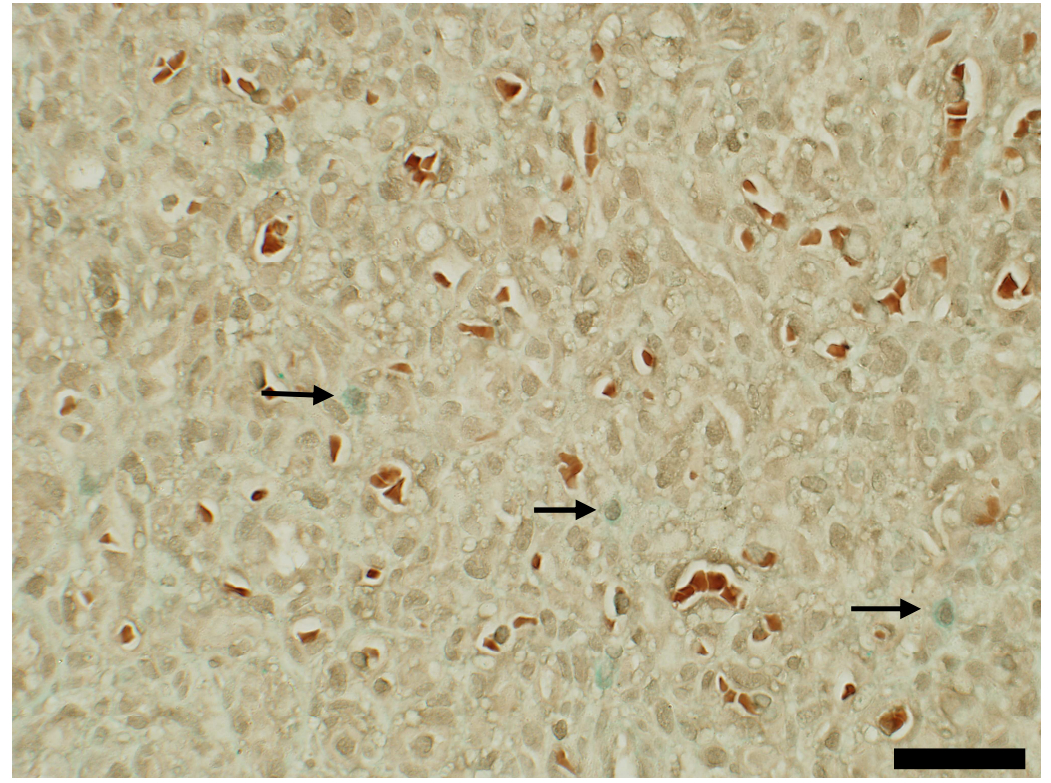

B

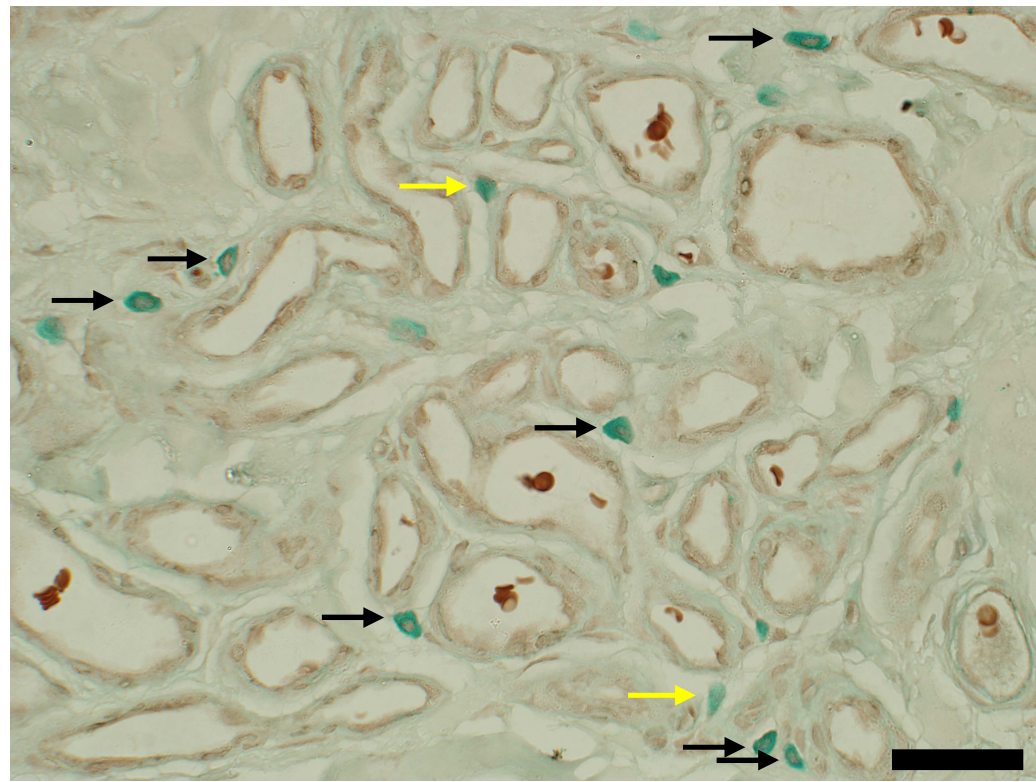

C

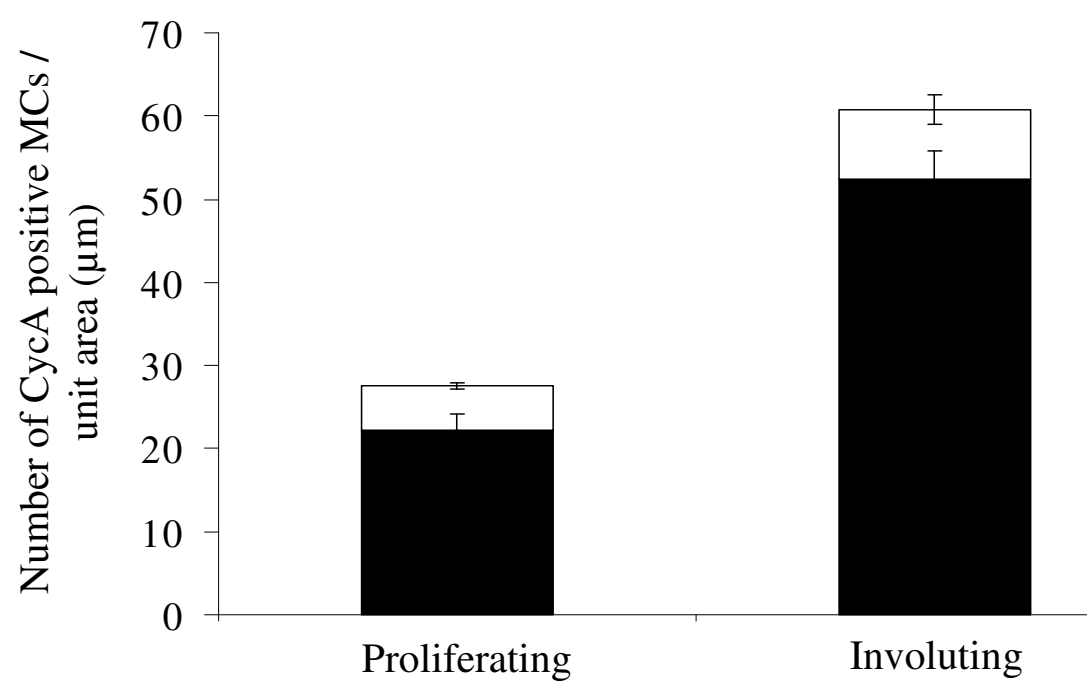




\subsection{DISCUSSION}

This is the first study to examine CycA expression in haemangioma. This study found that proliferating lesions expressed highest levels of CycA mRNA with decreased abundance in involuting and involuted haemangiomas. However, Western blotting studies showed that total protein levels of CycA did not differ between the phases. IHC confirmed this finding. Immunohistochemical staining showed that CycA immunoreactivity was detected in lesions from all three phases and that as a consequence of a decrease in cell number between phases, more CycA staining was present in proliferating lesions as compared to involuting and involuted lesions.

Although the findings from this study are purely descriptive, due to the presence of CycA in all phases of haemangioma together with its known function in protein remodelling and refolding as well as in EC proliferation and apoptosis, a model for the role of CycA in haemangioma was hypothesised and is depicted in Figure 6.5.

In this proposed model, a hypoxic event induces high expression and secretion of CycA from various cell types within the lesion. Secreted CycA then acts in a paracrine manner to induce further EC proliferation during the proliferative phase. With the initiation of involution, CycA functions as PPIase and participates in the tissue remodelling events that characterises the regression of haemangioma. The presence of CycA in MCs is of unclear significance. Perhaps the influx of MCs during the involuting phase still expressing $\mathrm{CycA}$ is required to maintain $\mathrm{CycA}$ protein levels within the lesion. A second role for CycA in the involuting phase of haemangioma may be in the induction of EC apoptosis. This is supported by the findings of Jin et al (2004) who showed that CycA can induce EC apoptosis similar to the proapoptotic effect of $\mathrm{TNF} \alpha$. However, further investigation is required to determine the precise role(s) of CycA in the pathogenesis of haemangioma. 


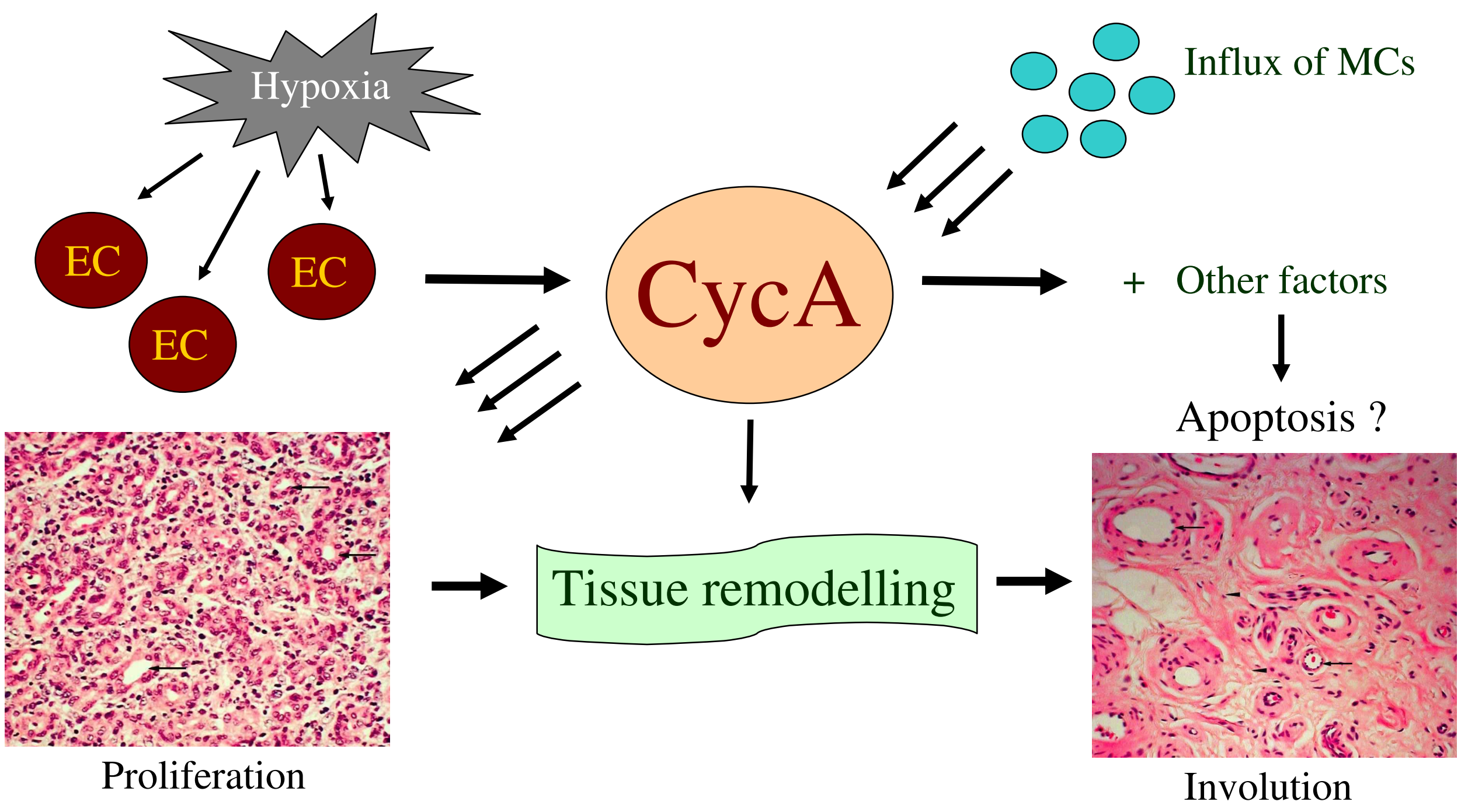

Figure 6.5: A role for CycA in haemangioma progression. See text for details. 


\section{CHAPTER 7: KEY FINDINGS AND FINAL CONCLUSIONS}

\subsection{THE SIGNIFICANCE OF THIS RESEARCH}

Over the past two decades, various studies have identified a number of cellular and extracellular markers associated with haemangioma. However, the molecular mechanisms that govern haemangioma proliferation and its subsequent involution are still largely unknown. This research aimed to enhance the current understanding of the molecular basis of haemangioma, as well as the complexity of cell signalling in the biology of this tumour. In this study, quantitative and qualitative differences in expression were investigated for a set of genes that were chosen because of their association with tumour susceptibility and angiogenesis. It was hoped that results of this research would identify novel markers that would lead to the development of more effective treatments. This study was also conducted in order to develop a working model for the biology of haemangioma. The following is a summary of some of the novel findings of this study.

\subsubsection{KEY FINDINGS}

\subsubsection{VEGF, VEGFRs AND STAT PROTEINS IN HAEMANGIOMA}

Using an in vitro model, that mimics as closely as possible the features of haemangioma, this study confirmed VEGF to be an important factor governing haemangioma proliferation. The addition of exogenous VEGF resulted in significant outgrowth of capillary-like structures from haemangioma explants. These results are consistent with the known role of VEGF as an angiogenic factor (Ferrara, 2000) as well as its high expression during the proliferative phase of haemangioma (Takahashi et al., 1994; Tan, 2001). In addition, proliferating lesions expressed highest levels of VEGFR2 as compared to involuting and involuted lesions. These findings confirm that 
VEGF/VEGFR-2 signalling is involved in the proliferation of haemangioma. In contrast, the presence of VEGFR-1 in all phases of haemangioma suggests that VEGFR-1 signalling may be required during the entire life cycle of haemangioma. As VEGFR-1 is involved in cellular differentiation (Neufeld et al., 1999), it is postulated that VEGFR-1 activation may be involved in transforming the highly cellular content of the proliferative phase into the fibro-fatty residuum of the involuting and involuted phases.

This study identified that STAT protein signalling is required for capillary-like outgrowth from explanted haemangioma tissues and for the proliferation of haemangioma tissue-derived cells. Of the seven members in the STAT family of transcription factors, STAT-3 expression was examined in haemangioma samples due to its association with tumour proliferation (Bromberg et al., 1998). Although there were high levels of STAT-3 in proliferating lesions, total abundance of STAT-3 per cell did not vary with the different phases. This suggests that STAT-3 abundance decreases with ongoing involution of haemangioma and that this decrease is concomitant with a reduction in cell number in involuting and involuted lesions. In addition, VEGF did not activate STAT-3 in cells purified from haemangioma lesions in vitro and activated STAT-3 did not correlate with proliferation in vivo. STAT-1 was also investigated due to its association with apoptosis. However, all lesions examined in this study showed low expression for STAT-1. These findings suggest that although STAT-3 and STAT-1 signalling may be occurring in haemangioma, these transcription factors are not critical regulators of haemangioma. However, preliminary experiments conducted in this study showed that STAT-5 activation is associated with VEGF signalling in haemangioma. Since STAT-5 is now known to be critical in cell transformation, its activators and down-stream targets in haemangioma biology warrants further investigation. 


\subsubsection{THE USE OF TRAIL AS TREATMENT FOR HAEMANGIOMA}

Pharmacological therapy is the mainstay treatment for haemangioma during the proliferative phase with steroids being the first line of treatment and IFN $\alpha$, the second choice, both of which were discovered serendipitously. These treatments are slow and are associated with serious side-effects. Surgery and laser therapy are indicated if pharmacological therapy fails. However, all these treatment modalities are empirical measures and leave definite sequelae (Tan, 2001). Therefore, one of the aims of this investigation was to use the in vitro haemangioma model (Tan et al., 2000 ) to help elucidate the mechanisms of action of existing therapies, such as IFN $\alpha$, and also identify novel agents for the treatment of haemangioma. Findings from this research identified TRAIL in combination with a low dose of IFNa as a potential treatment for proliferating haemangioma. Results showed that the combined effect of TRAIL with a low dose of IFN $\alpha$ acted synergistically and inhibited capillary-like outgrowth from haemangioma explants to a similar extent as the singly added high dose of IFN $\alpha$. Since TRAIL is known to be non-toxic to normal cells, this novel finding of its effect against haemangioma capillary-like outgrowth deserves further investigation.

\subsubsection{THE IDENTIFICATION OF STEM CELLS AND THEIR ROLE IN}

\section{HAEMANGIOMA PROGRESSION}

The proliferative phase of haemangioma is characterised by rapid proliferation of ECs with the accumulation of other cellular elements involved in angiogenesis including MCs, pericytes and macrophages (Takahashi et al., 1994). However, the most curious biological characteristic of haemangioma is the spontaneous regression. Although various cell types and cell-specific markers have been identified in haemangioma, the mechanisms of origin of the heterogeneous cell population, their post-natal presentation and their distinctive pattern of limited growth and involution are poorly understood. 
In this study, EPCs and MSCs were purified from haemangioma tissues. We postulate that abnormal stem cell development and differentiation underlies the aethiopathogenesis of haemangioma. Although EPCs and MSCs have been previously reported in haemangioma (Yu et al., 2004a; Yu et al., 2006), this study showed that the cellular structures that rapidly emanated from explanted proliferative haemangioma biopsies were MSCs as they readily differentiated into osteocytes and adipocytes in vitro. However, the cells obtained from the direct dissociation of proliferating lesions were characterised as immature EPCs. These EPCs then further differentiated into MSCs with continued culture in vitro. This de novo transdifferentiation process in vitro may be due to the culture of the EPCs in the absence of growth factors which were present in the original lesion. This study also found that the MSCs that differentiated from EPCs maintained their plasticity into adipocytes in vitro. Since adipocyte deposition is associated with the spontaneous involution of haemangioma, we hypothesise that this process of transdifferentiation from EPCs to MSCs and MSCs to adipocytes regulates the biology of haemangioma.

\subsubsection{HOW DO THE FINDINGS FROM THIS STUDY FIT IN WITH THE CURRENT KNOWLEDGE OF HAEMANGIOGENESIS?}

The high incidence of haemangioma in the head and neck region together with the solitary and segmental post-natal presentation of these lesions led Waner et al (2003) to suggest that developmental detriments may be influential in the pathogenesis of haemangioma.

To put the findings of this investigation into context, the developmental origins of the face are briefly described.

The face begins to form during the $4^{\text {th }}$ week of embryological development with the formation of the mesenchyme. The head mesenchyme is distinctive in that it is derived from neural crest cells rather than mesoderm (Risau \& Flamme, 1995). The neural crest cells that form the face migrate dorsolaterally from the nueral tube to 
produce five craniomesenchyme prominences consisting of a single frontonasal prominence, paired maxillary and paired mandibular prominences (Mitchell \& Sharma, 2005). These cranial neural crest cells are encoded with specific morphogenetic information prior to their migration (Gilbert, 1994). These prominences then differentiate into cartilage, bone, cranial neurons, glia and the connective tissues of the face including the corneal endothelium and stroma and the dermis and adipose tissue of the skin. However, the fate of the neural crest cells depends to a large degree upon where the cells migrate and settle and to the local environmental cues (Le Douarin et al., 2004).

Neural crest-derived mesenchyme unlike mesoderm-derived mesenchyme, do not possess endogenous angioblasts and must rely on in-migrating angioblasts and vascular sprouts for vascularisation (Noden, 1988). Angioblasts are the EC precursors that form the primitive vascular channels during embryonic development (Risau et al., 1988; Risau, 1997). Angioblast migration is particularly prominent in the head region implying the presence of increased levels of motility or chemotactic factors (Noden, 1991). Therefore, Waner et al (2003) proposed that haemangioma preferentially occured in regions where neural crest-associated angiogenic mechanisms were elevated.

The association between segmental haemangioma and PHACES syndrome deserves special consideration. Several of the associated anomalies, such as lingual thyroid and sternal clefting, have their origin between 6 and 8 weeks of gestational age, suggesting a so-called developmental-field defect (Frieden et al., 1996). Furthermore, the parotid glands are frequently involved by overlying plaque-like haemangiomas. These glands also begin their development at about 6 weeks of intra-uterine life. Waner et al (2003) suggested that the precursors of parotid hemangiomas may have reached the region during this early time frame, with proliferation held in check until latter in gestation or after birth. The authors also suggested that the differential patterns of tissue involvement seen in focal and segmental haemangioma may reflect the timing of 
deposition of haemangioma precursor cells, with earlier events resulting in segmental haemangioma and later events resulting in focal haemangioma. These ideas, although speculative, suggests that haemangioma is not just a tumour but may be a developmental problem associated with abnormal angiogenesis.

In order to explain the fascinating relationship between haemangioma and the placenta, Waner et al (2003) suggested that the regions of growing neuromesenchyma represented the 'fertile ground' for the implantation and proliferation of embolised placental cells or aberrant angioblasts by virtue of their inherent need to attract invading vascular precursors.

In view of these ideas and the findings from our investigation, we propose a model for the involvement of neural crest cells in the pathogenesis of haemangioma (Figure 7.1). In this model, some migrating neural crest cells possess abnormalities that may be caused by mutations (Figure 7.1-A). While normal neural crest-derived mesenchyma recruit angioblasts as part of the normal developmental process, the presence of abnormal neural crest cells leads to excessive recruitment of these cells resulting in the presence of excessive EPCs in the region of the haemangioma (Figure 7.1-B). We propose that these EPCs are recruited either from the developing vasculature or embolised from the placenta. A placental origin for these EPCs could explain the expression of various placental antigens by the ECs in haemangioma.

We hypothsise that EPC proliferation may be inhibited at this stage by passive immunity within the intra-uterine environment afforded by maternal circulation. During gestation, the maternal immune response against the foetus occurs in part by secretion of immuno-suppressors through the placenta (Jauniaux et al., 1995). Circulating maternal angiogenesis inhibitors have also been described during pregnanacy (Klauber et al., 1997; Levine et al., 2004). Thus, birth removes the intra-uterine suppression of angiogenesis and allows the proliferation of a pre-established lesion. This could explain the observed post-natal growth of these lesions. Alternatively, in the immediate post- 
natal period, an unknown factor could stimulate excessive angiogenesis in the region of the 'nascent' haemangioma. The idea that hypoxia may initiate and maintain haemangioma growth is supported by a number of clinical observations, although direct experimental data is not yet been available. For example, a blanched area of skin is usually noticed before the actual growth of the lesion. This may be an area of local ischaemia in the skin, caused by an unknown event that creates a hypoxic environment and thus, triggers growth factor expression. Studies have shown increased expression of IGF-2 and VEGF in response to hypoxia (Yu et al., 2004b; Levy et al., 1998; Stein et al., 1998; Tong et al., 2006). This could explain the high levels of these growth factors seen during the proliferating phase of haemangioma. Also observed on haemangioma ECs is GLUT-1, another hypoxia-inducible molecule (Loike et al., 1992).

We hypothesise that the high levels of VEGF and IGF-2, in turn, induces proliferation of the EPCs and results in the recruitment of support cells including MCs, pericytes and macrophages. At this stage the EPCs are protected from apoptosis due to OPG expression. All these events ultimately result in increased blood vessel formation that characterises the proliferative phase of haemangioma (Figure 7.1-C). Preliminary results suggest that STAT-5 is activated in response to VEGF.

However, in the early stages of the involuting phase, an unknown angiogenic inhibitory factor is induced which results in the down-regulation of growth factor levels within the lesion. The consequence of low levels of VEGF (and other growth factors) results in the differentiation of EPCs into MSCs. These MSCs, in turn, differentiate into adipocytes that form the fibro-fatty tissue seen in involuting and involuted lesions (Figure 7.1-D). The signal for MSC differentiation into adipocytes in vivo is unclear. We propose a role for insulin and Dexamethasone in the adipogenesis of haemangioma, as these factors are involved in adipocytic differentiation in vitro.

The trigger for involution has also been suggested to be due to the 'loss of protection' of haemangioma cells from immune surveillance mechanisms (Ritter et al., 
2003; Friedlander et al., 2005). Recent research has suggested that an immune response may be involved in the proliferation and involution of haemangioma. Ritter et al (2003) reported the expression of peripheral $\mathrm{T}$ cell surface markers, $\mathrm{CD} 3$ and $\mathrm{CD} 8$, and suggested a cytotoxic T cell phenotype in haemangioma. These authors also identified high levels of indoleamine 2, 3 dioxygenase (IDO) in proliferating haemangioma which decreased during involution. IDO is an enzyme involved in the tryptophan degradation Availability of tryptophan is critical to the function of $\mathrm{T}$ cells in promoting apoptosis (Frumento et al., 2002). Ritter et al (2003) therefore suggested that the high levels of IDO and concomitant low tryptophan concentrations in the proliferative phase delays the onset of involution. In retrospect, the trigger for involution could occur as a consequence of low IDO levels, high tryptophan concentrations and therefore increased T cell cyto-toxicity within the lesion.

Although a number of aspects in the model shown in Figure 7.1 are speculative, the possible link between neural crest cell development and the non-random facial presentation of haemangioma is an exciting notion that warrants further investigation.

\subsubsection{THE OVERALL SIGNIFICANCE OF THIS RESEARCH}

This investigation has resulted in an improved understanding of the cellular and molecular mechanisms regulating haemangioma proliferation and involution. The identification of an EPC to a MSC differentiation which may be responsible for governing the biology of haemangioma progression and the identification of TRAIL as potential therapy for haemangioma represent novel findings. From a clinician's perspective, the ultimate aim of haemangioma research is to acquire substantial understanding of haemangiogenesis that would allow the development of effective treatments for haemangioma. The results presented in this thesis have led to a better understanding of the pathogenesis of haemangioma with the potential to develop novel treatment strategies for this common tumour of infancy. 
A 4 weeks

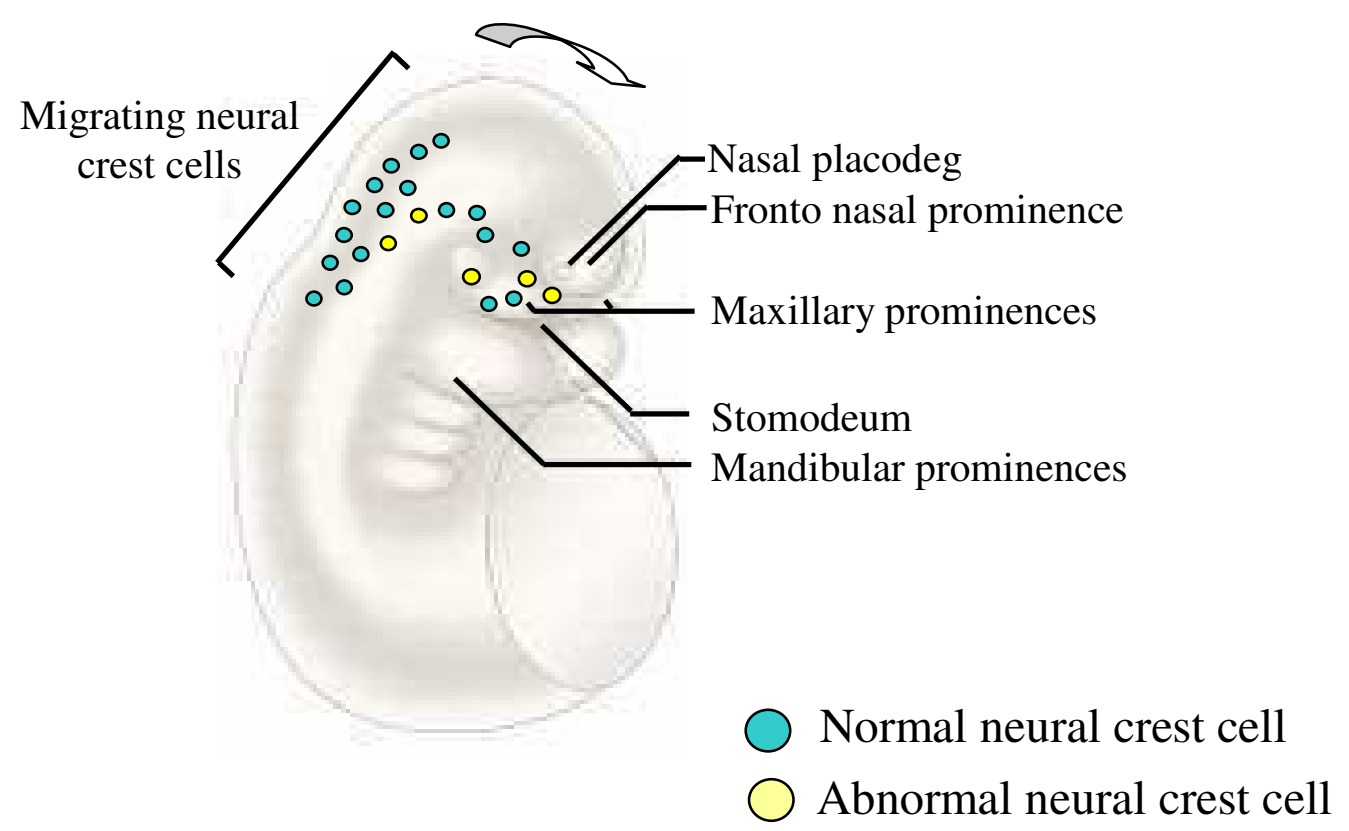

B

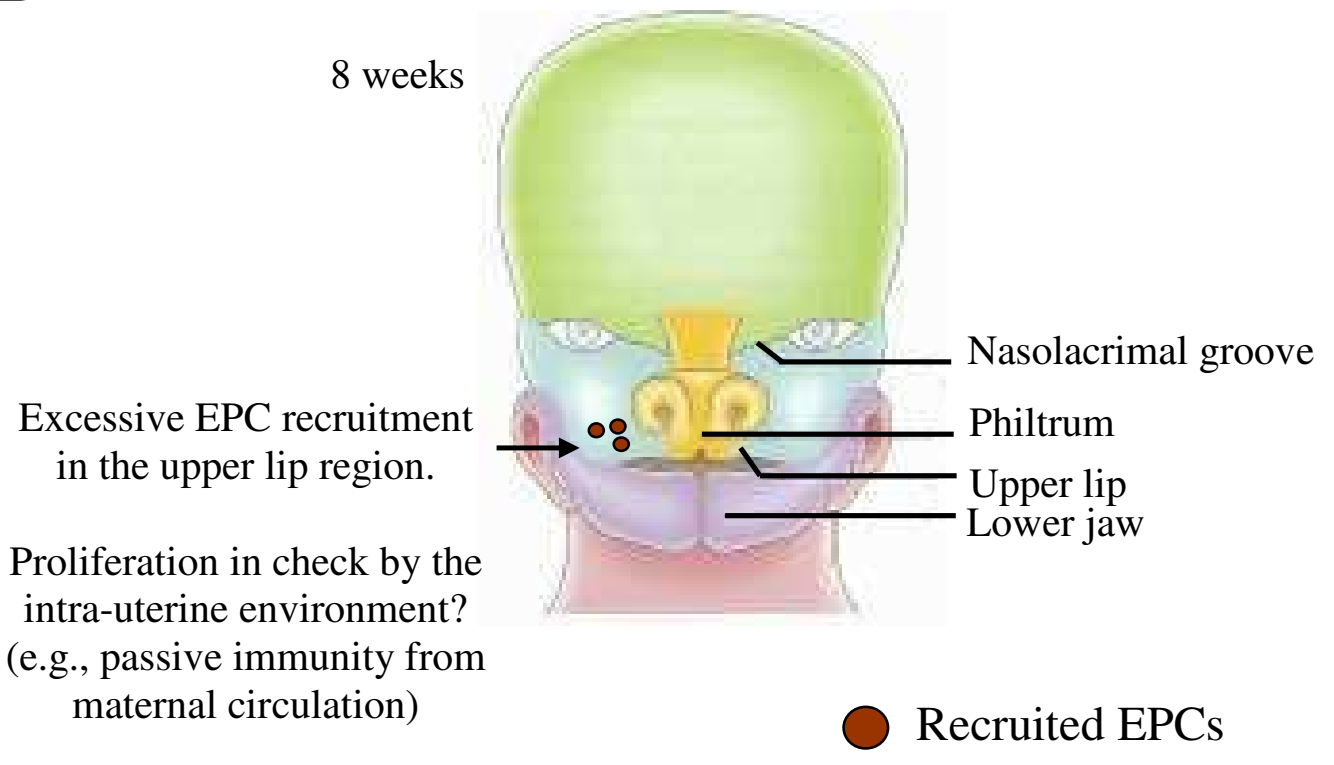

Figure 7.1: A proposed model for the development of haemangioma based on the speculative ideas and findings from this investigation. (see section 7.1.1.4 for details). 


\section{Proliferating phase}
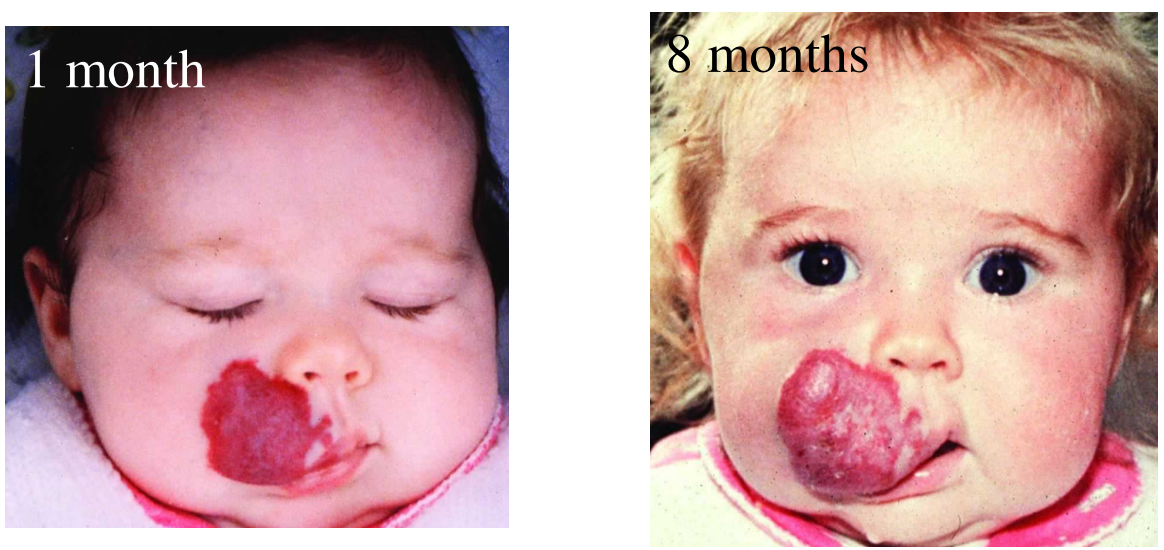

Hypoxia?

Loss of inhibitory factors

e.g., Loss of maternal immunity?

Low IFN- $\beta$ in the overlying dermis?

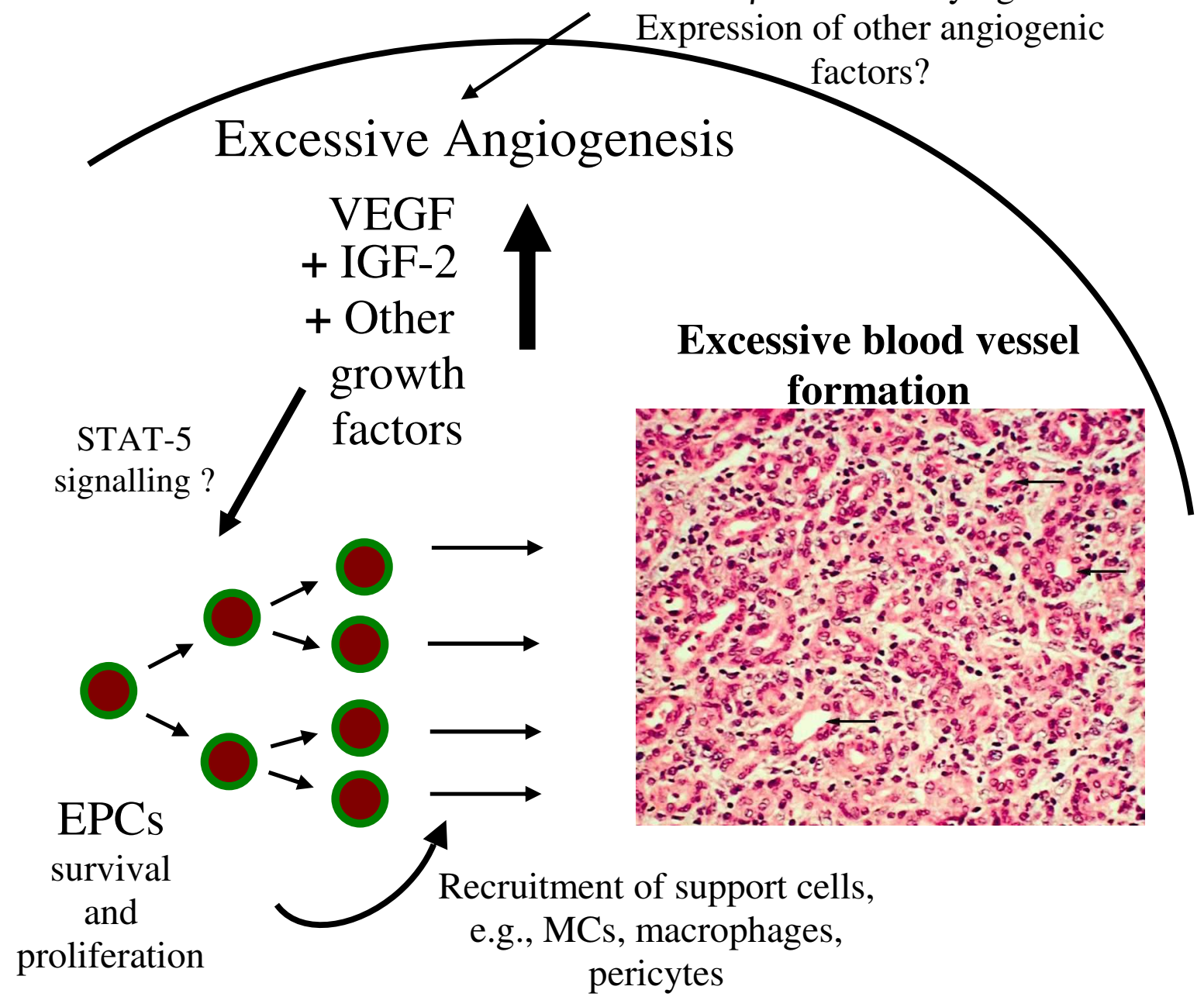

\section{OPG}

Figure 7.1: A proposed model for the development of haemangioma based on the speculative ideas and findings from this investigation. contd (see section 7.1.1.4 for details). 
D
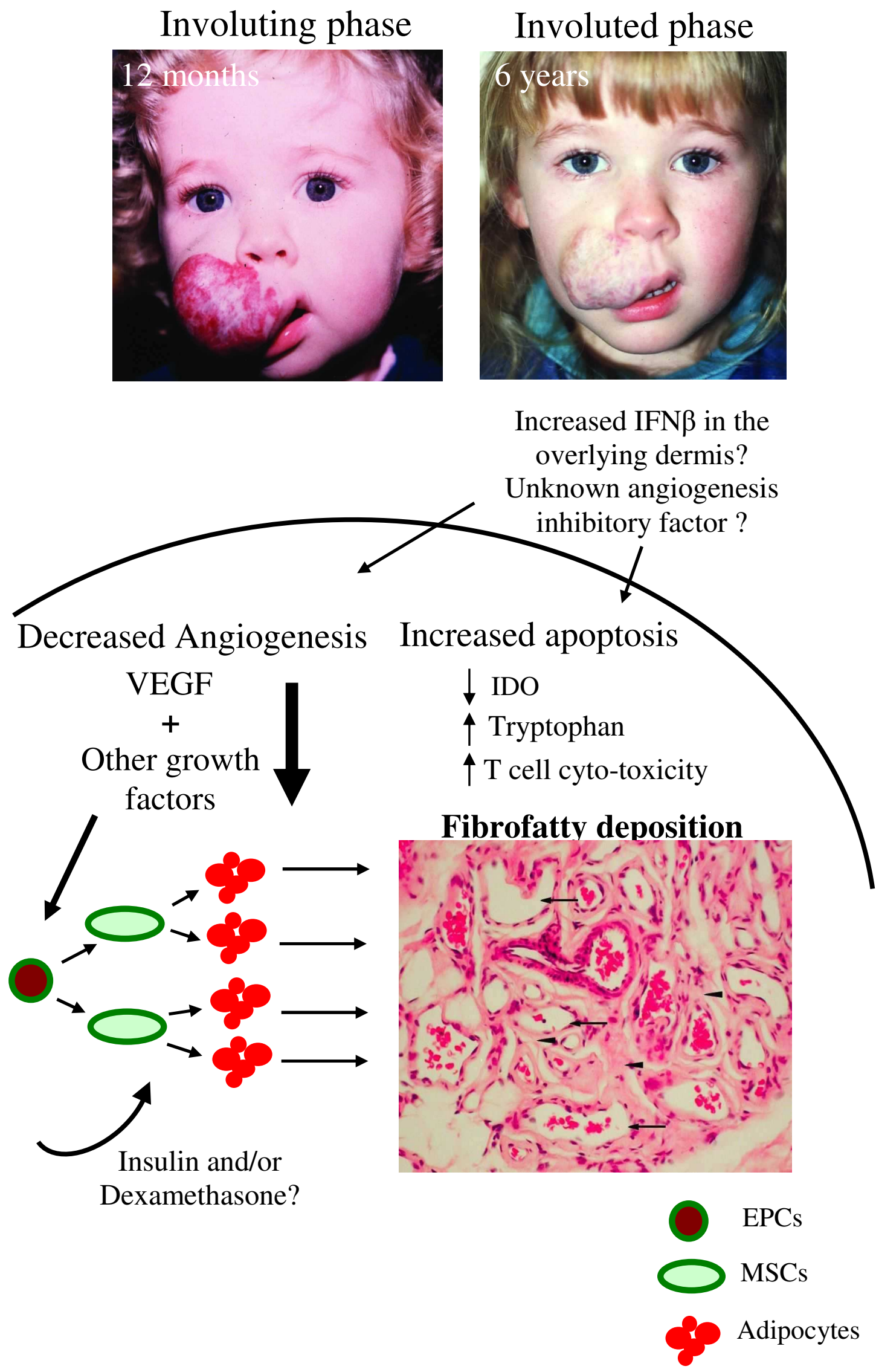

Figure 7.1: A proposed model for the development of haemangioma based on the speculative ideas and findings from this investigation. (contd) (see section 7.1.1.4 for details). 


\section{APPENDIX 1: CHAPTER 3}

\subsection{PRELIMINARY EXPERIMENTS}

\subsubsection{METHODS}

\subsubsection{QUANTITATIVE REAL TIME PCR}

In order to perform qRT-PCR accurately, a number of preliminary experiments were performed. These included (i) optimisation of PCR parameters for each primer set to yield PCR products of the predicted sizes without spurious non-specific bands and primer dimer, (ii) testing the efficiency of each primer set and (iii) identification of an appropriate house keeping gene to use as the internal reference gene. The importance of the house keeping gene and a brief introduction to qRT-PCR is discussed in section 2.1.1.1 (page 55).

1) Testing the PCR Efficiencies of Each Primer Pair: RNA purified from snap frozen normal skin was reverse transcribed into cDNA as described in sections 2.1.1.2.1 and 2.1.2 (pages 58 and 59). qRT-PCR was carried out as outlined in section 2.1.4 (page 65). PCR products were then separated by electrophoresis on $2.5 \%$ Agarose gels (See Appendix 4, section 4.1.4, page 295). PCR products were excised and purified from the gels as described in Appendix 4, section 4.1.5 (page 296).

Efficiencies for all the primer pairs used in this study were determined from diminishing dilutions of the respective purified PCR products. An initial 500-fold dilution of the purified PCR product was prepared from which eight-fold serial dilutions were prepared. $1 \mu \mathrm{l}$ of each dilution was then used as template and amplified in duplicate by qRT-PCR for each primer set. The threshold Ct was plotted against the Log of template concentrations and the slope of the line generated was used to calculate the Efficiency (E) according to the equation:

$$
E=10^{[-1 / \text { slope }]}-1(\text { Rasmussen, 2001) }
$$


Efficiencies for all the primer pairs were calculated to be approximately 2 which is the theoretical maximum (Rasmussen, 2001).

2) Validation of an Appropriate Internal Reference Gene: Two approaches were used to identify an appropriate internal reference gene for this study.

A) RNA was purified from six proliferating, eight involuting and seven involuted haemangioma biopsies and cDNA was synthesised using random hexamers as described in section 2.1.2 (page 59). GAPDH, CycA and 18SrRNA were amplified by qRT-PCR and the $\triangle \mathrm{Ct}$ values of GAPDH and CycA mRNA were calculated relative to $18 \mathrm{SrRNA}$ using the formula :

$$
\Delta \mathrm{Ct}=\mathrm{Ct}_{(\mathrm{GAPDH} \text { or } \mathrm{CycA})}-\mathrm{Ct}_{(18 \mathrm{SrRNA})}
$$

B) cDNA was also synthesised using Oligo(dT) $)_{20}$. qRT-PCR was performed for GAPDH and CycA and the absolute Ct values were calculated when $500 \mathrm{ng}$ of total RNA was used in the cDNA synthesis reaction.

\subsubsection{IMMUNOHISTOCHEMICAL OPTIMISATION}

HeLa cells, HUVECs, EOMA cells and placenta tissue sections were initially used to optimise the IHC staining procedures for the various antibodies used in this study. Cultured cells were grown onto $13 \mathrm{~mm}$ diameter borosilicate glass coverslips and cultured in their respective culture medium as described in section 2.1.6.2 (page 67). Immunostaining for STAT-3, p-STAT-3 (Tyr 705) and STAT-1 was performed on cultured HeLa cells fixed with 4\% PFA and immunostaining for VEGFR-2 was done on HUVECs and EOMA cells fixed with a mixture of acetone:methanol, as decribed in section 2.1.8.2.2 (page 74). Fixed cells were incubated with primary antibodies against STAT-3, p-STAT-3 (Tyr 705), STAT-1 and VEGFR-2 followed by incubation with

either an anti-rabbit DIG conjugate or an anti-mouse DIG conjugate followed by incubation with an anti-DIG-Rhodamine conjugate. Coverslips were stained in DAPI 
(see section 2.1.8.3, page 75) and mounted in Antifade. Control coverslips were included in each experiment in which the primary antibody was omitted.

Formalin fixed paraffin embedded placenta sections were deparaffinised, rehydrated and subjected to a sodium citrate antigen retrieval step as outlined in section 2.1.8.2.1 (page 74). Sections were then blocked and incubated overnight at $4^{\circ} \mathrm{C}$ with rabbit anti- GLUT-1 primary antibodies. Bound GLUT-1 antibody was detected with an anti-rabbit Cy3 conjugate. Slides were then stained in DAPI and mounted in Antifade.

\subsubsection{STATISTICS}

The non-parametric Kruskil Wallis by ranks test was used to identify changes in CycA and GAPDH mRNA expression with $500 \mathrm{ng}$ of RNA. This was followed by the multiple comparisons test to determine where the significant changes occurred. The $\mathrm{R}$ package was used to determine changes in mRNA expression relative to 18SrRNA. The linear mixed effects method of analysis was used to allow for repeated measures on subjects and for each transcript, a sequential bonferroni adjustment was made for multiple testing. Data is represented as relative fold changes in mRNA expression \pm s.e.m.

\subsubsection{RESULTS}

\subsubsection{PCR QUALITY CONTROL}

PCR efficiencies were calculated as described in section 1.1.1.1 (page 267). All qRT-PCR assays were linear over a 10,000-fold range with $\mathrm{Ct}$ values ranging between 12.5 and 37 cycles (Figure 1.1). The correlation coefficients were consistently $>0.98$ in each assay and each PCR amplification displayed an efficiency close to 2 (Figure 1.3). Melt-cure analyses were performed after every amplification to ensure the reactions were free from contaminating DNA. A typically melt-curve plot is shown in Figure 1.2. 


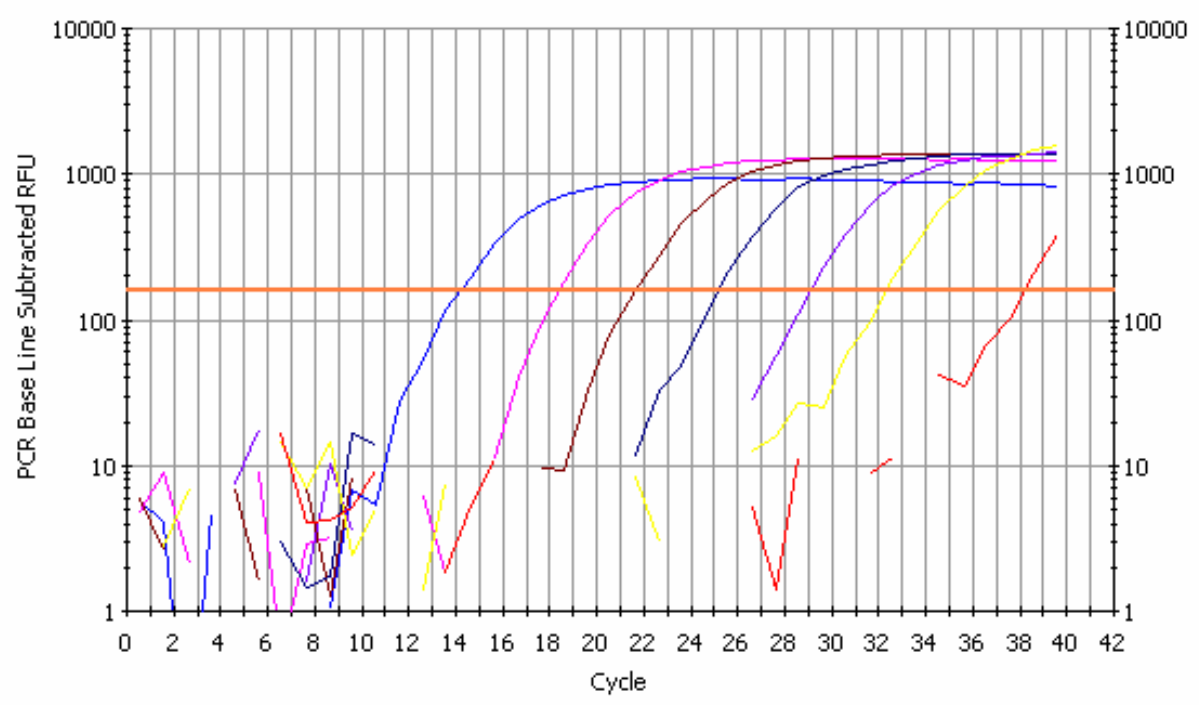

Figure 1.1: An example of the cycling numbers obtained during a typical real-time PCR amplification of serially diluted PCR template. The Ct values changed proportionally according to the serial dilution of the sample. The figure above shows the PCR curves obtained when serially diluted STAT-1 PCR product was amplified by qRT-PCR. Samples that had $\mathrm{Ct}$ values below 30 were considered positive when there was no evidence of amplification in the no-template negative controls. A maxiumum of $1 \mathrm{Ct}$ difference between duplicates was accepted.

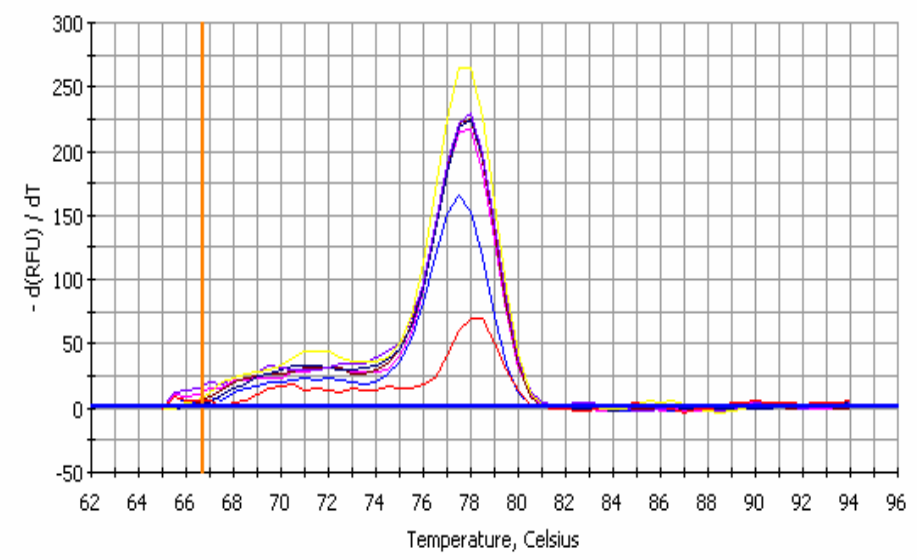

Figure 1.2: The melt-curve obtained at the end of a PCR amplification with serially diluted STAT-1 template. As the amplified product have the same length and are of the same nucleotide ratio, they have the same melting temperature. This can be observed by a series of peaks one on top of each other on the melt-curve. Absence of other peaks indicates the absence of spurious non-specific product formation. 
A

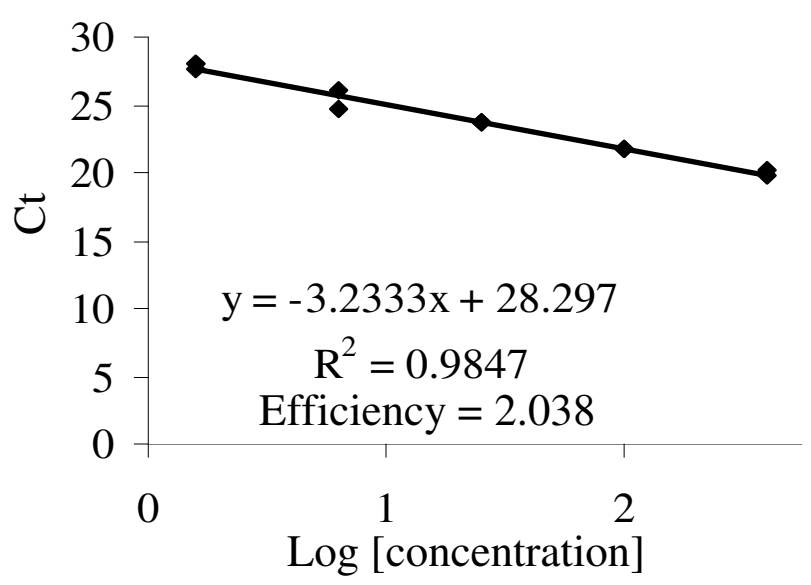

B

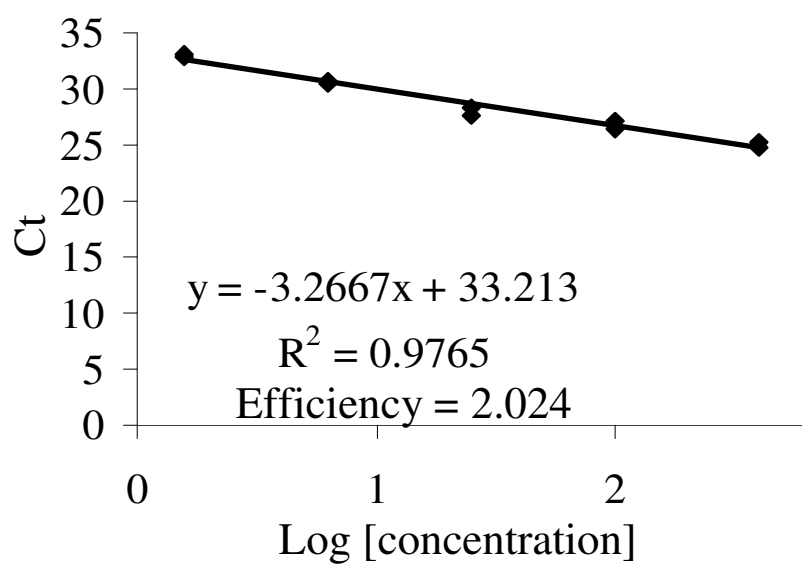

C

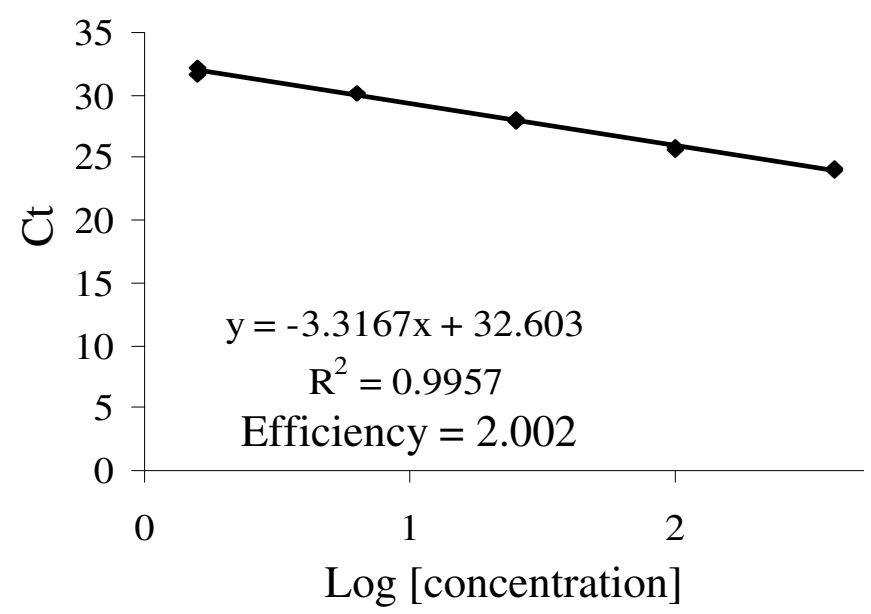

Figure 1.3: qRT-PCR primer pair efficiencies. The PCR amplification efficiencies using primers for STAT-1 (A), STAT-3 (B) and VEGFR-2 (C) were calculated as described in section 1.1.1.1. Serially diluted PCR product was amplified by qRT-PCR in duplicate. The slope of the line obtained by plotting the $\mathrm{Ct}$ value against Log [Concentration] was then used to calculate the efficiency of the PCR reaction. 


\subsubsection{VALIDATION OF AN APPROPRIATE INTERNAL REFERENCE GENE}

Primer pairs for four commonly used house keeping genes, 18SrRNA, GAPDH, CycA and HPRT, were designed for use in this investigation. An ideal reference gene would be one that is not differentially regulated across the three phases of haemangioma (proliferating, involuting and involuted) and is expressed at relatively similar levels as the gene of interest (Roche LC technical note 15/2002; Vandesompele et al., 2002).

Preliminary experiments showed that a neat cDNA sample (from normal skin) amplifies 18SrRNA at cycle $10(\mathrm{Ct}=10)$ whereas the test genes were not amplified until cycle 25 or later, representing an approximately 500,000-fold difference in abundance. Because the 18SrRNA transcript is far more abundant than all the test genes, it was not considered a suitable internal reference gene for this study. The use of HPRT as an internal reference was also discounted because of its late amplification in haemangioma samples.

To validate the use of GAPDH and CycA as internal reference genes, two different approaches were used. Absolute Ct values were calculated from proliferating, involuting and involuted haemangioma biopsies (eight per phase) for both genes when $500 \mathrm{ng}$ of total RNA was used for the cDNA synthesis reaction. CycA mRNA expression changed between phases (Figure 1.4-A), with significant differences between the proliferative and involuting phase $(p<0.01)$ and also between the proliferative and involuted phase $(p<0.01)$. However, no significant change in GAPDH mRNA expression between the phases was identified ( $p<0.34$, Figure 1.4-B). 

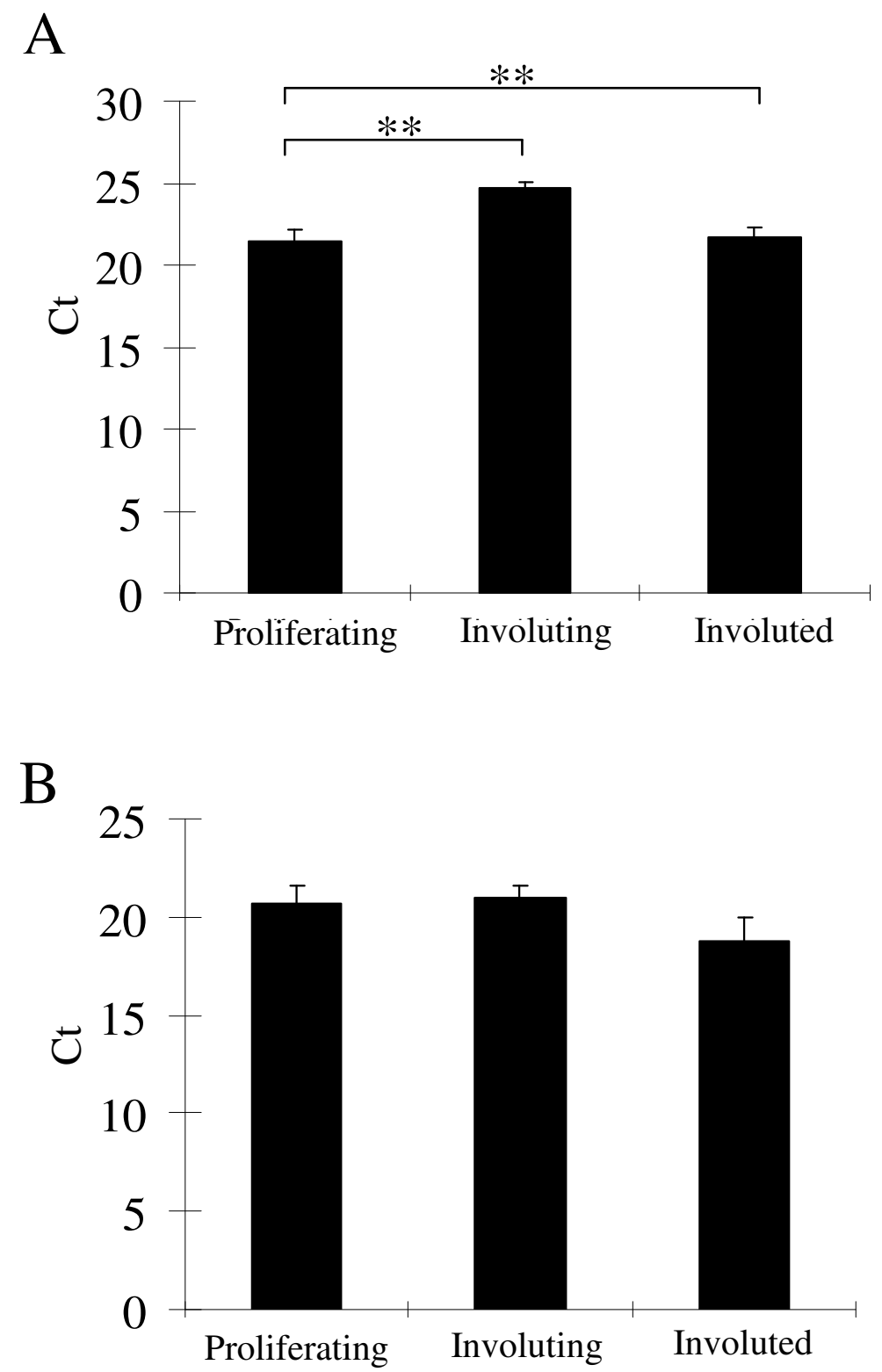

Figure 1.4: Validation of an appropriate internal house-keeping gene (Experiment 1). The $\mathrm{Ct}$ values obtained when qRT-PCR was performed with $500 \mathrm{ng}$ of starting mRNA from each sample, were used to detect differences in CycA (A) and GAPDH (B) mRNA expression across phases of haemangioma. (A) Significant changes in CycA mRNA levels was detected between the proliferative and involuting phase and between the proliferative and involuted phase $(* * p<0.01)$. (B) GAPDH mRNA levels did not vary between phases.

For the second approach, qRT-PCR was performed for GAPDH and CycA using 18SrRNA as the internal reference gene. The use of 18SrRNA was accepted in this case as it was found to be expressed in the same dynamic range as GAPDH and CycA. The $\triangle \mathrm{Ct}$ values for GAPDH and CycA mRNA were calculated relative to $18 \mathrm{SrRNA}$ using the formula $\Delta \mathrm{Ct}=\mathrm{Ct}{ }_{(\mathrm{GAPDH} \text { or } \mathrm{CycA})}-\mathrm{Ct}{ }_{(18 \mathrm{SrRNA})}$. Relative to 18SrRNA, GAPDH and 
CycA mRNA levels did not change between phases $(\mathrm{p}<0.1229$ and $\mathrm{p}<0.054$, respectively) (Figure 1.5). However, since the $p$ value obtained for CycA expression ( $\mathrm{p}$ $<0.054)$ was so close to the chosen threshold for significance, GAPDH was chosen as a more suitable reference gene for this study.
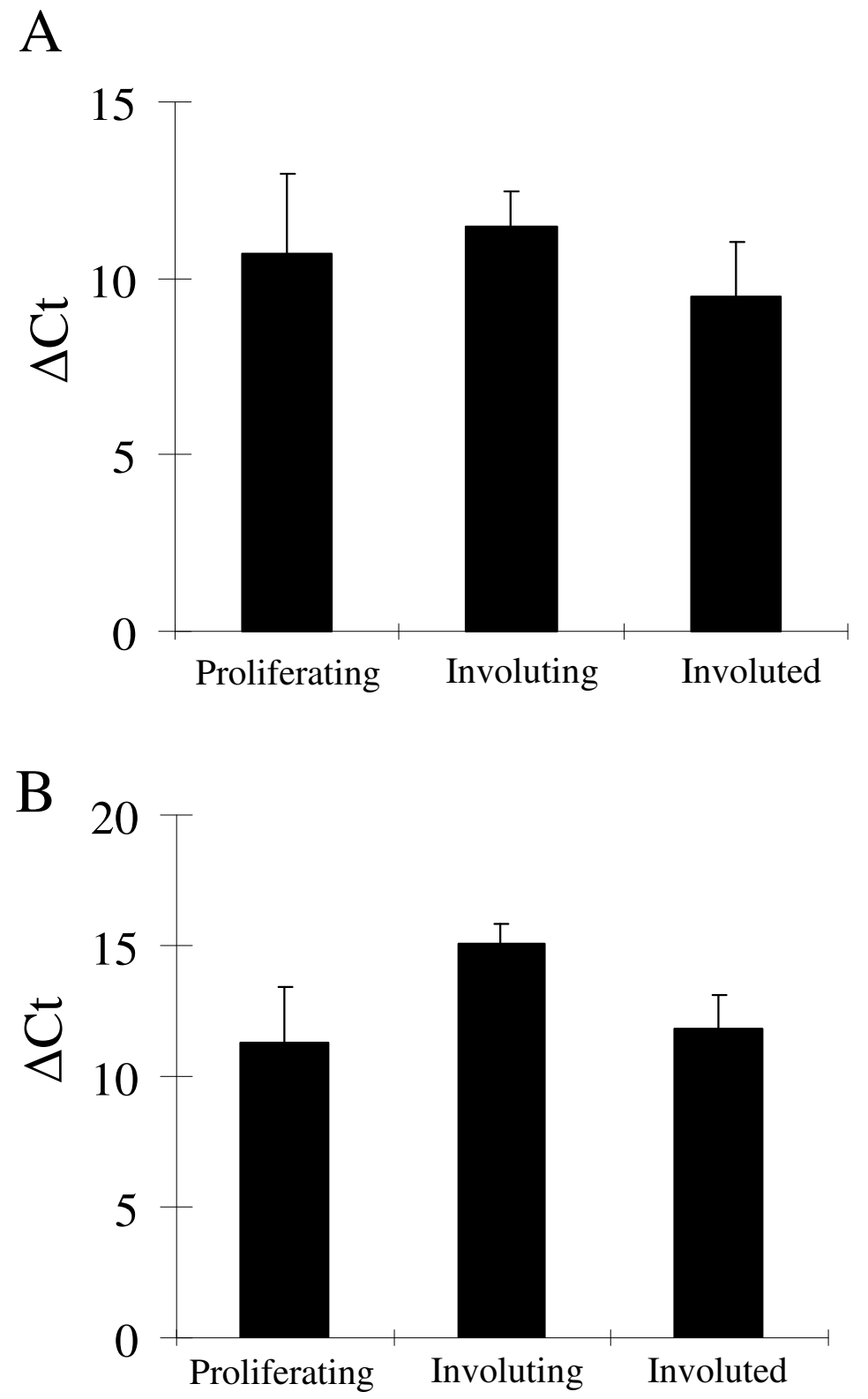

Figure 1.5:Validation of an appropriate internal house-keeping gene (Experiment 2). To detect changes in CycA (A) and GAPDH (B) mRNA between proliferating, involuting and involuted lesions, $\triangle \mathrm{Ct}$ values for $\mathrm{CycA}$ and GAPDH mRNA relative to 18SrRNA were analysed. Statistical analysis was performed using the $\mathrm{R}$ package as described in section 1.1.1.3. Relative to 18SrRNA, no statitstically significant change in CycA or GAPDH mRNA expression was identified across the phases. 


\subsubsection{IMMUNOHISTOCHEMICAL OPTIMISATION}

Paraffin embedded placenta sections, HeLa cells and EOMA cells were used at the start of this study to optimise the IHC staining procedures and to determine antibody specificity. Control slides in which the primary antibody was omitted showed no staining. Paraffin embedded tissue sections exhibited some background fluorescence, thought to be due to the autofluorescence of collagen fibres. A tissue bleaching step was therefore introduced into the immunostaining protocol using $\mathrm{NaBH}_{4}$ which effectively reduced the problem, allowing specific signal to be detected.

HeLa cells in culture expressed STAT-3 (Figure 1.6-A). However, STAT-1 and p-STAT-3 (Tyr 705) expression was low in these cells (Figure 1.6-C and -E, respectively). Treatment with IFN $\alpha-2 b$ for 10 min induced STAT-1 and p-STAT-3 (Tyr 705) expression (Figure 1.6-D and -F, respectively).

As expected, positive immunoreactivity was detected for VEGFR-2 and GLUT1 when IHC staining was performed on EOMA cells and placenta tissue sections respectively (Figure 1.7). Previous studies have shown that VEGFR-2 is expressed by EOMA cells (Ilan et al., 2003), and GLUT-1 is expressed in placenta (Hahn et al., 1998). 

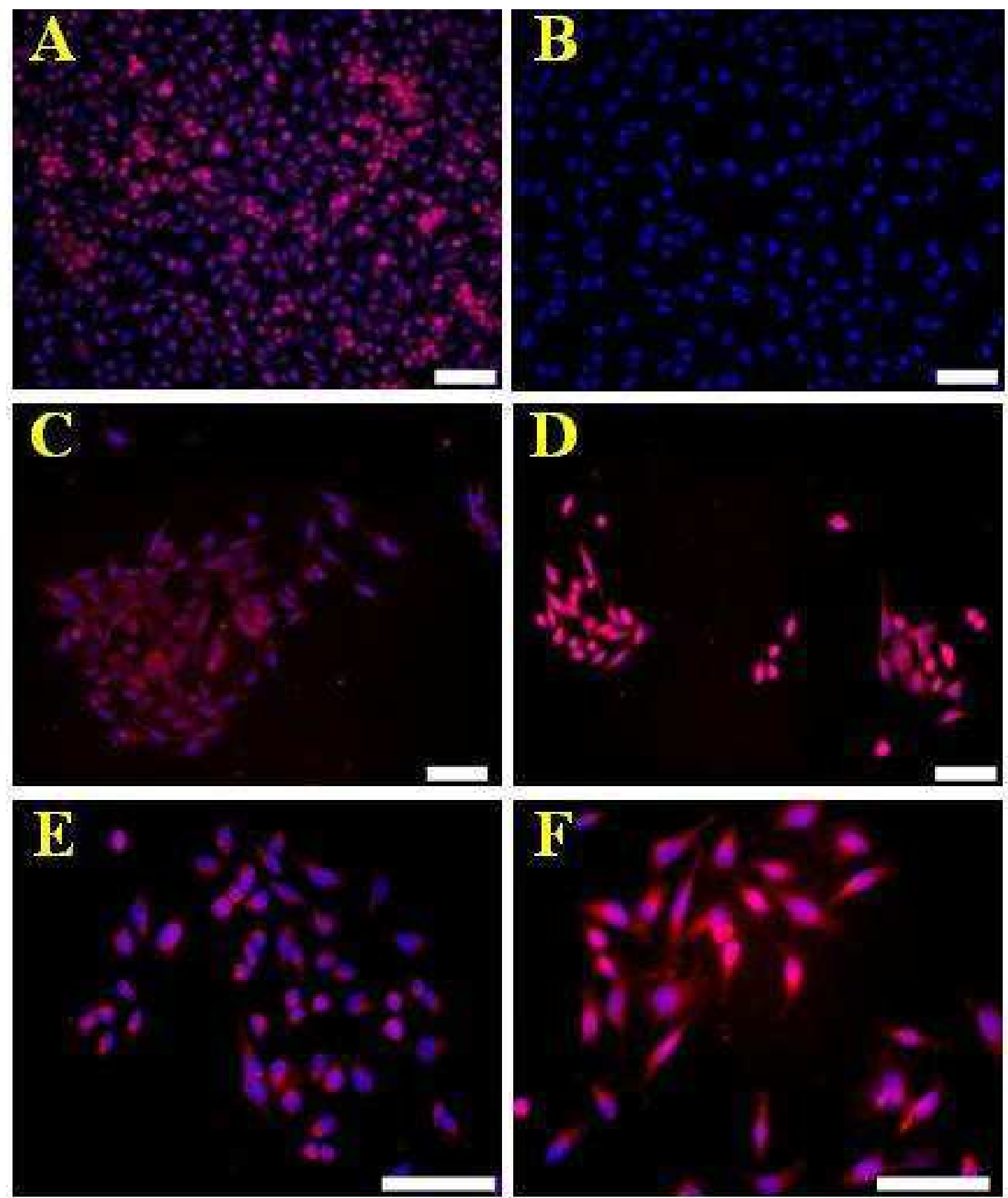

Figure 1.6: Immunohistochemical localisation of STAT-3, p-STAT-3 (Tyr 705) and STAT-1 on HeLa cells. Cells were fixed and stained with rabbit anti-STAT-3 (A), rabbit anti-p-STAT-3 (Tyr 705) (C\&D) or mouse anti-STAT-1 (E\&F) antibodies. Bound antibodies were detected with either an anti-rabbit DIG conjugate or an antimouse DIG conjugate followed by incubation with an anti-DIG-Rhodamine conjugate (red). Cell nuclei were counterstained with DAPI (blue). (A) STAT-3 was expressed by unstimulated HeLa cells growing in culture. IFN $\alpha-2 b(10,000 \mathrm{U} / \mathrm{ml})$ stimulation for 10 min induced both p-STAT-3 (Tyr 705) (D) and STAT-1 (F) expression as compared to unstimulated cells $(\mathbf{C} \& \mathbf{E}$, respectively). No staining was detected when the primary antibodies were omitted (B). Scale bars: $500 \mu \mathrm{m}$ (A\&B), $100 \mu \mathrm{m}(\mathrm{C} \& D), 50 \mu \mathrm{m}(\mathrm{E} \& \mathrm{~F})$. 

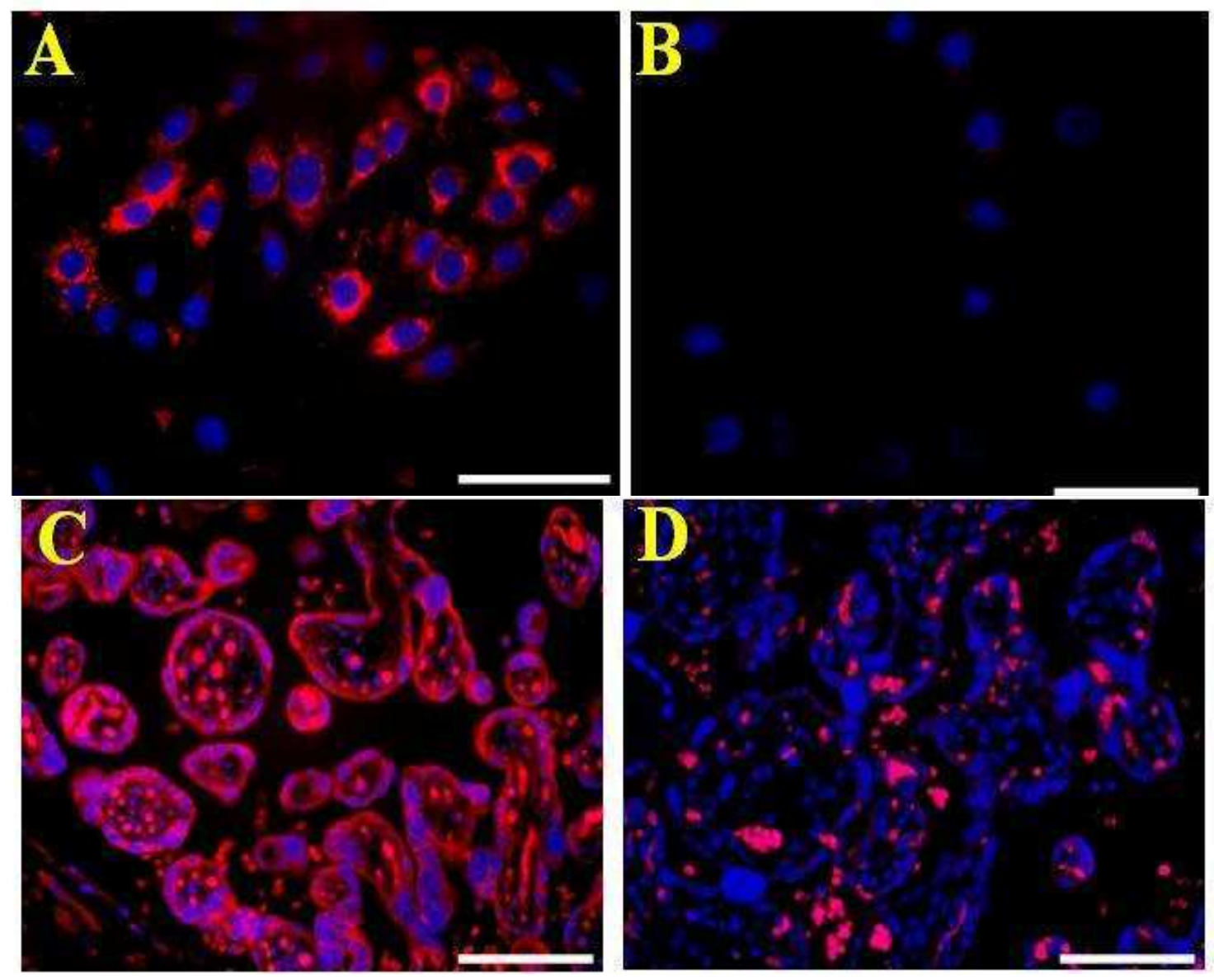

Figure 1.7: Immunohistochemical localisation of VEGFR-2 on EOMA cells and GLUT1 on placenta tissue sections. EOMA cells were fixed and stained with rabbit antiVEGFR-2 antibodies. Bound antibodies were detected with an anti-rabbit-DIG conjugate followed by an anti-DIG-Rhodamine conjugate (Panel A, red staining). Placenta sections were incubated with rabbit anti-GLUT-1 antibodies followed by an anti-rabbit $\mathrm{Cy} 3$ conjugate (Panel $\mathrm{C}$, red staining) for visualization. Cell nuclei were counter stained with DAPI (blue). No staining was detected in the negative controls (Panels B\&D, respectively). The red staining seen in Panel $\mathbf{D}$ are the autofluorescent blood cells. Scale bars: $50 \mu \mathrm{m}$.

\subsubsection{WESTERN BLOTTING}

\subsection{Lysate preparation, transfer conditions and antibody specificity}

In preliminary experiments, snap frozen normal skin and HeLa cells were used to optimise the Western blotting technique. Three lysis buffers were tested, (i) RIPA buffer, (ii) $50 \mathrm{mM}$ Tris-HCL, pH 6.8, $5 \mathrm{mM}$ EDTA and 2\%SDS, and (iii) RIPA buffer lysis buffer containing 2\% SDS and additional protease and phosphotase inhibitors. This modified RIPA buffer was chosen because it was highly reducing and effectively prevented the action of proteases and phosphotases from degrading the proteins within the samples. This allowed for long-term storage of the samples at $-80^{\circ} \mathrm{C}$ for several 
months. Proliferating haemangioma tissues had a higher concentration of proteins (570 $\mu \mathrm{g} / \mathrm{mg})$ than involuting and involuted haemangioma tissues $(114 \mu \mathrm{g} / \mathrm{mg}$ and $27 \mu \mathrm{g} / \mathrm{mg}$, respectively).

Skin was crushed to a powder in liquid nitrogen before the addition of the lysis buffer in order to maximise protein output as the skin tissue became tough and rubbery when solid pieces were put directly into the lysis buffers.

Complete transfer of proteins onto the PVDF membranes was a problem in this study. Therefore, a number of different combinations of the basic Western blotting transfer buffer components and transfer conditions were tested to ensure 90-95\% of the protein bands were transferred onto the membranes. Increasing transfer time and adding SDS along with methanol to the transfer buffer resulted in the transfer of $90-95 \%$ of the protein onto the PVDF membranes. Following transfer, gels were stained with Comassie Blue-R (See Appendix 4, section 4.2.5, page 298). In initial experiments, prior to the blocking step, the membranes were also stained with Ponceau S to detect the presence of the fractionated proteins. The recipe for the Ponceau S stain is given in Appendix 4, section 4.2.22 (page 301).

\subsection{Antibody quantitation}

In preliminary experiments, lysates prepared from normal infant skin and Hela cells, treated and untreated with IFN $\alpha-2 b(10,000 \mathrm{U} / \mathrm{ml})$, for $10 \mathrm{~min}$ were used to show the staining patterns for STAT-3, p-STAT-3 (Tyr 705), STAT-1, CycA and GAPDH antibodies. All antibodies showed immunoreactive bands at the appropriate sizes and also stained in a quantitative manner i.e., when increasing amounts of protein lysates were run on the NuPage MES gels, there was an increase in band intensity of the correct size on the membrane (Figure 1.8). Figure 1.8-B and $-\mathrm{C}$ show decreasing band intensities with a decrease in protein concentration as identified by densitometry analysis using the Image $\mathrm{J}$ software. These results indicate that this method of quantification can be used to measure protein changes in haemangioma. 
A
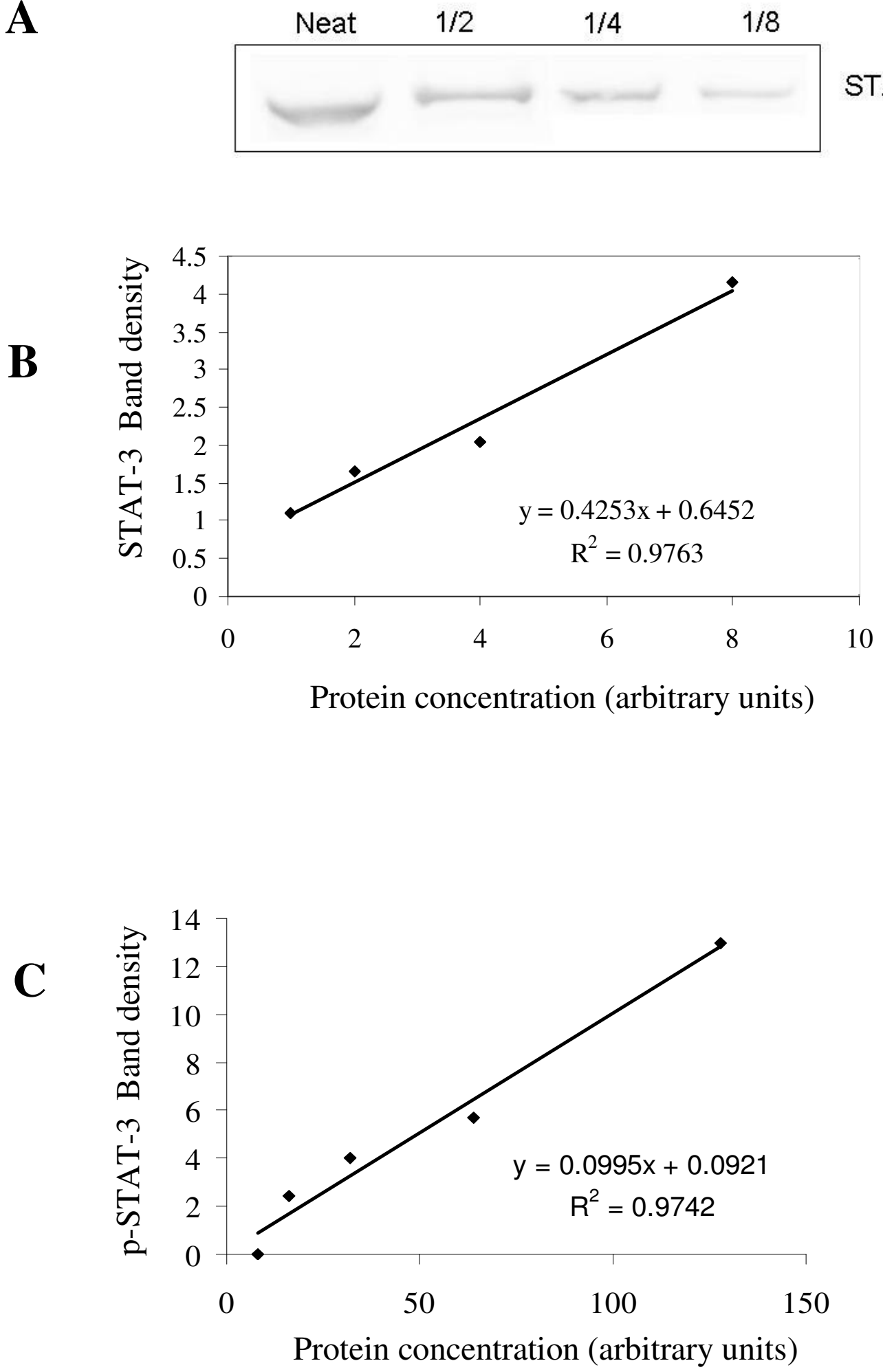

Figure 1.8: Antibody specificity by Western blotting. Decreasing amounts of total protein from HeLa cell lysates showed concomitant decrease in band intensity for STAT-3 (A). This decrease was quantifiable using the Image $\mathbf{J}$ program. Panel $\mathbf{B}$ and $\mathbf{C}$ show the decrease in STAT-3 (B) and p-STAT-3 (Tyr 705) (C) band densities obtained with decreasing amounts of total protein. 


\subsection{GENE EXPRESSION CHANGES IN HAEMANGIOMA}
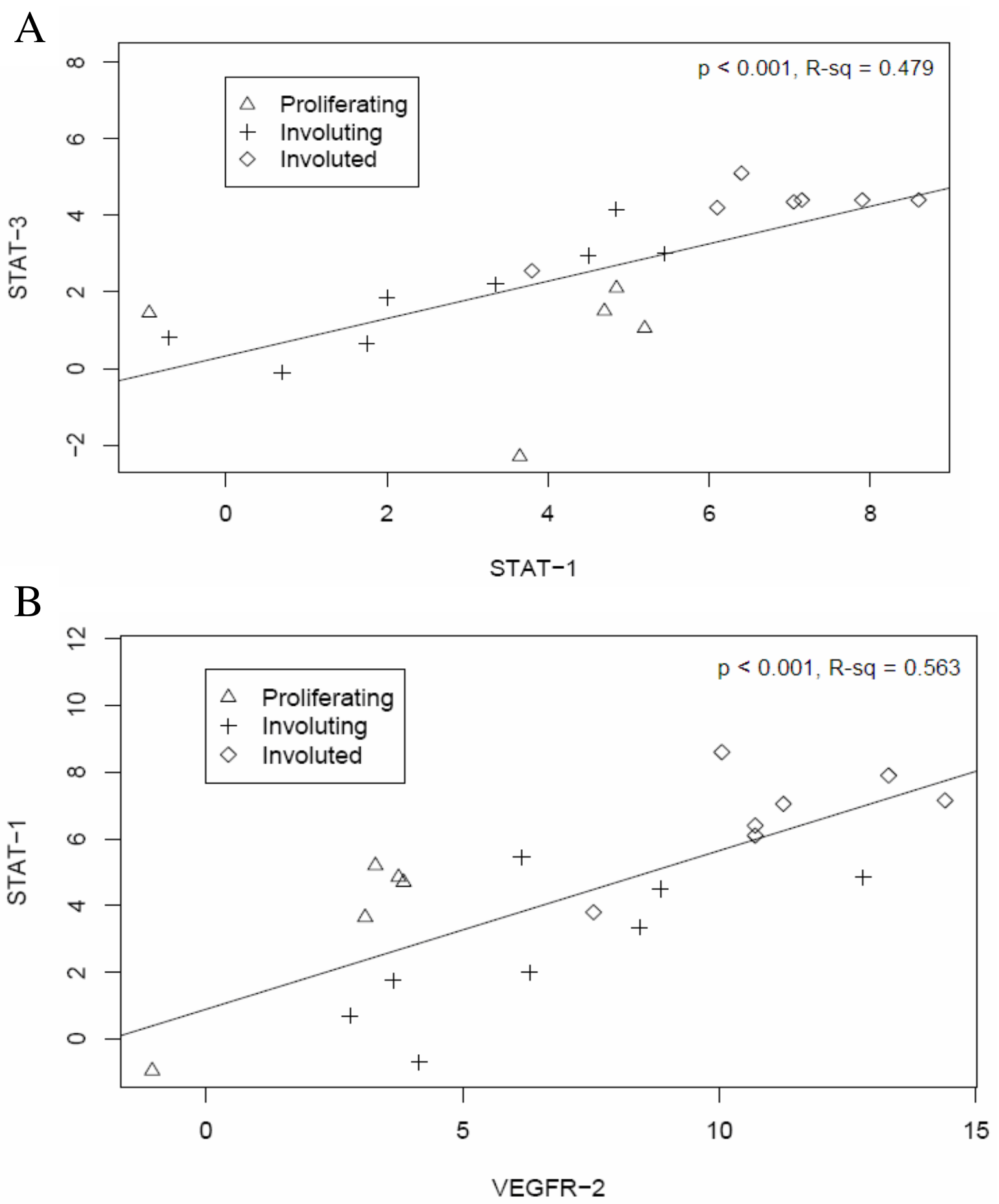

Figure 1.9: Correlation between STAT-3, STAT-1 and VEGFR-2 mRNA expression in proliferating, involuting and involuted haemangiomas. The mRNA levels for STAT-3, STAT-1 and VEGFR-2 were determined in haemangioma biopsies from the different clinical phases using qRT-PCR. The threshold value $(\mathrm{Ct})$ was determined for each gene and then expressed as a difference $(\Delta \mathrm{Ct})$ between that for the target gene and that for internal house-keeping gene, GAPDH. Low $\Delta \mathrm{Ct}$ values reflect greater mRNA expression. Proliferating haemangiomas $(\triangle)$ had greatest STAT-3 and VEGFR-2 expression compared with involuting $(+)$ and involuted $(\diamond)$ lesions while involuting lesions expressed slightly higher STAT-1 mRNA than proliferating and involuted lesions. (A) STAT-3 and STAT-1 mRNA expression were correlated (Spearman rho $\mathrm{r}=$ $0.692 ; p<0.001)$. (B) STAT-1 and VEGFR-2 mRNA expression were correlated (Spearman rho $\mathrm{r}=0.750 ; p<0.001$ ). 


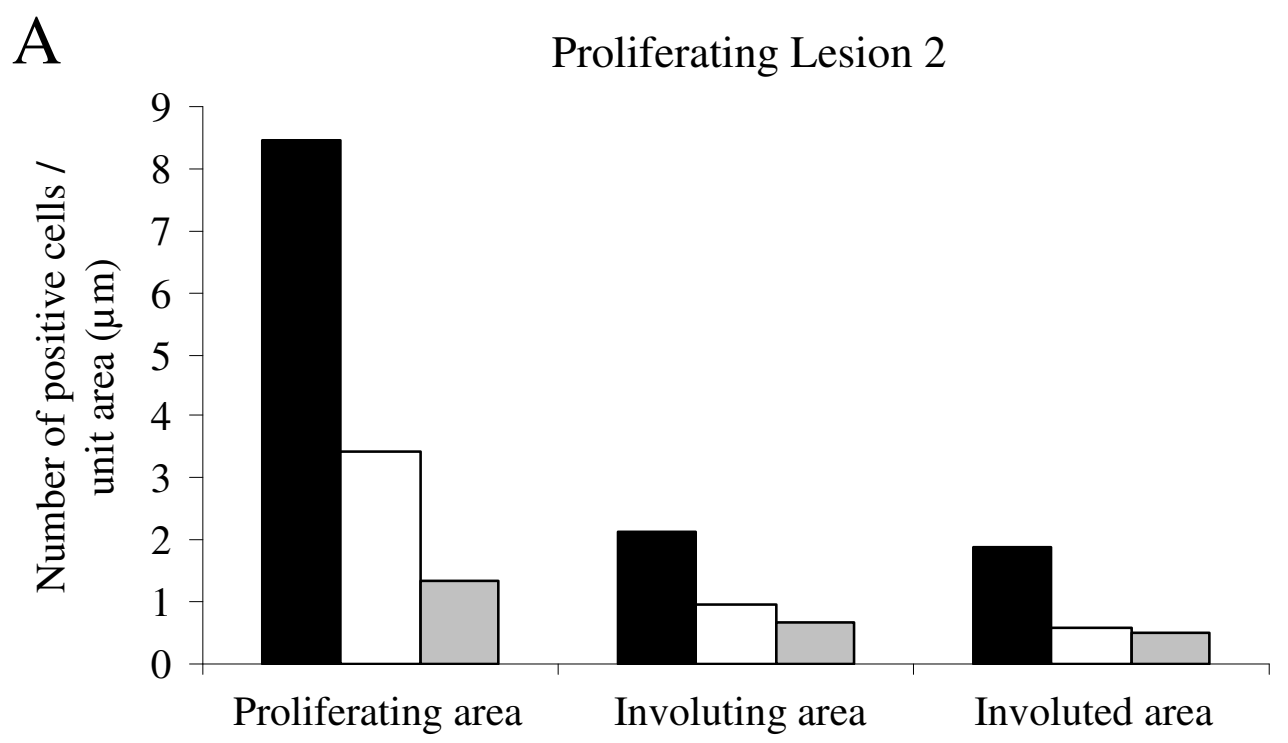

\begin{tabular}{llll}
\hline Lesion 2 & Proliferative area & Involuting area & Involuted area \\
\hline STAT-3 & $25.7 \%$ & $31.1 \%$ & $30.4 \%$ \\
PCNA & $17.8 \%$ & $11.4 \%$ & $7.7 \%$ \\
STAT3+PCNA & $8.0 \%$ & $5.2 \%$ & $3.9 \%$ \\
\hline
\end{tabular}

B

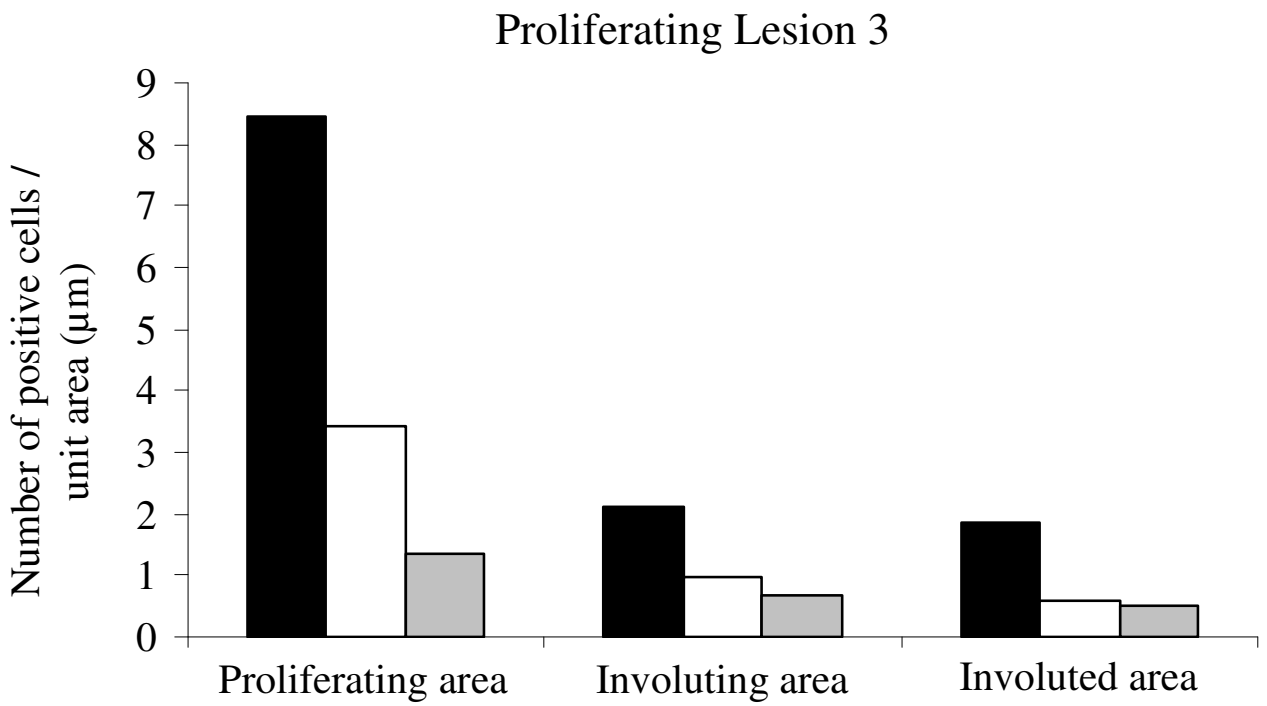

\begin{tabular}{llll}
\hline Lesion 3 & Proliferative area & Involuting area & Involuted area \\
\hline STAT-3 & $70.4 \%$ & $31.1 \%$ & $38.9 \%$ \\
PCNA & $27.3 \%$ & $13.3 \%$ & $24.5 \%$ \\
STAT3+PCNA & $12.7 \%$ & $4.9 \%$ & $10.6 \%$ \\
\hline
\end{tabular}

Figure 1.10: STAT-3 expression correlates with proliferation in proliferating haemangioma. Black columns show STAT-3 positive cells, white columns show PCNA positive cells and grey columns show number of cells that stain positive for STAT-3 and PCNA. 'Proliferating' areas within proliferating lesions contained the most number of STAT-3 positive cells which decreased in the 'involuting' and 'involuted' areas. 'Involuted' areas contained few STAT-3 and PCNA positive cells. Percentage of cells that stained positive for STAT-3, PCNA and those that expressed both STAT-3 and PCNA (STAT-3+PCNA) are given in the corresponding table below each graph. 


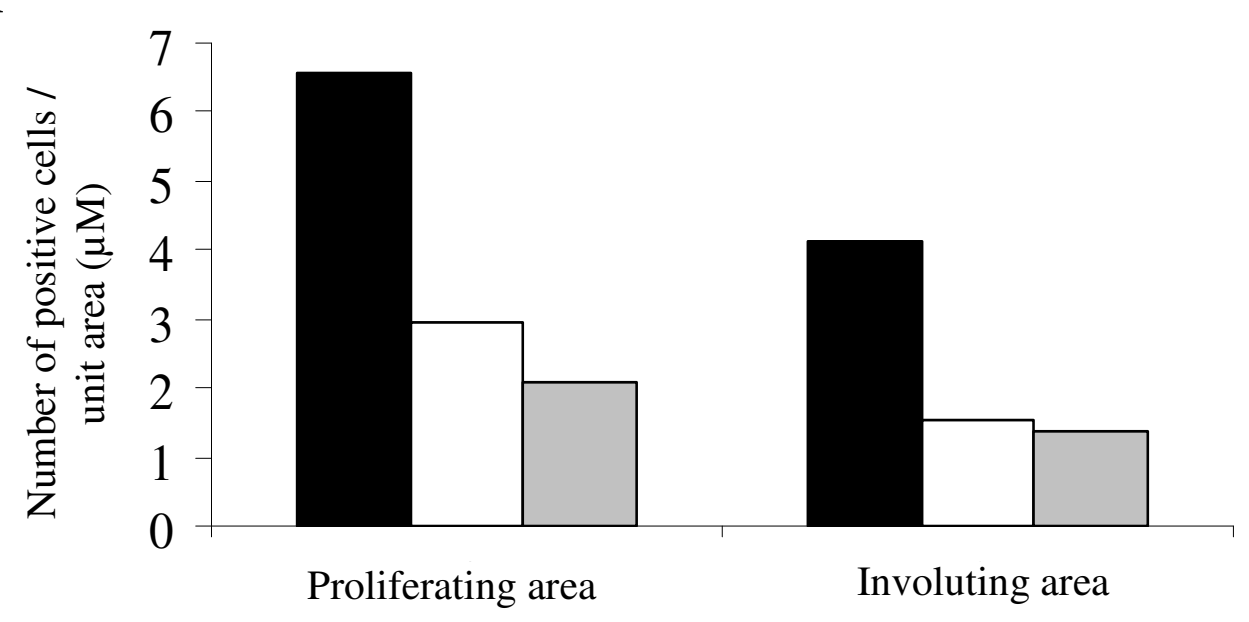

\begin{tabular}{lll}
\hline Lesion 2 & Proliferative area & Involuting area \\
\hline STAT-3 & $60.7 \%$ & $62.9 \%$ \\
PCNA & $27.3 \%$ & $23.5 \%$ \\
STAT3/PCNA & $19.4 \%$ & $21.1 \%$ \\
\hline
\end{tabular}

B Involuting Lesion 3

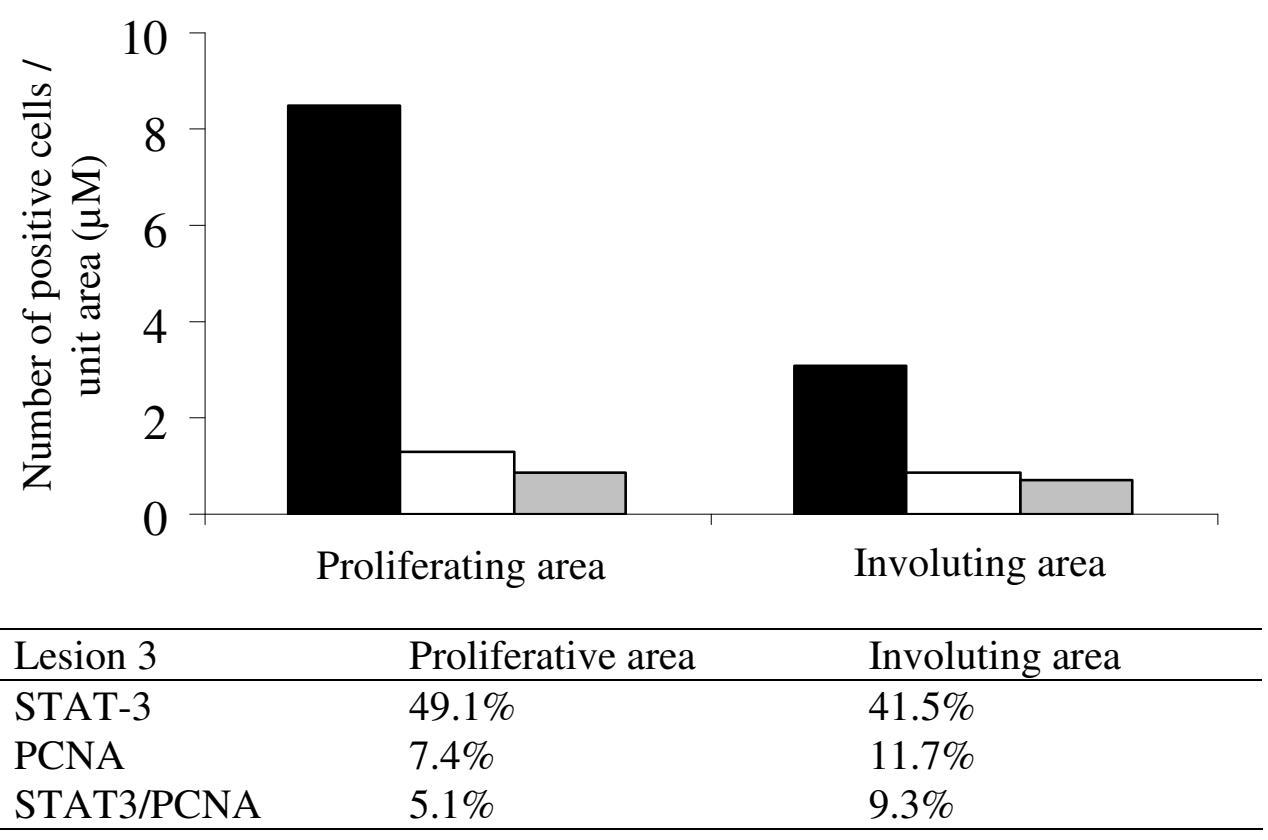

Figure 1.11: STAT-3 expression correlates with proliferation in involuting haemangioma. Black columns show STAT-3 positive cells, white columns show PCNA positive cells and grey columns show number of cells that stain positive for STAT-3 and PCNA. 'Proliferating' areas within an involuting lesion contained the most number of STAT-3 positive cells which decreased in the 'involuting' and 'involuted' areas. There were also fewer PCNA positive cells in the 'involuting' area as compared to the 'proliferative' area. The 'involuted' areas were devoid of any PCNA expression. 'Involuted' areas contained very few STAT-3 positive cells. Percentage of cells that stained positive for STAT-3, PCNA and those that expressed both STAT-3 and PCNA (STAT-3+PCNA) are given in the corresponding table below each graph. 


\section{APPENDIX 2: CHAPTER 4}
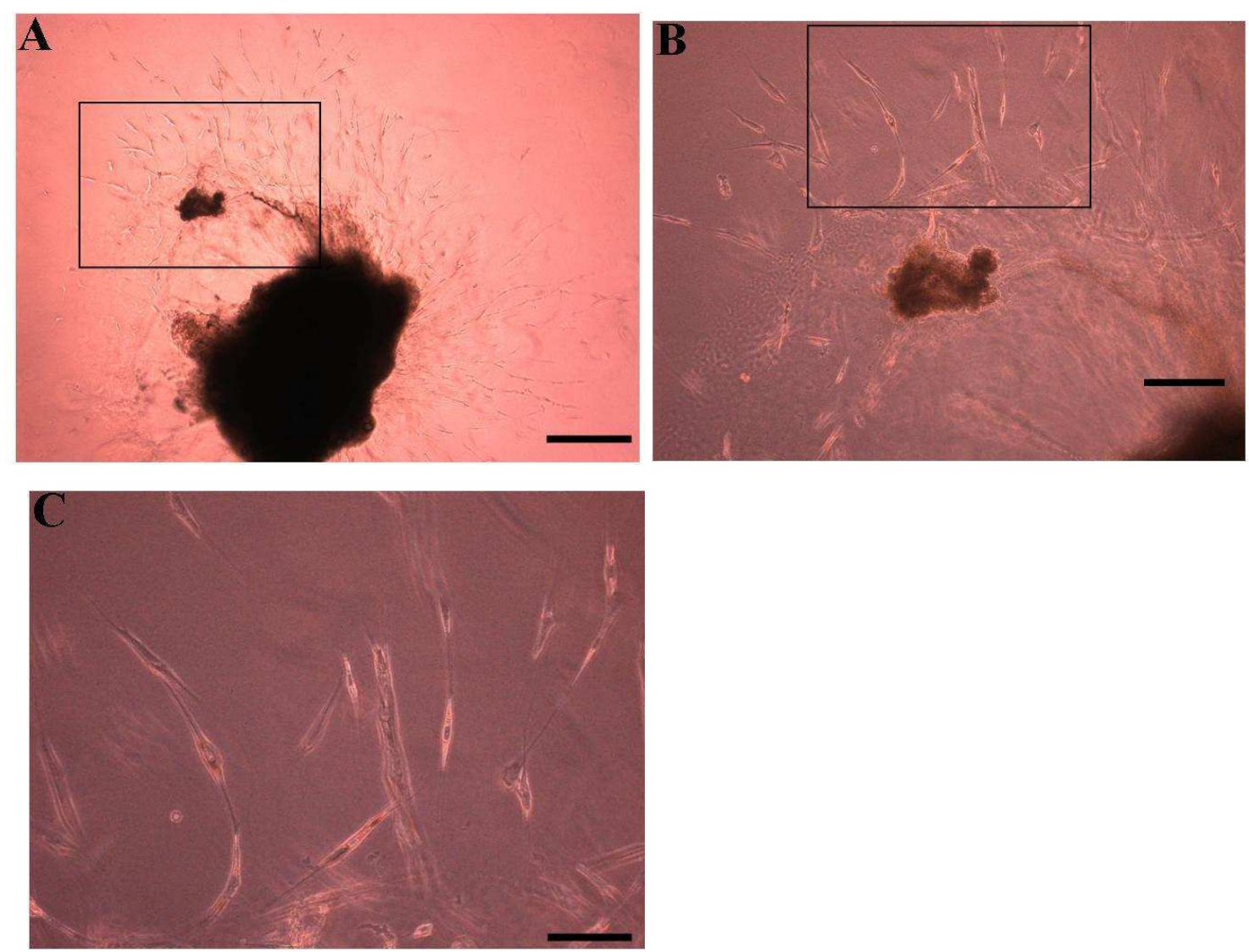

Figure 2.1: Haemangioma explant model (1). Tissue taken from a proliferative haemangioma was embedded in fibrin gel and cultured as described in sections 2.3.2 and 2.3.3. Photographs were taken on day 7 in culture. (A) Phase contrast image showing the entire tissue piece with capillary-like outgrowths emanating from the tissue perimeter. Panel $\mathbf{B}$ and $\mathbf{C}$ are the enlargements of the areas outlined in Panels $\mathbf{A}$ and $\mathbf{B}$, respectively. Scale bars: (A) $500 \mu \mathrm{m}$, (B) $200 \mu \mathrm{m}$ and (C) $100 \mu \mathrm{m}$. 

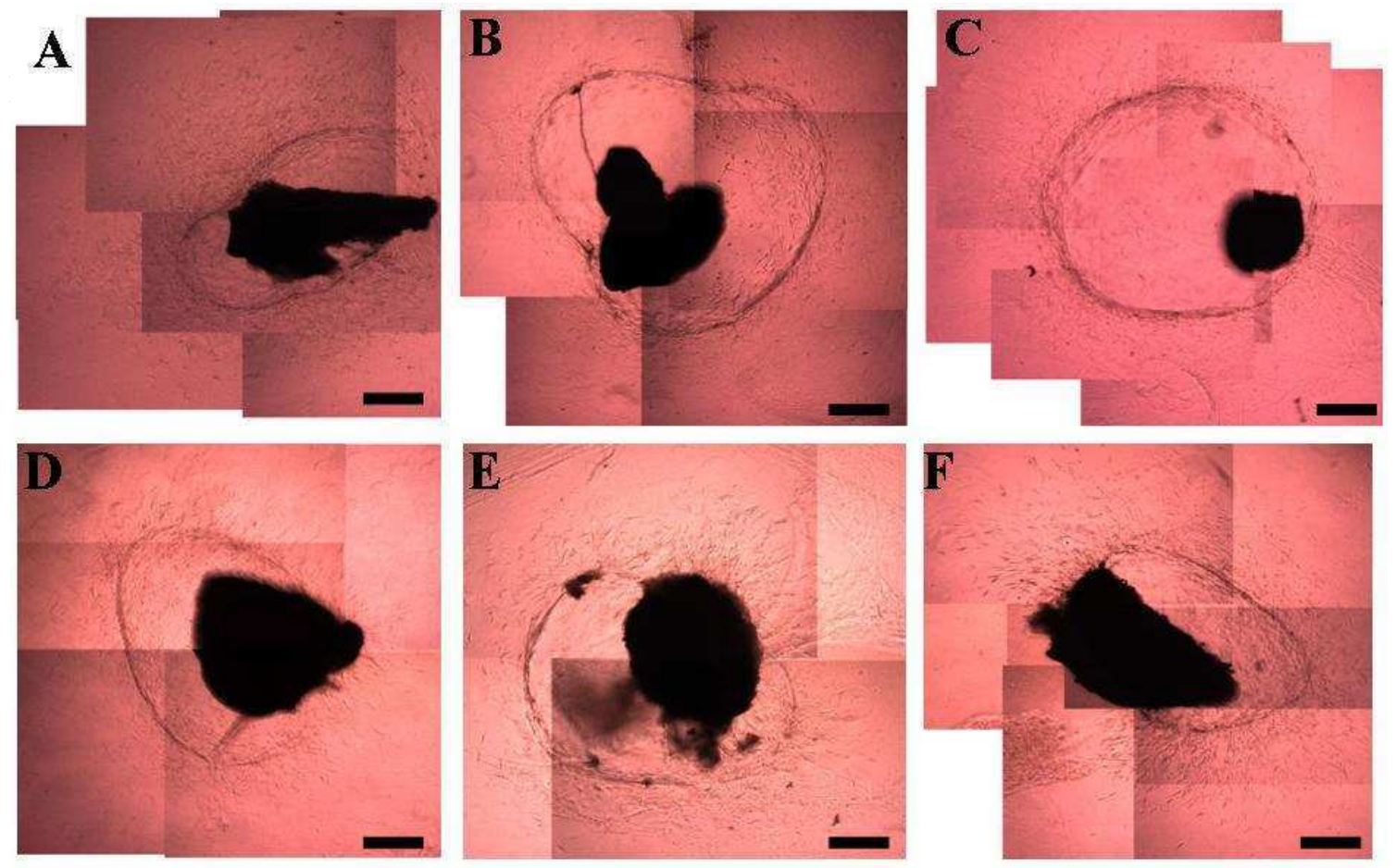

Figure 2.2: Haemangioma explant model (2). Proliferating haemangioma biopsies were cultured as described in sections 2.3.2 and 2.3.3. Panels $\mathbf{A}$ to $\mathbf{F}$ are the phase contrast images showing that the capillary-like structures follow a circular pattern of growth around the original tissue piece. Photographs were taken on day 14 in culture. Scale bars: $500 \mu \mathrm{m}$.
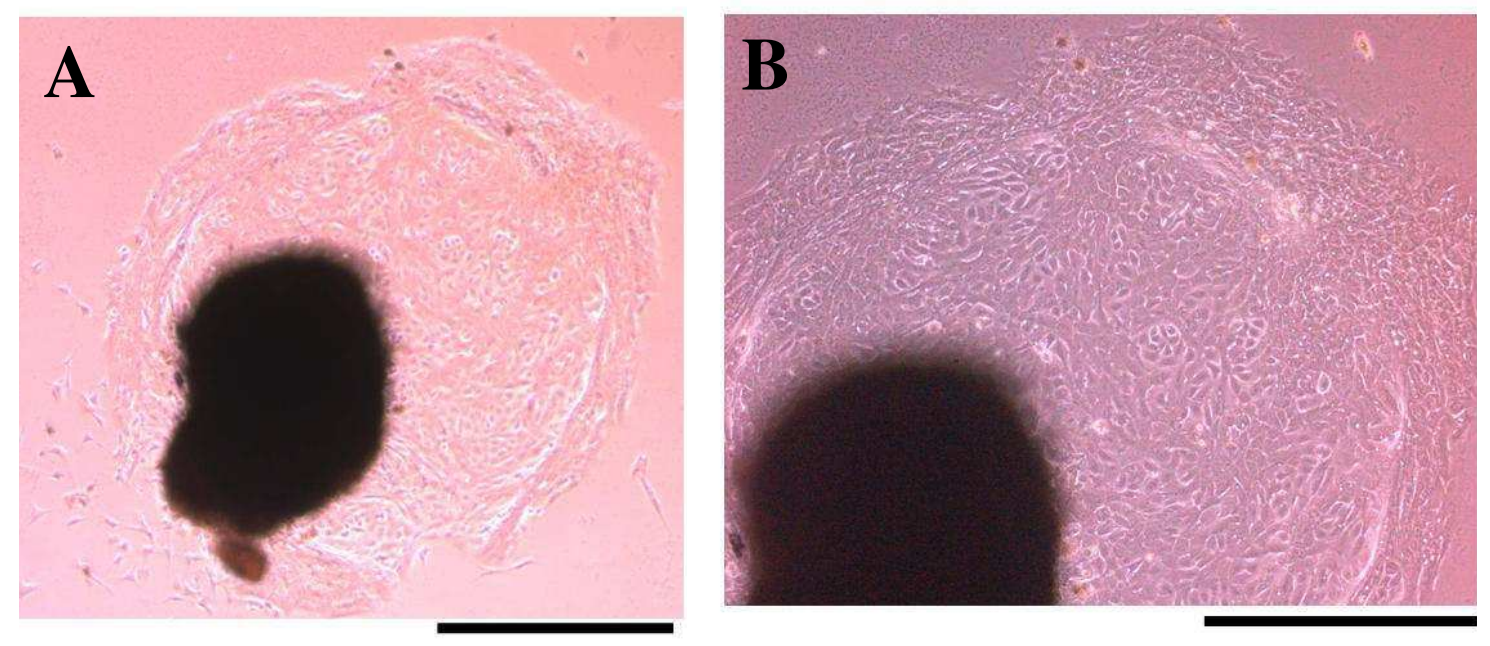

Figure 2.3: Phase contrast images showing the growth of a different cell type from haemangioma explants. $4-5 \%$ of cultured proliferating haemangioma explants gave rise to another cell type emanating from its surface. These cells exhibited the morphology similar to that of ECs. Photographs were taken on day 16 in culture. Scale bars: (A) $1000 \mu \mathrm{m}$ and (B) $500 \mu \mathrm{m}$. 


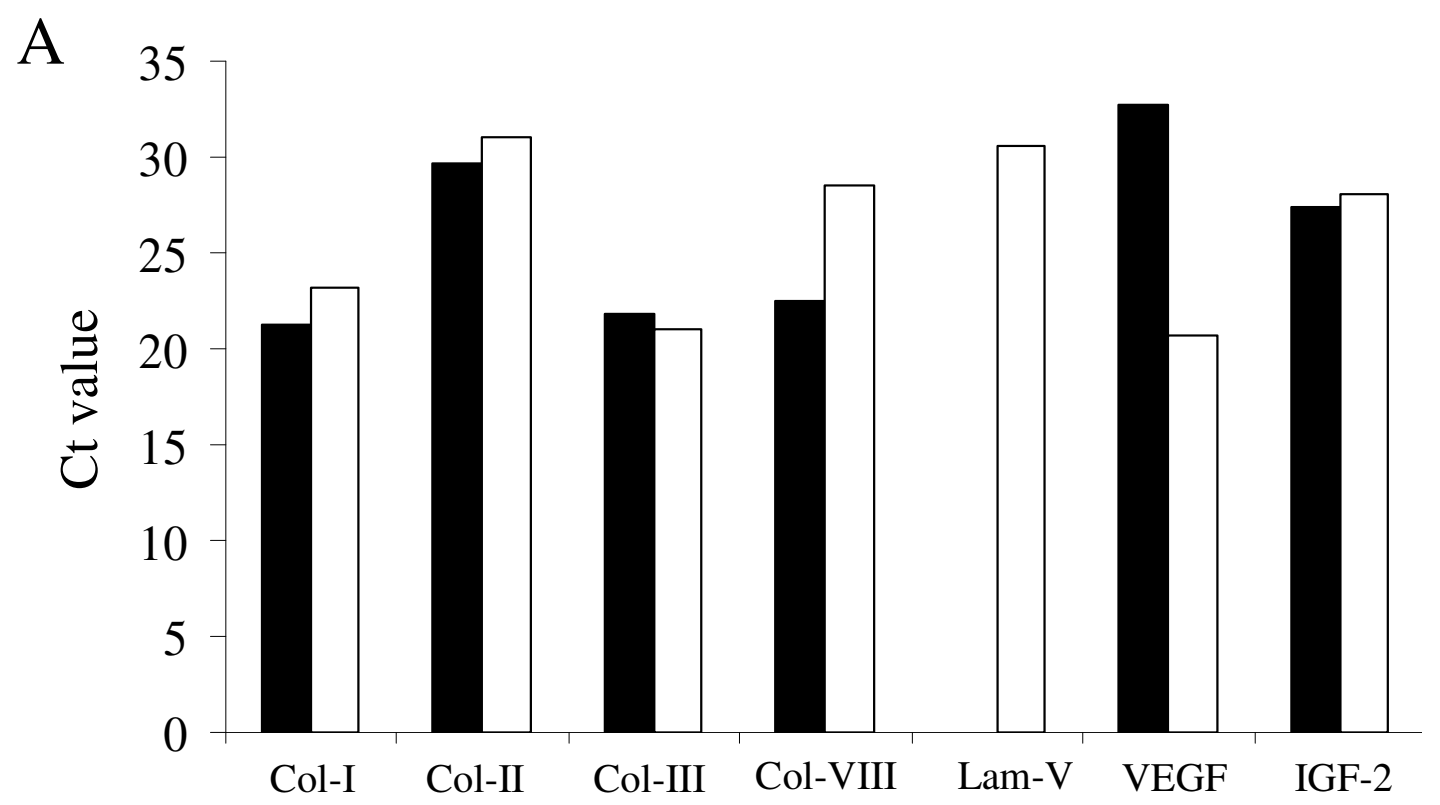

B

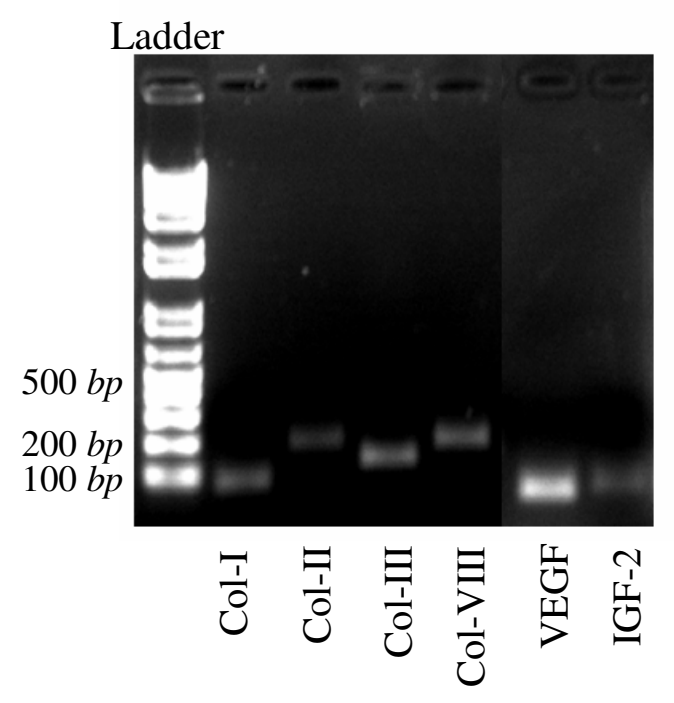

Figure 2.4: qRT-PCR results showing mRNA expression patterns for Collagen I, -II, -III, VIII, Laminin-V, IGF-2 and VEGF-2 in HaemDMSCs and their original biopsy of origin. (A) Black columns show mRNA expression in HaemDMSCs and white columns show expression in the tissue piece. HaemDMSCs expressed similar levels of mRNA for Collagen I (Col-I), -II, -III and IGF-2 as the original biopsy from which they originated. They expressed more mRNA for Col-VIII but lower levels of VEGF mRNA than the tissue. mRNA for Lamining V (Lam-V) was not detected in the HaemDMSCs. (B) An ethidium bromide stained $1 \%$ agarose gel confirming that that the amplified products were of the expected size. 


\begin{tabular}{|c|c|c|c|c|c|c|c|c|c|}
\hline Phase & Patient & Passage & Collagen-I & Collagen-II & Collagen-III & Collagen-VIII & Laminin-V & VEGF & IGF-2 \\
\hline Prolif & HaemDCs-A & 3 & 1.3 & 9.8 & 1.9 & 2.6 & - & 7.5 & 12.8 \\
\hline Prolif & HaemDCs-B & 3 & 6.2 & 13.3 & 4 & 4.4 & - & 10.1 & - \\
\hline Prolif & HaemDCs-C & 3 & 7 & 12.8 & 4.5 & 8.5 & - & 7.1 & 14.4 \\
\hline Prolif & HaemDCs-D & 3 & 6.5 & 13 & 5.4 & 5.7 & - & - & 11.3 \\
\hline Prolif & Tissue-A & NA & 5.1 & 12.9 & 2.9 & 10.4 & 12.5 & 2.6 & 10 \\
\hline \multirow[t]{3}{*}{ Prolif } & Tissue-B & NA & 3.7 & 9.9 & 1.8 & - & 9.3 & 2.9 & 9.6 \\
\hline & HUVECS & 12 & - & - & 12.2 & 6.6 & 13.6 & 10.6 & 13.1 \\
\hline & 3T3 Fibroblasts & & - & - & - & - & - & - & - \\
\hline
\end{tabular}

Table 2.1: mRNA characterisation of HaemDCs. qRT-PCR was used to detect mRNA transcripts for various markers in HaemDCs. The table shows the raw $\triangle \mathrm{Ct}$ values (relative to GAPDH) obtained from HaemDC isolates designated HaemDCs-A, HaemDCs-B, HaemDCs-C and HaemDCs-D. The original tissue pieces of HaemDCs-A and HaemDCs-B, HUVECs and 3T3 fibroblasts were also analysed in parallel. Key: '-' indicates no amplification showing absence of mRNA transcripts; NA: Not applicable. See section 4.3.3.3 for details. 


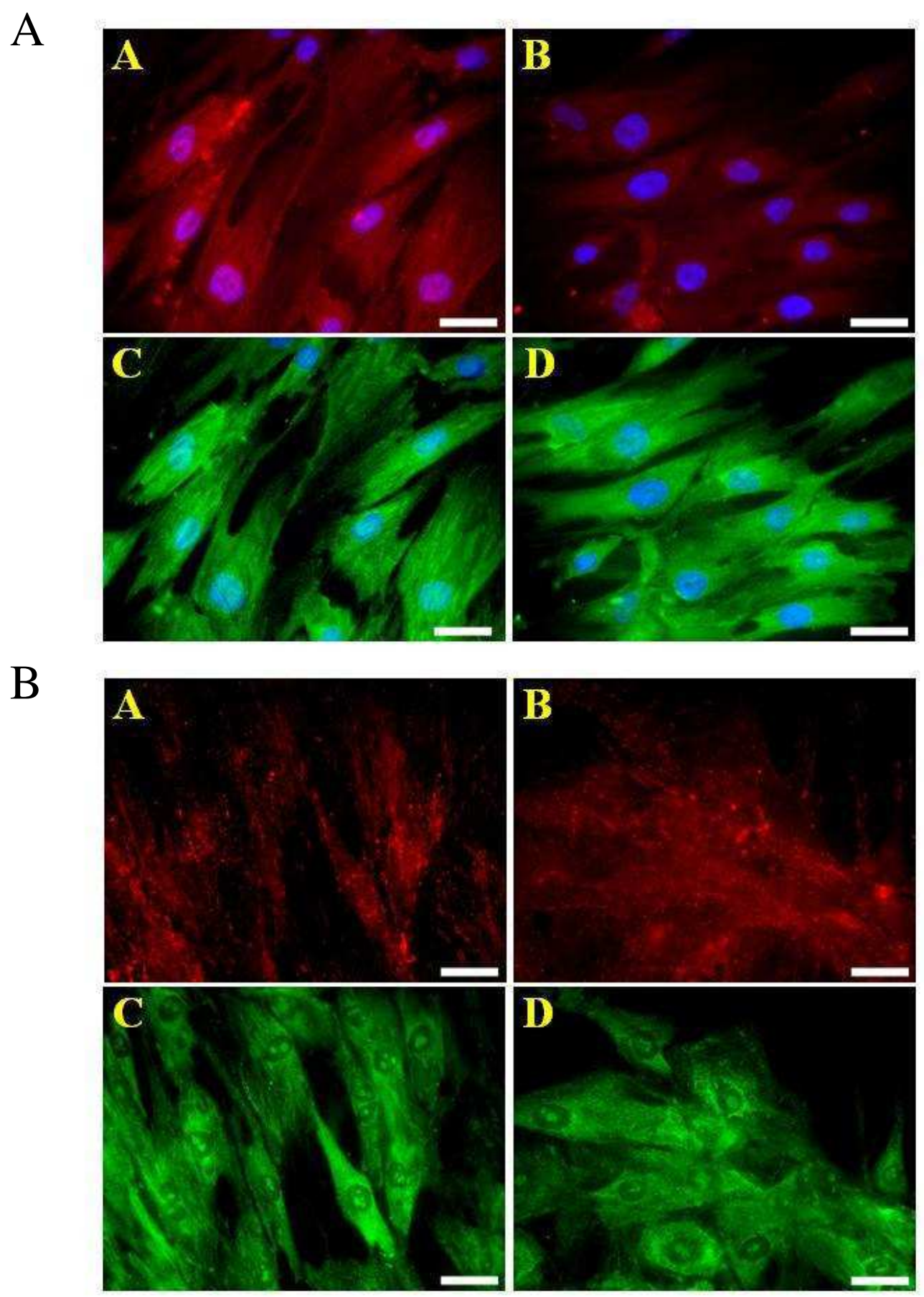

Figure 2.5: Adipocytic differentiation conditions does not induce the nuclear translocation of p-STAT proteins in HaemDMSCs. Immunolocalisation for STAT and p-STAT proteins was performed as described in section 2.3.14.1. Panel A: Immunolocalisation of p-STAT-3 (Tyr 705) (red) and STAT-3 (green) in HaemDMSCs before (A\&B, respectively) and after stimulation with adipocytic differentiation media for $24 \mathrm{hrs}(\mathbf{B} \& \mathbf{D}$, respectively). Cell nuclei were counterstained with DAPI (blue). Panel B: Immunolocalisation of p-STAT-5 (Tyr 695) (red) and STAT-5 (green) in HaemDMSCs before (A\&B, respectively) and after stimulation with adipocytic differentiation media for $24 \mathrm{hrs}(\mathbf{B} \& \mathbf{D}$, respectively). Scale bars: $20 \mu \mathrm{m}$. 


\subsection{PURIFICATION OF HAEMANGIOMA DERIVED ENDOTHELIAL CELLS}

Boye et al (2001) have purified ECs from fresh haemangioma biopsies using Ulexlectin coated magnetic beads. In this study, a similar protocol was used except that magnetic beads coated with antibodies to the EC specific marker, CD31 was used. However, in this study, purification of ECs from fresh haemangioma biopsies using this technique was unsuccessful. This may be due to several reasons, (i) low quantities of starting material. Boye et al (2001) did not specify the weight or volume of starting material that allowed successful isolation of ECs in their lab. (ii) different culture media and growth supplements. During this study, the quantity of haemangioma tissue available was a limiting factor. Cell recovery was low after the collagenase digestion and magnetic bead separation steps performed as described in section 2.3.16 (page 99). Similar to the conditions described by Boye et al (2001), this study utilised both fibronectin and gelatin coated dishes for cell culture. In addition, most of the additional supplements were added to the culture media with the exception of FGF-2. Another difference was that MCDB 131 minimal medium was used instead of the endothelial basal medium (EBM) medium described previously (Boye et al., 2001). Unfortunately, the few cells that survived the purification procedure did not survive passed the first passage to allow for immunohistochemical characterisation. Figure 2.7 shows the isolated cells in culture following bead purification from two haemangioma biopsies. 

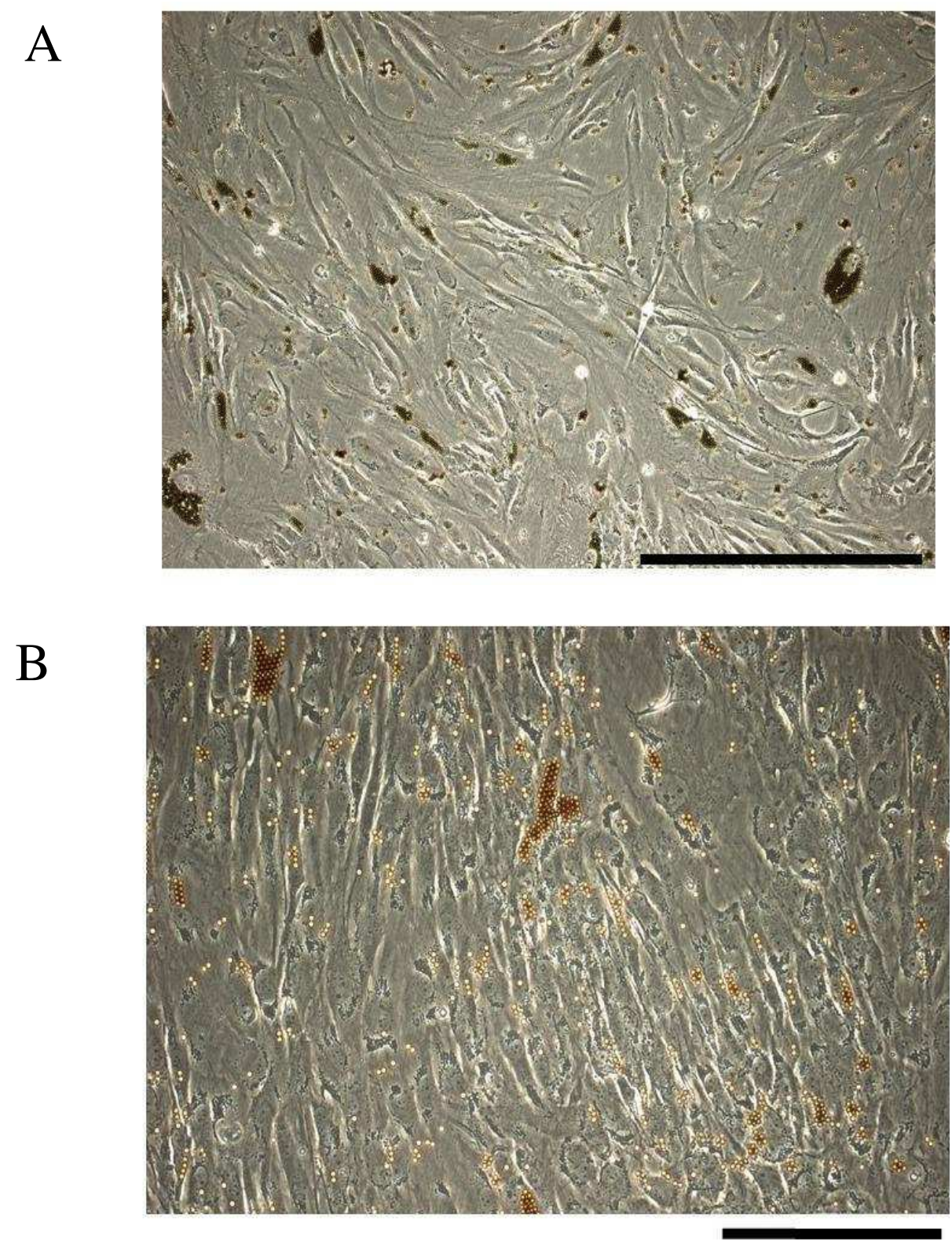

Figure 2.6: $\mathrm{CD} 31^{+}$cells isolated from two proliferating haemangioma biopsies. Photographs were taken on day 7 in culture. The beads used for purification remained attached to some of the cells in culture. Scale bars: (A) $500 \mu \mathrm{m}$, (B) $200 \mu \mathrm{m}$. 


\section{APPENDIX 3: CHAPTER 5}

\begin{tabular}{lcc|cc|cc|cc|cc|cc}
\hline & \multicolumn{2}{c|}{ Patient 1 } & \multicolumn{2}{|c|}{ Patient 2 } & \multicolumn{2}{|c|}{ Patient 3 } & \multicolumn{2}{|c|}{ Patient 4 } & \multicolumn{2}{|l}{ Patient 5 } & \multicolumn{2}{|c}{ Patient 6 } \\
& $\mathrm{T}$ & $\mathrm{C}$ & $\mathrm{T}$ & $\mathrm{C}$ & $\mathrm{T}$ & $\mathrm{C}$ & $\mathrm{T}$ & $\mathrm{C}$ & $\mathrm{T}$ & $\mathrm{C}$ & $\mathrm{T}$ & $\mathrm{C}$ \\
\hline TRAIL & 3.9 & $>16$ & 6.6 & $>16$ & 5.1 & $>16$ & 4.9 & $>16$ & 4.9 & 15.7 & 4.9 & $>16$ \\
DR4 & 5.9 & 9.5 & 11.5 & 9.4 & 7.1 & 11.5 & 7.6 & 12.4 & 7.9 & 13.5 & 6.8 & 11.8 \\
DR5 & 8.6 & 13.5 & 15.1 & 14.8 & 9.8 & 15.1 & 10.8 & 13 & 21 & 16.3 & 10.6 & 15.8 \\
DcR1 & 5.6 & 9 & 11.5 & 9.7 & 6.3 & 11.5 & 8.4 & 11.2 & 10.7 & 12.1 & 7.9 & 12.3 \\
DcR2 & 8.5 & 4.9 & 6.5 & 5.5 & 10.9 & 5.8 & 8.3 & 6.4 & 9.9 & 4.4 & 8.3 & 7.4 \\
OPG & 11.9 & 1.2 & 11.6 & 2.1 & 10.1 & 9.9 & 11.9 & 4.5 & 9.7 & 3.7 & 9.1 & 4.7 \\
\hline
\end{tabular}

Table 3.1: Differences in mRNA levels for TRAIL and TRAIL receptors in haemangioma tissue and HaemDMSCs. qRT-PCR was used to analyse mRNA expression levels of TRAIL and TRAIL receptors in proliferating haemangioma biopsies and in their respective HaemDMSC isolates. Values in the table are the raw $\triangle \mathrm{Ct}$ values obtained for each gene from the haemangioma tissue pieces $(\mathrm{T})$ and the HaemDMSCs isolates (C). 


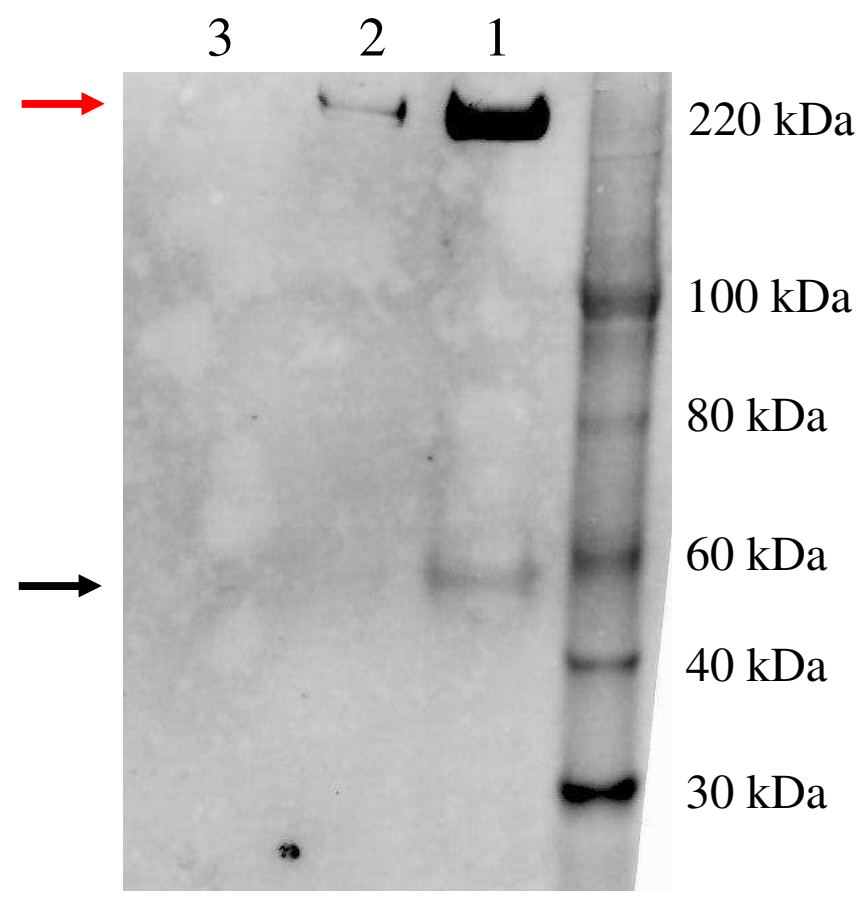

Figure 3.1: OPG protein expression in the media of HaemDMSCs conditioned over a 72-hour period. Lane 1: $40 \mu \mathrm{g}$ of total protein from conditioned media, Lane 2: $5 \mu \mathrm{g}$ of total protein from conditioned media Lane 3: contains an equivalent amount of concentrated unconditioned cell culture media. Following electrophoresis and transfer onto a PVDF membrane, the membrane was blocked with the mouse anti-OPG MAB8051 primary antibody. Lane 1 shows the presence of an immunoreactive band at $60 \mathrm{kDa}$ from the conditioned media of HaemDMSCs (black arrow). In addition, a band at $220 \mathrm{kDa}$ was also detected (red arrow) in Lanes 1 and 2. Both bands were not present in unconditioned media (Lane 3). 


\section{APPENDIX 4}

\subsection{GENERAL MOLECULAR BIOLOGY TECHNIQUES}

\subsubsection{RNA Precipitation}

Where necessary, RNA was precipitated by adding 2.5 times the volume $100 \%$ ethanol and $1 / 10$ sodium acetate $(\mathrm{pH} 5.2)$. This was left at $-20^{\circ} \mathrm{C}$ for a minimum of 20 min and centrifuged at $13,000 \mathrm{Xg}$ for $15 \mathrm{~min}$ at $4^{\circ} \mathrm{C}$. The pellet was air dried in a laminar flow hood for $10 \mathrm{~min}$ and resuspended in 10-15 $\mu$ l of nuclease-free, sterile $\mathrm{dH}_{2} \mathrm{O}$.

\subsubsection{PCR Amplification}

During PCR set-up, all reagents were kept on ice. Latex gloves, dedicated pipettors and barrier tips were used in a designated PCR product free room to prevent contamination by previously amplified material. Reactions were performed using thinwalled microamp (Invitrogen) PCR tubes in Perkin Elmer GeneAmp PCR system 2700 (version 2.07 software). All reagents were purchased from Invitrogen. Reactions followed the general protocol of using a cocktail mix of buffer, deoxynucleotide triphosphates (dNTPs) and $\mathrm{dH}_{2} \mathrm{O}$. The use of a cocktail of common reagents ensures that reactions contain identical reagent concentrations and delinearing larger volumes increase pipetting accuracy. All PCR reactions were performed alongside a no template (water) control and amplification from an equivalent amount of RNA that had not been reverse transcribed. The control was necessary to ensure contamination was not present. Amplification was verified by agarose gel electrophoresis of $5 \mu 1$ of PCR product and staining with ethidium bromide.

\subsubsection{PCR Reaction Using Taq DNA Polymerase}

cDNA synthesised from normal skin was used as template to optimize annealing conditions and primer concentrations for all primer sets used in this study. Optimum 
reaction conditions were obtained in $20 \mu$ f final volume with 1 X PCR Buffer $(20 \mathrm{mM}$ Tris-HCL (pH 8.4), $50 \mathrm{mM} \mathrm{KCL),} 1.5 \mathrm{mM} \mathrm{MgCl}_{2}, 200 \mu \mathrm{M}$ dGTP, dCTP, dATP and dTTP, $1 \mathrm{U}$ Taq polymerase (Invitrogen), $1 \mu \mathrm{cDNA}$ template with varying primer concentrations. A range of annealing temperatures was examined for each gene. The thermal cycling conditions used for the PCR amplification involved the following steps: an initial denaturation at $95^{\circ} \mathrm{C}$ for $2 \mathrm{~min}$, followed by 35 cycles of a 3 -cycle amiplification:denaturation at $95^{\circ} \mathrm{C}$ for $15 \mathrm{sec}$, annealing of primers to the template DNA initially carried out at $5^{\circ} \mathrm{C}$ below the $\mathrm{Tm}$ of the primer with the lowest $\mathrm{Tm}$ for 20 sec and extension at $72^{\circ} \mathrm{C}$ for $30 \mathrm{sec}$. This 35 cycle sequence was then followed by $72^{\circ} \mathrm{C}$ for $5 \mathrm{~min}$, and the reaction was then held at $4^{\circ} \mathrm{C}$. Tm of a given primer was calculated using the formula:

$$
\mathrm{Tm}=[3 \times \mathrm{G} / \mathrm{C}]+[2 \times \mathrm{A} / \mathrm{T}]
$$

The resulting PCR fragments were visualised by agarose gel electrophoresis as outlined in section 4.1.4. For each gene, primer concentration titrated to $0.1 \mu \mathrm{M}$ with an annealing temperature of $60^{\circ} \mathrm{C}$ produced a clean PCR fragment of the expected size with no visible non-specific products or primer dimers. Therefore, these conditions were used for all qRT-PCR amplifications (see section 2.1.4, page 65).

\subsubsection{Agarose Gel Electrophoresis}

DNA samples were mixed with $1 \mu 1$ of $6 \mathrm{x}$ agarose loading buffer (30\% glycerol (v/v) in $\mathrm{dH}_{2} \mathrm{O}, 0.025 \%$ bromophenol blue) and loaded into $1 \%$ agarose gels. The agarose gels were prepared by dissolving the appropriate amount of low melting point agarose (Sigma) in TAE buffer (40 mM Tris-acetate, $1 \mathrm{mM}$ EDTA, pH 8.0). The solution was heated in a microwave to facilitate dissolution of the agarose and then cooled by running the flask under cold water. The solution was then supplemented with $1 \mu \mathrm{g} / \mathrm{ml}$ of ethidium bromide. The molten agarose was transferred into a cassette and a plastic comb inserted to form the lanes for loading he DNA samples. After polymerisation, the gel was immersed in TAE and the comb carefully removed. A 100 
$b p$ ladder (Invitrogen) was used as a standard. Electrophoresis was performed at $100 \mathrm{~V}$ for $45 \mathrm{~min}$, or until the dye front was two-thirds of the way down the gel. Bands were observed under UV light and polaroid photographs taken.

\subsubsection{Purification of DNA Fragments from Agarose Gels.}

PCR products of the appropriate sizes were cut out of the agarose gel and purified using the QIAquick® gel extraction kit (Qiagen, GmbH, Germany). The slice was weighed, and $300 \mu 1$ of QG buffer (pH 7.5) was added for every $100 \mathrm{mg}$ of gel. The sample was incubated at $50^{\circ} \mathrm{C}$ until the gel slice had dissolved. Isopropanol (100 $\mu 1$ of 100\%) was added per $100 \mathrm{mg}$ of gel slice, and the sample was mixed. The sample was then loaded to the upper reservoir of a QIAquick ${ }^{\circledR}$ spin column provided in the kit placed in a $2 \mathrm{ml}$ collection tube and centrifuged in a table top centrifuge for $1 \mathrm{~min}$ at 10,000 X g. Flow-through was discarded and $750 \mu \mathrm{l}$ of PE buffer added. The solution was incubated for up to $5 \mathrm{~min}$ at room temperature before centrifugation for $1 \mathrm{~min}$ at $10,000 \mathrm{X}$ g. After discarding the flow-through again, the column was centrifuged once again to elute the residual flow-through. The column was then placed in a clean $1.5 \mathrm{ml}$ microcentrifuge tube, and $50 \mu \mathrm{l}$ of elution buffer (10 mM Tris-HCL, $\mathrm{pH} 8.5)$ was added to the centre of the QIAquick® membrane. The sample was left to stand for 5 min to increase the DNA yield and then eluted by centrifugation at 13,000 X $\mathrm{g}$ for $2 \mathrm{~min}$. Purified DNA was stored at $-20^{\circ} \mathrm{C}$ until required.

\subsubsection{Acetone Protein Precipitation}

To each sample, four times the volume of ice cold acetone was added. The tubes were vortexed and incubated overnight at $-20^{\circ} \mathrm{C}$. The tubes were centrifuged at 13,000 $\mathrm{X} \mathrm{g}$ for $10 \mathrm{~min}$ at $4^{\circ} \mathrm{C}$ and the supernatant was removed without disturbing the pellet. The uncapped tubes were airdried for 10-15 $\mathrm{min}$ on the bench top. The protein pellets were then resuspended in freshly prepared Western blotting lysis buffer. 


\subsection{STOCK SOLUTIONS, BUFFERS AND OTHER RECIPES}

4.2.1 Phosphate buffered saline (PBS) $0.15 \mathrm{M} \mathrm{pH} 7.4$

$\begin{array}{ll}\text { Reagents } & \mathrm{g} / \mathrm{L} \\ \mathrm{NaCl} & 0.8 \mathrm{~g} \\ \mathrm{KCL} & 0.2 \mathrm{~g} \\ \mathrm{Na}_{2} \mathrm{HPO}_{4} & 1.15 \mathrm{~g} \\ \mathrm{KHPO}_{4} & 0.2 \mathrm{~g}\end{array}$

PBS was made by dissolving the salts in $\mathrm{dH}_{2} \mathrm{O}$. The $\mathrm{pH}$ was adjusted to 7.4 using concentrated $\mathrm{HCL}$ or $\mathrm{NaOH}$

4.2.2 Tris buffered saline (TBS)

$\begin{array}{ll}\text { Reagents } & \mathrm{g} / \mathrm{L} \\ \text { Tris } & 2.42 \mathrm{~g} \\ \mathrm{NaCL} & 8 \mathrm{~g}\end{array}$

TBS was made by dissolving the salts in $\mathrm{dH}_{2} \mathrm{O}$. The $\mathrm{pH}$ was adjusted to 7.6 using concentrated HCL

\subsubsection{Tris Buffered Saline-Tween-20}

TBST was prepared by adding $1 \mathrm{ml}$ Tween-20 to $1 \mathrm{~L}$ of TBS buffer.

\subsubsection{RIPA Buffer (100 ml)}

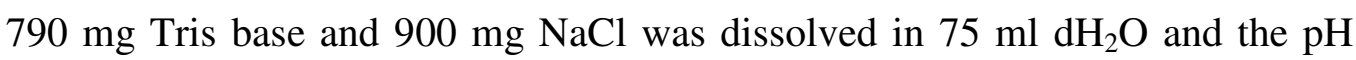
adjusted to $7.4 .10 \mathrm{ml}$ of NP-40 and $2.5 \mathrm{ml}$ of $10 \%$ Na-Deoxycholate was added and the solution was stirred until it turned colourless. $1 \mathrm{ml}$ of $100 \mathrm{mM}$ EDTA was added and the final volume adjusted to $100 \mathrm{ml}$ with $\mathrm{dH}_{2} \mathrm{O}$. The solution was aliquoted and stored at $20^{\circ} \mathrm{C}$. Final concentration: $50 \mathrm{mM}$ Tris- $\mathrm{HCl}(\mathrm{pH} 7.4), 1 \% \quad \mathrm{NP}-40,0.25 \%$ NaDeoxycholate, $150 \mathrm{mM} \mathrm{NaCl}, 1 \mathrm{mM}$ EDTA. 


\subsubsection{For Coomassie R-250 staining}

Comassie staining solution : $0.2 \mathrm{~g}$ Coomassie R-250, $10 \mathrm{ml}$ acetic acid, $100 \mathrm{ml}$

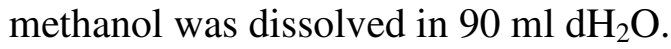

Destaining Solution: $10 \%$ acetic acid, $40 \%$ methanol and $50 \% \mathrm{dH}_{2} \mathrm{O}$.

\subsubsection{Western blot transfer buffer}

$\begin{array}{lc}\text { Reagents } & \mathrm{g} / \mathrm{L} \\ \text { Tris } & 3.03 \mathrm{~g} \\ \text { Glycine } & 14.4 \mathrm{~g} \\ \text { SDS } & 0.38 \mathrm{~g} \\ \text { Methanol } & 150 \mathrm{ml}\end{array}$

The reagents were combined and the volume made upto $1 \mathrm{~L}$ with $\mathrm{dH}_{2} \mathrm{O}$.

\subsubsection{Stripping Buffer}

$2 \mathrm{~g} \mathrm{SDS}, 750 \mu \mathrm{l} \beta$-mercaptoethanol and $10 \mathrm{ml}$ Tris-HCL (0.5 M, pH 6.8) was

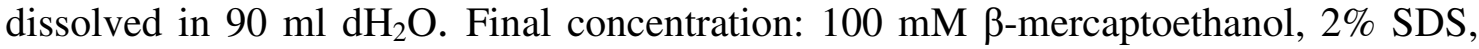
62.5 mM Tris-HCL pH 6.8.

\subsubsection{4\% Paraformaldehyde}

$4 \mathrm{~g}$ of PFA was added to $100 \mathrm{ml}$ PBS. A few drops of $\mathrm{NaOH}$ were added followed by gentle heat treatment in a microwave. Once dissolved the $\mathrm{pH}$ was adjusted to 7.1 using concentrated HCL.

\subsubsection{0 mM Sodium Citrate (pH 6.0)}

This solution was made up fresh for each use. $0.3 \mathrm{~g}$ of tri-sodium citrate was dissolved in $100 \mathrm{ml}$ of $\mathrm{dH}_{2} \mathrm{O}$. The $\mathrm{pH}$ was adjusted to 6.0 using a $1 \mathrm{M}$ HCL solution. 


\subsubsection{Csaba Stain}

Briefly, the alcian-blue-safranin solution (Csaba stain) was prepared by adding $900 \mathrm{mg}$ alcian blue, $45 \mathrm{mg}$ of safranin, $1.2 \mathrm{~g}$ of ferric ammonium sulphate to $250 \mathrm{ml}$ of 0.2 M sodium acetate buffer, $\mathrm{pH} 7.42$.

\subsubsection{Coating culture dishes with $1 \%$ gelatin}

$1 \mathrm{~g}$ of commercially available gelatin was dissolved in $100 \mathrm{ml}$ of PBS by gentle heating in a microwave. Once dissolved this solution was autoclaved and filter sterilised through a $0.2 \mu \mathrm{M}$ filter. For coating culture dishes, enough $1 \%$ gelatin was added to cover the entire surface and the dish was left for at least $20 \mathrm{~min}$ in the incubator. Immediately before use, the gelatin was removed and the culture dishes were rinsed once with sterile PBS.

\subsubsection{AG490 Stock Solution}

AG490 powder was dissolved in $100 \%$ DMSO to a final concentration of 340 mM. A stock solution of $1 \mathrm{mM}$ was prepared by diluting $15 \mu \mathrm{l}$ of AG490 (340 mM) into $5 \mathrm{ml}$ cell culture media. This solution was passed through a $0.2 \mu \mathrm{M}$ filter, aliquoted and stored at $-20^{\circ} \mathrm{C}$.

\subsubsection{FACS buffer}

$100 \mathrm{ml}$ PBS was combined with $100 \mathrm{mg}$ sodium azide.

\subsubsection{Alizarin Red Staining Solution}

$2 \mathrm{~g}$ of Alizarin red solution was dissolved in $100 \mathrm{ml}$ of $\mathrm{dH}_{2} \mathrm{O}$. The $\mathrm{pH}$ was adjusted to 4.1-4.3 with $10 \%$ ammonium hydroxide. This solution was stored at room temperature. 


\subsubsection{5 (A) Oil Red O stock stain}

$0.5 \mathrm{~g}$ of Oil red $\mathrm{O}$ (sigma) was dissolved in $100 \mathrm{ml}$ of isopropanol and was stored at room temperature.

\section{(B) Oil Red O working solution}

Oil red stock stain was dissolved in $\mathrm{dH}_{2} \mathrm{O}$ at a ratio of 3:2 and allowed to stand for a minimum of $10 \mathrm{~min}$. The solution was then passed through a $75 \mu \mathrm{M}$ cell strainer and used immediately.

\subsubsection{Glycerin Jelly Mounting Medium}

$1 \mathrm{~g}$ of Gelatin was dissolved in $6 \mathrm{ml}$ of $\mathrm{dH}_{2} \mathrm{O}$ to which $7 \mathrm{ml}$ of glycerol was then added. The mounting medium was stored at $4^{\circ} \mathrm{C}$

\subsubsection{Endothelial Cell Growth Supplement - For 3 mg/ml Stock Solution}

$15 \mathrm{mg}$ of ECGS powder was resuspended in $5 \mathrm{ml}$ pre-warmed PBS. To ensure dissolution, the vial was left at $37^{\circ} \mathrm{C}$ for $15 \mathrm{~min}$ and mixed at 5 min intervals. The solution was then filter sterilized through a $0.2 \mu \mathrm{M}$ filter, aliquoted and stored at $-20^{\circ} \mathrm{C}$ until required. ECGS was used in cell culture media at a final concentration of $15 \mu \mathrm{g} / \mathrm{ml}$ unless otherwise stated.

\subsubsection{0\% SDS Polyacrylamide Gels}

(2 gels-1.5 mm thick)

$\begin{array}{ll}\underline{\text { Reagents }} & \mathrm{g} / \mathrm{L} \\ \text { Acrylamide/bis (30\% stock) } & 6.66 \mathrm{ml} \\ 1.5 \mathrm{M} \text { Tris pH } 8.8 & 5 \mathrm{ml} \\ \mathrm{dH}_{2} \mathrm{O} & 8 \mathrm{ml} \\ 10 \% \text { SDS in } \mathrm{dH}_{2} \mathrm{O} & 200 \mu \mathrm{l} \\ \text { TEMED } & 10 \mu \mathrm{l} \\ 10 \% \text { ammonium persulphate } & 100 \mu \mathrm{l}\end{array}$

The solutions were added in order shown. TEMED and 10\% ammonium persulphate were added last to initiate polymerisation. The gel solution was added to the 
casting apparatus and overlayed with isopropanol. The gel was left to polymerise for 30 min. The isopropanol was removed and the stacking gel was then made and cast on top of the separating gel.

\subsubsection{4\% Stacking Gel}

(2 gels $1.5 \mathrm{~mm}$ thick)

$\begin{array}{ll}\text { Reagents } & \mathrm{g} / \mathrm{L} \\ \text { Acrylamide/bis (30\% stock) } & 1.33 \mathrm{ml} \\ 0.5 \mathrm{M} \text { Tris pH } 6.8 & 2.5 \mathrm{ml} \\ \mathrm{dH}_{2} \mathrm{O} & 6.1 \mathrm{ml} \\ 10 \% \text { SDS in } \mathrm{dH}_{2} \mathrm{O} & 100 \mu \mathrm{l} \\ \text { TEMED } & 10 \mu \mathrm{l} \\ 10 \% \text { ammonium persulphate } & 50 \mu \mathrm{l}\end{array}$

\subsubsection{Protein Gel Loading Buffer (10X)}

To make $20 \mathrm{ml}$ loading buffer, $11.3 \mathrm{ml}$ Glycerol, $2.3 \mathrm{~g}$ SDS and $10 \mathrm{mg}$ bromophenol blue was dissolved in $8.76 \mathrm{ml}$ of Tris-Hcl (0.5 M, $\mathrm{pH} 6.8)$. Buffer was stored in aliquots at $-20^{\circ} \mathrm{C}$.

\subsubsection{X SDS Page Running Buffer (For 1 Litre)}

$3.03 \mathrm{~g}$ Tris base, $14.4 \mathrm{~g}$ Glycine, $1 \mathrm{~g}$ SDS was dissolved in $1 \mathrm{~L}$ of $\mathrm{dH}_{2} \mathrm{O}$.

\subsubsection{Ponceau S Stain}

Ponceau S Staining Solution was made up with $0.1 \%$ (w/v) Ponceau S 5\% (v/v) acetic acid dissolved in $\mathrm{dH}_{2} \mathrm{O}$.

\subsubsection{Western Blotting Apparatus}

\begin{tabular}{|c|l|}
\hline Bottom & Grey Side of Cassette \\
& Fibre pad \\
& 3 MM Filter Paper \\
& Electrophoresed Gel \\
& PVDF Membrane \\
& 3 MM Filter Paper \\
& Fibre pad \\
& Clear Side of Cassette \\
\hline
\end{tabular}




\subsubsection{Sodium Orthovanadate}

A $200 \mathrm{mM}$ solution of sodium orthovanadate was prepared in $\mathrm{dH}_{2} \mathrm{O}$ and the $\mathrm{pH}$ was adjusted to 10.0 using either $1 \mathrm{M} \mathrm{NaOH}$ or $1 \mathrm{M} \mathrm{HCl}$. The yellow solution was boiled in a microwave until it turned colourless after which it was allowed to cool to room temperature. The $\mathrm{pH}$ was adjusted to 10.0 and the solution was boiled. These steps were repeated until the solution remained colourless and the $\mathrm{pH}$ stabilised at $\mathrm{pH}$ 10.0. Activated sodium orthovanadate was stored in $20 \mu 1$ aliquots at $-20^{\circ} \mathrm{C}$ until required. 


\section{REFERENCES}

Abedin, M., Tintut, Y. and Demer, L. L. (2004). Mesenchymal stem cells and the artery wall. Circ Res 95, 671-676.

Adams, D. M. and Lucky, A. W. (2006). Cervicofacial vascular anomalies I. Hemangiomas and other benign vascular tumors. Semin Pediatr Surg 2, 124-132.

Akashi K, Traver D, Miyamoto T, Weissman IL. (2000). A clonogenic common myeloid progenitor that gives rise to all myeloid lineages. Nature 404, 193-197.

Akira, S. (2000). Roles of STAT3 defined by tissue-specific gene targeting. Oncogene $19,2607-2611$.

Al Buainian, H., Verhaeghe, E., Dierckxsens, L. and Naeyaert, J. M. (2003). Early treatment of hemangiomas with lasers. A review. Dermatology 206, 370-373.

Al-Khaldi, A., Eliopoulos, N., Martineau, D., Lejeune, L., Lachapelle, K. and Galipeau, J. (2003). Postnatal bone marrow stromal cells elicit a potent VEGF-dependent neoangiogenic response in vivo. Gene Ther 10, 621-629.

Allain, F., Vanpouille, C., Carpentier, M., Slomianny, M. C., Durieux, S. and Spik, G. (2002). Interaction with glycosaminoglycans is required for cyclophilin B to trigger integrin-mediated adhesion of peripheral blood $\mathrm{T}$ lymphocytes to extracellular matrix. Proc Natl Acad Sci USA 99, 2714-2719.

Almasan, A and Ashkenazi, A. (2003). Apo2L/TRAIL: apoptosis signaling, biology, and potential for cancer therapy. Cytokine Growth Factor Rev 14, 337-348.

Almeida-Porada, G., El-Shabrawy, D., Porada, C. and Zanjani, E. D. (2002). Differentiative potential of human metanephric mesenchymal cells. Exp Hematol 30, 1454-1462.

Amir, J., Metzker, A., Krikler, R. and Reisner, S. H. (1986). Strawberry hemangioma in preterm infants. Pediatr Dermatol 3, 331-332.

Andl, C. D, Mizushima, T., Oyama, K., Bowser, M., Nakagawa, H. and Rustgi, A. K. (2004). EGFR-induced cell migration is mediated predominantly by the JAK-STAT pathway in primary esophageal keratinocytes. Am J Physiol Gastrointest Liver Physiol 287, G1227-12237.

Anfosso, F., Bardin, N., Frances, V., Vivier, E., Camoin-Jau, L., Sampol, J. and DignatGeorge, F. (1998). Activation of human endothelial cells via S-endo-1 antigen (CD146) stimulates the tyrosine phosphorylation of focal adhesion kinase p125(FAK). J Biol Chem 273, 26852-26856.

Anfosso, F., Bardin, N., Vivier, E., Sabatier, F., Sampol, J. and Dignat-George, F. (2001). Outside-in signaling pathway linked to CD146 engagement in human endothelial cells. J Biol Chem 276, 1564-1569.

Annabi, B., Lee, Y. T., Turcotte, S., Naud, E., Desrosiers, R. R., Champagne, M., Eliopoulos, N., Galipeau, J. and Béliveau, R. (2003). Hypoxia promotes murine bonemarrow-derived stromal cell migration and tube formation. Stem Cells 21, 337-347. 
Antonelli-Orlidge, A., Saunders, K. B., Smith, S. R. and D'Amore, P. A. (1989). An activated form of transforming growth factor beta is produced by cocultures of endothelial cells and pericytes. Proc Natl Acad Sci USA 86, 4544-4548.

Arizono, Y., Yoshikawa, H., Naganuma, H., Hamada, Y., Nakajima, Y. and Tasaka, K. (2003). A mechanism of resistance to TRAIL/Apo2L-induced apoptosis of newly established glioma cell line and sensitisation to TRAIL by genotoxic agents. $\mathrm{Br} \mathbf{J}$ Cancer 88, 298-306.

Asahara, T., Murohara, T., Sullivan, A., Silver, M., Van der Zee, R., Li, T., Witzenbichler, B., Schatteman, G. and Isner, J. M. (1997). Isolation of putative progenitor endothelial cells for angiogenesis. Science 275, 964-967.

Asahara, T., Masud,a H., Takahashi, T., Kalka, C., Pastore, C., Silver, M., Kearne, M., Magner, M. and Isner, J. M. (1999). Bone marrow origin of endothelial progenitor cells responsible for postnatal vasculogenesis in physiological and pathological neovascularization. Circ Res 85, 221-228.

Ashkenazi, A. and Dixit, V. M. (1998). Death receptors: signaling and modulation. Science. 281, 1305-1308.

Ashkenazi, A., Pai, R. C., Fong, S., Leung, S., Lawrence, D. A., Marsters, S. A., Blackie, C., Chang, L., McMurtrey, A. E., Hebert, A., DeForge, L., Koumenis, I. L., Lewis, D., Harris, L., Bussiere, J., Koeppen, H., Shahrokh, Z. and Schwall, R. H. (1999). Safety and antitumor activity of recombinant soluble Apo2 ligand. J Clin Invest 104, 155-162.

Ashkenazi, A. (2002). Targeting death and decoy receptors of the tumour-necrosis factor superfamily. Nat Rev Cancer 2, 420-430.

Atkins, G. J., Bouralexis ,S., Evdokiou, A., Hay, S., Labrinidis, A., Zannettino, A. C., Haynes, D. R. and Findlay, D. M. (2002). Human osteoblasts are resistant to Apo2L/TRAIL-mediated apoptosis. Bone 31, 448-456.

Augustin, H. G., Braun, K., Telemenakis, I., Modlich, U. and Kuhn, W. (1995). Ovarian angiogenesis. Phenotypic characterization of endothelial cells in a physiological model of blood vessel growth and regression. Am J Pathol 147, 339-351.

Banes, A. K., Shaw, S., Jenkins, J., Redd, H., Amiri, F., Pollock, D. M. and Marrero, M. B. (2004). Angiotensin II blockade prevents hyperglycemia-induced activation of JAK and STAT proteins in diabetic rat kidney glomeruli. Am J Physiol Renal Physiol 286, F653-659.

Bardin, N., Frances, V., Lesaule, G., Horschowski, N., George, F. and Sampol, J. (1996). Identification of the S-Endo 1 endothelial-associated antigen. Biochem Biophys Res Commun 218, 210-216.

Bardin, N., Anfosso, F., Masse, J. M., Cramer, E., Sabatier, F., Le Bivic, A., Sampol, J. and Dignat-George, F. (2001). Identification of CD146 as a component of the endothelial junction involved in the control of cell-cell cohesion. Blood 98, 3677-3684.

Barlow, C. F., Priebe, C. J., Mulliken, J. B., Barnes, P. D., Mac Donald, D., Folkman, J. and Ezekowitz, R. A. (1998). Spastic diplegia as a complication of interferon Alfa-2a treatment of hemangiomas of infancy. J Pediatr 132, 527-530. 
Bartoli, M., Gu, X., Tsai, N. T., Venema, R. C., Brooks, S. E., Marrero, M. B. and Caldwell, R. B. (2000). Vascular endothelial growth factor activates STAT proteins in aortic endothelial cells. J Biol Chem 275, 33189-33192.

Bartoli, M., Platt, D., Lemtalsi, T., Gu, X., Brooks, S. E., Marrero, M. B. and Caldwell, R. B. (2003). VEGF differentially activates STAT3 in microvascular endothelial cells. FASEB J. 17, 1562-1564.

Becker, S., Groner, B. and Muller, C. W. (1998). Three-dimensional structure of the Stat3beta homodimer bound to DNA. Nature 394, 145-151.

Belloni, P. N. and Tressler, R. J. (1990). Microvascular endothelial cell heterogeneity: interactions with leukocytes and tumor cells. Cancer Metastasis Rev 8, 353-389.

Berard, M., Sordello, S., Ortega, N., Carrier, J. L., Peyri, N., Wassef, M., Bertrand, N., Enjolras, O., Drouet, L. and Plouet, J. (1997). Vascular endothelial growth factor confers a growth advantage in vitro and in vivo to stromal cells cultured from neonatal hemangiomas. Am J Pathol 150, 1315-1326.

Bereta, J and Bereta, M. (1995). Stimulation of glyceraldehyde-3-phosphate dehydrogenase mRNA levels by endogenous nitric oxide in cytokine-activated endothelium. Biochem Biophys Res Commun 217, 363-369.

Berg, J. N., Walter, J. W., Thisanagayam, U., Evans, M., Blei, F., Waner, M., Diamond, A. G., Marchuk, D. A. and Porteous, M. E. (2001). Evidence for loss of heterozygosity of $5 \mathrm{q}$ in sporadic haemangiomas: are somatic mutations involved in haemangioma formation? J Clin Pathol 54, 249-252.

Bernatchez, P. N., Soker, S. and Sirois, M. G. Vascular endothelial growth factor effect on endothelial cell proliferation, migration, and platelet-activating factor synthesis is Flk-1-dependent.(1994). J Biol Chem 274, 31047-31054.

Bhatia, P., Taylor, W. R., Greenberg, A. H. and Wright JA. (1994). Comparison of glyceraldehyde-3-phosphate dehydrogenase and 28S-ribosomal RNA gene expression as RNA loading controls for northern blot analysis of cell lines of varying malignant potential. Anal Biochem 216, 223-226.

Bianchi, L., Canton, C., Bini, L., Orlandi, R., Menard, S., Armini, A., Cattaneo, M., Pallini, V., Bernardi, L. R. and Biunno, I. (2005). Protein profile changes in the human breast cancer cell line MCF-7 in response to SEL1L gene induction. Proteomics 5, 2433-2442.

Bielenberg, D. R., Bucana, C. D., Sanchez, R., Mulliken, J. B., Folkman, J. and Fidler, I. J. (1999). Progressive growth of infantile cutaneous hemangiomas is directly correlated with hyperplasia and angiogenesis of adjacent epidermis and inversely correlated with expression of the endogenous angiogenesis inhibitor, IFN-beta. Int $\mathbf{J}$ Oncol 14, 401-408.

Bjarnason, R., Wickelgren, R., Hermansson, M., Hammarqvist, F., Carlsson, B. and Carlsson, L. M. (1998). Growth hormone treatment prevents the decrease in insulin-like growth factor I gene expression in patients undergoing abdominal surgery. J Clin Endocrinol Metab 83, 1566-1572. 
Blei, F., Walter, J., Orlow, S. J. and Marchuk, D. A. (1998). Familial segregation of hemangiomas and vascular malformations as an autosomal dominant trait. Arch Dermatol 134, 718-722.

Boesiger, J., Tsai, M., Maurer, M., Yamaguchi, M., Brown, L. F., Claffey, K. P., Dvorak, H. F. and Galli, S. J. (1998). Mast cells can secrete vascular permeability factor/ vascular endothelial cell growth factor and exhibit enhanced release after immunoglobulin E-dependent upregulation of fc epsilon receptor I expression. J Exp Med 188, 1135-1145.

Bolton, W. E., Mikulka, W. R., Healy, C. G., Schmittling, R. J. and Kenyon, N. S. (1992). Expression of proliferation associated antigens in the cell cycle of synchronized mammalian cells. Cytometry 13, 117-126.

Bonni, A., Sun, Y., Nadal-Vicens, M., Bhatt, A., Frank, D. A., Rozovsky, I., Stahl, N., Yancopoulos, G. D. and Greenberg, M. E. (1997). Regulation of gliogenesis in the central nervous system by the JAK-STAT signaling pathway. Science 278, 477-483.

Boon, L. M., Enjolras, O. and Mulliken, J. B. (1996). Congenital hemangioma: evidence of accelerated involution. J Pediatr 128, 329-335.

Boon, L. M., MacDonald, D. M. and Mulliken, J. B. (1999). Complications of systemic corticosteroid therapy for problematic hemangioma. Plast Reconstr Surg 104, 16161623.

Borden, E. C., Hogan, T. F. and Voelkel, J. G. (1982). Comparative antiproliferative activity in vitro of natural interferons alpha and beta for diploid and transformed human cells. Cancer Res 42, 4948-4953.

Bouralexis, S., Findlay, D. M., Atkins, G. J., Labrinidis, A., Hay, S. and Evdokiou, A. (2003). Progressive resistance of BTK-143 osteosarcoma cells to Apo2L/TRAILinduced apoptosis is mediated by acquisition of DcR2/TRAIL-R4 expression: resensitisation with chemotherapy. Br J Cancer 89, 206-214.

Bowers, R. E., Graham, E. A. and Tominson, K. M. (1960). The natural history of the strawberry nevus. Arch Dermatol 82, 59-72.

Bowman, T., Garcia, R., Turkson, J. and Jove, R. (2000). STATs in oncogenesis. Oncogene 19, 2474-2488.

Boye, E., Yu, Y., Paranya, G., Mulliken, J. B., Olsen, B. R. and Bischoff, J. (2001). Clonality and altered behavior of endothelial cells from hemangiomas. J Clin Invest 107, 745-752.

Boyer, A. S., Ayerinskas, I. I., Vincent, E. B., McKinney, L. A., Weeks, D. L., Runyan, R. B. (1999). TGFbeta2 and TGFbeta3 have separate and sequential activities during epithelial-mesenchymal cell transformation in the embryonic heart. Dev Biol 208, 530545.

Bree, A. F., Siegfried, E., Sotelo-Avila, C. and Nahass, G. (2001). Infantile hemangiomas: speculation on placental trophoblastic origin. Arch Dermatol 137, 573577. 
Breier, G., Albrecht, U., Sterrer, S. and Risau, W. (1992). Expression of vascular endothelial growth factor during embryonic angiogenesis and endothelial cell differentiation. Development 114, 521-532.

Breier, G. (2000). Angiogenesis in embryonic development--a review. Placenta 21, Suppl A:S11-5.

Briscole, J., Kohlhuber, F. and Mullerz, M. (1996). JAKs and STATs branch out. Trends Cell Biol 6, 336-340.

Bromberg, J. F., Horvath, C. M., Wen, Z., Schreiber, R. D. and Darnell, J. E. Jr. (1996). Transcriptionally active Stat 1 is required for the antiproliferative effects of both interferon alpha and interferon gamma. Proc Natl Acad Sci USA 93, 7673-7678.

Bromberg $_{\mathrm{a}}$, J. F., Horvath, C. M., Besser, D., Lathem, W. W. and Darnell, J. E. Jr. (1998). Stat3 activation is required for cellular transformation by v-src. Mol Cell Biol $18,2553-2558$.

Bromberg, J. F., Wrzeszczynska, M. H., Devgan, G., Zhao, Y., Pestell, R. G., Albanese, C. and Darnell, J. E. Jr. (1999). Stat3 as an oncogene. Cell 98, 295-303.

Bromberg, J. F. (2001). Activation of STAT proteins and growth control. Bioessays 23, 161-169.

Bromberg, J. F. (2002). Stat proteins and oncogenesis. J Clin Invest 109, 1139-1142.

Brown, L. F., Tognazzi, K., Dvorak, H. F. and Harrist, T. J. (1996). Strong expression of kinase insert domain-containing receptor, a vascular permeability factor/vascular endothelial growth factor receptor in AIDS-associated Kaposi's sarcoma and cutaneous angiosarcoma. Am J Pathol 148, 1065-1074.

Brown, K. J., Maynes, S. F., Bezos, A., Maguire, D. J., Ford, M. D. and Parish, C. R. (1996). A novel in vitro assay for human angiogenesis. Lab Invest 75, 539-555.

Bucay, N., Sarosi, I., Dunstan, C. R., Morony, S., Tarpley, J., Capparelli, C., Scully, S., Tan, H. L., Xu, W., Lacey, D. L., Boyle, W. J. and Simonet, W. S. (1998). Osteoprotegerin-deficient mice develop early onset osteoporosis and arterial calcification. Genes Dev, 1260-1268.

Buckland J. (2002). Haematopoiesis: The origin of HSCs. Nature Reviews Immunology 2,461 .

Buettner, R., Mora, L. B. and Jove, R. (2002). Activated STAT signaling in human tumors provides novel molecular targets for therapeutic intervention. Clin Cancer Res 8, 945-954.

Burger, M., Hartmann, T., Burger, J. A. and Schraufstatter, I. (2005). KSHV-GPCR and CXCR2 transforming capacity and angiogenic responses are mediated through a JAK2STAT3-dependent pathway. Oncogene 24, 2067-2075.

Burke, W. M., Jin, X., Lin, H. J., Huang, M., Liu, R., Reynolds, R. K. and Lin, J. (2001). Inhibition of constitutively active Stat3 suppresses growth of human ovarian and breast cancer cells. Oncogene 20, 7925-7934. 
Burman, D., Mansell, P. W. and Warin, R. P. (1967). Miliary haemangiomata in the newborn. Arch Dis Child 42, 193-197.

Burton, B. K., Schulz, C. J., Angle, B. and Burd, L. I. (1995). An increased incidence of haemangiomas in infants born following chorionic villus sampling (CVS). Prenat Diagn $15,209-214$.

Bustin, S. A. (2000). Absolute quantification of mRNA using real-time reverse transcription polymerase chain reaction assays. J Mol Endocrinol 25, 169-193.

Calo, V., Migliavacca, M., Bazan, V., Macaluso, M., Buscemi, M., Gebbia, N. and Russo, A. (2003). STAT proteins: from normal control of cellular events to tumorigenesis. J Cell Physiol 197, 157-168.

Camenisch, T. D., Schroeder, J. A., Bradley, J., Klewer, S. E. and McDonald, J. A. (2002). Heart-valve mesenchyme formation is dependent on hyaluronan-augmented activation of ErbB2-ErbB3 receptors. Nat Med 8, 850-855.

Camp, H. S., Ren, D. and Leff, T. (2002). Adipogenesis and fat-cell function in obesity and diabetes. Trends Mol Med 8, 442-447.

Campagnoli, C., Roberts ,I. A., Kumar, S., Bennett, P. R., Bellantuono, I., Fisk, N. M. (2001). Identification of mesenchymal stem/progenitor cells in human first-trimester fetal blood, liver, and bone marrow. Blood 98, 2396-2402.

Carmeliet $_{\mathrm{a}}$, P., Mackman, N., Moons, L., Luther, T., Gressens, P., Van Vlaenderen, I., Demunck, H., Kasper, M., Breier, G. and Evrard, P. (1996). Role of tissue factor in embryonic blood vessel development. Nature 383, 73-75.

Carmeliet, $\mathrm{P}_{\mathrm{b}}$., Ferreira, V., Breier, G., Pollefeyt, S., Kieckens, L., Gertsenstein, M., Fahrig, M., Vandenhoeck, A., Harpal, K. and Eberhardt, C. (1996). Abnormal blood vessel development and lethality in embryos lacking a single VEGF allele. Nature 380, 435-439.

Carmeliet, P and Luttun, A. (2001). The emerging role of the bone marrow-derived stem cells in (therapeutic) angiogenesis. Thromb Haemost 86, 289-297.

Catlett-Falcone, R., Landowski, T. H., Oshiro, M. M., Turkson, J., Levitzki, A., Savino, R., Ciliberto, G., Moscinski, L., Fernández-Luna, J. L., Nuñez, G., Dalton, W. S. and Jove, R. (1999). Constitutive activation of Stat3 signaling confers resistance to apoptosis in human U266 myeloma cells. Immunity 1, 105-115.

Ceradini, D. J., Kulkarni, A. R., Callaghan, M. J., Tepper, O. M., Bastidas, N., Kleinman, M. E., Capla, J. M., Galiano, R. D., Levine, J. P. and Gurtner. G. C. (2004). Progenitor cell trafficking is regulated by hypoxic gradients through HIF-1 induction of SDF-1. Nat Med 10, 858-864.

Chan, K. S., Carbajal, S., Kiguchi, K., Clifford, J., Sano, S. and DiGiovanni, J. (2004). Epidermal growth factor receptor-mediated activation of Stat3 during multistage skin carcinogenesis. Cancer Res 64, 2382-2389.

Chang, E., Boyd, A., Nelson, C. C., Crowley, D., Law, T., Keough, K. M., Folkman, J., Ezekowitz, R. A. and Castle, V. P. (1997). Successful treatment of infantile hemangiomas with interferon-alpha-2b. J Pediatr Hematol Oncol 19, 237-244. 
Chang, T. J., Juan, C. C., Yin, P. H., Chi, C. W. and Tsay, H. J. (1998). Up-regulation of beta-actin, cyclophilin and GAPDH in N1S1 rat hepatoma. Oncol Rep 5, 469-471.

Chang, J., Most, D., Bresnick, S., Mehrara, B., Steinbrech, D. S., Reinisch, J., Longaker, M. T. and Turk, A. E. (1999). Proliferative hemangiomas: analysis of cytokine gene expression and angiogenesis. Plast Reconstr Surg 103, 1-9.

Charnock-Jones, D. S., Kaufmann, P. and Mayhew, T. M. (2004). Aspects of human fetoplacental vasculogenesis and angiogenesis. I. Molecular regulation Placenta 25, 103-113.

Chatterjee-Kishore. M., Van den Akker, F. and Stark, G. R. (2000). Association of STATs with relatives and friends. Trends Cell Biol 10, 106-111.

Chawla-Sarkar, M., Leaman, D. W. and Borden, E. C. (2001). Preferential induction of apoptosis by interferon (IFN)-beta compared with IFN-alpha2: correlation with TRAIL/Apo2L induction in melanoma cell lines. Clin Cancer Res 7, 1821-1831.

Chawla-Sarkar, M., Lindner, D. J., Liu, Y. F., Williams, B. R., Sen, G. C., Silverman, R. H. and Borden, E. C. (2003). Apoptosis and interferons: role of interferon-stimulated genes as mediators of apoptosis. Apoptosis 8, 237-249.

Chen, Q., Gong, B., Mahmoud-Ahmed, A. S., Zhou, A., His, E. D., Hussein, M. and Almasan, A. (2001). Apo2L/TRAIL and Bcl-2-related proteins regulate type I interferon-induced apoptosis in multiple myeloma. Blood 98, 2183-2192.

Chen, H., Ye, D., Xie, X., Chen, B. and Lu, W. (2004). VEGF, VEGFRs expressions and activated STATs in ovarian epithelial carcinoma. Gynecol Oncol 94, 630-635.

Chen Z, Han ZC. (2007). STAT3: a critical transcription activator in angiogenesis. Med Res Rev 28, 185-200.

Cheng, L., Liang, J. and Tang, S. (1999). [The study on the role of apoptosis suppressive gene bcl-2 in the pathogenesis of hemangioma]. Zhonghua Zheng Xing Shao Shang Wai Ke Za Zhi 15, 35-36.

Cheung, D. S., Warman, M. L. and Mulliken, J. B. (1997). Hemangioma in twins. Ann Plast Surg 38, 269-274.

Chin, Y. E., Kitagawa, M., Kuida, K., Flavell, R. A. and Fu, X. Y. (1997). Activation of the STAT signaling pathway can cause expression of caspase 1 and apoptosis. Mol Cell Biol 17, 5328-5337.

Chin, Y. E., Kitagaw,a M., Kuida, K., Flavell, RA. and Fu, X. Y. (1997). Activation of the STAT signaling pathway can cause expression of caspase 1 and apoptosis. Mol Cell Biol 17, 5328-5337.

Chinnaiyan, A. M., Prasad, U., Shankar, S., Hamstra, D. A., Shanaiah, M., Chenevert, T. L., Ross, B. D. and Rehemtulla, A. Combined effect of tumor necrosis factor-related apoptosis-inducing ligand and ionizing radiation in breast cancer therapy. Proc Natl Acad Sci USA 97, 1754-1759.

Choi, E. A., Lei, H., Maron, D. J., Wilson, J. M., Barsoum, J., Fraker, D. L., El-Deiry, W. S. and Spitz, F. R. (2003). Stat1-dependent induction of tumor necrosis factor- 
related apoptosis-inducing ligand and the cell-surface death signaling pathway by interferon beta in human cancer cells. Cancer Res 63, 5299-52307.

Cohen, R. C. and Myers, N. A. (1986). Diagnosis and management of massive hepatic hemangiomas in childhood. J Pediatr Surg 21, 6-9.

Cohen, M. M. Jr. (2002). Vasculogenesis, angiogenesis, hemangiomas, and vascular malformations. Am J Med Genet 108, 265-274.

Collin-Osdoby, P., Rothe, L., Anderson, F., Nelson, M., Maloney, W. and Osdoby, P. (2001). Receptor activator of NF-kappa B and osteoprotegerin expression by human microvascular endothelial cells, regulation by inflammatory cytokines, and role in human osteoclastogenesis. J Biol Chem 276, 20659-20672.

Cooper, A. G. and Bolande, R. P. (1965). Multiple Hemangiomas in an Infant with Cardiac Hypertrophy. Postmortem Angiographic Demonstration of the Arteriovenous Fistulae. Pediatrics 35, 27-35.

Cross, S. S., Yang, Z., Brown, N. J., Balasubramanian, S. P., Evans, C. A., Woodward, J. K., Neville-Webbe, H. L., Lippitt, J. M., Reed, M. W., Coleman, R. E. and Holen, I. (2006). Osteoprotegerin (OPG)--a potential new role in the regulation of endothelial cell phenotype and tumour angiogenesis? Int J Cancer 118, 1901-1908.

Cundy, T., Hegde, M., Naot, D., Chong, B., King, A., Wallace, R., Mulley, J., Love, D. R., Seidel, J., Fawkner, M., Banovic, T., Callon, K. E., Grey, A. B., Reid, I. R., Middleton-Hardie, C. A. and Cornish, J. (2002). A mutation in the gene TNFRSF11B encoding osteoprotegerin causes an idiopathic hyperphosphatasia phenotype. Hum Mol Genet 11, 2119-2127.

Dai, X., Sayama, K., Shirakata, Y., Hanakawa, Y., Yamasaki, K., Tokumaru, S., Yang, L., Wang, X., Hirakawa, S., Tohyama, M., Yamauchi, T., Takashi, K., Kagechika, H. and Hashimoto, K. (2007). STAT5a/PPARgamma pathway regulates involucrin expression in keratinocyte differentiation. J Invest Dermatol 127, 1728-1735.

Darnell, J. E. Jr., Kerr, I. M. and Stark, G. R. (1994). Jak-STAT pathways and transcriptional activation in response to IFNs and other extracellular signaling proteins. Science 264, 1415-1421

Darnell, J. E. Jr. (1997). STATs and gene regulation. Science 277, 1630-1635.

Davidovich, I. A., Levenson, A. S. and Chernokhvostov, V. V. (2004). Overexpression of DcR1 and survivin in genetically modified cells with pleiotropic drug resistance. Cancer Lett 211, 189-197.

Davis, S and Yancopoulos, G, D. (1999). The angiopoietins: Yin and Yang in angiogenesis. Curr Top Microbiol Immunol 237, 173-185.

Degli-Esposti, M. A., Dougall, W. C., Smolak, P. J., Waugh, J. Y., Smith, C. A. and Goodwin, R. G. (1997). The novel receptor TRAIL-R4 induces NF-kappaB and protects against TRAIL-mediated apoptosis, yet retains an incomplete death domain. Immunity 7, 813-820.

Degli-Esposti, M. (1999). To die or not to die--the quest of the TRAIL receptors. J Leukoc Biol 65, 535-542. 
Deng, J., Hua, K., Lesser, S. S. and Harp, J. B. (2000). Activation of signal transducer and activator of transcription-3 during proliferative phases of 3T3-L1 adipogenesis. Endocrinology. 2000 141, 2370-2376.

Deng, Y., Lin, Y. and Wu, X. (2002). TRAIL-induced apoptosis requires Baxdependent mitochondrial release of Smac/DIABLO. Genes Dev 16, 33-45.

Delahunt, B., Bethwaite, P. B., Nacey, J. N. and Ribas, J. L. (1993). Proliferating cell nuclear antigen (PCNA) expression as a prognostic indicator for renal cell carcinoma: comparison with tumour grade, mitotic index, and silver-staining nucleolar organizer region numbers. J Pathol 170, 471-477.

DeLisser, H. M., Newman, P. J. and Albelda, S. M. (1994). Molecular and functional aspects of PECAM-1/CD31. Immunol Today 15, 490-495.

Demeestere, I., Gervy, C., Centner, J., Devreker, F., Englert, Y. and Delbaere, A. (2004). Effect of insulin-like growth factor-I during preantral follicular culture on steroidogenesis, in vitro oocyte maturation, and embryo development in mice. Biol Reprod 70, 1664-1669.

Dethlefsen, S. M., Butterfield, C. E. and Ausprunk, D. H. (1986). Structural changes induced in capillary endothelial cells by growth factors: altered distribution of cytoskeletal elements and organelles in cells spreading in the presence of tumor conditioned medium and hypothalamus derived growth factor. Tissue Cell 18, 827-837.

Devine, S. M., Bartholomew, A. M., Mahmud, N., Nelson, M., Patil, S., Hardy, W., Sturgeon, C., Hewett, T., Chung, T., Stock, W., Sher, D., Weissman, S., Ferrer, K., Mosca, J., Deans, R., Moseley, A. and Hoffman, R. (2001). Mesenchymal stem cells are capable of homing to the bone marrow of non-human primates following systemic infusion. Exp Hematol 29, 244-255.

Devine, M. J., Mierisch, C. M., Jang, E., Anderson, P. C. and Balian G. (2002). Transplanted bone marrow cells localize to fracture callus in a mouse model. J Orthop Res 20, 1232-1239.

Devine, S. M., Cobbs, C., Jennings, M., Bartholomew, A. and Hoffman R. (2003). Mesenchymal stem cells distribute to a wide range of tissues following systemic infusion into nonhuman primates. Blood 101, 2999-3001.

Dhir, R., Ni, Z., Lou, W., DeMiguel, F., Grandis, J. R. and Gao AC. (2002). Stat3 activation in prostatic carcinomas Prostate 51, 241-246.

Dipietro $_{a}$, L. A. and Polverini, P. J. (1993). Angiogenic macrophages produce the angiogenic inhibitor thrombospondin 1. Am J Pathol 143, 678-684.

Dipietro $_{b}$, L. A. and Polverini, P. J. (1993). Role of the macrophage in the positive and negative regulation of wound neovascularization. Behring Inst Mitt, 238-247.

Dosanjh, A., Chang, J., Bresnick, S., Zhou, L., Reinisch, J., Longake,r M. and Karasek, M. (2000). In vitro characteristics of neonatal hemangioma endothelial cells: similarities and differences between normal neonatal and fetal endothelial cells. J Cutan Pathol 27, 441-450. 
Dosquet, C., Coudert, M. C., Wassef, M., Enjolras, O. and Drouet, L. (1998). Importance of bFGF ("basic fibroblast growth factor") for diagnosis and treatment of hemangiomas. Ann Dermatol Venereol 125, 313-316.

Drevs, J., Hofmann, I., Hugenschmidt, H., Wittig, C., Madjar, H., Müller, M., Wood, J., Martiny-Baron, G., Unger, C. and Marmé D. (2000). Effects of PTK787/ZK 222584, a specific inhibitor of vascular endothelial growth factor receptor tyrosine kinases, on primary tumor, metastasis, vessel density, and blood flow in a murine renal cell carcinoma model. Cancer Res 60, 4819-4824.

Drolet BA, Esterly NB, Frieden IJ. (1999). Hemangiomas in children. N Engl J Med 341, 173-181.

Dubois-Stringfellow, N., Kolpack-Martindale, L., Bautch, V. L. and Azizkhan, R. G. (1994). Mice with hemangiomas induced by transgenic endothelial cells. A model for the Kasabach-Merritt syndrome. Am J Pathol 144, 796-806.

Dubois, J., Hershon, L., Carmant, L., Bélanger, S., Leclerc, J. M. and David, M. (1999). Toxicity profile of interferon alfa- $2 b$ in children: A prospective evaluation. J Pediatr 135, $782-785$.

Durbin, J. E., Hackenmiller, R., Simon, M. C. and Levy, D. E. (1996). Targeted disruption of the mouse Stat1 gene results in compromised innate immunity to viral disease. Cell 84, 443-450.

Dvorin, E. L., Wylie-Sears, J., Kaushal, S., Martin, D. P. and Bischoff J. (2003). Quantitative evaluation of endothelial progenitors and cardiac valve endothelial cells: proliferation and differentiation on poly-glycolic acid/poly-4-hydroxybutyrate scaffold in response to vascular endothelial growth factor and transforming growth factor beta1. Tissue Eng 9, 487-493.

Dwek, R. A. (1998). Biological importance of glycosylation. Dev. Biol. Stand 96, $43-$ 47.

Dyduch, G., Okoń, K. and Mierzyński W. (2004). Benign vascular proliferations--an immunohistochemical and comparative study. Pol J Pathol 55, 59-64.

Edmondson, H. A. (1956). Differential diagnosis of tumors and tumor-like lesions of liver in infancy and childhood. AMA J Dis Child 91, 168-186.

Ehrbar, M., Metters, A., Zammaretti, P., Hubbell, J. A. and Zisch, A. H. (2005). Endothelial cell proliferation and progenitor maturation by fibrin-bound VEGF variants with differential susceptibilities to local cellular activity. J Control Release 101, 93-109.

Eichmann, A., Marcelle, C., Breant, C. and Le Douarin, N. M. (1993). Two molecules related to the VEGF receptor are expressed in early endothelial cells during avian embryonic development. Mech Dev 42, 33-48.

El-Adawi, H., Deng, L., Tramontano, A., Smith, S., Mascareno, E., Ganguly, K., Castillo, R. and El-Sherif, N. (2003). The functional role of the JAK-STAT pathway in post-infarction remodeling. Cardiovasc Res 57, 129-138. 
Engvall, E., Earwicker, D., Haaparanta, T., Ruoslahti, E. and Sanes, J. R. (1990). Distribution and isolation of four laminin variants; tissue restricted distribution of heterotrimers assembled from five different subunits. Cell Regul 1, 731-740.

Enjolras, O., Riche, M. C., Merland, J. J. and Escande, J. P. (1990). Management of alarming hemangiomas in infancy: a review of 25 cases. Pediatrics 85, 491-498.

Enjolras, O. and Mulliken, J. B. (1997). Vascular tumors and vascular malformations (new issues). Adv Dermatol 13, 375-423.

Erices, A., Conget, P. and Minguell JJ. (2000). Mesenchymal progenitor cells in human umbilical cord blood. Br J Haematol 109, 235-242.

Ezekowitz, R. A., Mulliken, J. B. and Folkman, J. (1992). Interferon alfa-2a therapy for life-threatening hemangiomas of infancy. N Engl J Med 326, 1456-1463.

Faraggiana, T., Crescenzi, A. and Marinozzi V. (1989). Presence of an alphagalactolipid on the cell surfaces of endothelial cells of human kidney. Histochem J 21, 235-240.

Fargeas, C. A., Corbeil, D. and Huttner, W. B. (2003). AC133 antigen, CD133, prominin-1, prominin-2, etc.: prominin family gene products in need of a rational nomenclature. Stem Cells 21, 506-508.

Fawcett, S. L., Grant, I., Hall, P. N., Kelsall, A. W. and Nicholson JC. (2004). Vincristine as a treatment for a large haemangioma threatening vital functions. Br $\mathrm{J}$ Plast Surg 57, 168-171.

Ferrara, N., Houck, K., Jakeman, L. and Leung, D. W. (1992). Molecular and biological properties of the vascular endothelial growth factor family of proteins. Endocr Rev 13, 18-32.

Ferrara, N., Carver-Moore, K., Chen, H., Dowd, M., Lu, L., O'Shea, K. S., PowellBraxton, L., Hillan, K. J. and Moore, M. W. (1996). Heterozygous embryonic lethality induced by targeted inactivation of the VEGF gene. Nature 380, 439-442.

Ferrara, N. (2000). Vascular endothelial growth factor and the regulation of angiogenesis. Recent Prog Horm Res 55, 15-35.

Finn, M. C., Glowacki, J. and Mulliken, J. B. (1983). Congenital vascular lesions: clinical application of a new classification. J Pediatr Surg 18, 894-900.

Finnegan, M. C., Goepel, J. R., Hancock, B. W. and Goyns, M. H. (1993). Investigation of the expression of housekeeping genes in non-Hodgkin's lymphoma. Leuk Lymphoma 10, 387-393.

Flamme, I. and Risau, W. (1992). Induction of vasculogenesis and hematopoiesis in vitro. Development 116, 435-439.

Floyd, Z. E. and Stephens, J. M. (2003). STAT5 promotes adipogenesis in nonprecursor cells and associates with the glucocorticoid receptor during adipocyte differentiation. Diabetes 52, 308-314. 
Folkman, J. and Cotran, R. (1976). Relation of vascular proliferation to tumor growth. Int Rev Exp Pathol 16, 207-248.

Folkman, J. and Klagsbrun, M. (1987). Angiogenic factors. Science 235, 442-447.

Folkman, J. and D'Amore, P. A. (1996). Blood vessel formation: what is its molecular basis? Cell 87, 1153-1155.

Folkman, J. (1997). Angiogenesis and angiogenesis inhibition: an overview. Exs 79, 18.

Fong, G. H., Rossant, J., Gertsenstein, M. and Breitman, M. L. (1995). Role of the Flt-1 receptor tyrosine kinase in regulating the assembly of vascular endothelium. Nature $376,66-70$.

Fong, G. H., Zhang, L., Bryce, D. M. and Peng, J. (1999). Increased hemangioblast commitment, not vascular disorganization, is the primary defect in flt-1 knock-out mice. Development 126, 3015-3025

Forsythe, J. A., Jiang, B. H., Iyer, N. V., Agani, F., Leung, S. W., Koos, R. D., andSemenza, G. L. (1996). Activation of vascular endothelial growth factor gene transcription by hypoxia-inducible factor 1 . Mol Cell Biol 16, 4604-4613

Foss, D. L., Baarsch, M. J. and Murtaugh, M. P. (1998). Regulation of hypoxanthine phosphoribosyltransferase, glyceraldehyde-3-phosphate dehydrogenase and beta-actin mRNA expression in porcine immune cells and tissues. Anim Biotechnol 9, 67-78.

Freeman, W. M., Walker, S. J. and Vrana, K. E. (1999). Quantitative RT-PCR: pitfalls and potential. Biotechniques. 26, 112-122, 124-125.

French, L. E., Chonn, A., Ducrest, D., Baumann, B., Belin, D., Wohlwend, A., Kiss, J. Z., Sappino, A. P., Tschopp, J. and Schifferli, J. A. (1993). Murine clusterin: molecular cloning and mRNA localization of a gene associated with epithelial differentiation processes during embryogenesis. J Cell Biol 122, 1119-1130.

Frid, M. G., Kale, V.A. and Stenmark, K. R. (2002). Mature vascular endothelium can give rise to smooth muscle cells via endothelial-mesenchymal transdifferentiation: in vitro analysis. Circ Res 90, 1189-1196.

Frieden, I. J., Reese, V. and Cohen, D. (1996). PHACE syndrome. The association of posterior fossa brain malformations, hemangiomas, arterial anomalies, coarctation of the aorta and cardiac defects, and eye abnormalities. Arch Dermatol 132, 307-311.

Friedlander, S. F., Ritter, M. R. and Friedlander M. (2005). Recent progress in our understanding of the pathogenesis of infantile hemangiomas. Lymphat Res Biol 3, 219225.

Friesel, R., Komoriya, A. and Maciag, T. (1987). Inhibition of endothelial cell proliferation by gamma-interferon. J Cell Biol 104, 689-696.

Froehner, S. C. (1998). Gathering glycine receptors at synapses. Science 282, 1277 1279. 
Frumento, G., Rotondo, R., Tonetti, M., Damonte, G., Benatti, U. and Ferrara, G. B. (2002). Tryptophan-derived catabolites are responsible for inhibition of $\mathrm{T}$ and natural killer cell proliferation induced by indoleamine 2,3-dioxygenase. J Exp Med 19, 459468.

Fukuchi, Y., Nakajima, H., Sugiyama, D., Hirose, I., Kitamura, T. and Tsuji, K. (2004). Human placenta-derived cells have mesenchymal stem/progenitor cell potential. Stem Cells 22, 649-658.

Funamoto M, Fujio Y, Kunisada K, Negoro S, Tone E, Osugi T, Hirota H, Izumi M, Yoshizaki K, Walsh K, Kishimoto T, Yamauchi-Takihara K. (2000). Signal transducer and activator of transcription 3 is required for glycoprotein 130-mediated induction of vascular endothelial growth factor in cardiac myocytes. J Biol Chem 275, 10561-10566.

Fruman, D. A., Klee, C. B., Bierer, B. E. and Burakoff, S. J. (1992). Calcineurin phosphatase activity in T lymphocytes is inhibited by FK 506 and cyclosporin A. Proc Natl Acad Sci USA 89, 3686-3690.

Fuchs, S., Baffour, R., Zhou, Y. F., Shou, M., Pierre, A., Tio, F. O., Weissman, N. J., Leon, M. B., Epstein. S. E. and Kornowski, R. (2001). Transendocardial delivery of autologous bone marrow enhances collateral perfusion and regional function in pigs with chronic experimental myocardial ischemia. J Am Coll Cardiol 37, 1726-1732.

Fulda, S., Wick, W., Weller, M. and Debatin, K. M. (2002). Smac agonists sensitize for Apo2L/TRAIL- or anticancer drug-induced apoptosis and induce regression of malignant glioma in vivo. Nat Med 8, 808-815.

Fuller, K., Wong, B., Fox, S., Choi, Y. and Chambers, T. J. (1998). TRANCE is necessary and sufficient for osteoblast-mediated activation of bone resorption in osteoclasts. J Exp Med 188, 997-1001.

Furchgott, R. F. and Zawadzki, J. V. (1980). The obligatory role of endothelial cells in the relaxation of arterial smooth muscle by acetylcholine. Nature 288, 373-376.

Furumatsu, T., Shen, Z. N., Kawai, A., Nishida, K., Manabe, H., Oohashi, T., Inoue, H. and Ninomiya, Y. (2003). Vascular endothelial growth factor principally acts as the main angiogenic factor in the early stage of human osteoblastogenesis. J Biochem 133, 633-639.

Galat. A. (1993). Peptidylproline cis-trans-isomerases Immunophilins. Eur. J. Biochem $216,689$.

Gambero, S., Canalli, A. A., Traina, F., Albuquerque, D. M., Saad, S. T., Costa, F. F. and Conran, N. (2006). Therapy with hydroxyurea is associated with reduced adhesion molecule gene and protein expression in sickle red cells with a concomitant reduction in adhesive properties. Eur J Haematol 78, 144-51.

Garcia, R., Bowman, T. L., Niu, G., Yu, H., Minton, S., Muro-Cacho, C. A., Cox, C. E., Falcone, R., Fairclough, R., Parsons, S., Laudano, A., Gazit, A., Levitzki, A., Kraker, A. and Jove, R. (2001). Constitutive activation of Stat3 by the Src and JAK tyrosine kinases participates in growth regulation of human breast carcinoma cells. Oncogene 20, 2499-2513. 
Garlanda, C. and Dejana, E. (1997). Heterogeneity of endothelial cells. Specific markers. Arterioscler Thromb Vasc Biol 17, 1193-1202.

Garmendía G, Miranda N, Borroso S, Longchong M, Martínez E, Ferrero J, Porrero P, López-Saura P. (2001). Regression of infancy hemangiomas with recombinant IFNalpha 2b. J Interferon Cytokine Res 21, 31-38.

Garzon, M. C., Enjolras, O. and Frieden, I. J. (2000). Vascular tumors and vascular malformations: evidence for an association. J Am Acad Dermatol 42, 275-279.

Gehling, U. M., Ergün, S., Schumacher, U., Wagener, C., Pantel, K., Otte, M., Schuch, G., Schafhausen, P., Mende, T., Kilic, N., Kluge, K., Schäfer, B., Hossfeld, D. K and Fiedler, W. (2000). In vitro differentiation of endothelial cells from AC133-positive progenitor cells. Blood 95, 3106-3112.

Gerber, H. P., Hillan, K. J., Ryan, A. M., Kowalski, J., Keller, G. A., Rangell, L., Wright, B. D., Radtke, F., Aguet, M. and Ferrara, N. (1999). VEGF is required for growth and survival in neonatal mice. Development 126, 1149-1159.

Gilbert, S. F. Developmental Biology. 4th ed. Massachusetts: Sinauer Associates, (1994).

Giulietti, A., Overbergh, L., Valckx, D., Decallonne, B., Bouillon, R. and Mathieu, C. (2001). An overview of real-time quantitative PCR: applications to quantify cytokine gene expression. Methods 25, 386-401

Glowacki, J. and Mulliken, J. B. (1982). Mast cells in hemangiomas and vascular malformations. Pediatrics 70, 48-51.

Glukhova, M. A., Frid, M. G. and Koteliansky, V. E. (1991). Phenotypic changes of human aortic smooth muscle cells during development and in the adult vessel. Am $\mathbf{J}$ Physiol 261, 78-80.

Gong, B. and Almasan, A. (2000). Apo2 ligand/TNF-related apoptosis-inducing ligand and death receptor 5 mediate the apoptotic signaling induced by ionizing radiation in leukemic cells. Cancer Res 60, 5754-5760.

Gonzalez-Crussi, F., Chou, P. and Crawford, S. E. (1991). Congenital, infiltrating giantcell angioblastoma. A new entity? Am J Surg Pathol 15, 175-183.

Gordon, J. R. and Galli, S. J. (1990). Mast cells as a source of both preformed and immunologically inducible TNF-alpha/cachectin. Nature 346, 274-276.

Grandis, J. R., Drenning, S. D., Zeng, Q., Watkins, S. C., Melhem, M. F., Endo, S., Johnson, D. E., Huang, L., He, Y. and Kim, J. D. (2000). Constitutive activation of Stat3 signaling abrogates apoptosis in squamous cell carcinogenesis in vivo. Proc Natl Acad Sci USA 97, 4227-4232.

Grataroli, R., Vindrieux, D., Gougeon, A. and Benahmed, M. (2002). Expression of tumor necrosis factor-alpha-related apoptosis-inducing ligand and its receptors in rat testis during development. Biol Reprod 66, 1707-1715.

Green, DR. (2000). Apoptotic pathways: paper wraps stone blunts scissors. Cell 102, 14. 
Griffith, T. S., Chin, W. A., Jackson, G. C., Lynch, D. H. and Kubin, M. Z. (1998). Intracellular regulation of TRAIL-induced apoptosis in human melanoma cells. J Immunol 161, 2833-2840.

Griffith, T. S., Wiley, S. R., Kubin, M. Z., Sedger, L. M., Maliszewski, C. R. and Fanger, N. A. (1999). Monocyte-mediated tumoricidal activity via the tumor necrosis factor-related cytokine, TRAIL. J Exp Med 189, 1343-1354.

Grupen, C. G., Nagashima, H. and Nottle, M. B. (1997). Role of epidermal growth factor and insulin-like growth factor-I on porcine oocyte maturation and embryonic development in vitro. Reprod Fertil Dev 9, 571-575.

Gresser. I. (1997). Wherefore interferon? J Leukoc Biol 61, 567-574.

Gumbiner, B. M. (1996). Cell adhesion: the molecular basis of tissue architecture and morphogenesis. Cell 84, 345-57.

Gutterman, J. U. (1994). Cytokine therapeutics: lessons from interferon alpha. Proc Natl Acad Sci USA 91, 1198-1205.

Haas, T. L. and Madri, J. A. (1999). Extracellular matrix-driven matrix metalloproteinase production in endothelial cells: implications for angiogenesis. Trends Cardiovasc Med 9, 70.

Hahn, T., Hartmann, M., Blaschitz, A., Skofitsch, G., Graf, R., Dohr, G. and Desoye, G. (1995).Localisation of the high affinity facilitative glucose transporter protein GLUT 1 in the placenta of human, marmoset monkey (Callithrix jacchus) and rat at different developmental stages. Cell Tissue Res 280, 49-57.

Hahn, T., Barth, S., Weiss, U., Mosgoeller, W. and Desoye, G. (1998). Sustained hyperglycemia in vitro down-regulates the GLUT1 glucose transport system of cultured human term placental trophoblast: a mechanism to protect fetal development? FASEB J $12,1221-1231$.

Haik, B. G., Karcioglu, Z. A., Gordon, R. A. and Pechous, B. P. (1994). Capillary hemangioma (infantile periocular hemangioma) Surv Ophthalmol 38, 399-426.

Hamano, K., Li, T. S., Kobayashi, T., Kobayashi, S., Matsuzaki, M. and Esato, K. (2000). Angiogenesis induced by the implantation of self-bone marrow cells: a new material for therapeutic angiogenesis. Cell Transplant 9, 439-443.

Hamilton, G. S. and Steiner, J. P. (1998). Immunophilins: beyond immunosuppression. J Med Chem 41, 5119-5143.

Hamlat, A., Adn, M., Pasqualini, E., Brassier, G. and Askar, B. (2005).

Pathophysiology of capillary haemangioma growth after birth. Med Hypotheses 64, 1093-1096.

Hanahan, D. and Folkman, J. (1996). Patterns and emerging mechanisms of the angiogenic switch during tumorigenesis. Cell 86, 353-364.

Hanahan, D. (1997). Signaling vascular morphogenesis and maintenance. Science 277, 48-50. 
Hansen-Smith, F., Egginton, S., Zhou, A. L. and Hudlicka, O. (2001). Growth of arterioles precedes that of capillaries in stretch-induced angiogenesis in skeletal muscle. Microvasc Res 62, 1-14.

Harik, S. I., Hall, A. K., Richey, P., Andersson, L., Lundahl, P. and Perry, G. (1993). Ontogeny of the erythroid/HepG2-type glucose transporter (GLUT-1) in the rat nervous system. Brain Res Dev Brain Res 72, 41-49.

Hasan $_{a}$, Q., Ruger, B. M., Tan, S. T., Gush, J. and Davis, P. F. (2000). Clusterin/apoJ expression during the development of hemangioma. Hum Pathol 31, 691-697.

Hasan $_{b}$, Q., Tan, S. T., Gush, J., Peters, S. G. and Davis, P. F. (2000). Steroid therapy of a proliferating hemangioma: histochemical and molecular changes. Pediatrics 105, 117 120.

Hasan, Q., Tan, S. T., Gush, J. and Davis, P. F. (2001). Altered mitochondrial cytochrome $\mathrm{b}$ gene expression during the regression of hemangioma. Plast Reconstr Surg 108, 1471-6; discussion 1477-1478.

Hasan, Q., Tan, S. T., Xu, B. and Davis, P. F. (2003). Effects of five commonly used glucocorticoids on haemangioma in vitro. Clin Exp Pharmacol Physiol 30, 140-1444.

Hattori, R., Maulik, N., Otani, H., Zhu, L., Cordis, G., Engelman, R. M., Siddiqui, M. A. and Das, D. K. (2001). Role of STAT3 in ischemic preconditioning. J Mol Cell Cardiol 33, 1929-1936.

Haynes, D. R., Barg, E., Crotti, T. N., Holding, C., Weedon, H., Atkins, G. J., Zannetino, A., Ahern, M. J., Coleman, M., Roberts-Thomson, P. J., Kraan, M., Tak, P. P. and Smith, M. D. (2003). Osteoprotegerin expression in synovial tissue from patients with rheumatoid arthritis, spondyloarthropathies and osteoarthritis and normal controls. Rheumatology 42, 123-134.

Heid, C. A., Stevens, J., Livak, K. J. and Williams, P. M. (1996). Real time quantitative PCR. Genome Res 6, 986-994.

Hendrix, M. J., Seftor, E. A., Hess, A. R. and Seftor, R. E. (2003). Vasculogenic mimicry and tumour-cell plasticity: lessons from melanoma. Nat Rev Cancer 3, 411421.

Hilbe, W., Dirnhofer, S., Oberwasserlechner, F., Schmid, T., Gunsilius, E., Hilbe, G., Wöll, E. and Kähler, C. M. (2004). CD133 positive endothelial progenitor cells contribute to the tumour vasculature in non-small cell lung cancer. J Clin Pathol 57, 965-969.

Hildano, A. and Nakajima, S. (1972). Earliest features of the strawberry mark in the newborn. Br J Dermatol 87, 138-144.

Hiraishi, K., Suzuki, K., Hakomori, S. and Adachi, M. (1993). Le(y) antigen expression is correlated with apoptosis (programmed cell death). Glycobiology 3, 381-390.

Hirano, T., Yasukawa, K., Harada, H., Taga, T., Watanabe, Y., Matsuda, T., Kashiwamura, S., Nakajima, K., Koyama, K. and Iwamatsu, A. (1986). Complementary DNA for a novel human interleukin (BSF-2) that induces B lymphocytes to produce immunoglobulin. Nature 324, 73-76. 
Hirschi, K. K., Rohovsky, S. A., Beck, L. H., Smith, S. R. and D'Amore, P. A. (1999). Endothelial cells modulate the proliferation of mural cell precursors via platelet-derived growth factor-BB and heterotypic cell contact. Circ Res 84, 298-305.

Hofbauer, L. C. and Schoppet, M. (2004). Clinical implications of the osteoprotegerin/RANKL/RANK system for bone and vascular diseases. JAMA 292, 490-495.

Holden, K. R. and Alexander, F. (1970). Diffuse neonatal hemangiomatosis. Pediatrics 46, 411-421.

Holen, I., Croucher, P. I., Hamdy, F. C. and Eaton, C. L. (2002). Osteoprotegerin (OPG) is a survival factor for human prostate cancer cells. Cancer Res 62, 1619-1623.

Hong, X., Jiang, F., Kalkanis, S. N., Zhang, Z. G., Zhang, X. P., DeCarvalho, A. C., Katakowski, M., Bobbitt, K., Mikkelsen, T. and Chopp, M. (2006). SDF-1 and CXCR4 are up-regulated by VEGF and contribute to glioma cell invasion. Cancer Lett 236, 3945.

Hopfel-Kreiner, I. (1980). Histogenesis of hemangiomas - An ultrastructural study on capillary and cavernous hemangiomas of the skin. Pathol Res Pract 170, 70-90.

Horiguchi, A., Oya, M., Marumo, K. And Murai, M. (2002). STAT3, but not ERKs, mediates the IL-6-induced proliferation of renal cancer cells, ACHN and 769P. Kidney Int 6, 926-938.

Horvath, C. M. (2000). STAT proteins and transcriptional responses to extracellular signals. Trends Biochem Sci 25, 496-502.

Hoshino, A. and Fujii, H. (2007). Temporal regulation of Stat5 activity in determination of cell differentiation program. Biochem Biophys Res Commun 358, 914-919.

Hotta, T., Suzuki, H., Nagai, S., Yamamoto, K., Imakiire, A., Takada, E., Itoh, M. and Mizuguchi, J. (2003). Chemotherapeutic agents sensitize sarcoma cell lines to tumor necrosis factor-related apoptosis-inducing ligand-induced caspase- 8 activation, apoptosis and loss of mitochondrial membrane potential. J Orthop Res 21, 949-957.

Howard, G. C and Bethell, D. R. Basic Methods in Antibody Production and characterisation. Califormia: CRC Press, 2001.

Huang, C., Cao, J., Huang, K. J., Zhang, F., Jiang, T., Zhu, L. and Qiu, Z. J. (2006). Inhibition of STAT3 activity with AG490 decreases the invasion of human pancreatic cancer cells in vitro. Cancer Sci 97, 1417-1423.

Hunt, T. K., Knighton, D. R., Thakral, K. K., Goodson, W. H., 3rd and Andrews, W. S. (1984). Studies on inflammation and wound healing: angiogenesis and collagen synthesis stimulated in vivo by resident and activated wound macrophages. Surgery 96, 48-54.

Hur, J., Yoon, C. H., Kim, H. S., Choi, J. H., Kang, H. J., Hwang, K. K., Oh, B. H., Lee, M. M. and Park, Y. B. (2004). Characterization of two types of endothelial progenitor cells and their different contributions to neovasculogenesis. Arterioscler Thromb Vasc Biol 24, 288-293. 
Hurvitz, C. H., Alkalay, A. L., Sloninsky, L., Kallus, M. and Pomerance, J. J. (1986) Cyclophosphamide therapy in life-threatening vascular tumor. J Pediatr 109, 360.

Ihara, S., Nakajima, K., Fukada, T., Hibi, M., Nagata, S., Hirano, T. and Fukui, Y. (1997). Dual control of neurite outgrowth by STAT3 and MAP kinase in PC12 cells stimulated with interleukin-6. Embo J 16, 5345-5352.

Ilan, N., Tucker, A. and Madri, J. A. (2003). Vascular endothelial growth factor expression, beta-catenin tyrosine phosphorylation, and endothelial proliferative behavior: a pathway for transformation? Lab Invest 83, 1105-1115.

Issik, F. F., Rand, R. P., Gruss, J. S., Benjamin, D. and Alpers, C. E. (1996). Monocyte chemoattractant protein-1 mRNA expression in hemangiomas and vascular malformations. J Surg Res 61, 71-76.

Iwata, J., Sonobe, H., Furihata, M., Ido, E. and Ohtsuki, Y. (1996). High frequency of apoptosis in infantile capillary haemangioma. J Pathol 4, 403-408.

Jackson, I. T., Carreño, R., Potparic, Z. and Hussain, K. Hemangiomas, vascular malformations, and lymphovenous malformations: classification and methods of treatment. (1993). Plast Reconstr Surg 91, 1216-1230.

Jackson, K. A., Majka, S. M., Wang, H., Pocius, J., Hartley, C. J., Majesky, M. W., Entman, M. L., Michael, L. H., Hirschi, K. K. and Goodell, MA. (2001). Regeneration of ischemic cardiac muscle and vascular endothelium by adult stem cells. J Clin Invest 107, 1395-1402.

Jang, Y. C., Arumugam, S., Ferguson, M., Gibran, N. S. and Isik, F. F. (1998). Changes in matrix composition during the growth and regression of human hemangiomas. J Surg Res 80, 9-15.

Jansson, T. and Illsley, N. P. (1993). Osmotic water permeabilities of human placental microvillous and basal membranes. J Membr Biol 132, 147-155.

Jauniaux, E., Jurkovic, D., Gulbis, B., Liesnard, C., Lees, C. and Campbell, S. (1995). Materno-fetal immunoglobulin transfer and passive immunity during the first trimester of human pregnancy. Hum Reprod 10, 3297-300.

Jiang, Y., Vaessen, B., Lenvik, T., Blackstad, M., Reyes, M. and Verfaillie, C. M. (2002). Multipotent progenitor cells can be isolated from postnatal murine bone marrow, muscle, and brain. Exp Hematol 30, 896-904.

Jenne, D. E. and Tschopp, J. (1992). Clusterin: the intriguing guises of a widely expressed glycoprotein. Trends Biochem Sci 17, 154-159.

Jia, J., Zhang, W. F. and Zhao, Y. F. (2007). Monocytes: a possible source of hemangioma endothelial cells. Med Hypotheses 68, 98-100.

Jin, Z. G., Melaragno, M. G., Liao, D. F., Yan, C., Haendeler, J., Suh, Y. A., Lambeth, J. D. and Berk, B. C. (2002). Cyclophilin A is a secreted growth factor induced by oxidative stress. Circ Res 87, 789-796. 
Jin, Z. G., Lungu, A. O., Xie, L., Wang, M., Wong, C. and Berk, B.C. (2004). Cyclophilin A is a proinflammatory cytokine that activates endothelial cells. Arterioscler Thromb Vasc Biol 24, 1186-1191.

Johns, T. G., Mackay, I. R., Callister, K. A., Hertzog, P. J., Devenish, R. J. and Linnane, A. W. (1992). Antiproliferative potencies of interferons on melanoma cell lines and xenografts: higher efficacy of interferon beta. J Natl Cancer Inst 84, 1185-1190.

Johnson, H. M., Torres, B. A., Green, M. M,, Szente, B. E., Siler, K. I., Larkin, J. and Subramaniam, P. S. (1998). Cytokine-receptor complexes as chaperones for nuclear translocation of signal transducers. Biochem Biophys Res Commun 244, 607-614.

Jones, D. T., Ganeshaguru, K., Mitchell, W. A., Foroni, L., Baker, R. J., Prentice, H. G., Mehta, A. B. andWickremasinghe, R. G. (2003). Cytotoxic drugs enhance the ex vivo sensitivity of malignant cells from a subset of acute myeloid leukaemia patients to apoptosis induction by tumour necrosis factor receptor-related apoptosis-inducing ligand. Br J Haematol 121, 713-720.

Jundt, G., Berghäuser, K. H., Termine, J. D. and Schulz, A. (1987). Osteonectin--a differentiation marker of bone cells. Cell Tissue Res 248, 409-415.

Junqueira, L. C., Bignolas, G. and Brentani, R. R. (1979). Picrosirius staining plus polarization microscopy, a specific method for collagen detection in tissue sections. Histochem J 11, 447-455.

Kaipainen, A., Korhonen, J., Mustonen, T., van Hinsbergh, V. W., Fang, G. H., Dumont, D., Breitman, M. and Alitalo, K. (1995). Expression of the fms-like tyrosine kinase 4 gene becomes restricted to lymphatic endothelium during development. Proc Natl Acad Sci U S A 92, 3566-70.

Kalka, C., Masuda, H., Takahashi, T., Kalka-Moll, W. M., Silver, M., Kearney, M., Li, T., Isner, J. M. and Asahara, T. (2000). Transplantation of ex vivo expanded endothelial progenitor cells for therapeutic neovascularization. Proc Natl Acad Sci USA 97, 34223427.

Kalka, C., Tehrani, H., Laudenberg, B., Vale, P. R., Isner, J. M., Asahara, T. and Symes, J. F. (2000). VEGF gene transfer mobilizes endothelial progenitor cells in patients with inoperable coronary disease. Ann Thorac Surg 70, 829-834.

Kanzaki, H., Chiba, M., Sato, A., Miyagawa, A., Arai, K., Nukatsuka, S. and Mitani, H. (2006). Cyclical tensile force on periodontal ligament cells inhibits osteoclastogenesis through OPG induction. J Dent Res 85, 457-462.

Kaplan, D. H., Shankaran, V., Dighe, A. S., Stockert, E., Aguet, M., Old, L. J. and Schreiber, R. D. (1998). Demonstration of an interferon gamma-dependent tumor surveillance system in immunocompetent mice. Proc Natl Acad Sci U S A 95, 75567561.

Kataoka, H., Takakura, N., Nishikawa, S., Tsuchida, K., Kodama, H., Kunisada, T., Risau, W., Kita, T. and Nishikawa, S. I. (1997). Expressions of PDGF receptor alpha, cKit and Flk1 genes clustering in mouse chromosome 5 define distinct subsets of nascent mesodermal cells. Dev Growth Differ 39, 729-740. 
Katz, H. R., Kaye, R. E. and Austen, K. F. (1991). Mast cell biochemical and functional heterogeneity. Transplant Proc 23, 2900-2904.

Kawamoto, A., Gwon, H. C., Iwaguro, H., Yamaguchi, J. I., Uchida, S., Masuda, H., Silver, M., Ma, H., Kearney, M., Isner, J. M. and Asahara, T. (2001). Therapeutic potential of ex vivo expanded endothelial progenitor cells for myocardial ischemia. Circulation 103, 634-637.

Keane, M. M., Ettenberg, S. A., Nau, M. M., Russell, E.K. and Lipkowitz, S. (1999).

Chemotherapy augments TRAIL-induced apoptosis in breast cell lines. Cancer Res 59, 734-741.

Kelley, S. K. and Ashkenazi, A. (2004). Targeting death receptors in cancer with Apo2L/TRAIL. Curr Opin Pharmacol 4, 333-339.

Kerr, I. M., Costa-Pereira, A. P., Lillemeier, B. F. and Strobl, B. (2003). Of JAKs, STATs, blind watchmakers, jeeps and trains. FEBS Lett 546, 1-5.

Kim, K. J., Li, B., Winer, J., Armanini, M., Gillett, N., Phillips, H. S. and Ferrara, N. (1993). Inhibition of vascular endothelial growth factor-induced angiogenesis suppresses tumour growth in vivo. Nature 362, 841-844.

Kim, J. W., Kim, S. Y., Park, S. Y., Kim, Y. M., Kim, J. M., Lee, M. H., Ryu, H. M. (2004). Mesenchymal progenitor cells in the human umbilical cord. Ann Hematol 83, 733-738.

Kirby, M. L. and Waldo, K. L. (1995). Neural crest and cardiovascular patterning. Circ Res 77, 211-215.

Kischkel, F. C., Lawrence, D. A., Chuntharapai, A., Schow, P., Kim, K. J. and Ashkenazi, A. (2000). Apo2L/TRAIL-dependent recruitment of endogenous FADD and caspase- 8 to death receptors 4 and 5. Immunity 12, 611-620.

Khan, Z. A., Melero-Martin, J. M., Wu, X., Paruchuri, S., Boscolo, E., Mulliken, J. B. and Bischoff, J. (2006). Endothelial progenitor cells from infantile hemangioma and umbilical cord blood display unique cellular responses to endostatin. Blood 108, 915921.

Kivell, B. M. (2003). Phd Thesis.

Klauber, N., Rohan, R.M., Flynn, E. et al. (1997) Critical components of the female reproductive pathway are suppressed by the angiogenesis inhibitor AGM-1470. Nature Med., 3, 443-446.

Kleinman, M. E., Greives, M. R., Churgin, S. S., Blechman, K. M., Chang, E. I., Ceradini, D. J., Tepper, O. M. and Gurtner, G. C. (2007). Hypoxia-induced mediators of stem/progenitor cell trafficking are increased in children with hemangioma. Arterioscler Thromb Vasc Biol 27, 2664-2670.

Knight, M. J., Riffkin, C. D., Muscat, A. M., Ashley, D. M. and Hawkins, C. J. (2001). Analysis of FasL and TRAIL induced apoptosis pathways in glioma cells. Oncogene 20, 5789-5798.

Koblizek, T. I., Weiss, C., Yancopoulos, G. D., Deutsch, U. and Risau, W. (1998). Angiopoietin-1 induces sprouting angiogenesis in vitro. Curr Biol 8, 529-532. 
Koch, A. E., Polverini, P. J. and Leibovich, S. J. (1986). Stimulation of neovascularization by human rheumatoid synovial tissue macrophages. Arthritis Rheum 29, 471-479.

Koch, A. E., Polverini, P. J., Kunkel, S. L., Harlow, L. A., DiPietro, L. A., Elner, V. M., Elner, S. G. and Strieter, R. M. (1992). Interleukin-8 as a macrophage-derived mediator of angiogenesis. Science 258, 1798-1801.

Kocher, A. A., Schuster, M. D., Szabolcs, M. J., Takuma, S., Burkhoff, D., Wang, J., Homma, S., Edwards, N. M. and Itescu, S. (2001). Neovascularization of ischemic myocardium by human bone-marrow-derived angioblasts prevents cardiomyocyte apoptosis, reduces remodeling and improves cardiac function. Nat Med 7, 430-436.

Kofron, J. L., Kuzmic, P., Kishore, V., Colón-Bonilla, E. and Rich, D. H. (1991). Determination of kinetic constants for peptidyl prolyl cis-trans isomerases by an improved spectrophotometric assay. Biochemistry 30, 6127-6134.

Körbling, M., Estrov, Z. and Champlin, R. (2003). Adult stem cells and tissue repair. Bone Marrow Transplant 32, S23-24.

Korpelainen, E. I. and Alitalo, K. (1998). Signaling angiogenesis and lymphangiogenesis. Curr Opin Cell Biol 10, 159-164.

Korpelainen, E. I., Karkkainen, M., Gunji, Y., Vikkula, M. and Alitalo, K. (1999). Endothelial receptor tyrosine kinases activate the STAT signaling pathway: mutant Tie2 causing venous malformations signals a distinct STAT activation response. Oncogene $18,1-8$.

Klauber, N., Rohan, R. M., Flynn, E. and D'Amato, R. J. (1997). Critical components of the female reproductive pathway are suppressed by the angiogenesis inhibitor AGM1470. Nat Med 3, 443-6.

Kleinman, M. E., Tepper, O. M., Capla, J. M., Bhatt, K. A., Ceradini, D. J., Galiano, R. D., Blei, F., Levine, J. P. and Gurtner, G. C. (2003). Increased circulating AC133+ CD34+ endothelial progenitor cells in children with hemangioma. Lymphat Res Biol 1, 301-307.

Kraling, B. M., Razon, M. J., Boon, L. M., Zurakowski, D., Seachord, C., Darveau, R. P., Mulliken, J. B., Corless, C. L. and Bischoff, J. (1996). E-selectin is present in proliferating endothelial cells in human hemangiomas. Am J Pathol 148, 1181-1191.

Krause, D. S., Fackler, M. J., Civin, C. I. and May, W. S. (1996). CD34: structure, biology, and clinical utility. Blood 87, 1-13.

Kumar, A., Commane, M., Flickinger, T. W., Horvath, C. M. and Stark, G. R. (1997). Defective TNF-alpha-induced apoptosis in STAT1-null cells due to low constitutive levels of caspases. Science 278, 1630-1632.

Kumar-Sinha, C., Varambally, S., Sreekumar, A. and Chinnaiyan, A. M. (2002). Molecular cross-talk between the TRAIL and interferon signaling pathways. J Biol Chem 277, 575-585. 
Kurki, P., Vanderlaan, M., Dolbeare, F., Gray, J. and Tan, E. M. (1986). Expression of proliferating cell nuclear antigen (PCNA)/cyclin during the cell cycle. Exp Cell Res 166, 209-219.

Kuwabara, T. and Cogan, D. G. (1963). Retinal vascular patterns. VI. Mural cells of the retinal capillaries. Arch Ophthalmol 69, 492-502.

Kuzu, I., Bicknell, R., Harris, A. L., Jones, M., Gatter, K. C. and Mason, D. Y. (1992). Heterogeneity of vascular endothelial cells with relevance to diagnosis of vascular tumours. J Clin Pathol 45, 143-148.

Lacey, D. L., Timms, E., Tan, H. L., Kelley, M. J., Dunstan, C. R., Burgess, T., Elliott, R., Colombero, A., Elliott, G., Scully, S., Hsu, H., Sullivan, J., Hawkins, N., Davy, E., Capparelli, C., Eli, A., Qian, Y. X., Kaufman, S., Sarosi, I., Shalhoub, V., Senaldi, G., Guo, J., Delaney, J. and Boyle, W. J. (1998). Osteoprotegerin ligand is a cytokine that regulates osteoclast differentiation and activation. Cell 93, 165-176.

Lang, I., Hartmann, M., Blaschitz, A., Dohr, G., Skofitsch, G. and Desoye, G. (1993). Immunohistochemical evidence for the heterogeneity of maternal and fetal vascular endothelial cells in human full-term placenta. Cell Tissue Res 274, 211-218.

Leaman, D. W., Chawla-Sarkar, M., Vyas, K., Reheman, M., Tamai, K., Toji, S. and Borden, E. C. (2002). Identification of X-linked inhibitor of apoptosis-associated factor1 as an interferon-stimulated gene that augments TRAIL Apo2L-induced apoptosis. J Biol Chem 277, 28504-28511.

LeBlanc, H., Lawrence, D., Varfolomeev, E., Totpal, K., Morlan, J., Schow, P., Fong, S., Schwall, R., Sinicropi, D. and Ashkenazi, A. (2002). Tumor-cell resistance to death receptor--induced apoptosis through mutational inactivation of the proapoptotic Bcl-2 homolog Bax. Nat Med 8, 274-281.

LeBlanc, H. N. and Ashkenazi, A. (2003). Apo2L/TRAIL and its death and decoy receptors. Cell Death Differ 10, 66-75.

Le Blanc, K., Rasmusson, I., Götherström, C., Seidel, C., Sundberg, B., Sundin, M., Rosendahl, K., Tammik, C. and Ringdén, O. (2004). Mesenchymal stem cells inhibit the expression of CD25 (interleukin-2 receptor) and CD38 on phytohaemagglutininactivated lymphocytes. Scand J Immunol 60, 307-315.

Le Douarin, N. M., Creuzet, S., Couly, G. and Dupin, E. (2004). Neural crest cell plasticity and its limits. Development 131, 4637-4650.

Lee, M., Kim, H., Jeon, D., Hwang, I., Choi, B., Myung, K., Choi, Y., Paik, S. and Baik, M. (1996). Iron metabolism-related genes and mitochondrial genes are induced during involution of mouse mammary gland. Biochem Biophys Res Commun 224, 164 168.

Lee, R. H., Kim, B., Choi, I., Kim, H., Choi, H. S., Suh, K., Bae, Y. C. and Jung, J. S. (2004). Characterization and expression analysis of mesenchymal stem cells from human bone marrow and adipose tissue. Cell Physiol Biochem 14, 311-324

Leibovich, S. J., Polverini, P. J., Shepard, H. M., Wiseman, D. M., Shively, V. and Nuseir, N. (1987). Macrophage-induced angiogenesis is mediated by tumour necrosis factor-alpha. Nature 329, 630-632. 
Leibovich, S. J. and Wiseman, D. M. (1988). Macrophages, wound repair and angiogenesis. Prog Clin Biol Res 266, 131-145.

Leivo, I. and Engvall, E. (1988). Merosin, a protein specific for basement membranes of Schwann cells, striated muscle, and trophoblast, is expressed late in nerve and muscle development. Proc Natl Acad Sci USA 85, 1544-1548.

Lemmink, H. H., Tuyt, L., Knol, G., Krikke, E. and Vellenga, E. (2001). Identification of LIL-STAT in monocytic leukemia cells and monocytes after stimulation with interleukin-6 or interferon- $\gamma$. Blood 98, 3849-3852.

Leppilampi, M., Koistinen, P., Savolainen, E. R., Hannuksela, J., Parkkila, A. K., Niemelä, O., Pastoreková, S., Pastorek, J., Waheed, A., Sly, W. S., Parkkila, S. and Rajaniemi, H. (2002). The expression of carbonic anhydrase II in hematological malignancies. Clin Cancer Res 8, 2240-2245.

Leung, D. W., Cachianes, G., Kuang, W. J., Goeddel, D. V. and Ferrara, N. (1989). Vascular endothelial growth factor is a secreted angiogenic mitogen. Science 246, 13061309.

Leverkus, M., Neumann, M., Mengling, T., Rauch, C. T., Bröcker, E. B., Krammer, P. H. and Walczak, H. (2000). Regulation of tumor necrosis factor-related apoptosisinducing ligand sensitivity in primary and transformed human keratinocytes. Cancer Res 60, 553-559

Levine, R. J., Maynard, S. E., Qian, C., Lim, K. H., England, L. J., Yu, K. F., Schisterman, E. F., Thadhani, R., Sachs, B. P., Epstein, F. H., Sibai, B. M., Sukhatme, V. P. and Karumanchi, S. A. (2004). Circulating angiogenic factors and the risk of preeclampsia. N Engl J Med 350, 672-83.

Levy, N. S., Chung, S., Furneaux, H. and Levy, A. P. (1998). Hypoxic stabilization of vascular endothelial growth factor mRNA by the RNA-binding protein HuR. J Biol Chem 273, 6417-6423.

Li, Q., Yu, Y., Bischoff, J., Mulliken, J. B. and Olsen, B. R. (2003). Differential expression of CD146 in tissues and endothelial cells derived from infantile haemangioma and normal human skin. J Pathol 201, 296-302.

Lindahl, P., Johansson, B. R., Leveen, P. and Betsholtz, C. (1997). Pericyte loss and microaneurysm formation in PDGF-B-deficient mice. Science 277, 242-245.

Liu, J., Chen, C. M. and Walsh, C. T. (1991). Human and Escherichia coli cyclophilins: sensitivity to inhibition by the immunosuppressant cyclosporin A correlates with a specific tryptophan residue. Biochemistry 30, 2306-2310.

Liu, X., Yue, P., Khuri, F. R. and Sun, S. Y. (2005). Decoy receptor 2 (DcR2) is a p53 target gene and regulates chemosensitivity. Cancer Res 65, 9169-9175.

Liu, G., Yuan, X., Zeng, Z., Tunici, P., Ng, H., Abdulkadir, I. R., Lu, L., Irvin, D., Black, K. L. and Yu, J. S. (2006). Analysis of gene expression and chemoresistance of CD133+ cancer stem cells in glioblastoma. Mol Cancer 5, 67. 
Livak, K. J. and Schmittgen, T. D. (2001). Analysis of relative gene expression data using real-time quantitative PCR and the 2(-Delta Delta C(T)) Method. Methods 25, 402-408.

Lloyd, K. O. (1987). Philip Levine award lecture. Blood group antigens as markers for normal differentiation and malignant change in human tissues. Am J Clin Pathol 87, 129-139.

Loike, J. D., Cao, L., Brett, J., Ogawa, S., Silverstein, S. C. and Stern, D. (1992). Hypoxia induces glucose transporter expression in endothelial cells. Am J Physiol 263, C326-C333.

Lovell, M. J. and Mathur, A. (2004). The role of stem cells for treatment of cardiovascular disease. Cell Prolif 37, 67-87.

Lu, W., Chen, H., Yel, F., Wang, F. and Xie, X. (2006). VEGF induces phosphorylation of STAT3 through binding VEGFR2 in ovarian carcinoma cells in vitro. Eur J Gynaecol Oncol 27, 363-369.

Luna, L.G. Manual of Histologic Staining Methods of the Armed Forces Institute of Pathology. New York: McGraw-Hill (1992).

Lyden, D., Hattori, K., Dias, S., Costa, C., Blaikie, P., Butros, L., Chadburn, A., Heissig, B., Marks, W., Witte, L., Wu, Y., Hicklin, D., Zhu, Z., Hackett, N. R., Crystal, R. G., Moore, M. A., Hajjar, K. A., Manova, K., Benezra, R. and Rafii, S. (2001). Impaired recruitment of bone-marrow-derived endothelial and hematopoietic precursor cells blocks tumor angiogenesis and growth. Nat Med 7, 1194-1201.

Ma, F. Y., Anderson, G. M., Gunn, T. D., Goffin, V., Grattan, D. R. and Bunn, S. J. (2005). Prolactin specifically activates signal transducer and activator of transcription $5 \mathrm{~b}$ in neuroendocrine dopaminergic neurons. Endocrinology 146, 5112-5119.

MacFarlane, M., Harper, N., Snowden, R. T., Dyer, M. J., Barnett, G. A., Pringle, J. H. and Cohen, G. M. (2002). Mechanisms of resistance to TRAIL-induced apoptosis in primary B cell chronic lymphocytic leukaemia. Oncogene 21, 6809-6818.

Maisonpierre, P. C., Suri, C., Jones, P. F., Bartunkova, S., Wiegand, S. J., Radziejewski, C., Compton, D., McClain, J., Aldrich, T. H., Papadopoulos, N. et al. (1997). Angiopoietin-2, a natural antagonist for Tie2 that disrupts in vivo angiogenesis. Science 277, 55-60.

Majka, S. M., Jackson, K. A., Kienstra, K. A., Majesky, M. W., Goodell, M. A. and Hirschi, K. K. (2003). Distinct progenitor populations in skeletal muscle are bone marrow derived and exhibit different cell fates during vascular regeneration. J Clin Invest 111, 71-79.

Malan, E. Vascular Malformations (Angiodysplasias). (1974). Milan, Italy: Carlo Erba Foundation, 1-19.

Mallory, J. C., Crudden, G., Oliva, A., Saunders, C., Stromberg, A. and Craven, R. J. (2005). A novel group of genes regulates susceptibility to antineoplastic drugs in highly tumorigenic breast cancer cells. Mol Pharmacol 68, 1747-1756. 
Malyankar, U. M., Scatena, M., Suchland, K. L., Yun, T. J., Clark, E. A. and Giachelli, C. M. (2004). Osteoprotegerin is an alpha vbeta 3-induced, NF-kappa B-dependent survival factor for endothelial cells. J Biol Chem 275, 20959-20962.

Mancini, A. J. and Smoller, B. R. (1996). Proliferation and apoptosis within juvenile capillary hemangiomas. Am J Dermatopathol 18, 505-14.

Mantych $_{\mathrm{a}}$, G. J., James, D. E. and Devaskar, S. U. (1993). Jejunal/kidney glucose transporter isoform (Glut-5) is expressed in the human blood-brain barrier. Endocrinology 132, 35-40.

Mantych $_{b}$, G. J., Sotelo-Avila, C. and Devaskar, S. U. (1993). The blood-brain barrier glucose transporter is conserved in preterm and term newborn infants. J Clin Endocrinol Metab 77, 46-9.

Marchuk, D. A. (2001). Pathogenesis of hemangioma. J Clin Invest 107, 665-6.

Margileth, A. M. and Museles, M. (1965). Cutaneous hemangiomas in children. Diagnosis and conservative management. Jama 194, 523-526.

Marler, J. J., Fang, J., Requena-Kasserjian, Y., Fishman, S. J., Folkman, J., Moses M. (2002). "Increased incidence of matrix metalloproteinases in the urine of children with vascular anomalies". The $13^{\text {th }}$ International Workshop on Vascualr Anomalies Montreal, Canada. pp.7.

Marsters, S. A., Sheridan, J. P., Pitti, R. M., Huang, A., Skubatch, M., Baldwin, D., Yuan, J., Gurney, A., Goddard, A. D., Godowski, P. and Ashkenazi A. (1997). A novel receptor for Apo2L/TRAIL contains a truncated death domain. Curr Biol 7, 1003-1006.

Masters, J. R. (2002). HeLa cells 50 years on: the good, the bad and the ugly. Nature Reviews Cancer 2, 315-319.

Martin-Padura, I., De Castellarnau, C., Uccini, S., Pilozzi, E., Natali, P. G., Nicotra, M. R., Ughi, F., Azzolini, C., Dejana, E. and Ruco, L. (1995). Expression of VE (vascular endothelial)-cadherin and other endothelial-specific markers in haemangiomas. J Pathol $175,51-57$.

Mascareno, E., El-Shafei, M., Maulik, N., Sato, M., Guo, Y., Das, D. K. and Siddiqui, M. A. (2001). JAK/STAT signaling is associated with cardiac dysfunction during ischemia and reperfusion. Circulation 104, 325-329.

Mayer, H., Bertram, H., Lindenmaier, W., Korff, T., Weber, H. and Weich. H. (2005). Vascular endothelial growth factor (VEGF-A) expression in human mesenchymal stem cells: autocrine and paracrine role on osteoblastic and endothelial differentiation. J Cell Biochem 95, 827-839.

Mazière. C., Conte, M. A. and Mazière, J. C. (2001). Activation of JAK2 by the oxidative stress generated with oxidized low-density lipoprotein. Free Radic Biol Med $31,1334-1340$.

Meng, R. D., McDonald, E. R., Sheikh, M. S., Fornace, A. J. and El-Deiry, W. S. (2000). The TRAIL decoy receptor TRUNDD (DcR2, TRAIL-R4) is induced by adenovirus-p53 overexpression and can delay TRAIL-, p53-, and KILLER/DR5dependent colon cancer apoptosis. Mol Ther 1, 130-144. 
Meininger, C. J. and Zetter, B. R. (1992). Mast cells and angiogenesis. Semin Cancer Biol 3, 73-79.

Meininger, C. J., Brightman, S. E., Kelly, K. A. and Zetter, B. R. (1995). Increased stem cell factor release by hemangioma-derived endothelial cells. Lab Invest 72, 166-173.

Melero-Martin, J. M., Khan, Z. A., Picard, A., Wu, X., Paruchuri, S. and Bischoff, J. (2007). In vivo vasculogenic potential of human blood-derived endothelial progenitor cells. Blood 109, 4761-4768.

Meraz, M. A., White, J. M., Sheehan, K. C., Bach, E. A., Rodig, S. J., Dighe, A. S., Kaplan, D. H., Riley, J. K., Greenlund, A. C., Campbell, D., Carver-Moore, K., DuBois, R. N., Clark, R., Aguet, M. and Schreiber, R. D. (1996). Targeted disruption of the Stat 1 gene in mice reveals unexpected physiologic specificity in the JAK-STAT signaling pathway. Cell 84, 431-442.

Mérino, D., Lalaoui, N., Morizot, A., Schneider, P., Solary, E. and Micheau, O. (2006). Differential inhibition of TRAIL-mediated DR5-DISC formation by decoy receptors 1 and 2. Mol Cell Biol 26, 7046-7055.

Metcalfe, D. D., Baram, D. and Mekori, Y. A. (1997). Mast cells. Physiol Rev 77, 10331079.

Metry, D.W., Dowd, C. F., Barkovich, A. J. and Frieden, I. J. (2001). The many faces of PHACE syndrome. J Pediatr 1, 117-123.

Miao, L., Yi, P., Wang, Y. and Wu, M. (2003). Etoposide upregulates Bax-enhancing tumour necrosis factor-related apoptosis inducing ligand-mediated apoptosis in the human hepatocellular carcinoma cell line QGY-7703. Eur J Biochem 270, 2721-2731.

Mignatti, P., Tsuboi, R., Robbins, E. and Rifkin, D. B. (1989). In vitro angiogenesis on the human amniotic membrane: requirement for basic fibroblast growth factor-induced proteinases. J Cell Biol 108, 671-682.

Migone, T. S., Lin, J. X., Cereseto, A., Mulloy, J. C., O'Shea, J. J., Franchini, G. and Leonard, W. J. (1995). Constitutively activated Jak-STAT pathway in $\mathrm{T}$ cells transformed with HTLV-I. Science 269, 79-81.

Millauer, B., Shawver, L. K., Plate, K. H., Risau, W. and Ullrich, A. (1994). Glioblastoma growth inhibited in vivo by a dominant-negative Flk-1 mutant. Nature 367: 576-579.

Millauer, B., Longhi, M. P., Plate, K. H., Shawver, L. K., Risau, W., Ullrich, A. and Strawn, L. M. (1996). Dominant-negative inhibition of Flk-1 suppresses the growth of many tumor types in vivo. Cancer Res 56, 1615-1620.

Minamide, S., Naora, H., Adachi, M. and Okano, A. (1995). Apoptosis as a mechanism of skin renewal: $\mathrm{Le}(\mathrm{y})$-antigen expression is involved in an early event of a cell's commitment to apoptosis. Histochem Cell Biol 103, 339-343

Miracle, A., Ankley, G. and Lattier, D. (2006). Expression of two vitellogenin genes (vg1 and vg3) in fathead minnow (Pimephales promelas) liver in response to exposure to steroidal estrogens and androgens. Ecotoxicol Environ Saf 63, 337-342. 
Mitchell, B. and Sharma, R. Embryology: an illustrated colour text. Edinburgh: Churchill Livingstone, 2005.

Mitsiades, C. S., Treon, S. P., Mitsiades, N., Shima, Y., Richardson, P., Schlossman, R., Hideshima, T. and Anderson, K. C. (2001). TRAIL/Apo2L ligand selectively induces apoptosis and overcomes drug resistance in multiple myeloma: therapeutic applications. Blood 98, 795-804.

Miyashita, T., Kawakami, A., Nakashima, T., Yamasaki, S., Tamai, M., Tanaka, F., Kamachi, M., Ida, H., Migita, K., Origuchi, T., Nakao, K. and Eguchi, K. (2004). Osteoprotegerin (OPG) acts as an endogenous decoy receptor in tumour necrosis factorrelated apoptosis-inducing ligand (TRAIL)-mediated apoptosis of fibroblast-like synovial cells. Clin Exp Immunol 137, 430-436.

Mizuno, A., Amizuka, N., Irie, K., Murakami, A., Fujise, N., Kanno, T., Sato, Y., Nakagawa, N., Yasuda, H., Mochizuki, S., Gomibuchi, T., Yano, K., Shima, N., Washida, N., Tsuda, E., Morinaga, T., Higashio, K. and Ozawa, H. (1998). Severe osteoporosis in mice lacking osteoclastogenesis inhibitory factor/osteoprotegerin. Biochem Biophys Res Commun. 247, 610-615.

Morinaga, T., Nakagawa, N., Yasuda, H., Tsuda, E. And Higashio, K. (1998). Cloning and characterization of the gene encoding human osteoprotegerin/osteoclastogenesisinhibitory factor. Eur J Biochem 254, 685-691.

Morrison, B. H., Bauer, J. A., Hu, J., Grane, R. W., Ozdemir, A. M., Chawla-Sarkar, M., Gong, B., Almasan, A., Kalvakolanu, D. V. and Lindner, D. J. (2002). Inositol hexakisphosphate kinase 2 sensitizes ovarian carcinoma cells to multiple cancer therapeutics. Oncogene 21, 1882-1889.

Mosca, J. D., Hendricks, J. K., Buyaner, D., Davis-Sproul, J., Chuang, L. C., Majumdar, M. K., Chopra, R., Barry, F., Murphy, M., Thiede, M. A., Junker, U., Rigg, R. J., Forestell, S. P., Böhnlein, E., Storb, R. and Sandmaier, B. M. (2000). Mesenchymal stem cells as vehicles for gene delivery. Clin Orthop Relat Res 379 Suppl, S71-90.

Montesano, R., Vassalli, J. D., Baird, A., Guillemin, R. and Orci, L. (1986). Basic fibroblast growth factor induces angiogenesis in vitro. Proc Natl Acad Sci USA 83, 7297-72301.

Morris, A. and Zvetkova, I. (1997). Cytokine research: the interferon paradigm. J Clin Pathol 50, 635-639.

Morrison, B.H., Bauer, J.A, Hu, J., Grane, R. W., Ozdemir, A. M., Chawla-Sarkar, M., Gong, B., Almasan, A., Kalvakolanu, D. V. and Lindner, D. J. (2002). Inositol hexakisphosphate kinase 2 sensitizes ovarian carcinoma cells to multiple cancer therapeutics. Oncogene 21, 1882-1889.

Mullikena, J. B. and Glowacki, J. (1982). Hemangiomas and vascular malformations in infants and children: a classification based on endothelial characteristics. Plast Reconstr Surg 69, 412-422.

Mulliken, J. B., Zetter, B. R. and Folkman, J. (1982). In vitro characteristics of endothelium from hemangiomas and vascular malformations. Surgery 92, 348-353. 
Mulliken, J. B. Vascular Birthmarks: Hemangiomas and Vascular Malformations. Philidelphia: W.B. Saunders (1988).

Mulliken, J. B., Boon, L. M., Takahashi, K., Ohlms, L. A., Folkman, J. and Ezekowitz, A. B. (1995). Pharmacological therapy for endangering hemangiomas. Current Opinion in Dermatol, 109-113.

Mulliken, J. B. Enjolras, O. (2004). Congenital hemangiomas and infantile hemangioma: missing links. J Am Acad Dermatol 6, 875-882.

Mustonen, T. and Alitalo, K. (1995). Endothelial receptor tyrosine kinases involved in angiogenesis. J Cell Biol 129, 895-898.

Nagaya, N., Fujii, T., Iwase, T., Ohgushi, H., Itoh, T., Uematsu, M., Yamagishi, M., Mori, H., Kangawam, K. and Kitamura, S. (2004). Intravenous administration of mesenchymal stem cells improves cardiac function in rats with acute myocardial infarction through angiogenesis and myogenesis. Am J Physiol Heart Circ Physiol 287, 670-676.

Naka, T., Sugamura, K., Hylander, B. L., Widmer, M. B., Rustum, Y. M. and Repasky, E. A. (2002). Effects of tumor necrosis factor-related apoptosis-inducing ligand alone and in combination with chemotherapeutic agents on patients' colon tumors grown in SCID mice. Cancer Res 62, 5800-5806.

Nakamura, H. (1988). Electron microscopic study of the prenatal development of the thoracic aorta in the rat. Am J Anat 181, 406-418.

Nakamura, T. (2000). Apoptosis and expression of Bax/Bcl-2 proteins in pyogenic granuloma: a comparative study with granulation tissue and capillary hemangioma. $\mathbf{J}$ Cutan Pathol 27, 400-405.

Nanbu-Wakao, R., Morikawa, Y., Matsumura, I., Masuho, Y., Muramatsu, M. A., Senba, E. and Wakao, H. (2002). Stimulation of 3T3-L1 adipogenesis by signal transducer and activator of transcription 5. Mol Endocrinol 16, 1565-1576.

Nehls, V. and Drenckhahn, D. (1991). Heterogeneity of microvascular pericytes for smooth muscle type alpha-actin. J Cell Biol 113, 147-154.

Neville-Webbe, H. L., Cross, N. A., Eaton, C. L., Nyambo, R., Evans, C. A., Coleman, R. E. and Holen, I. (2004). Osteoprotegerin (OPG) produced by bone marrow stromal cells protects breastcancer cells from TRAIL-induced apoptosis. Breast Cancer Res Treat 86, 269-279.

Neufeld, G., Cohen, T., Gengrinovitch, S. and Poltorak, Z. (1999). Vascular endothelial growth factor (VEGF) and its receptors. FASEB J 13, 9-22.

Nguyen, M., Strubel, N. A. and Bischoff, J. (1993). A role for sialyl Lewis-X/A glycoconjugates in capillary morphogenesis. Nature 365, 267-269.

Nguyen, V. A., Furhapter, C., Romani, N., Weber, F. and Sepp, N. (2004). Infantile hemangioma is a proliferation of beta 4-negative endothelial cells adjacent to HLA-DRpositive cells with dendritic cell morphology. Hum Pathol 35, 739-744. 
Nicosia, R. F. and Ottinetti, A. (1990). Growth of microvessels in serum-free matrix culture of rat aorta. A quantitative assay of angiogenesis in vitro. Lab Invest 63, 115122.

Niculescu, F., Soane, L., Badea, T., Shin, M. and Rus, H. (1999). Tyrosine phosphorylation and activation of Janus kinase 1 and STAT3 by sublytic C5b-9 complement complex in aortic endothelial cells. Immunopharmacol 42, 187-193.

Nielsen, M., Kaestel, C. G., Eriksen, K. W., Woetmann, A., Stokkedal, T., Kaltoft, K., Geisler, C., Ropke, C. and Odum, N. (1999). Inhibition of constitutively activated Stat3 correlates with altered Bcl-2/Bax expression and induction of apoptosis in mycosis fungoides tumor cells. Leukemia 13, 735-738.

Noden, D. M. (1988). Interactions and fates of avian craniofacial mesenchyme. Development 103, 121-140.

Noden, D. M. (1991). Cell movements and control of patterned tissue assembly during craniofacial development. J Craniofac Genet Dev Biol 11, 192-213.

Noonan, D. M. and Hassell, J. R. (1993). Perlecan, the large low-density proteoglycan of basement membranes: structure and variant forms. Kidney Int 43, 53-60.

Norrby, K., Jakobsson, A. and Sorbo, J. (1990). Quantitative angiogenesis in spreads of intact rat mesenteric windows. Microvasc Res 39, 341-348.

Norrby, K. (1994). Basic fibroblast growth factor and de novo mammalian angiogenesis. Microvasc Res 48, 96-113.

North, P. E., Waner, M., Mizeracki, A. and Mihm, M. C., Jr. (2000). GLUT1: a newly discovered immunohistochemical marker for juvenile hemangiomas. Hum Pathol 31, $11-22$.

North, P. E., Waner, M., Mizeracki, A., Mrak, R. E., Nicholas, R., Kincannon, J., Suen, J. Y. and Mihm, M. C., Jr. (2001). A unique microvascular phenotype shared by juvenile hemangiomas and human placenta. Arch Dermatol 137, 559-570.

North, P. E., Waner, M. and Brodsky, M. C. (2002). Are infantile hemangiomas of placental origin? Ophthalmology 109, 633-634.

Ogino, S. and Redline, R. W. (2000). Villous capillary lesions of the placenta: distinctions between chorangioma, chorangiomatosis, and chorangiosis. Hum Pathol 31, 945-954.

Ohlms, L. A., Jones, D. T., McGill, T. J. and Healy, G. B. (1994). Interferon alfa-2a therapy for airway hemangiomas. Ann Otol Rhinol Laryngol 103, 1-8.

Okuyama, H., Krishnamachary, B., Zhou, Y. F., Nagasawa, H., Bosch-Marce, M. and Semenza, GL. (2006).Expression of vascular endothelial growth factor receptor 1 in bone marrow-derived mesenchymal cells is dependent on hypoxia-inducible factor 1 . $\mathrm{J}$ Biol Chem 281, 15554-15563.

Onji, M. (1999). In what types of diseases does anti-carbonic anhydrase II antibodypositivity have clinical significance? J Gastroenterol 34, 431-432. 
Orlic, D., Kajstura, J., Chimenti, S., Bodine, D. M., Leri, A. and Anversa. P. (2001). Transplanted adult bone marrow cells repair myocardial infarcts in mice. Ann N Y Acad Sci 938, 221-229.

Orlidge, A. and D'Amore, P. A. (1987). Inhibition of capillary endothelial cell growth by pericytes and smooth muscle cells. J Cell Biol 105, 1455-1462.

Ortiga-Carvalho, T. M., Oliveira, K. J., Soares, B. A. and Pazos-Moura, C. C. 2002. The role of leptin in the regulation of TSH secretion in the fed state: in vivo and in vitro studies. Journal of Endocrinology 174,121-125.

Oshima, K., Yanase, N., Ibukiyama, C., Yamashina, A., Kayagaki, N., Yagita, H. and Mizuguchi, J. (2001). Involvement of TRAIL/TRAIL-R interaction in IFN-alphainduced apoptosis of Daudi B lymphoma cells. Cytokine 14, 193-201.

Ossina, N. K., Cannas, A., Powers, V. C., Fitzpatrick, P. A., Knight, J. D., Gilbert, J. R., Shekhtman, E. M., Tomei, L. D., Umansky, S. R. and Kiefer, M. C. (1997). Interferongamma modulates a p53-independent apoptotic pathway and apoptosis-related gene expression. J Biol Chem 272, 16351-16357.

Osugi T, Oshima Y, Fujio Y, Funamoto M, Yamashita A, Negoro S, Kunisada K, Izumi M, Nakaoka Y, Hirota H, Okabe M, Yamauchi-Takihara K, Kawase I, Kishimoto T. (2002). Cardiac-specific activation of signal transducer and activator of transcription 3 promotes vascular formation in the heart. J Biol Chem 277, 6676-6681.

Oswald, J., Boxberger, S., Jørgensen, B., Feldmann, S., Ehninger, G., Bornhäuser, M. and Werner, C. (2004). Mesenchymal stem cells can be differentiated into endothelial cells in vitro. Stem Cells 22, 377-384.

Pack, G. T and Miller, T.R. (1950). Hemangiomas; classification, diagnosis and treatment. Angiology 1, 405-426.

Pardridge, W. M., Boado, R. J. and Farrell, C. R. (1990). Brain-type glucose transporter (GLUT-1) is selectively localized to the blood-brain barrier. Studies with quantitative western blotting and in situ hybridization. J Biol Chem 265, 18035-18040.

Park, S., Tepper, O. M., Galiano, R. D., Capla, J. M., Baharestani, S., Kleinman, M. E., Pelo, C. R., Levine, J. P. and Gurtner, G. C. (2004). Selective recruitment of endothelial progenitor cells to ischemic tissues with increased neovascularization. Plast Reconstr Surg 113, 284-293

Parkkila, A. K., Herva, R., Parkkila, S. and Rajaniemi, H. (1995). Immunohistochemical demonstration of human carbonic anhydrase isoenzyme II in brain tumours. Histochem J 27, 974-982.

Paruchuri, S., Yang, J. H., Aikawa, E., Melero-Martin, J. M., Khan, Z. A., Loukogeorgakis, S., Schoen, F. J. and Bischoff, J.. Human pulmonary valve progenitor cells exhibit endothelial/mesenchymal plasticity in response to vascular endothelial growth factor-A and transforming growth factor-beta2. Circ Res 99, 861-869.

Pitti, R. M., Marsters, S. A., Ruppert, S., Donahue, C. J., Moore, A. and Ashkenazi, A. (1996). Induction of apoptosis by Apo-2 ligand, a new member of the tumor necrosis factor cytokine family. J Biol Chem 271, 12687-12690. 
Parums, D. V., Cordell, J. L., Micklem, K., Heryet, A. R., Gatter, K. C. and Mason, D. Y. (1990). JC70: a new monoclonal antibody that detects vascular endothelium associated antigen on routinely processed tissue sections. J Clin Pathol 43, 752-757.

Pasyk, K. A., Cherry, G. W., Grabb, W. C. and Sasaki, G. H. (1984). Quantitative evaluation of mast cells in cellularly dynamic and adynamic vascular malformations. Plast Reconstr Surg 73, 69-77.

Paweletz, N. and Knierim, M. (1989). Tumor-related angiogenesis. Crit Rev Oncol Hematol 9, 197-242.

Payne, M. M., Moyer, F., Marcks, K. M. and Trevaskis, A. E. (1966). The precursor to the hemangioma. Plast Reconstr Surg 38, 64-67.

Peichev, M., Naiyer, A. J, Pereira, D., Zhu, Z., Lane, W. J., Williams, M., Oz, M. C., Hicklin, D. J., Witte, L., Moore, M. A. and Rafii, S. (2000). Expression of VEGFR-2 and $\mathrm{AC} 133$ by circulating human $\mathrm{CD} 34(+)$ cells identifies a population of functional endothelial precursors. Blood 95, 952-958

Pepper, M. S., Ferrara, N., Orci, L. and Montesano, R. (1992). Potent synergism between vascular endothelial growth factor and basic fibroblast growth factor in the induction of angiogenesis in vitro. Biochem Biophys Res Commun 189, 824-831.

Peters, K. G., De Vries, C. and Williams, L. T. (1993). Vascular endothelial growth factor receptor expression during embryogenesis and tissue repair suggests a role in endothelial differentiation and blood vessel growth. Proc Natl Acad Sci U S A 90, 8915-8919.

Petersen, B. H., Rapaport, R., Henry, D. P., Huseman, C. and Moore, W. V. (1990). Effect of treatment with biosynthetic human growth hormone $(\mathrm{GH})$ on peripheral blood lymphocyte populations and function in growth hormone-deficient children. J Clin Endocrinol Metab 6, 1756-1760.

Phinney, D. G. (2002). Building a consensus regarding the nature and origin of mesenchymal stem cells. J Cell Biochem Suppl 38, 7-12.

Pinheiro, J. C and Bates, D. M. Mixed effects models in S and S-PLUS. New York: Springer publishers, 2000.

Pittenger, M. F., Mackay, A. M., Beck, S. C, Jaiswal, R. K., Douglas, R., Mosca, J. D., Moorman, M. A., Simonetti, D. W., Craig, S. and Marshak, D. R. (1999). Multilineage potential of adult human mesenchymal stem cells. Science 284, 143-147.

Pittenger, M. F and Martin, B. J. (2004). Mesenchymal stem cells and their potential as cardiac therapeutics. Circ Res 95, 9-20.

Plank, M. J and Sleeman, B. D. (2003). A reinforced random walk model of tumour angiogenesis and anti-angiogenic strategies. Math Med Biol 2, 35-81.

Plate, K. H., Breier, G., Weich, H. A., Mennel, H. D. and Risau, W.(1994). Vascular endothelial growth factor and glioma angiogenesis: coordinate induction of VEGF receptors, distribution of VEGF protein and possible in vivo regulatory mechanisms. Int J Cancer 59, 520-529. 
Planat-Benard, V., Silvestre, J. S., Cousin, B., André, M., Nibbelink, M., Tamarat, R. (2004) Plasticity of human adipose lineage cells toward endothelial cells: physiological and therapeutic perspectives. Circulation 109, 656-663.

Pollack, I. F., Erff, M. and Ashkenazi, A. (2001). Direct stimulation of apoptotic signaling by soluble Apo21/tumor necrosis factor-related apoptosis-inducing ligand leads to selective killing of glioma cells. Clin Cancer Res 7, 1362-1369.

Polverini, P. J. and Leibovich, S. J. (1984). Induction of neovascularization in vivo and endothelial proliferation in vitro by tumor-associated macrophages. Lab Invest 51, 635642.

Porstmann, T., Ternynck, T. and Avrameas, S. (1985). Quantitation of 5-bromo-2deoxyuridine incorporation into DNA: an enzyme immunoassay for the assessment of the lymphoid cell proliferative response. J Immunol Methods 82, 169-179.

Pratt, G. A. (1953). Birthmarks in infants. Arch. Dermatol 67: 302-305.

Price, P. A., June, H. H., Buckley, J. R. and Williamson, M. K. (2001). Osteoprotegerin inhibits artery calcification induced by warfarin and by vitamin D. Arterioscler Thromb Vasc Biol 21, 1610-1616.

Price, M. J., Chou, C. C., Frantzen, M., Miyamoto, T., Kar, S., Lee, S., Shah, P. K., Martin, B. J., Lill, M., Forrester, J. S., Chen, P. S. and Makkar, R. R. (2006). Intravenous mesenchymal stem cell therapy early after reperfused acute myocardial infarction improves left ventricular function and alters electrophysiologic properties. Int J Cardiol 111, 231-239.

Pritzker, L. B., Scatena, M. and Giachelli, C. M. (2004). The role of osteoprotegerin and tumor necrosis factor-related apoptosis-inducing ligand in human microvascular endothelial cell survival. Mol Biol Cell 15, 2834-2841.

Pulford, K., Ralfkiaer, E., MacDonald, S. M., Erber, W. N., Falini, B., Gatter, K. C. and Mason, D. Y. (1986). A new monoclonal antibody (KB61) recognizing a novel antigen which is selectively expressed on a subpopulation of human B lymphocytes. Immunology 57, 71-76.

Qu, Z., Liebler, J. M., Powers, M. R., Galey, T., Ahmadi, P., Huang, X. N., Ansel, J. C., Butterfield, J. H., Planck, S. R. and Rosenbaum, J. T. (1995). Mast cells are a major source of basic fibroblast growth factor in chronic inflammation and cutaneous hemangioma. Am J Pathol 147, 564-573.

Quinn, T. P., Peters, K. G., De Vries, C., Ferrara, N. and Williams, L. T. (1993). Fetal liver kinase 1 is a receptor for vascular endothelial growth factor and is selectively expressed in vascular endothelium. Proc Natl Acad Sci U S A 90, 7533-7537.

Quirici, N., Soligo, D., Caneva, L., Servida, F., Bossolasco, P. and Deliliers, G. L. (2001). Differentiation and expansion of endothelial cells from human bone marrow CD133(+) cells. Br J Haematol 115, 186-194.

Rafii, S., Meeus, S., Dias, S., Hattori, K., Heissig, B., Shmelkov, S., Rafii, D. and Lyden, D. (2002). Contribution of marrow-derived progenitors to vascular and cardiac regeneration. Semin Cell Dev Biol 13, 61-67. 
Rafii, S. and Lyden, D. (2003). Therapeutic stem and progenitor cell transplantation for organ vascularization and regeneration. Nat Med 9, 702-712.

Rafii, S., Avecilla, S., Shmelkov, S., Shido, K., Tejada, R., Moore, M. A., Heissig, B. and Hattori, K. (2003). Angiogenic factors reconstitute hematopoiesis by recruiting stem cells from bone marrow microenvironment. Ann N Y Acad Sci. 996, 49-60.

Ram, P. T., Horvath, C. M. and Iyengar, R. (2000). Stat3-mediated transformation of NIH-3T3 cells by the constitutively active Q205L Galphao protein. Science 287, 142144.

Ramp, U., Caliskan, E., Mahotka, C., Krieg, A., Heikaus, S., Gabbert, H. E. and Gerharz, CD. (2003). Apoptosis induction in renal cell carcinoma by TRAIL and gamma-radiation is impaired by deficient caspase-9 cleavage. $\mathrm{Br} \mathrm{J}$ Cancer 88, 18001807.

Ramsdell, A. F. and Markwald, R. R. (1997). Induction of endocardial cushion tissue in the avian heart is regulated, in part, by TGFbeta-3-mediated autocrine signaling. Dev Biol 188, 64-74.

Rasmussen, R. Quantification on the LightCycler. In Rapid Cycle Real-Time PCR, Methods and Applications. New york: Springer press, 2001

Ravi. R. and Bedi, A. (2002). Requirement of BAX for TRAIL/Apo2L-induced apoptosis of colorectal cancers: synergism with sulindac-mediated inhibition of Bclx(L). Cancer Res 62, 1583-1587.

Razon, M. J., Kraling, B. M., Mulliken, J. B. and Bischoff, J. (1998). Increased apoptosis coincides with onset of involution in infantile hemangioma. Microcirculation $5,189-195$.

Redonnet, A., Bonilla, S., Noël-Suberville, C., Pallet, V., Dabadie, H., Gin, H. and Higueret, P. (2002). Relationship between peroxisome proliferator-activated receptor gamma and retinoic acid receptor alpha gene expression in obese human adipose tissue. Int J Obes Relat Metab Disord 26, 920-927.

Reese, V., Frieden, I. J., Paller, A. S., Esterly, N. B., Ferriero, D., Levy, M. L., Lucky, A. W., Gellis, S. E. and Siegfried, E. C. (1993). Association of facial hemangiomas with Dandy-Walker and other posterior fossa malformations. J Pediatr 122, 379-384.

Reyes, M., Dudek, A., Jahagirdar, B., Koodie, L., Marker, P. H. and Verfaillie, C. M. (2002). Origin of endothelial progenitors in human postnatal bone marrow. J Clin Invest $109,337-346$.

Reyes, M., Lund, T., Lenvik, T., Aguiar, D., Koodie, L. and Verfaillie, C. M. (2001). Purification and ex vivo expansion of postnatal human marrow mesodermal progenitor cells. Blood 98, 2615-2625.

Rettig, W. J., Garin-Chesa, P., Healey, J. H., Su, S. L., Jaffe, E. A. and Old, L. J. (1992). Identification of endosialin, a cell surface glycoprotein of vascular endothelial cells in human cancer. Proc Natl Acad Sci U S A 89, 10832-10836. 
Ririe, K. M., Rasmussen, R. P. and Wittwer, C. T.(1997). Product differentiation by analysis of DNA melting curves during the polymerase chain reaction. Anal Biochem $245,154-160$.

Risau, W., Sariola, H., Zerwes, H. G., Sasse, J., Ekblom, P., Kemler, R. and Doetschman, T. (1988). Vasculogenesis and angiogenesis in embryonic-stem-cellderived embryoid bodies. Development 102, 471-478.

Risau, W. and Flamme, I. (1995). Vasculogenesis. Annu Rev Cell Dev Biol 11, 73-91.

Risau, W. (1997). Mechanisms of angiogenesis. Nature 386, 671-674.

Ritter, M. R., Dorrell, M. I., Edmonds, J., Friedlander, S. F. and Friedlander, M. (2002). Insulin-like growth factor 2 and potential regulators of hemangioma growth and involution identified by large-scale expression analysis. Proc Natl Acad Sci U S A 99, 7455-7460.

Ritter, M. R., Moreno, S. K., Dorrell, M. I., Rubens, J., Ney, J., Friedlander, D. F., Bergman, J., Cunningham, B. B., Eichenfield, L., Reinisch, J., Cohen, S., Veccione, T., Holmes, R., Friedlander, S. F. and Friedlander, M. (2003). Identifying potential regulators of infantile hemangioma progression through large-scale expression analysis: a possible role for the immune system and indoleamine 2,3 dioxygenase (IDO) during involution. Lymphat Res Biol 1, 291-9.

Ritter, M. R., Reinisch, J., Friedlander, S. F. and Friedlander, M. (2006). Myeloid cells in infantile hemangioma. Am J Pathol 168, 621-628.

Robb, L., Lyons, I., Li, R., Hartley, L., Köntgen, F., Harvey, R. P., Metcalf, D. and Begley, C. G. (1995). Absence of yolk sac hematopoiesis from mice with a targeted disruption of the scl gene. Proc Natl Acad Sci USA 92, 7075-7079.

Robb, L., Elwood, N. J., Elefanty, A. G., Köntgen, F., Li, R., Barnett, L. D. and Begley, C. G. (1996). The scl gene product is required for the generation of all hematopoietic lineages in the adult mouse. EMBO J 15, 4123-4129.

Rollins, B. J., Yoshimura, T., Leonard, E. J. and Pober, J. S. (1990). Cytokine-activated human endothelial cells synthesize and secrete a monocyte chemoattractant, MCP-1/JE. Am J Pathol 136, 1229-1233.

Rombouts, W. J. and Ploemacher, R. E. (2003). Primary murine MSC show highly efficient homing to the bone marrow but lose homing ability following culture. Leukemia 17, 160-170.

Rose'Meyer, R. B., Mellick, A. S., Garnham, B. G., Harrison, G. J., Massa, H. M. and Griffiths, L. R. (2003). The measurement of adenosine and estrogen receptor expression in rat brains following ovariectomy using quantitative PCR analysis. Brain Res Brain Res Protoc 11, 9-18.

Rosenblum, M. G., Yung, W. K., Kelleher, P. J., Ruzicka, F., Steck, P. A., Borden, E. C. (1990). Growth inhibitory effects of interferon-beta but not interferon-alpha on human glioma cells: correlation of receptor binding, 2',5'-oligoadenylate synthetase and protein kinase activity. J Interferon Res 10, 141-151. 
Ruger, B., Dunbar, P. R., Hasan, Q., Sawada, H., Kittelberger, R., Greenhill, N. and Neale, T. J. (1994). Human mast cells produce type VIII collagen in vivo. Int J Exp Pathol 75, 397-404.

Rutherford, M. S., Witsell, A. and Schook, L. B. (1993) Mechanisms generating functionally heterogenous macrophages: chaos revisted. J Leukoc Biol 53, 602-618.

Sadler, T. W. Langman's Medical Embryology. Baltimore: Williams \& Wilkins (1985).

Sado, Y., Kagawa, M., Naito, I., Ueki, Y., Seki, T., Momota, R., Oohashi, T. and Ninomiya, Y. (1998). Organization and expression of basement membrane collagen IV genes and their roles in human disorders. J Biochem 123, 767-776.

Sage, E. H. (1992). Secretion of SPARC by endothelial cells transformed by polyoma middle $\mathrm{T}$ oncogene inhibits the growth of normal endothelial cells in vitro. Biochem Cell Biol 70, 579-592.

Sahagun, G., Moore, S. A., Fabry, Z., Schelper, R. L. and Hart, M. N. (1989).Purification of murine endothelial cell cultures by flow cytometry using fluorescein-labeled griffonia simplicifolia agglutinin. Am J Pathol 134, 1227-1232.

Sahni, M., Ambrosetti, D. C., Mansukhani, A., Gertner, R., Levy, D. and Basilico, C. (1999). FGF signaling inhibits chondrocyte proliferation and regulates bone development through the STAT-1 pathway. Genes Dev 13, 1361-1366.

Saito, T., Kuang, J. Q., Bittira, B., Al-Khaldi, A. and Chiu, R. C. (2002). Xenotransplant cardiac chimera: immune tolerance of adult stem cells. Ann Thorac Surg 74, 19-24.

Salvesen, G. S. (2002). Caspases and apoptosis. Essays Biochem 38, 9-19.

Sano, S., Itami, S., Takeda, K., Tarutani, M., Yamaguchi, Y., Miura, H., Yoshikawa, K., Akira, S. and Takeda, J. (1999). Keratinocyte-specific ablation of Stat3 exhibits impaired skin remodeling, but does not affect skin morphogenesis. Embo J 18, 46574668.

Sandra, F., Hendarmin, L., Nakao, Y., Nakamura, N. and Nakamura, S. (2005). TRAIL cleaves caspase- $8,-9$ and -3 of AM-1 cells: a possible pathway for TRAIL to induce apoptosis in ameloblastoma. Tumour Biol 26, 258-264.

Sato, T. N., Tozawa, Y., Deutsch, U., Wolburg-Buchholz, K., Fujiwara, Y., GendronMaguire, M., Gridley, T., Wolburg, H., Risau, W. and Qin, Y. (1995). Distinct roles of the receptor tyrosine kinases Tie-1 and Tie-2 in blood vessel formation. Nature 376, 7074.

Sato, K., Hida, S., Takayanagi, H., Yokochi, T., Kayagaki, N., Takeda, K., Yagita, H., Okumura, K., Tanaka, N., Taniguchi, T. and Ogasawara, K. (2001). Antiviral response by natural killer cells through TRAIL gene induction by IFN-alpha/beta. Eur J Immunol 31, 3138-3146.

Schmid, H., Cohen, C. D., Henger, A., Irrgang, S., Schlöndorff, D. and Kretzler, M. (2003). Validation of endogenous controls for gene expression analysis in microdissected human renal biopsies. Kidney Int 1, 356-360. 
Schindler, C., Shuai, K., Prezioso, V. R. and Darnell, J. E., Jr. (1992). Interferondependent tyrosine phosphorylation of a latent cytoplasmic transcription factor. Science 257, 809-13.

Schmittgen, T. D. and Zakrajsek, B. A. (2000). Effect of experimental treatment on housekeeping gene expression: validation by real-time, quantitative RT-PCR. J Biochem Biophys Methods 46, 69-81.

Schoen, F. J. and Cotran, R. S. Pathologic Basis of Disease. Philidelphia: WB Saunders Co (1999).

Schwartz, L. B. (1994). Mast cells: function and contents. Curr Opin Immunol 6, 91-97.

Sedmak, D. D., Davis, D. H., Singh, U., van de Winkel, J. G. and Anderson, C. L. (1991). Expression of IgG Fc receptor antigens in placenta and on endothelial cells in humans. An immunohistochemical study. Am J Pathol 138, 175-181.

Seki, Y., Kai, H., Shibata, R., Nagata, T., Yasukawa, H., Yoshimura, A. and Imaizumi, T. (2000). Role of the JAK/STAT pathway in rat carotid artery remodeling after vascular injury. Circ Res 87, 12-18.

Seko, Y., Fujimura, T., Taka, H., Mineki, R., Murayama, K. and Nagai, R. (2004). Hypoxia followed by reoxygenation induces secretion of cyclophilin A from cultured rat cardiac myocytes. Biochem Biophys Res Commun 317, 162-168.

Selvey, S., Thompson, E. W., Matthaei, K., Lea, R. A., Irving, M. G. and Griffiths, L. R. (2001). Beta-actin--an unsuitable internal control for RT-PCR. Mol Cell Probes 15, 307-311.

Sers, C., Riethmuller, G. and Johnson, J. P. (1994). MUC18, a melanoma-progression associated molecule, and its potential role in tumor vascularization and hematogenous spread. Cancer Res 54, 5689-5694.

Sewry, C. A., Philpot, J., Sorokin, L. M., Wilson, L. A., Naom, I., Goodwin, F., D'Alessandro, M., Dubowitz, V. and Muntoni, F. (1996). Diagnosis of merosin (laminin-2) deficient congenital muscular dystrophy by skin biopsy. Lancet 347, 582584.

Shalaby, F., Rossant, J., Yamaguchi, T. P., Gertsenstein, M., Wu, X. F., Breitman, M. L. and Schuh, A. C. (1995). Failure of blood-island formation and vasculogenesis in Flk-1-deficient mice. Nature 376, 62-66.

Sheir, D., Butler, J and Lewis, R. Hole's Human Anatomy. Boston: McGraw Hill (2004).

Sherry, B., Yarlett, N., Strupp, A. and Cerami, A. (1992). Identification of cyclophilin as a proinflammatory secretory product of lipopolysaccharide-activated macrophages. Proc Natl Acad Sci USA 89, 3511-3515.

Shi, Q., Rafii, S., Wu, M. H., Wijelath, E. S., Yu, C., Ishida, A., Fujita, Y., Kothari, S., Mohle, R., Sauvage, L. R., Moore, M. A., Storb, R. F. and Hammond, W. P.(1998). Evidence for circulating bone marrow-derived endothelial cells. Blood 92, 362-367. 
Shi, X., Zhang, H., Paddon, H., Lee, G., Cao, X. and Pelech, S. (2002). Phosphorylation of STAT3 serine-727 by cyclin-dependent kinase 1 is critical for nocodazole-induced mitotic arrest. Biochemistry 45, 5857-5867.

Shigeno, M., Nakao, K., Ichikawa, T., Suzuki, K., Kawakami, A., Abiru, S., Miyazoe, S., Nakagawa, Y., Ishikawa, H., Hamasaki, K., Nakata, K., Ishii, N. and Eguchi, K. (2003). Interferon-alpha sensitizes human hepatoma cells to TRAIL-induced apoptosis through DR5 upregulation and NF-kappa B inactivation. Oncogene 22, 1653-1662.

Shih, I. M. and Kurman, R. J. (1996). Expression of melanoma cell adhesion molecule in intermediate trophoblast. Lab Invest 75, 377-388.

Shipman, C. M. and Croucher, P. I. (2003). Osteoprotegerin is a soluble decoy receptor for tumor necrosis factor-related apoptosis-inducing ligand/Apo2 ligand and can function as a paracrine survival factor for human myeloma cells. Cancer Res. 2003 Mar 1;63(5):912-916.

Shivapurkar, N., Toyooka, S., Toyooka, K. O., Reddy, J., Miyajima, K., Suzuki, M., Shigematsu, H., Takahashi, T., Parikh, G., Pass, H. I., Chaudhary, P. M. and Gazdar, A. F. (2004). Aberrant methylation of trail decoy receptor genes is frequent in multiple tumor types. Int J Cancer 109, 786-792.

Simonet, W. S., Lacey, D. L., Dunstan, C. R., Kelley, M., Chang, M. S., Lüthy, R., Nguyen, H. Q., Wooden, S., Bennett, L., Boone, T., Shimamoto, G., DeRose, M., Elliott, R., Colombero, A., Tan, H. L.,Trail, G., Sullivan, J., Davy, E., Bucay, N., Renshaw-Gegg, L., Hughes, T. M., Hill, D., Pattison, W., Campbell, P., Sander, S., Van, G., Tarpley, J., Derby, P., Lee, R. and Boyle, W. J. (1997). Osteoprotegerin: a novel secreted protein involved in the regulation of bone Density. Cell 89, 309-319.

Silva, G. V., Perin, E. C., Dohmann, H. F., Borojevic, R., Silva, S. A., Sousa, A. L., Assad, J. A., Vaughn, W. K., Mesquita, C. T., Belém, L., Carvalho, A. C., Dohmann, H. J., Barroso do Amaral, E., Coutinho, J., Branco, R., Oliveira, E. and Willerson, J. T. (2004). Catheter-based transendocardial delivery of autologous bone-marrow-derived mononuclear cells in patients listed for heart transplantation. Tex Heart Inst J 31, 214219.

Simpson, J. R. (1959). Natural history of cavernous haemangiomata. Lancet 2, 10571059.

Sinclair, A. M., Göttgens, B., Barton, L. M., Stanley, M. L., Pardanaud, L., Klaine, M., Gering, M., Bahn, S., Sanchez, M., Bench, A. J., Fordham, J. L., Bockamp, E. and Green, A. R. (1999). Distinct 5' SCL enhancers direct transcription to developing brain, spinal cord, and endothelium: neural expression is mediated by GATA factor binding sites. Dev Biol 209, 128-142.

Smyth, M. J., Takeda, K., Hayakawa, Y., Peschon, J. J., van den Brink, M. R. and Yagita, H. (2003). Nature's TRAIL--on a path to cancer immunotherapy. Immunity 18, $1-6$.

Song, J. I. and Grandis, J. R. STAT signaling in head and neck cancer. (2000). Oncogene 19, 2489-2495. 
Spanaus, K. S., Schlapbach, R. and Fontana, A. (1998). TNF-alpha and IFN-gamma render microglia sensitive to Fas ligand-induced apoptosis by induction of Fas expression and down-regulation of Bcl-2 and Bcl-xL. Eur J Immunol 28, 4398-4408.

Spierings, D. C., de Vries, E. G., Vellenga, E., van den Heuvel, F. A., Koornstra, J. J., Wesseling, J., Hollema, H. and de Jong, S. (2004). Tissue distribution of the death ligand TRAIL and its receptors. J Histochem Cytochem 52, 821-831.

Spiller, J. C., Sharma, V., Woods, G. M., Hall, J. C. and Seidel, F. G. (1992). Diffuse neonatal hemangiomatosis treated successfully with interferon alfa-2a. J Am Acad Dermatol 27, 102-104.

Sprick, M. R., Weigand, M. A., Rieser, E., Rauch, C. T., Juo, P., Blenis, J., Krammer, P. H. and Walczak, H. (2000). FADD/MORT1 and caspase- 8 are recruited to TRAIL receptors 1 and 2 and are essential for apoptosis mediated by TRAIL receptor 2 . Immunity 12, 599-609.

Stark, G. R., Kerr, I. M., Williams, B. R., Silverman, R. H. and Schreiber, R. D. (1998). How cells respond to interferons. Annu Rev Biochem 67, 227-264.

Stephanou, A. and Latchman, D. S. (2003). STAT-1: a novel regulator of apoptosis. Int J Exp Pathol 84, 239-244.

Strawn, L. M., McMahon, G., App, H., Schreck, R., Kuchler, W. R., Longhi, M. P., Hui, T. H., Tang, C., Levitzki, A., Gazit, A., Chen, I., Keri, G., Orfi, L., Risau, W., Flamme, I., Ullrich, A., Hirth, K. P. and Shawver, L. K. (1996). Flk-1 as a target for tumor growth inhibition. Cancer Res 56, 3540-3545.

Stein, I., Itin, A., Einat, P., Skaliter, R., Grossman, Z. and Keshet, E. (1998). Translation of vascular endothelial growth factor mRNA by internal ribosome entry: implications for translation under hypoxia. Mol Cell Biol 18, 3112-3119.

Stephens, J. M., Morrison, R. F. and Pilch, P. F. (1996). The expression and regulation of STATs during 3T3-L1 adipocyte differentiation. J Biol Chem 271, 10441-10444.

Stephens, J. M., Morrison, R. F., Wu, Z. and Farmer, S. R. (1999). PPARgamma liganddependent induction of STAT1, STAT5A, and STAT5B during adipogenesis. Biochem Biophys Res Commun 262, 216-222.

Su, W. C., Kitagawa, M., Xue, N., Xie, B., Garofalo, S., Cho, J., Deng, C., Horton, W. A. and Fu, X. Y. (1997). Activation of Stat1 by mutant fibroblast growth-factor receptor in thanatophoric dysplasia type II dwarfism. Nature 386, 288-292.

Sunderkotter, C., Steinbrink, K., Goebeler, M., Bhardwaj, R. and Sorg, C. (1994). Macrophages and angiogenesis. J Leukoc Biol 55, 410-422.

Supuran, C. T. and Scozzafava, A. (2005). Carbonic anhydrase inhibitors--Part 94. 1,3,4-thiadiazole-2-sulfonamidederivatives as antitumor agents? Eur J Med Chem 35, 867-874

Suri, C., Jones, P. F., Patan, S., Bartunkova, S., Maisonpierre, P. C., Davis, S., Sato, T. N. and Yancopoulos, G. D. (1996). Requisite role of angiopoietin-1, a ligand for the TIE2 receptor, during embryonic angiogenesis. Cell 87, 1171-1180. 
Sutherland, F. W., Perry, T. E., Yu, Y., Sherwood, M. C., Rabkin, E., Masuda, Y., Garcia, G. A., McLellan, D. L., Engelmayr, G. C., Sacks, M. S., Schoen, F. J., Mayer, J. E. Jr. (2005). From stem cells to viable autologous semilunar heart valve. Circulation $111,2783-2791$.

Takahashi, K., Mulliken, J. B., Kozakewich, H. P., Rogers, R. A., Folkman, J. and Ezekowitz, R. A. (1994). Cellular markers that distinguish the phases of hemangioma during infancy and childhood. J Clin Invest 93, 2357-2364.

Takahashi, T. and Shibuya, M. (1997). The $230 \mathrm{kDa}$ mature form of KDR/Flk-1 (VEGF receptor-2) activates the PLC- $\gamma$ pathway and partially induces mitotic signals in NIH3T3 fibroblasts. Oncogene 14, 2079-2089.

Takeda, K., Noguchi, K., Shi, W., Tanaka, T., Matsumoto, M., Yoshida, N., Kishimoto, T. and Akira, S. (1997). Targeted disruption of the mouse Stat3 gene leads to early embryonic lethality. Proc Natl Acad Sci USA 94, 3801-3804.

Takigawa, M., Nishida, Y., Suzuki, F., Kishi, J., Yamashita, K. and Hayakawa, T. (1990). Induction of angiogenesis in chick yolk-sac membrane by polyamines and its inhibition by tissue inhibitors of metalloproteinases (TIMP and TIMP-2). Biochem Biophys Res Commun 171, 1264-1271.

$\operatorname{Tan}_{\mathrm{a}}$ ST, Velickovic M, Ruger BM, Davis PF. (2000). Cellular and extracellular markers of hemangioma. Plast Reconstr Surg 3, 529-538.

$\mathrm{Tan}_{\mathrm{b}}$, S. T., Hasan, Q., Velickovic, M., Ruger, B. M., Davis, R. P. and Davis, P. F. (2000). A novel in vitro human model of hemangioma. Mod Pathol 13, 92-99.

Tan S. T. (2001). Phd Thesis.

Tan, S. T., Wallis, R. A., He, Y. and Davis, P. F. (2004). Mast cells and hemangioma. Plast Reconstr Surg 113, 999-1011.

Tang, Y. L., Zhao, Q., Zhang, Y. C., Cheng, L., Liu, M., Shi, J., Yang, Y. Z., Pan, C., Ge, J., Phillips, M. I. (2004). Autologous mesenchymal stem cell transplantation induce VEGF and neovascularization in ischemic myocardium. Regul Pept 117, 3-10.

Tang, L., Lin, Z. and Li, Y. M. (2006). Effects of different magnitudes of mechanical strain on Osteoblasts in vitro. Biochem Biophys Res Commun 344, 122-128.

Tenniswood, M. P., Guenette, R. S., Lakins, J., Mooibroek, M., Wong, P. and Welsh, J. E. (1992). Active cell death in hormone-dependent tissues. Cancer Metastasis Rev 11, 197-220.

Tepper, O. M., Capla, J. M., Galiano, R. D., Ceradini, D. J., Callaghan, M. J., Kleinman, M. E. and Gurtner, G. C. (2005). Adult vasculogenesis occurs through in situ recruitment, proliferation, and tubulization of circulating bone marrow-derived cells. Blood 105, 1068-1077.

Tepper, O. M., Sealove, B. A., Murayama, T. and Asahara, T. (2003). Newly emerging concepts in blood vessel growth: recent discovery of endothelial progenitor cells and their function in tissue regeneration. J Investig Med 51, 353-359. 
Thakral, K. K., Goodson, W. H., 3rd and Hunt, T. K. (1979). Stimulation of wound blood vessel growth by wound macrophages. J Surg Res 26, 430-436.

Thellin, O., Zorzi, W., Lakaye, B., De Borman, B., Coumans, B., Hennen, G., Grisar, T., Igout, A. and Heinen, E. (1999). Housekeeping genes as internal standards: use and limits. J Biotechnol 75, 291-295.

Tilton $_{\mathrm{a}}$, R. G., Kilo, C. and Williamson, J. R. (1979). Pericyte-endothelial relationships in cardiac and skeletal muscle capillaries. Microvasc Res 18, 325-335.

Tilton $_{\mathrm{b}}$, R. G., Kilo, C., Williamson, J. R. and Murch, D. W. (1979). Differences in pericyte contractile function in rat cardiac and skeletal muscle microvasculatures. Microvasc Res 18, 336-352.

Todaro, G. J. and Green, H. (1963). Quantitative studies of the growth of mouse embryo cells in culture and their development into established lines. J Cell Biol 17, 299-313.

Tomanek, R. J. and Schatteman, G. C. (2000). Angiogenesis: new insights and therapeutic potential. Anat Rec 261, 126-135.

Tong, Q., Zheng, L., Lin, L., Li, B., Wang, D., Huang, C. and Li, D. (2006). VEGF is upregulated by hypoxia-induced mitogenic factor via the PI-3K/Akt-NF-kappaB signaling pathway. Respir Res 7, 37.

Totzke, G., Schulze-Osthoff, K. and Jänicke, R. U. (2003). Cyclooxygenase-2 (COX-2) inhibitors sensitize tumor cells specifically to death receptor-induced apoptosis independently of COX-2 inhibition. Oncogene 22, 8021-8030.

Tropel, P., Noël, D., Platet, N., Legrand, P., Benabid, A. L. and Berger, F. (2004). Isolation and characterisation of mesenchymal stem cells from adult mouse bone marrow. Exp Cell Res 295, 395-406.

Tschopp, J., Irmler, M. and Thome, M. (1998). Inhibition of fas death signals by FLIPs. Curr Opin Immunol 10, 552-558.

Tsuda, E., Goto, M., Mochizuki, S., Yano, K., Kobayashi, F., Morinaga, T. and Higashio, K. (1997). Isolation of a novel cytokine from human fibroblasts that specifically inhibits osteoclastogenesis. Biochem Biophys Res Commun 234, 137-142.

Tu, W., Cheung, P. T. and Lau, Y. L. (1999). IGF-I increases interferon-gamma and IL6 mRNA expression and protein production in neonatal mononuclear cells. Pediatr Res $46,748-754$.

Tuan, R. S., Boland, G. and Tuli, R. (2003). Adult mesenchymal stem cells and cellbased tissue engineering. Arthritis Res Ther 5, 32-45.

Turkson, J., Bowman, T., Garcia, R., Caldenhoven, E., De Groot, R. P. and Jove, R. (1998). Stat3 activation by Src induces specific gene regulation and is required for cell transformation. Mol Cell Biol 18, 2545-2552.

Vachon, P. H., Loechel, F., Xu, H., Wewer, U. M. and Engvall, E. (1996). Merosin and laminin in myogenesis; specific requirement for merosin in myotube stability and survival. J Cell Biol 134, 1483-1497. 
Valdembri D, Serini G, Vacca A, Ribatti D, Bussolino F. (2002). In vivo activation of JAK2/STAT-3 pathway during angiogenesis induced by GM-CSF. FASEB J 16, 225227.

Vandesompele, J., De Preter, K., Pattyn, F., Poppe, B., Van Roy, N., De Paepe, A. and Speleman, F.. (2002). Accurate normalization of real-time quantitative RT-PCR data by geometric averaging of multiple internal control genes. Genome Biol 3, 34.

Van Noesel, M. M., van Bezouw, S., Salomons, G. S., Voûte, P. A., Pieters, R., Baylin, S. B., Herman, J. G. and Versteeg, R. (2002). Tumor-specific down-regulation of the tumor necrosis factor-related apoptosis-inducing ligand decoy receptors DcR1 and DcR2 is associated with dense promoter hypermethylation. Cancer Res 62, 2157-2161.

Veikkola, T. and Alitalo, K. (1999). VEGFs, receptors and angiogenesis. Semin Cancer Biol 9, 211-220.

Veikkola, T., Karkkainen, M., Claesson-Welsh, L. and Alitalo, K. (2000). Regulation of angiogenesis via vascular endothelial growth factor receptors. Cancer Res 60, 203-212.

Veikkola, T. and Alitalo, K. (1999). VEGFs, receptors and angiogenesis. Semin Cancer Biol 9, 211-220.

Vial, T. and Descotes, J. (1994). Clinical toxicity of the interferons. Drug Saf 10, 115150.

Vindrieux, D., Devonec, M., Benahmed, M. and Grataroli, R. (2002). Identification of tumor necrosis factor-alpha-related apoptosis-inducing ligand (TRAIL) and its receptors in adult rat ventral prostate. Mol Cell Endocrinol 198, 115-121.

Vikkula, M., Boon, L. M., Mulliken, J. B. and Olsen, B. R. (1998). Molecular basis of vascular anomalies. Trends Cardiovasc Med 8, 281-292.

Villanova, M., Malandrini, A., Toti, P., Salvestroni, R., Six, J., Martin, J. J. and Guazzi, G. C. (1996). Localization of merosin in the normal human brain: implications for congenital muscular dystrophy with merosin deficiency. J Submicrosc Cytol Pathol 28, $1-4$.

Villars, F., Bordenave, L., Bareille, R. and Amédée, J. (2000). Effect of human endothelial cells on human bone marrow stromal cell phenotype: role of VEGF? J Cell Biochem 79, 672-685.

Vittet, D., Prandini, M. H., Berthier, R., Schweitzer, A., Martin-Sisteron, H., Uzan, G. and Dejana, E. (1996). Embryonic stem cells differentiate in vitro to endothelial cells through successive maturation steps. Blood 88, 3424-3431.

Voit, T., Fardeau, M. and Tome, F. M. (1994). Prenatal detection of merosin expression in human placenta. Neuropediatrics 25, 332-333.

Volpert, O., Jackson, D., Bouck, N. and Linzer, D. I. (1996). The insulin-like growth factor II/mannose 6-phosphate receptor is required for proliferin-induced angiogenesis. Endocrinology 137, 3871-3876.

Wakabayashi, M., Shiro, T., Seki, T., Nakagawa, T., Itoh, T., Imamura, M., Shiozaki, Y., Inoue, K. and Okamura, A. (1995). Lewis Y antigen expression in hepatocellular carcinoma. An immunohistochemical study. Cancer 75, 2827-2835. 
Walter, J. W., Blei, F., Anderson, J. L., Orlow, S. J., Speer, M. C. and Marchuk, D. A. (1999). Genetic mapping of a novel familial form of infantile hemangioma. Am J Med Genet 82, 77-83.

Walter, J. W., North, P. E., Waner, M., Mizeracki, A., Blei, F., Walker, J. W., Reinisch, J. F. and Marchuk, D. A. (2002). Somatic mutation of vascular endothelial growth factor receptors in juvenile hemangioma. Genes Chromosomes Cancer 33, 295-303.

Walczak, H., Degli-Esposti, M. A., Johnson, R. S., Smolak, P. J., Waugh, J. Y., Boiani, N., Timour, M. S., Gerhart, M. J., Schooley, K. A., Smith, C. A., Goodwin, R. G. and Rauch, C. T. (1997). TRAIL-R2: a novel apoptosis-mediating receptor for TRAIL. EMBO J 16, 5386-5397.

Walczak, H., Miller, R. E., Ariail, K., Gliniak, B., Griffith, T. S., Kubin, M., Chin, W., Jones, J., Woodward, A., Le, T., Smith, C., Smolak, P., Goodwin, R. G., Rauch, C. T., Schuh, J. C. and Lynch, D. H. (1999). Tumoricidal activity of tumor necrosis factorrelated apoptosis-inducing ligand in vivo. Nat Med 5, 157-163.

Walczak, H., Bouchon, A., Stahl, H. and Krammer, P. H. (2000). Tumor necrosis factor-related apoptosis-inducing ligand retains its apoptosis-inducing capacity on Bcl2- or Bcl-xL-overexpressing chemotherapy-resistant tumor cells. Cancer Res 60, 30513057.

Waterman, E. A., Cross, N. A., Lippitt, J. M., Cross, S. S., Rehman, I., Holen, I., Hamdy, F. C. and Eaton, C. L. (2007). The antibody MAB8051 directed against osteoprotegerin detects carbonic anhydrase II: implications for association studies with human cancers. Int J Cancer 121, 1958-1966.

Waner, M., North, P. E., Scherer, K. A., Frieden, I. J., Waner, A. and Mihm, M. C. Jr. (2003). The nonrandom distribution of facial hemangiomas. Arch Dermatol 7, 869-875.

Wang, B., Zhuang, F. L., Zhang, P. F., Zhang, S. and Lin, H. (2003). Expression of apoptosis and Bcl-2, Bax in hemangioma and vascular malformations. Zhonghua Zheng Xing Wai Ke Za Zhi 19, 347-349.

Wang, H., Zhang, Y., Toratani, S. and Okamoto, T. (2004). Transformation of vascular endothelial cells by a point mutation in the Tie2 gene from human intramuscular haemangioma. Oncogene 23, 8700-8704

Wang, Y., Barbacioru, C., Hyland, F., Xiao, W., Hunkapiller, K. L., Blake, J., Chan, F., Gonzalez, C., Zhang, L. and Samaha, R. R. (2006). Large scale real-time PCR validation on gene expression measurements from two commercial long-oligonucleotide microarrays. BMC Genomics 7, 59.

Wen, J., Ramadevi, N., Nguyen, D., Perkins, C., Worthington, E. and Bhalla, K. (2000). Antileukemic drugs increase death receptor 5 levels and enhance Apo-2L-induced apoptosis of human acute leukemia cells. Blood 96, 3900-3906.

White, C. W., Wolf, S. J., Korones, D. N., Sondheimer, H. M., Tosi, M. F. and Yu, A. (1991). Treatment of childhood angiomatous diseases with recombinant interferon alfa2a. J Pediatr 118, 59-66.

Williams, R. L., Risau, W., Zerwes, H. G., Drexler, H., Aguzzi, A. and Wagner, E. F. (1989). Endothelioma cells expressing the polyoma middle $\mathrm{T}$ oncogene induce hemangiomas by host cell recruitment. Cell 57, 1053-1063. 
Witzenbichler, B., Maisonpierre, P. C., Jones, P., Yancopoulos, G. D. and Isner, J. M. (1998). Chemotactic properties of angiopoietin-1 and -2, ligands for the endothelialspecific receptor tyrosine kinase Tie2. J Biol Chem 273, 18514-18521.

Wong, P., Pineault, J., Lakins, J., Taillefer, D., Leger, J., Wang, C. and Tenniswood, M. (1993). Genomic organization and expression of the rat TRPM-2 (clusterin) gene, a gene implicated in apoptosis. J Biol Chem 268, 5021-5031.

Wong, P., Taillefer, D., Lakins, J., Pineault, J., Chader, G. and Tenniswood, M. (1994). Molecular characterization of human TRPM-2/clusterin, a gene associated with sperm maturation, apoptosis and neurodegeneration. Eur J Biochem 221, 917-925.

Worle, H., Maass, E., Kohler, B. and Treuner, J. (1999). Interferon alpha-2a therapy in haemangiomas of infancy: spastic diplegia as a severe complication. Eur J Pediatr 158, 344.

Wu, R., Sun, S. and Steinberg, BS. (2003). Requirement of STAT3 Activation for Differentiation of Mucosal Stratified Squamous Epithelium. Mol Med 9, 77-84.

Wu, X., Rabkin-Aikawa, E., Guleserian, K, J., Perry, T. E., Masuda, Y., Sutherland, F. W., Schoen, F. J., Mayer, J. E. Jr. and Bischoff, J. (2004). Tissue-engineered microvessels on three-dimensional biodegradable scaffolds using human endothelial progenitor cells. Am J Physiol Heart Circ Physiol 287, H480-487.

Wu, X., Huang, L., Zhou, Q., Song, Y., Li, A., Jin, J. and Cui B.(2005). Mesenchymal stem cells participating in ex vivo endothelium repair and its effect on vascular smooth muscle cells growth. Int J Cardiol 105, 274-282.

Xu, Q., Leiva, M. C., Fischkoff, S. A., Handschumacher, R. E. and Lyttle, C. R. (1992). Leukocyte chemotactic activity of cyclophilin. J Biol Chem 267, 11968-11971.

Xu, Z. W., Kleeff, J., Friess, H., Büchler, M. W. and Solioz, M. (2003). Synergistic cytotoxic effect of TRAIL and gemcitabine in pancreatic cancer cells. Anticancer Res 23, 251-258.

Yahata, Y., Shirakata, Y., Tokumaru, S., Yamasaki, K., Sayama, K., Hanakawa, Y., Detmar, M. and Hashimoto, K. (2003). Nuclear translocation of phosphorylated STAT3 is essential for vascular endothelial growth factor-induced human dermal microvascular endothelial cell migration and tube formation. J Biol Chem 278, 40026-40031.

Yamamoto, T., Kita, M., Kimura, I., Oseko, F., Terauchi, R., Takahashi, K., Kubo, T. and Kanamura, N. (2006). Mechanical stress induces expression of cytokines in human periodontal ligament cells. Oral Dis 12, 171-175.

Yamashita, J., Itoh, H., Hirashima, M., Ogawa, M., Nishikawa, S., Yurugi, T., Naito, M., Nakao, K. and Nishikawa, S. (2000).Flk1-positive cells derived from embryonic stem cells serve as vascular progenitors. Nature 408, 92-96.

Yamaura, G., Turoczi, T., Yamamoto, F., Siddqui, M. A., Maulik, N. and Das, D. K. (2003). STAT signaling in ischemic heart: a role of STAT5A in ischemic preconditioning. Am J Physiol Heart Circ Physiol 285, H476-H482.

Yang, H., Li, M., Chai, H., Yan, S., Lin, P., Lumsden, A. B., Yao, Q. and Chen, C. (2005). Effects of cyclophilin A on cell proliferation and gene expressions in human vascular smooth muscle cells and endothelial cells. J Surg Res 123, 312-319. 
Yang, J., Chatterjee-Kishore, M., Staugaitis, S. M., Nguyen, H., Schlessinger, K., Levy, D. E. and Stark, G. R. (2005). Novel roles of unphosphorylated STAT3 in oncogenesis and transcriptional regulation. Cancer Res 65, 939-947.

Yasuda, H., Shima, N., Nakagawa, N., Yamaguchi, K., Kinosaki, M., Mochizuki, S., Tomoyasu, A., Yano, K., Goto, M., Murakami, A., Tsuda, E., Morinaga, T., Higashio, K., Udagawa, N., Takahashi, N. and Suda, T. (1998). Osteoclast differentiation factor is a ligand for osteoprotegerin/osteoclastogenesis-inhibitory factor and is identical to TRANCE/RANKL. Proc Natl Acad Sci USA 95, 3597-3602.

Yin, J. L., Shackel, N. A., Zekry, A., McGuinness, P. H., Richards, C., Putten, K. V., McCaughan, G. W., Eris, J. M. and Bishop, G. A. (2001). Real-time reverse transcriptase-polymerase chain reaction (RT-PCR) for measurement of cytokine and growth factor mRNA expression with fluorogenic probes or SYBR Green I. Immunol Cell Biol 79, 213-221.

Yoshimura, T., Yuhki, N., Moore, S. K., Appella, E., Lerman, M. I. and Leonard, E. J. (1989). Human monocyte chemoattractant protein-1 (MCP-1). Full-length cDNA cloning, expression in mitogen-stimulated blood mononuclear leukocytes, and sequence similarity to mouse competence gene JE. FEBS Lett 244, 487-493.

Yoshiura, K., Nakaoka, T., Nishishita, T., Sato, K., Yamamoto, A., Shimada, S., Saida, T., Kawakami, Y., Takahashi, T. A., Fukuda, H., Imajoh-Ohmi, S., Oyaizu, N. and Yamashita, N. (2005). Carbonic anhydrase II is a tumor vessel endothelium-associated antigen targeted by dendritic cell therapy. Clin Cancer Res 11, 8201-8207.

Yu, Y., Varughese, J., Brown, L. F., Mulliken, J. B. and Bischoff, J. (2001). Increased Tie2 expression, enhanced response to angiopoietin-1, and dysregulated angiopoietin-2 expression in hemangioma-derived endothelial cells. Am J Pathol 159, 2271-2280.

Yu, Y., Flint, A. F., Mulliken, J. B., Wu, J. K. and Bischoff, J. (2004a). Endothelial progenitor cells in infantile hemangioma. Blood 103, 1373-1375.

Yu, Y., Wylie-Sears, J., Boscolo, E., Mulliken, J. B. and Bischoff, J. (2004b). Genomic imprinting of IGF2 is maintained in infantile hemangioma despite its high level of expression. Mol Med 10, 117-123

Yu, Y., Fuhr, J., Boye, E., Gyorffy, S., Soker, S., Atala, A., Mulliken, J. B. and Bischoff, J. (2006). Mesenchymal stem cells and adipogenesis in hemangioma involution. Stem Cells 24, 1605-1612.

Yun, T. J., Chaudhary, P. M., Shu, G. L., Frazer, J. K., Ewings, M. K., Schwartz, S. M., Pascual, V., Hood, L. E. and Clark, E. A. (1998). OPG/FDCR-1, a TNF receptor family member, is expressed in lymphoid cells and is up-regulated by ligating CD40. J Immunol 161, 6113-6121.

Yun, T. J., Tallquist, M. D., Aicher, A., Rafferty, K. L., Marshall, A. J., Moon, J. J., Ewings, M. E., Mohaupt, M., Herring, S. W. and Clark, E. A. (2001). Osteoprotegerin, a crucial regulator of bone metabolism, also regulates B cell development and function. $\mathrm{J}$ Immunol 166, 1482-1491.

Zachary, I. and Gliki, G. (2001). Signaling transduction mechanisms mediating biological actions of the vascular endothelial growth factor family. Cardiovasc Res 49, 568-581. 
Zamzami, N., Hirsch, T., Dallaporta, B., Petit, P. X. and Kroemer, G. (1997). Mitochondrial implication in accidental and programmed cell death: apoptosis and necrosis. J Bioenerg Biomembr 29, 185-193.

Zannettino, A. C., Holding, C. A., Diamond, P., Atkins, G. J., Kostakis, P., Farrugia, A., Gamble, J., To, L. B., Findlay, D. M. and Haynes, D. R. (2005). Osteoprotegerin (OPG) is localized to the Weibel-Palade bodies of human vascular endothelial cells and is physically associated with von Willebrand factor. J Cell Physiol 204, 714-723.

Zetter, B. R. (1988). Angiogenesis. State of the art. Chest 93, 159S-166S.

Zhang, J. and Snyder, S. H. (1992). Nitric oxide stimulates auto-ADP-ribosylation of glyceraldehyde-3-phosphate dehydrogenase. Proc Natl Acad Sci USA 89, 9382-9385.

Zhang, Y., Turkson, J., Carter-Su, C., Smithgall, T., Levitzki, A., Kraker, A., Krolewski, J. J, Medveczky, P. and Jove, R. (2000). Activation of Stat3 in v-Srctransformed fibroblasts requires cooperation of Jak1 kinase activity. J Biol Chem 275, 24935-24944.

Zhang, L., Wang, W., Qi, Z. L., Dong, J. S., Song, H. D., Yang, J. S., Lin, X. X. Indentification of differentially expressed genes in proliferating and involuting hemangiomas by cDAN microarray analysis of gene-expression profiles. Zhonghua Zheng Xing Wai Ke Za Zhi 19, 452-455

Zhang, L., Lin, X., Wang, W., Zhuang, X., Dong, J., Qi, Z. and Hu, Q. (2005). Circulating level of vascular endothelial growth factor in differentiating hemangioma from vascular malformation patients. Plast Reconstr Surg 116, 200-204.

Zhao, L., Melenhorst, J. J. and Hennighausen, L. (2002). Loss of interleukin 6 results in delayed mammary gland involution: a possible role for mitogen-activated protein kinase and not signal transducer and activator of transcription 3. Mol Endocrinol 16, 29022912.

Zhong, H. and Simons, J. W. (1999). Direct comparison of GAPDH, beta-actin, cyclophilin, and 28S rRNA as internal standards for quantifying RNA levels under hypoxia. Biochem Biophys Res Commun 259, 523-526.

Zhong, H., De Marzo, A. M., Laughner, E., Lim, M., Hilton, D. A., Zagzag, D., Buechler, P., Isaacs, W. B., Semenza, G. L. and Simons, J. W. (1999). Overexpression of hypoxia-inducible factor 1alpha in common human cancers and their metastases. Cancer Res 59, 5830-5835.

Zhu, Z. M., Kojima, N., Stroud, M. R., Hakomori, S. and Fenderson, B. A. (1995). Monoclonal antibody directed to Le(y) oligosaccharide inhibits implantation in the mouse. Biol Reprod 52, 903-912.

Zide, B. M., Glat, P. M., Stile, F. L. and Longaker, M. T. (1997). Vascular lip enlargement: Part I. Hemangiomas--tenets of therapy. Plast Reconstr Surg 100, 16641673.

Ziegler, B. L., Valtieri, M., Porada, G. A., De Maria, R., Müller, R., Masella, B., Gabbianelli, M., Casella, I., Pelosi, E., Bock, T., Zanjani, E. D. and Peschle, C. (1999). KDR receptor: a key marker defining hematopoietic stem cells. Science 285, 15531558 . 
Zong, C. S., Chan, J., Levy, D. E., Horvath, C., Sadowski, H. B. and Wang, L. H. (2000). Mechanism of STAT3 activation by insulin-like growth factor I receptor. J Biol Chem 275, 15099-15105.

Zvaifler, N. J., Marinova-Mutafchieva, L., Adams, G., Edwards, C. J., Moss, J., Burger, J. A. and Maini, R. N. (2000). Mesenchymal precursor cells in the blood of normal individuals. Arthritis Res 2, 477-488. 